\title{
Distributed optimization, control and learning in multiagent networks
}

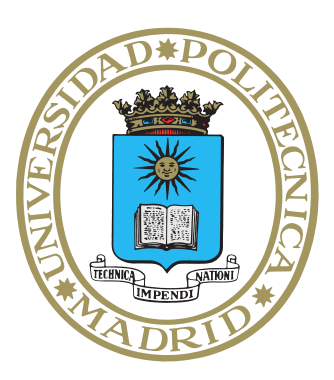

Sergio Valcárcel Macua, MSc.

Supervisor: Prof. Santiago Zazo Bello, PhD.

Dept. Senales, Sistemas y Radiocomunicaciones

Escuela Técnica Superior de Ingenieros de Telecomunicación Universidad Politécnica de Madrid

This dissertation is submitted for the degree of Doctor of Philosophy 



\begin{abstract}
It is estimated that, in the next decade, there will be tens of billions of interconnected devices in the world, each one sensing, streaming and processing data. In order to manage such huge amount of data, traditional architectures - where a fusion center gather all the data - may not satisfy performance specifications or cost constraints (e.g., data privacy, resilience, scalability or communication cost). Distributed computing is an interesting alternative that consists in moving the data processing to the deviceswhich become intelligent agents - so that the fusion-center is completely avoided. This thesis proposes and evaluates algorithms for two complementary multiagent scenarios.

First, we consider cooperative distributed algorithms, where the nodes interact with each other to solve a social problem. Even if each agent has only access to very few data, it can approximate the performance of a centralized architecture through cooperation. In this context, we propose distributed component analysis methodsincluding principal component analysis, factor analysis and linear discriminant analysisbased on the consensus-averaging scheme. We also propose and analyze an off-policy reinforcement learning algorithm, where the agents explore the state-set independently and share some intermediate results (not the samples) with their neighbors in order to evaluate a common target policy. Finally, we introduce a distributed implementation of the cross-entropy method for black-box global (nonconvex) optimization, where the objective is unknown to the agents.

The second scenario consists in dynamic potential games. This is a class of statebased time-varying games, where the agents influence each other and compete for a shared resource, so that they have to find an equilibrium. These kind of games can be formalized as multiobjective optimal control problems, which are generally difficult to solve. We extend previous analysis for these kind of games and guarantee existence of equilibrium under mild conditions. In addition, we propose a framework for finding - or even learning with reinforcement learning methods - an equilibrium strategy. We also study the applicability of this kind of games with a number of examples.
\end{abstract}




\section{Resumen}

Se ha estimado que en el mundo habrá decenas de miles de millones de dispositivos interconectados en las próximas décadas, cada uno obteniendo datos del entorno, procesándolos y transmitiéndolos en tiempo real. Para manejar esta ingente cantidad de datos, las arquitecturas tradicionales, en las que existe un centro de fusión recopilando las muestras de todos los dispositivos, podría no satisfacer requisitos de rendimiento y coste de operación de estas redes (por ejemplo, privacidad de los datos, robustez frente a fallos o el coste de transmitir los datos desde cada dispositivo). La computación distribuida es una alternativa interesante que consiste en no utilizar un nodo central de fusión de datos y en que la computación la hagan exclusivamente los nodos de la red. Esta tesis propone y evalúa algoritmos para dos escenarios multiagente complementarios.

Primero, consideramos algoritmos distribuidos cooperativos, en los que los agentes interaccionan para solucionar un problema común. Incluso si cada agente tiene acceso a un pequeño conjunto de datos, al cooperar con sus vecinos puede obtener rendimiento similar al que tendría una arquitectura centralizada. En este contexto, proponemos métodos de análisis de componentes distribuidos - incluyendo "principal component analysis", "factor analysis" y "linear discriminant analysis"- basados en un esquema del tipo "consensus-averaging". También proponemos y analizamos un algoritmo de "off-policy reinforcement learning", que permite que los agentes exploren el espacio de estados de forma independiente y compartan sus estimadores intermedios (nunca las muestras obtenidas durante la exploración) con sus vecinos para evaluar una política objetivo común. Finalmente, introducimos un algoritmo distribuido tipo "cross-entropy" para optimización global (no convexa) de funciones objetivo desconocidas ("black-box").

El segundo escenario consiste en juegos potenciales dinámicos. Se trata de una clase de juegos en los que el estado del entorno varía con el tiempo, en función de las acciones de los agentes, y en los que los agentes compiten por un recurso común. Este tipo de juegos puede formalizarse como problemas de control óptimo multiobjetivo, los cuáles son difíciles de abordar. Nuestro análisis generaliza resultados anteriores, garantizando la existencia de un punto de equilibrio del sistema, bajo condiciones muy generales, y proporcionando un método para encontrarlo - o incluso aprenderlo mediante técnicas de "reinforcement learning". También estudiamos la aplicación de este tipo de juegos con varios ejemplos de ingeniería de comunicaciones. 
To Angeles and Horacio 



\section{Acknowledgements}

This thesis would not have been possible without the collaboration and support of many wonderful people.

Firstly, I would like to express my deepest gratitude to my supervisor Professor Santiago Zazo for his unconditional support in all stages of this thesis. His guidance, confidence, generosity and patience have made this thesis possible. It has been a great pleasure to be able to work together and I am sure we will continue collaborating in future projects.

I would also like to express special gratitude to Professor Ali H. Sayed for generously adopting me like one of his students at UCLA, and for teaching me his high research standards, which have been crucial to the quality of this work.

I warmly thank Pavle Belanovic for introducing me to consensus techniques and for being the best postdoc - both personally and professionally - that a junior graduate student could imagine.

A special thanks also goes to Jianshu Chen, from whom I have learned very much about convergence issues and with whom it has been a pleasure to collaborate since we met. I also want to thank Xiaochuan Zhao, Shang-Kee Ting, Zaid J. Towfic, Sheng-Yuan Tu, Chung-Kai Yu, and Mohammad-Reza Gholami for being such a great hosts during my visit at the Adaptive Systems Laboratory (ASL) at UCLA. I really learned and enjoyed the time there.

I would like to thank Professor Matilde Sánchez-Fernández for her generosity and for the fruitful collaboration. I am also grateful to Professor Mariano García Otero for being in my committee and for always being available for insightful discussions on any statistical issue. I also appreciate Professor José Ignacio Ronda for discussions on multiple mathematical issues.

I would like to thank Professors Philippe Preux and Alessandro Lazaric for hosting me at SequeL team at INRIA Lille - Nord Europe, as well as Gergely Neu, Amir Sani, Marta Soare, Daniele Calandriello, Michal Valko, Balázs Szörényi, Prashanth LA, Hadrien Glaude and all the people there for sharing your time with me and for the 
interesting discussions on bandits, reinforcement learning and life. I specially thank Professor Olivier Pietquin for reviewing this thesis and being in my committee.

I also appreciate Professors Jesús Pérez Arriaga and Gerhard Neumann for reviewing this thesis and being in my committee, as well as Professors Narciso García Santos, Antonio Artés Rodríguez, Víctor Elvira Arregui and Juan José Murillo Fuentes for being in my committee.

Warm thanks to Marcos Sainz for designing the multimachine implementation of DCE and, more importantly, for being my spiritual buddy.

Thanks to the members of the Signal Processing Applications Group (GAPS) at UPM, who have contributed to my personal and professional development, especially to Adrian Población, Marta Martínez, Benjamín Béjar and Iñigo Arrazola for sharing the spiritual journey, to Javier Zazo for the intense discussions and for teaching me so much about optimization, to Carlos Moreno León and Johan Samuel Romero Peña for being the best Master students that I could have advised, to Jorge del Val for your enthusiasm and the exciting discussions, to Juan Parras Moral for sharing mathematical insights as well as excellent olive oil, to Jose Luis Blanco Murillo and Virginia Yagüe for helping me when I struggled with some technical question, to Nelson Dopico and Carlos Gil Soriano for pioneering the work on the sensor-network testbed, to José María Sanz for the jokes, and to many others with whom I have interacted during these years.

I would also like to thank Professor Fernando de la Torre for giving me the chance to start my research career and for encouraging me to pursue a $\mathrm{PhD}$.

Thanks to my dear aunts Aurea, Isabel and Pepa for their tender support and concerns, urging me to finish this thesis.

Finally, I want to say thanks, with all my heart, to my love Marta, to my beloved parents Angeles and Horacio, to my loving siblings Sara and Angel, and to my yoga master Paramahansa Yogananda for your unconditional love, for inspiring me, for all the important things that I have learned from you and for sharing this life together. 


\section{Contents}

List of Figures xiii

List of Tables $\quad$ xix

1 Introduction 1

1.1 Motivation .......................... 1

1.2 Problems under study . . . . . . . . . . . . . . . . 6

1.3 Summary of contributions . . . . . . . . . . . . . . . 9

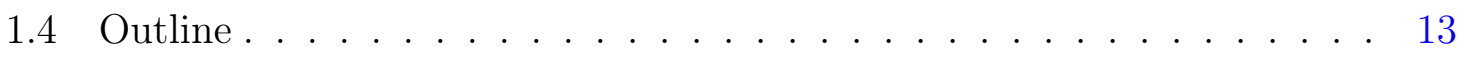

1.5 Notation . . . . . . . . . . . . . . . . . . . 14

2 Distributed component analysis methods using average consensus 15

2.1 Introduction . . . . . . . . . . . . . . . . . . . . . 15

2.2 Background on component analysis methods . . . . . . . . . . . . 17

2.2.1 Principal Component Analysis . . . . . . . . . . . . . 17

2.2.2 PCA in the context of latent variable models . . . . . . . . . . 18

2.2.3 Static Linear Gaussian models . . . . . . . . . . . . . . 20

2.2.4 Fisher Linear Discriminant Analysis . . . . . . . . . . . . . . 22

2.3 Background on consensus algorithms . . . . . . . . . . . . . . . . 29

2.3.1 The consensus problem . . . . . . . . . . . . 30

2.3.2 Convergence of consensus algorithm . . . . . . . . . . . . . 32

2.3.3 Consensus in higher dimensions . . . . . . . . . . . . . . . 35

2.4 Distributed estimation . . . . . . . . . . . . . . . . 37

2.5 Distributed PCA and SLGM algorithms . . . . . . . . . . . . . . . . 39

2.5.1 Distributed PCA from consensus in covariance matrix . . . . . . 40

2.5.2 Distributed PCA as an EM algorithm . . . . . . . . . . . 40

2.5.3 Distributed Factor Analysis . . . . . . . . . . . . . . . . . . 42

2.5.4 Distributed Probabilistic PCA . . . . . . . . . . . . . 44 
2.6 Distributed LDA algorithms . . . . . . . . . . . . . . . 45

2.6.1 Distributed LDA from consensus in scatter matrices . . . . . . . 46

2.6 .2 Distributed iterative LDA . . . . . . . . . . . . . 47

2.6.3 Distributed regularized LDA for undersampled data . . . . . . . 49

2.7 Experiments . . . . . . . . . . . . . . . . . . . . . . 51

2.7.1 Experiments with distributed PCA . . . . . . . . . 51

2.7.2 Experiments with distributed LDA . . . . . . . . . . 55

2.8 Extension to other component analysis methods . . . . . . . . . . . . 59

2.8.1 Component analysis methods as optimization problems . . . . . 59

2.8.2 Distributed optimization strategies . . . . . . . . . . . . 60

2.8.3 A diffusion-based algorithm for distributed component analysis methods . . . . . . . . . . . . . . 62

2.9 How to implement doubly-stochastic combination matrices . . . . . . 62

2.9.1 Basic design for tackling temporary link failures . . . . . . . . . 62

2.9.2 Extension for dealing with permanent topology changes . . . . . 64

2.10 Conclusions . . . . . . . . . . . . . . . . . . . . 65

$3 \quad$ Distributed policy evaluation under multiple behavior strategies $\quad 67$

3.1 Introduction . . . . . . . . . . . . . . . . . . . . . 67

3.1.1 Related works . . . . . . . . . . . . . . . 70

3.2 Preliminaries . . . . . . . . . . . . . . . . 72

3.2.1 Markov decision processes (MDP) . . . . . . . . . . 72

3.2.2 Value function . . . . . . . . . . . . . . . . . 73

3.2.3 Approximate value function as a saddle-point problem . . . . . 75

3.2.4 Primal-dual stochastic optimization . . . . . . . . . . . . . . 80

3.3 Multi-agent learning . . . . . . . . . . . . . . . . . 82

3.4 Performance analysis . . . . . . . . . . . . . . . . . . . . . . . . . 87

3.4 .1 Data model . . . . . . . . . . . . . . . . 87

3.4.2 Existence and uniqueness of solution . . . . . . . . . . 88

3.4.3 Error recursion . . . . . . . . . . . . . . . . . . 89

3.4 .4 Convergence in the mean . . . . . . . . . . . . . . . . 91

3.4.5 Mean-square stability . . . . . . . . . . . . . . . . . . 92

3.4.6 Mean-square performance . . . . . . . . . . . . . . 95

3.4 .7 Bias analysis . . . . . . . . . . . . . . . . . 9 97

3.5 Simulations . . . . . . . . . . . . . . . . . . . 100

3.6 Extensions of DGTD . . . . . . . . . . . . . . . . . 103

3.6.1 Approaching the actual target . . . . . . . . . . 105 
3.6.2 Distributed off-policy control algorithms . . . . . . . . . . 106

3.6.3 Diffusion algorithms for transfer learning . . . . . . . . . . . . 110

3.7 Conclusions . . . . . . . . . . . . . . . . . . . . . . . . 111

3.8 Appendix: Proof of Theorem $3.1 \ldots \ldots$. . . . . . . . . . . . . 112

4 Distributed Cross-Entropy method for black-box global optimization 119

4.1 Introduction . . . . . . . . . . . . . . . . . . . . . . . . . . 119

4.1.1 Related works . . . . . . . . . . . . . . . . . . . 121

4.1 .2 Contributions . . . . . . . . . . . . . . . . . . . 123

4.2 Problem Setting . . . . . . . . . . . . . . . . . . . . . 124

4.3 Preliminaries . . . . . . . . . . . . . . . . . 126

4.3.1 Bayesian filter optimization $(\mathrm{BFO}) \ldots \ldots$. . . . . . . 127

4.3.2 BFO with surrogate sampling distribution . . . . . . . . . . 130

4.3.3 Particle filter optimization (PFO) with projected importance re-sampling step . . . . . . . . . . . . . . . . . . . 134

4.4 Distributed Cross-Entropy (DCE) Algorithm . . . . . . . . . . . . . . 137

4.4.1 Distributed surrogate sampling distribution . . . . . . . . . 138

4.4.2 Distributed stochastic approximation . . . . . . . . . . . . . 140

4.5 Convergence Analysis for the Single Agent Case . . . . . . . . . . . . . 145

4.5.1 Convergence of single agent Bayesian filter optimization . . . . . 145

4.6 Convergence Analysis for a Network of Agents . . . . . . . . . . . . . . 151

4.6.1 Convergence of diffusion damping BFO . . . . . . . . . . . . . 152

4.6.2 Almost sure agreement among DCE agents . . . . . . . . . . . . 155

4.6.3 Almost sure convergence of DCE agents . . . . . . . . . . 165

4.7 Numerical Experiments . . . . . . . . . . . . . . . . . . . . . . . 169

4.7.1 Split computational load vs. performance . . . . . . . . . . 172

4.7.2 Enhanced exploration of DCE . . . . . . . . . . . . . . . 181

4.8 Discussion on the robustness of DCE . . . . . . . . . . . . . . . . . . . 184

4.8.1 Monte Carlo approximation error . . . . . . . . . . . . . 184

4.8.2 Gradient ascent on the space of distributions . . . . . . . . . . . 184

4.9 Conclusion and Future Work . . . . . . . . . . . . . . . . . . . . . . 188

5 Dynamic potential games with constraints 191

5.1 Introduction . . . . . . . . . . . . . . . . . . . . . . . . 191

5.2 Problem setting . . . . . . . . . . . . . . . . . . 195

5.3 Overview of static potential games . . . . . . . . . . . . . . . . 199

5.4 Open loop dynamic potential games . . . . . . . . . . . . . . . . 202 
5.5 Open loop stochastic dynamic potential games . . . . . . . . . . . . . . 210

5.5.1 Problem setting for open loop stochastic dynamic games . . . . 210

5.5.2 Open Loop analysis of SDPGs . . . . . . . . . . . . . . . . . . 212

5.6 Closed loop stochastic dynamic potential games with parametric policies 216

5.6.1 Problem setting for closed loop stochastic dynamic games . . . . 216

5.6.2 Closed loop analysis of SDPGs with parametric policies . . . . . 222

5.7 Network flow control . . . . . . . . . . . . . . . . . 233

5.7.1 Network flow dynamic game and related OCP . . . . . . . . . 234

5.7.2 Finite horizon approximation and simulation results . . . . . . . 236

5.8 Dynamic Multiple Access Channel . . . . . . . . . . . . . . . . . . . . 239

5.8.1 Multiple access channel DPG and related OCP . . . . . . . 239

5.8 .2 Simulation results . . . . . . . . . . . . . . . . . 240

5.9 Optimal Scheduling . . . . . . . . . . . . . . . . . . . 241

5.9.1 Proportional fair and equal rate games and related OCP . . . . 242

5.9.2 Solving the OCP with dynamic programming . . . . . . . . 244

5.10 Reinforcement learning for DPG . . . . . . . . . . . . . . . . 249

5.10 .1 Learning parametric policies for MAC problem . . . . . . . . 250

5.11 Conclusions . . . . . . . . . . . . . . . . . . . 252

6 Conclusions $\quad 255$

$\begin{array}{ll}\text { Bibliography } & 259\end{array}$ 


\section{List of Figures}

2.1 PCA vs LDA. PCA finds the most representative directions. LDA minimize the within class covariance matrices, while also maximizing the mean distance between classes preserve. . . . . . . . . . . . . 26

2.2 Example of cooperative networks considered in this chapter. The whole network is displayed together with a zoom over the up-right corner. The zoomed area displays the neighborhood of agent $k \ldots \ldots 31$

2.3 Global matrix $Y$ with a total of $T$ samples, distributed unevenly over $N$ agents. . . . . . . . . . . . . . . 37

2.4 Sparse network of 200 agents with average degree $5 \ldots \ldots$. . . . . . 52

2.5 Performance of PCA in isolation and with cooperation via consensus using CB-DPCA, showing the convergance of all agents towards the centralized solution. The subspaces found by the agents are shown as thin continuous lines; while the subspace found by the traditional (centralized) PCA algorithm is shown as a larger and thicker broken line. 53

2.6 Inherent cost-performance tradeoff for CB-DPCA, cost being the volume of information transmitted, and performance represented by the reduction of the sin of the angle between the locally-estimated and the central (optimal) subspace . . . . . . . . . . . . . 54

2.7 Performance of PCA in isolation and with cooperation via consensus using CB-DPCA. Images (c), (e), and (g) illustrate how a agent asymptotically converges to the centralized solution given in image (b). Images (d), (f), and (h) show the same for another agent in the network. . . . . 56

2.8 Performance of LDA in isolation (left); and with cooperation via consensus using CB-DLCA, for $R=10$ iterations in each Average Consensus subroutine, showing the convergence of all agents towards the centralized solution (right) . . . . . . . . . . . . . . . 57 
2.9 Accuracy vs. cost trade-off for the CB-DLDA algorithm where the accuracy is measured as the sin of the angle between local and centralized subspaces for the results displayed in Figure 2.8 . . . . . . . . . . . . 58

2.10 Histogram of classification success rates for ULDA and CB-DULDA by consensus alignment. Results averaged over 100 independent experiments. The success rate of the centralized solution is represented as a vertical dashed line. . . . . . . . . . . . . . . . . . . . . . . . . .

2.11 Example 1. A chain of agents running WFS, where the link between the two agents in the left end of the chain is faulty. The iteration number ( $i$ ) of the estimate (e.g., $w_{k, i}$ ) is written inside each circle. At time $t=1$, agent $j$ cannot update because info from agent $k$ is missing. Similarly, although agent $k$ has info from agent $l$, it misses the estimate from agent $j$, so it cannot update its estimate. On the other hand, agents $l$ and $m$ have all the information from their neighborhoods. Nevertheless, at time $t=2$, agent $l$ misses $w_{k, 1}$ so it has to stop. agent $m$ have both $w_{l, 1}$ and $w_{m, 1}$ so it can compute $w_{m, 2}$. At time $t=3$, agent $m$ is missing $w_{l, 2}$ so it is forced to stop too and, hence, the whole network has to either wait until the link between $j$ and $k$ works again (some retransmission finally reaches the agent) or until a retransmission timeout, which they understand as a permanent topology change so they safely remove each other from their neighbor tables. . . . . . . . . . . . . . . . . 6 64

2.12 Robustness of WFS. We begin with a chain of 4 agents, with values $\{2,3,4,5\}$, respectively. Then, we add up to 6 more agents, one at a time, with values $\{6,7,8,9,10,11\}$, respectively. Note that WFS is able to achieve the sample average of all the available agents at each time (resulting in 6.5 when all agents are included in this case) . . . . . . . . 66

3.1 Example of the network of collaborative agents considered in this paper. Each agent operates in similar but independent environments, so that the agents do not influence each other. The agents follow different behavior policies $\left(\phi_{k} \neq \phi_{l}, \forall k, l \in \mathcal{N}, k \neq l\right)$, which are different from the target policy $\pi$ that they aim to estimate; we assume $\pi$ is the same target for all agents. . . . . . . . . . . . . . . . . 83 
3.2 Problem representation. Network of 15 randomly connected agents. States are numbered from left to right and from down to top, so that the bottom-left corner corresponds with $s=1$, and the top-right corner with $s=S=400$. The agents are attracted towards the center of a region each (green circle) at states $\{395,368, \ldots, 21\}$. The predator's area is the rectangle $5 \leq$ column $\leq 20,8 \leq$ row $\leq 15$. The reward at every time step is -1 , except when crossing the predator's area where it becomes -15 , and when eating the food when it rises to +20 . The 64 features are obtained with 8 Gaussian basis functions per row and per column and with standard deviation 0.005, equidistantly located at positions (yellow stars) that are computed by taking the grid as a continuous unit-square. . . . . . . . . . . . . . . . 102

3.3 Value vectors for myopic (top, $\pi_{1}$ ) and detour (bottom, $\pi_{2}$ ) policies for each state of the grid. The left column shows the exact value vectors computed using (3.10). The myopic policy shows a valley because the agents have many chances of entering into the predator's area, so the expected reward will be negative. For the detour policy, the agent has little chance of entering into the predator's region, so the expected value will be near zero in this area. . . . . . . . . . . . . . . . . 103

3.4 Value vectors for myopic (top, $\pi_{1}$ ) and detour (bottom, $\pi_{2}$ ) policies for each state of the grid. The left column shows the off-policy, linear approximation achieved by a centralized station that has gathered all samples from all agents. The middle column shows the estimated value vector obtained with our diffusion GTD algorithm for some agent (chosen at random, the result is very similar for all agents though) that only uses samples from its own non-exploratory policy (i.e., moving towards its attractive point). By including the diffusion step, every agent is able to reproduce the main features of the surfaces (valleys, hills and flat areas). The right column shows the same value vectors but estimated with no cooperation (for a node that did not diverge). . . . . . . . . 104 
3.5 Projected Bellman error (i.e., $J_{\mathrm{PB}}$ ) given by Eq. (3.21) for centralized, diffusion and non-cooperative solutions. Results are obtained with the following parameters. We set the long-term reward to $\gamma=0.95$, which we have found to make $v^{\pi_{1}}$ very different from $v^{\pi_{2}}$ for the specific values of $\pi_{1}$, $\pi_{2}$ and rewards used during the experiments. For both cooperative and non-cooperative nodes, the step-size and step-size ratio are $\mu=1 \times 10^{-3}$ and $\eta=0.1$, which adequately trade bias and convergence-rate to illustrate the behavior of the algorithm. We assume every agent takes one sample at every time-step. Since a central station would have $N$ times more samples than the rest of the agents, we set its step-size equal to $\mu / N$. Results are averaged over 50 independent experiments. . . . . 105

4.1 Black-box optimization. . . . . . . . . . . . . . . 126

4.2 Example of increasing likelihood functions. The left side shows the proportional likelihood (4.14), which is proportional to the queried value $J(x)$. Note that we have considered that $J(x)$ may be negative, so it has been shifted to ensure $\epsilon^{\text {prop }}+J(x) \geq 0$. The right side shows the smooth indicator likelihood function (4.15) for $\epsilon=100$ and $\gamma=0.6 J_{\max } \cdot 130$

4.3 Illustration of the shape of some of the test objective functions for their first two dimensions, $x(1)$ and $x(2) \ldots \ldots \ldots \ldots$. . . . . . . 173

4.4 Influence of the degree on the performance of DCE. We can see worstcase (dashed red), average (solid black) and best-case (dotted blue) over 30 independent trials for each neighborhood size. . . . . . . . . . . . 176

4.5 Convergence of SACE (left column) and DCE (right column) with simulation parameters listed in Table 4.1. The graphs display the worst (solid top), average (dashed) and best (solid down) results among all trials (and all agents for DCE). Results have been averaged over 200 independent trials (and over all agents for DCE) in each case. . . . . . 178

4.6 Continues Figure 4.5 . . . . . . . . . . . . . . . . . . . . . . . . . . . . 179

4.7 Continues Figure 4.6. . . . . . . . . . . . . . . . . . . 180

4.8 Convergence for $M$-dimensional Rastrigin, Ackley and Pinter functions. Similar to Figures 4.5-4.7, the graphs display the worst (solid top), average (dashed) and best (solid down) results among all trials (and all agents for DCE). Results have been averaged over 200 independent trials in each case. For DCE, results have been also averaged over all agents. . . . . . . . . . . . . . . . . . 183 
4.9 Original vs. model-based domain. The left side shows the Garland function $J(x)$, which has multiple extrema such that algorithm prone to get trapped in local minima. The right side shows the model-based function $\mathbb{E}_{f_{w}}[J(x)]$ for a Normal surrogate sampling distribution $f_{w}(\cdot)=$ $\mathcal{N}\left(\cdot ; \mu, \sigma^{2}\right)$. Note that the bigger the variance, the smoother the modebased objective. . . . . . . . . . . . . . . . . 185

5.1 Open loop dynamic games. All agents operate in the same environment and influence each other. The problem can be seen as a multiobjective optimal control problem with coupled constraints. . . . . . . . . . . . 198

5.2 Network scenario for two users and two layers of relay nodes. agent $b_{1}$ aims to transmit to destination $d_{1}$, while $b_{2}$ aims to transmit to destination $d_{2}$. They have to share relay nodes $n_{1}, \ldots, n_{4}$. We denote the $L=6$ aggregated flows as $L_{1}, \ldots, L_{6}$. . . . . . . . . . . . . 234

5.3 Network flow control with $N=2$ agents, $S=4$ relay nodes and $A_{1}=A_{2}=4$ available paths per node, so that $A=A_{1}+A_{2}=8$. (Top) Aggregated flow rates at $L_{1}, \ldots, L_{6}$. (Middle) Flow for each of the $A=8$ available paths. (Bottom) Battery level in each of the $S=4$ relay nodes.238

5.4 Dynamic multiple access scenario with $N=4$ users. (Top) Evolution of transmitter power. (Bottom) Transmission rate for every user. The equilibrium is a schedule with order determined by each user's channel gain.

5.5 Periodic time variation of the channel coefficients $\left|h_{k, t}\right|^{2}$ for $N=2$ users. All possible combination of coefficients are included in a window of $T=20$ time steps. . . . . . . . . . . . . . . . . . 247

5.6 Proportional fair scheduling problem for $N=2$ users. (Top) Transmitter power $u_{k, i}$. (Bottom) Average rate $x_{k, i}$ given by (5.216). Both users achieve near maximum average rates for their channel coefficients $\left|h_{k, i}\right|^{2} .248$

5.7 Equal rate problem for $N=2$ users. (Top) Transmitter power $u_{k, i}$. (Bottom) Average rate $x_{k, i} / i$ (recall that $x_{k, i}$ given by (5.219) denotes accumulated rate). User 1 reduces its average rate to match that of User 2, regardless of having higher channel coefficient. . . . . . . . . . . . 248

5.8 Results for the mac problem (5.227). We display the convergence of the TRPO and DDPG algorithms as well as the result obtained with the convex optimization solver). . . . . . . . . . . . . . . . . . . 252 



\section{List of Tables}

2.1 Minimum, mean and maximum success rate for all agents in the network for single-agent ULDA (which is equivalent to CB-DULDA with $R=0$ ) and CB-DULDA with $R \in\{1,2,5,10\}$ consensus iterations. . . . . . . 58

2.2 Neighbor table for agent $k$ of Figure 2.2. . . . . . . . . . . . . 63

4.1 Simulation parameters for each objective function. . . . . . . . . . . . . 174

4.2 Simulation results for SACE and DCE with simulation parameters listed in Table 4.1. Results are in number of experiments over 100 where the convergence error was less than $\Delta . \ldots \ldots \ldots \ldots$. . . . . . . 177

4.3 Simulation parameters for Dejong 5th. . . . . . . . . . . . 180

4.4 Percentage of trials that converged to an error smaller than $\Delta=10^{-8}$ for SACE and DCE (with multiple topologies) for Dejong 5th function, $J^{1)}$, and for multiple number of initial samples, $S_{0}$. Results have been averaged over 200 independent trials with simulation parameters listed in Table 4.3. . . . . . . . . . . . . . . . . . . . . . . 181

4.5 Simulation parameters for $M=100$-dimensional problems. . . . . . . . 182

4.6 Percentage of trials that converged to an error smaller than $\Delta=10^{-3}$ for SACE and DCE for $M=100$-dimensional problems. Results have been averaged over 200 independent trials with simulation parameters listed in Table 4.5 . . . . . . . . . . . . . . . . . . . 182 



\section{Chapter 1}

\section{Introduction}

We start introducing philosophical and practical motivations for studying distributed multiagent computing scenarios. Next, we present the problems that will be studied in the following chapters. Then, we summarize the main contributions of this work and enumerate the publications that resulted from the development of this thesis. Finally, we include the outline and some notation.

\subsection{Motivation}

What is intelligence? The Wikipedia entry on intelligence states the following [316]:

"Intelligence has been defined in many different ways including as one's capacity for logic, understanding, self-awareness, learning, emotional knowledge, planning, creativity and problem solving. It can be more generally described as the ability to perceive information, and to retain it as knowledge to be applied towards adaptive behaviors within an environment or context."

Building intelligent systems that could help us to make better decisions and increase our productivity promises to impact every industry and hopefully change our world for the better [154]. However, the key underlying principles that make us intelligent are still unknown. For instance, is there a single algorithm that causes intelligence - the so named cortical hypothesis $[118,117,155]$ ? Or rather, does it emerge from the combination of a whole suit of complex architectures? We still don't know. Fortunately, step by step, advances from philosophy to physics to neuroscience to computer science are allowing us to solve small parts of the puzzle and even to embed some degree of intelligence in multiple artificial systems that range from, e.g., self-driving cars [292] to 
recognizing forms in images from unlabeled data [158] to playing the board game Go at the human master level [263].

One remarkable discovery in the history of neuroscience is that the nervous system plays an important role in animal intelligence (this has not been always accepted, e.g., the famous Greek philosopher Aristotle believed that the heart was the center of intellect, while the brain was a radiator for cooling the blood [17, p.5]). Although the origin of the nervous system is still a mystery, or in words of Rita Levi-Montalcini "a problem that will remain unsolved" [161, p. 33], at least we know that living microorganisms populated our planet much earlier than the nervous system appeared. Hence, we can consider the nervous system as the result of an evolutionary process, i.e., an effective solution provided by natural evolution for better adaptation to the environment. From this point of view, if we take the more general definition of intelligence given above, could we see evolution as an intelligent process at the species level that embeds adaptation capabilities in the individual organisms but that also transcends them? In such case, where do the roots of the intelligence at the species level lie in the individual organisms? We can distinguish two main approaches for theories that aim to respond this kind of questions: those that see intelligence as an emergent phenomenon and those that see it as an intrinsic property.

One example of the emergent point of view is to consider an intelligent organism simply as a "vehicle" for its genes to adapt better to the environment. This is exposed by Richard Dawkins with his theory of the "selfish genes":

"It is finally time to return to the problem with which we started, to the tension between individual organism and gene as rival candidates for the central role in natural selection... One way of sorting this whole matter out is to use the terms 'replicator' and 'vehicle'. The fundamental units of natural selection, the basic things that survive or fail to survive, that form lineages of identical copies with occasional random mutations, are called replicators. DNA molecules are replicators. They generally, for reasons that we shall come to, gang together into large communal survival machines or 'vehicles'." [65]

For Dawkins, the genes are non-adaptive systems, with hard-wired rules for interacting with their environment. Nevertheless, some of these rules allow them to organize into intelligent systems that are able to learn and adapt their behaviors. If this is the case, the main question is how animal intelligence can emerge from mechanistic molecules. Marvin Minsky states: 
"How can intelligence emerge from non-intelligence? To answer that, we'll show that you can build a mind from many little parts, each mindless by itself. I'll call "Society of Mind" this scheme in which each mind is made of many smaller processes. These we'll call agents. Each mental agent by itself can only do some simple thing that needs no mind or thought at all. Yet when we join these agents in societies - in certain very special ways - this leads to true intelligence." [192, prologue]

The other point of view, in which intelligence is an intrinsic property of the constituent elements of any intelligent system, is described by the physicist Freeman Dyson as follows:

"It appears that mind, as manifested by the capacity to make choices, is to some extent inherent in every electron." [85] (see also [84], [61, p. 185])

Here, Dyson suggests that intelligence might lie in the roots of matter and, therefore, everything material can enjoy some degree of intelligence. This is very different from the emergent point of view and suppose that higher intelligence is built from the interaction of lower intelligent elements. The physicist David Bohm also suggests that intelligence is an intrinsic part of reality, such that matter and mind (or ultimately consciousness) form an "unbroken wholeness", an "undivided flowing movement" [41]. Interestingly, recent studies on meditation-and-brain research with fMRI and EEG study the interaction between mind and brain. In particular, these studies show how long term meditation is correlated with changes in grey matter volume [174], reduced activity in the mind-wandering and self-referential thoughts centers of the brain [49], enhanced connectivity between brain regions [175], and increased ability to change brain function and activate specific brain circuitries [176]. From these results, physician Deepak Chopra considers that the mind, being able to change the brain, should be rooted beyond the nervous system:

"Neuroscience has already shown that the brain scans of advanced Buddhist monks are very different from the norm. The biggest discovery was that general activity in the prefrontal cortex was very intense-more intense, in fact, that ever observed before-a change that came about after years of meditation on compassion. As it happens, the prefrontal cortex is the brain center for compassion, among other higher functions. In this case, it would be inaccurate to say that the brain change itself. First, the monks had the intention to be compassionate; they meditated upon it for years, and their 
brain followed suit. (...) We can't shift in a spiritual direction unless the brain shifts, too, and it's our desire that alters the material landscape of the brain, not vice versa." [61, pp. 183-185]

In summary, while for the emergent point of view, intelligence emerges from the interaction of non-intelligent elements; for the intrinsic point of view, higher intelligence is expressed through the combination of less but already intelligent elements. We remark that both (apparently very different) points of view agree in that systems that exhibit sophisticated intelligent behavior are the result of the interaction of smaller-maybe mindless, maybe mindful-elements.

Modern artificial neural networks with multiple hidden layers - also known as deep learning networks - have achieved remarkable success in multiple domains (see, e.g., recent books $[75,106]$ and references therein). These deep learning networks are composed of multiple computational units, named neurons, structured in layers, such that the neurons in one layer perform a very simple computation of the output of the neurons in the preceding layer. The simple computation performed by the neurons is typically a non-linear mapping of the weighted sum of their inputs. Thus, we can say that each individual neuron is not adaptive at all: it behaves mechanistically. Nevertheless, the weights given by each neuron to the outputs of the previous layer are free parameters that can be optimized. For instance, we can perform (nonlinear) regression between the input and the output of the whole network by adjusting the weights of all neurons, such that they minimize the prediction error. Thus, although the individual neurons remain non-adaptive, the network is able to learn and adapt to the training data. This is a paradigmatic example of the emergent approach to intelligence, which, as we said, is achieving state-of-the-art results in multiple applications.

Although the emergent paradigm is scientifically appealing and we have successful application examples of emergent systems (like the mentioned deep learning architectures), and even though the intrinsic paradigm seems very speculative, the fact is that intrinsically intelligent systems (i.e., formed by intelligent elements) are much more quotidian, if not pervasive, in our daily life [102]. Consider the group decisions made by, e.g., a basketball team, a group of researchers discussing a theory, or a company developing a complex engineering project. In these cases, similar to the emergent approach, the network of computational elements is able to yield results that no one of the constituents could yield by itself; however, the computing elements here are humans, which are already intelligent by themselves. Indeed, recent studies suggest that we have a biologically-based "honest signaling" systems, evolved from ancient primate signaling mechanisms, that reveal our attitudes toward the people we interact 
with and that is important to group decision making [223]. Other examples of familiar intrinsic systems include swarms of foraging ants or bees, flocks of birds reducing air friction, wolf or dolphin packs haunting, etc. In the context of our discussion, a logic conclusion is that if there is a single key algorithm for all levels of intelligence (as the cortical hypothesis suggests), and we have familiar animal and human societal evidence of intelligent systems formed by intelligent parts, then such single key algorithm should follow the intrinsic approach.

The previous discussion motivates our area of study. In particular, as opposed to the emergent approach, we study algorithms for two multiagent scenarios: i) a network of intelligent agents that cooperate to solve a social problem, and ii) a set of intelligent competing agents that have to find an equilibrium between their strategies.

Apart of the philosophical motivation discussed above, distributed algorithms on networks of cooperative agents are also attractive from an engineering point of view. Several studies estimates that there will be tens of billions of devices connected to the so named "Internet of things" (IoT) [87, 235, 305]. This implies a huge amount of geographically distributed data that could be useful and valuable for innovative applications of artificial intelligence. However, the cost of gathering such amount of data at a single central fusion center may be prohibitive. Consider, for instance, a wireless sensor-and-actuator network (WSAN) composed of small devices with limited communication range and limited batteries. If the intelligence of the system is only located at the central fusion center, every device has to route its sensing data to such location. This implies that the nodes closer to the gateway have to forward packets from farther nodes; but this extra communication cost can deplete their own batteries quickly. Moreover, the centralized scheme is sensitive against link failure or attack, since a hacker could block the whole network by compromising the critical routing nodes. Finally, the fact that all nodes have to transmit their own data puts their privacy at risk. On the other hand, a distributed system, consisting of a network of devices able to sense, process and act in the environment without any central coordination, offers a more scalable, robust and secure alternative.

Moreover, many real-life problems can be described by a set of agents competing for a common resource (e.g., wireless users sharing the same radio channel, companies sharing the market, routers maximizing their throughput over a common network infrastructure, etc.). From an engineering point of view, we should be able to find equilibrium strategies between the conflicting interests of the agents. But this is usually challenging, especially for dynamic problems with coupled constraints on the agents' strategies. 


\subsection{Problems under study}

"Somewhat strangely for a book that purports to be rigorous, we will not give a precise definition of a multiagent system. The reason is that many competing, mutually inconsistent answers have been offered in the past. Indeed, even the seemingly simpler question - What is a (single) agent?has resisted a definitive answer."

Y. Shoham and K. Leyton-Brown [262, p.xiii]

Before introducing the specific scenarios under study, we provide our custom definitions for the terms multiagent networks and distributed algorithms.

We consider a multiagent network a set of multiple interconnected autonomous agents that have learning and adaptive capabilities. The nodes in the network represent agents and the links represent the communication allowed between them. In particular, if two agents can exchange some information (i.e., they can share some estimate or one agent can see other agent's action or reward), we assume there is a link between them. If the communication flows in both directions, we say that the link is undirected; otherwise we say it is directed. Moreover, from the network topology point of view, we distinguish between:

- Fully connected: Every node is linked with with every other node in the network.

- Strongly connected: There is at least one path between every pair of nodes.

Apart of the topology, another relevant feature of the system is the attitude of the agents. We distinguish between:

- Cooperative: All agents cooperate to solve a global (social) problem.

- Competitive: All agents are selfish and aim to solve their own problems.

- Coalitions: Each agent can cooperate with some agents and compete with others.

The term distributed algorithm has been generically used to denote a network of processing units that perform computations over a shared or distributed set of variables (including, e.g., training dataset, parameter vector, etc.) but it can refer to different degrees of decentralized computation. In this thesis we use the customary convention of distinguishing between parallel, decentralized, and distributed scenarios:

- Parallel: Each processing unit processes its own slice of information, and the final results are gathered at the output. Performing algebraic operations [304], 
solving fixed-point equations [27] or even optimizing nonconvex problems [52] can be done very efficiently in such architectures. Moreover, game theoretic scenarios - like the dynamic games studied in Chapter 5-are well modeled with this approach.

- Decentralized: Data is spatially dispersed (i.e., partially available at each node) and some local processing is performed before the intermediate results are passed on to a single fusion center, which produces the final result. Examples of this master-slave architecture are given in [288] and, more recently, in [132]).

- Distributed: Data is spatially dispersed and locally processed, and emphasizes that there is no master, or coordinator, or central fusion node, as opposed to the decentralized case. Instead, the computing units cooperate with their neighbors (i.e., only single-hop communication is allowed) by sharing only the intermediate results (i.e., no data is shared). Hence, the final result is available at all the nodes when the process stops - as opposed to the parallel case, where each node only had one slice of the result. The algorithms presented in Chapters 2-4 are of this kind.

In this thesis, we consider: i) distributed scenarios, where strongly-connected networks of intelligent agents cooperate to solve a social problem; and ii) parallel scenarios, where a fully-connected network of selfish competing agents share a common resource. No coalitional or decentralized scenarios will be considered. We approach the cooperative distributed scenario with consensus-diffusion algorithms (e.g., [211, 14, 138, 269, 270, 54, 249, 247, 200, 261, 76]), and we model the parallel competitive scenario with dynamic potential games (e.g., [264, 196, 70, 181, 122, 81]).

Consensus-diffusion algorithms, have proved to be a powerful tool for building cooperative distributed algorithms. Examples of applications include filtering [209], detection [12], classification [91, 285], convex optimization [201, 56], nonconvex optimization [76], and many others. We say that consensus-diffusion algorithms are cooperative in the sense that the agents tend to reach a consensus on some social objective that is a combination of their individual objectives. To distinguish between consensus and diffusion we use another customary convention. In this thesis, when we talk about consensus, we mainly refer to average-consensus algorithms, in which the nodes achieve consensus on the average of their datasets (this is described in Chapter 2). When we talk about diffusion, we mean a first order optimization algorithm with an extra combination step that is similar to the average-consensus step. Again, this is a customary convention. In the literature, we can also find the so named consensus 
first-order optimization algorithm that is slightly different from the first-order diffusion variant. Actually, there are several variants of both diffusion and consensus first-order algorithms, which can be expressed in a more general unifying form (see, e.g., [249, 247] for an excellent discussion on their differences and similarities). It has been shown in $[249,247,291]$ that the dynamics of diffusion gradient descent networks leads to enhanced stability and lower mean-square-error (MSE) than consensus counterparts. Therefore, whenever we propose a distributed optimization algorithm, we will only focus on diffusion strategies. The specific distributed cooperative scenarios under study in this thesis are the following:

- In Chapter 2, we use average-consensus algorithms to propose distributed implementations of classical feature extraction methods, including principal component analysis (PCA), factor analysis (FA) and linear discriminant analysis (LDA).

- In Chapter 3, we apply diffusion optimization to reinforcement learning, in particular we propose a distributed algorithm for off-policy evaluation in a scenario where the agents explore the environment with different behavior policies and learn to predict a common target policy together. We assume that the agents operate in similar but independent environments, so that they do not influence each other with their actions; but still they can benefit from learning together.

- Chapter 4 proposes a diffusion-based stochastic-approximation particle-filter algorithm, named distributed-cross-entropy where a network of agents cooperate for optimizing an unknown nonconvex function.

The parallel competitive scenario is studied in Chapter 5. We consider a fullyconnected network of agents that compete to maximize their individual rewards in a time-varying environment, which we assume can be characterized by a discrete-time dynamical system equipped with a set of states and a state-transition equation. This is formally expressed as a noncooperative dynamic game. A dynamic game starts at an initial state. Then, the agents take some action, based on the current state of the game, and receive some reward values. Then, the game moves to another state. This sequence of state-transitions is repeated at every time step over a (possibly) infinite time horizon. We consider the case in which the aim of each agent is to find the strategy (i.e., sequence of actions) that maximizes its long term cumulative reward, given other agents' strategies. Thus, a game can be represented as a set of coupled optimal-control-problems (OCPs), or equivalently as a multiobjective OCP, which is difficult to solve in general. As opposed to Chapter 3, Chapter 5 studies the optimal 
control problem when the agents operate in the same environment and, therefore, influence each other with their actions. We focus on a class of games, named dynamic potential games (DPG), that appear often in applications where the agents share some common resource (e.g., oligopolies, exploitation of natural resources, communication channel, etc.) $[264,196,70,181,122,81]$.

Our goal studying the mentioned scenarios is to understand the essential aspects of multiagent networks and distributed computation (like global vs. local datasets, local estimators of global magnitudes, cooperative vs. non-cooperative vs. competitive agents, solution concepts for cooperative and competitive scenarios, etc.) and to provide concrete methods for their solution.

\subsection{Summary of contributions}

The specific research contributions of this thesis are:

- Development of distributed component analysis methods, using consensus-averaging techniques, that asymptotically match the centralized performance and that can be used for standard signal processing applications, like compression or classification, even for large, sparse networks of agents.

- Development of a diffusion-based distributed gradient-temporal-difference (DGTD) algorithm for off-policy reinforcement learning that extends the single-agent gradient-temporal-difference (GTD) due to [277, 275, 178, 177] to cooperative multi-agent networks. As a byproduct of this derivation, we show that the GTD algorithm, motivated as a two time-scales stochastic approximation in [275], is indeed a stochastic Arrow-Hurwicz algorithm applied to the dual problem of the original formulation. This is a novel interesting result by itself, since it gives insight on how the algorithm works and could lead to future extensions (after publishing this result in Dec. 2013 with reference [297], the same saddle-point derivation was independently rediscovered two years later in July 2016 by reference [170]). In addition, we analyze the steady-state and transient behavior of the proposed distributed algorithm, deriving closed-form expressions that characterize the network performance for sufficiently small constant step-sizes, which generalize previous analysis for the single-agent GTD algorithm. We note that the proposed DGTD introduces a novel family of distributed primal-dual algorithms that has been later analyzed by other authors [285]. We also discuss a number of extensions of the proposed distributed-GTD, including a cooperative 
actor-critic method for off-policy learning of the optimal policy (i.e., the control problem).

- Development of a diffusion-based distributed-cross-entropy (DCE) algorithm that matches (can even outperform) the performance of the original stochastic approximation single-agent-cross-entropy (SACE) method [128]. Convergence analysis of DCE that generalizes previous analysis of single-agent related frameworks due to $[128,108,340]$. In addition to the algorithmic and theoretical contributions, we discuss how diffusion-based distributed stochastic approximation algorithms behave on non-convex problems, and establish a connection between cross-entropy and continuation optimization schemes (like those presented analyzed in $[194,119])$.

- Analysis of deterministic and stochastic dynamic-potential-games (DPGs) with continuous state-action variables and constrained state-action sets in the general nonreduced form. We start by an open-loop (OL) analysis of deterministic DPGs, then we extend the OL results to stochastic DPGs and, finally, we provide a simplified closed-loop (CL) analysis of stochastic DPGs for parametric policies. In all cases, we introduce a related single-objective control problem and show that the solution of this single-objective problem is a Nash equilibrium of the game. The results generalize previous analysis due to [68-70, 122, 105, 81] and are presented in a form that is the natural extension of the analysis for static potential games $[264,196]$. We remark the importance of dealing with constraints, as they are required for many applications; and the relevance of including a CL analysis, since CL policies can yield optimal performance in stochastic settings. Another contribution is to show that the proposed framework can be applied to multiple communication engineering problems. Finally, we show that it is possible to approximate a CL Nash equilibrium of the game by interaction with a simulated environment, without explicit knowledge of the problem, by using modern deep-reinforcement learning techniques.

The publications resulting from this thesis are the following:

Chapter 2:

- [293] Valcarcel Macua, S., Belanovic, P., and Zazo, S. (2010). Consensus-based distributed principal component analysis in wireless sensor networks. In IEEE Int. Workshop on Signal Processing Advances in Wireless Communications (SPAWC), pages 1-5. 
- [294] Valcarcel Macua, S., Belanovic, P., and Zazo, S. (2011). Distributed linear discrimi- nant analysis. In IEEE Int. Conference on Acoustics, Speech and Signal Processing (ICASSP), pages 3288-3291.

- [20] Belanovic, P., Valcarcel Macua, S., and Zazo, S. (2012b). Distributed static linear Gaussian models using consensus. Neural Networks, 34:96-105.

- [299] Valcarcel Macua, S., Leon, C. M., Romero, J. S., Pereira, S. S., Zazo, J., Pages-Zamora, A., López-Valcarce, R., and Zazo, S. (2014). How to implement doubly-stochastic matrices for consensus-based distributed algorithms. In IEEE Sensor Array and Multichannel Signal Processing Workshop (SAM), pages 333-336.

- [19] Belanovic, P., Valcarcel Macua, S., and Zazo, S. (2012a). Distributed primary user identification from imprecise location information. In Int. Conference on Systems, Signals and Image Processing (IWSSIP), pages 89-92.

- [21] Belanovic, P., Valcarcel Macua, S., and Zazo, S. (2012c). Location-aided distributed primary user identification in a cognitive radio scenario. In IEEE Int. Conference on Acoustics, Speech and Signal Processing (ICASSP), pages 3761-3764.

Chapter 3:

- [297] Valcarcel Macua, S., Chen, J., Zazo, S., and Sayed, A. H. (2015. Also available as arXiv:1312.7606, Dec. 2013). Distributed policy evaluation under multiple behavior strategies. IEEE Transactions on Automatic Control, 60(5):1260-1274.

- [296] Valcarcel Macua, S., Chen, J., Zazo, S., and Sayed, A. H. (2013a). Cooperative off-policy prediction of markov decision processes in adaptive networks. In IEEE Int. Conference on Acoustics, Speech and Signal Processing (ICASSP), pages 4539-4543.

- [295] Valcarcel Macua, S., Belanovic, P., and Zazo Bello, S. (2012). Diffusion gradient temporal difference for cooperative reinforcement learning with linear function approximation. In Cognitive information workshop (CIP).

Chapter 4: 
- [302] Valcarcel Macua, S., Zazo, S., and Zazo, J. (2015). Distributed blackbox optimizaion of nonconvex functions. In IEEE Int. Conference on Acoustics, Speech and Signal Processing (ICASSP).

- Valcarcel Macua, S., Sainz, M., Chen, J., and Zazo, S. (2017), Distributed Cross-Entropy for Black-Box Global Optimization. Journal paper in preparation.

\section{Chapter 5:}

- [333] Zazo, S., Valcarcel Macua, S., Sánchez-Fernández, M., and Zazo, J. (2016d). Dynamic potential games with constraints: Fundamentals and applications in communications. IEEE Transactions on Signal Processing, 64(14):3806-3821.

- [303] Valcarcel Macua, S., Zazo, S., and Zazo, J. (2016). Learning in constrained stochastic dynamic potential games. In IEEE Int. Conference on Acoustics, Speech and Signal Processing (ICASSP), pages 4568-4572.

- [300] Closed-Loop Stochastic Dynamic Potential Games with Parametric Policies and Constraints. Valcarcel Macua, S., Zazo, J., and Zazo, S. (2016a) Learning, Inference and Control of Multi-Agent Systems Workshop at Neural Information Processing (NIPS).

Other publications:

- [332] Zazo, J., Zazo, S., and Valcarcel Macua, S. (2016b). Robust worst-case analysis of demand-side management in smart grids. IEEE Transactions on Smart Grid.

- [329] Zazo, J., Valcarcel Macua, S., Zazo, S., Pérez, M., Pérez-Álvarez, I., Jiménez, E., Cardona, L., Brito, J. H., and Quevedo, E. (2016a). Underwater electromagnetic sensor networks, part II: Localization and network simulations. Sensors, 16(12):2176.

- [331] Zazo, J., Zazo, S., and Valcarcel Macua, S. (2016c). Non-monotone quadratic potential games with single quadratic constraints. In IEEE Int. Conference on Acoustics, Speech and Signal Processing (ICASSP), pages 4373-4377.

- [79] Dopico, N. I., Bejar, B., Valcarcel Macua, S., Belanovic, P., and Zazo, S. (2011). Improved animal tracking algorithms using distributed kalman-based filters. In European Wireless - Sustainable Wireless Technologies, pages 1-8. 
- [330] Zazo, J., Zazo, S., and Valcárcel Macua, S. (2014). Distributed cognitive radio systems with temperature-interference constraints and overlay scheme. In European Signal Processing Conference (EUSIPCO), pages 855-859.

- [301] Valcarcel Macua, S. and Zazo, S. (2014). Some applications of distributed signal processing. In XXIV National Simp. of Int. Union of Radio Science (URSI).

- [218] Parras, J., del Val, J., Zazo, S., Zazo, J., and Valcarcel Macua, S. (2016). A new approach for solving anti-jamming games in stochastic scenarios as pursuitevasion games. In IEEE Statistical Signal Processing Workshop (SSP), pages $1-5$.

- [74] del Val, J., Zazo, S., Valcarcel Macua, S., Zazo, J., and Parras, J. (2016). Optimal attack and defence of large scale networks using mean field theory. In European Signal Processing Conference (EUSIPCO), pages 973-977.

\subsection{Outline}

The outline of this thesis is as follows:

- Chapter 2 introduces average-consensus as a basic form of cooperation. We show how average-consensus can be used for computing the first and second order moments from a distributed dataset and to build a number of cooperative component analysis methods. We finish the chapter pointing to distributed optimization as a framework for developing more general analysis methods.

- Chapter 3 uses distributed optimization for cooperative policy evaluation. We derive the gradient-temporal-difference (GTD) algorithm in a novel primal-dual manner and show how to implement it in a distributed manner, even when the agents follow different policies. We provide a detailed analysis of the algorithm. We finish the chapter pointing to interesting extensions, like distributed off-policy actor-critic algorithms or generalizing across similar domains.

- Chapter 4 uses distributed stochastic approximation for cooperative global search. We provide novel results for the particle filter optimization framework and perform numerical experiments to compare with the centralized solution. 
- Chapter 5 deals with the non-cooperative scenario. We present dynamic games and show conditions for these games being potential. Moreover, we provide open-loop and a preliminary closed-loop analysis of general dynamic potential games (stochastic, described in nonreduced form and with explicit constraints).

- Chapter 6 provides some conclusions.

\subsection{Notation}

We indicate some general notation that is shared along chapters. More specific notation is included when necessary, as well some additional remarks at the beginning of each chapter.

Lower case letters are used to denote both scalar values and vectors. Matrices are denoted by upper case letters. Boldface notation denotes random variables (e.g., $s$ is a realization for $\boldsymbol{s}$ ). For time varying scalar variables, the time index $i$ is expressed between parenthesis (e.g., $r(i)$ ); while for time varying vectors or matrices, the time index is set as a subscript (e.g., $x_{i}$ ). Moreover, whenever a variable is specific to some agent $k$ we add a subscript (e.g., $x_{k, i}$ means the variable corresponding to agent $k$ at time $i$ ).

All vectors are column vectors. Superscript $\cdot{ }^{\top}$ denotes transposition. The identity matrix of size $M$ is denoted by $I_{M}$, the null matrix of size $M \times L$ is denoted by $0_{M \times L}$, and $\mathbb{1}_{M}$ and $0_{M}$ stand for vectors of ones and zeros of length $M$, respectively. The Kronecker product operation is denoted by $\otimes$. The spectrum, $m$-th eigenvalue, maximum eigenvalue and spectral radius of a matrix are denoted by $\lambda(\cdot), \lambda_{m}(\cdot), \lambda_{\max }(\cdot)$ and $\rho(\cdot)=\left|\lambda_{\max }(\cdot)\right|$, respectively. The operator $\operatorname{col}\{\cdot\}$ stacks vectors (or matrices) into a long vector (or a tall matrix), we sometimes write:

$$
\operatorname{col}\left\{x_{1}, \ldots, x_{N}\right\} \triangleq\left[\begin{array}{c}
x_{1} \\
\vdots \\
x_{N}
\end{array}\right]=\left(x_{k}\right)_{k=1}^{N}
$$

Operator $\operatorname{diag}\{\cdot\}$ creates a diagonal matrix (a block-diagonal matrix) from a given vector (a set of square matrices). The Euclidean norm is given by $\|y\|^{2} \triangleq y^{\top} y$; and the weighted Euclidean (semi)norm is given by $\|y\|_{D}^{2} \triangleq y^{\top} D y$, where $D$ is a positive (semi)definite matrix. The expected value operator is denoted by $\mathbb{E}[\cdot]$; the expected value operator with respect to probability distribution $d$ is denoted by $\mathbb{E}_{d}[\cdot]$. 


\section{Chapter 2}

\section{Distributed component analysis methods using average consensus}

\subsection{Introduction}

In this chapter, we illustrate the principles underlying cooperative fully distributed algorithms. We consider a very simple strategy, known as consensus averaging, that is used for proposing distributed implementations of a number of component analysis (CA) methods.

Principal component analysis (PCA) is one of the most fundamental and best known feature extraction algorithms [220, 125, 136], dating back to the 1930s. Since then it has enjoyed tremendous success in many diverse fields, inspiring numerous variations and extensions. We examine the processing and communications (intra- and inter-node) aspects of performing PCA, static linear Gaussian models (SLGMs) and linear discriminant analysis (LDA), and propose average-consensus based distributed implementations for all of them. As we will see, SLGMs include principal component analysis (PCA), as well as two closely related algorithms, factor analysis (FA) and probabilistic PCA (PPCA).

At the time we performed this research, there existed various partially distributed implementations of PCA. These earlier approaches focused on saving part of the multihop communication cost by either local computations [141] or aggregation services [229, $10,159]$, but they still relied on a fusion center for merging the local results. Reference [326] presented a distributed distance matrix estimation algorithm for non-linear manifold learning and dimensionality reduction; it built on a distributed implementation of the power method [250], which was also based on a standard average consensus algorithm, but it required a post-consensus flooding of intermediate results, involving 
multiple-hops to get the information to every agent in the network, which prevents from scaling to large networks. Up to the best of our knowledge, the consensusbased algorithms presented here were the first fully distributed component analysis algorithms that required neither fusion center, nor flooding, and still were able to achieve near optimal results just by local (1-hop) neighborhood communications, and with communication cost that is independent on the network size and on the number of data samples, being able to scale to arbitrarily large networks and large datasets. Many other related distributed PCA have appeared after publishing the results included in this chapter (see, e.g., [327, 24, 111, 167, 45, 89, 222, 318, 197, 166, 283]). Moreover, some of these more modern works extend the consensus averaging approach used here, while others present novel distributed implementations; some of them are presented in a heuristic fashion similar to here, while others include rigorous convergence analysis. A complete comparison with all of these more modern distributed component analysis methods is out of the scope of this introduction, since our main purpose is to present a number a contributions that, we hope, will gently introduce the reader to the more sophisticated fully distributed cooperative algorithms of Chapters 3 and 4 .

Our first contribution is a method for distributed estimation of the first two moments of a multi-dimensional data set, when the agents have different number of samples. This method is based on the well-studied average consensus algorithms.

The second contribution of this article is a direct application of the previous idea to systematicaly develop a set of fully distributed component analysis methods. We begin with two distributed algorithms to perform PCA. The first is a direct method, deriving local approximations of the sample covariance matrix of the global data set, and hence the dominant eigenvectors and the principal subspace spanned by these. The other is an iterative method, based on an expectation maximization (EM) procedure. Both algorithms asymptotically converge to the centralized solution given by classical PCA. Then, we introduce distributed implementations of factor analysis (FA) and probabilistic principal component analysis (PPCA). Finally, we present three distributed implementations for linear discriminant analysis, namely a direct method that follows the same idea of estimating the covariance matrix in a distributed manner and then computing the eigenvectors locally; a distributed coordinate descent method; and uncorrelated-LDA, which implicitly adds some regularization to the covariance matrix to be able to operate on undersampled datasets.

The third contribution is to illustrate the behavior of these algorithms with some experimental examples of their use over large, sparse networks with distributed datasets, which represent the most difficult setting. 
For simplicity, we do not examine here convergence or the influence of noisy links, time-varying topologies, and delay and quantization effects. They have been extensively analyzed in the literature (see, e.g., $[212,46,253,252,213,139]$ ) in generic terms, and their influence on our algorithms is beyond the scope of this introductory chapter.

Publications. The results described in this chapter resulted in the following publications [293, 294, 20, 299, 19, 21].

Notation. In this chapter, $Y \in \mathbb{R}^{M \times T}$ denotes the observed dataset, which is a matrix where there are $T$ data samples (columns) of length $M$ each; and $W$ is the matrix with columns spanning the subspace obtained with each of the component analysis methods that will be described.

\subsection{Background on component analysis methods}

The basic idea underlying component analysis (CA) methods (also named subspace methods) is to project the data (or its non-linear expansion in a feature space [260]) into a lower dimensional subspace, keeping those relevant components that are optimal for a given task (e.g. classification, estimation). In other words, CA methods are linear and non linear methods for "manifold learning", i.e. they find a low-dimensional basis for describing high-dimensional observed data, uncovering the intrinsic dimensionality.

CA methods have been being applied for many tasks, like data compression, overcoming the curse of dimensionality with high-dimensional data (which usually requires a large number of samples to build accurate models (see e.g. [38])), de-noising, setting reasonable distance metrics (e.g. between two frames in a picture), etc.

There have been some papers seeking for a a common framework to unify CA methods. Reference [239] showed how Principal Component Analysis (PCA), Factor Analysis (FA) and Probabilistic Principal Component Analysis (P-PCA) can be derived from the point of view of static linear Gaussian models. Reference [321] proposed a unifying view of PCA, Linear Discriminant Analysis (LDA), and some other methods using graph theory. More recently, reference [66] introduced a very general framework of PCA, LDA and many others, as well as their weighted and kernel extensions.

\subsubsection{Principal Component Analysis}

PCA was first introduced by Pearson in 1901, as a methodology for linear regression, and later, in 1933, independently derived and given its current name by Hotelling, as a technique for the analysis of correlation between many random variables. For a detailed 
review on PCA see e.g. [136] and references therein. The two most common derivations of PCA involve finding the lower-dimensional subspace: (a) where the variance of the data is maximized, or (b) where the linear projection minimizes the mean square error of the distance between the original and the projected data. Both derivations result in similar principal components (PC), i.e. similar basis of the subspace.

Let $Y \triangleq\left(y_{1}, \ldots, y_{T}\right)$ be a global dataset of $T$ independent and identically distributed (i.i.d.) samples. Each sample is $M$-dimensional, such that $Y \in \mathbb{R}^{M \times T}$. The standard single-agent PCA requires that the agent has access to the full data set $Y$, so that it can obtain the principal subspace $W \in \mathbb{R}^{M \times L}$ directly by getting the sample data covariance matrix $\Sigma$, and then its $L$ dominant eigenvectors, as summarized in Algorithm 2.1.

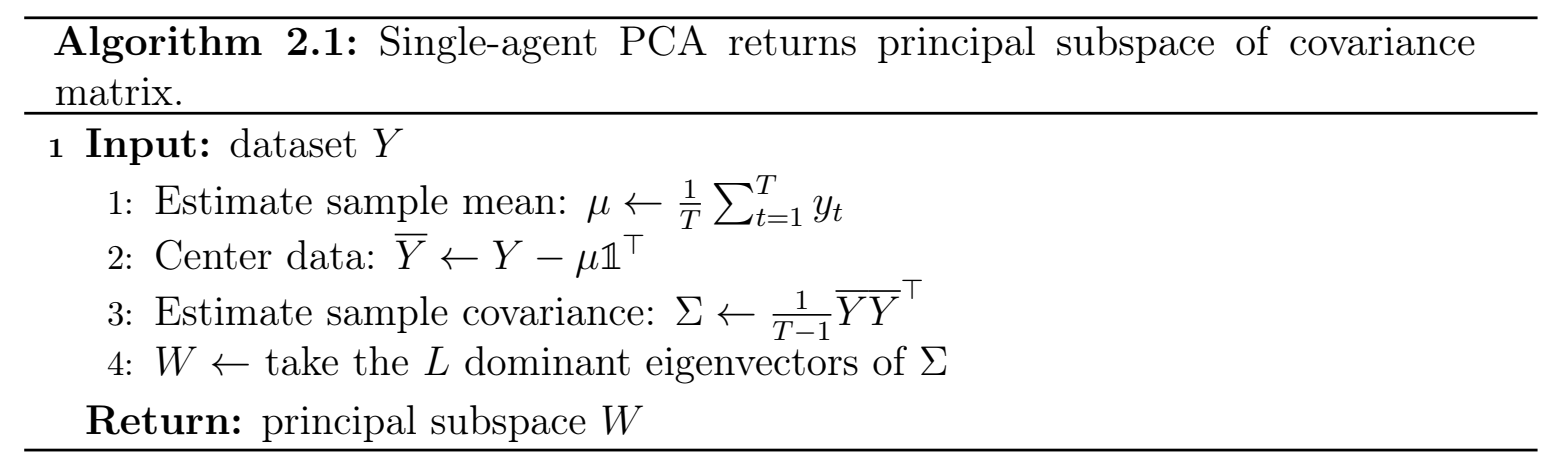

\subsubsection{PCA in the context of latent variable models}

References $[238,284]$ proposed to think of the observed data as the maximum likelihood solution of a probabilistic latent variable model. First, introduce an explicit latent variable $\boldsymbol{x} \in \mathbb{R}^{L}$ that corresponds to the principal component subspace (recall that boldface notation denotes random variables). Let us define a Gaussian prior distribution over the latent variable $\boldsymbol{x} \sim \mathcal{N}\left(0_{L}, Q\right)$. The observable data is modeled by random variable $\boldsymbol{y} \in \mathbb{R}^{M}$. We assume that the observable data is generated by a linear transformation of the latent variable plus some some random noise $\boldsymbol{\epsilon} \sim \mathcal{N}\left(0_{M}, R\right)$ :

$$
\boldsymbol{y}=W \boldsymbol{x}+\boldsymbol{\epsilon}
$$

Hence, the conditional distribution of the observable data is also Gaussian, of the form $\boldsymbol{y} \mid \boldsymbol{x} \sim \mathcal{N}\left(0_{M}, W Q W^{\top}+R\right)$ In this setting, we can think that the observable dataset is generated by choosing a value for the latent variable and then sampling the observed variable conditioned on this latent value. Taking the limit $\boldsymbol{\epsilon} \rightarrow 0$ in (2.1), then it 
results the standard PCA model [238]:

$$
Y=W X
$$

where the columns of $W$ span the $L$-dimensional subspace $(L \leq M)$ which maximizes the variance of the projection of $Y$ onto that space. Under this assumption, the maximum likelihood estimate for $W$ is the set of $L$ dominant eigenvectors of the sample covariance matrix of the global data set $Y$ (see [284]). Hence, based on this probabilistic latent variable model with zero observation noise, it is possible to find an iterative EM like algorithm that converges to a maximum independent on the distribution of the observed data set. By linking EM with block coordinate descent, reference [66] uses the results from [13] to argue that there is a unique global maximum, so that convergence to the global solution is guaranteed, up to saddle points if the data is ill posed. In general, the EM algorithm is a two steps iterative technique for computing maximum likelihood solutions. In the E-step, it computes sufficient statistics, while in the $M$-step, the parameters of the model are estimated. For a detailed review on EM see, e.g., [185].

The single-agent EM algorithm for PCA presented in [238, 284], that we denote as EM-PCA, is shown in Algorithm 2.2. At each iteration, the E-step projects the data onto a lower dimensional subspace, while the $M$-step seeks for an update of the subspace which could minimize the mean square error of the distance between the original and the projected data. Thus, the E-step is given by

$$
X=\left(W^{\top} W\right)^{-1} W^{\top} Y
$$

and the $M$-step is given by

$$
W=Y X^{\top}\left(X X^{\top}\right)^{-1}
$$

If the data is not zero mean, we have to center the samples by subtracting the mean prior to the EM loop composed of (2.3)-(2.4).

It is worth noting that both the columns of $W$ and the dominant $L$ eigenvectors of the sample covariance matrix span the same principal subspace. However, they are not equal. In fact the columns of $W$ are not orthogonal. In the case orthogonality is required, reference [1] showed that adding very simple lower/upper triangularization operators to the E/M steps, the EM loop will output directly an orthogonal basis of the principal subspace, similar (up to rotation) to the one found in Algorithm 2.1. 


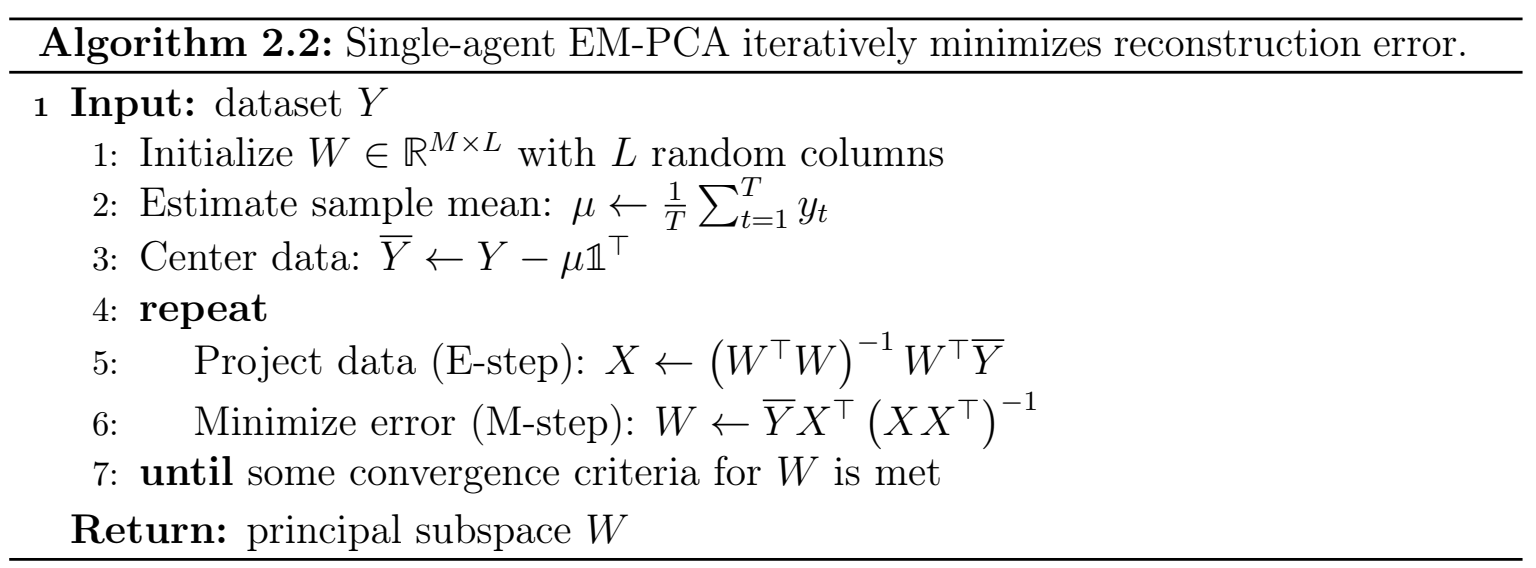

Although the model (2.1) was assumed zero mean data, we have included the data centering step in Algorithm 2.2 for completeness. We do the same in the following subsection.

\subsubsection{Static Linear Gaussian models}

Roweis and Ghahramani [239] noted that the approach of a latent variable model could be extended to many other methods, both static and dynamic, (e.g. PCA, P-PCA, FA, Hidden Markov Models and Kalman filters, among others). In particular, they showed that FA and P-PCA can be derived from the same generative static linear Gaussian model described in Section 2.2.2, just by not vanishing but constraining the covariance matrix $R$ that controls the observation noise.

\section{Factor Analysis}

For instance, by restricting $R$ to be diagonal, we recover a standard statistical model known as maximum likelihood factor analysis. The unknown latent variables $X$ are called the factors in this context; the matrix $W$ is called the factor loading matrix, and the diagonal elements of $R$ are often known as the uniqueness (for a detailed review on FA, see, e.g., [236, 239]). The main difference between FA and PCA is that, instead of assuming that most of the total variance of a variable is important and in common with other observed variables, FA allows for a considerable amount of uniqueness to be present in the data. As reference [239] shows, the learning algorithm for $W$ and $R$ is exactly an EM algorithm. During the E-step of FA, the factors are obtained as the mean, $X$, and covariance, $Q$, of the latent data distribution using parameters, $W$ and 
$R$, from the latest iteration:

$$
\begin{aligned}
& X=W^{\top}\left(W W^{\top}+R\right)^{-1} Y \\
& Q=I_{L}-W^{\top}\left(W W^{\top}+R\right)^{-1} W
\end{aligned}
$$

During the $M$-step, the maximization of the updated moments yields the new parameter values for $W$ and $R$ :

$$
\begin{aligned}
W & =Y X^{\top}\left(X X^{\top}+T Q\right)^{-1} \\
R & =\text { diagelements }\left[\frac{1}{T-1}\left(Y Y^{\top}-W X \bar{Y}^{\top}\right)\right]
\end{aligned}
$$

where we recall that $T$ denotes the number of samples and operator diagelements[.] outputs a diagonal matrix of the same size and with same diagonal elements as the input (i.e., it discharges all off-diagonal entries of the input). We remark that we have followed the model described in Section 2.2.2, which implies that the dataset is zero mean. The steps for FA are summarized in Algorithm 2.3.

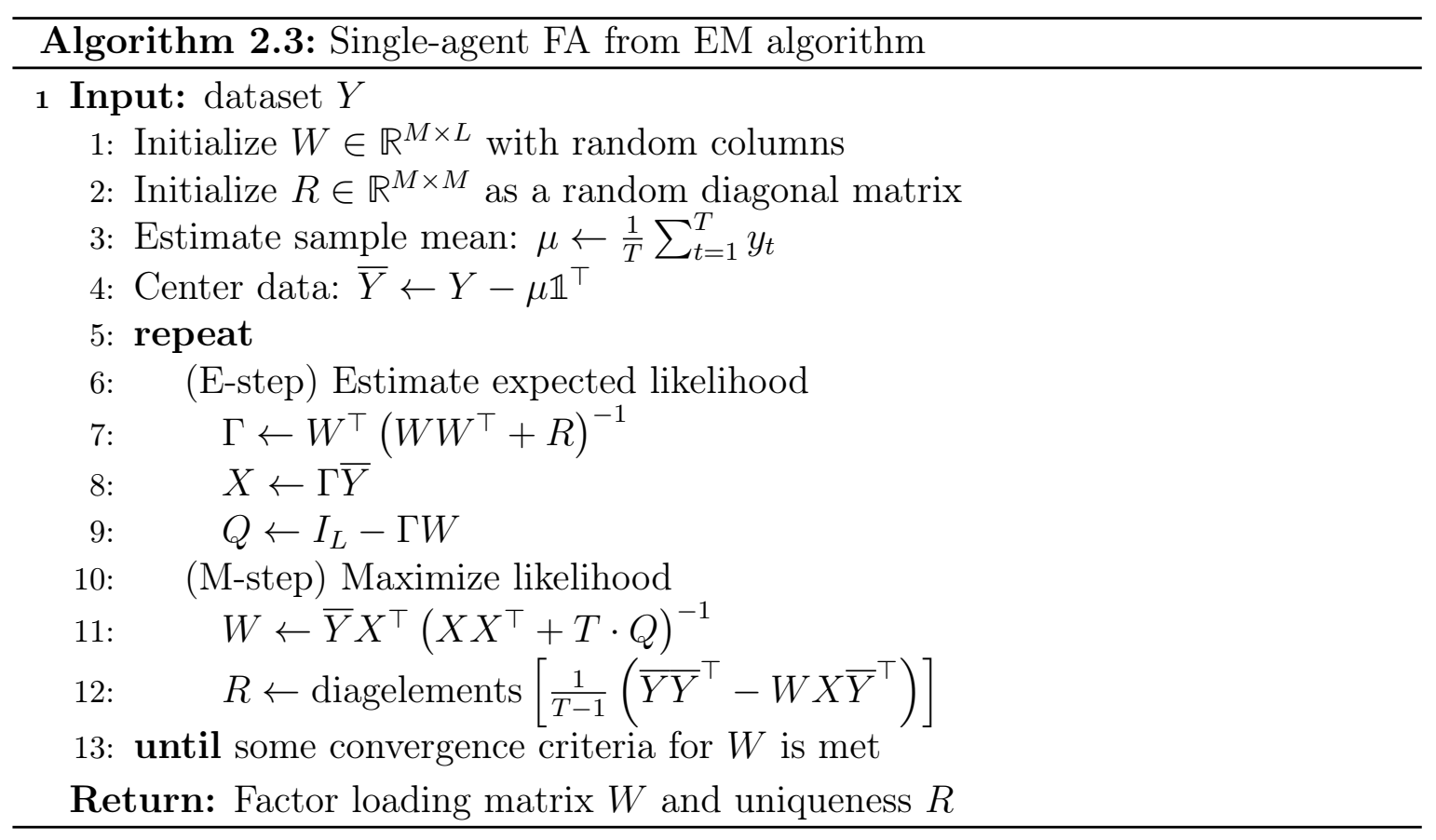

\section{Probabilistic PCA}

As we have seen in section 2.2.2, standard PCA results from the generative model defined by (2.1)) when taking the observation error zero. Similar to FA, if we allow the 
covariance of the observed noise to be a multiple of the identity matrix $R \sim N(0, \alpha \mathcal{I})$, then we now have a fully probabilistic model for PCA. This causes that the observation noise is updated as follows:

$$
\alpha=\frac{1}{(T-1) M} \operatorname{Tr}\left[\overline{Y Y}^{\top}-W X \bar{Y}^{\top}\right]
$$

Reference $[238,284]$ introduced an iterative EM algorithm for P-PCA, which is reproduced in Algorithm 2.4 (we follow the formulation from [238]).

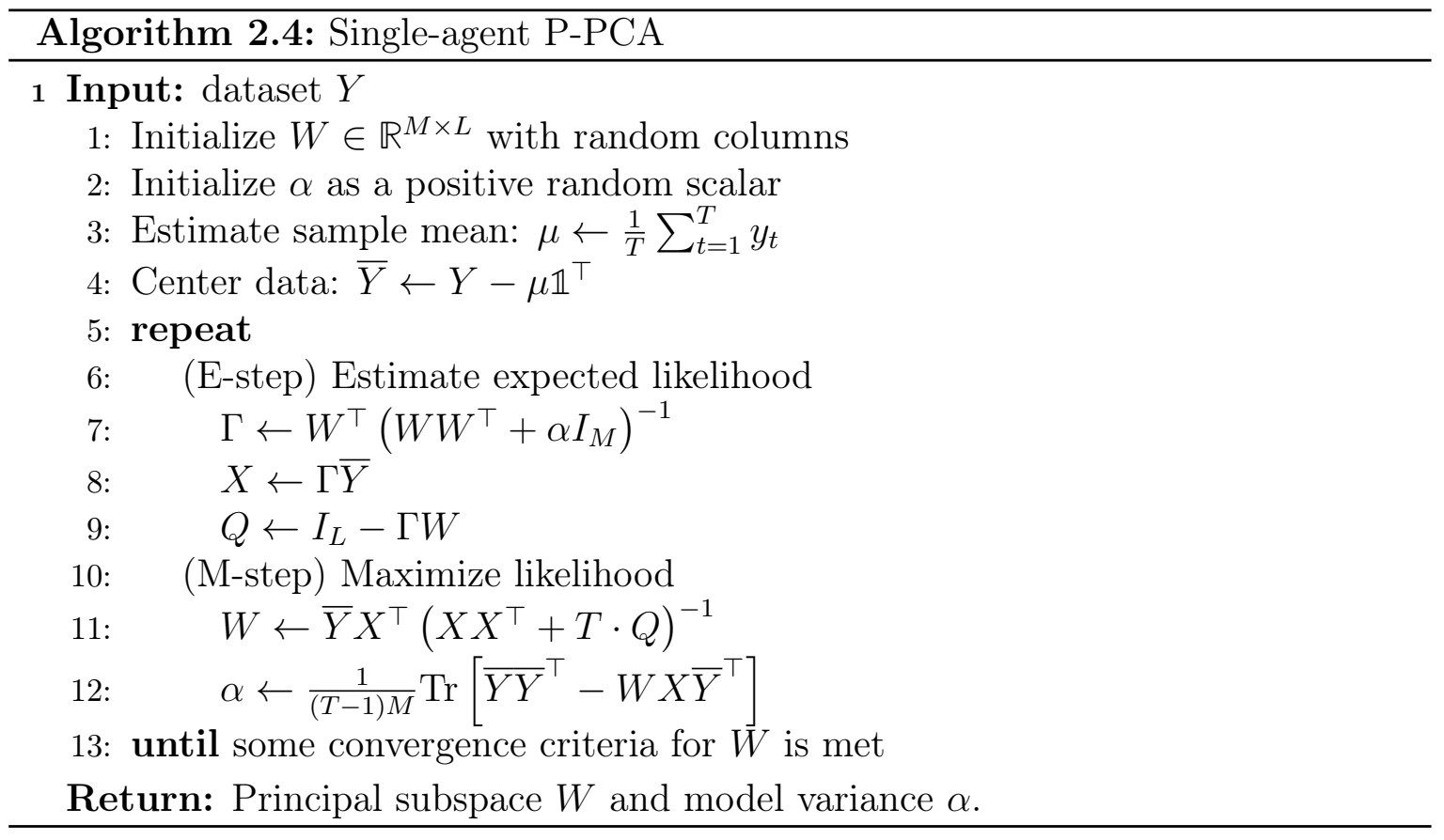

P-PCA offers some interesting applications. For instance, it can deal with missing data, provided that it is missing at random, by marginalizing over the distribution of the unobserved variables. Furthermore, we can use a Bayesian approach in order to estimate the number of most relevant principal components [38].

\subsubsection{Fisher Linear Discriminant Analysis}

In classification, a discriminant is a function that takes an input vector $y$ and assigns it to one of $D$ classes, denoted $\left\{B_{1}, \ldots, B_{D}\right\}$. We assume that the classes are disjoint, so that each input is assigned to one and only one class. Hence, the input space is thereby divided into decision regions whose boundaries are called decision surfaces. Moreover, we shall assume that the dimensionality of the input space is greater than the number of classes, such that $D<M$. 
Suppose we take the $M$-dimensional input vector $y$ and project it down to a lower $L$-dimensional space $(L \leq M)$ using

$$
x=W^{\top} y
$$

We are interested in a special subspace where, on one hand, the separation between the means of the projected classes is maximum, while, on the other hand, the variance within each class is minimum, thereby minimizing the class overlap [38].

Suppose we are given a training dataset consisting of $T$ data points lying in an $M$-dimensional vector space, and their corresponding labels. We express the set of data points as a matrix $Y \triangleq\left(y_{t}\right)_{t=1}^{T} \in \mathbb{R}^{M \times T}$, with columns $y_{t} \in \mathbb{R}^{M}$; and the set of labels as an indicator matrix $G \in \mathbb{R}^{T \times D}$, with elements $g_{t d} \in\{0,1\}$, such that $g_{t d}=1$ if the corresponding sample $y_{t}$ belongs to class $d$, and 0 otherwise [67]. Since the classes are disjoint, we have $\sum_{d=1}^{D} g_{t d}=1$. By slightly abusing notation, we write $t \in B_{d}$ to indicate that $y_{t}$ belongs to class $d$ :

$$
\left\{t \in B_{d}\right\} \triangleq\left\{y_{t}, t=1, \ldots, T: g_{t d}=1\right\}
$$

We define the within-class covariance matrix for class $d$ as:

$$
\Psi_{d} \triangleq \sum_{t \in B_{d}}\left(y_{t}-\mu_{d}\right)\left(y_{t}-\mu_{d}\right)^{\top}
$$

where $\mu_{d}$ is the sample mean of the points in class $B_{d}$, and being $T_{d}$ the amount of points in that class:

$$
\mu_{d} \triangleq \frac{1}{T_{d}} \sum_{t \in B_{d}} y_{t}
$$

The number of points in each class can be easily obtained from the diagonal of the gram matrix of $G$ :

$$
\left(T_{d}\right)_{d=1}^{D}=\text { diagelements }\left[G^{\top} G\right]
$$

or equivalently, we write: $T_{d}=\left[G^{\top} G\right]_{d d}$. The total within-class covariance matrix $\Sigma_{w}$ that we aim to minimize is given by the sum of the $D$ within-class covariance matrices:

$$
\Sigma_{w} \triangleq \frac{1}{T-1} \sum_{d=1}^{D} \Psi_{d}
$$


We can use the average distance between the means of the classes as a measure of the separation between classes:

$$
\Sigma_{b} \triangleq \frac{1}{T-1} \sum_{d=1}^{D} T_{d}\left(\mu_{d}-\mu\right)\left(\mu_{d}-\mu\right)^{\top}
$$

where $\mu$ is the sample mean of all the points in the dataset:

$$
\mu \triangleq \frac{1}{T} \sum_{t=1}^{T} y_{t}
$$

Note that $\Sigma_{b}$ is the sum of $D$ rank 1 matrices. But, due to (2.17), it is clear that:

$$
q \triangleq \operatorname{rank}\left[\Sigma_{b}\right] \leq D-1
$$

Reference [94] showed that the optimal subspace that minimizes (2.15) and maximizes (2.16) can be found as the solution of the following problem:

$$
\underset{W \in \mathbb{R}^{M \times L}}{\operatorname{maximize}} \operatorname{Tr}\left[\left(W^{\top} \Sigma_{w} W\right)^{-1} W^{\top} \Sigma_{b} W\right]
$$

Maximizing (2.19) can be seen as a general eigenvalue problem (GEP) with solution given by

$$
W^{\star}=\operatorname{eig}_{L}\left[\Sigma_{w}^{-1} \Sigma_{b}\right]
$$

where $\operatorname{eig}_{L}[\cdot]$ denotes the operator that takes the eigenvectors associated with the $L$ highest eigenvalues (see, e.g., [94, 66]). This result is known as Fisher's linear discriminant. Although strictly it is not a discriminant but rather a specific choice of direction for projection of the data down to an embedded subspace, which is spanned by the eigenvectors corresponding with the $L$ highest eigenvalues. From (2.18), we have the constraint that $L \leq q$.

Interestingly, it can be shown that if we replace the within class covariance, $\Sigma_{w}$, with the total covariance of all data points, denoted $\Sigma$ and given by (2.22) below, then we obtain another problem that is equivalent to (2.19):

$$
\underset{W \in \mathbb{R}^{M \times L}}{\operatorname{maximize}} \operatorname{Tr}\left[\left(W^{\top} \Sigma W\right)^{-1} W^{\top} \Sigma_{b} W\right]
$$


where

$$
\Sigma \triangleq \frac{1}{T-1} \sum_{t=1}^{T}\left(y_{t}-\mu\right)\left(y_{t}-\mu\right)^{\top}
$$

In other words, minimizing the total covariance is equivalent to minimizing the withinclass covariance. And the solution to (2.21) is the same as (2.20), but can be computed in another form derived from this alternative formulation:

$$
W^{\star}=\operatorname{eig}_{L}\left[\Sigma^{-1} \Sigma_{b}\right]
$$

For simplicity, we will prefer this alternative formulation when deriving distributed implementations of LDA.

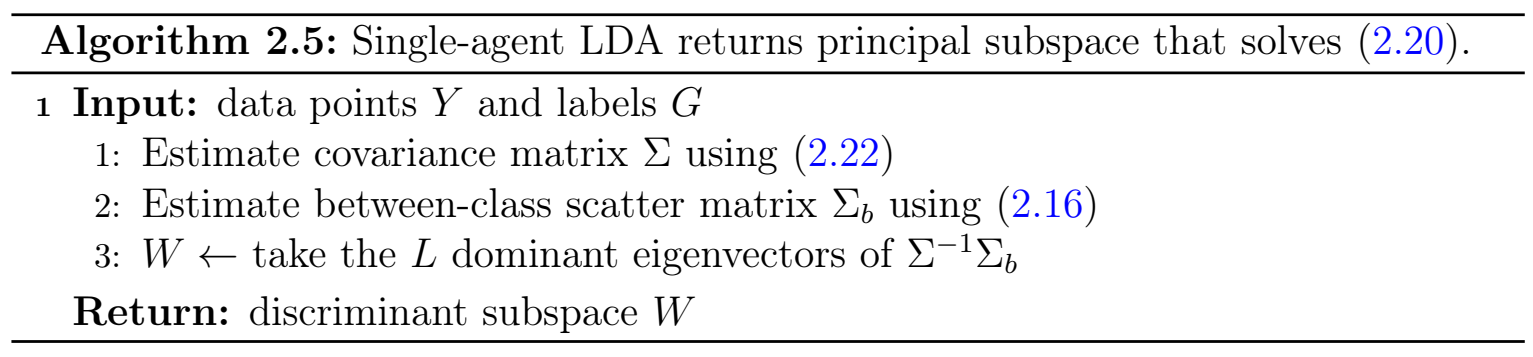

The eigen-formulation in (2.20) reminds PCA, in the sense that it can be also derived as the eigenvectors of the sample covariance matrix. However, while PCA captures the directions of maximum covariance, LDA takes into account the within classes and between classes scatter matrices. Thus, LDA allows us to separate the classes better in the subuspace (see Figure 2.1).

\section{Iterative LDA}

Together with many other component analysis methods, LDA can also be derived in the general framework due to [66] as the optimal solution of the following optimization problem, which is equivalent to (2.19) and (2.21):

$$
\underset{W \in \mathbb{R}^{M \times L}, A \in \mathbb{R}^{D \times L}}{\operatorname{maximize}} J_{\text {lda }}(W, A) \triangleq\left\|\left(G^{\top} G\right)^{-1 / 2}\left(G^{\top}-A W^{\top} \bar{Y}\right)\right\|_{F}^{2}
$$

where $\|X\|_{F} \triangleq \operatorname{Tr}\left\|X^{\top} X\right\|^{2}=\operatorname{Tr}\left[\left\|X X^{\top}\right\|^{2}\right]$ is the squared Frobenius norm for any matrix $X, G$ is the label indicator matrix defined in the previous subsection, $A$ is a generative matrix for the column space of $G$, and $\bar{Y}$ denotes the centered set of training 


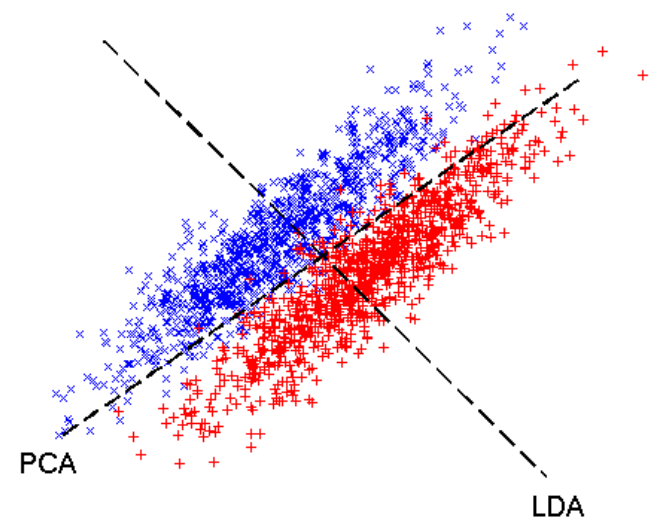

Figure 2.1 PCA vs LDA. PCA finds the most representative directions. LDA minimize the within class covariance matrices, while also maximizing the mean distance between classes preserve.

data points:

$$
\bar{Y} \triangleq Y-\mu \mathbb{1}^{\top}
$$

To show that (2.24) is equivalent to (2.21), we only have to optimize $J(W, A)$ over $A$, which can be solved in closed form by setting the gradient equal to zero:

$$
\begin{aligned}
\nabla_{A} J_{\text {lda }}(W, A) & =\nabla_{A} \operatorname{Tr}\left[\left(G^{\top} G\right)^{-1}\left(G^{\top}-A W^{\top} \bar{Y}\right)\left(G^{\top}-A W^{\top} \bar{Y}\right)^{\top}\right] \\
& =\left(G^{\top} G\right)^{-1}\left(A W^{\top} \overline{Y Y}^{\top} W-G^{\top} \bar{Y}^{\top} W\right) \\
& =0_{D \times L}
\end{aligned}
$$

Hence, we have

$$
A=G^{\top} \bar{Y}^{\top} W\left(W^{\top} \overline{Y Y}^{\top} W\right)^{-1}
$$

By substituting (2.27) in (2.24), we can remove the dependence on $A$, which yields:

$$
J_{\text {lda }}(W) \propto \operatorname{Tr}\left[\left(W^{\top} \overline{Y Y}^{\top} W\right)^{-1} W^{\top} \bar{Y} G\left(G^{\top} G\right)^{-1} G^{\top} \bar{Y}^{\top} W\right]
$$

Note that $\Sigma=\frac{1}{T-1} \overline{Y Y}^{\top}$ and $\Sigma_{b}=\frac{1}{T-1} \bar{Y} G\left(G^{\top} G\right)^{-1} G^{\top} \bar{Y}^{\top}$. Hence, (2.28) and, thus, (2.24), are equivalent to (2.21). 
The benefit from (2.24) is that it allows for an iterative solution through alternating least squares (ALS), which consists in optimizing for $A$ and $W$, alternatively (this is more usually known as block coordinate descent). As we have seen, the solution can be obtained in closed form by taking $\nabla_{A} J_{\mathrm{lda}}(W, A)=0_{D \times L}$ and $\nabla_{W} J_{\mathrm{lda}}(W, A)=0_{M \times L}$, which yields the following update equations:

$$
\begin{aligned}
A & =G^{\top} \bar{Y}^{\top} W\left(W^{\top} \overline{Y Y}^{\top} W\right)^{-1} \\
W & =\left(\overline{Y Y}^{\top}\right)^{-1} \bar{Y} G\left(G^{\top} G\right)^{-1} A\left(A^{\top}\left(G^{\top} G\right)^{-1} A\right)^{-1}
\end{aligned}
$$

This iterative procedure is summarized in Algorithm 2.6. As reference [66] points out, the optimization of (2.24) "avoids the small sample size problem and can be a numerically efficient for large amounts of high dimensional data."

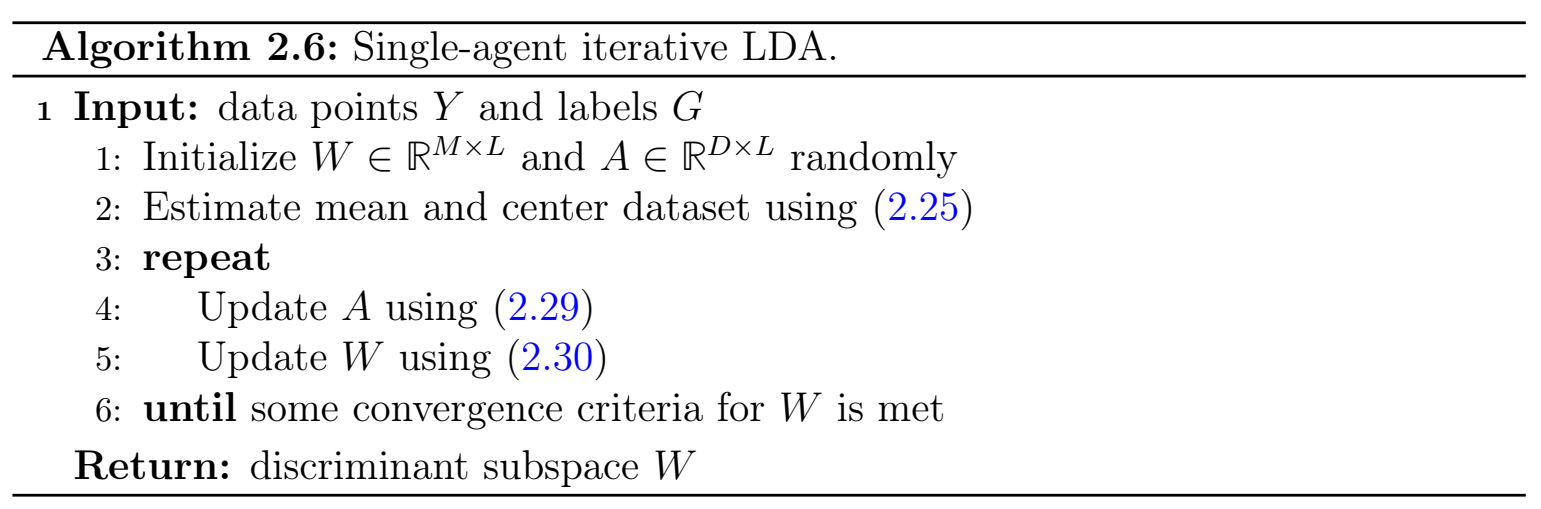

\section{Regularized LDA for undersampled data}

When dealing with data sets whose dimensionality is comparable to the number of samples, the within class and between classes scatter matrices may be singular and solutions based on eigen-decomposition or iterative ALS can have high variance. One way of surmounting this problem is by adding some bias to the data towards the plausible set of parameter values, which can be seen as a form of regularization. One interesting case of this form of regularization is Uncorrelated LDA (ULDA) (see, e.g., [134, 324, 325]), which solves the following objective:

$$
\operatorname{maximize}_{W \in \mathbb{R}^{M \times L}} \operatorname{Tr}\left[\left(W^{\top} \Sigma W\right)^{+} W^{\top} \Sigma_{b} W\right]
$$

where $(\cdot)^{+}$denotes the pseudoinverse of a matrix (see, e.g., [103, Sec. 5.5.4]). Note that (2.31) seems actually a natural extension of (2.21) when $\Sigma$ is singular. 
Let $Z$ be a matrix that simultaneously diagonalize $\Sigma, \Sigma_{b}$ and $\Sigma_{w}$, i.e.,

$$
\begin{gathered}
Z^{\top} \Sigma Z=\Delta \\
Z^{\top} \Sigma_{b} Z=\Delta_{b} \\
Z^{\top} \Sigma_{w} Z=\Delta_{w}
\end{gathered}
$$

such that $\Delta, \Delta_{b}$ and $\Delta_{w}$ are diagonal matrices. The solution to (2.31) is given by the first $q$ columns of $Z$ (see, e.g., [324]), where $q$ is the rank of $\Sigma_{b}$, as given by (2.18). The adjective "uncorrelated" in the name comes from the observation that the projected covariance matrices in (2.32)-(2.34) are diagonal, meaning that the transformation yields uncorrelated features.

In order to find $Z$, ULDA performs the following steps. The first step consists in estimating the mean and centering the dataset using (2.25) to obtain $\bar{Y}$. Then, ULDA defines matrices $H$ and $H_{b}$ :

$$
\begin{aligned}
H & \triangleq \frac{1}{\sqrt{T}} \bar{Y} \\
H_{b} & \triangleq \frac{1}{\sqrt{T}}\left[\frac{\mu_{1}-\mu}{\sqrt{T_{1}}}, \ldots, \frac{\mu_{D}-\mu}{\sqrt{T_{D}}}\right]
\end{aligned}
$$

such that they satisfy:

$$
\begin{aligned}
\Sigma & =H H^{\top} \\
\Sigma_{b} & =H_{b} H_{b}^{\top}
\end{aligned}
$$

Working with $H$ saves the computation of the whole covariance matrix. Then, it computes the reduced singular value decomposition (SVD) of $H$ to find its eigenvalues and left eigenvectors:

$$
H=U \Delta_{H} V^{\top}
$$

where $U$ and $V$ are orthogonal and $\Delta_{H}$ is diagonal. These terms are then used to build:

$$
B \triangleq \Delta_{H}^{-1} U^{\top} H_{b}
$$

This matrix is again decomposed using the SVD as:

$$
B=P \tilde{\Delta}_{b} Q^{\top}
$$


where $P$ and $Q$ are orthogonal and $\tilde{\Delta}_{b}$ is diagonal. Then, we have:

$$
\Delta_{H}^{-1} U^{\top} \Sigma_{b} U \Delta_{H}=\Delta_{H}^{-1} U^{\top} H_{b} H_{b}^{\top} U \Delta_{H}=P \tilde{\Delta}_{b} Q^{\top} Q \tilde{\Delta}_{b}^{\top} P^{\top}=P \tilde{\Delta}_{b}^{2} P^{\top}
$$

Now, matrix $P$ is used to find the simultaneous diagonalization matrix:

$$
Z=U \Delta_{H}^{-1} P
$$

So that:

$$
Z^{\top} \Sigma_{b} Z=P^{\top} P \tilde{\Delta}_{b}^{2} P^{\top} P=\tilde{\Delta}_{b}^{2}=\Delta_{b}
$$

Finally, the discriminant subspace, $W$, is obtained by taking the first $L \leq q$ columns of $Z$. These steps are summarized in Algorithm 2.7.

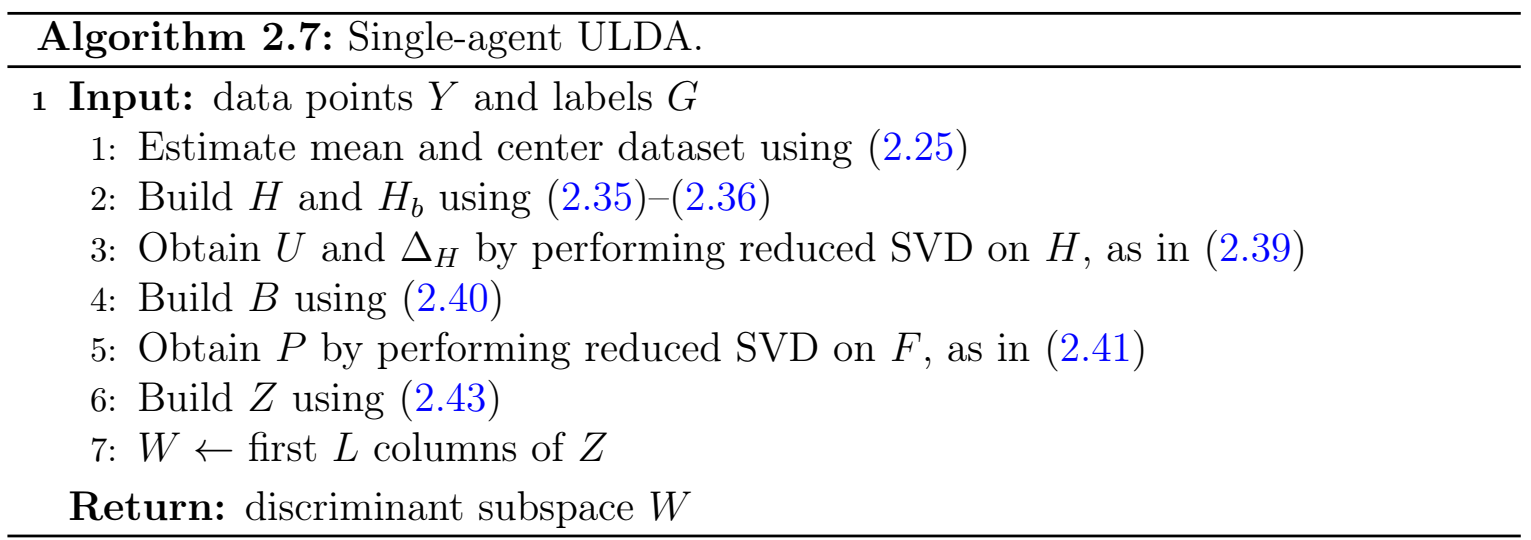

\subsection{Background on consensus algorithms}

Many real life systems are made up of independent agents communicating and working together towards a common goal. Examples include a football team, a group of medical experts treating a patient, a pack of predators choosing a prey, a flock of flying birds minimizing total air resistance, a colony of ants foraging, a swarm of robots, and a network of agents measuring a common parameter.

One usually desired feature for such systems is the ability to reach agreement. Ideally, the agents should be able to agree on a common value in a decentralized fashion, i.e. without the need for a central entity which has a global view of the system. This also implies that only local communication between the agents is required. Other desirable properties are privacy, such that no observation data is exchanged, 
and scalability, such that the communication cost remains constant with the size of the network - which is achieved by allowing only within-neighborhood communication, since the cost only grows with the number of neighbors.

Average consensus algorithms [211] fulfill all the requirements above and have hence been a focus of intense study in many disciplines of science in the last decades. They were first studied already in the 60's in the field of management science and statistics [73]. The significant potential of these algorithms in distributed computing has attracted attention from the control theory community (see e.g. [287, 131, 320, 62]) since the early 80's. The advent of wireless sensor networks (WSN) sparked renewed interest in consensus algorithms from the signal processing community (see e.g. $[14,253,252,210,244])$, with specific modifications to suit this new setting. In last years, much work has shifted from consensus to more general distributed optimization methods, where an intermediate step that reminds the average-consensus is used (see, e.g., $[18,270,54,249,247,203,261,76]$ and references therein).

In Chapters 3 and 4 of this thesis, we will consider the more general distributed optimization framework (in particular, we will use first order diffusion optimization methods for convex and non-convex optimization). However, in this chapter we are only concerned on standard average-consensus for reaching agreement on the average of some observed value.

\subsubsection{The consensus problem}

We consider a network of $N$ connected agents measuring a common parameter (e.g, a set of motes measuring the temperature), in which every agent aims to estimate the average of the measurements of all the agents across the network, but it only has access to its own observation. The network of forms a connected graph (i.e., there is at least one path between every pair of agents). For simplicity, assume that the agents take one single observation, denoted $y_{k} \in \mathbb{R}$, where $k=1, \ldots, N$ stands for the agent index. Let $w^{\star}$ denote the average that the agents aim to estimate:

$$
w^{\star} \triangleq \frac{1}{N} \sum_{k=1}^{N} y_{k}
$$

The network is modeled by a graph where the nodes are the agents, and edges represent communication links. We say that two nodes can communicate with each other, if and only if they are neighbors. More formally, the network is represented by a non-negative connectivity matrix $C=\left[c_{l k}\right]$ of size $N \times N$, with elements $c_{l k} \geq 0$, for 


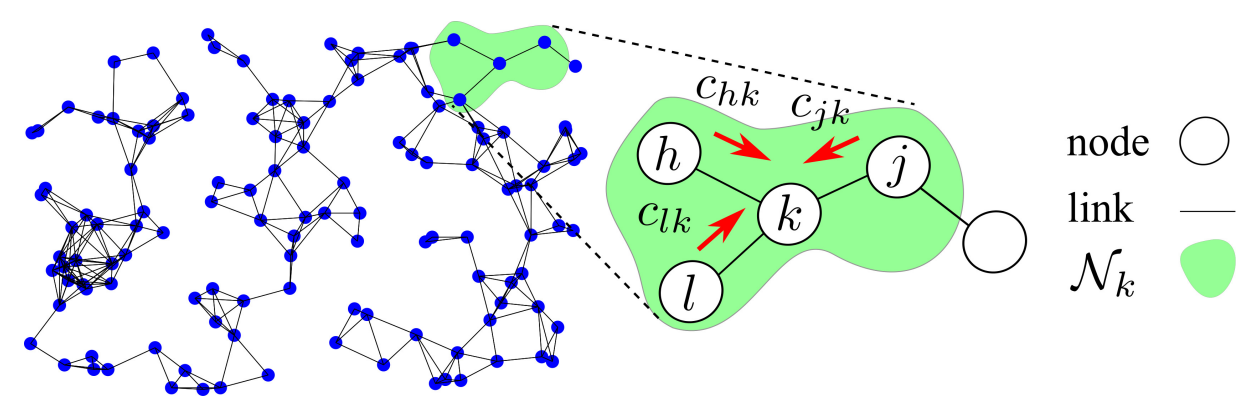

Figure 2.2 Example of cooperative networks considered in this chapter. The whole network is displayed together with a zoom over the up-right corner. The zoomed area displays the neighborhood of agent $k$.

all $l, k=1, \ldots, N$, such that the element $c_{l k}$ represents the weight given by agent $k$ to the information coming from agent $l$. Each agent $k$ is only allowed to communicate within its own neighborhood $\mathcal{N}_{k}$, which is defined as the set of agents that are directly connected with agent $k$, including itself:

$$
\mathcal{N}_{k} \triangleq\left\{l: c_{l k}>0\right\}_{l=1}^{N}
$$

In other words, we say that agents $k$ and $l$ are neighbors if and only if $c_{l k}>0$. See Figure 2.2.

Let $w_{k, i}$ denote the estimate of the average for agent $k$ at time $i$, which is initialized with the agent's initial measurement, i.e.,

$$
w_{k, 0}=y_{k}
$$

The consensus algorithm consists in all agents performing the following recursion:

$$
w_{k, i+1}=\sum_{l \in \mathcal{N}_{k}} c_{l k} w_{l, i}
$$

The steps for the consensus algorithm are detailed in Algorithm 2.8.

We say that (2.48) is a fully distributed recursion for three main reasons:

- Each agent only shares information with its neighbors (i.e., only single-hop communication);

- Data is distributed among the agents;

- and no sample data is shared, rather only intermediate estimates are exchanged, preserving data privacy. 


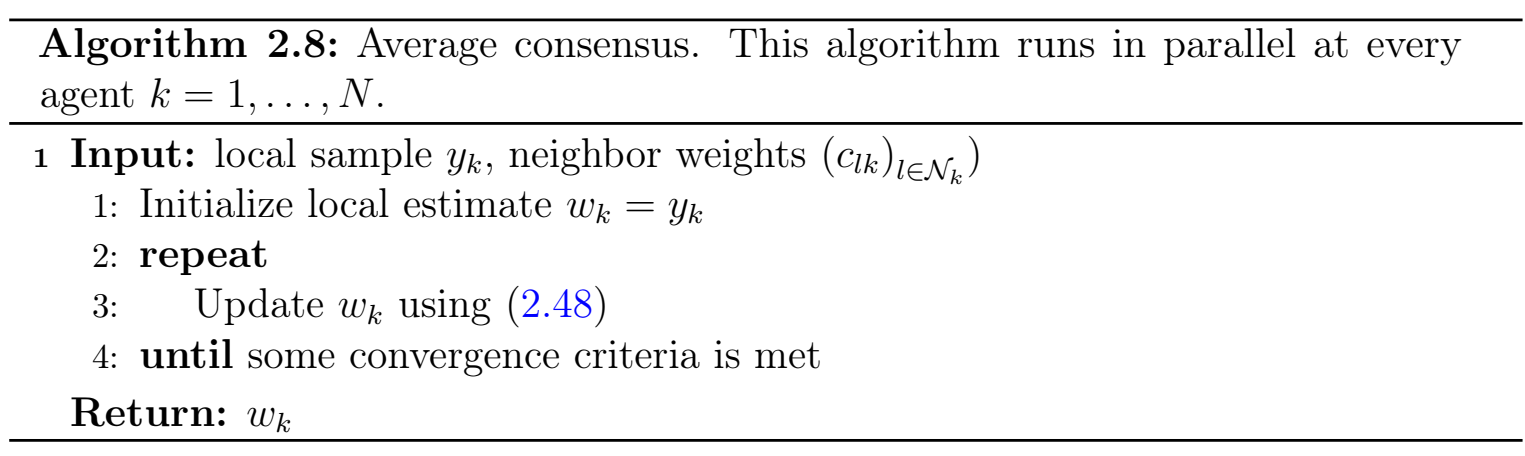

The following subsection shows that distributed recursion (2.48) actually converges to the network average.

\subsubsection{Convergence of consensus algorithm}

By introducing the vector of length $N$ with entries the individual estimates of all agents, given by

$$
w_{i} \triangleq\left(w_{k, i}\right)_{k=1}^{N}=\left[\begin{array}{c}
w_{1, i} \\
\vdots \\
w_{N, i}
\end{array}\right] \in \mathbb{R}^{N}
$$

we can express (2.48) as a network recursion for all agents in compact form: Suppose that the agents perform the following recursion:

$$
w_{i+1}=C^{\top} w_{i}
$$

In order to study the evolution of the network recursion (2.50), we assume that the combination matrix $C$ is designed to satisfy the following conditions

$$
\begin{gathered}
\sum_{l \in \mathcal{N}_{k}} c_{l k}=1, \quad \sum_{l \in \mathcal{N}_{k}} c_{k l}=1 \\
\exists k \in[1, \ldots, N]: c_{k k}>0
\end{gathered}
$$

Conditions in (2.51) imply that $C$ is doubly stochastic and can be equivalently expressed as:

$$
\mathbb{1}^{\top} C=\mathbb{1}^{\top}, \quad C \mathbb{1}=\mathbb{1}
$$


Condition (2.52) means that at least one agent gives some positive weight (credit) to its own information. More technically, it implies that the stochastic matrix $C$ is aperiodic (i.e., if we think in $C$ as the state transition probability matrix of some Markov chain, then $c_{k k}$ would be the probability of returning in state $k$ ). Recall that we have assumed that the network is connected (i.e., there is at least one path between every pair of agents), which is equivalent to say that $C$ is irreducible. In addition, being $C$ a square, nonnegative and irreducible matrix, we conclude that it is also primitive (see, e.g., [162, Prop. 1.7]). Hence, since $C$ is doubly stochastic, we conclude that 1 is the Perron-Frobenius eigenvalue of $C$, and $\mathbb{1}$ may be taken as the corresponding right Perron-Frobenius eigenvector (see, e.g., [258, pp.118]). Finally, the Perron-Frobenius theorem yields the following result:

$$
\lim _{i \rightarrow \infty} C^{i}=\lim _{i \rightarrow \infty}\left(C^{\top}\right)^{i}=\frac{1}{N} \mathbb{1} \mathbb{1}^{\top}
$$

and the rate of approach to the limit is geometric (see, e.g., [124, Theorem 8.5.1], [258, Theorem 4.2]).

Proposition 1. For a connected graph with a combination matrix $C$ that satisfies (2.51)-(2.52), we have

$$
\lim _{i \rightarrow \infty} w_{k, i+1}=w^{\star}
$$

Proof: Using the Perron-Frobenius theory described above, we can easily obtain the convergence point of network recursion (2.50):

$$
\lim _{i \rightarrow \infty} w_{i+1}=\lim _{i \rightarrow \infty} C^{\top} w_{i}=\lim _{i \rightarrow \infty}\left(C^{\top}\right)^{i} w_{0}=\mathbb{1} \frac{1}{N} \sum_{k=1}^{N} y_{k}=\mathbb{1} w^{\star}=\left[\begin{array}{c}
w^{\star} \\
\vdots \\
w^{\star}
\end{array}\right]
$$

One final remark on the combination matrix $C$ is that ensuring that conditions (2.51)-(2.52) hold in a real distributed computing scenario is not trivial. First, it would be desirable that the agents are able to find the weight values $c_{l k}$ by themselves, in a fully distributed manner (i.e., by local within-neighborhood communication and with agents having no knowledge of the network topology beyond its own neighborhood). Second, the agents have to surmount communications impairments (e.g., noisy links) and keep the updates synchronized. 
It turns out that there are several works showing how to obtain valid weights in a distributed manner (see, e.g., [246, Table 14.1]). One widely used example is the Metropolis rule [116, 189, 320], which is described as follows:

$$
c_{l k}= \begin{cases}\frac{1}{\max \left[\left|\mathcal{N}_{k}\right|,\left|\mathcal{N}_{l}\right|\right]}, & \text { if } k \neq l \text { are neighbors } \\ 1-\sum_{m \in \mathcal{N}_{k} \backslash\{k\}} c_{m k}, & \text { if } k=l \\ 0, & \text { otherwise }\end{cases}
$$

In addition, there are more sophisticated policies for building combination matrices that aim to optimize the convergence time or minimize the estimation error (see, e.g., [46, Sec. IV], [246, Secs. 14.2-14.5] or [90]).

Nevertheless, when communications or synchronization among agents are not perfect, even theoretically well designed combination matrices become nondoubly-stochastic, resulting into biased results. In particular, left-stochasticity (i.e., $C^{\top} \mathbb{1}=\mathbb{1}$ ) means that the weights given by each agent to all its neighbors add up to one. This condition is easy to enforce even when some packets are lost, because the agent could recalculate the weights at every iteration giving positive weight only to the packets that were successfully received - including its own - (of course, the convergence analysis would be more complicated, since the combination matrix would be time-varying in such case, but the point is that the agent has all the information it needs to allocate the weights). On the other hand, ensuring right stochasticity (i.e., $C \mathbb{1}=\mathbb{1}$ ) is more challenging because each row depends on the whole neighborhood. It means that the weights given by the neighbors of some agent to the information coming from that agent must add up to one. In a real scenario (e.g., under wireless communications), this condition may be difficult to satisfy. Consider that some agent broadcasts a packet, which is only received by a subset of its neighbors. Those neighbors that received the packet can give positive weight and compute (2.48). However, those that did not receive the packet must set the corresponding weight to zero and, thus, the right stochastic condition will not hold. Therefore, the algorithm will converge to a biased result.

Besides dealing with lossy links, another issue is how to keep synchronization among neighbors, so they update its estimate at the same rate. Consider a simple network of just two agents, $k$ and $l$, which are performing periodic consensus iterations. Assume the clock of $k$ has a drift so, eventually, it will be out of synch and, although it may receive data coming from agent $l$, it will not transmit any packet. In this case, agent $k$ has all the required information to perform the combination step. On the other hand, agent $l$ is still waiting for the data coming from agent $k$, so it can not update 
its estimate at that iteration. It means that, during the update of $k$, the combination matrix $C$ is only left stochastic, as opposed to doubly stochastic, and, again, the asymptotic result will be biased.

There are few implementations of distributed algorithms [234, 216, 144, 145]. However, they are sensitive to asynchronous and noisy communications. In Section 2.9, we propose a simple protocol for guaranteeing that the combination matrix is doublystochastic at every iteration, independently of the amount of packet loss and the lack of synchronization among agents. The protocol is also robust against permanent changes in the topology (e.g., because of agent failure or after adding new agents). In addition, in Chapters 4-3, we will propose novel distributed algorithms for which we can ensure convergence even with left-stochastic matrices. For now, let us simply assume that conditions (2.51)-(2.52) hold.

\subsubsection{Consensus in higher dimensions}

It is of course possible to extend the averaging procedure from the scalar case given above, to a vector or a matrix at each agent.

In the vector case, each agent starts with a vector of $M$ components, i.e., $y_{k} \in \mathbb{R}^{M}$. Let the average vector that the agents aim to estimate be given by

$$
w^{\star} \triangleq \frac{1}{N} \sum_{k=1}^{N} y_{k} \in \mathbb{R}^{M}
$$

The estimates of are now vectors of length $M$, i.e., $w_{k} \in \mathbb{R}^{M}, k=1, \ldots, N$. For the network estimate, we can follow (2.49) and aggregate all individual estimates in a long vector of length $N M$ :

$$
w_{i} \triangleq\left(w_{k, i}\right)_{k=1}^{N} \in \mathbb{R}^{N M}
$$

where we set $w_{k, 0}=y_{k}$. Let us replicate the combination in a long block-diagonal matrix of the form:

$$
\mathcal{C} \triangleq C \otimes I_{M}
$$

Then, we can express the network recursion in compact form:

$$
w_{i+1}=\mathcal{C} w_{i}
$$


Extending Proposition 1 to the vector case is trivial, so we can conclude:

$$
\lim _{i \rightarrow \infty} w_{i}=\lim _{i \rightarrow \infty} \mathcal{C}^{i} w_{0}=\left[\begin{array}{c}
w^{\star} \\
\vdots \\
w^{\star}
\end{array}\right] \in \mathbb{R}^{N M}
$$

Thus, similar to the scalar case $(2.55)$, we have: $\lim _{i \rightarrow \infty} w_{k, i}=w^{\star}$, where $w^{\star}$ is now the vector defined by (2.58).

For the matrix case, we consider that each agent starts with a matrix $Y_{k}$ of size $M \times Q$. The average matrix that the agents aim to estimate is then given by

$$
W^{\star} \triangleq \frac{1}{N} \sum_{k=1}^{N} Y_{k} \in \mathbb{R}^{M \times Q}
$$

Let the matrix $W_{k, i} \in \mathbb{R}^{M \times Q}$ denote the estimate of $W^{\star}$ at agent $k$ at time $i$. In this case, we find convenient to represent the set of local estimates as a tall network matrix of size $N M \times Q$ :

$$
W_{i} \triangleq\left(W_{k, i}\right)_{k=1}^{N}=\left[\begin{array}{c}
W_{1, i} \\
\vdots \\
W_{N, i}
\end{array}\right] \in \mathbb{R}^{N M \times Q}
$$

Using the extended block diagonal combination matrix $\mathcal{C}$ given by $(2.60)$, we obtain the following network recursion:

$$
W_{i+1}=\mathcal{C} W_{i}
$$

Again, we use Proposition 1 to conclude that:

$$
\lim _{i \rightarrow \infty} W_{i}=\lim _{i \rightarrow \infty} \mathcal{C}^{i} W_{0}=\left[\begin{array}{c}
W^{\star} \\
\vdots \\
W^{\star}
\end{array}\right] \in \mathbb{R}^{N M \times Q}
$$

which is equivalent to say that every agent converges to the average matrix:

$$
\lim _{i \rightarrow \infty} W_{k, i}=W^{\star}
$$

The average consensus algorithm for vector and matrices is similar to Algorithm 2.8 . 


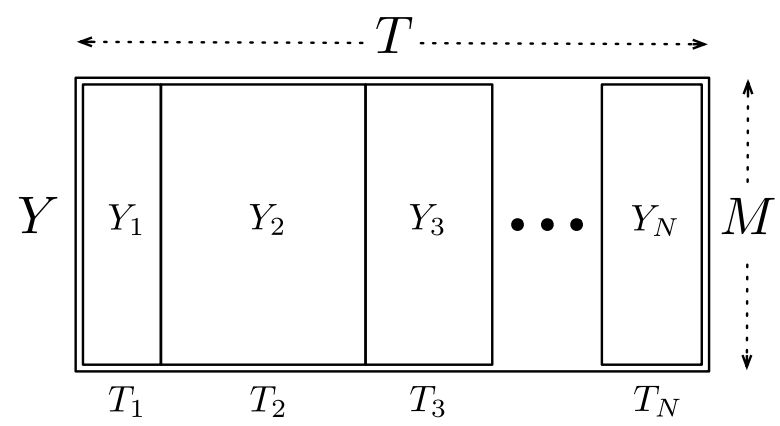

Figure 2.3 Global matrix $Y$ with a total of $T$ samples, distributed unevenly over $N$ agents.

\subsection{Distributed estimation}

In this section, we present a set of simple methods for distributed estimation of the first and second order moments of a distributed multivariate dataset.

Let $Y \triangleq\left(y_{1}, \ldots, y_{T}\right)$ be a global dataset of $T$ independent and identically distributed (i.i.d.) sample vectors of length $M$. The dataset is not available at any one location, but rather is partially available at each agent. In particular, each agent $k$ has the knowledge of a block of $T_{k}$ samples of $X$. The agents do not overlap samples, so that the total number of samples is given by

$$
T=\sum_{k=1}^{N} T_{k}
$$

Let $y_{k, t} \in \mathbb{R}^{M}$ denote the $t^{t h}$ data sample at agent $k$. The set of data samples available at agent $k$ is then

$$
Y_{k} \triangleq\left(y_{k, 1}, \ldots, y_{k, T_{k}}\right) \in \mathbb{R}^{M \times T_{k}}
$$

Hence, the global data set can be expressed in matrix form as

$$
Y \triangleq\left(Y_{1}, \ldots, Y_{N}\right)
$$

This setting is depicted in Figure 2.3.

The agents aim to estimate the sample mean and sample covariance of the global dataset, given by

$$
\mu \triangleq \frac{1}{T} \sum_{t=1}^{T} y_{t}
$$




$$
\Sigma \triangleq \frac{1}{T-1} \sum_{t=1}^{T}\left(y_{t}-\mu\right)\left(y_{t}-\mu\right)^{\top}
$$

Here, we propose a process to approximate these moments with three consensus algorithms.

In the first step, every agent calculates the average number of samples, that we denote as $\bar{T}=T / N$ by running the consensus algorithm for the mean number of samples per agent. Let $\bar{T}_{k, 0}=T_{k}$. Then, every agent performs the following iterates

$$
\bar{T}_{k, i+1}=\sum_{l \in \mathcal{N}_{k}} c_{l k} \bar{T}_{l, i}
$$

By using the result (2.55) from Proposition 1, we have that all agents asymptotically converge to

$$
\bar{T}^{\star} \triangleq \frac{1}{N} \sum_{n=1}^{N} \bar{T}_{k, 0}=\frac{1}{N} \sum_{n=1}^{N} T_{k}=\frac{1}{N} T=\bar{T}
$$

From here, every agent can derive in isolation (i.e., without any communication) the globally weighted local mean, which is defined as

$$
\bar{\mu}_{k} \triangleq \frac{1}{\bar{T}^{\star}} \sum_{t=1}^{T_{k}} y_{k, t}
$$

The mean of the global set $Y$ can be estimated by performing average consensus algorithm on these weighted local means, which-again from (2.55) - converges to

$$
\mu^{\star} \triangleq \frac{1}{N} \sum_{k=1}^{N} \bar{\mu}_{k}=\frac{1}{N} \sum_{k=1}^{N} \frac{1}{\bar{T}^{\star}} \sum_{t=1}^{T_{k}} y_{k, t}=\frac{1}{T} \sum_{k=1}^{N} \sum_{t=1}^{T_{k}} y_{k, t}=\mu
$$

where we used the simple arithmetical fact that $1 /(N \bar{T})=1 / T$. Thus, every agent $k$ can use (2.76) to center its local data set, denoted $\bar{Y}_{k}$ :

$$
\bar{Y}_{k} \triangleq Y_{k}-\mu \mathbb{1}^{\top}
$$

The final step consists in performing average consensus on the local products $\bar{Y}_{k} \bar{Y}_{k}^{\top}$, so that all agents converge to an approximation of the global sample covariance matrix:

$$
\Sigma^{\star} \triangleq \frac{1}{N} \sum_{k=1}^{N} \bar{Y}_{k} \bar{Y}_{k}^{\top}
$$




$$
\begin{aligned}
& =\frac{1}{N} \sum_{k=1}^{N} \frac{1}{\bar{T}^{\star}} \sum_{t=1}^{T_{k}}\left(y_{k, t}-\mu\right)\left(y_{k, t}-\mu\right)^{\top} \\
& =\frac{1}{T} \sum_{k=1}^{N} \sum_{t=1}^{T_{k}}\left(y_{k, t}-\mu\right)\left(y_{k, t}-\mu\right)^{\top}
\end{aligned}
$$

We say that (2.78) is an approximation of the sample covariance, since (2.80) is slightly biased due to the fact that we use the sample mean for centering the data, instead of the actual mean of the data. This makes the denominator of the consensus result be $T$ instead of considering the Bessel's correction $T-1$ :

$$
\Sigma^{\star}=\frac{T-1}{T} \Sigma
$$

Clearly, the approximation converges to the actual sample covariance when $T$ is large:

$$
\lim _{T \rightarrow \infty} \Sigma^{\star}=\lim _{T \rightarrow \infty} \frac{T-1}{T} \Sigma=\Sigma
$$

Hence, by performing average consensus iterations over the average number of samples (2.74), over the locally weighted means (2.76) and over the local covariances (2.78), every agent can estimate the first and second order moments in a fully distributed manner, even when they have access to different number of samples.

In the following sections, we use these results systematically to propose fully distributed implementations of the methods discussed in Section 2.2.

\subsection{Distributed PCA and SLGM algorithms}

In this section we consider a network of agents with a distributed dataset, as described by equations (2.68), (2.69) and (2.70) in Section 2.4.

In a centralized implementation, a fusion center (central station) requires gathering all the data samples, from all the agents. With this access to the full data set, the fusion center can perform the single-agent algorithms already described. The challenge, then, is to be able for every agent to approximate the same principal subspace that a fusion would achieve, but without such fusion center, only by local single-hop within neighborhood communications.

In this section, we use $\mu$ and $\Sigma$ to denote the sample mean and sample covariance of the full dataset, given by (2.71)-(2.72), respectively.

All algorithms presented in this section are proposed to run locally by each agent. 


\subsubsection{Distributed PCA from consensus in covariance matrix}

To distribute the single-agent PCA method, described by Algorithm 2.1, every agent should first obtain the global mean and use it to center its local dataset. Then, each agent can start with its globally weighted local covariance matrix and proceed iteratively applying consensus until asymptotically converging to the approximation of the global sample covariance matrix of the full dataset.

Once the consensus estimate of the global covariance matrix is available, each agent can locally obtain its own approximation to the global PCA as the subspace spanned by the dominant eigenvectors of its estimate of the global covariance. This subspace will be arbitrarily close, depending on the number of consensus iterations, to that spanned by the dominant eigenvectors of the actual global covariance. This is the base of Algorithm 2.9, denoted Consensus Based Distributed PCA (CB-DPCA) this algorithm was published in $[293,20]$. Note that this procedure includes three consensus loops, which may require several iterations each. In practice, we will show with numerical simulations that relatively few consensus iterations are enough to obtain sound results. Finally, we remark that the scaling factor $\frac{T-1}{T}$ has no influence on the eigenvectors of $\Sigma$. Thus, the principal subspace obtained from $\Sigma^{\star}$ is the same as the one from $\Sigma$.

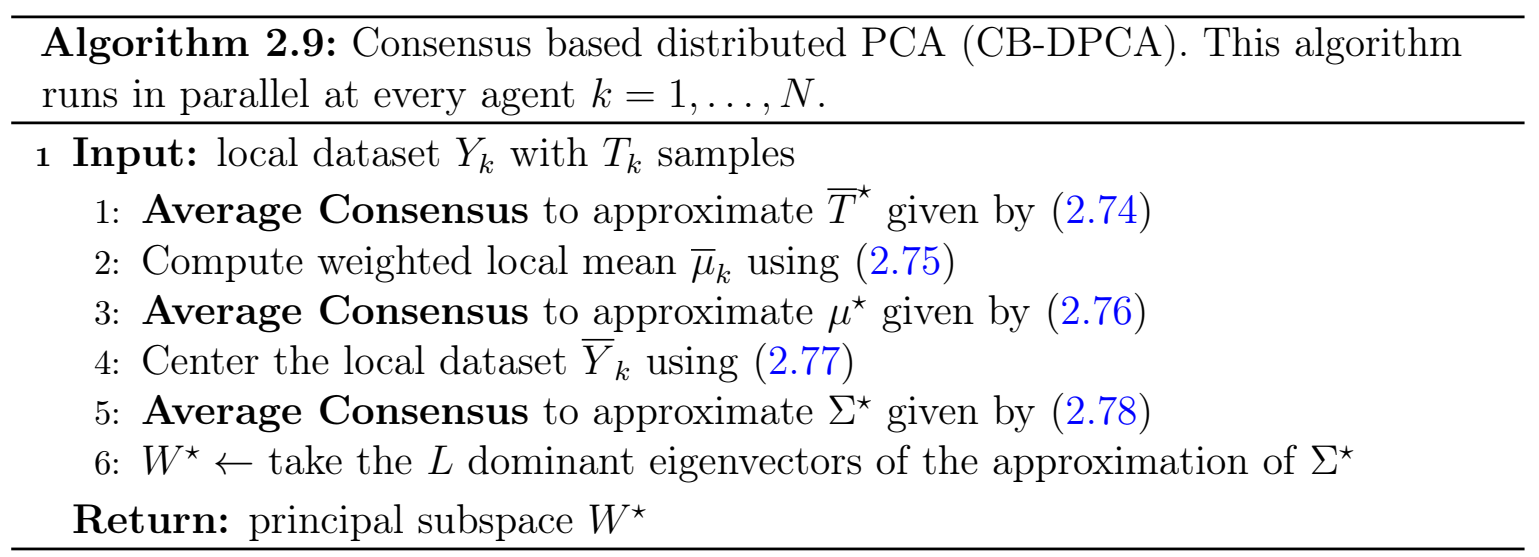

Although $W^{\star}$ is computed locally, it only depends on the shared variable $\Sigma^{\star}$, so that we use superscript star to denote that $W^{\star}$ will be also approximately the same for every agent.

\subsubsection{Distributed PCA as an EM algorithm}

In many real applications (like ambient intelligence, with cheap sensors ubiquitously deployed) agents will likely offer low storing and processing resources; at least compared 
with the amount of continuously collected data that they may have to manage. Hence, an iterative algorithm to compute $W$ will be often desirable.

It is important to note that, after centering the dataset, just as all the samples in the local dataset, $Y_{k}$, are independent of each other, but together form the global data set $Y$, so are the local projections, $X_{k}$, in turn forming the global projected data set $X$. In other words, $X$ has the same block-wise structure as $Y$. Thus, given the global projection matrix, $W^{\star}$, each agent can in isolation derive its part of the global projected data set:

$$
\begin{aligned}
Y_{k} & =W^{\star} X_{k} \\
X_{k} & =\left(W^{\star \top} W^{\star}\right)^{-1} W^{\star \top} Y_{k}
\end{aligned}
$$

Hence, in order to distribute the EM algorithm for PCA, given by equations (2.3)-(2.4), we first note that, assuming the same estimate $W^{\star}$ is available at all the agents at the start of the iteration, each agent can carry out the E-step in isolation and derive its own portion of the projections $X_{k}$ using (2.82). For the $M$-step, we can define the local variables

$$
\begin{gathered}
\Delta_{k} \triangleq Y_{k} X_{k}^{\top} \\
\Gamma_{k} \triangleq X_{k} X_{k}^{\top}
\end{gathered}
$$

and use them as inputs into an embedded average consensus algorithm, whose results asymptotically approach:

$$
\begin{aligned}
& \Delta^{\star} \triangleq \frac{1}{N} \sum_{k=1}^{N} Y_{k} X_{k}^{\top}=Y Y^{\top} \\
& \Gamma^{\star} \triangleq \frac{1}{N} \sum_{k=1}^{N} X_{k} X_{k}^{\top}=X X^{\top}
\end{aligned}
$$

The agents then use these shared estimates to locally update the subspace:

$$
\begin{aligned}
W^{\star} & =\Delta^{\star}\left(\Gamma^{\star}\right)^{-1} \\
& =\left(\sum_{k=1}^{N} Y_{k} X_{k}^{\top}\right)\left(\sum_{k=1}^{N} X_{k} X_{k}^{\top}\right)^{-1} \\
& =Y X^{\top}\left(X X^{\top}\right)^{-1}
\end{aligned}
$$


which equals that calculated by (2.4). Equations (2.84)-(2.87) are the base of the proposed Consensus Based EM Distributed PCA (CB-EM-DPCA), as it is shown in Algorithm 2.10, which asymptotically matches the results of both the E and M steps of the centralized EM algorithm for PCA at every iteration - this algorithm was published in $[20]$.

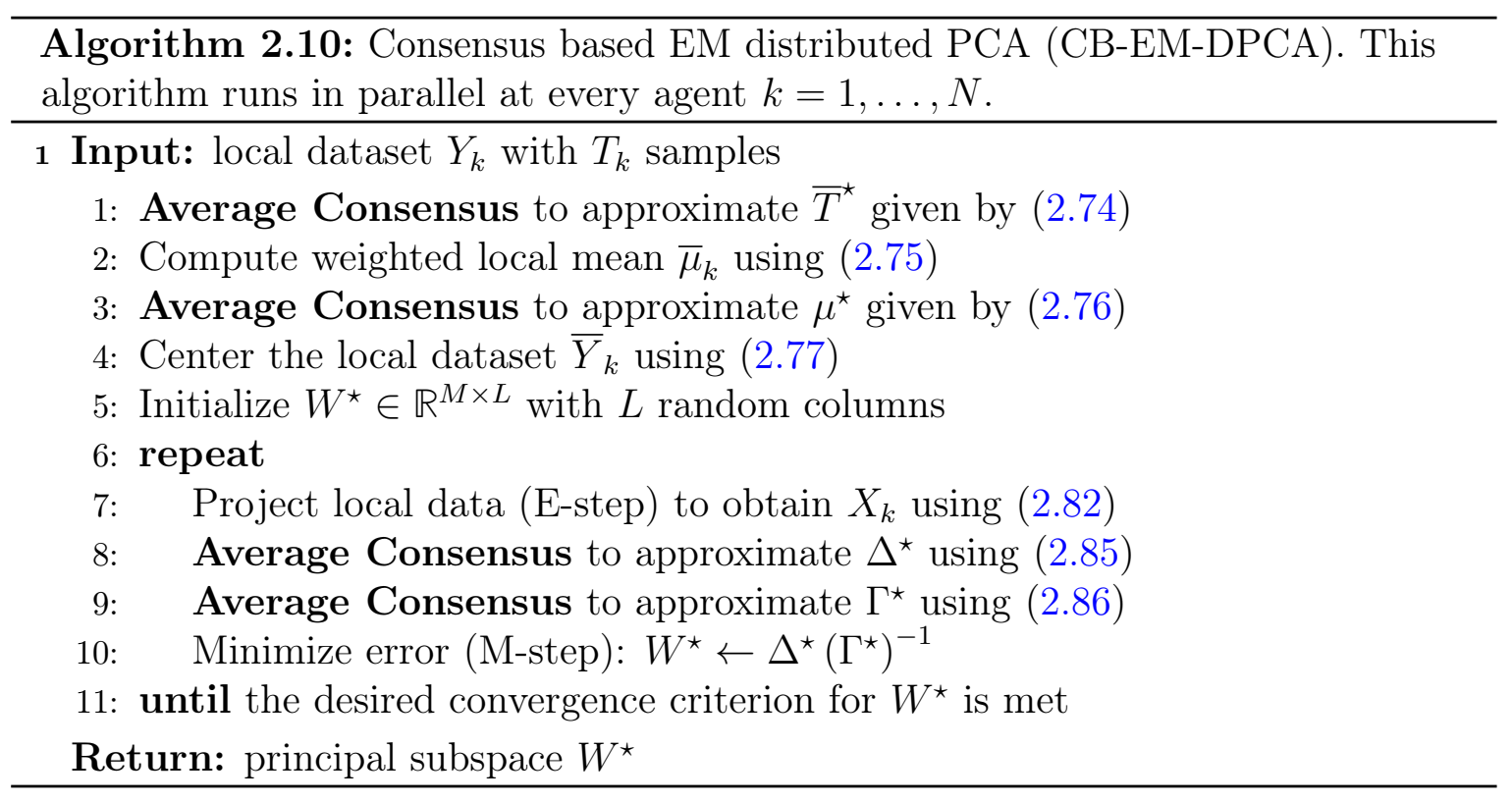

Although CB-EM-DPCA can be computationally more tractable than CB-DPCA, especially when $M \gg L$ (i.e, the input dimensionality is much larger than the output dimensionality), since the inversion matrix is over the covariance of the (lower dimensional) latent data. We remark that the main handicap of this algorithm is that there are two average consensus algorithms embedded in the main loop (Lines 6-11). This means that the iterative within neighborhood communication involved by consensus for exchanging estimates has to be repeated multiple times.

\subsubsection{Distributed Factor Analysis}

In order to distribute the EM algorithm for FA, we follow the same approach as in CB-EM-DPCA (Algorithm 2.10). Previous to starting the EM loop, the agents run consensus algorithms to obtain the average number of samples, $\bar{T}^{\star}$, and the consensus estimate of the global covariance matrix, $\Sigma^{\star}$. 
Assuming the same $W^{\star}$ and $R^{\star}$ are available at all the agents at the start of every new iteration, then the E-step given by (2.5)-(2.6) can be performed locally:

$$
\begin{aligned}
& X_{k}=W^{\star \top}\left(W^{\star} W^{\star \top}+R^{\star}\right)^{-1} Y_{k} \\
& Q^{\star}=I_{L}-W^{\star \top}\left(W^{\star} W^{\star \top}+R^{\star}\right)^{-1} W^{\star}
\end{aligned}
$$

where, since (2.89) only depends on consensus variables $\left(W^{\star}\right.$ and $\left.R^{\star}\right)$, we use superscript star to indicate that $Q^{\star}$ will be also shared by all agents.

However, the $M$-step involves communications among neighbors. Again, we split $W$ into terms: $\Delta_{k}$, which is similar to $(2.83)$, and

$$
\Gamma_{k} \triangleq X_{k} X_{k}^{\top}+Q^{\star}
$$

Then, the agents run a couple of average consensus algorithms, one on $\Delta_{k}$ and the other on the covariance term of (2.90), to asymptotically obtain:

$$
\begin{gathered}
\Delta^{\star} \triangleq \frac{1}{N \bar{T}^{\star}} \sum_{k=1}^{N} Y_{k} X_{k}^{\top} \\
\eta^{\star} \triangleq \frac{1}{N \bar{T}^{\star}} \sum_{k=1}^{N} X_{k} X_{k}^{\top}
\end{gathered}
$$

With these variables, they can locally compute:

$$
\begin{aligned}
W^{\star} & =\Delta^{\star}\left(\eta^{\star}+Q^{\star}\right)^{-1} \\
& =\frac{1}{T}\left(\sum_{k=1}^{N} Y_{k} X_{k}^{\top}\right)\left(\frac{1}{T} \sum_{k=1}^{N} X_{k} X_{k}^{\top}+Q^{\star}\right)^{-1} \\
& =\left(\sum_{k=1}^{N} Y_{k} X_{k}^{\top}\right)\left(\sum_{k=1}^{N} X_{k} X_{k}^{\top}+T \cdot Q^{\star}\right)^{-1} \\
& =Y X\left(X X^{\top}+T \cdot Q^{\star}\right)^{-1} \\
R^{\star} & =\text { diagelements }\left[\Sigma^{\star}-W^{\star} \Delta^{\star \top}\right] \\
& =\text { diagelements }\left[\frac{1}{T}\left(Y Y^{\top}-W^{\star} X Y^{\top}\right)\right]
\end{aligned}
$$

where, although $W^{\star}$ and $R^{\star}$ are locally computed, we used superscript star notation to remark that these variables are approximately the same for all agents. Note that, apart of the asymptotic approximation, the only difference between FA, given by (2.5)-(2.8), and DFA, given by (2.88)-(2.94), is that (2.94) is scaled by $1 / T$ instead of $1 /(T-1)$. 
In practice, this scale factor is usually negligible, especially for large $T$. This algorithm was published in [20].

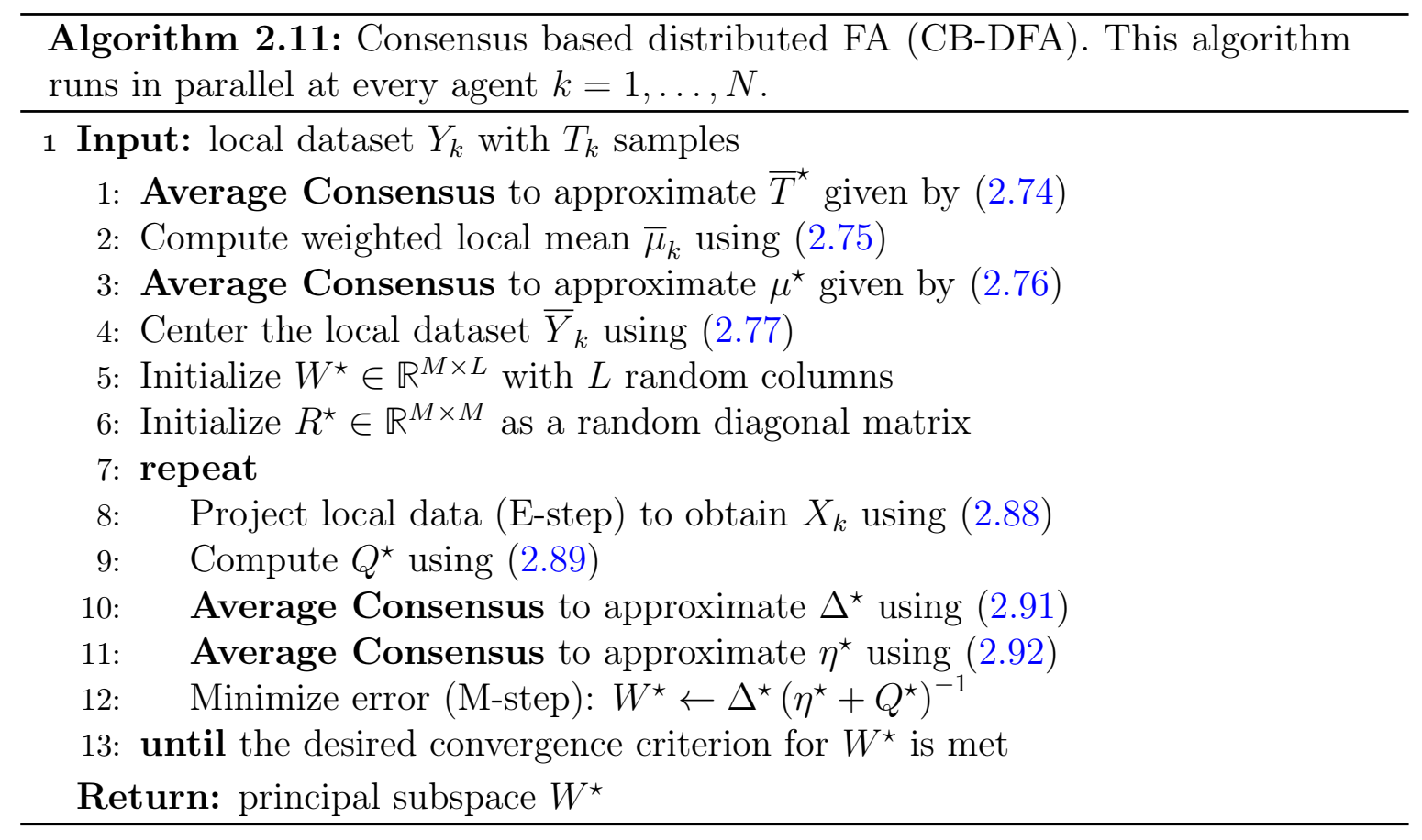

\subsubsection{Distributed Probabilistic PCA}

As we have seen in (2.9), the only difference between PPCA and FA is how to update the observed noise. Hence, we can follow the very same approach as CB-DFA to introduce a distributed EM algorithm to compute PPCA.

For the E-step, the only difference consists in replacing $R^{\star}$ with $\alpha^{\star} I_{M}$ :

$$
\begin{aligned}
& X_{k}=W^{\star \top}\left(W^{\star} W^{\star \top}+\alpha^{\star} I_{M}\right)^{-1} Y_{k} \\
& Q^{\star}=I_{L}-W^{\star \top}\left(W^{\star} W^{\star \top}+\alpha^{\star} I_{M}\right)^{-1} W^{\star}
\end{aligned}
$$

where we assume that all agents have access to $\alpha^{\star}$ at the start of every iteration. As explained for the previous algorithms, although locally computed, we use superscript star to indicate that $\alpha^{\star}$ is shared by all agents.

Similar to FA, for the $M$-step, the agents compute $\Delta^{\star}$ and $\eta^{\star}$ using (2.91)-(2.92) and compute

$$
W^{\star}=\Delta^{\star}\left(\eta^{\star}+Q^{\star}\right)^{-1}
$$


The difference lies in the update of the covariance noise:

$$
\begin{aligned}
\alpha^{\star} & =\frac{1}{M} \operatorname{Tr}\left[\Sigma^{\star}-W \Delta^{\star \top}\right] \\
& =\frac{1}{T M} \operatorname{Tr}\left[Y Y^{\top}-W X Y^{\top}\right]
\end{aligned}
$$

which is the consensus approximation of (2.9) with the difference in the scaling factor $1 /(T M)$ instead of $1 /((T-1) M)$.

The steps of Consensus Based Distributed Probabilistic PCA (CB-DPPCA) are detailed in Algorithm 2.12. This algorithm was published in [20].

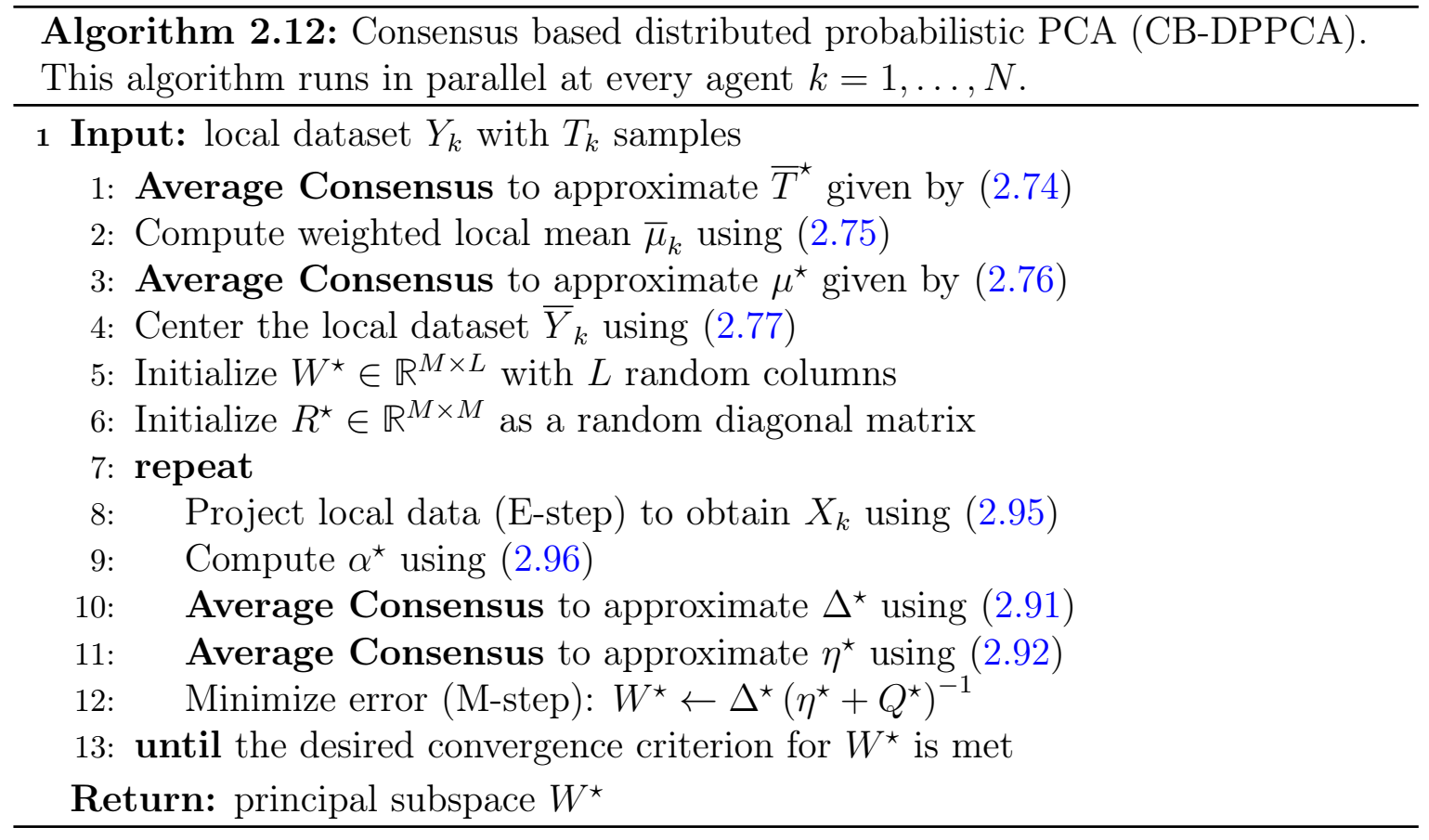

\subsection{Distributed LDA algorithms}

In this section, we consider the same model as in Section 2.2.4. But here we assume that the dataset has been split into $N$ local datasets, each one available at a single agent. Let

$$
\begin{aligned}
& Y \triangleq\left(Y_{k}\right)_{k=1}^{N} \in \mathbb{R}^{M \times T} \\
& G \triangleq\left(G_{k}\right)_{k=1}^{N}
\end{aligned}
$$


denote the global set of data points and the corresponding global indicator matrix of size $T \times D$ indicating the labels for the points in $Y$. We recall that $D$ is the number of classes, where each class is denoted by $B_{d}, d=1, \ldots, D$. The local matrix of data points available at agent $k$ and its corresponding indicator matrix are given by:

$$
\begin{aligned}
& Y_{k} \triangleq\left(y_{k, t}\right)_{t=1}^{T_{k}} \in \mathbb{R}^{M \times T_{k}} \\
& G_{k} \triangleq\left(g_{k, t}^{\top}\right)_{t=1}^{T_{k}}
\end{aligned}
$$

where the size of $G_{k}$ is $T_{k} \times D$, and $g_{k, t}$ is a binary vector of length $D$ that indicates the class of point $y_{k, t}$. In other words, the nonzero position in the $t$-th row of $G_{k}$ gives the class of the data point in the $t$-th column of $Y_{k}$. Let $g_{k, t, d}$ denote the $d$-th position of vector $g_{k, t}$, such that $g_{k, t}=\left(g_{k, t, d}\right)_{d=1}^{D}$. It is clear that sample $y_{k, t}$ belongs to class $B_{d}$ if and only if $g_{k, t, d}=1$.

\subsubsection{Distributed LDA from consensus in scatter matrices}

For distributing Algorithm 2.5, the agents have to estimate the total covariance and the between class scatter matrices. Once these matrices are available, they can locally compute the subspace.

We have seen that the average number of samples, the global mean and the global covariance matrix - denoted $\bar{T}^{\star}, \mu^{\star}$ and $\Sigma^{\star}$, respectively - can be approximated by running consensus on the local data sets, as given by (2.74), (2.76) and (2.78).

In order to compute the mean of each class, denoted $\mu_{d k}$, each agent has to build a new set of data matrices $Y_{k d}$, one for each class, containing all samples that belong to class $d$ :

$$
Y_{k d} \triangleq\left(y_{k, t}\right)_{t \in B_{d}}
$$

where, we have adapted (2.11) to the local dataset notation, such that $t \in B_{d}$ indicates that $y_{k, t}$ belongs to class $d$. The agents can estimate the global between-class scatter matrix as follows. Let $T_{k d}=\left|\left\{t \in B_{d}\right\}\right|$ denote the number of samples belonging to class $d$ in the $k$-th agent's local dataset, such that $Y_{k d} \in \mathbb{R}^{M \times T_{k, d}}$. Every agent can run average consensus to obtain the average number of samples per class:

$$
\bar{T}_{d}^{\star} \triangleq \frac{1}{N} \sum_{k=1}^{N} T_{k d}
$$


Then, they can compute the mean of their local datasets:

$$
\mu_{k d} \triangleq \frac{1}{\bar{T}_{d}^{\star}} \sum_{t \in B_{d}} y_{k, t}
$$

By running consensus on (2.105), they achieve the global mean for each class:

$$
\mu_{d}^{\star} \triangleq \frac{1}{N} \sum_{k=1}^{N} \frac{1}{\bar{T}_{d}^{\star}} \sum_{t \in B_{d}} y_{k, t}
$$

It is apparent that (2.106) asymptotically matches the same magnitude for the global dataset given by (2.13). Once all agents have $\mu^{\star}, \bar{T}_{d}^{\star}$ and $\mu_{d}^{\star}$ available, they can locally estimate the between-class scatter matrix:

$$
\begin{aligned}
\Sigma_{b}^{\star} & \triangleq \frac{1}{\bar{T}^{\star}} \sum_{d=1}^{D} \bar{T}_{d}^{\star}\left(\mu_{d}^{\star}-\mu^{\star}\right)\left(\mu_{d}^{\star}-\mu^{\star}\right)^{\top} \\
& =\frac{1}{\bar{T}^{\star}} \sum_{d=1}^{D} \frac{1}{N} \sum_{k=1}^{N} T_{k d}\left(\mu_{d}^{\star}-\mu^{\star}\right)\left(\mu_{d}^{\star}-\mu^{\star}\right)^{\top} \\
& =\frac{1}{T} \sum_{d=1}^{D} T_{d}\left(\mu_{d}-\mu\right)\left(\mu_{d}-\mu\right)^{\top}
\end{aligned}
$$

where we used the fact that the total number of data points belonging to class $B_{d}$ in the global dataset, denoted $T_{d}$, satisfies:

$$
T_{d} \triangleq \sum_{k=1}^{N} T_{k d}
$$

Note that the consensus estimate (2.107) approximates the actual between-class covariance matrx, $\Sigma_{b}$, given by (2.16), up to the already discussed $T /(T-1)$ scale factor.

These steps form the Consensus Based Distributed LDA algorithm, summarized in Algorithm 2.13, which was published in [294].

\subsubsection{Distributed iterative LDA}

In order to optimize (2.24) in a distributed fashion using average consensus algorithms, we follow the same ALS approach presented in (2.29)-(2.30). 


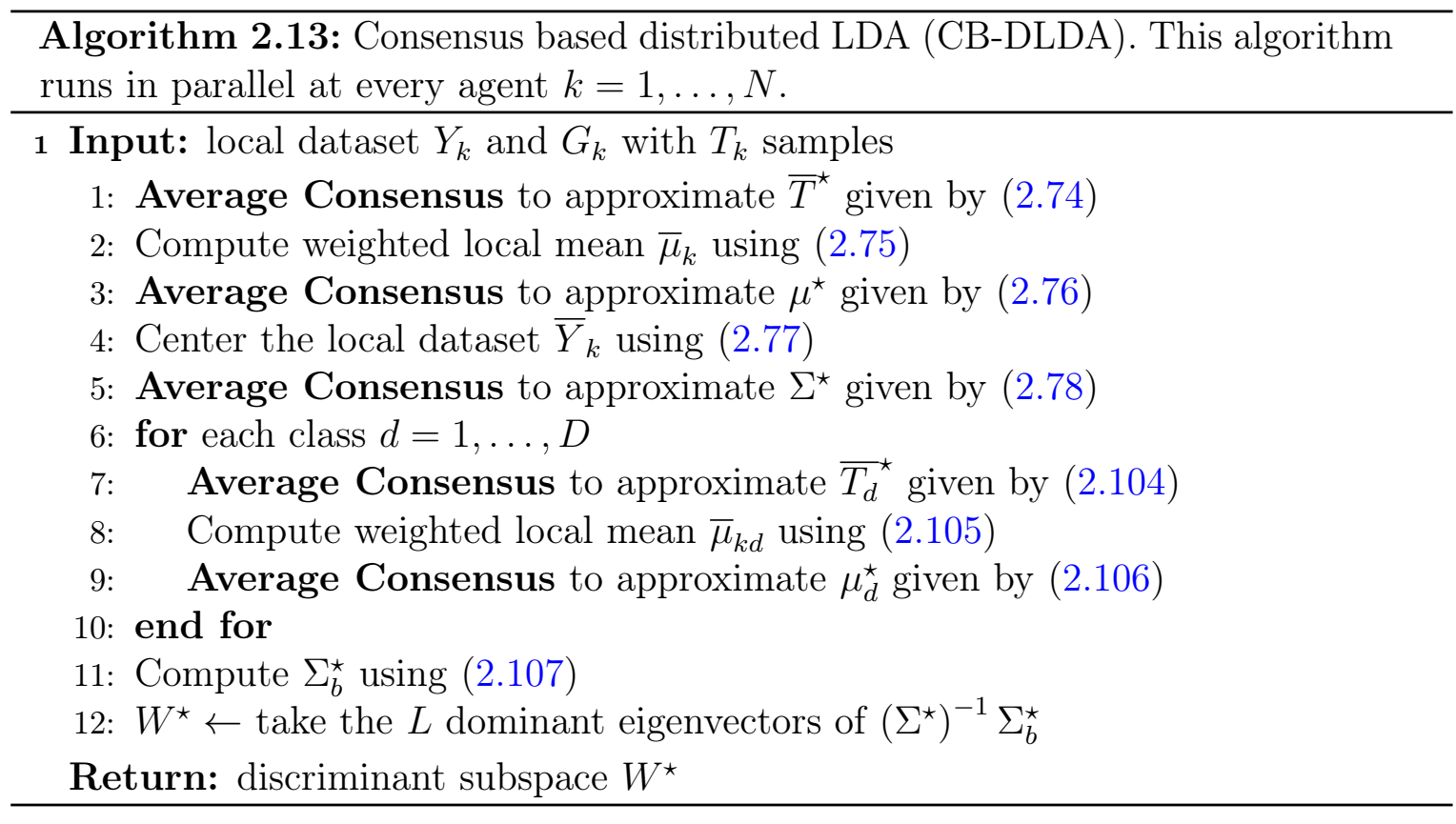

At every iteration, we run average consensus to obtain the following three factors:

$$
\begin{aligned}
& \Delta^{\star} \triangleq \frac{1}{N} \sum_{k=1}^{N} \bar{Y}_{k} G_{k} \\
& \Gamma^{\star} \triangleq \frac{1}{N} \sum_{k=1}^{N} G_{k}^{\top} G_{k} \\
& \Xi^{\star} \triangleq \frac{1}{N} \sum_{k=1}^{N} \bar{Y}_{k} \bar{Y}_{k}^{\top}
\end{aligned}
$$

Once the agents have share these variables, they can locally compute the updates:

$$
\begin{aligned}
A^{\star} & =\Delta^{\star^{\top}} W^{\star}\left(W^{\star \top} \Xi^{\star} W^{\star}\right)^{-1} \\
W^{\star} & =\left(\Xi^{\star}\right)^{-1} \Delta^{\star}\left(\Gamma^{\star}\right)^{-1} A^{\star}\left(A^{\star \top}\left(\Gamma^{\star}\right)^{-1} A^{\star}\right)^{-1}
\end{aligned}
$$

We remark that, although (2.112)-(2.113) are computed locally, we include superscript star to emphasize that $A^{\star}$ and $W^{\star}$ are shared by all agents. Moreover, it is straightforward to see that the factors $1 / N$ cancel out in $(2.112)-(2.113)$, so that $A^{\star}$ and $W^{\star}$ are asymptotically (with the number of iterations of the embedded average consensus subroutines) equal to (2.29)-(2.30).

These steps form the Consensus Based Distributed LDA algorithm, summarized in Algorithm 2.13, which was published in [294]. 


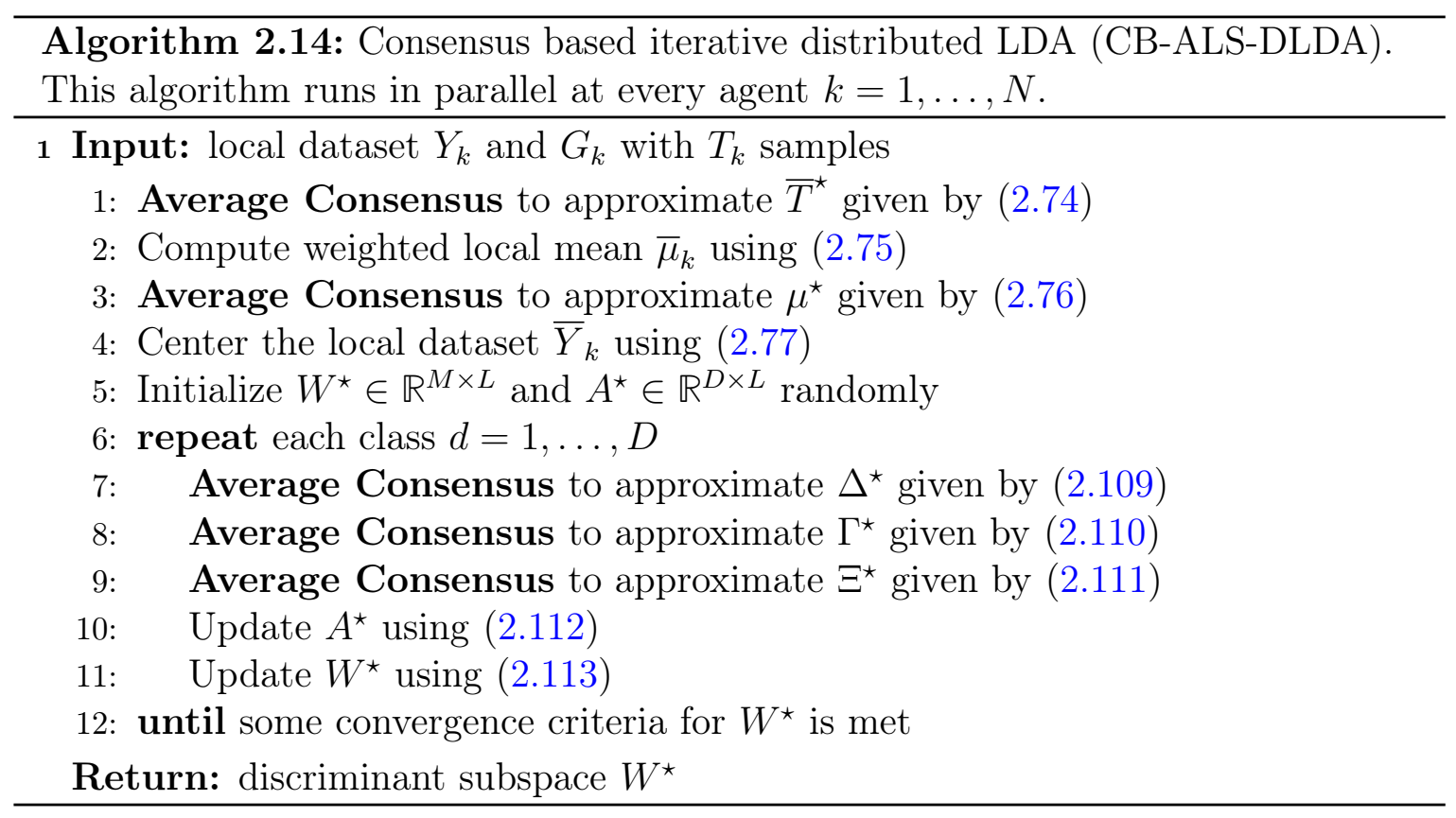

\subsubsection{Distributed regularized LDA for undersampled data}

Implementing regularization as adding variance to the global sample covariance matrix in a distributed fashion can be easily embedded in CB-LDA.

In order to avoid having to choose the regularization parameter (i.e., the amount of variance added by each agent), ULDA can be implemented in a distributed manner too. However, we note that the matrices $H$ and $H_{b}$, given by (2.35)-(2.36), are not easily estimated with average consensus for the agents, since they require the whole data set. Fortunately, we can reformulate ULDA using the total covariance and between-classes scatter matrices, $\Sigma$ and $\Sigma_{b}$, which can be easily estimated, as we have seen.

Assume that $\Sigma^{\star}$, the consensus estimate of $\Sigma$, is available at all agents. Then, they can diagonalize it:

$$
\Sigma^{\star}=U^{\star} \Upsilon^{\star} U^{\star \top}
$$

where we use superscript star to emphasize that, since $\Sigma^{\star}$ has been estimated by all agents, $U^{\star}$ and $\Upsilon^{\star}$ are also shared by all agents (up to consensus estimation error due to finite number of iterations), even when they have been computed locally. From (2.114), each agent can build:

$$
H^{\star}=U^{\star} \Upsilon^{\star \frac{1}{2}}
$$


such that a relationship similar to $(2.37)$ holds. Note that $U^{\star}$ is orthogonal and $\Upsilon^{\star \frac{1}{2}}$ is diagonal. Moreover, the equivalence with the diagonal term of (2.39) is clear:

$$
\Upsilon^{\star \frac{1}{2}}=\Delta_{H}
$$

Thus, as opposed to (2.39), it is not needed to compute the reduced SVD of $H^{\star}$ here, since its has same left eigenvectors as $\Sigma^{\star}$ :

$$
U^{\star \top} H^{\star}=U^{\star \top} U^{\star} \Upsilon^{\star \frac{1}{2}}=\Upsilon^{\star \frac{1}{2}}
$$

We repeat the process to obtain $H_{b}$ from $\Sigma_{b}^{\star}$. First, the agents decompose $\Sigma_{b}^{\star}$ :

$$
\Sigma_{b}^{\star}=U_{b}^{\star} \Upsilon_{b}^{\star} U_{b}^{\star \top}
$$

and build a matrix that satisfies (2.38):

$$
H_{b}^{\star}=U_{b}^{\star} \Upsilon_{b}^{\star \frac{1}{2}}
$$

From (2.116) and (2.117), we can build a matrix similar to (2.40), but now from variables available at all agents:

$$
B^{\star}=\Upsilon^{\star-\frac{1}{2}} U^{\star \top} U_{b}^{\star} \Upsilon_{b}^{\star \frac{1}{2}}
$$

Then, similar to (2.41), the agents decompose $B^{\star}$ :

$$
B^{\star}=P^{\star} \tilde{\Delta}_{b}^{\star} Q^{\star \top}
$$

and use $P$ to find the simultaneous diagonalization matrix:

$$
Z^{\star}=U^{\star} \Upsilon^{\star-\frac{1}{2}} P^{\star}
$$

So that:

$$
\begin{aligned}
Z^{\star \top} \Sigma_{b}^{\star} Z^{\star} & =Z^{\star \top} H_{b}^{\star} H_{b}^{\star \top} Z^{\star} \\
& =P^{\star \top} \Upsilon^{\star-\frac{1}{2}} U^{\star \top} U_{b}^{\star} \Upsilon_{b}^{\star \frac{1}{2}} \Upsilon_{b}^{\star \frac{1}{2}} U_{b}^{\star \top} U^{\star} \Upsilon^{\star-\frac{1}{2}} P^{\star} \\
& =P^{\star \top} B^{\star} B^{\star \top} P^{\star} \\
& =P^{\star \top} P^{\star} \tilde{\Delta}_{b}^{\star} Q^{\star \top} Q^{\star} \tilde{\Delta}_{b}^{\star} P^{\star \top} P^{\star} \\
& =\tilde{\Delta}_{b}^{\star 2}
\end{aligned}
$$


Finally, the discriminant subspace, $W^{\star}$, is obtained by taking the first $L \leq q$ columns of $Z^{\star}$. These steps are summarized in Algorithm 2.15, which was published in [294].

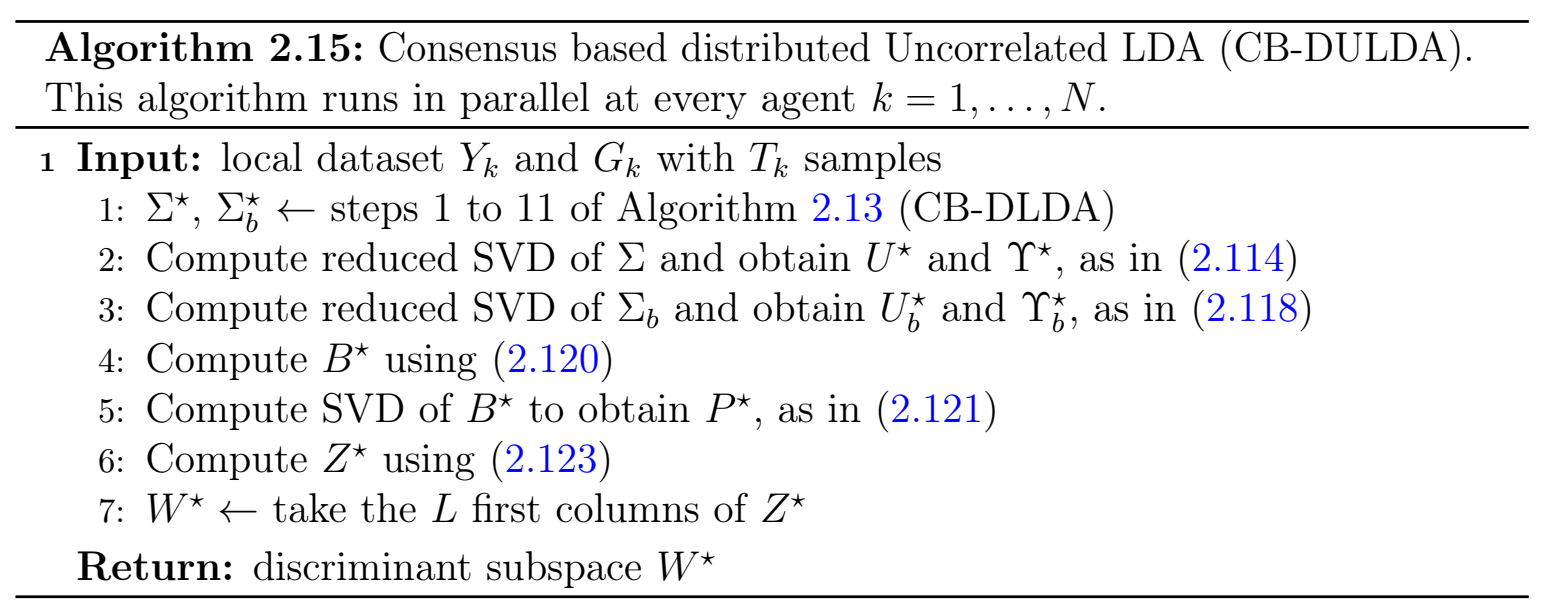

\section{$2.7 \quad$ Experiments}

In this section we illustrate the distributed algorithms described above in two blocks of experiments.

In the first block of experiments, we test distributed PCA on two simulated scenarios: a simple toy problem in which we examine the inherent properties of the proposed algorithms; and an image compression problem, in order to demonstrate their applicability to traditional more realistic applications.

In the second block of experiments, we test distributed LDA also on two scenarios: a toy example where we see the convergence of the locally calculated subspaces to the global (centralized) result; and a more realistic application where distributed LDA is used as an intermediate step to project local data onto a discriminant subspace, before training a K nearest neighbor (KNN) classifier. Then, the effectiveness of the consensus on the subspaces in the LDA step is shown in terms of the improvement in the performance of the classifier.

\subsubsection{Experiments with distributed PCA}

In the scenarios in this subsection, we use a relatively large and sparse randomly deployed Euclidean network of 200 agents, where each agent has a random degree - the number of neighbors is usually known as degree in the graph-theory literature-, such that the average degree is 5, see Figure 2.4(a). The topology aims to represent a 


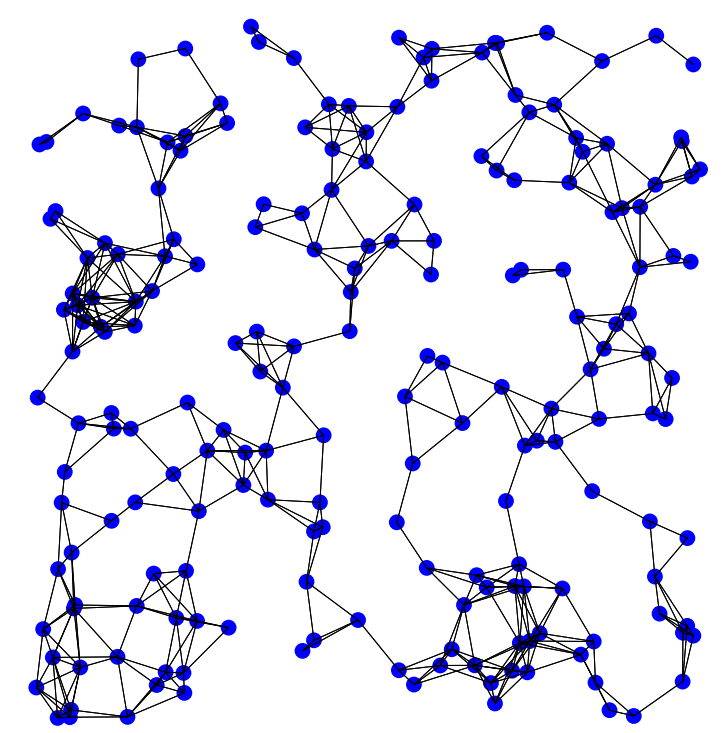

Figure 2.4 Sparse network of 200 agents with average degree 5.

random wireless sensor network, where the connectivity between each pair of agents is determined by their Euclidean distance.

\section{Toy problem}

In this example, the input data is embedded in a 2-dimensional space, i.e. $M=2$, as e.g. when every agent in a sensor network is an agent equipped with two sensors (e.g., temperature and humidity), apart of the CPU and communication capabilities. Meanwhile the subspace is 1-dimensional, $L=1$. We repeated the experiment with different data distributions. For each trial, a distributed data set of 400 samples is drawn from a mixture of 4 Gaussians with random mean (over the range $[(0,0),(3,3)]$ ) and random covariance matrix (with variance for each dimension over the range $[0,9]$ ), are randomly allocated among the agents. Every agent has taken a different and random number of samples, between one and four each, $T_{k} \in\{1,2,3,4\}$. A small number of samples in each agent allows us to highlight the performance of the algorithm even in such a low-dimensional problem. In general, when each agent has very many samples, they are independently able to estimate the subspace well, making collaboration redundant. In our extremely simple toy example, we need in the order of $T_{k} \approx 2$ samples/agent to keep in a range where cooperation is meaningful, for illustration purposes.

The weight matrix, $C$, is filled (in a fully distributed manner) using MetropolisHasting weights [116, 320, 95]. 


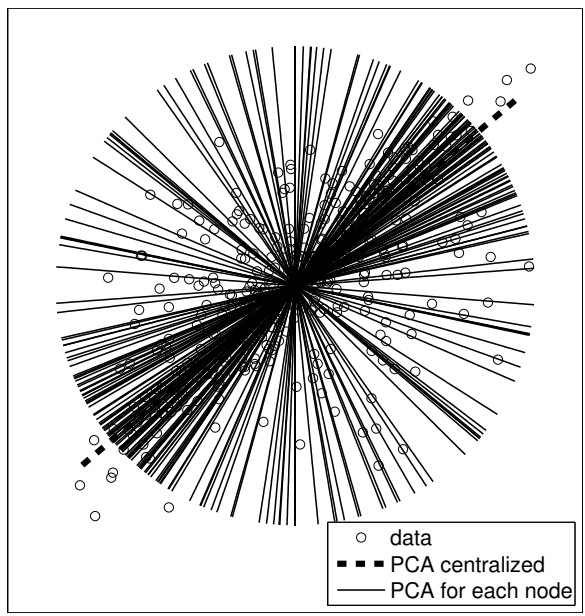

(a) without cooperation

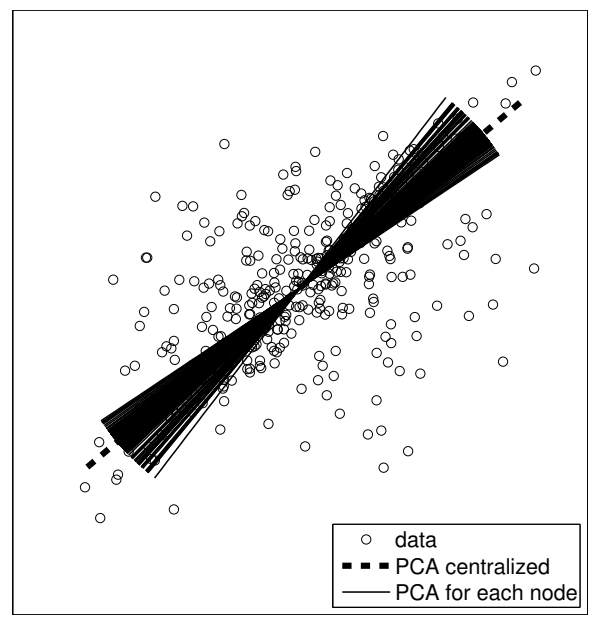

(b) with 10 consensus iterations

Figure 2.5 Performance of PCA in isolation and with cooperation via consensus using CB-DPCA, showing the convergance of all agents towards the centralized solution. The subspaces found by the agents are shown as thin continuous lines; while the subspace found by the traditional (centralized) PCA algorithm is shown as a larger and thicker broken line.

Figure 2.5 shows the performance of the CB-DPCA algorithm for one experiment, with each agent functioning in isolation (a); and with cooperation, after 10 consensus iterations (b). In both diagrams the subspace found each agent, which only has access to its local dataset, is shown as a continuous line; while the subspace found by the traditional (centralized) PCA algorithm, which has access to the entire data set, is shown as a larger and thicker broken line. It can be seen clearly that the cooperation among the agents which is built into the CB-DPCA algorithm leads not only to significant alignment among the agents subspaces, but also to the centralized solution.

A natural performance/cost trade-off exists in every consensus based algorithm, due to its iterative nature. The effects of this trade-off are shown in Figure 2.6. As expected, the communication cost grows linearly with the number of iterations of the consensus algorithm, simply because each iteration demands the exchange of a given volume of data among all the neighbors in the network.

On the other hand, the angle between the resulting subspaces and the centralized solution drops monotonically (in the mean) with each iteration. In Figure 2.6, we show the mean and maximum sin of this angle for all agents in the network. We can see that the subspaces computed by all agents in the network approximate the centralized solution more closely with each additional consensus iteration. 


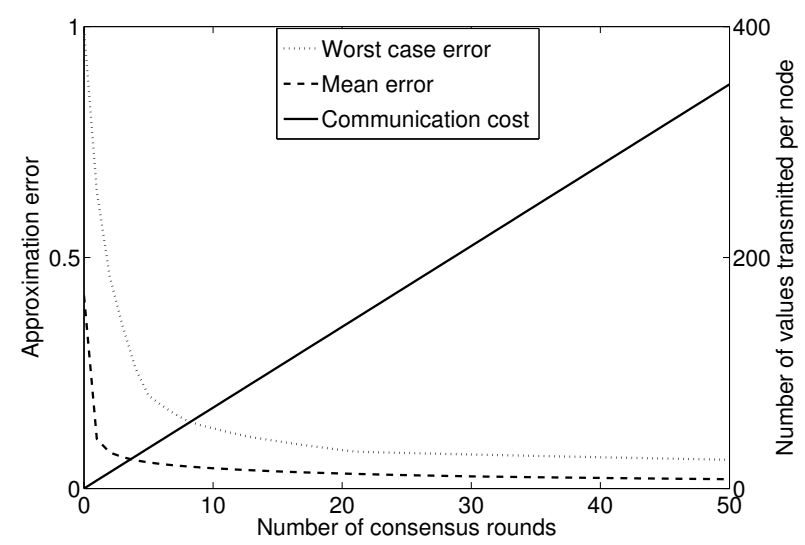

Figure 2.6 Inherent cost-performance tradeoff for CB-DPCA, cost being the volume of information transmitted, and performance represented by the reduction of the sin of the angle between the locally-estimated and the central (optimal) subspace

Although both examples are shown for the CB-DPCA algorithm, equivalent figures, behavior and conclusions are also valid for CB-EM-DPCA, CB-DPPCA and CB-DFA algorithms.

\section{Image compression}

Now, we consider one of the typical applications of PCA: image compression. We use the Lena image at $512 \times 512$ resolution and 8 bit grayscale. The image has been divided into 441 blocks of $24 \times 24$ pixels. Those blocks have been vectorized (aggregating columns) as points lying in a 576 dimensional subspace. Thus, the vectorized blocks are considered the samples of the dataset: $Y \in \mathbb{R}^{576 \times 441}$.

Each agent randomly draws an arbitrary number of samples over the range $T_{k} \in$ $\{1, \ldots, 6\}$. We consider CB-DPCA. Hence, as explained in Algorithm 2.9, every agent first computes its own sample covariance matrix, which is then shared iteratively with its neighbors a given number of consensus iterations. After the iterative collaboration, each agent will locally compute the 20 first principal components based on its resulting estimate of the global sample covariance matrix.

Figure 2.7(a) displays the original Lena image, while 2.7(b) shows the compression that a fusion center would achieve in a centralized manner, with access to the whole dataset. The compressed image is obtained by projecting the dataset into the principal subspace and then mapping the projected points back to the original base. Let $\bar{Y}_{c}$ denote the compressed data (obtained from the centered dataset). And let $\widehat{Y}$ denote 
the reconstructed dataset. Then, the projection-reconstruction process is given by:

$$
\begin{aligned}
\bar{Y}_{c} & =W^{\top} \bar{Y} \\
\widehat{Y} & =W \bar{Y}_{c}+\mu \mathbb{1}^{\top}
\end{aligned}
$$

Figures 2.7(c) and 2.7(d) show the compression that two random agents, named denoted "agent 1" and "agent 2", could achieve in isolation, i.e. with no cooperation with their neighbors. Let $R$ denote the number of consensus iterations. Then in Figures 2.7(e) and 2.7(f), we show the noticeable improvement achieved when agents share their estimates even for just $R=1$ consensus round. Finally, Figures 2.7(g) and 2.7(h) show how both agents asymptotically converge towards the same global solution after $R=50$ consensus iterations.

\subsubsection{Experiments with distributed LDA}

In the two scenarios in this subsection, we use an Euclidean network with connectivity similar to Figure 2.4, with average degree 5, but with $N=100$ agents. The reason for making the network smaller is simply to obtain more illustrative results in the second scenario. We remark that the results are qualitatively the same for larger or smaller $N$ though.

\section{Toy example}

The network is provided with a distributed data set of $T=600$ 2-dimensional samples, i.e., $M=2$ and $Y \in \mathbb{R}^{2 \times 600}$. Each sample belongs to one of $D=3$ classes, with all classes being Gaussian distributed with the same covariance but different mean. Each agent receives a random number of samples from the data set, drawn from a discrete uniform distribution over the range $T_{k} \in\{1,2,3,4\}$ samples.

Figure 2.8 shows the performance of the D-LDA algorithm with each agent operating in isolation (left); and with cooperation, after 10 consensus iterations (right). The results are similar to those obtained for PCA in Figure 2.5. In both diagrams the subspace found by the traditional (centralized) LDA algorithm, which has access to the entire data set, is shown as a larger, thicker, broken line. It can be clearly seen that the cooperation among the agents, which is built into the CB-DLDA algorithm, leads not only to significant alignment among the agents' subspaces, but also to the centralized solution. 


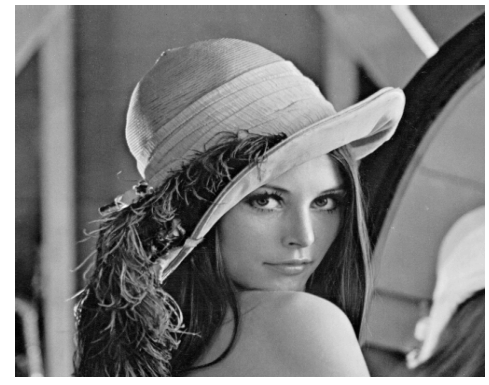

(a) original

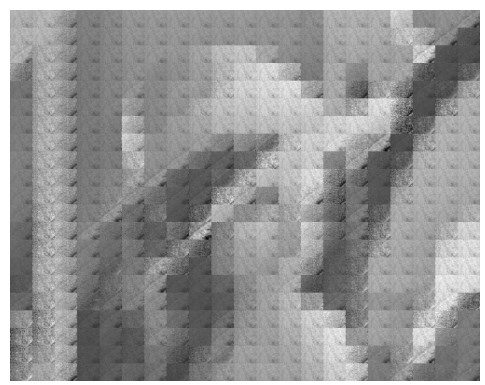

(c) agent 1: isolation

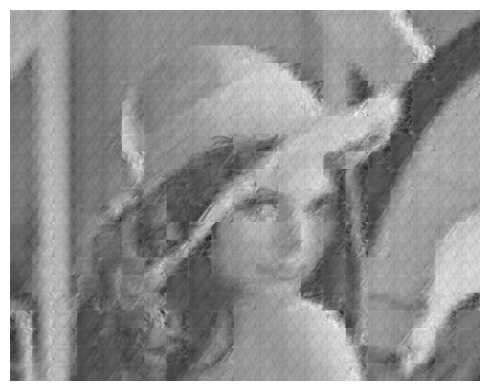

(e) agent 1: $R=1$

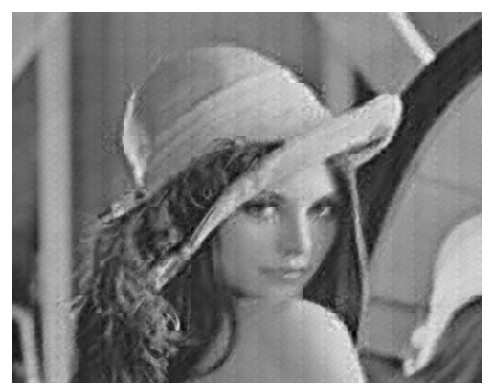

(g) agent 1: $R=50$

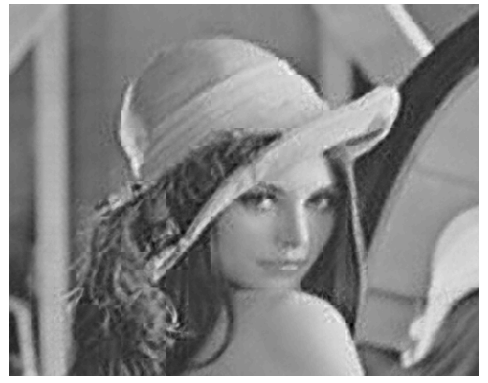

(b) compressed, fusion center

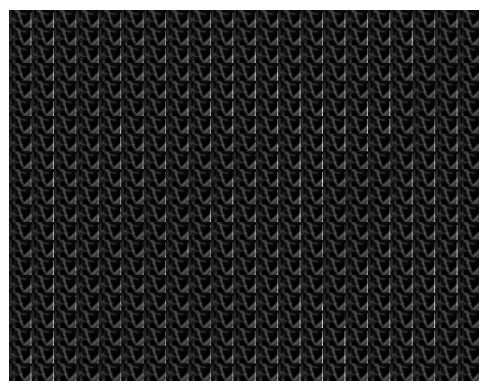

(d) agent 2: isolation

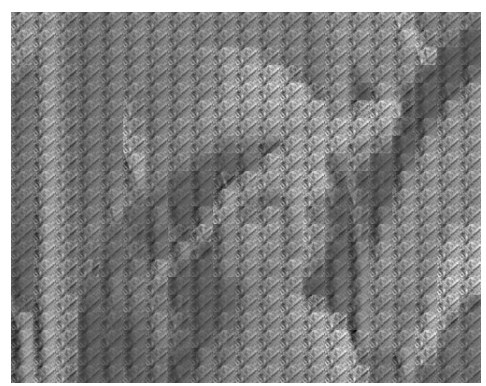

(f) agent 2: $R=1$

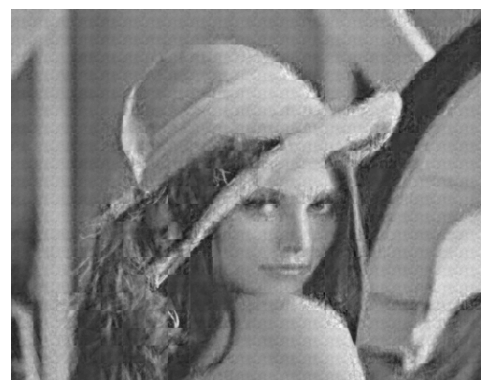

(h) agent 2: $R=50$

Figure 2.7 Performance of PCA in isolation and with cooperation via consensus using CB-DPCA. Images (c), (e), and (g) illustrate how a agent asymptotically converges to the centralized solution given in image (b). Images (d), (f), and (h) show the same for another agent in the network. 


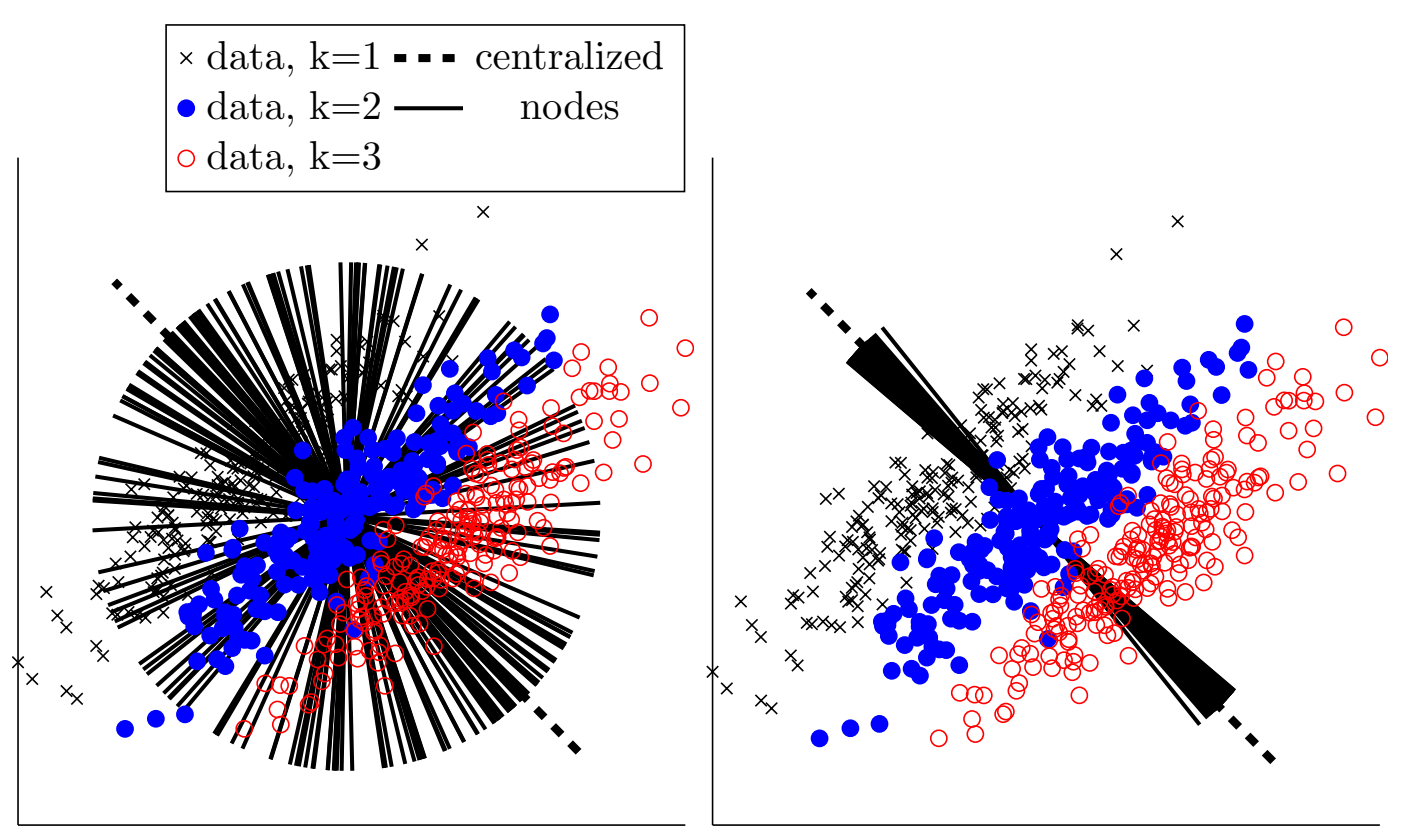

Figure 2.8 Performance of LDA in isolation (left); and with cooperation via consensus using CB-DLCA, for $R=10$ iterations in each Average Consensus subroutine, showing the convergence of all agents towards the centralized solution (right).

Again, a natural performance/cost trade-off exists in the CB-DLDA algorithm, due to the iterative nature of the underlying average consensus algorithm. The effects of this trade-off are shown in Figure 2.9. Similar to CB-DPCA, the communication cost grows linearly with the number of iterations of the consensus algorithm; while the more number of consensus iterations, the closer to the centralized solution the local subspaces computed by all the agents network will be.

\section{Distributed classification}

In this experiment, we use the D-ULDA algorithm to train a KNN classifier. We applied this distributed classifier to the Landsat data set [92], whose $T=4435$ training samples and 2000 test samples of $M=36$-dimensional data belonging to $D=6$ classes have been distributed randomly over the same network as in the toy example.

Figure 2.10 shows the performance of the classifier when no interaction among the agents is allowed, thus effectively restricting each agent to build the discriminant subspace with the local data only, as well as the case when $R=10$ consensus iterations are performed for each Consensus Averaging subroutine of Algorithm 2.15 (CB-DULDA). Once the agents have estimated the discriminant subspace (in isolation with ULDA or through cooperation with CB-DULDA), they used the projected data to train a local 


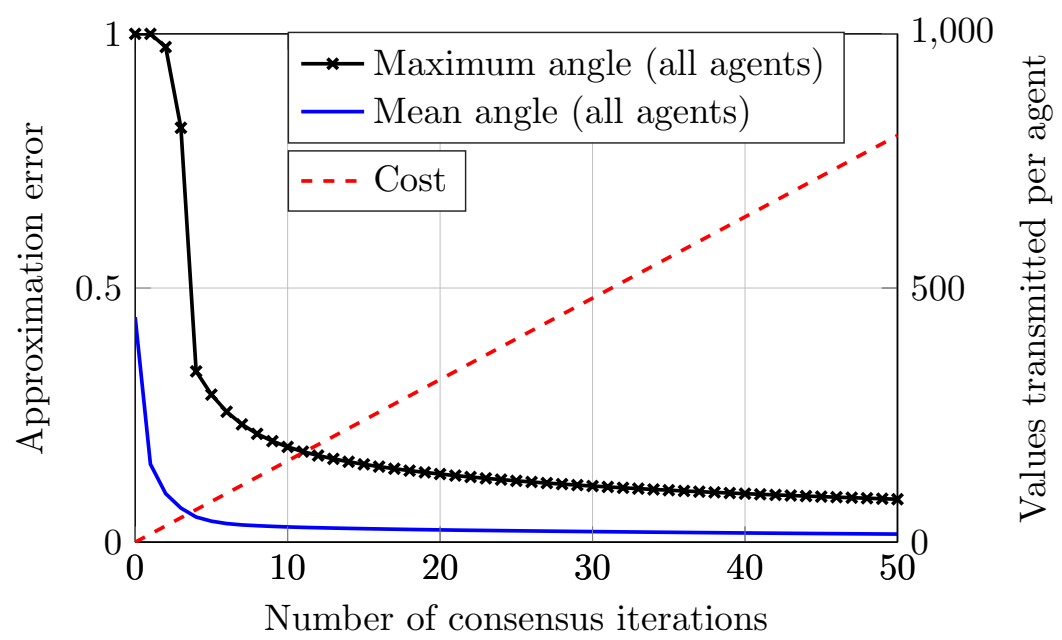

Figure 2.9 Accuracy vs. cost trade-off for the CB-DLDA algorithm where the accuracy is measured as the sin of the angle between local and centralized subspaces for the results displayed in Figure 2.8.

Table 2.1 Minimum, mean and maximum success rate for all agents in the network for single-agent ULDA (which is equivalent to CB-DULDA with $R=0$ ) and CB-DULDA with $R \in\{1,2,5,10\}$ consensus iterations.

\begin{tabular}{|l|c|c|c|c|c|c|}
\hline & $R=0$ & $R=1$ & $R=2$ & $R=5$ & $R=10$ & $R=100$ \\
\hline$\rho_{\min }$ & $6.5 \%$ & $28.4 \%$ & $32.1 \%$ & $34.7 \%$ & $36.6 \%$ & $38.6 \%$ \\
\hline$\rho_{\text {mean }}$ & $42.2 \%$ & $64.4 \%$ & $67.1 \%$ & $69.6 \%$ & $70.4 \%$ & $71.4 \%$ \\
\hline$\rho_{\max }$ & $77.4 \%$ & $91.7 \%$ & $93.1 \%$ & $94.4 \%$ & $94.6 \%$ & $95 \%$ \\
\hline
\end{tabular}

KNN classifier. The curves are shown as histograms of the proportion of the agents performing the classification of their local samples with the given accuracy. Table The centralized solution, which has access to the entire data set, classifies with $\rho_{\text {cent }}=85.1 \%$ success rate. The mean success rate for the isolated agents is $\rho_{\text {mean }, R=0}=42.2 \%$, while the mean success rate for the cooperative agents, after just $R=5$ consensus iterations of the embedded average consensus subroutines is $\rho_{\text {mean }, R=5}=69.6 \%$. Clearly, the subspace alignment afforded by the consensus alignment for only $R=5$ iterations leads to a significant mean improvement in the performance of the KNN classifier, of more than $27 \%$. The results have been averaged over 100 independent experiments, each one with different network topology and different allocation of samples to the agents.

We remark that performance saturates at about $71 \%$ and improves very little even for large number of consensus iterations. The missing performance with respect to the 


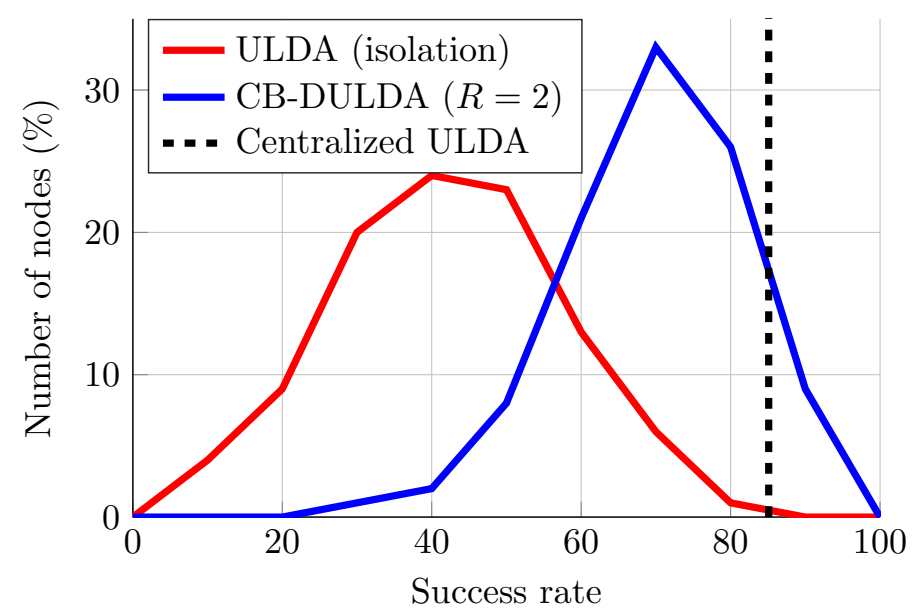

Figure 2.10 Histogram of classification success rates for ULDA and CB-DULDA by consensus alignment. Results averaged over 100 independent experiments. The success rate of the centralized solution is represented as a vertical dashed line.

centralized case is given by:

$$
\rho_{\text {cent }}-\rho_{\text {mean }, R=10}=85.1-71.4=13.4 \%
$$

The reason for this gap is that the agents are training their local KNN classifiers with very few samples. In other words, although CB-DULDA approximates ULDA correctly, we have not included a distributed implementation of KNN, so that the agents train with local classifiers that rest performance with respect to the centralized KNN.

\subsection{Extension to other component analysis methods}

Although all the proposed distributed methods are based on average-consensus, we could have followed another approach. In this section we show that several component analysis methods (including PCA, P-PCA, FA and LDA, but also many others) can be formulated as an optimization problem. Thus, we could use distributed optimization algorithms to solve them in a cooperative and fully distributed manner.

\subsubsection{Component analysis methods as optimization problems}

Recall that the LDA subspace could be obtained by solving optimization problem (2.24). It turns out that the subspace provided by many other component analysis methods can be also expressed as the solution of some optimization problems. In particular, reference [66] introduced the so named least-squares weighted kernel reduced 
rank regression (LS-WKRRR) problem as a general framework that includes several component analysis methods as particular cases, including the classical PCA, P-PCA, FA and LDA methods discussed so far, but also their weighted and kernel extensions, as well as canonical correlation analysis, nonlinear embedding methods (like Laplacian eigenmaps and locality preserving projection), k-means and spectral clustering or even nonnegative matrix factorization. This general problem is formally described as follows:

$$
\underset{A \in \mathbb{R}^{D \times L}, W \in \mathbb{R}^{M \times L}}{\operatorname{maximize}} J(A, W) \triangleq\left\|\Gamma_{r}\left(\Phi-A W^{\top} \Upsilon\right) \Gamma_{c}\right\|_{F}^{2}
$$

where $\Phi=\phi(G)$ and $\Upsilon=\varphi(Y)$ map the dataset to a feature space using kernel methods; and $\Gamma_{r}$ and $\Gamma_{c}$ denote different weights for the features and samples, respectively. In other words, the LS-WKRRR framework unifies and expresses all the mentioned methods as particular instances of a common optimization problem (e.g., for LDA (2.24), we have $\Gamma_{r}=\left(G^{\top} G\right)^{-1 / 2}, \Gamma_{c}=I, \Phi=G^{\top}$ and $\left.\Upsilon=\bar{Y}\right)$.

\subsubsection{Distributed optimization strategies}

In Chapters 3 and 4 of this thesis, we will show a general approach for distributed optimization over networks and apply it for reinforcement learning and global search. There are several strategies that can be used for distributed optimization, such as incremental (see, e.g., [199, 231, 205, 135, 39, 172]), diffusion (e.g, [56, 58, 248, 57, 249, 36, 246, 247]) and consensus (e.g., [289, 212, 201, 232, 270]) strategies.

In an incremental strategy, the algorithm has first to determine a cyclic trajectory that visits all agents in the network. Then, the agents update their estimate sequentially. If some node or link fails, a new cycle has to be computed, which is a combinatorial problem. Both diffusion and consensus strategies surmount these problems.

Diffusion strategies combine the average-consensus update that we have seen in previous sections with a gradient descent update (or more generally with a stochasticapproximation) to perform distributed optimization. There are several possible variants, one especially effective and simple is the so named adapt-then-combine (ATC) diffusion algorithm [56, 58, 57], which consists of two-steps: the adaptation step, at which every agent updates its own intermediate estimate independently of the other agents; and the combination step, at which every agent combines its neighbors' estimates. For instance, if the objective function to be minimized is denoted $J: \mathbb{R}^{M} \rightarrow \mathbb{R}$, the ATC updates would be as follows:

$$
\widehat{w}_{k, i+1}=w_{k, i}+\mu \nabla_{w} J\left(w_{k, i}\right)
$$




$$
w_{k, i+1}=\sum_{l \in \mathcal{N}_{k}} c_{l k} \widehat{w}_{l, i+1}
$$

These two ATC steps can be grouped in a single equation as follows:

$$
w_{k, i+1}=\sum_{l \in \mathcal{N}_{k}} c_{l k}\left(w_{l, i}+\mu \nabla_{w} J\left(w_{l, i}\right)\right)
$$

Consensus strategies also combine average-consensus and gradient descent (or stochastic approximation), but they are intertwined them in a single step. For instance, for the objective function $J$, the consensus-optimization updates would be as follows:

$$
w_{k, i+1}=\sum_{l \in \mathcal{N}_{k}} c_{l k} w_{l, i}+\mu_{i+1} \nabla_{w} J\left(w_{k, i}\right)
$$

The main difference between ATC (2.130) and consensus (2.131) is that the latter combines local and in-neighborhood information asymmetrically (the local gradient is not multiplied by $c_{l k}$ ).

Consensus strategies have been successfully applied to the solution of static optimization problems, where the objective does not drift with time They have been studied largely under diminishing step-size conditions to ensure agreement among cooperating agents (that is the reason why we have included the time subscript in $\mu_{i+1}$ in (2.131)). Diffusion strategies, on the other hand, have been proved to be particularly apt at endowing networks with continuous adaptation and learning abilities to enable tracking of drifting conditions.

It has been shown in [291] that the dynamics of diffusion networks leads to enhanced stability and lower mean-square-error (MSE) than consensus networks. In particular, the analysis in $[249,247,291]$ shows that the asymmetrical combination of local data and in-neighborhood information typical of consensus networks can make the state of consensus networks grow unbounded even when all individual agents would be mean stable in isolation. This behavior does not happen in diffusion networks, in which local and external information are symmetrically combined by construction, which ends up enhancing the stability of the network. For these reasons, in the reminder of this thesis, whenever we have to derive a cooperative distributed algorithms we will only focus on diffusion strategies. 


\subsubsection{A diffusion-based algorithm for distributed component analysis methods}

We could follow the distributed optimization approach and use ATC for minimizing (2.127). In this case, instead of using average-consensus to approximate the terms of the EM or ALS methods, we could use (2.128)-(2.129) to compute the gradient in each of the variables, $A$ and $W$, in a kind of distributed block-coordinate descent, to optimize the specific instance of (2.127) corresponding to any of the particular component analysis methods mentioned above:

$$
\begin{aligned}
\widehat{A}_{k, i+1} & =A_{k, i}-\alpha_{A} \nabla_{A} J\left(A_{k, i}, W_{k, i}\right) \\
\widehat{W}_{k, i+1} & =W_{k, i}-\alpha_{W} \nabla_{W} J\left(A_{k, i}, W_{k, i}\right) \\
A_{k, i} & =\sum_{l \in \mathcal{N}_{k}} c_{l k} \widehat{A}_{l, i} \\
W_{k, i} & =\sum_{l \in \mathcal{N}_{k}} c_{l k} \widehat{W}_{l, i}
\end{aligned}
$$

where $J$ is the objective function in (2.24), $\nabla_{A}$ and $\nabla_{W}$ denote the gradients with respect to $A$ and $W$, respectively, and $\alpha_{A}$ and $\alpha_{W}$ are step-size for each recursion. Nevertheless, we leave this idea as future research and we will use this kind of diffusion ATC distributed optimization for reinforcement learning and global optimization problems.

\subsection{How to implement doubly-stochastic combination matrices}

\subsubsection{Basic design for tackling temporary link failures}

In order to tolerate packet loss, asynchronous transmissions and other impairments of real environments, and avoid biased results of the distributed algorithm, some sort of coordination among neighbors becomes necessary, so they can give total unit weight to agent $k$ at every iteration (thus, making the combination matrix row stochastic). We propose an efficient and easy to implement mechanism, coined Wait-For-the-Slowest (WFS) protocol. The idea underlying WFS is simple: synchronization at the application layer (rather than at the MAC level). In particular, every agent has a table where it stores an iteration counter for each of its neighbors, so it waits until this table is filled with the correct information before performing the combination step. This mechanism 
Table 2.2 Neighbor table for agent $k$ of Figure 2.2.

\begin{tabular}{|c|c|c|}
\hline $\mathcal{N}$. & $|\mathcal{N}|$. & $a_{\cdot k}$ using Metropolis rule $(2.57)$ \\
\hline$h$ & 3 & $a_{h k}=1 / 4$ \\
\hline$j$ & 3 & $a_{j k}=1 / 4$ \\
\hline$l$ & 7 & $a_{l k}=1 / 7$ \\
\hline$k$ & 4 & $a_{k k}=1-(1 / 4+1 / 4+1 / 7)=5 / 14$ \\
\hline
\end{tabular}

works efficiently under the assumptions that every agent knows its neighbors before performing the consensus iterations. Indeed, WFS is composed of two main stages: an initial setup and the operation during the consensus loop.

The setup stage is executed only once, at the beginning of the algorithm, with the purpose of building the combination matrix. During this initial stage, every agent broadcasts 'discovery' messages with some useful local information (e.g., $\left|\mathcal{N}_{k}\right|$ for the Metropolis rule (2.57)). Let us say that agent $k$ receives a discovery message from agent $l$, then, it checks whether agent $l$ was already in its neighbor list; if it was not, another row is added to the table; otherwise, just the degree $|\mathcal{N}$.$| and the weight a_{\cdot k}$ are updated (see Table 2.2 for an example of neighbor table). At the end of the setup stage, every agent has stored its neighbor table, where the weight column can be thought of as a column of the combination matrix.

Once the agents know their neighborhoods and have set the corresponding combination weights, they start the consensus stage. When some event (which could be asynchronous, such as the arrival of a new packet or the availability of a new sample, or periodic, in synch with an internal iteration timer) triggers the combination step (2.48), the agent has to be sure that it has the right estimates. Let us say that agent $k$ wants to update $w_{k, i}$, then it should combine $w_{l, i-1}$ for all $l \in \mathcal{N}_{k}$, instead of any other $w_{l, t}$ with $t \neq i-1$. If such information is not available, the agent patiently waits for a retransmission of the missing information. Note that when one agent stops updating its estimate (because it misses information from any of its neighbors), then the rest of its neighbors will also wait for it. Therefore, the difference in the iteration number between two neighbors is at most 1 (e.g., $w_{k, i}$ and $w_{l, i-1}$ ). Thus, at every iteration, every agent has to transmit two parameters, namely, the two most recent estimates (i.e., $w_{k, i}$ and $\left.w_{k, i-1}\right)$. This process is illustrated with an example in Figure 2.11.

We remark that we have successfully implemented this protocol in two different settings:

1. A wireless sensor network, where each agent is an embedded devices (we used commercial off-the-shelf Econotag motes, equipped with MC13224 System-on- 


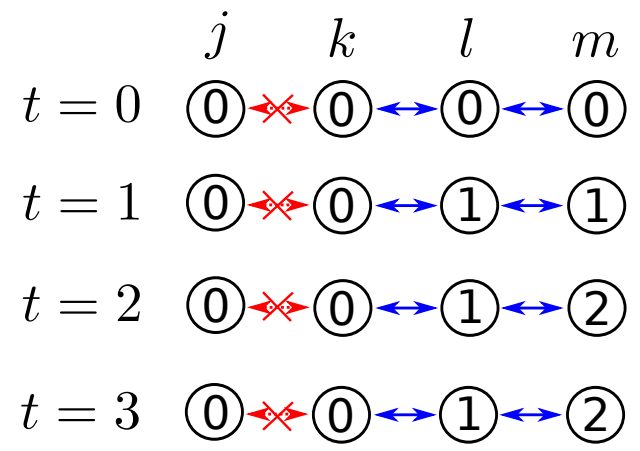

Figure 2.11 Example 1. A chain of agents running WFS, where the link between the two agents in the left end of the chain is faulty. The iteration number $(i)$ of the estimate (e.g., $w_{k, i}$ ) is written inside each circle. At time $t=1$, agent $j$ cannot update because info from agent $k$ is missing. Similarly, although agent $k$ has info from agent $l$, it misses the estimate from agent $j$, so it cannot update its estimate. On the other hand, agents $l$ and $m$ have all the information from their neighborhoods. Nevertheless, at time $t=2$, agent $l$ misses $w_{k, 1}$ so it has to stop. agent $m$ have both $w_{l, 1}$ and $w_{m, 1}$ so it can compute $w_{m, 2}$. At time $t=3$, agent $m$ is missing $w_{l, 2}$ so it is forced to stop too and, hence, the whole network has to either wait until the link between $j$ and $k$ works again (some retransmission finally reaches the agent) or until a retransmission timeout, which they understand as a permanent topology change so they safely remove each other from their neighbor tables.

Chip, which offers an ARM7 microcontroller at 24MHz with $96 \mathrm{~KB}$ RAM and an integrated 802.15.4 radio, everything controlled through the event-driven ContikiOS operating system) and the WFS performed as expected and efficiently, being able to complete one iteration per second in different topologies, including a fully connected network of 10 devices (see demonstration video at [298]);

2. A datacenter, where we run a multithread-multimachine implementation of the distributed cross-entropy algorithm described in Chapter 4, where the agents are threads allocated in (possibly) multiple machines and we use WFS to synchronize the agents (open source Java code available at [243]).

\subsubsection{Extension for dealing with permanent topology changes}

Although the mechanism explained above is robust against temporary link failures (e.g., due to fading, collisions...) and asynchronous communications (e.g., because of clock drifts, different transmission rates...), two extensions are required in order to tackle permanent changes in the topology: a link-timer that measures how long a link has 
been inactive, and a dynamic neighbor table that can be updated during the consensus stage.

When one agent receives a packet from one of its neighbors, it resets the link-timer. If one link fails permanently (e.g., due to agent failure), the timer of each of the affected agents will reach a tunable timeout period, hence, they will assume that the link will not recover anymore (at least in the short term) and remove it from their tables. The iteration number is a form of acknowledgement that the information is diffusing properly. Therefore, every agent keeps transmitting its relevant parameters as described in Sec. 2.9.1. Note that if the link-failures divide the network in two or more islands, then WFS will still work in each of the islands, but they may independently converge to different results.

When a new agent is added to the network after the network has entered the consensus stage, the other agents will not take it into account (they will continue giving zero weight). Nevertheless, if the agents are able to update their neighbor tables every time they receive a packet from an unknown neighbor, even during the consensus stage, then the network will be able to integrate new agents on the fly. When the new agent boots, it enters the setup-stage, in which it broadcasts discovery messages and listens to discover its neighbors. During this stage, apart of storing its neighbors' identity, it also keeps their iteration number. At the end of this stage, the agent sets its iteration number equal to the highest iteration number among its neighbors. This way, the new agent will be included in new iterations of the consensus algorithm without biasing earlier uncompleted iterations in its neighborhood.

Figure 2.12 shows an example of the robustness of WFS against noisy links, asynchronous updates and permanent changes in the topology.

\subsection{Conclusions}

In this chapter we presented a method for estimation of means and covariance and scatter matrices, when the agents have local datasets with different amount of samples, using average consensus. We used this method to introduce two distributed algorithms to perform PCA. Both algorithms require no fusion center and rely only on low-volume local (1-hop) communications. This in turn makes them scalable and robust against link failure. Moreover, the data is never exchanged, guaranteeing privacy for the local datasets. Furthermore, we gave two extensions of the presented algorithms to distribute two closely related techniques: FA and PPCA, as well as three distributed implementations of LDA. The approach we followed in all cases, combining local 


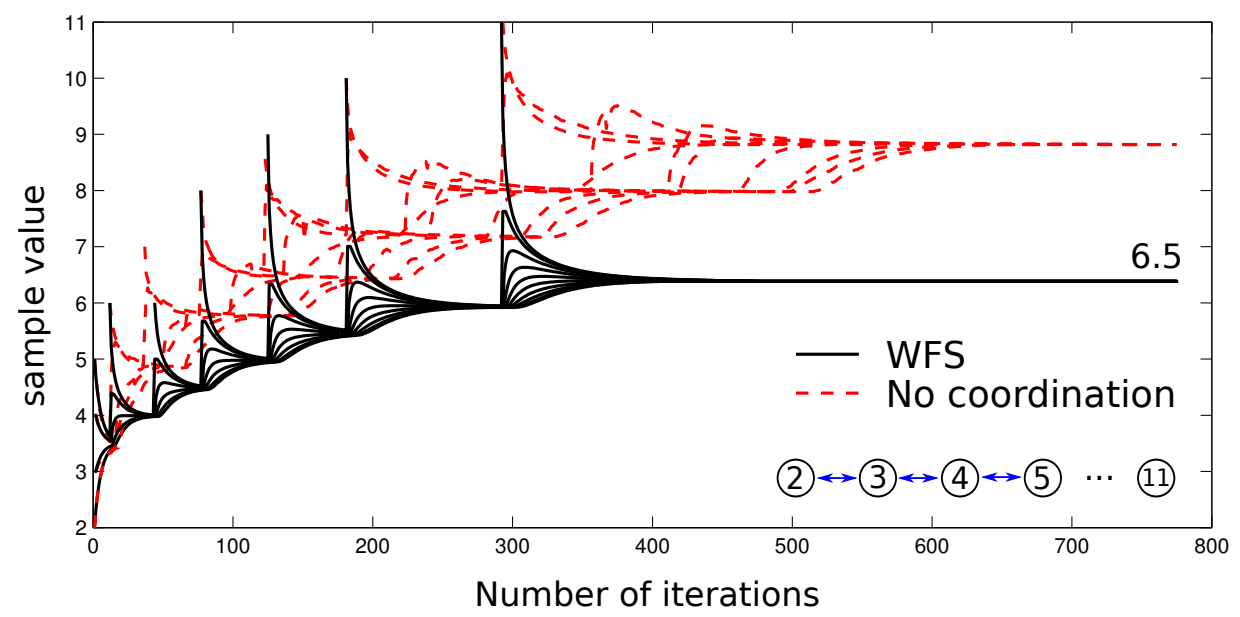

Figure 2.12 Robustness of WFS. We begin with a chain of 4 agents, with values $\{2,3,4,5\}$, respectively. Then, we add up to 6 more agents, one at a time, with values $\{6,7,8,9,10,11\}$, respectively. Note that WFS is able to achieve the sample average of all the available agents at each time (resulting in 6.5 when all agents are included in this case).

computations with the distributed estimation of means and scatter matrices, is very systematic and can be applied to other component analysis methods. We have illustrated the performance of our algorithms on simulated toy examples as well as in more realistic applications, like image compression and distributed classification, with a discussion on the convergence and the inherent cost-performance trade-off.

Finally, we have presented distributed optimization and discussed a promising approach for developing numerous component analysis methods, which consists in applying distributed block-coordinate descent on the LS-WKRRR problem. Nevertheless, we leave this idea as future research. In the next chapters, we use distributed optimization for two problems, namely reinforcement learning and black-box global optimization. 


\section{Chapter 3}

\section{Distributed policy evaluation under multiple behavior strategies}

\subsection{Introduction}

In this chapter, we consider the problem in which a network of autonomous agents collaborate to predict the response of the environment to their actions. The network forms a connected graph, where there is at least one path between every pair of nodes. The agents learn locally from their individual interactions with the environment and share knowledge with their neighbors (only direct neighborhood communication is allowed). We assume the environment can be modeled as a Markov decision process (MDP). But the actual state of the environment is unknown for the agents. Instead, they just have access to a feature vector that represents the state. Such feature representation is convenient in problems with very large state dimensions, since it is computationally more efficient to work with features of smaller dimension than the size of the original state-space.

In the scenario under study in this chapter, every agent takes actions according to an individual policy, which is possibly different from that of every other agent. The objective of the agents is to assess the response of the environment to a common hypothetical behavior, the target policy, which is the same for every agent but different from the actual behavior policies they are following. This problem of predicting the response to a target policy different from the behavior policy is commonly referred as off-policy learning (see, e.g., [274, Sec. 5.6],,[227, 215]). Off-policy learning has shown useful in several scenarios: 
1. when the target policy is risky and some actions could be costly (e.g., choosing the advertisement to show a user visiting a website [280]) or even dangerous (e.g., determining the medical treatment for a patient [279]), so we can estimate the response to the target policy from more conservative behavior policies;

2. when having many different predictions in parallel from a single stream of data is useful for extracting a feature representation of the environment [276, 71, 195];

3. if the agents have to learn from historical data (e.g., from human demonstration), where the learned target policies are different from the policy used for obtaining the data.

4. in hierarchically structured environments, where it is convenient to predict the response of the environment for multiple policies (e.g., skills for achieving subgoals at different time-spans) from a single data stream.

The predictions by the agents are made in the form of value functions, which account for the expected long-term accumulated reward from any given initial state and by following some policy $[228,274,28]$. If the value function is higher for one policy than for another, we can expect that following one policy will yield higher return than the other in the long term. Recently, value functions have been extended to predict other signals, different from the reward. In particular, references [276, 71, 195] showed how value functions were able to predict robot sensory data (e.g., the sudden change in an inertial measurement unit when the robot collides with the wall).

In this chapter we focus on learning value functions from off-policy streaming data, where the environment data is represented with features. At the moment of researching in this topic, there were few methods with convergence guarantees under this setting. The gradient-temporal-difference (GTD) algorithm is one of these methods [177]. GTD was originally proposed for the single agent scenario in [277, 275], and derived by means of the stochastic optimization of a suitable cost function. The main advantages of this single-agent GTD are its low complexity (so that it can work even with large feature vectors) and its convergence guarantees (for diminishing step-sizes) under the off-policy setting. In Section 3.3 we apply diffusion strategies to develop a distributed GTD algorithm that extends the single-agent GTD to multi-agent networks. As discussed in Chapter 2, there are several distributed strategies that can be used for this purpose, such as incremental (see, e.g., [199, 231, 205, 135, 39, 172]), consensus (e.g., [289, 212, 201, 232, 270]) and diffusion (e.g, [56, 58, 248, 57, 249, 36, 246, 247]) strategies. In a incremental strategy, the algorithm has first to determine a cyclic 
trajectory that visits all agents in the network. Then, the agents update their estimate sequentially. If some node or link fails, a new cycle has to be computed, which is a combinatorial problem. Both diffusion and consensus strategies surmount these problems. Consensus strategies have been successfully applied to the solution of static optimization problems, where the objective does not drift with time. They have been studied largely under diminishing step-size conditions to ensure agreement among cooperating agents. Diffusion strategies, on the other hand, have been proved to be particularly apt at endowing networks with continuous adaptation and learning abilities to enable tracking of drifting conditions. There are several forms of diffusion; recent overviews appear in [248, 249, 247]. It has been shown in [291] that the dynamics of diffusion networks leads to enhanced stability and lower mean-square-error (MSE) than consensus networks. In particular, the analysis in [249, 247, 291] shows that consensus networks combine local data and in-neighborhood information asymmetrically, which can make the state of consensus networks grow unbounded even when all individual agents are mean stable in isolation. This behavior does not happen in diffusion networks, in which local and external information are symmetrically combined by construction, which ends up enhancing the stability of the network. For these reasons, we focus in the remainder of this chapter on the derivation of a diffusion strategy for GTD over multi-agent networks. As a byproduct of this derivation, we show that the GTD algorithm, motivated as a two time-scales stochastic approximation in [275], is indeed a stochastic Arrow-Hurwicz algorithm applied to the dual problem of the original formulation. This is a novel interesting result by itself, since it gives insight on how the algorithm works and could lead to future extensions (we note that after publishing this result in Dec. 2013 [297], the same saddle-point derivation was independently rediscovered and published two years later in July 2016 by reference [170]).

The convergence analysis of reinforcement learning algorithms is usually challenging even for the single-agent case, and studies are often restricted to the case of diminishing step-sizes [277, 275, 178]. For a distributed algorithm, the analysis becomes more demanding because the estimation process at each node is influenced by the estimates at the other nodes, so the error propagates across the network. Another difficulty in the distributed case is that the agents may follow different behavior policies and, thus, their individual cost functions could have different minimizers. In Section 3.4, we will analyze the steady-state and transient behavior of the proposed distributed algorithm, deriving closed-form expressions that characterize the network performance for sufficiently small constant step-sizes. We employ constant, as opposed to decaying step-sizes, because we are interested in distributed solutions that are able to continually adapt and learn. 
The performance analysis will reveal that when the agents follow the same behavior policy, they will be able to find an unbiased estimator for the centralized solution. On the other hand, when the agents behave differently, they will approach, up to some bias, the solution of a convex combination of their individual problems. This bias is proportional to the step-size, so it becomes negligible when the step-size is sufficiently small. One important benefit that results when the agents behave differently is that, although the agents do not directly share their samples, the in-network experience becomes richer in a manner that the diffusion strategy is able to exploit. In particular, in the reinforcement learning literature (see, e.g., [274] for an intuitive introduction to RL; [31] and [30, Vol 2, Ch. 6] for a more formal perspective; [278, 51] for reviews of modern RL algorithms; and [314] for a survey on recent developments in the field), it is customary to assume that the behavior policy must allow the learning agent to visit every possible state infinitely often. We will relax this assumption and show that the distributed algorithm is able to perform well even when the individual agents only visit small portions of the state-space, as long as there are other agents that explore the remaining regions. Therefore, even though none of the agents can find the optimal estimate of the value function by itself, they can achieve it through cooperation. This is an interesting capability that emerges from the networked solution.

In this chapter we consider a setting in which the agents can communicate and cooperate with their neighbors, but they operate without influencing each other. This setup is meaningful in many real applications. Consider, for example, a water purification plant controlled and monitored by a wireless actuator-sensor network, in which each device is attached to a different water-tank. The quality of the water (e.g., the amount of bacteria) in one tank will be influenced by the decisions (e.g., delivering some amount of chlorine) made by the device controlling that tank, independently of what other devices do. Still, since all water tanks behave similarly under similar circumstances, the devices in the network can benefit from sharing their individual knowledge. In Chapter 5, we will consider a complementary setting, in which the action of one agent can influence the environment response for every other agent.

\subsubsection{Related works}

There are several insightful works in the literature that address issues pertaining to distributed learning albeit under different scenarios and conditions than what is studied in this article. For example, the work in [140] proposes a useful algorithm, named QD-learning, which is a distributed implementation of Q-learning using consensus-based stochastic approximation. The diffusion strategy proposed herein is different in several 
respects. QD-learning asymptotically solves the optimal control problem, learning the policy that maximizes the long-term reward of the agents. Here, we focus on predicting the long-term reward for a given policy, which is an important part of the control problem. However, QD-learning is developed in [140] under the assumption of perfectknowledge of the state. Here, we study the case in which the agents only know a feature representation of the state, which is used to build a parametric approximation of the value function, allowing us to tackle large problems, for which Q-learning schemes can diverge [11, 290]. Finally, we enforce constant step-sizes in order to enable continuous adaptation and learning. In comparison, the analysis in [140] employs a diminishing step-size that dies out as time progresses and, therefore, turns off adaptation and is not able to track concept drifts in the data.

Another related work [34] analyzes the performance of cooperative distributed asynchronous estimation of linearly approximated value functions using standard temporal difference (TD), but it is well known that TD learning with parametric approximation schemes can diverge when the agents learn off-policy [11, 290]. In addition, although the algorithm in [34] is distributed, in the sense that there is no fusion center, it requires full connectivity (i.e., every node must be able to exchange information with every other node in the network), which is a restrictive assumption that prevents the algorithm from large-scale deployments. In this article, we focus on fully distributed solutions that only require the network of agents to be connected (but not necessarily fully connected). Other related-but more heuristic - approaches include $[254,309]$.

After we publish the results presented in this chapter, more recent works have presented related algorithms and analysis. The primal-dual interpretation of the single agent GTD was independently rediscovered and presented in reference [170] — more than two years after our results were published. Very recently, reference [271] has extended the analysis for time-varying graphs of a simplified version of the proposed distributed GTD algorithm, in which all agents follow the same behavior policy. Moreover, the diffusion-based distributed stochastic Arrow-Hurwicz algorithm presented here-which is useful for other strongly-convex quadratic problems beyond policy evaluation - was later generalized to more general convex problems by reference [286].

Publications. The results described in this chapter resulted in the following publications [297, 296, 295].

Notation. The state of the environment and the action taken by an agent are denoted by $s$ and $a$, respectively. With a slight abuse of notation, $s(i)$ and $a(i)$ denote the state and action variables at time $i$. Moreover, whenever a variable is specific to 
some agent $k$ we add a subscript (e.g., $s_{k}(i)=s$ means that the environment seen by agent $k$ is at state $s$ at time $i$ ). Apart from states and actions, some other relevant magnitudes are $x_{k, i}, r_{k}(i)$ and $w_{k, i}$, which denote the feature vector representing the state, the received scalar reward signal, and the estimated parameter vector by agent $k$ at time $i$, respectively. Operator vec[.] stacks the columns of a matrix, one beneath the other, into a long vector. The expected value with respect to probability distribution $d$ is denoted by $\mathbb{E}_{d}[\cdot]$; we use multiple sub-indexes (e.g., $\left.\mathbb{E}_{d, \phi, \mathcal{P}}[\cdot]\right)$ when the expectation is taken with regard to multiple distributions.

\subsection{Preliminaries}

We start by enumerating the variables that define a Markov decision process (MDP). Then, in Subsection 3.2.2, we formalize the notion of prediction with value functions that measure the expected response of the environment. Although value functions can be computed in close form as a function of the state, when the state set is finite, in Subsection 3.2.3, we will show that it is more convenient -especially when the statespace of the MDP is large- to express the value function as a parametric function of some features that represent the state. When the state set is continuous, a parametric approximation of the value function makes the problem tractable. We consider a linear parametric approximation of the value function and show how finding the optimal parameter can be formulated as a saddle-point problem that arises from minimizing a suitable cost function. In Subsection 3.2.4, we introduce a stochastic version of the Arrow-Hurwitz algorithm to find the parameter directly from samples, without any knowledge of the underlying statistics of the environment. For the single-agent case, this algorithm reduces to the gradient-temporal-difference (GTD2) introduced in [275] but is motivated here from a primal-dual perspective rather than by using a two-rate stochastic approximation argument. This primal-dual formulation will be useful for the study of multi-agent networks since it leads to a meaningful in-network stationary distribution that combines the individual stationary distributions of the agents.

\subsubsection{Markov decision processes (MDP)}

We consider Markov decision processes (MDP) that are characterized by (see, e.g., $[228,28])$ :

- a finite set of states $\mathbb{S}$ of size $S \triangleq|\mathbb{S}|$;

- a finite set of actions $\mathbb{A}$ of size $A \triangleq|A|$; 
- the kernel of transition probabilities $\mathcal{P}\left(s^{\prime} \mid s, a\right)$, which gives the probability of going from one state $s$ to another state $s^{\prime}$, given an action $a$;

- and the reward function $r: \mathbb{S} \times \mathbb{A} \times \mathbb{S} \rightarrow \mathbb{R}$ that the agent aims to predict, which is associated with every transition, such that $r\left(s, a, s^{\prime}\right)$ denotes the reward received by a generic agent for the transition from $s$ to $s^{\prime}$ after taking action $a$.

The agents aim to predict the response of their environment when they follow some stationary policy $\pi$, such that $\pi(a \mid s)$ stands for the probability of an agent choosing action $a$ when the environment is at state $s$. We assume that the finite-state Markov chain resulting from the MDP is irreducible and aperiodic under any policy of interest. Thus, it has a unique positive stationary probability distribution of visiting each state (see, e.g., [228, App. A] [162]) denoted by the vector

$$
d^{\pi} \triangleq\left[\begin{array}{c}
d^{\pi}(1) \\
\vdots \\
d^{\pi}(S)
\end{array}\right]
$$

where

$$
d^{\pi}(s) \triangleq \lim _{i \rightarrow \infty} \mathbb{P}(s(i)=s \mid s(0), \boldsymbol{a}(t)=a(t) \sim \pi(\cdot \mid s(t)), \quad \forall s(0) \in \mathbb{S}, t=0, \ldots, i
$$

such that $d^{\pi}(s)>0, s=1, \ldots S$, and $\sum_{s=1}^{S} d^{\pi}(s)=1$. The state transition probabilities of the Markov chain, from initial state $s$ to destination $s^{\prime}$ are given by

$$
p_{s s^{\prime}}^{\pi} \triangleq \sum_{a \in \mathbb{A}} \mathcal{P}\left(s^{\prime} \mid s, a\right) \pi(a \mid s)
$$

Let $P^{\pi}$ the state-transition probability matrix of size $S \times S$ that collects the $p_{s s^{\prime}}^{\pi}$ as its $\left(s, s^{\prime}\right)$-th entry.

\subsubsection{Value function}

In order to make predictions of the reward signal, we use state value functions, $v: \mathbb{S} \rightarrow \mathbb{R}$, which provide the expected cumulative sum of the reward, weighted by an exponentially-decaying time window $[28,195,228,274]$. This time window spans from $i=0$ to $i=\infty$, but it has an effective length controlled by a constant $\gamma \in(0,1)$, which trades short-sighted $(\gamma \rightarrow 0)$ vs. long-term planning $(\gamma \rightarrow 1)$. The value function for 
target policy $\pi$, starting from some initial state $s \in \mathbb{S}$ at time $i$, is defined as:

$$
v^{\pi}(s) \triangleq \mathbb{E}_{\pi, \mathcal{P}}\left[\sum_{t=1}^{\infty} \gamma^{t-1} \boldsymbol{r}(i+t) \mid \boldsymbol{s}(i)=s\right]
$$

where

$$
\boldsymbol{r}(i+1) \triangleq r(\boldsymbol{s}(i), \boldsymbol{a}(i), \boldsymbol{s}(i+1))
$$

and the expectation is taken with regard to all possible state-transitions:

$$
\mathbb{E}_{\pi, \mathcal{P}}\left[r\left(s, \boldsymbol{a}, \boldsymbol{s}^{\prime}\right)\right]=\sum_{a \in \mathbb{A}} \pi(a \mid s) \sum_{s^{\prime} \in \mathbb{S}} \mathcal{P}\left(s^{\prime} \mid s, a\right) r\left(s, a, s^{\prime}\right)
$$

Note that $\boldsymbol{a}(i)$ is random because it is drawn from a probability distribution $\pi$, which together with the probabilistic transition dictated by $\mathcal{P}$, leads to a random future state $\boldsymbol{s}(i+1)$.

Let $s^{\prime}$ denote the destination state after transitioning from $s$. Introduce the shorthand $r^{\pi}(s)$ to denote the expected reward that can be collected over the next transition when the agent is currently at state $s$ :

$$
r^{\pi}(s) \triangleq \mathbb{E}_{\pi, \mathcal{P}}\left[r\left(s, \boldsymbol{a}, \boldsymbol{s}^{\prime}\right)\right]
$$

where $\boldsymbol{s}^{\prime}$ is the random arriving state after transition from $s$. Then, some algebra will show that we can write (3.4) as a fixed point equation, known as Bellman equation $[228,274,28]$ :

$$
\begin{aligned}
v^{\pi}(s) & =\mathbb{E}_{\pi, \mathcal{P}}\left[\boldsymbol{r}(i+1)+\gamma \boldsymbol{r}(i+2)+\gamma^{2} \boldsymbol{r}(i+3)+\ldots \mid \boldsymbol{s}(i)=s\right] \\
& =\mathbb{E}_{\pi, \mathcal{P}}[\boldsymbol{r}(i+1) \mid \boldsymbol{s}(i)=s]+\gamma \mathbb{E}_{\pi, \mathcal{P}}[\boldsymbol{r}(i+2)+\gamma \boldsymbol{r}(i+3)+\ldots \mid \boldsymbol{s}(i)=s] \\
& =r^{\pi}(s)+\gamma \mathbb{E}_{\pi, \mathcal{P}}\left[\sum_{t=1}^{\infty} \gamma^{t-1} \boldsymbol{r}(i+1+t) \mid \boldsymbol{s}(i)=s\right] \\
& =r^{\pi}(s)+\gamma \mathbb{E}_{\pi, \mathcal{P}}\left[v^{\pi}\left(s^{\prime}\right) \mid \boldsymbol{s}(i+1)=s^{\prime}\right] \\
& =r^{\pi}(s)+\gamma \sum_{s^{\prime} \in \mathbb{S}} \sum_{a \in \mathbb{A}} \mathcal{P}\left(s^{\prime} \mid s, a\right) \pi(a \mid s) v^{\pi}\left(s^{\prime}\right) \\
& =r^{\pi}(s)+\gamma \sum_{s^{\prime} \in \mathbb{S}} p_{s s^{\prime}}^{\pi} v^{\pi}\left(s^{\prime}\right)
\end{aligned}
$$


Let $v^{\pi}$ and $r^{\pi}$ be the vectors of length $S$ that collect the values $v^{\pi}(s)$ and $r^{\pi}(s)$ for all $s \in \mathbb{S}$, respectively:

$$
v^{\pi} \triangleq\left[\begin{array}{c}
v^{\pi}(1) \\
\vdots \\
v^{\pi}(S)
\end{array}\right] \in \mathbb{R}^{S}, \quad r^{\pi} \triangleq\left[\begin{array}{c}
r^{\pi}(1) \\
\vdots \\
r^{\pi}(S)
\end{array}\right] \in \mathbb{R}^{S}
$$

Then, Eq. (3.8) can be written in vector form as the linear system of equations:

$$
\left(I_{S}-\gamma P^{\pi}\right) v^{\pi}=r^{\pi}
$$

We shall refer to $v^{\pi}$ as the value vector. There are two challenges when we aim to obtain $v^{\pi}$ from (3.10). The first challenge is that the size of the state-space can be very large (e.g., the chess game has $10^{47}$ possible states, making (3.10) computationally intractable). The second challenge arises when the agents do not know anything about the environment, thus $P^{\pi}$ and $r^{\pi}$ are unavailable. In the following subsections we review how to address these two issues.

\subsubsection{Approximate value function as a saddle-point problem}

For the single agent scenario, references [277, 275] introduced efficient algorithms with convergence guarantees under general conditions. The algorithms save on computations by relying on features that span a space of much lower dimensionality than the size of the original state space. More formally, let $x: \mathbb{S} \rightarrow \mathbb{R}^{M}$ be some mapping from states to features, such that $x(s)$ gives the feature vector of length $M \ll S$ that represents the state $s$. Now, it would be efficient to approximate the original value function $v^{\pi}(s)$ as a parametric function of $x(s)$, for some parameter vector $w \in \mathbb{R}^{M}$. When this is done, the problem of making a prediction (i.e., estimating the value vector $v^{\pi}$ ) becomes equivalent to seeking a parameter vector $w^{\star}$ that is optimal in a certain sense. Among many parametrizations, a linear approximation of the form

$$
v^{\pi}(s) \approx x(s)^{\top} w
$$

has been extensively studied in the literature (see, e.g., [290, 251, 96, 97]) and it is promising mainly because it leads to solutions with low computational demands. Moreover, it is expected that if one chooses the mapping of features carefully, then the linear approximation model will generally provide good results (see, e.g., [187, 217, $151,328,179,42,22])$. Note that we can rewrite (3.11) as the linear projection onto a 
basis given by $M$ functions $z_{m}: \mathbb{S} \rightarrow \mathbb{R}, m=1, \ldots, M$ :

$$
x(s)^{\top} w=\sum_{m=1}^{M} z_{m}(s) w_{m}
$$

such that $w \triangleq\left(w_{m}\right)_{m=1}^{M}$, and the feature vector for state $s$ is given by

$$
x(s)^{\top}=\left[z_{1}(s), \ldots, z_{M}(s)\right]^{\top} \in \mathbb{R}^{M}
$$

By slightly abusing of notation, let the vector of the basis function for every state be denoted by

$$
z_{m}=\left[\begin{array}{c}
z_{m}(1) \\
\vdots \\
z_{m}(S)
\end{array}\right] \in \mathbb{R}^{S}
$$

Let $X$ be the matrix of size $S \times M$ formed by stacking the transposed feature vectors $x(s)^{\top}$ on top of each other or, equivalently, having the basis function vectors $z_{m}$ as columns:

$$
X \triangleq\left[\begin{array}{c}
x(1)^{\top} \\
\vdots \\
x(S)^{\top}
\end{array}\right]=\left[\begin{array}{ccc}
z_{1}(1) & \ldots & z_{M}(1) \\
\vdots & \ldots & \vdots \\
z_{1}(S) & \ldots & z_{M}(S)
\end{array}\right] \in \mathbb{R}^{S \times M}
$$

Then, the linear approximation (3.11) can be expressed in vector form as:

$$
v^{\pi} \approx X w
$$

By modeling the value function in the form (3.16), we solve for $w$ by using the approximation (3.16) in (3.10). Doing so leads to the approximate Bellman equation:

$$
X w=r^{\pi}+\gamma P^{\pi} X w
$$

We assume that the features available for the agents constitute a linearly independent set of basis functions, which effectively represent the states. Thus, $X$ is full column rank by construction. However, the fixed point equation (3.17) may not have a solution $w$ in general because the right-hand side need not lie in the range space of $X$, which we denote by $\mathbb{X}$. To address this issue, one approach is to solve instead the projected 
Bellman equation [290]:

$$
X w=\Pi\left(r^{\pi}+\gamma P^{\pi} X w\right)
$$

where $\Pi$ is a projection operator onto $\mathbb{X}$. Since $\mathbb{X}$ is a subspace of $\mathbb{R}^{M}$, the projection operator with respect to some metric norm $\|\cdot\|_{D}$ is defined as:

$$
\Pi x \triangleq \arg \min _{\bar{x} \in \mathbb{K}}\|x-\bar{x}\|_{D}^{2}
$$

where $D$ is a symmetric positive-definite matrix. The matrix $\Pi$ is given by

$$
\Pi=X\left(X^{\top} D X\right)^{-1} X^{\top} D
$$

Therefore, for different choices of $D$, we have different projection operators, each of which can be used to project the right side of (3.18) onto the range space of $X$. However, some choices for $D$ will lead to simpler solutions, as we will reveal in Subsection 3.2.4.

Equation (3.18) is now an over-determined consistent linear system of equations. To solve for $w$, reference [275] considered the weighted least-squares problem:

$$
\underset{w}{\operatorname{minimize}} \quad J_{\mathrm{PB}}(w) \triangleq\left\|\Pi\left(r^{\pi}+\gamma P^{\pi} X w\right)-X w\right\|_{D}^{2}
$$

where the cost function $J_{\mathrm{PB}}(w)$ is referred to as the projected Bellman error. Since $X w$ already lies in $\mathbb{X}$ and $D$ is positive definite, it can be verified that

$$
\begin{aligned}
J_{\mathrm{PB}}(w) & =\left\|\Pi r^{\pi}-\Pi\left(I_{S}-\gamma P^{\pi}\right) X w\right\|_{D}^{2} \\
& =\left(r^{\pi}-\left(I_{S}-\gamma P^{\pi}\right) X w\right)^{\top} \Pi^{\top} D \Pi\left(r^{\pi}-\left(I_{S}-\gamma P^{\pi}\right) X w\right) \\
& =\left(X^{\top} D r^{\pi}-B w\right)^{\top}\left(X^{\top} D X\right)^{-1}\left(X^{\top} D r^{\pi}-B w\right)
\end{aligned}
$$

where we have introduced:

$$
B \triangleq X^{\top} D\left(I_{S}-\gamma P^{\pi}\right) X
$$

Using (3.22), it can also be verified that the solution $w^{\star}$ that minimizes $J_{\mathrm{PB}}(w)$ satisfies the following normal equations [245]:

$$
B^{\top}\left(X^{\top} D X\right)^{-1} B w^{\star}=B^{\top}\left(X^{\top} D X\right)^{-1} X^{\top} D r^{\pi}
$$


Since $\left\|P^{\pi}\right\|_{\infty}=1$ and $\gamma<1$, we can bound the spectral radius of $\gamma P^{\pi}$ by

$$
\rho\left(\gamma P^{\pi}\right) \leq\left\|\gamma P^{\pi}\right\|_{\infty}=\gamma<1
$$

Thus, the inverse $\left(I_{S}-\gamma P^{\pi}\right)^{-1}$ exists. In addition, since the matrices $D$ and $X$ have full rank and full column rank, respectively, by assumption, we conclude that matrix $B$ is invertible, so the minimizer $w^{\star}$ is given by

$$
w^{\star} \triangleq \arg \min _{w \in \mathbb{R}^{M}} J_{\mathrm{PB}}=\left(X^{\top} D\left(I_{S}-\gamma P^{\pi}\right) X\right)^{-1} X^{\top} D r^{\pi}
$$

If the quantities $\left\{P^{\pi}, r^{\pi}\right\}$ were known, one would proceed to solve (3.26) and determine the desired vector $w^{\star}$ and the sought-after value vector $v^{\pi}$ from (3.16). However, we want the agents to learn $v^{\pi}$ without any prior knowledge of the environment. In other words, we cannot assume $P^{\pi}$ and $r^{\pi}$ are known. For this reason, we need to develop an alternative solution method. In the process of doing so, first for a single-agent, we shall arrive at the same gradient temporal difference method of [275] albeit by using a fundamentally different approach involving a primal-dual argument. The approach will subsequently enable us to generalize to a fully distributed solution, involving multiple agents.

So let us continue with the single-agent case for now. Our first step relies on relating Eq. (3.22) to the saddle-point conditions of a convex optimization problem. Indeed, minimizing $J_{\mathrm{PB}}(w)$ in $(3.21)$ is equivalent to the following quadratic programming problem:

$$
\begin{aligned}
\underset{\varepsilon, w}{\operatorname{minimize}} & \frac{1}{2} \varepsilon^{\top}\left(X^{\top} D X\right)^{-1} \varepsilon \\
\text { s.t. } & \varepsilon=X^{\top} D r^{\pi}-B w
\end{aligned}
$$

where we have introduced the splitting variable $\varepsilon$. Since problem (3.27) is convex and satisfies Slater's condition [48], strong duality holds and the primal and dual optimal values are attained and equal and they form a saddle-point of the Lagrangian. Specifically, the Lagrangian of (3.27) is

$$
L(\varepsilon, w, \theta)=\frac{1}{2}\|\varepsilon\|_{\left(X^{\top} D X\right)^{-1}}^{2}+\theta^{\top}\left(X^{\top} D r^{\pi}-B w-\varepsilon\right)
$$


where $\theta$ is the Lagrange multiplier. By minimizing $L(\varepsilon, w, \theta)$ over $\varepsilon$ and $w$, we obtain that the dual function is $g(\theta)=-\infty$ unless $B^{\top} \theta=0_{M}$, in which case we have

$$
g(\theta)=-\frac{1}{2} \theta^{\top} X^{\top} D X \theta+\theta^{\top} X^{\top} D r^{\pi}
$$

Therefore, the dual problem of (3.27) consists in maximizing (3.29) subject to the constraint $B^{\top} \theta=0_{M}$. Maximizing $g(\theta)$ is equivalent to minimizing $-g(\theta)$, so that we can express the dual problem of (3.27) as follows:

$$
\begin{array}{cl}
\underset{\theta}{\operatorname{minimize}} & \frac{1}{2} \theta^{\top} X^{\top} D X \theta-\theta^{\top} X^{\top} D r^{\pi} \\
\text { s.t. } & B^{\top} \theta=0_{M}
\end{array}
$$

The main reason to solve (3.30) instead of the primal problem (3.27) is that the dual formulation removes the inverse in the weighting matrix, $X^{\top} D X$. This transformation brings two benefits. First, in Sec. 3.2.4, we will see that it is straightforward to optimize (3.30) from samples. Second, as it is explained in Sections 3.3 and 3.4.2, problem (3.30) leads to a distributed algorithm in which the agents are able to combine their individual experience to solve the problem.

Had we assumed $P^{\pi}$ and $r^{\pi}$ to be known, problem (3.30) would be trivial, with unique solution $\theta=0_{M}$, since this is the only vector that satisfies the constraint. In other words, the optimal dual variable of the primal problem must be $\theta=0$, and this is something that we can readily infer from the constraint of the dual problem. However, since we do not assume any prior knowledge, we are going to employ instead a primal-dual algorithm that leads to an iterative stochastic-approximation mechanism to learn from samples. First, we derive the Lagrangian of (3.30) as

$$
\begin{aligned}
L(\theta, w) & =\frac{1}{2} \theta^{\top} X^{\top} D X \theta-\theta^{\top} X^{\top} D r^{\pi}+w^{\top} B^{\top} \theta \\
& =\theta^{\top} X^{\top} D\left(\frac{1}{2} X \theta+\left(I_{S}-\gamma P^{\pi}\right) X w-r^{\pi}\right)
\end{aligned}
$$

where $w$ denotes the Lagrange multiplier. We use the same notation $w$ to denote the dual variable for (3.31) because it can be verified that by computing the dual of the dual problem (3.30) we recover the original problem (3.21), which is equivalent to (3.27). Thus, the optimal dual variable $w^{\star}$ of (3.31) is also the optimal solution to (3.21). To find a saddle-point $\left\{\theta^{\star}, w^{\star}\right\}$ of the Lagrangian (3.31) we alternate between applying gradient descent to $L(\theta, w)$ with respect to $\theta$ and gradient ascent with respect 
to $w$ :

$$
\begin{aligned}
\theta_{i+1} & =\theta_{i}-\mu_{\theta} X^{\top} D\left(X \theta_{i}+\left(I_{S}-\gamma P^{\pi}\right) X w_{i}-r^{\pi}\right) \\
w_{i+1} & =w_{i}+\mu_{w} X^{\top}\left(I_{S}-\gamma P^{\pi}\right)^{\top} D X \theta_{i}
\end{aligned}
$$

where $\mu_{\theta}$ and $\mu_{w}$ are positive step-sizes.

Construction (3.32a)-(3.32b) is the well-known Arrow-Hurwicz algorithm (see, e.g., [23], [225, Ch. 9.3.3] and [8, Ch. 10]).

\subsubsection{Primal-dual stochastic optimization}

As mentioned before, since the agents do not have prior knowledge of the environment, we need to replace (3.32a)-(3.32b) by constructions that do not depend on the quantities $\left\{P^{\pi}, r^{\pi}\right\}$. In order to find the solution directly from samples, we need to convert these gradient iterations into stochastic approximations. The selection of an appropriate weighted norm $\|\cdot\|_{D}$ in (3.21) now becomes relevant. If we choose a weighting matrix $D$ that represents the probability distribution of visiting each state, then we can express the terms that appear in (3.32a)-(3.32b) as expectations that we can substitute with their sample estimates. We proceed to explain the details.

Recall that the state-visitation probability depends on the agent's policy. When sampling off-policy, an agent acts following a behavior policy $\phi$ that differs from the target policy $\pi$ (for which it wants to estimate the value vector) and, therefore, the sampled stationary state-visitation probability induced by $\phi$ will be different from the stationary state-visitation probability induced by $\pi$. If we assume that the stateprocess induced by the behavior policy is a stationary Markov chain, then there is a unique stationary distribution of visiting each state with all its entries being strictly positive and, therefore, after sampling long enough, the agent will visit all possible state transitions, albeit perhaps at a very different frequency of the stationary distribution that would have been induced by the target policy. This is usually referred as having the agent follow an exploratory policy. Note that this stationary assumption can be easily satisfied when the agent follows a stochastic policy with non-zero probability of making every action at every state (i.e., $\phi(a \mid s)>0, \forall(s, a) \in \mathbb{S} \times \mathbb{A}$ ).

Let us set the weighting matrix in (3.21) equal to the state-visitation probability induced by the behavior policy (which we emphasize with the corresponding superscript):

$$
D \triangleq D^{\phi} \triangleq \operatorname{diag}\left\{d^{\phi}\right\}
$$


Equations (3.32a)-(3.32b) depend on $P^{\pi}$ and $r^{\pi}$, meaning that the agent aims to predict the value vector along the expected trajectory that would have been induced by the target policy $\pi$. However, the state-visitation distribution of this trajectory, $d^{\pi}$, does not match the distribution of the samples actually gathered by the agent, given by $d^{\phi}$. Importance sampling [153, Ch. 9.7] is a technique for estimating properties of a particular distribution, while only having samples generated from a different distribution. Let us introduce importance weights that measure the dissimilarity between the target $(\pi)$ and behavior $(\phi)$ policies:

$$
\xi(a, s) \triangleq \frac{\pi(a \mid s)}{\phi(a \mid s)}
$$

By using importance sampling, reference [178] showed that we can write the gradient inside (3.32a) in terms of moment values of the behavior policy as follows:

$$
\begin{aligned}
X^{\top} D^{\phi} & \left(X \theta_{i}+\left(I_{S}-\gamma P^{\pi}\right) X w_{i}-r^{\pi}\right) \\
= & \sum_{s \in \mathbb{S}} d^{\phi}(s) x(s)\left(x(s)^{\top} \theta_{i}+\left(x(s)^{\top}-\gamma \sum_{s^{\prime} \in \mathbb{S}} p_{s s^{\prime}}^{\pi} x\left(s^{\prime}\right)^{\top}\right) w_{i}\right. \\
& \left.-\sum_{a \in \mathbb{A}} \sum_{s^{\prime} \in \mathbb{S}} \mathcal{P}\left(s^{\prime} \mid s, a\right) \pi(a \mid s) r\left(s, a, s^{\prime}\right)\right) \\
= & \sum_{s \in \mathbb{S}} \sum_{a \in \mathbb{A}} \sum_{s^{\prime} \in \mathbb{S}} \mathcal{P}\left(s^{\prime} \mid s, a\right) \pi(a \mid s) d^{\phi}(s) x(s)\left(x(s)^{\top} \theta_{i}+\left(x(s)-\gamma x\left(s^{\prime}\right)\right)^{\top} w_{i}-r\left(s, a, s^{\prime}\right)\right) \\
= & \sum_{s \in \mathbb{S}} \sum_{a \in \mathbb{A}} \sum_{s^{\prime} \in \mathbb{S}} \mathcal{P}\left(s^{\prime} \mid s, a\right) \phi(a \mid s) \xi(a, s) d^{\phi}(s) \\
& x(s)\left(x(s)^{\top} \theta_{i}+\left(x(s)-\gamma x\left(s^{\prime}\right)\right)^{\top} w_{i}-r\left(s, a, s^{\prime}\right)\right) \\
= & \mathbb{E}_{d^{\phi}, \phi, \mathcal{P}}\left[\boldsymbol{x}(s)\left(\boldsymbol{x}(s)^{\top} \theta_{i}+\left(\boldsymbol{x}(s)-\gamma \boldsymbol{x}\left(s^{\prime}\right)\right)^{\top} w_{i}-r\left(\boldsymbol{s}, \boldsymbol{a}, \boldsymbol{s}^{\prime}\right)\right) \xi(\boldsymbol{a}, \boldsymbol{s})\right]
\end{aligned}
$$

Similarly, we can express the gradient inside (3.32b) as

$$
X^{\top}\left(I_{S}-\gamma P^{\pi}\right)^{\top} D^{\phi} X \theta_{i}=\mathbb{E}_{d^{\phi}, \phi, \mathcal{P}}\left[\left(\boldsymbol{x}(s)-\gamma \boldsymbol{x}\left(s^{\prime}\right)\right) \boldsymbol{x}(s)^{\top} \xi(\boldsymbol{a}, \boldsymbol{s})\right] \theta_{i}
$$

The agent does not know these expected values though. Rather, at every time-step, the agent observes transitions of the form $\left\{x_{i}, a(i), x_{i+1}, r(i+1)\right\}$, where $x_{i} \triangleq x(s(i))$ denotes the feature vector observed at time $i$.

In addition, the agent knows both its behavior policy $\phi$ and the target policy $\pi$ that it wants to evaluate so it can compute the importance weight. Nevertheless, in an actual implementation, the agent need not know the states but just features, hence, the actual policies must be conditioned on the feature vectors. Slightly abusing notation, 
we introduce the importance weight that the node computes at every time step:

$$
\xi(i) \triangleq \frac{\pi\left(a(i) \mid x_{i}\right)}{\phi\left(a(i) \mid x_{i}\right)} \approx \frac{\pi(a(i) \mid s(i))}{\phi(a(i) \mid s(i))} \triangleq \xi(a(i), s(i))
$$

Since a sample of a random variable is an unbiased estimator of its expected value, we can build a pair of stochastic approximation recursions from (3.32a)-(3.32b) and $(3.35)-(3.36)$ :

$$
\begin{aligned}
\theta_{i+1} & =\theta_{i}-\mu_{\theta} x_{i}\left(x_{i}^{\top} \theta_{i}+\left(x_{i}-\gamma x_{i+1}\right)^{\top} w_{i}-r(i+1)\right) \xi(i) \\
w_{i+1} & =w_{i}+\mu_{w}\left(x_{i}-\gamma x_{i+1}\right) x_{i}^{\top} \theta_{i} \xi(i)
\end{aligned}
$$

Recursions (3.38a)-(3.38b) coincide with the single-agent gradient-temporal difference (GTD2) algorithm, which was derived in [275] using a different approach. The above derivation from (3.27) to (3.38b) shows that GTD2 is a stochastic Arrow-Hurwicz algorithm applied to the dual problem of (3.21). As we will see in the following sections, the proposed primal-dual derivation — which is an interesting result by itselfis convenient for a multi-agent formulation, since it leads to a meaningful in-network state-visitation distribution that combines the individual stationary distributions of the agents, thus overcoming non-exploratory individual behavior policies.

We remark that we have only considered importance sampling for the policy, but not for the state-visitation probability distribution. The reason is that, while $\pi(a \mid s)$ and $\phi(a \mid s)$ are known to the agent, the state-visitation probabilities, $d^{\pi}$ and $d^{\phi}$, are unknown a priori. As we will see in Sec. 3.4.2, this discrepancy between state-visitation probabilities will change the problem. We might consider doing importance sampling on estimates of these visitation probabilities, but this would imply other issues and is left for future study. Interestingly, as explained in Sec. 3.6, we can take advantage of the cooperative exploration of the network to compensate this discrepancy, in a manner that is very different from the importance sampling approach.

\subsection{Multi-agent learning}

We now consider a network of $N$ connected agents that operate in similar but independent MDPs. The state-space $\mathbb{S}$, action-space $\mathbb{A}$, and transition probabilities $\mathcal{P}$ are the same for every node, but their actions do not influence each other. Thus, the transition probabilities seen by each agent $k$ are only determined by its own actions, $a_{k}(i) \in \mathbb{A}$, 


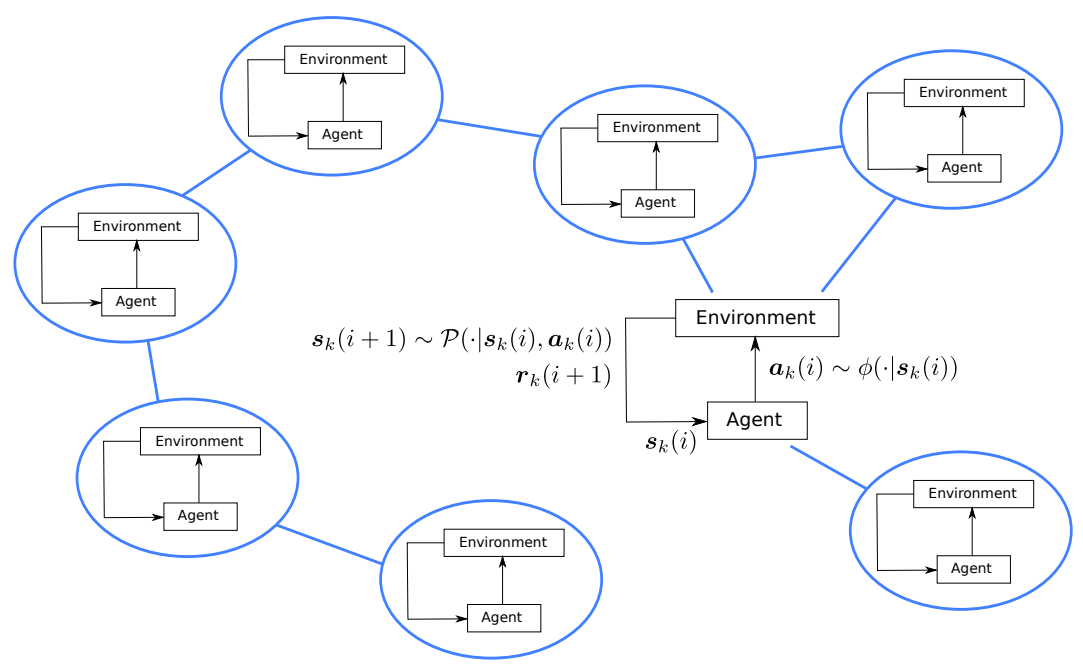

Figure 3.1 Example of the network of collaborative agents considered in this paper. Each agent operates in similar but independent environments, so that the agents do not influence each other. The agents follow different behavior policies $\left(\phi_{k} \neq \phi_{l}, \forall k, l \in \mathcal{N}\right.$, $k \neq l$ ), which are different from the target policy $\pi$ that they aim to estimate; we assume $\pi$ is the same target for all agents.

and the previous state of its environment, $s_{k}(i) \in \mathbb{S}$ :

$$
s_{k}(i+1) \sim \mathcal{P}\left(\cdot \mid s_{k}(i), a_{k}(i)\right), \quad k=1 \ldots N
$$

This assumption is convenient because it makes the problem stationary without forcing each agent to know the actions and feature vectors of every other agent in the network. The agents aim to predict the response of their environment to a common target policy $\pi$ while they follow different behavior policies, denoted by $\phi_{k}(a \mid s)$ each. We remark that if the stationary distributions of the behavior and target policies are very different, or if the behavior policies are not exploratory enough, (e.g., the agents are practically constrained to explore small regions of the state-space), then their individual estimates may have high variance.

Motivated by recent results on network behavior in [58, 247], we note that, through collaboration, each agent may contribute to the network with its own experience. Let $D^{\phi_{k}}$ be the diagonal matrix that represents the stationary state-visitation distribution induced by $\phi_{k}$ for agent $k$. We then introduce the following global problem in place of 
(3.30) with $D$ substituted by $D^{\phi_{k}}$ :

$$
\begin{aligned}
\underset{\theta}{\operatorname{minimize}} & \sum_{k=1}^{N} \tau_{k}\left(\frac{1}{2} \theta^{\top} X^{\top} D^{\phi_{k}} X \theta-\theta^{\top} X^{\top} D^{\phi_{k}} r^{\pi}\right) \\
\text { s.t. } & \sum_{k=1}^{N} \tau_{k}\left(X^{\top} D^{\phi_{k}}\left(I_{S}-\gamma P^{\pi}\right) X\right)^{\top} \theta=0
\end{aligned}
$$

where

$$
\tau=\left[\tau_{1}, \ldots, \tau_{N}\right]^{\top}
$$

is a vector of non-negative parameters, whose purpose is to weight the contribution of each agent's local problem to the global problem, such that:

$$
\begin{aligned}
\tau_{k} & \geq 0, \quad k=1, \ldots, N \\
\sum_{k=1}^{N} \tau_{k} & =1
\end{aligned}
$$

Since the dual problem (3.30) removes the inverse of the weighting matrices $X^{\top} D^{\phi_{k}} X$, we can introduce the in-network stationary distribution

$$
D^{\bar{\phi}} \triangleq \sum_{k=1}^{N} \tau_{k} D^{\phi_{k}}
$$

Note that solving the aggregated problem (3.40) is effectively solving the single-agent problem (3.30) with $D$ replaced by $D^{\bar{\phi}}$. The Lagrangian of (3.40) can be expressed as

$$
L(\theta, w)=\sum_{k=1}^{N} \tau_{k} L_{k}(\theta, w)
$$

where the individual Lagrangians are given by

$$
\begin{aligned}
L_{k}(\theta, w) & =\theta^{\top} X^{\top} D^{\phi_{k}}\left(\frac{1}{2} X \theta+\left(I_{S}-\gamma P^{\pi}\right) X w-r^{\pi}\right) \\
& =\theta^{\top} \mathbb{E}_{d^{\phi_{k}, \phi_{k}, \mathcal{P}}}\left[\boldsymbol{x}(s)\left(\frac{1}{2} \boldsymbol{x}(s)^{\top} \theta+\left(\boldsymbol{x}(s)-\gamma \boldsymbol{x}\left(s^{\prime}\right)\right)^{\top} w-r\left(\boldsymbol{s}, \boldsymbol{a}, \boldsymbol{s}^{\prime}\right)\right) \xi(\boldsymbol{a}, \boldsymbol{s})\right]
\end{aligned}
$$

which are similar to (3.31) but with stationary distribution $D^{\phi_{k}}$. 
In order to find the global saddle-point of the aggregate Lagrangian (3.45) in a cooperative and stochastic manner, we apply diffusion strategies [248, 249, 247]. We choose the adapt-then-combine (ATC) diffusion variant for distributed optimization over networks $[56,58,57]$. The algorithm consists of two-steps: the adaptation step, at which every agent updates its own intermediate estimate independently of the other agents; and the combination step, at which every agent combines its neighbors' estimates. Similar to the derivation of the single-agent algorithm (3.38a)-(3.38b), we can express the gradient of the individual Lagrangians (3.46) in terms of moment values (i.e., replacing $D^{\phi}$ by $D^{\phi_{k}}$ into (3.35)-(3.36)). We then follow a primal-dual approach and apply ATC twice:

1. We apply ATC for minimizing $L_{k}(\theta, w)$ in (3.46) over $\theta$ through stochastic gradient descent:

$$
\begin{aligned}
\widehat{\theta}_{k, i+1} & =\theta_{k, i}-\mu_{\theta} x_{k, i}\left(x_{k, i}^{\top} \theta_{k, i}+\left(x_{k, i}-\gamma x_{k, i+1}\right)^{\top} w_{k, i}-r_{k}(i+1)\right) \xi_{k}(i) \\
\theta_{k, i+1} & =\sum_{l \in \mathcal{N}_{k}} c_{l k} \widehat{\theta}_{l, i+1}
\end{aligned}
$$

2. We apply ATC again for maximizing $L_{k}(\theta, w)$ in (3.46) over $w$ through stochastic gradient ascent:

$$
\begin{aligned}
& \widehat{w}_{k, i+1}=w_{k, i}+\mu_{w}\left(x_{k, i}-\gamma x_{k, i+1}\right) x_{k, i}^{\top} \theta_{k, i} \xi_{k}(i) \\
& w_{k, i+1}=\sum_{l \in \mathcal{N}_{k}} c_{l k} \widehat{w}_{l, i+1}
\end{aligned}
$$

where $r_{k}(i+1)$ is the reward obtained by agent $k$ at time $i+1, \mathcal{N}_{k}$ stands for the neighborhood of agent $k$ (i.e., the set of agents that are able to communicate with agent $k$ in a single hop, including $k$ itself), $\hat{\theta}$ and $\hat{w}$ correspond to the locally adapted estimates, and $\theta$ and $w$ correspond to the combined estimates for the adapt-then-combine strategy.

The combination coefficients $\left\{c_{l k}\right\}$ define the weights on the links in the network and can be chosen freely by the designer, as long as they satisfy:

$$
\begin{aligned}
& c_{l k} \geq 0, \quad \sum_{l \in \mathcal{N}_{k}} c_{l k}=1 \\
& c_{l k}=0 \text { if } l \notin \mathcal{N}_{k} \\
& c_{k k}>0 \text { for at least one agent } k
\end{aligned}
$$


Let $C \triangleq\left(c_{l k}\right)_{l, k=1}^{N}$ be the combination matrix. Then, condition (3.48) implies that $C$ is left-stochastic, which is a relaxation of the conditions (2.51) presented in Chapter 2. Condition (3.49) relates the information combination process with the underlying topology, so that agents can only give positive weight to the information coming from their neighbors (i.e., only within neighborhood information is considered). Condition (3.50) means that there is at least one agent that trusts its local measurements and is able to perform its own adaptation step (i.e., we say that $C$ is aperiodic/acyclic). We also assume that the topology of the network is connected, meaning that there is at least one path between any pair of nodes (i.e., we say that $C$ is irreducible) and that the combination matrix $C$ remains fixed over time. Therefore, conditions (3.48)-(3.50) are sufficient (not necessary) to ensure that $C$ is a primitive matrix [258, Theorem 1.4] (i.e., there exists $j>0$ such that all entries of $C^{j}$ are strictly positive). It follows from the Perron-Frobenius Theorem that $C$ has a unique eigenvalue at one, while all other eigenvalues are strictly inside the unit circle (see, e.g., [258, Theorem 1.1] [124, Theorem 8.4.4]). We normalize the entries of the eigenvector that is associated with the eigenvalue at one to add up to one and refer to it as the Perron eigenvector of $C$. All its entries will be strictly positive. We we will show in Sect. 3.4.7 that the values for $\left\{\tau_{k}\right\}$ turn out to be determined by this Perron eigenvector.

Iterations (3.47a)-(3.47d) constitute the proposed diffusion off-policy GTD (DGTD) algorithm, which we remark is a fully distributed algorithm because the combination step is taken only over $\mathcal{N}_{k}$. DGTD is summarized in Algorithm 3.1.

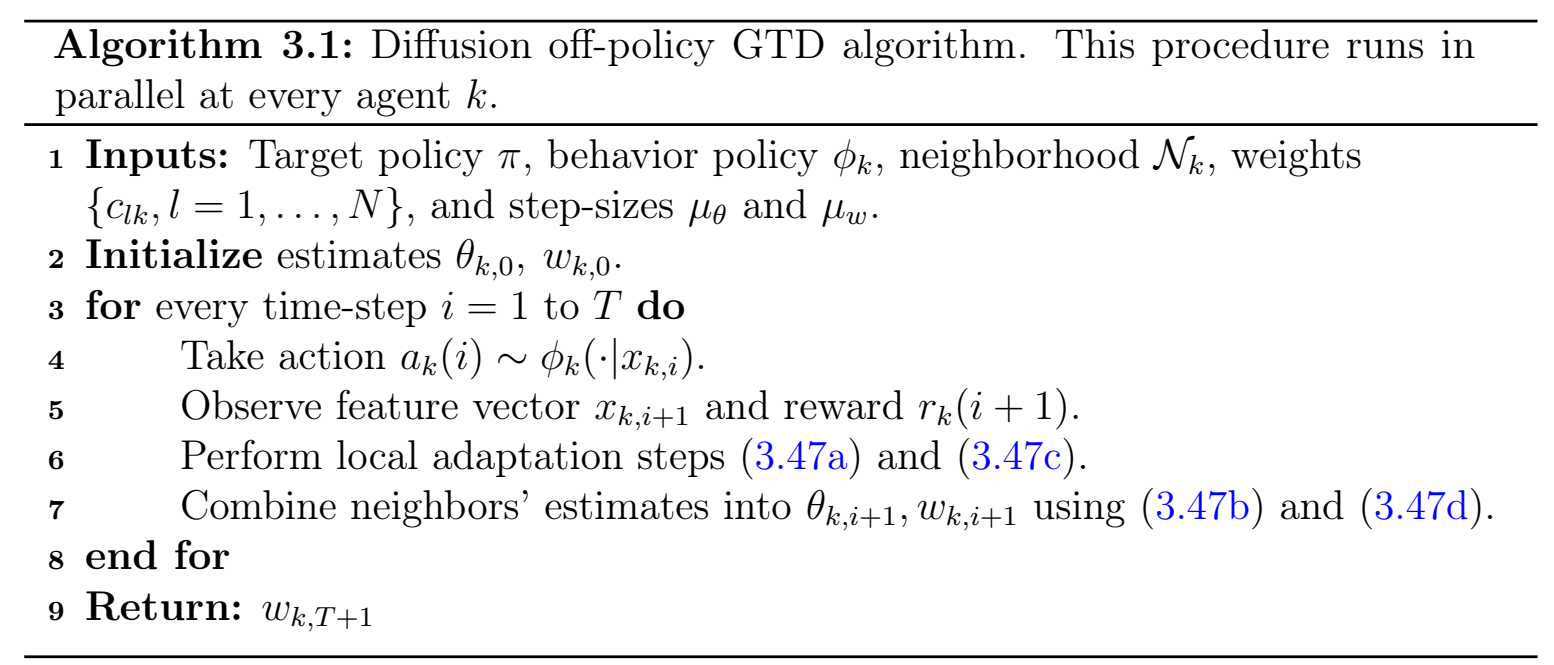

In the following section, we study the behavior of the DGTD. The analysis can be applied to GTD, since this can be seen as a particular case of DGTD with one single agent. 


\subsection{Performance analysis}

In this section we analyze the existence and uniqueness of the optimal solution to the multi-agent learning problem (3.40). We extend the energy conservation arguments of $[53,56,57,248]$ to perform a mean-square-error (MSE) analysis of the diffusion GTD algorithm (3.47a)-(3.47d) and provide convergence guarantees under sufficiently small step-sizes. We also obtain closed form expressions of the mean-square-deviation (MSD) and analyze the bias of the algorithm. We will rely on some reasonable conditions on the data, as explained next.

\subsubsection{Data model}

To begin with, we model the quantities appearing in (3.47a)-(3.47d) as instantaneous realizations of random variables, which we denote by using boldface notation. We aggregate the variables into vectors of length $2 M$ each:

$$
\begin{gathered}
\boldsymbol{\alpha}_{k, i} \triangleq\left[\begin{array}{c}
\boldsymbol{\theta}_{k, i} \\
\boldsymbol{w}_{k, i}
\end{array}\right], \quad \boldsymbol{\psi}_{k, i} \triangleq\left[\begin{array}{c}
\widehat{\boldsymbol{\theta}}_{k, i} \\
\widehat{\boldsymbol{w}}_{k, i}
\end{array}\right] \\
\boldsymbol{g}_{k, i+1} \triangleq\left[\begin{array}{c}
-\eta \boldsymbol{x}_{k, i} \boldsymbol{\xi}_{k}(i) \boldsymbol{r}_{k}(i+1) \\
0_{M}
\end{array}\right]
\end{gathered}
$$

where we are now writing $\mu_{w} \triangleq \mu$ and $\mu_{\theta} \triangleq \eta \mu_{w}$, such that $\eta>0$ is the step-size ratio between the two adaptation steps. We further introduce the following $2 M \times 2 M$ coefficient matrix:

$$
\boldsymbol{G}_{k, i+1} \triangleq\left[\begin{array}{cc}
\eta \boldsymbol{x}_{k, i} \boldsymbol{x}_{k, i}^{\top} \boldsymbol{\xi}_{k}(i) & \eta \boldsymbol{x}_{k, i}\left(\boldsymbol{x}_{k, i}-\gamma \boldsymbol{x}_{k, i+1}\right)^{\top} \boldsymbol{\xi}_{k}(i) \\
-\left(\boldsymbol{x}_{k, i}-\gamma \boldsymbol{x}_{k, i+1}\right) \boldsymbol{x}_{k, i}^{\top} \boldsymbol{\xi}_{k}(i) & 0_{M \times M}
\end{array}\right]
$$

Then, the diffusion algorithm (3.47a)-(3.47d) with stochastic variables can be expressed as the following pair of stochastic equations:

$$
\begin{aligned}
\boldsymbol{\psi}_{k, i+1} & =\boldsymbol{\alpha}_{k, i}-\mu\left(\boldsymbol{G}_{k, i+1} \boldsymbol{\alpha}_{k, i}+\boldsymbol{g}_{k, i+1}\right) \\
\boldsymbol{\alpha}_{k, i+1} & =\sum_{l \in \mathcal{N}_{k}} c_{l k} \boldsymbol{\psi}_{l, i+1}
\end{aligned}
$$

We assume the following conditions for (3.54a)-(3.54b): 
Assumption 3.1. The state transitions $\left\{\left(s_{k}(i), s_{k}(i+1)\right)\right\}$ visited by each agent $k$ are independent and identically distributed (i.i.d.) samples, with initial states $\left\{s_{k}(i)\right\}$ drawn from the stationary distribution $d^{\phi_{k}}$.

Assumption 3.2. There is some positive probability that every state is visited by at least one agent, thus $D^{\bar{\phi}}$ in (3.44) is positive-definite.

Assumption 3.3. The feature matrix $X$ and the expected reward signal $r^{\pi}$ are bounded from below and from above.

Assumption 3.4. The feature matrix $X$ is full column rank.

Assumption 3.5. The combination matrix $C=\left(c_{l k}\right)_{l, k=1}^{N}$ is left-stochastic and primitive and satisfies (3.49).

Given the sequence of states visited by each agent $\left\{s_{k}(1), s_{k}(2), \ldots, s_{k}(i), \ldots\right\}$, the segments that start and end at the same state are independent of one another. When the Markov chain that defines these state transitions has short mixing time, these segments tend to be short (see, e.g., [162]). Assumption 3.1 approximates these independent segments with sequences of just one step. This is a customary approximation (see, e.g., $[43,277,275])$ that simplifies the analysis because the tuples $\left\{\boldsymbol{x}_{k, i}, \boldsymbol{a}_{k}(i), \boldsymbol{x}_{k, i+1}, \boldsymbol{r}_{k}(i)\right\}$ become i.i.d. samples, rendering $\boldsymbol{G}_{k, i+1}$ and $\boldsymbol{g}_{k, i+1}$ independent of $\boldsymbol{\alpha}_{k, i}$. Assumption 3.2 refers to a property of the network. For a single-agent algorithm, the agent should visit every state with positive probability; otherwise it may not be able to approach the value function. Here, we impose the milder condition that every state must be visited by at least one agent. Assumption 3.3 holds for most practical implementations, and will be used in the stability analysis. Assumption 3.4 means that the basis functions $\left\{z_{1}, \ldots z_{M}\right\}$ form a basis (i.e., they are linear independent). Sufficient conditions for Assumption 3.5 are (3.48)-(3.50).

\subsubsection{Existence and uniqueness of solution}

Solving the aggregated dual problem (3.40) is equivalent to finding the saddle-points $\left\{w^{o}, \theta^{\circ}\right\}$ of the global Lagrangian (3.45). A saddle-point of the Lagrangian must satisfy (see, e.g., [48, Sec. 5.4]):

$$
L\left(\theta^{o}, w^{o}\right)=\min _{\theta} \max _{w} L(\theta, w)=\max _{w} \min _{\theta} L(\theta, w)
$$

These conditions are equivalent to the following system of linear equations:

$$
\nabla_{\theta} L(\theta, w)=X^{\top} D^{\bar{\phi}}\left(X \theta-r^{\pi}+\left(I_{S}-\gamma P^{\pi}\right) X w\right)=0_{M}
$$




$$
\nabla_{w} L(\theta, w)=X^{\top}\left(I_{S}-\gamma P^{\pi}\right)^{\top} D^{\bar{\phi}} X \theta=0_{M}
$$

To find the saddle-point $\left\{\theta^{o}, w^{o}\right\}$, we solve for $\theta$ in (3.57) first. Since Assumption 3.2 establishes that $D^{\bar{\phi}}$ is full-rank, we recall from (3.24)-(3.26) that $X^{\top} D^{\bar{\phi}}\left(\gamma P^{\pi}-I_{S}\right) X$ is invertible and, hence, $\theta^{\circ}=0_{M}$. Then, substituting $\theta^{\circ}$ into (3.56) yields:

$$
w^{o}=\left(X^{\top} D^{\bar{\phi}}\left(I_{S}-\gamma P^{\pi}\right) X\right)^{-1} X^{\top} D^{\bar{\phi}} r^{\pi}
$$

Equation (3.58) therefore illustrates one clear benefit of cooperation. If the behavior policy of some agent prevents him from exploring the entire state-space, then some of the entries of its corresponding $d^{\phi_{k}}$ will be zero and the agent may be unable to estimate the value vector on its own. Nevertheless, as long as any other agent in the network can visit these unexplored states, the matrix $D^{\bar{\phi}}$ will be positive-definite, guaranteeing the existence and uniqueness of a solution $w^{\circ}$.

We remark that the off-policy solution $w^{o}$ in (3.58) is in fact an approximation to the on-policy solution that the agents wish to predict, which is given by (3.26) when $D \triangleq D^{\pi}$ :

$$
w^{\pi}=\left(X^{\top} D^{\pi}\left(I_{S}-\gamma P^{\pi}\right) X\right)^{-1} X^{\top} D^{\pi} r^{\pi}
$$

That is, the obtained solution (3.58) is still an approximation of (3.59) because $D^{\bar{\phi}}$ is not necessarily the same as $D^{\pi}$. However, it is interesting to realize that, by using diffusion strategies, the agents can estimate the exact on-policy solution if the scalars $\left\{\tau_{k}\right\}$ could be set to satisfy

$$
\sum_{k=1}^{N} \tau_{k} d^{\phi_{k}}=d^{\pi} \quad \Leftrightarrow \quad w^{o}=w^{\pi}
$$

This idea is left for future work, as explained in Sec. 3.6.1.

In the next subsections, we analyze the conditions that allow diffusion GTD to converge to (3.58).

\subsubsection{Error recursion}

We introduce the following error measures, which measure the difference between the estimates $\left\{\boldsymbol{\alpha}_{k, i}, \boldsymbol{\psi}_{k, i}\right\}$ at time $i$ and the optimal solution $\alpha^{o}=\left(\theta^{o}, w^{o}\right)$ for each agent $k$ :

$$
\tilde{\boldsymbol{\psi}}_{k, i} \triangleq \alpha^{o}-\boldsymbol{\psi}_{k, i}
$$




$$
\tilde{\boldsymbol{\alpha}}_{k, i} \triangleq \alpha^{o}-\boldsymbol{\alpha}_{k, i}
$$

Then, subtracting both sides of (3.54a)-(3.54b) from $\alpha^{o}$, we obtain

$$
\begin{aligned}
\tilde{\boldsymbol{\psi}}_{k, i+1} & =\tilde{\boldsymbol{\alpha}}_{k, i}+\mu\left(\boldsymbol{G}_{k, i+1} \boldsymbol{\alpha}_{k, i}+\boldsymbol{g}_{k, i+1}\right) \\
& =\tilde{\boldsymbol{\alpha}}_{k, i}+\mu\left(\boldsymbol{G}_{k, i+1} \boldsymbol{\alpha}_{k, i}+\boldsymbol{G}_{k, i+1} \alpha^{o}-\boldsymbol{G}_{k, i+1} \alpha^{o}+\boldsymbol{g}_{k, i+1}\right) \\
& =\left(I_{2 M}-\mu \boldsymbol{G}_{k, i+1}\right) \tilde{\boldsymbol{\alpha}}_{k, i}+\mu\left(\boldsymbol{G}_{k, i+1} \alpha^{o}+\boldsymbol{g}_{k, i+1}\right)
\end{aligned}
$$

Using the fact that $c_{l k}=0$ if $l \notin \mathcal{N}_{k}$, the error recursion for the combination step becomes

$$
\tilde{\boldsymbol{\alpha}}_{k, i}=\sum_{l \in \mathcal{N}_{k}} c_{l k} \tilde{\boldsymbol{\psi}}_{l, i}=\sum_{l=1}^{N} c_{l k} \tilde{\boldsymbol{\psi}}_{l, i}
$$

We collect the error variables from across the network into block vectors of size $2 M N$ :

$$
\begin{aligned}
& \tilde{\boldsymbol{\psi}}_{i} \triangleq\left(\tilde{\boldsymbol{\psi}}_{k, i}\right)_{k=1}^{N} \\
& \tilde{\boldsymbol{\alpha}}_{i} \triangleq\left(\tilde{\boldsymbol{\alpha}}_{k, i}\right)_{k=1}^{N}
\end{aligned}
$$

Let $\mathcal{C}$ and $\boldsymbol{R}_{i}$ be matrices of size $2 M N \times 2 M N$ defined by

$$
\begin{aligned}
\mathcal{C} & \triangleq C \otimes I_{2 M} \\
\boldsymbol{\mathcal { R }}_{i} & \triangleq \operatorname{diag}\left\{\boldsymbol{G}_{1, i}, \ldots, \boldsymbol{G}_{N, i}\right\}
\end{aligned}
$$

and let $\mathcal{G}_{i}$ be the matrix of size $2 M N \times 2 M$ defined by

$$
\boldsymbol{\mathcal { G }}_{i} \triangleq\left(\boldsymbol{G}_{k, i},\right)_{k=1}^{N}
$$

We also introduce the vectors of length $2 M N$ :

$$
\begin{aligned}
\boldsymbol{g}_{i} & \triangleq\left(\boldsymbol{g}_{k, i}\right)_{k=1}^{N} \\
\boldsymbol{n}_{i} & \triangleq \mathcal{G}_{i} \alpha^{o}+\boldsymbol{g}_{i}
\end{aligned}
$$

Then, the individual error recursions in (3.61)-(3.62) lead to the following network relations:

$$
\begin{aligned}
& \tilde{\boldsymbol{\psi}}_{i+1}=\left(I_{2 M N}-\mu \boldsymbol{\mathcal { R }}_{i+1}\right) \tilde{\boldsymbol{\alpha}}_{i}+\mu \boldsymbol{n}_{i+1} \\
& \tilde{\boldsymbol{\alpha}}_{i+1}=\mathcal{C}^{\top} \tilde{\boldsymbol{\psi}}_{i+1}
\end{aligned}
$$


which can be grouped together into the following network recursion:

$$
\tilde{\boldsymbol{\alpha}}_{i+1}=\mathcal{C}^{\top}\left(I_{2 M N}-\mu \boldsymbol{\mathcal { R }}_{i+1}\right) \tilde{\boldsymbol{\alpha}}_{i}+\mu \mathcal{C}^{\top} \boldsymbol{n}_{i+1}
$$

This recursion shows how the error dynamics evolves over the network over time.

\subsubsection{Convergence in the mean}

Introduce the following expected values for each agent:

$$
\begin{aligned}
& G_{k} \triangleq \mathbb{E}\left[\boldsymbol{G}_{k, i}\right]=\left[\begin{array}{cc}
\eta X^{\top} D^{\phi_{k}} X & \eta X^{\top} D^{\phi_{k}}\left(I_{S}-\gamma P^{\pi}\right) X \\
-X^{\top}\left(I_{S}-\gamma P^{\pi}\right)^{\top} D^{\phi_{k} X} & 0_{M \times M}
\end{array}\right] \\
& g_{k} \triangleq \mathbb{E}\left[\boldsymbol{g}_{k, i}\right]=\left[\begin{array}{c}
-\eta X^{\top} D^{\phi_{k}} r^{\pi} \\
0_{M}
\end{array}\right]
\end{aligned}
$$

Since Assumption 3.1 implies that the variables $\boldsymbol{\mathcal { R }}_{i+1}$ and $\tilde{\boldsymbol{\alpha}}_{i}$ are independent of each other, then by taking expectations of both sides of (3.74) we obtain

$$
\mathbb{E}\left[\tilde{\boldsymbol{\alpha}}_{i+1}\right]=\mathcal{C}^{\top}\left(I_{2 M N}-\mu \mathcal{R}\right) \mathbb{E}\left[\tilde{\boldsymbol{\alpha}}_{i}\right]+\mu \mathcal{C}^{\top}\left(\mathcal{G} \alpha^{o}+g\right)
$$

where

$$
\begin{aligned}
\mathcal{R} & \triangleq \mathbb{E}\left[\boldsymbol{\mathcal { R }}_{i}\right]=\operatorname{diag}\left\{G_{1}, \ldots, G_{N}\right\} \\
\mathcal{G} & \triangleq \mathbb{E}\left[\mathcal{G}_{i}\right]=\left(G_{k}\right)_{k=1}^{N} \\
g & \triangleq \mathbb{E}\left[\boldsymbol{g}_{i}\right]=\left(g_{k}\right)_{k=1}^{N}
\end{aligned}
$$

Therefore, the convergence of (3.77) is guaranteed when the matrix $\mathcal{C}^{\top}\left(I_{2 M N}-\mu \mathcal{R}\right)$ is stable.

Theorem 3.1 (Mean convergence). For the data model of Section 3.4.1, there exists small enough step-sizes, say $0<\mu<\mu^{o}$ (for some $\mu^{o}>0$ given by (3.196) in Appendix 3.8), such that the matrix $\mathcal{C}^{\top}\left(I_{2 M N}-\mu \mathcal{R}\right)$ is stable and, therefore, the mean-error recursion (3.77) is stable for every agent $k=1, \ldots, N$ and converges to the bias value given by

$$
\tilde{\alpha}_{\infty} \triangleq \lim _{i \rightarrow \infty} \mathbb{E}\left[\tilde{\boldsymbol{\alpha}}_{i}\right]=\left(I_{2 M N}-\mathcal{C}^{\top}\left(I_{2 M N}-\mu \mathcal{R}\right)\right)^{-1} \mu \mathcal{C}^{\top}\left(\mathcal{G} \alpha^{o}+g\right)
$$

Proof: See Appendix in Section 3.8. 
As it is explained in Appendix 3.8, the value $\mu^{o}$ only depends on the inputs of the algorithm, namely data-samples (state-features, actions and rewards), the weightedtopology matrix $C$, the cost-weights $\left\{\tau_{k}\right\}$, and the step-size ratio parameter $\eta$.

\subsubsection{Mean-square stability}

Although the error vector converges in the mean, we still need to ensure that it has bounded fluctuations around its fixed point value. To do so, we study the evolution and steady-state value of the variance. Note that real-vector-valued random variables are simply measurable, real-vector-valued functions defined on the sample space, which is a complete vector space where we can define the following norm (see, e.g., [169, Theorem A.11]):

$$
\left\|\tilde{\boldsymbol{\alpha}}_{i}\right\| \triangleq\left(\mathbb{E}\left[\sum_{m=1}^{M}\left|\tilde{\boldsymbol{\alpha}}_{i}(m)\right|^{2}\right]\right)^{1 / 2}
$$

where $\tilde{\boldsymbol{\alpha}}_{i}(m)$ denotes the $m$-th component of $\tilde{\boldsymbol{\alpha}}_{i}$. Moreover, we can define inner products of the form:

$$
\begin{gathered}
\left\|\tilde{\boldsymbol{\alpha}}_{i}\right\|^{2} \triangleq \mathbb{E}\left[\tilde{\boldsymbol{\alpha}}_{i}^{\top} \tilde{\boldsymbol{\alpha}}_{i}\right] \\
\left\|\tilde{\boldsymbol{\alpha}}_{i}\right\|_{\Sigma}^{2} \triangleq \mathbb{E}\left[\tilde{\boldsymbol{\alpha}}_{i}^{\top} \Sigma \tilde{\boldsymbol{\alpha}}_{i}\right]
\end{gathered}
$$

We remark that (3.83) is the error variance, which we aim to study in this section.

By computing the weighted inner product of both sides of (3.74) - using an arbitrary positive (semi)definite weighting matrix $\Sigma$ that we are free to choose - and applying the expectation operator, we obtain the following variance relation:

$$
\begin{aligned}
\left\|\tilde{\boldsymbol{\alpha}}_{i+1}\right\|_{\Sigma}^{2} & =\left\|\tilde{\boldsymbol{\alpha}}_{i}\right\|_{\Sigma^{\prime}}^{2}+\left\|\mu \mathcal{C}^{\top} \boldsymbol{n}_{i+1}\right\|_{\Sigma}^{2}+2 \mathbb{E}\left[\mu \boldsymbol{n}_{i+1}^{\top} \mathcal{C} \Sigma \mathcal{C}^{\top}\left(I_{2 M N}-\mu \boldsymbol{\mathcal { R }}_{i+1}\right) \tilde{\boldsymbol{\alpha}}_{i}\right] \\
& =\left\|\tilde{\boldsymbol{\alpha}}_{i}\right\|_{\Sigma^{\prime}}^{2}+2 b_{\Sigma}^{\top} \mathbb{E}\left[\tilde{\boldsymbol{\alpha}}_{i}\right]+\operatorname{Tr}\left(\mu^{2} \Sigma \mathcal{C}^{\top} \mathcal{R}_{n} \mathcal{C}\right)
\end{aligned}
$$

where we have introduced the following shorthands:

$$
\begin{aligned}
\Sigma^{\prime} \triangleq & \mathbb{E}\left[\left(I_{2 M N}-\mu \boldsymbol{\mathcal { R }}_{i+1}^{\top}\right) \mathcal{C} \Sigma \mathcal{C}^{\top}\left(I_{2 M N}-\mu \boldsymbol{\mathcal { R }}_{i+1}\right)\right] \\
= & \left(I_{2 M N}-\mu \mathcal{R}^{\top}\right) \mathcal{C} \Sigma \mathcal{C}^{\top}\left(I_{2 M N}-\mu \mathcal{R}\right) \\
& +\mu^{2} \mathbb{E}\left[\left(\boldsymbol{\mathcal { R }}_{i+1}-\mathcal{R}\right)^{\top} \mathcal{C} \Sigma \mathcal{C}^{\top}\left(\boldsymbol{\mathcal { R }}_{i+1}-\mathcal{R}\right)\right] \\
b_{\Sigma} \triangleq & \mu \mathbb{E}\left[\left(I_{2 M N}-\mu \boldsymbol{\mathcal { R }}_{i+1}^{\top}\right) \mathcal{C} \Sigma \mathcal{C}^{\top} \boldsymbol{n}_{i+1}\right] \\
\mathcal{R}_{n} \triangleq & \mathbb{E}\left[\boldsymbol{n}_{i} \boldsymbol{n}_{i}^{\top}\right]=\mathbb{E}\left[\left(\mathcal{G}_{i} \alpha^{o}+\boldsymbol{g}_{i}\right)\left(\boldsymbol{G}_{i} \alpha^{o}+\boldsymbol{g}_{i}\right)^{\top}\right]
\end{aligned}
$$


Let us vectorize matrix $\Sigma$ into a vector of length $2 M N$ :

$$
\sigma \triangleq \operatorname{vec}(\Sigma)
$$

Using the following Kronecker product property [245, Lemma B.8]:

$$
\operatorname{vec}(Y \Sigma Z)=\left(Z^{\top} \otimes Y\right) \operatorname{vec}(\Sigma)
$$

we can vectorize $\Sigma^{\prime}$ in (3.86) and find that its vector form is related to $\Sigma$ via the following linear relation:

$$
\sigma^{\prime} \triangleq \operatorname{vec}\left(\Sigma^{\prime}\right)=\mathcal{F} \sigma
$$

where the matrix $\mathcal{F}$ is given by

$$
\begin{aligned}
\mathcal{F} \triangleq & \left(\left(I_{2 M N}-\mu \mathcal{R}^{\top}\right) \mathcal{C}\right) \otimes\left(\left(I_{2 M N}-\mu \mathcal{R}^{\top}\right) \mathcal{C}\right) \\
& +\mu^{2} \mathbb{E}\left[\left(\left(\boldsymbol{\mathcal { R }}_{i+1}^{\top}-\mathcal{R}^{\top}\right) \mathcal{C}\right) \otimes\left(\left(\boldsymbol{\mathcal { R }}_{i+1}^{\top}-\mathcal{R}^{\top}\right) \mathcal{C}\right)\right]
\end{aligned}
$$

Furthermore, using this property:

$$
\operatorname{Tr}(\Sigma Y)=\left(\operatorname{vec}\left[Y^{\top}\right]\right)^{\top} \sigma
$$

we can rewrite (3.85) as:

$$
\left\|\tilde{\boldsymbol{\alpha}}_{i+1}\right\|_{\sigma}^{2}=\left\|\tilde{\boldsymbol{\alpha}}_{i}\right\|_{\mathcal{F} \sigma}^{2}+2 \sigma^{\top} \mathcal{U} \mathbb{E}\left[\tilde{\boldsymbol{\alpha}}_{i}\right]+h^{\top} \sigma
$$

where

$$
\begin{aligned}
\mathcal{U} & \triangleq \mu \mathbb{E}\left[\left(\mathcal{C}^{\top} \boldsymbol{n}_{i+1}\right) \otimes\left(\mathcal{C}^{\top}\left(I_{2 M N}-\mu \boldsymbol{R}_{i+1}\right)\right)\right] \\
h & \triangleq \mu^{2} \operatorname{vec}\left[\mathcal{C}^{\top} \mathcal{R}_{n} \mathcal{C}\right]
\end{aligned}
$$

In (3.94) we are using the notation $\|x\|_{\sigma}^{2}$ to represent $\|x\|_{\Sigma}^{2}$. Note that (3.94) is not a true recursion because the weighting matrices corresponding to $\sigma$ and $\mathcal{F} \sigma$ are different. Moreover, recursion (3.94) is coupled with the mean-error recursion (3.77). To study the convergence of (3.94) we will expand it into a state-space model following [245, 3]. Let $L \triangleq 2 M N$ and let $p(x)$ denote the characteristic polynomial of the $L^{2} \times L^{2}$ matrix 
$\mathcal{F}$, given by

$$
p(x) \triangleq \operatorname{det}(x I-\mathcal{F})=x^{L^{2}}+p_{L^{2}-1} x^{L^{2}-1}+\ldots+p_{0}
$$

By the Cayley-Hamilton Theorem [245], we know that every matrix satisfies its characteristic equation (i.e., $p(\mathcal{F})=0$ ), so that

$$
\mathcal{F}^{L^{2}}=-p_{0} I_{L^{2}}-p_{1} \mathcal{F}-\ldots-p_{L^{2}-1} \mathcal{F}^{L^{2}-1}
$$

Replacing $\sigma$ in (3.94) by $\mathcal{F}^{j} \sigma, j=0, \ldots, L^{2}-1$, we can derive the following state-space model:

$$
\begin{aligned}
& \underbrace{\left[\begin{array}{c}
\left\|\tilde{\boldsymbol{\alpha}}_{i+1}\right\|_{\sigma}^{2} \\
\left\|\tilde{\boldsymbol{\alpha}}_{i+1}\right\|_{\mathcal{F} \sigma}^{2} \\
\vdots \\
\left\|\tilde{\boldsymbol{\alpha}}_{i+1}\right\|_{\mathcal{F}^{2}-1}^{2}
\end{array}\right]}_{\mathcal{W}_{i+1}}=\underbrace{\left[\begin{array}{ccccc}
0 & 1 & 0 & \cdots & 0 \\
0 & 0 & 1 & \cdots & 0 \\
\vdots & & \ddots & 0 \\
0 & 0 & 0 & \cdots & 1 \\
-p_{0}-p_{1}-p_{2} \cdots & \cdots & -p_{L^{2}-1}
\end{array}\right]}_{\mathcal{T}} \underbrace{\left[\begin{array}{c}
\left\|\tilde{\boldsymbol{\alpha}}_{i}\right\|_{\sigma}^{2} \\
\left\|\tilde{\boldsymbol{\alpha}}_{i}\right\|_{\mathcal{F} \sigma}^{2} \\
\vdots \\
\left\|\tilde{\boldsymbol{\alpha}}_{i}\right\|_{\mathcal{F} L^{2}-1}^{2}
\end{array}\right]}_{\mathcal{W}_{i}} \\
& +2 \underbrace{\left[\begin{array}{c}
\sigma^{\top} \mathcal{U} \\
\sigma^{\top} \mathcal{F U} \\
\vdots \\
\sigma^{\top} \mathcal{F}^{L^{2}-1} \mathcal{U}
\end{array}\right]}_{\mathcal{Q}} \mathbb{E}\left[\tilde{\boldsymbol{\alpha}}_{i}\right]+\underbrace{\left[\begin{array}{c}
h^{\top} \sigma \\
h^{\top} \mathcal{F} \sigma \\
\vdots \\
h^{\top} \mathcal{F}^{L^{2}-1} \sigma
\end{array}\right]}_{\mathcal{Y}}
\end{aligned}
$$

Introduce

$$
n \triangleq \mathbb{E}\left[\boldsymbol{n}_{i}\right]=\mathcal{G} \alpha^{o}+g
$$

We combine (3.99) with the mean-recursion (3.77) and rewrite them more compactly as:

$$
\left[\begin{array}{c}
\mathcal{W}_{i+1} \\
\mathbb{E}\left[\tilde{\boldsymbol{\alpha}}_{i+1}\right]
\end{array}\right]=\left[\begin{array}{cc}
\mathcal{T} & 2 \mathcal{Q} \\
0 & \mathcal{C}^{\top}\left(I_{2 M N}-\mu \mathcal{R}\right)
\end{array}\right]\left[\begin{array}{c}
\mathcal{W}_{i} \\
\mathbb{E}\left[\tilde{\boldsymbol{\alpha}}_{i}\right]
\end{array}\right]+\left[\begin{array}{c}
\mathcal{Y} \\
\mathcal{C}^{\top} n
\end{array}\right]
$$

We are ready to state conditions for mean-square stability. 
Theorem 3.2 (Mean-square stability). Assume the step-size parameter $\mu$ is sufficiently small so that terms that depend on higher-order powers of $\mu$ can be ignored. Then, for the data model of Section 3.4.1, there exists $0<\mu_{\mathrm{MS}}^{o} \leq \mu^{o}$ (for $\mu^{o}$ used in Theorem 3.1 and given by (3.196) in Appendix 3.8), such that when $0<\mu<\mu_{\mathrm{MS}}^{o}$, the variance recursion (3.101) is mean-square stable.

Proof: Observe that the stability of the joint recursion (3.101) is equivalent to the stability of the matrices $\mathcal{T}$ and $\mathcal{C}^{\top}\left(I_{2 M N}-\mu \mathcal{R}\right)$, which is further equivalent to the following conditions on their spectral radii:

$$
\left\{\begin{array}{l}
\rho\left(\mathcal{C}^{\top}\left(I_{2 M N}-\mu \mathcal{R}\right)\right)<1 \\
\rho(\mathcal{T})<1
\end{array}\right.
$$

The first condition is the same mean-stability condition that was discussed in Theorem 3.1. For the second condition, we note from (3.99) that $\mathcal{T}$ is in companion form, and it is known that its eigenvalues are the roots of $p(x)$, which are also the eigenvalues of $\mathcal{F}$. Therefore, a necessary and sufficient condition for the stability of $\mathcal{T}$ is the stability of the matrix $\mathcal{F}$. When the step-sizes are small enough, the last term in (3.92) can be ignored since it depends on $\mu^{2}$ and we can write

$$
\mathcal{F} \approx\left(\mathcal{C}^{\top}\left(I_{2 M N}-\mu \mathcal{R}\right)\right)^{\top} \otimes\left(\mathcal{C}^{\top}\left(I_{2 M N}-\mu \mathcal{R}\right)\right)^{\top}
$$

which is stable if $\mathcal{C}^{\top}\left(I_{2 M N}-\mu \mathcal{R}\right)$ is stable.

We remark that $0<\mu_{\mathrm{MS}}^{o} \leq \mu^{o}$ is chosen to dismiss higher-order powers of $\mu$, and that $\mu^{o}$ (see Appendix 3.8) only depends on the inputs of the algorithm (i.e., data-samples, the weighted-topology matrix $C$, the weights $\left\{\tau_{k}\right\}$ and the parameter $\eta$ ).

\subsubsection{Mean-square performance}

Taking the limit of both sides of (3.94) we obtain:

$$
\lim _{i \rightarrow \infty}\left\|\tilde{\boldsymbol{\alpha}}_{i+1}\right\|_{\sigma}^{2}=\lim _{i \rightarrow \infty}\left\|\tilde{\boldsymbol{\alpha}}_{i}\right\|_{\mathcal{F} \sigma}^{2}+2 \sigma^{\top} \mathcal{U} \lim _{i \rightarrow \infty} \mathbb{E}\left[\tilde{\boldsymbol{\alpha}}_{i}\right]+h^{\top} \sigma
$$

Theorem 3.1 guarantees that

$$
\lim _{i \rightarrow \infty} \mathbb{E}\left[\tilde{\boldsymbol{\alpha}}_{i}\right]=\tilde{\alpha}_{\infty}
$$


Hence, the steady-state variance recursion in (3.104) leads to

$$
\lim _{i \rightarrow \infty}\left\|\tilde{\boldsymbol{\alpha}}_{i}\right\|_{\sigma}^{2}=q^{\top}(I-\mathcal{F})^{-1} \sigma
$$

where

$$
q \triangleq h+2 \mathcal{U} \tilde{\alpha}_{\infty}
$$

Result (3.106) is useful because it allows us to derive several performance metrics through the proper selection of the free weighting parameter vector $\sigma$ (or, equivalently, the parameter matrix $\Sigma$ ). For example, the network mean-square-deviation (MSD) is defined as the average of the MSD of all the agents in the network:

$$
\begin{aligned}
\mathrm{MSD}^{\text {network }} & \triangleq \lim _{i \rightarrow \infty} \frac{1}{N} \sum_{k=1}^{N}\left\|\tilde{\boldsymbol{\alpha}}_{k, i}\right\|^{2} \\
& =\lim _{i \rightarrow \infty}\left\|\tilde{\boldsymbol{\alpha}}_{i}\right\|_{\frac{1}{N} I_{2 M N}}^{2}
\end{aligned}
$$

Choosing the weighting matrix in (3.106) as $\Sigma=I_{2 M N} / N$, we get:

$$
\mathrm{MSD}^{\text {network }}=\frac{1}{N} q^{\top}(I-\mathcal{F})^{-1} \operatorname{vec}\left(I_{2 M N}\right)
$$

We can also obtain the MSD of any particular node $k$, as

$$
\mathrm{MSD}_{k} \triangleq \lim _{i \rightarrow \infty}\left\|\tilde{\boldsymbol{\alpha}}_{i}\right\|_{\mathcal{J}_{k}}^{2}
$$

where $\mathcal{J}_{k}$ is a block-diagonal matrix of $N$ blocks of size $2 M \times 2 M$, such that all blocks in the diagonal are zero except for block $k$ which is the identity matrix. Then, we can express

$$
\mathrm{MSD}_{k}=\lim _{i \rightarrow \infty}\left\|\tilde{\boldsymbol{\alpha}}_{k}\right\|_{\mathcal{J}_{k}}^{2}
$$

Following the same procedure as with the network MSD, we obtain the MSD for node $k$ :

$$
\operatorname{MSD}_{k}=q^{\top}(I-\mathcal{F})^{-1} \operatorname{vec}\left(\mathcal{J}_{k}\right)
$$




\subsubsection{Bias analysis}

We showed in (3.58) that, under Assumption 3.2, there exists a unique solution $\alpha^{o}$ for the global optimization problem (3.40). On the other hand, the error recursion (3.77) converges in the mean-square sense to some bias value $\tilde{\alpha}_{\infty}$. Now, we examine under which conditions $\tilde{\alpha}_{\infty}$ is small when the step-size is small. The analysis of the bias value $\tilde{\alpha}_{\infty}$ in (3.81) is similar to the examination developed in [57, Theorem 3] for multi-objective optimization. The main difference lies in the fact that we do not assume the matrix $\mathcal{R}$ in (3.78) to be symmetric.

Theorem 3.3 (Bias at small step-size). Consider the data model of Section 3.4.1, where the combination matrix $C$ satisfies Assumption 3.5 (i.e., it is primitive leftstochastic). Suppose the Perron eigenvector that corresponds to the eigenvalue of $C$ at one is equal to the vector of weights $\left\{\tau_{k}\right\}$ in the global problem (3.40) (i.e., $\tau^{\top} C=\tau^{\top}$ and $\left.\tau^{\top} \mathbb{1}_{N}=1\right)$. Assume further that the step-size $\mu$ is sufficiently small to ensure mean-square stability. Then, it holds that

$$
\tilde{\alpha}_{\infty}=O(\mu)
$$

Proof: We follow an argument similar to [57, 336]. It suffices to show that $\lim _{\mu \rightarrow 0} \frac{\left\|\tilde{\alpha}_{\infty}\right\|}{\mu}=\varepsilon_{o}$, where $\varepsilon_{o}$ is a constant independent of $\mu$. Substituting (3.153), (3.155) and (3.165) into (3.81) yields

$$
\tilde{\alpha}_{\infty}=\mu\left(Y_{C} \otimes I_{2 M}\right)\left(I_{2 M N}-\mathcal{S}\right)^{-1}\left(Y_{C}^{-1} \otimes I_{2 M}\right) \mathcal{C}^{\top}\left(\mathcal{G} \alpha^{o}+g\right)
$$

Expanding (3.67), (3.152) and (3.158) into (3.114) leads to

$$
\tilde{\alpha}_{\infty}=\mu\left(Y_{C} \otimes I_{2 M}\right)\left(I_{2 M N}-\mathcal{S}\right)^{-1}\left(J_{C} \otimes I_{2 M}\right)\left[\begin{array}{c}
\left(p^{\top} \otimes I_{2 M}\right)\left(\mathcal{G} \alpha^{o}+g\right) \\
\left(Y_{C}^{l} \otimes I_{2 M}\right)\left(\mathcal{G} \alpha^{o}+g\right)
\end{array}\right]
$$

From now on, assume that the weights used in (3.40) in defining the global cost are the entries of the Perron eigenvector of $C$, i.e.:

$$
p \triangleq \tau
$$


Then, the first row of the last term in (3.115) stands for the saddle-point conditions of the Lagrangian of the global problem (3.45):

$$
\begin{aligned}
\left(\tau^{\top} \otimes I_{2 M}\right)\left(\mathcal{G} \alpha^{o}+g\right) & =\sum_{k=1}^{N} \tau_{k}\left(G_{k} \alpha^{o}+g_{k}\right) \\
& =\left[\begin{array}{c}
\eta X^{\top} D^{\bar{\phi}}\left(X \theta^{o}+\left(I_{S}-\gamma P^{\pi}\right) X w^{o}-r^{\pi}\right) \\
-X^{\top}\left(I_{S}-\gamma P^{\pi}\right)^{\top} D^{\bar{\phi}} X \theta^{o}
\end{array}\right] \\
& =0_{2 M}
\end{aligned}
$$

Therefore, expanding $J_{C}$ into (3.115) yields

$$
\tilde{\alpha}_{\infty}=\mu\left(Y_{C} \otimes I_{2 M}\right)\left(I_{2 M N}-\mathcal{S}\right)^{-1}\left[\begin{array}{c}
0_{2 M} \\
\left(J_{C}^{0} \otimes I_{2 M}\right)\left(Y_{C}^{l} \otimes I_{2 M}\right)\left(\mathcal{G} \alpha^{o}+g\right)
\end{array}\right]
$$

From (3.165), we know that the upper-left block of $I_{2 M N}-\mathcal{S}$ is given by $\mu \bar{G}$. By using [23, Theorem 3.6], we establish that the similar matrix $\bar{G}_{\sqrt{\eta}}$ in (3.187) is invertible. Therefore, $\bar{G}$ is also invertible so we can use the following relation

$$
\left[\begin{array}{ll}
Z_{11} & Z_{12} \\
Z_{21} & Z_{22}
\end{array}\right]^{-1}=\left[\begin{array}{cc}
Z_{11}^{-1}+Z_{11}^{-1} Z_{12} U Z_{21} Z_{11}^{-1} & -Z_{11}^{-1} Z_{12} U \\
-U Z_{21} Z_{11}^{-1} & U
\end{array}\right]
$$

where $U=\left(Z_{22}-Z_{21} Z_{11}^{-1} Z_{12}\right)^{-1}$, to write

$$
\left(I_{2 M N}-\mathcal{S}\right)^{-1}=\left[\begin{array}{ll}
H_{11} & H_{12} \\
H_{21} & H_{22}
\end{array}\right]
$$

In this way, relation (3.118) simplifies to

$$
\tilde{\alpha}_{\infty}=\mu\left(Y_{C} \otimes I_{2 M}\right)\left[\begin{array}{l}
H_{12}\left(J_{C}^{0} Y_{C}^{l} \otimes I_{2 M}\right)\left(\mathcal{G} \alpha^{o}+g\right) \\
H_{22}\left(J_{C}^{0} Y_{C}^{l} \otimes I_{2 M}\right)\left(\mathcal{G} \alpha^{o}+g\right)
\end{array}\right]
$$

The only terms in (3.121) that depend on $\mu$ are $H_{12}$ and $H_{22}$. In the limit, when $\mu \rightarrow 0$ these two terms become independent of $\mu$ :

$$
\begin{aligned}
& \lim _{\mu \rightarrow 0} H_{12}=-\bar{G}^{-1} \mathcal{E}_{12}\left(I_{2 M N}-J_{C}^{0} \otimes I_{2 M}\right)^{-1} \\
& \lim _{\mu \rightarrow 0} H_{22}=\left(I_{2 M N}-J_{C}^{0} \otimes I_{2 M}\right)^{-1}
\end{aligned}
$$

Hence, we can conclude that $\lim _{\mu \rightarrow 0} \frac{\left\|\tilde{\alpha}_{\infty}\right\|}{\mu}=\varepsilon_{o}$. 
We remark that the bias in (3.113) comes from agents following different behavior policies, which means that they are solving different optimization problems, with different minimizer each. When they use diffusion strategies, the combination step pulls them toward the global solution. Nevertheless, the adaptation step pushes each agent towards the minimizer of its individual cost function. Note, however, that if all agents followed the same behavior policy, their individual optimization problems would be identical, therefore, both the adaptation and the combination steps would pull them toward the global solution and their fixed-point estimates would be unbiased with respect to the solution of the global optimization problem (3.40). This case is studied in the following corollary.

Corollary 3.1 (Bias for a common behavior policy). Let Assumptions 3.1-3.3 hold. If every agent follows the same behavior policy $\phi$ (i.e., $\left.\phi_{k}=\phi, k=1, \ldots, N\right)$, then it holds that

$$
\tilde{\alpha}_{\infty}=0
$$

Proof: Under the same behavior policy, the following magnitudes would be also the same for every agent $k=1, \ldots, N$ :

$$
\begin{gathered}
G_{k} \triangleq \bar{G} \\
g_{k} \triangleq \bar{g}
\end{gathered}
$$

Hence, the saddle-point conditions of the Lagrangian of the global problem (3.45) would not depend on the combination weights $\left\{\tau_{k}\right\}$ :

$$
\sum_{k=1}^{N} \tau_{k}\left(\bar{G} \alpha^{o}+\bar{g}\right)=\bar{G} \alpha^{o}+\bar{g}=0_{2 M}
$$

Therefore, if all the agents followed the same behavioral policy, then

$$
\mathcal{G} \alpha^{o}+g=0_{2 M N}
$$

and, from (3.81), we would conclude that $\tilde{\alpha}_{\infty}=0$. 


\subsection{Simulations}

In this section we illustrate the performance of the proposed D-GTD cooperative algorithm on a toy example, compared to two alternative approaches:

- Noncooperative: the same set of agents running the single agent GTD algorithm.

- Centralized: a central node gathering all the samples from every agent in the network and running the single agent GTD algorithm.

The example is as follows. Consider a group of animals foraging in a 2D-world (see Figure 3.2). The group forms a network of $N=15$ agents with arbitrarily connected topology and neighborhood size varying between 2 and 9 , i.e., $|\mathcal{N}|_{k} \in\{2,3, \ldots, 9\}$ . The weights of the communication links (i.e., the $c_{l k}$ elements of $C$ ) are obtained independently by each node following an averaging rule [40, 248], such that equal weight is given to any member of the neighborhood, including itself:

$$
c_{l k}=\frac{1}{\left|\mathcal{N}_{k}\right|}, \quad \forall l \in \mathcal{N}_{k}
$$

We remark that this rule leads to a left (rather than doubly) stochastic combination matrix $C$ that satisfies (3.48)-(3.50). We assume that the combination matrix $C$-and, hence, the network topology-remains fixed.

The world is a continuous bounded square, discretized into 20 rows and 20 columns. which amounts to $20 \times 20=400$ possible locations. The agents move in four possible directions, namely, $\mathbb{A}=\{$ north, south, east, west $\}$. The probability of moving along the direction of the action is one, unless there is a wall in the direction of the agent, in such case, the agent remains in the same position with probability one. Apart of the moving agents, the rest of the environment remains static.

We consider the case in which the agents are territorial and tend to settle in different regions each. In other words, the behavior policies of the agents $\left\{\phi_{k}\right\}$ are all different and constrain exploration to some regions of the state-space. At every time-step, each agent is attracted to the state at the center of its territory (green circle) with 0.8 probability, and it moves in a different direction with 0.2 probability.

The reward function is the same for all agents; and the reward obtained by each agent at every time-step only depends on its own position. At every time step, the agents move and consume some energy (i.e., they receive some negative reward). In the north-east corner of the world, there is food, which the agents understand as positive reward. However, there is a large area below the food with a predator that is harmful 
to go through, so agents receive large negative reward if they visit these states. The predator's area is the rectangle $5 \leq$ column $\leq 20,8 \leq$ row $\leq 15$. The reward at every time step is -1 , except when crossing the predator's area where it becomes -15 , and when eating the food when it rises to +20 .

Since the agents do not influence each other, we can equivalently consider that each agent is operating in its own MDP, which is equal to the MDP of every other agent. Thus, the state space of each agent's MDP is $\mathbb{S}=\{1, \ldots, 400\}, S=400$, and the state is the location of the agent. But this state is unknown. Instead, each agent self-localizes itself in the grid by sensing a Gaussian radial basis function of its distance to each of the $8 \times 8=64$ fixed markers. These markers (depicted by a yellow star in the picture) are equidistantly located per row and per column in the grid. The mean, $\mu_{m}$, of each Gaussian basis functions is the location of the marker and its standard deviation $\sigma_{m}=0.005$ for $m=1, \ldots, 8$. In other words, let $y_{m}$ denote the position of marker $m$, and let $o\left(s_{k}(i)\right)$ denote the location of agent $k$ at time $i$ (we say the location of an agent at state is the geometric center of the square corresponding with $s_{k}(i)$ in the continuous grid). Then, the basis functions and features are given by

$$
\begin{aligned}
z_{m}\left(s_{k}(i)\right) & =\exp \left(\frac{-1}{\sigma_{m}^{2}}\left(o\left(s_{k}(i)\right)-y_{m}\right)^{\top}\left(o\left(s_{k}(i)\right)-y_{m}\right)\right) \\
x_{k, i} & =\left(z_{m}\left(s_{k}(i)\right)\right)_{m=1}^{M}
\end{aligned}
$$

We consider a policy-selection problem in which the agents want to choose the policy that maximizes their long-term expected accumulated reward. Since the agents are getting negative reward at every time step (because of energy consumption) they want to know how to reach the food, while losing as less energy as possible. A natural policy, denoted $\pi_{1}$, could be to go straight to the food with high (0.8) probability and low (0.2) probability of going in another direction, but then the agents would face the harmful predator and the total expected reward may be low. Thus, we say that $\pi_{1}$ is a myopic policy. Another more insightful policy, denoted $\pi_{2}$, could be to take a detour and avoid the predator's area with very high (0.95) probability. Nevertheless, if the detour takes too long, then the agents would consume too much energy and it may not be worth trying. In order to evaluate which policy is better (myopic $\pi_{1}$ or detour $\pi_{2}$ ), the agents have to learn the value vector of each candidate-policy from samples. If the agents were learning on-policy, they would have to follow one candidate policy for long enough so they could apply stochastic optimization over the samples, then they would have to start again but following the other candidate policy. In other words, on-policy learning does not allow to reuse samples while evaluating different policies. 
The benefit of the off-policy formulation is that the agents can evaluate several target policies in parallel from a single data stream.

Since the agents only have samples of state-transitions in their respective territories, it is difficult for them to predict the value vector for each of the target policies $\left(\pi_{1}\right.$ and $\pi_{2}$ ). However, since they sample complementary regions of the state-space, they can collaborate, applying diffusion strategies, to learn the value vector of the two target policies and evaluate which one is better.

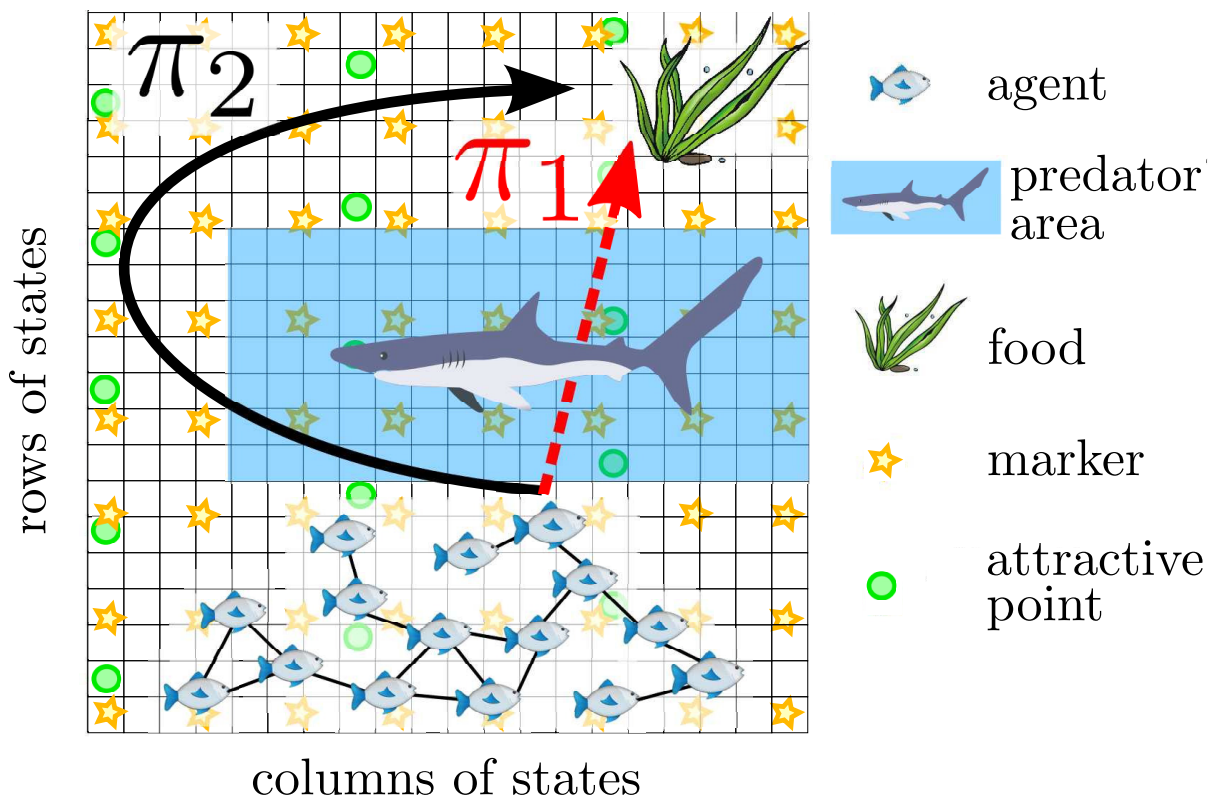

Figure 3.2 Problem representation. Network of 15 randomly connected agents. States are numbered from left to right and from down to top, so that the bottom-left corner corresponds with $s=1$, and the top-right corner with $s=S=400$. The agents are attracted towards the center of a region each (green circle) at states $\{395,368, \ldots, 21\}$. The predator's area is the rectangle $5 \leq$ column $\leq 20,8 \leq$ row $\leq 15$. The reward at every time step is -1 , except when crossing the predator's area where it becomes -15 , and when eating the food when it rises to +20 . The 64 features are obtained with 8 Gaussian basis functions per row and per column and with standard deviation 0.005, equidistantly located at positions (yellow stars) that are computed by taking the grid as a continuous unit-square.

Figure 3.3 shows the exact value vector and its linear approximation, where the parameter has been obtained in closed form through (3.58), for the myopic and detour policies under the off-policy setting. Figure 3.4 shows their approximation with parameters found by the centralized algorithm, by a single agent (chosen at random) running the proposed cooperative diffusion GTD algorithm, and by a single non-cooperative agent (also chosen at random among those non-cooperatives agents that 


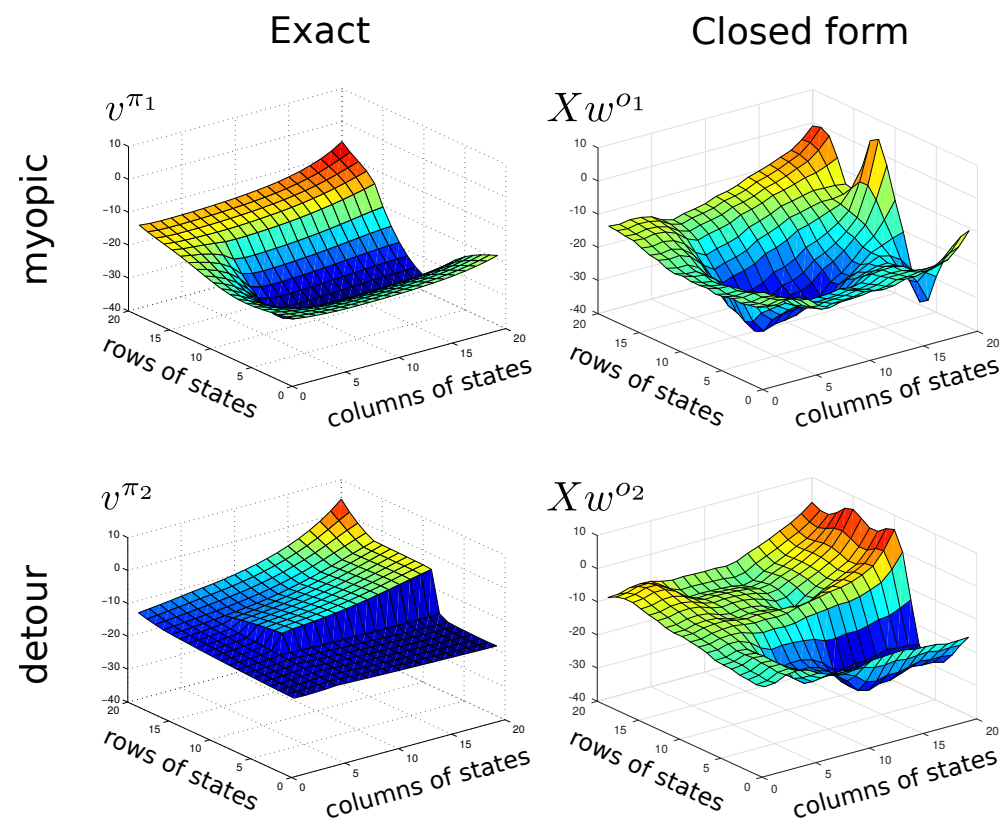

Figure 3.3 Value vectors for myopic (top, $\pi_{1}$ ) and detour (bottom, $\pi_{2}$ ) policies for each state of the grid. The left column shows the exact value vectors computed using (3.10). The myopic policy shows a valley because the agents have many chances of entering into the predator's area, so the expected reward will be negative. For the detour policy, the agent has little chance of entering into the predator's region, so the expected value will be near zero in this area.

have not diverged), under the constrained-exploration off-policy multi-agent setting explained above.

Figure 3.5 shows the learning curve of the algorithm. Since the agents have only samples from small portions of the state-space, the non-cooperative algorithm may diverge. On the other hand, when the agents cooperate (i.e., communicate their estimates to their neighbors), the diffusion algorithm allows them to benefit from the experience from other agents in the network, and they approach the same solution as a centralized architecture (i.e., with a fusion center that gathers all the samples from every node) would achieve but more efficiently, by communicating only within neighborhoods.

\subsection{Extensions of DGTD}

In this section we discuss some preliminary ideas on how to extend the current results. First, we remark that the single agent GTD2 algorithm approaches a solution that can be different from the actual target, and show that DGTD could be used for making 

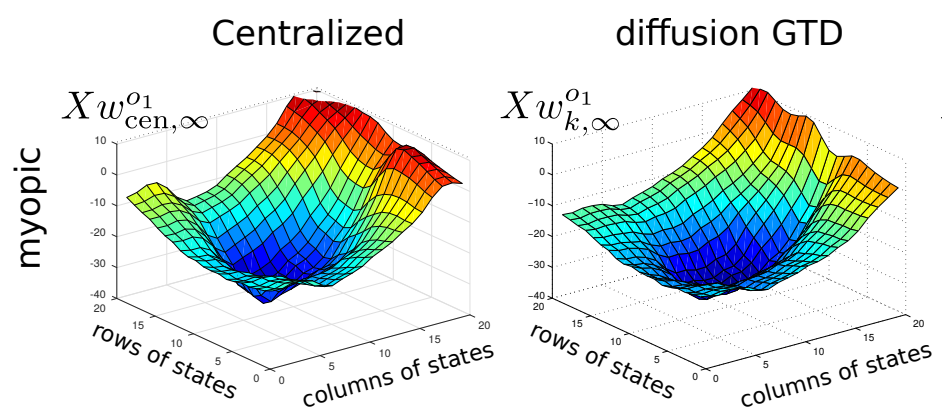

\section{Non-cooperative}
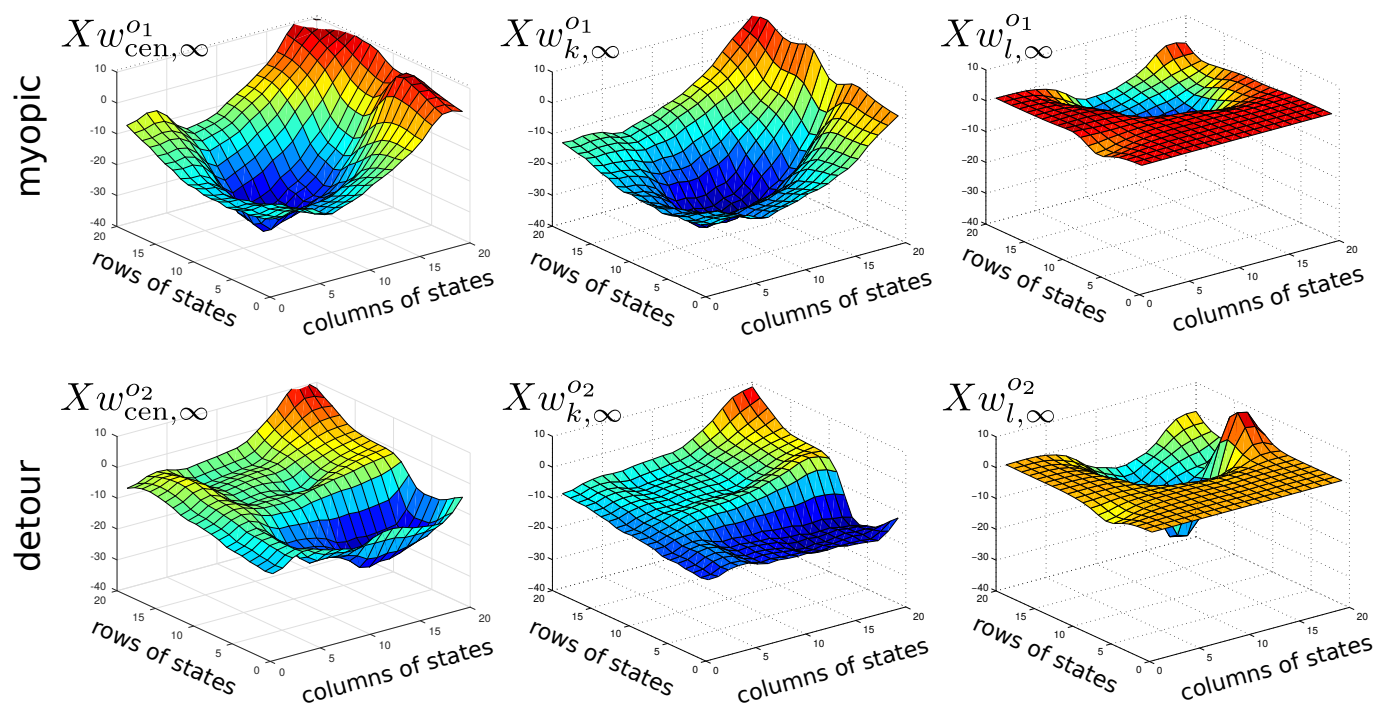

Figure 3.4 Value vectors for myopic (top, $\pi_{1}$ ) and detour (bottom, $\pi_{2}$ ) policies for each state of the grid. The left column shows the off-policy, linear approximation achieved by a centralized station that has gathered all samples from all agents. The middle column shows the estimated value vector obtained with our diffusion GTD algorithm for some agent (chosen at random, the result is very similar for all agents though) that only uses samples from its own non-exploratory policy (i.e., moving towards its attractive point). By including the diffusion step, every agent is able to reproduce the main features of the surfaces (valleys, hills and flat areas). The right column shows the same value vectors but estimated with no cooperation (for a node that did not diverge). 

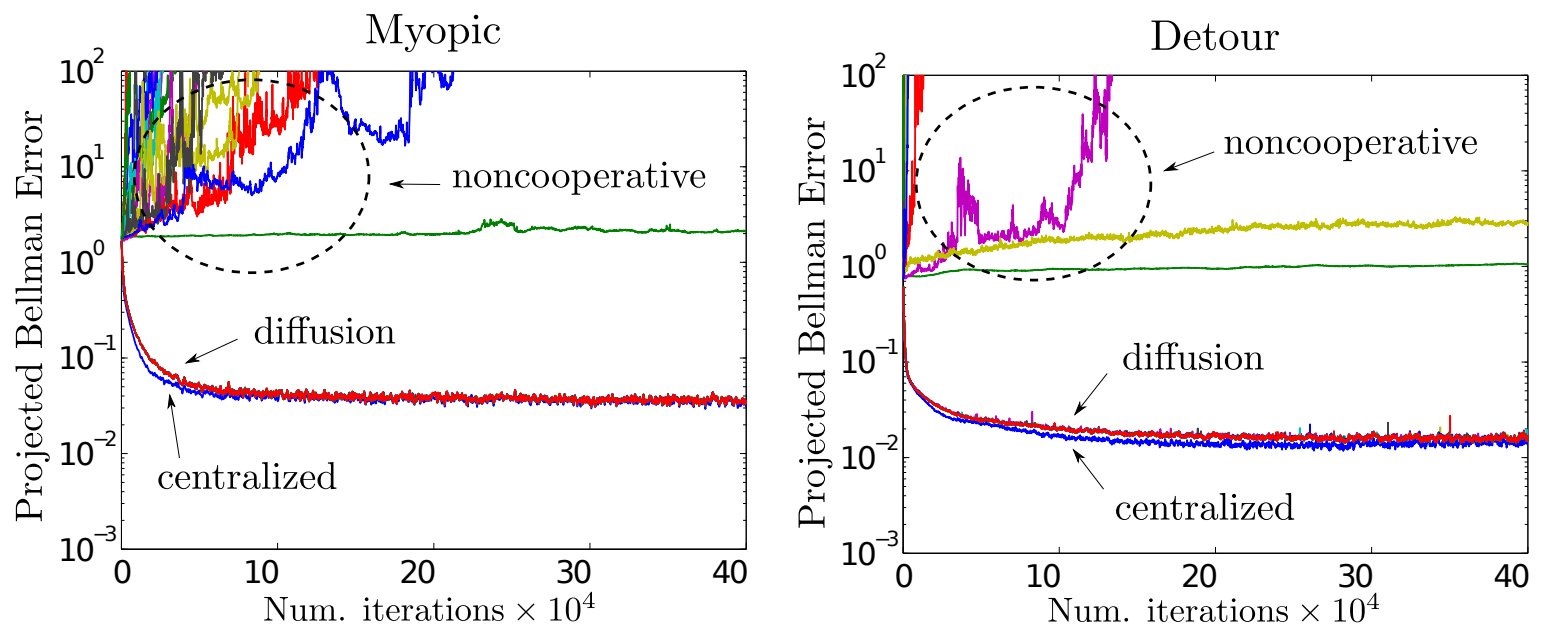

Figure 3.5 Projected Bellman error (i.e., $J_{\mathrm{PB}}$ ) given by Eq. (3.21) for centralized, diffusion and non-cooperative solutions. Results are obtained with the following parameters. We set the long-term reward to $\gamma=0.95$, which we have found to make $v^{\pi_{1}}$ very different from $v^{\pi_{2}}$ for the specific values of $\pi_{1}, \pi_{2}$ and rewards used during the experiments. For both cooperative and non-cooperative nodes, the step-size and step-size ratio are $\mu=1 \times 10^{-3}$ and $\eta=0.1$, which adequately trade bias and convergence-rate to illustrate the behavior of the algorithm. We assume every agent takes one sample at every time-step. Since a central station would have $N$ times more samples than the rest of the agents, we set its step-size equal to $\mu / N$. Results are averaged over 50 independent experiments.

the finding the exact target. Second, we show that DGTD can be naturally extended to distributed off-policy control with a diffusion-based distributed actor-critic method. Finally, we conjecture that diffusion-based distributed algorithms should be useful for some forms of transfer learning, like combining experience from multiple instances of a family of problems to tackle other instances that have never seen before.

\subsubsection{Approaching the actual target}

As explained in Sec. 3.4.2, even when using importance weights, the primal-dual off-policy solution, $w^{o}$, is different from the on-policy solution, $w^{\pi}$, that the agents wish to predict. The reason is that $D^{\bar{\phi}}$ can be different from $D^{\pi}$, as it is apparent from (3.58)-(3.59):

$$
\begin{aligned}
& w^{o}=\left(X^{\top} D^{\bar{\phi}}\left(I_{S}-\gamma P^{\pi}\right) X\right)^{-1} X^{\top} D^{\bar{\phi}} r^{\pi} \\
& w^{\pi}=\left(X^{\top} D^{\pi}\left(I_{S}-\gamma P^{\pi}\right) X\right)^{-1} X^{\top} D^{\pi} r^{\pi}
\end{aligned}
$$


This problem arises also in the original single-agent GTD algorithm. And, to the best of our knowledge, no technique has been proposed to prevent such difference.

Interestingly, diffusion strategies allow the agents to estimate the exact on-policy solution, such that $w^{o}=w^{\pi}$, by setting the scalars $\left\{\tau_{k}\right\}$ to satisfy

$$
\sum_{k=1}^{N} \tau_{k} d^{\phi_{k}}=d^{\pi}
$$

In general, since the linear system of equations in (3.60) may not be consistent, we will be interested in selecting the scalars $\left\{\tau_{k}\right\}$ that correspond to solving:

$$
\min _{\left\{\tau_{k}\right\}}\left\|d^{\pi}-\sum_{k=1}^{N} \tau_{k} d_{k}^{\phi}\right\|^{2}
$$

As indicated in Sec. 3.3, the weight vector $\tau$ corresponds with the Perron eigenvector of the combination matrix $C$. Reference [336] showed that, given a network topology, it is always possible to construct a matrix $C$ such that it has a given Perron eigenvector by using a procedure due to Hastings [116]. It is an interesting problem to develop an algorithm that obtains the optimal set of weights that minimizes (3.135) in a distributed manner. In addition, we remark that this idea could be useful for the single-agent setting too, by understanding that instead of having multiple agents, we have a single agent that uses DGTD for fusing trajectories obtained from multiple policies.

\subsubsection{Distributed off-policy control algorithms}

As we have already discussed, diffusion algorithms allow for efficient distributed implementations of first-order stochastic gradient descent algorithms, and some interesting properties emerge that were not present in the original single-agent algorithms. This is the case, e.g., of the enhanced robustness against local optimum presented in Chapter 4 or the possibility of approaching the online target described above in Sec. 3.6.1.

So far in this chapter, we have focused on predicting the value function for a given policy. In this subsection, we remark that the proposed DGTD algorithm can be extended for the control problem, i.e, for finding the optimal policy that maximizes the expected long term reward. In particular, we show that it could be used for a distributed implementation of the off-policy actor-critic (Off-PAC) algorithm due to [72]. The underlying idea of actor-critic methods is to combine an actor that optimizes the policy and a critic that evaluates the policy (see, e.g., [110, 150, 35],[278, Sec. 4.4] 
and references therein). In other words, the policy evaluation provided by the critic is the merit figure that guides the optimization process performed by the actor.

In order to optimize the policy, actor-critic methods usually rely stochastic gradient ascent on a set of smoothly parameterized policies. Let $\pi_{u}$ denote a parametric policy with parameter $u \in \mathbb{U} \subseteq \mathbb{R}^{U}$, where $U$ is the length of the parameter vector, and let $\Omega^{u} \triangleq\left\{\pi_{u}: u \in \mathbb{U}\right\}$ the set of parametric policies. For simplicity, we assume that $\mathbb{U}=\mathbb{R}^{U}$. Similar to the off-policy prediction problem, the off-policy control problem distinguishes between the behavior policy $\phi$ and the target optimal policy $\pi_{u}$ that we aim to learn. The single-agent Off-PAC is motivated from the following optimization problem:

$$
\underset{u}{\operatorname{maximize}} J(u)
$$

where the objective is the weighted sum of the value function induced by the parametric policy, $\pi_{u}$, with weights given by the stationary distribution induced by the behavior policy, $\phi$ :

$$
J(u) \triangleq \sum_{s \in \mathbb{S}} d^{\phi}(s) v^{\pi_{u}}(s)
$$

The actor improves the policy by performing stochastic gradient ascent on $J(u)$. Similar to (3.16), Off-PAC assumes that the value function is linearly approximated. Thus, after some smart manipulations, reference [72] arguments that $\nabla_{u} J(u)$ can be approximated as follows:

$$
\nabla_{u} J(u) \approx\left(r_{i+1}-\left(x_{i}-\gamma x_{i+1}\right)^{\top} w_{i}\right) \frac{\nabla_{u} \pi_{u}(a(i) \mid s(i))}{\pi_{u}(a(i) \mid s(i))} \xi(i)
$$

where $\xi(i)$ is given by (3.37). When the policy is the Gibbs distribution, we have

$$
\frac{\nabla_{u} \pi_{u}(a(i) \mid s(i))}{\pi_{u}(a(i) \mid s(i))}=y_{s a}-\sum_{a^{\prime} \in \mathbb{A}} \pi\left(a^{\prime} \mid s\right) y_{s a}
$$

where $y_{s a}$ are state-action-features given by

$$
y_{s a}^{\top} \triangleq\left[z_{1}^{\prime}(s, a), \ldots, z_{U}^{\prime}(s, a)\right]
$$

for some basis functions $z_{m}^{\prime}: \mathbb{S} \times \mathbb{A} \rightarrow \mathbb{R}, m=1, \ldots, U$. The state-action-features basis functions, $\left\{z_{m}^{\prime}\right\}_{m=1}^{U}$, are usually related to the state-features basis functions $\left\{z_{m}\right\}_{m=1}^{M}$ (we remark that they could be unrelated though). In particular, we usually have 
$M<U$, since $M$ features are used to approximate the state and we require some extra features to approximate the action.

The critic (i.e., policy evaluation step) consists in updating parameter $w_{i}$ that is used for approximating the value function: $v^{\pi_{u}}=X w_{i}$. Since this is an off-policy prediction problem with linear feature approximation, Off-PAC leverages gradient temporal difference methods and perform gradient descent on the projected Bellman error, $J_{\mathrm{PB}}(w)$, given by (3.22). The single-agent Off-PAC algorithm proposed by reference [72] uses a sophisticated variation of the GTD algorithm that includes eligibility traces (being able to trade-off bias and variance in the Monte Carlo estimate of the value function). Nevertheless, for the sake of simplicity, we consider the GTD algorithm described in Sec. 3.2.4.

In summary, the (variation of the) single-agent Off-PAC considered here is given by the following equations:

$$
\begin{aligned}
\theta_{i+1} & =\theta_{i}-\mu_{\theta} x_{i}\left(x_{i}^{\top} \theta_{i}+\left(x_{i}-\gamma x_{i+1}\right)^{\top} w_{i}-r(i+1)\right) \xi(i) \\
w_{i+1} & =w_{i}+\mu_{w}\left(x_{i}-\gamma x_{i+1}\right) x_{i}^{\top} \theta_{i} \xi(i) \\
u_{i+1} & =u_{i}+\mu_{u}\left(r_{i+1}-\left(x_{i}-\gamma x_{i+1}\right)^{\top} w_{i}\right) \frac{\nabla_{u} \pi_{u}(a(i) \mid s(i))}{\pi_{u}(a(i) \mid s(i))} \xi(i)
\end{aligned}
$$

where (3.141a)-(3.141b) constitute the critic - which is exactly the primal-dual update GTD algorithm given by (3.38a)-(3.38b) - and the actor is the stochastic gradient for updating the policy parameter given by (3.141c).

Now, we propose some preliminary ideas for multi-agent off-policy control from multiple behavior policies. Let $\phi_{k}$ denote each agent's behavior policy, $k=1, \ldots, N$. Let each agent's individual objective, $J_{k}: \mathbb{U} \rightarrow \mathbb{R}$, be given by

$$
J_{k}(u) \triangleq \sum_{s \in \mathbb{S}} d^{\phi_{k}}(s) v^{\pi_{u}}(s)
$$

Introduce a a common global objective, $J^{\text {glob }}: \mathbb{U} \rightarrow \mathbb{R}$, that is a convex combination of all agents' individual objectives:

$$
J^{\text {glob }}(u) \triangleq \sum_{k=1}^{N} \tau_{k} J_{k}(u)
$$


where $\left(\tau_{k}\right)_{k=1}^{N}$ are non-negative combination weights (similar to (3.41)) Then, we can formulate the following cooperative optimization problem:

$$
\underset{u}{\operatorname{maximize}} J^{\text {glob }}(u)
$$

By extending the gradient approximation (3.138) to the global objective, we have:

$$
\nabla_{u} J^{\mathrm{glob}}(u) \approx \sum_{k=1}^{N} \tau_{k}\left(r_{k, i+1}-\left(x_{k, i}-\gamma x_{k, i+1}\right)^{\top} w_{i}\right) \frac{\nabla_{u} \pi_{u}\left(a_{k}(i) \mid s_{k}(i)\right)}{\pi_{u}\left(a_{k}(i) \mid s_{k}(i)\right)} \xi_{k}(i)
$$

Then, we can propose a distributed off-policy actor-critic (D-Off-PAC) as follows: the distributed critic performs the DGTD algorithm given by (3.47a)-(3.47d) for cooperative off-policy evaluation; while the distributed actor performs ATC for cooperative optimization of (3.144). This D-Off-PAC method is summarized with the following equations:

$$
\begin{aligned}
\widehat{\theta}_{k, i+1} & =\theta_{k, i}-\mu_{\theta} x_{k, i}\left(x_{k, i}^{\top} \theta_{k, i}+\left(x_{k, i}-\gamma x_{k, i+1}\right)^{\top} w_{k, i}-r_{k}(i+1)\right) \xi_{k}(i) \\
\theta_{k, i+1} & =\sum_{l \in \mathcal{N}_{k}} c_{l k} \widehat{\theta}_{l, i+1} \\
\widehat{w}_{k, i+1} & =w_{k, i}+\mu_{w}\left(x_{k, i}-\gamma x_{k, i+1}\right) x_{k, i}^{\top} \theta_{k, i} \xi_{k}(i) \\
w_{k, i+1} & =\sum_{l \in \mathcal{N}_{k}} c_{l k} \widehat{w}_{l, i+1} \\
\widehat{u}_{k, i+1} & =u_{k, i}+\mu_{u}\left(r_{k, i+1}-\left(x_{k, i}-\gamma x_{k, i+1}\right)^{\top} w_{k, i}\right) \frac{\nabla_{u} \pi_{u}\left(a_{k}(i) \mid s_{k}(i)\right)}{\pi_{u}\left(a_{k}(i) \mid s_{k}(i)\right)} \xi_{k}(i) \\
u_{k, i+1} & =\sum_{l \in \mathcal{N}_{k}} c_{l k} \widehat{u}_{l, i+1}
\end{aligned}
$$

We remark that D-Off-PAC is a preliminary result that should be further investigated. In particular, convergence analysis and numerical experiments are required to understand the benefits of the proposed cooperation scheme for the control problem.

Moreover, while DGTD was theoretically well grounded from a primal-dual perspective, D-Off-PAC — and actor-critic methods in general - are usually derived from the (perhaps more engineering) approximate policy iteration approach. The problem of deriving (distributed) actor-critic algorithms from a theoretically sound primal-dual approach is a future line of research.

Finally, since diffusion-based distributed off-policy policy evaluation algorithms allow to combine experience from different agents, we consider whether distributed 
off-policy control algorithms can be used for some form of transfer learning. This is briefly discussed in the following subsection.

\subsubsection{Diffusion algorithms for transfer learning}

The individual objectives, $J_{k}$, given by (3.142) establish that the agents can follow multiple behavior policies, $\phi_{k}$, but that they aim to find the same optimal target policy that maximizes a common value function, $v^{\pi_{u}}$. The optimal value function is determined by the structure of the state-transition and reward functions. Therefore, Eq. (3.142) implicitly assumes that all agents operate in the same MDP.

A generalization of (3.142) is to allow the agents to optimize multiple value functions. More formally, consider the following individual objectives:

$$
J_{k}(u) \triangleq \sum_{s \in \mathbb{S}} d^{\phi_{k}}(s) v_{k}^{\pi_{u}}(s), \quad k=1, \ldots, N
$$

where each individual value function, $v_{k}^{\pi_{u}}$, has now superscript $k$ to indicate that it is specific to each agent. Having multiple value functions imply that the agents can operate in different MDPs. In order to find a (parametric) policy that simultaneously optimize each agent's objective, we formulate the following multiobjective problem:

$$
\underset{u}{\operatorname{maximize}} \quad J^{\mathrm{vec}}(u)
$$

where $J^{\text {vec }}: \mathbb{U} \rightarrow \mathbb{R}^{N}$ denotes the vector-valued objective that includes all agents' individual objectives:

$$
J^{\mathrm{vec}}(u) \triangleq\left(J_{k}(u)\right)_{k=1}^{N}
$$

Let us define an optimal point for multiobjective problems as a point that is simultaneously optimal for all subproblems. First, note that the vector-valued comparison $J^{\mathrm{vec}}\left(u^{\prime}\right) \geq J^{\mathrm{vec}}(u)$ holds for two points $u^{\prime}, u \in \mathbb{U}$ if and only $J_{k}\left(u^{\prime}\right) \geq J_{k}(u)$ for all $k=1, \ldots, N$.

Definition 3.1. A point $u^{\star} \in \mathbb{U}$ is optimal, if $J^{\mathrm{vec}}\left(u^{\star}\right) \geq J^{\mathrm{vec}}(u), \forall u \in \mathbb{U}$, where the comparison is component-wise, i.e. $J_{k}\left(u^{\star}\right) \geq J_{k}(u)$ for all $k=1, \ldots, N$.

Nevertheless, most vector optimization problems do not have such optimal points. In this case, another (more relaxed) common solution concept is that of Pareto optimality (or efficiency), which can be defined as follows (see, e.g., [86, pp. 24, 48]). 
Definition 3.2. A point $u^{\star} \in \mathbb{U}$ is called strongly Pareto optimal if there is no other $u \in \mathbb{U}$ such that $J^{\mathrm{vec}}(u) \geq J^{\mathrm{vec}}\left(u^{\star}\right)$.

Definition 3.3. A point $u^{\star} \in \mathbb{U}$ is called weakly Pareto optimal if there is no other $u \in \mathbb{U}$ such that $J^{\operatorname{vec}}(u)>J^{\operatorname{vec}}\left(u^{\star}\right)$.

Now, similar to (3.144), we can define a global objective that is a convex combination of all agents' individual objectives:

$$
J^{\text {glob }}(u) \triangleq \sum_{k=1}^{N} \tau_{k} J_{k}(u)=\sum_{k=1}^{N} \tau_{k} \sum_{s \in \mathbb{S}} d^{\phi_{k}}(s) v_{k}^{\pi_{u}}(s)
$$

and formulate the following cooperative optimization problem:

$$
\underset{u}{\operatorname{maximize}} J^{\mathrm{glob}}(u)
$$

Then, it can be shown that the solutions of problem (3.151) with nonnegative (positive) weights, $\tau_{k}$, are always weakly (strongly) Pareto optimal points of (3.148) (see, e.g., [48, Sec. 4.7.4], [86, Sec. 3.1]).

Problem (3.148) is very related to transfer learning. For instance, suppose that the $N$ individual objectives, $\left\{J_{1}(u), \ldots, J_{N}(u)\right\}$, refer to $N$ members of the same family of problems (e.g., the classic mountain-car problem [274, Example 8.2] with different high and slope parameters or different Atari 2600 games). Then, the solution to the cooperative problem (3.148) will yield a policy that is Pareto optimal. Intuitively speaking, this Pareto optimal policies might be used as efficient starting points when learning a new member of the same problem family that has never been seen before. In other words, we conjecture that the agents might be able to transfer knowledge from one problem to another by using our diffusion-based distributed off-policy algorithm.

\subsection{Conclusions}

In this chapter we considered a network of agents, with an arbitrary connected topology, cooperating to predict the response of their environment. Each prediction is expressed as a numerical value given by the corresponding value function. Focusing on problems with very large state-spaces-where computing the exact value function would be infeasible-we proposed a distributed strategy based on diffusion adaptation to promote a decentralized temporal difference solution, which is an extension of the original single-agent GTD2 in [275], but which we have derived using a very different, novel manner. 
The proposed diffusion GTD (DGTD) maintains the efficiency of the single-agent GTD2 [275], with linear complexity in both computation time and memory footprint. With diffusion GTD, the agents cooperate to improve the stability and accuracy of their prediction. In particular, they are able to approach the optimal solution through cooperation, even when none could do it in isolation. We remark that cooperation is fully distributed with communications only within each agent's neighborhood; neither fusion-center, nor multi-hop communications are required.

We provided conditions that guarantee convergence of the proposed diffusion GTD and derived performance bounds for sufficiently small step-sizes. These results generalize the original asymptotic results of the single agent GTD2. Constant step-sizes are a desirable feature for an adaptive network, since it allows the network to learn continuously, and to track concept drifts in the data.

Finally, we included some interesting lines of research, namely estimating the exact on-policy solution, deriving off-policy control algorithms or applying diffusion for transfer learning.

In this chapter we have assumed that the agents can communicate and cooperate with their neighbors, but they operate without influencing each other state-transitions and reward. In the following chapter we tackle an alternative, complementary problem in which a set of agents operate in a common MDP and the state-transitions and their individual rewards depend on the policies followed by other agents and they compete to maximize their value functions.

\subsection{Appendix: Proof of Theorem 3.1}

To study the spectrum of $\mathcal{C}^{\top}\left(I_{2 M N}-\mu \mathcal{R}\right)$, we express the combination coefficient matrix in its Jordan canonical form:

$$
C^{\top}=Y_{C} J_{C} Y_{C}^{-1}
$$

Using the property $(U \otimes V)(Y \otimes Z)=(U Y) \otimes(V Z)$, we obtain

$$
\begin{aligned}
\mathcal{C}^{\top}\left(I_{2 M N}-\mu \mathcal{R}\right)= & \left(Y_{C} \otimes I_{2 M}\right)\left(J_{C} \otimes I_{2 M}\right) \\
& \left(I_{2 M N}-\mu\left(Y_{C}^{-1} \otimes I_{2 M}\right) \mathcal{R}\left(Y_{C} \otimes I_{2 M}\right)\right)\left(Y_{C}^{-1} \otimes I_{2 M}\right)
\end{aligned}
$$

so that, by similarity,

$$
\lambda\left(\mathcal{C}^{\top}\left(I_{2 M N}-\mu \mathcal{R}\right)\right)=\lambda\left(\left(J_{C} \otimes I_{2 M}\right)\left(I_{2 M N}-\mu \mathcal{E}\right)\right)
$$


where

$$
\mathcal{E} \triangleq\left(Y_{C}^{-1} \otimes I_{2 M}\right) \mathcal{R}\left(Y_{C} \otimes I_{2 M}\right)
$$

As stated in Section 3.3, conditions (3.48)-(3.50) ensure that $C^{\top}$ is a primitive rightstochastic matrix. Hence, from the Perron-Frobenius Theorem [124], the Jordan canonical form of $C$ can be expressed as

$$
J_{C}=\operatorname{diag}\left\{1, J_{C}^{0}\right\}
$$

where all the eigenvalues of $J_{C}^{0}$ are strictly inside the unit circle. Moreover, since $C^{\top}$ is right-stochastic it has one right-eigenvector of all ones associated with its unit eigenvalue, and its corresponding left eigenvector, $p$, has positive entries (i.e., $p_{k}>0,1 \leq k \leq N$ ):

$$
C^{\top} \mathbb{1}_{N}=\mathbb{1}_{N}, \quad p^{\top} C^{\top}=p^{\top}, \quad \mathbb{1}_{N}^{\top} p=1
$$

We therefore decompose

$$
Y_{C}^{-1}=\operatorname{col}\left\{p^{\top}, Y_{C}^{l}\right\}, \quad Y_{C}=\left[\mathbb{1}_{N} Y_{C}^{r}\right]
$$

and partition $\mathcal{E}$ as

$$
\begin{aligned}
& \mathcal{E}=\left[\begin{array}{cc}
\bar{G} & \mathcal{E}_{12} \\
\mathcal{E}_{21} & \mathcal{E}_{22}
\end{array}\right] \\
& \bar{G} \triangleq\left(p^{\top} \otimes I_{2 M}\right) \mathcal{R}\left(\mathbb{1}_{N} \otimes I_{2 M}\right)=\sum_{k=1}^{N} p_{k} G_{k} \\
& \mathcal{E}_{12} \triangleq\left(p^{\top} \otimes I_{2 M}\right) \mathcal{R}\left(Y_{C}^{r} \otimes I_{2 M}\right) \\
& \mathcal{E}_{21} \triangleq\left(Y_{C}^{l} \otimes I_{2 M}\right) \mathcal{R}\left(\mathbb{1}_{N} \otimes I_{2 M}\right) \\
& \mathcal{E}_{22} \triangleq\left(Y_{C}^{l} \otimes I_{2 M}\right) \mathcal{R}\left(Y_{C}^{r} \otimes I_{2 M}\right)
\end{aligned}
$$

Introduce the following shorthand in (3.154):

$$
\mathcal{S} \triangleq\left(J_{C} \otimes I_{2 M}\right)\left(I_{2 M N}-\mu \mathcal{E}\right)
$$

Then, expanding (3.156) and (3.159)-(3.163) into (3.164) we have

$$
\mathcal{S}=\left[\begin{array}{cc}
I_{2 M}-\mu \bar{G} & -\mu \mathcal{E}_{12} \\
-\mu\left(J_{C}^{0} \otimes I_{2 M}\right) \mathcal{E}_{21} & \left(J_{C}^{0} \otimes I_{2 M}\right)\left(I_{L}-\mu \mathcal{E}_{22}\right)
\end{array}\right]
$$


where

$$
L \triangleq 2 M(N-1)
$$

Using the same technique proposed in $[338,59]$, we appeal to eigenvalue perturbation analysis to examine the spectral radius of (3.165). We introduce the $N \times N$ diagonal matrix

$$
\Omega_{N}^{\epsilon} \triangleq \operatorname{diag}\left\{\epsilon, \epsilon^{2}, \epsilon^{3}, \ldots, \epsilon^{N}\right\}
$$

with parameter $\epsilon>0$. Let the Jordan canonical form of $\bar{G}$ be given by:

$$
J_{\bar{G}}=Y_{\bar{G}}^{-1} \bar{G} Y_{\bar{G}}
$$

Introduce a similarity transformation with parameters $\epsilon, \beta$, and $\sigma$ :

$$
\Phi=\left[\begin{array}{cc}
Y_{\bar{G}} \Omega_{2 M}^{\epsilon} & 0_{2 M \times L} \\
0_{L \times 2 M} & \frac{\sqrt{\mu}}{\sigma} \Omega_{N-1}^{\beta} \otimes I_{2 M}
\end{array}\right]
$$

We apply the similarity transformation $(3.169)$ to $\mathcal{S}$ :

$$
\Phi^{-1} \mathcal{S} \Phi=\left[\begin{array}{cc}
I_{2 M}-\mu J_{\bar{G}}^{\epsilon} & -\frac{\mu \sqrt{\mu}}{\sigma} \mathcal{S}_{12} \\
-\sigma \sqrt{\mu} \mathcal{S}_{21} & J_{C}^{0 \beta} \otimes I_{2 M}-\mu \mathcal{S}_{22}
\end{array}\right]
$$

where

$$
\begin{aligned}
& \mathcal{S}_{12} \triangleq\left(\Omega_{2 M}^{\epsilon}\right)^{-1} Y_{\bar{G}}^{-1} \mathcal{E}_{12}\left(\Omega_{N-1}^{\beta} \otimes I_{2 M}\right) \\
& \mathcal{S}_{21} \triangleq\left(\left(\left(\Omega_{N-1}^{\beta}\right)^{-1} J_{C}^{0}\right) \otimes I_{2 M}\right) \mathcal{E}_{21} Y_{\bar{G}} \Omega_{2 M}^{\epsilon} \\
& \mathcal{S}_{22} \triangleq\left(\left(\left(\Omega_{N-1}^{\beta}\right)^{-1} J_{C}^{0}\right) \otimes I_{2 M}\right) \mathcal{E}_{22}\left(\Omega_{N-1}^{\beta} \otimes I_{2 M}\right)
\end{aligned}
$$

and $J_{\bar{G}}^{\epsilon}$ and $J_{C}^{0 \beta}$ have the same form as the (upper triangular) Jordan canonical forms $J_{\bar{G}}$ and $J_{C}^{0}$, except that the unit entries are replaced by $\epsilon$ and $\beta$, respectively. By applying Gerschgorin theorem [124] to (3.170), we can identify the regions where the eigenvalues of $\mathcal{S}$ should lie:

$$
\left|\lambda(\mathcal{S})-\left(1-\mu \lambda_{m}(\bar{G})\right)\right| \leq \mu \epsilon+\frac{\mu \sqrt{\mu}}{\sigma} \sum_{q=1}^{L}\left|\left[\mathcal{S}_{12}\right]_{m q}\right|
$$




$$
\left|\lambda(\mathcal{S})-\left(\lambda_{k+1}(C)-\mu\left[\mathcal{S}_{22}\right]_{m m}\right)\right| \leq \beta+\sigma \sqrt{\mu} \sum_{q=1}^{2 M}\left|\left[\mathcal{S}_{21}\right]_{m q}\right|+\mu \sum_{\substack{q=1 \\ q \neq m}}^{L}\left|\left[\mathcal{S}_{22}\right]_{m q}\right|
$$

where $[\cdot]_{m q}$ stands for the element at row $m$ and column $q$ of a matrix. Although we use the same subscript $m$ in both equations, note that $1 \leq m \leq 2 M$ in (3.174) while $1 \leq m \leq L$ in (3.175); in addition, recall that $C$ is an $N \times N$ matrix, hence, we use subscript $k=\operatorname{ceil}\{m /(2 M)\}$, where ceil $\{\cdot\}$ rounds a real number to its nearest greater or equal integer.

We are looking for sufficient conditions that guarantee that the mean recursion (3.77) converges for small step-size. Recall from (3.154) that (3.77) converges when $|\lambda(\mathcal{S})|<1$. Let us solve for $|\lambda(\mathcal{S})|$ in $(3.174)$ first. Based on the following inequality:

$$
z|-| y|\leq| z-y \mid
$$

we obtain:

$$
\begin{aligned}
|\lambda(\mathcal{S})|-\left|1-\mu \lambda_{m}(\bar{G})\right| & \leq\left|\lambda(\mathcal{S})-\left(1-\mu \lambda_{m}(\bar{G})\right)\right| \\
& \leq \mu \epsilon+\frac{\mu \sqrt{\mu}}{\sigma} \chi_{(12)}
\end{aligned}
$$

where

$$
\chi_{(12)} \triangleq \sum_{q=1}^{L}\left|\left[\mathcal{S}_{12}\right]_{m q}\right|
$$

Therefore, we have

$$
|\lambda(\mathcal{S})| \leq \mu \epsilon+\frac{\mu \sqrt{\mu}}{\sigma} \chi_{(12)}+\left|1-\mu \lambda_{m}(\bar{G})\right|
$$

Since $\mathcal{S}, \bar{G}$ and $C$ are not generally guaranteed to be symmetric, their eigenvalues may be complex. Using the fact that

$$
1-z \leq\left(1-\frac{1}{2} z\right)^{2}, \quad \forall z \in \mathbb{R}
$$

we obtain:

$$
\left|1-\mu \lambda_{m}(\bar{G})\right| \leq 1-\mu \operatorname{Re}\left\{\lambda_{m}(\bar{G})\right\}+\frac{\mu^{2}}{2}\left|\lambda_{m}(\bar{G})\right|^{2}
$$


where $\operatorname{Re}\{\cdot\}$ denotes the real part of a complex number. Combining (3.177), (3.179) and (3.181), the stability condition implied by (3.174) requires finding small step-sizes $\mu$ such that

$$
|\lambda(\mathcal{S})| \leq \mu \epsilon+\frac{\mu \sqrt{\mu}}{\sigma} \chi_{(12)}+1-\mu \operatorname{Re}\left\{\lambda_{m}(\bar{G})\right\}+\frac{\mu^{2}}{2}\left|\lambda_{m}(\bar{G})\right|^{2}<1
$$

which is equivalent to requiring

$$
\mu\left(\mu \frac{\left|\lambda_{m}(\bar{G})\right|^{2}}{2}+\sqrt{\mu} \frac{\chi_{(12)}}{\sigma}+\epsilon-\operatorname{Re}\left\{\lambda_{m}(\bar{G})\right\}\right)<0
$$

Sufficient conditions on $\mu$ that satisfy (3.183) are

$$
\mu \frac{\left|\lambda_{m}(\bar{G})\right|^{2}}{2}+\sqrt{\mu} \frac{\chi_{(12)}}{\sigma}+\epsilon-\operatorname{Re}\left\{\lambda_{m}(\bar{G})\right\}<0
$$

which leads to

$$
0<\mu<\left(\frac{-\frac{\chi_{(12)}}{\sigma}+\sqrt{\left(\frac{\chi_{(12)}}{\sigma}\right)^{2}+2\left|\lambda_{m}(\bar{G})\right|^{2}\left(\operatorname{Re}\left\{\lambda_{m}(\bar{G})\right\}-\epsilon\right)}}{\left|\lambda_{m}(\bar{G})\right|^{2}}\right)^{2}
$$

where, in order to guarantee that the term inside the square root in the right side of (3.185) is positive, we choose

$$
0<\epsilon<\min _{1 \leq m \leq 2 M} \operatorname{Re}\left\{\lambda_{m}(\bar{G})\right\}
$$

We now show that $\operatorname{Re}\left\{\lambda_{m}(\bar{G})\right\}$ is always positive. If we transform $\bar{G}$ into a similar matrix:

$$
\begin{aligned}
\bar{G}_{\sqrt{\eta}} & \triangleq\left[\begin{array}{cc}
I_{M} & 0_{M \times M} \\
0_{M \times M} & \sqrt{\eta} I_{M}
\end{array}\right] \bar{G}\left[\begin{array}{cc}
I_{M} & 0_{M \times M} \\
0_{M \times M} & \frac{1}{\sqrt{\eta}} I_{M}
\end{array}\right] \\
& =\left[\begin{array}{cc}
\sqrt{\eta} X^{\top} D^{\bar{\phi}} X & X^{\top} D^{\bar{\phi}}\left(I_{S}-\gamma P^{\pi}\right) X \\
-X\left(I_{S}-\gamma P^{\pi}\right)^{\top} D^{\bar{\phi}} X^{\top} & 0_{M \times M}
\end{array}\right]
\end{aligned}
$$

and use [23, Theorem 3.6] on $\bar{G}_{\sqrt{\eta}}$, we can establish that $\operatorname{Re}\left\{\lambda_{m}(\bar{G})\right\}>0$. 
Now, we solve for $|\lambda(\mathcal{S})|$ from (3.175). Let us abbreviate the sums in the right side of $(3.175)$ as

$$
\chi_{(21)} \triangleq \sum_{q=1}^{2 M}\left|\left[\mathcal{S}_{21}\right]_{m q}\right|, \quad \chi_{(22)} \triangleq \sum_{\substack{q=1 \\ q \neq m}}^{L}\left|\left[\mathcal{S}_{22}\right]_{m q}\right|
$$

In a manner similar to (3.177), we have

$$
|\lambda(\mathcal{S})|-\left|\lambda_{k+1}(C)-\mu\left[\mathcal{S}_{22}\right]_{m m}\right| \leq \beta+\sigma \sqrt{\mu} \chi_{(21)}+\mu \chi_{(22)}
$$

Using (3.189) and the fact

$$
|z-y| \leq|z|+|y|
$$

yields the following condition on $\mu$ for stability:

$$
\sqrt{\mu}\left(\sqrt{\mu}\left(\chi_{(22)}+\left|\left[\mathcal{S}_{22}\right]_{m m}\right|\right)+\sigma \chi_{(21)}\right)<1-\left|\lambda_{k+1}(C)\right|-\beta
$$

The following conditions on the step-size are jointly sufficient to satisfy (3.191):

$$
\begin{gathered}
0<\sqrt{\mu}<1-\left|\lambda_{k+1}(C)\right|-\beta \\
0<\sqrt{\mu}<\frac{1-\sigma \chi_{(21)}}{\chi_{(22)}+\left|\left[\mathcal{S}_{22}\right]_{m m}\right|}
\end{gathered}
$$

From the Perron-Frobenius, we know that $\lambda_{k+1}(C)<1$, for $1 \leq k \leq N-1$. Moreover, Assumption 3.3 guarantees that any element of $\mathcal{S}$ is bounded from below and above. Therefore, there exist parameters $0<\beta<1-\left|\lambda_{k+1}(C)\right|$ and $0<\sigma<1 / \chi_{(21)}$ that make the right side of (3.192) and (3.193) positive, respectively. Hence, we can square both inequalities and obtain the following conditions:

$$
\begin{aligned}
& 0<\mu<\left(1-\left|\lambda_{k+1}(C)\right|-\beta\right)^{2} \\
& 0<\mu<\left(\frac{1-\sigma \chi_{(21)}}{\chi_{(22)}+\left|\left[\mathcal{S}_{22}\right]_{m m}\right|}\right)^{2}
\end{aligned}
$$

Let bound (3.185), (3.194) and (3.195) by

$$
\mu^{o}=\min \left\{\left(1-\left|\lambda_{k+1}(C)\right|-\beta\right)^{2},\left(\frac{1-\sigma \chi_{(21)}}{\chi_{(22)}+\left|\left[\mathcal{S}_{22}\right]_{j j}\right|}\right)^{2}\right.
$$




$$
\left.\left(\frac{-\frac{\chi_{(12)}}{\sigma}+\sqrt{\left(\frac{\chi_{(12)}}{\sigma}\right)^{2}+2\left|\lambda_{m}(\bar{G})\right|^{2}\left(\operatorname{Re}\left\{\lambda_{m}(\bar{G})\right\}-\epsilon\right)}}{\left|\lambda_{m}(\bar{G})\right|^{2}}\right)^{2}\right\}
$$

for $1 \leq m \leq 2 M, 1 \leq k \leq N-1$ and $1 \leq j \leq L$. We conclude that if the step-size $0<\mu<\mu^{o}$, then diffusion GTD is mean-stable.

As a final remark, note that $\mu^{o}$ depends on the eigenvalues of $\bar{G}$, the eigenvalues of the weighted-topology matrix $C$, and the constructed matrix $\mathcal{S}$ in (3.165) (note that $\sigma, \epsilon$ and $\beta$ are similarity parameters, and the terms $\chi_{(22)}$ and $\chi_{(22)}$ are defined in (3.188) simply as a short hand of sums of the elements in $\mathcal{S}$ ). Recall that $\bar{G}$ is given by (3.160) as the weighted sum of the individual $G_{k}$, which only depend on the data samples, the importance weights and the step-size ratio parameter $\eta$. Finally, recall that $\mathcal{S}$ depends on the Jordan canonical form of $C$ and $\mathcal{R}$, where the latter is defined in (3.78) from the individual $G_{k}$. Thus, all the terms involved in $\mu^{o}$ are input data to the algorithm. 


\section{Chapter 4}

\section{Distributed Cross-Entropy method for black-box global optimization}

\subsection{Introduction}

We tackle the problem of optimizing an unknown objective function - with unknown gradient - that can only be evaluated through point-wise observations, i.e., the objective function is like a black-box in which we enter candidate solutions and obtain output values. The objective has none or little structure, for instance, it can be nondifferentiable and have multiple extrema. Thus, we consider global optimization methods that try to escape from local maxima and converge to the global solution.

Mother nature is full of black-box global optimization problems and natural evolution has been able to solve many of them. These problems also abound in science and engineering applications, and researchers have developed many bio-inspired algorithms that can solve them efficiently (e.g., genetic algorithms, particle swarm, etc.). In this work we propose a novel algorithm that combines three features of many biological systems: i) cooperation among multiple agents, ii) the survival of the fittest guiding principle, and iii) a divide and conquer approach.

A network of cooperative agents can explore the solution space simultaneously, obviously more efficiently than any agent by itself, and achieve consensus on where the optimal solution lies. Our proposed cooperative exploration strategy brings two important practical benefits: i) the computational load can be distributed so that the agents solve the problem simultaneously, while keeping communication overhead per agent independent of the network size; and ii) the result can outperform a standard centralized architecture where the fusion center has as many computationally resources as the whole network of cooperating agents. 
In the proposed algorithm, each agent runs a particle filter to update its belief (distribution) of where the solution lies. At every iteration, every agent broadcasts some statistics of its posterior distribution to its neighbors. Then, it merges its own statistics with those coming from its neighbors. Each agent's statistics are estimated from samples (i.e., particles) drawn from its current belief distribution: most of the particles will be discarded and only a small fraction of the samples that have achieved the highest output values will survive and guide the evolution of the posterior distribution in the next iteration.

The goal of particle filter optimization (PFO) is to find a belief distribution that allocates all its probability density to the set of optimal solutions. In other words, instead of directly searching the optimal set of solutions, the agents search in the space of distributions over the solution space. More specifically, PFO aims to find the belief distribution that maximizes the expected output value. That is, instead of directly maximizing the objective function, PFO seeks the distribution that maximizes the expected value of the original objective. This idea of searching in the space of distributions (i.e., probabilistic models) is known as model-based optimization (see, e.g., survey [129]), and we refer to the expectation of the original objective function as the model-based objective function. Suppose we are searching in the space of multivariate normal distributions. Hence, the optimization of the model-based objective is over the space of sufficient statistics. Suppose further, that the covariance matrix is given; then we only have to find the optimal mean, which lies in the same input space as the search of the original objective. By construction, the model-based objective as a function of the mean is smoother than the original objective. Indeed, the larger the variance of the normal distribution, the smoother the model-based objective. By initializing PFO with a high variance distribution, the initial model-based objective becomes very smooth, and the survival of the fittest heuristic should lead the belief toward its optimal set. In practice, survival of the fittest tends to be a too greedy approach and it is required to introduce some damping in the posterior so that the Monte Carlo noise introduced by the particle approximation (of the actual belief distribution) can be averaged. In any case (with or without damping), by reducing the variance of the posterior, a less smoothed model-based objective is revealed, which is closer to the original objective. Thus, we can see that PFO divides the original problem into a sequence of gradually refined problems that can be solved more easily (conquered) than the original problem. 


\subsubsection{Related works}

The term model-based method (MBM) was coined by [344] to emphasize algorithms in which the candidate solutions are generated from a distribution over the solution space, as opposed to instance-based methods in which candidate solutions are generated directly from the solution space. Examples of well known instance-based algorithms are simulated annealing [147], genetic algorithms [101, 268], tabu search [100] or the more modern simultaneous optimistic optimization [198]. Well known MBMs include antcolony optimization [80, 265], cross-entropy (CE) [240, 152], model-reference adaptive search (MRAS) [127], annealing adaptive search [130], estimation of distribution algorithms (EDA) [221, 335], covariance matrix adaptation evolution strategy (CMAES) $[115,114]$, or natural evolution strategies (NES) [315]. Although they may have been derived from different approaches, recently, reference [340] showed that some of these MBMs (e.g., CE and MRAS) can be expressed as instances of PFO.

The idea of introducing damping in $\mathrm{CE}$ as a heuristic for trading-off exploration and exploitation was originally proposed by [152]. More recently, reference [128] proposed a well theoretically grounded stochastic approximation for introducing damping into the model update, which has been used for designing more sophisticated MBMs, beyond CE, e.g., [341].

CMA-ES is almost identical to CE with the main differences being that the covariance update formula includes terms from previous iterations as well as a damping form that exploits correlations between consecutive steps (the so named "evolution path", which reminds some form of momentum techniques from gradient descent algorithms) [114]. NES is also very similar to CE and CMA-ES. The main difference with CMA-ES is that the damping in NES follows gradient ascent, along the natural gradient (instead of the "evolution path" mentioned above). The natural gradient is a second order method that normalizes the update with respect to uncertainty, preventing oscillations, premature convergence, and other undesired effects derive from, e.g., bad scaling in the parametrization [315].

Interestingly, MBMs have enjoyed much popularity in the context of reinforcement learning, where they are used to search the parametric policies that maximizes the return (see Sections 3.6.2 and 5.6.2 regarding the use of parametric policies in reinforcement learning). For instance, CE was successfully used for policy search in [180], and has shown state of the art performance in difficult tasks like the Tetris game [282]. CMA-ES has become very popular for policy search [121, 241]. Policy learning by weighting exploration and returns (PoWER) [148] is an MBM specifically designed for policy search. However, PoWER is a simplified version of the other MBM discussed above, 
since only the mean of the policy distribution is learned, while the covariance is a parameter controlled by the use. PoWER also follows the natural gradient. Relative entropy policy search (REPS) [224] is another MBM that has been designed for reinforcement learning. Two interesting particularities of REPS are: i) the model is a Gibbs-Boltzmann distribution, as opposed to the standard normal distribution; and ii) it includes a sophisticated mechanism to evaluate the fitness of each candidate policy model (which implies solving an optimization problem at every iteration). Policyimprovement-with-path-integrals (PI2) [281] is another MBM specifically designed for policy search, and also very related to CMA-ES. A line by line comparison of CMA-ES and PI2 can be found in [273], which shows that, while CMA-ES is a general black-box-optimization algorithm that is applied to reinforcement learning by taking the total reward of each episode as objective value, PI2 is able to use reward information obtained at every time-step of the episode.

In this paper, we propose a general distributed damped PFO framework and a fully distributed implementation of CE as specific instance of such framework. Similar to previous chapters, we say that the algorithm is fully distributed in the sense that we only assume local communication between neighbors over a connected graph. No central agent fusing information, or load balancing server or any kind of master or coordinator agent is required. All agents have the same role and run the same algorithm to cooperatively learn the parameters of the probabilistic model. In particular, we use the same distributed stochastic approximation techniques that we used in Chapter 3 for extending the stochastic-approximation single-agent-CE (SACE) algorithm due to [128] to a network of cooperative agents.

As discussed in Section 3.1 of previous chapter, there are three main strategies for implementing distributed stochastic approximations, namely incremental (see, e.g., [199, 231, 205, 135, 39, 172]), consensus (e.g., [289, 212, 201, 232, 270]) and diffusion (e.g, [56, 58, 248, 57, 249, 36, 246, 247]) strategies. In a incremental strategy, the algorithm has first to determine a cyclic trajectory that visits all agents in the network. Then, the agents update their estimate sequentially. If some agent or link fails, a new cycle has to be computed, which is a combinatorial problem. Both diffusion and consensus strategies surmount these problems, but diffusion enhances stability and performance with respect to consensus algorithms. Therefore, we only focus on diffusion-based stochastic approximation algorithms in this paper.

DCE can be related to many other global optimization methods. A standard heuristic for solving global optimization problems consists in running a sequence of instances of a local optimization algorithm, the idea is to wait until an instance has 
converged and then start another instance from a different initial condition, so that each instance explores a different region of the solution space. DCE extends this idea to cooperative exploration, where multiple networked agents start from different initial conditions, so that they explore multiple regions of the solution space simultaneously.

DCE can be also seen as a meta-evolutionary algorithm. Each agent draws its own population of samples and performs some truncation-selection mechanism to guide the model update toward the most promising samples. In addition, at another level, the network of agents can be seen as a population of algorithms, one per agent, with a more involved evolutionary mechanism, with the network topology influencing the manner in which the individual algorithms are combined.

The idea of solving a sequence of problems, implicit in MBM, is very related to continuation or graduated optimization algorithms [194, 119]. In Section 4.8, we use this connection to discuss some insights on why the proposed DCE has enhanced robustness against local maxima with respect to SACE.

PFO can be seen as a Monte Carlo approximation of Bayesian filter optimization (BFO) (see Section 4.3.1), where the agents aim to find some optimal distribution over the solution space. This is different from other Bayesian optimization (BO) algorithms that estimate a distribution over the space of objective functions using Gaussian processes (see, e.g., [50, 259] and references therein).

Finally, many algorithms have been proposed for distributed convex optimization under different scenarios (like noisy links, changing topologies or asynchronous iterations, see, e.g., [201, 47, 56, 343, 57, 55, 337-339, 319]). Nevertheless, there are much fewer studies on distributed nonconvex optimization. Although there are some useful theoretical works [36, 232] analyzing the convergence of diffusion and consensus first-order methods over nonconvex cost functions (indeed, we reference results from [36] in this paper), first-order methods are not effective for optimizing black-box multidimensional multi-extrema objectives.

\subsubsection{Contributions}

The main algorithmic contribution of this work is the diffusion-based distributedCE (DCE) algorithm, which offers four main benefits: i) networked agents increases robustness against local maxima by reducing their local Monte Carlo noise and exploring the solution space more efficiently, even showing superior performance than the singleagent algorithm for the same total number of evaluated samples (see Section 4.7.2); ii) the computation load is distributed among the agents; iii) data is never exchanged so privacy is preserved; and $i v$ ) the algorithm scales to large networks by tying 
communication costs per agent to the neighborhood size, instead of the network size, and with inherent stability against agent or link failure.

We also provide some relevant theoretical contributions. In particular, we relax conditions from previous analysis of the single-agent BFO framework due to [340]; then we use this result to show convergence of the a damping generalization of BFO to the global optimum; and, finally, we show convergence to the optimal solution for the cooperative multi-agent BFO setting. Moreover, we prove a conjecture due to [108] and generalize their analysis of the single-agent $\mathrm{CE}$ algorithm for discrete optimization to continuous domains and for a large family of likelihood functions under mild conditions. Finally, we extend almost sure convergence analysis of the stochastic approximation single-agent SACE from [128] to the cooperative multiagent setting. As a byproduct, we also extend a result on almost sure agreement from [36] to the case of left-stochastic combination matrices, which should be useful for more general distributed stochastic approximations.

In addition to the algorithmic and theoretical contributions, we give novel insights on how diffusion-based distributed stochastic approximation algorithms behave on non-convex problems, on the relationship of SACE with continuation optimization schemes, and on the use of the proposed DCE for multiobjective optimization problems.

Publications. A publication with the results described in this chapter is currently under review. Some preliminary results were included in [302].

Notation. We change notation with respect to Chapters 3-5. In particular, we use $\pi$ to denote importance weights (instead of policy) and $\xi$ to denote Monte Carlo noise (instead of importance weight). In this chapter, we use over-line notation, ${ }^{-}$, to emphasize that some variable is the result of the network weighted average of each agent's variable.

\subsection{Problem Setting}

Consider a network of $N$ agents that cooperate to solve the following problem in a fully distributed manner:

$$
\underset{x \in \mathbb{R}^{M}}{\operatorname{maximize}} J(x)
$$

where $J: \mathbb{R}^{M} \rightarrow \mathbb{R}$ is an unknown, deterministic, continuous, possibly nonconvex and non-differentiable objective function and $M$ is the problem dimensionality. We assume 
that $J$ is upper bounded, i.e., there exists some scalar $J_{\max }$ such that

$$
J(x) \leq J_{\max }, \quad \forall x \in \mathbb{R}^{M}
$$

Let $\mathbb{K}^{\star}$ be the set of optimal solutions to problem (4.1), i.e.,

$$
\mathbb{X}^{\star} \triangleq \arg \max _{x \in \mathbb{R}^{M}} J(x)
$$

The search is initialized over a non-empty compact set (usually a box) $\mathcal{X} \subset \mathbb{R}^{M}$, but the agents are allowed to evaluate samples beyond this initial search set. We assume that this initial set contains the optimal set, i.e., $\mathbb{X}^{\star} \subset \mathbb{X}$.

Instead of directly tackling problem (4.1), we use a model-based approach, which consists in modelling the optimal solution by a probabilistic model $g(x)$, so that we have to find a probabilistic distribution that assigns most of its probability density to the set of optimal solutions of problem (4.1). This means that, although $J(x)$ is a deterministic output variable, we will consider $x$ as a realization of a random variable $\boldsymbol{x}$, so that problem (4.1) is transformed into a new model-based problem:

$$
\underset{g \in \mathcal{G}}{\operatorname{maximize}} \mathbb{E}_{g}[J(\boldsymbol{x})] \triangleq \int J(x) g(x) d x
$$

where $\mathcal{G}$ is the space of distributions. Let $g^{\star}$ denote an optimal distribution that solves $(4.4)$ :

$$
g^{\star} \triangleq \arg \max _{g \in \mathcal{G}} \mathbb{E}_{g}[J(\boldsymbol{x})]
$$

Every optimal distribution satisfies:

$$
\int_{\swarrow^{\star}} g^{\star}(x) d x=1
$$

In the case that $J$ has a unique maximizer $x^{\star} \in \mathbb{R}^{M}$, such that $\mathbb{X}^{\star}=\left\{x^{\star}\right\}$, the optimal distribution $g^{\star}$ will be unique and given by a Dirac delta function [7, Sec. 1.11] over $x^{\star}$, yielding $\mathbb{E}_{g}[J(\boldsymbol{x})]=J\left(x^{\star}\right)$. In general, there may be several (even infinite) distributions that solve (4.5). In any case, we will not search for one particular optimal distribution, rather, we will search for any distribution for which (4.6) holds. Note that if we draw a sample $x$ from some optimal distribution $g^{\star}$ that satisfies (4.6), then it holds that $\mathbb{P}\left(\boldsymbol{x}=x^{\star} \in X^{\star}\right)=1$. Therefore, as long as we can find any optimal distribution $g^{\star}$ by solving the new problem (4.4), we could find the optimal solution to (4.1) equivalently. 


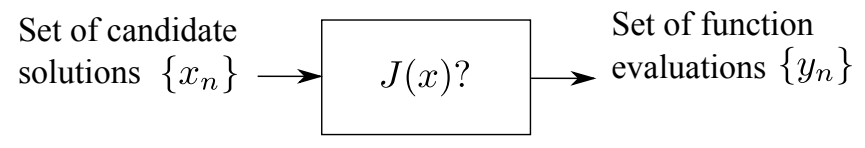

Figure 4.1 Black-box optimization.

Similar to Chapters 2 and 3, the network of agents forms a connected graph (i.e., by connected we mean that there is at least one path between every pair of agents, and they are not required to be connected directly in one hop). This graph is represented by a non-negative connectivity matrix $C=\left[c_{l k}\right]$ of size $N \times N$, such that the element $c_{l k}$ represents the weight given by agent $k$ to the information coming from agent $l$. Each agent $k$ is only allowed to communicate within its own neighborhood $\mathcal{N}_{k}$, which is defined as the set of agents that are directly connected with agent $k$, including itself. Since no routing or packet forwarding is allowed, we have $c_{l k}=0$ if agent $l \notin \mathcal{N}_{k}$. Indeed, we can formally define the neighborhood of agent $k$ as $\mathcal{N}_{k} \triangleq\left\{l: c_{l k}>0\right\}_{l=1}^{N}$, i.e., the set of all $l$ with positive weight.

We remark that the agents know nothing of the objective beforehand (neither its analytical form, nor its gradient). Rather, the objective function is like a "blackbox", which the agents could query its value $J(x)$ for a particular input solution $x$. At every iteration $i$, the information available to each agent includes a private data source and the shared estimates from its neighbors. The private data source includes candidate solution and output value pairs, which are never shared with others during the estimation process.

\subsection{Preliminaries}

In this section, we review previous developments on single-agent particle filter optimization, which will be the base for our derivation of a distributed algorithm. First, we introduce Bayesian filter optimization to obtain a recursive rule for updating the belief distribution, and discuss the likelihood function as a design parameter that must be chosen such that it guides the exploration of the objective function. Second, since drawing samples from an arbitrary belief distribution may be difficult, we introduce a family of surrogate sampling distributions and show how to project the belief over such family efficiently. We also introduce importance sampling so that the belief update recursion remains the same even when using a surrogate sampling distribution. Finally, we review a particle filter that is able to obtain a sequence of sampling distributions 
efficiently, as well as how to update a sequence of likelihood functions and how to estimate the the importance sampling weight from samples.

\subsubsection{Bayesian filter optimization (BFO)}

An MBM follows the following template. At every time $i$, we sample a candidate solution from our previous belief and query the objective function to obtain an output value:

$$
\begin{aligned}
\boldsymbol{x}_{i} & \sim g_{i-i} \\
\boldsymbol{y}_{i} & =J\left(\boldsymbol{x}_{i}\right), \quad i=0,1, \ldots
\end{aligned}
$$

Then, we use the observed output value to update the belief. This process is repeated until convergence, hopefully to the optimal density $g^{\star}$ given by (4.5).

We now show that this MBM idea of updating the belief so that it approaches the optimal density $g^{\star}$ could be expressed as a Bayesian filter [340]. Specifically, the optimal solution is modeled as a latent random variable to be estimated, and the output values are modeled as noisy measurements of the optimal value, where the randomness comes from our uncertain of the true solution. More formally, consider the following static state-space model:

$$
\begin{aligned}
\boldsymbol{x} & \sim g^{\star} \\
\boldsymbol{y}_{i} & =J(\boldsymbol{x})-\boldsymbol{\nu}_{i}, \quad i=0,1, \ldots
\end{aligned}
$$

where $\boldsymbol{x}$ is the latent variable that represents the optimal solution to be estimated, which will be fixed over time; $J(\boldsymbol{x})$ denotes the value at the optimal solution; and $\boldsymbol{\nu}_{i} \sim h_{\nu}$ is a random variable that models our uncertainty of where the solution lies. In other words, we consider the belief $g_{i-i}$ to be an approximation to $g^{\star}$ and split the queried value $J\left(\boldsymbol{x}_{i}\right)$ into two terms: the actual optimal value, $J(\boldsymbol{x})$, and some error, $\boldsymbol{\nu}_{i}$, due to the belief approximation.

Let $g_{i}$ denote the posterior probability (i.e., our belief of $\boldsymbol{x}$ given the observed function values by time $i, y_{0: i} \triangleq\left\{J\left(x_{0}\right), \ldots, J\left(x_{i}\right)\right\}$, which are evaluated at the set of previous candidate solutions, $\left\{x_{0}, \ldots, x_{i}\right\}$ :

$$
g_{i}(x)=p\left(x \mid y_{0: i}\right)
$$


Using Bayes rule, the $g_{i}(x)$ can be computed recursively as

$$
g_{i}(x)=p\left(x \mid y_{0: i-1}, y_{i}\right)=\frac{p\left(y_{i} \mid x\right) p\left(x \mid y_{0: i-1}\right)}{p\left(y_{i} \mid y_{0: i-1}\right)}=\frac{p\left(y_{i} \mid x\right) g_{i-1}(x)}{\int p\left(y_{i} \mid x\right) g_{i-1}(x) d x}
$$

We refer to this method as Bayesian-filter-optimization (BFO).

We remark that the likelihood depends on the latent (optimal) variable, rather than on the set of observed candidate solutions, $\left\{x_{0}, \ldots, x_{i}\right\}$. The reason is that, once we reformulated the problem as (4.9)-(4.10), we remove completely the observed candidate solutions from the formulation. Rather, there is only the latent variable, $\boldsymbol{x}$, that represents the optimal solution, with distribution $g^{\star}$. Indeed, we introduce the uncertainty, $\boldsymbol{\nu}_{i}$, as an artifact to model the fact that the latent distribution, $g^{\star}$, is unknown, such that: $\nu_{i}=J\left(\boldsymbol{x}=x \in \mathbb{K}^{\star}\right)-J\left(x_{i}\right)$.

Recursion (4.12) is useful because it suggests an algorithm for iteratively updating the belief. However, there are some major challenges. First of all, the likelihood $p\left(y_{i} \mid x\right)$ is unknown because the analytical form of $J(x)$ is unknown. Second, sampling from an arbitrary belief distribution could be difficult, thus it is more convenient to sample from a surrogate distribution that is as "close" as possible to the original belief; and we will use importance sampling to compensate the divergence between the actual belief and the surrogate distribution. Finally, we have to implement a recursion on the sampling distribution that can be efficiently approximated from samples, including the importance weights. In the rest of this subsection we address the issue of not knowing the likelihood function, as we proceed to explain.

An interesting observation we can make is that each particular form of likelihood function will lead to a particular stochastic process $\left\{\left(\boldsymbol{x}_{i}, \boldsymbol{y}_{i}\right)\right\}_{i=0}^{\infty}$, where $\boldsymbol{x}_{i} \sim g_{i}$ and $\boldsymbol{y}_{i}=J\left(\boldsymbol{x}_{i}\right)$. Now, if we take a different perspective and treat the likelihood function as a design option, then for each choice of the likelihood function we are indeed designing a particular algorithm of generating a stochastic process that, hopefully, could converge to the solution. We remark that, in this case, our choice of the likelihood function may not be the same as the ground truth likelihood. Nevertheless, we will show in Sec. 4.6 that when the chosen likelihood satisfies the property that the value of $p\left(y_{i} \mid x\right)$ at $y_{i}=J_{\max }$ is greater than any other feasible value, i.e.,

$$
p\left(y_{i} \mid x\right)<p\left(J_{\max } \mid x\right), \quad \forall y_{i}<J_{\max }
$$

then the generated sequence $\left\{\left(x_{0}, y_{0}\right),\left(x_{1}, y_{1}\right),\left(x_{2}, y_{2}\right), \ldots\right\}$ will converge to the true solution $\left(x^{\star}, J_{\max }\right)$. The intuition behind the formal convergence proofs provided in Section 4.6 is that if we interpret the Bayesian filter (4.12) as a guided random 
exploration of the unknown objective function, then condition (4.13) means that our designed likelihood function will direct the stochastic process to generate $y_{i}$ samples that are closer to $J_{\max }$, which intuitively implies that the $x_{i}$ samples must be generated by distributions that are closer to $g^{\star}$. Now, since we do not know where $J_{\max }$ lies, one practical way of ensuring that property (4.13) holds is to make $p\left(y_{i} \mid x\right)$ a monotonically increasing function over the entire interval of $y_{i}$. This distribution is well defined since, according to (4.3), we have that $y_{i} \in\left(-\infty, J_{\max }\right]$, so that $p\left(y_{i} \mid x\right)$ has upper-bounded support and can be properly normalized. Interestingly, an increasing likelihood function resembles the idea of survival of the fittest used in evolutionary algorithms [101].

Note that the likelihood $p\left(y_{i} \mid x\right)$ is in both the numerator and denominator of (4.12). Thus, we can replace $p\left(y_{i} \mid x\right)$ with any non-negative and monotonically increasing function $h:\left(-\infty, J_{\max }\right] \rightarrow \mathbb{R}_{+}$, without taking care of the normalization factor (where $\mathbb{R}_{+}$denotes the set of nonnegative real numbers). Examples of monotonically increasing likelihood functions are the proportional likelihood and the smooth indicator functions.

The proportional likelihood allocates its probability mass proportional to the output value as follows

$$
h^{\text {pro }}(J(x)) \triangleq J(x)
$$

We remark that the likelihood must be nonnegative. Hence, in order to (4.14) be a valid distribution, we must shift $J(x)$ with some constant value $\epsilon_{\text {pro }}$ so that $J(x)+\epsilon_{\text {pro }} \geq 0$, $\forall x \in \mathbb{R}^{M}$. The problem is that it requires $J$ to be lower bounded and the knowledge of such lower bound.

A second choice is the smooth indicator function [341], which is a smooth strictly increasing version of the indicator function that gives low probability density to the values that are below some threshold, $\gamma$, and high probability density to the values that are above the threshold:

$$
h^{\mathrm{smo}}(J(x) ; \gamma, \epsilon) \triangleq \frac{1}{1+e^{-\epsilon(J(x)-\gamma)}}
$$

where $\epsilon \gg 0$ is a parameter that determines the slope of the function. The threshold, $\gamma$, is usually set to a high quantile of the distribution of output values, so that most of the density of $h^{\text {smo }}$ is around $J_{\max }$, which is required for fast convergence as we will see in Section 4.5. Moreover, note that $h^{\text {smo }}$ is non-negative by construction, so that it can be used as a valid unnormalized likelihood distribution without any knowledge of $J$. These two reasons - being able to allocate most density around $J_{\max }$ and being 
non-negative - make the smooth indicator function our preferred choice for Bayesian filter optimization.

Proportional likelihood

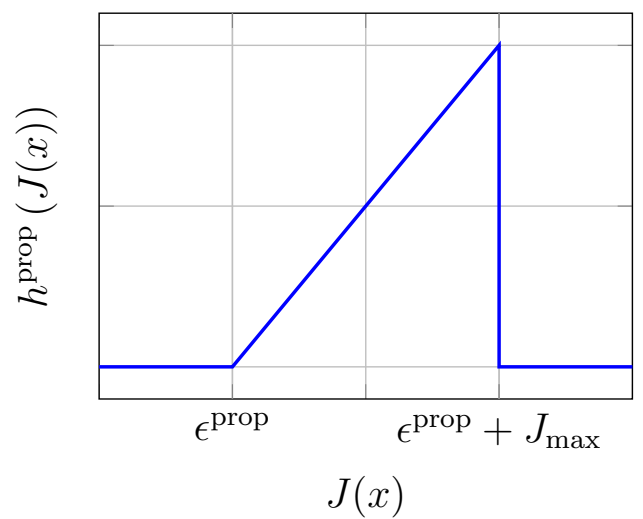

Smooth indicator likelihood

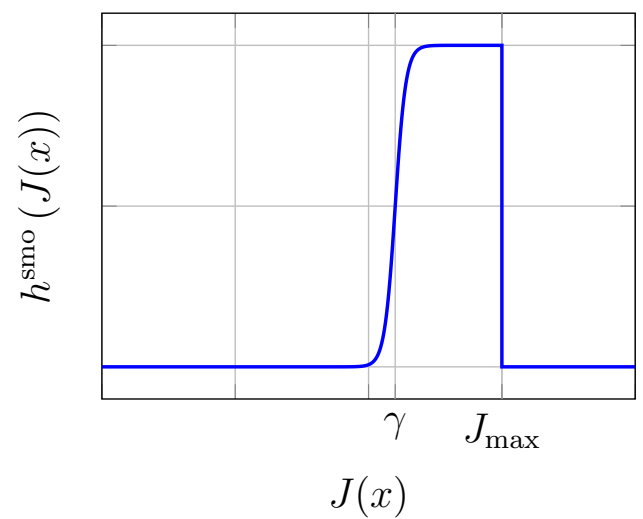

Figure 4.2 Example of increasing likelihood functions. The left side shows the proportional likelihood (4.14), which is proportional to the queried value $J(x)$. Note that we have considered that $J(x)$ may be negative, so it has been shifted to ensure $\epsilon^{\text {prop }}+J(x) \geq 0$. The right side shows the smooth indicator likelihood function (4.15) for $\epsilon=100$ and $\gamma=0.6 J_{\max }$.

As we have already advanced, in Section 4.5, we show that likelihoods like (4.14) or (4.15) guarantee asymptotic convergence of the posterior distribution towards the global optimal. Nevertheless, computing the exact posterior at every iteration is a challenging task in general. Hence, in order to build practical algorithms, we will rely on a particle filter - sequential Monte Carlo - approximation of (4.12). A particle filter requires drawing samples (i.e., particles) from the current belief at every iteration. Since sampling from arbitrary distributions is usually difficult, in the following subsection, we introduce a surrogate distribution that makes the sampling process easier.

\subsubsection{BFO with surrogate sampling distribution}

Reference [340] found that the Cross Entropy (CE) algorithm [152] can be interpreted as a particle filter implementation of (4.12) where, instead of sampling from the belief distribution, particles are drawn from a surrogate sampling distribution, $f_{w}$. We follow the same idea here and choose the sampling distribution to belong to a parametrized family of distributions $\left\{f_{w}(\cdot)\right\}_{w \in \mathbb{W}}$, where $w$ is the parameter and $\mathbb{W}$ is the parameter set.

We want the sampling distribution to be as similar as possible to the belief distribution. The similarity between distribution $f_{w}(x)$ and any distribution $g(x)$ can be 
measured by Kullback-Leibler (KL) divergence, defined as

$$
\mathcal{D}\left(g, f_{w}\right) \triangleq \mathbb{E}_{g}\left[\log \frac{g(\boldsymbol{x})}{f_{w}(\boldsymbol{x})}\right]
$$

Intuitively, minimizing (4.16) could be interpreted as projecting the belief $g_{i}(x)$ onto the parametrized family of distributions. More technically, the parameter $w$ that minimizes (4.16) is also the maximum likelihood estimate (MLE) of the values drawn from $g$ under model $f_{w}$ (see, e.g., [38, p. 57]).

We choose the surrogate sampling distribution $f_{w}$ to belong to the natural exponential family (NEF) of distributions (see, e.g., [153, App. D.1]), which can be expressed as

$$
f_{w}(x) \triangleq e^{w^{\top} \phi(x)-Z(w)}
$$

where $w \in \mathbb{W}$ is called the natural parameter, $\phi(x)$ is known as the natural statistics and $Z(w): \mathbb{W} \rightarrow \mathbb{R}$ ensures normalization:

$$
Z(w) \triangleq \log \int e^{w^{\top} \phi(x)} d x
$$

Note that $Z(w)$ is strictly convex and differentiable. The natural parameter set, $\mathbb{W}$, is the open convex set given by

$$
\mathbb{W} \triangleq\{w: Z(w)<\infty\}
$$

We only consider NEF distributions for which there is a one-on-one mapping between $w$ and the expected value of the natural statistics:

$$
\mathbb{E}_{f_{w}}[\phi(\boldsymbol{x})] \triangleq \int \phi(x) f_{w}(x) d x
$$

Therefore, $f_{w}$ can be equivalently parameterized with $\mathbb{E}_{f_{w}}[\phi(\boldsymbol{x})]$, which is known as mean parameter. Let $\Theta$ denote the set of mean parameters that can be obtained with a valid natural parameter:

$$
\Theta \triangleq\left\{\mathbb{E}_{f_{w}}[\phi(\boldsymbol{x})]: w \in \mathbb{W}\right\}
$$


Then, we can introduce the mapping between natural and mean parameters as $F$ : $\operatorname{int}(\Theta) \rightarrow \mathbb{W}$, such that

$$
w=F\left(\mathbb{E}_{f_{w}}[\phi(\boldsymbol{x})]\right)
$$

Indeed, it is well known that the inverted mapping $F^{-1}$ is actually obtained as the gradient of the normalization term (see, e.g., [153, App. D.1], [208]):

$$
\mathbb{E}_{f_{w}}[\phi(\boldsymbol{x})]=F^{-1}(w)=\nabla_{w} Z(w)
$$

For example, for the multivariate normal distribution with sufficient statistics given by its mean $\mu$ and covariance $\Sigma$, the natural statistics, $\phi(x): \mathbb{R}^{M} \rightarrow \mathbb{R}^{M+M^{2}}$, are composed of all first and second order terms:

$$
\phi(x)=\left[\begin{array}{c}
x \\
x x^{\top}
\end{array}\right]
$$

The mean parameter corresponds to the mean vector and correlation matrix of the Gaussian distribution:

$$
\mathbb{E}_{f_{w}}[\phi(\boldsymbol{x})]=\mathbb{E}_{f_{w}}\left[\begin{array}{c}
\boldsymbol{x} \\
\boldsymbol{x} \boldsymbol{x}^{\top}
\end{array}\right]=\left[\begin{array}{c}
\mu \\
\Sigma+\mu \mu^{\top}
\end{array}\right] \in \mathbb{R}^{M+M^{2}}
$$

and the mapping $F$ that transforms the mean parameter to the natural parameter is given by

$$
w=\left[\begin{array}{c}
\Sigma^{-1} \mu \\
-\frac{1}{2} \Sigma^{-1}
\end{array}\right] \in \mathbb{R}^{M+M^{2}}
$$

where both $\mathbb{W}$ and $\Theta$ are the Cartesian product of $\mathbb{R}^{M}$ and a positive semidefinite cone.

There are two main reasons to choose the NEF: First, it is easy to sample from many members of the NEF (e.g., the multivariate normal distribution). Second, when projected according to (4.16), the expected value of $\phi(x)$ under both $f_{w}$ and $g$ are the same. To see this, let

$$
w^{o} \triangleq \arg \min _{w \in \mathbb{W}} \mathcal{D}\left(g, f_{w}\right)
$$


which requires that $\left.\nabla_{w} \mathcal{D}\left(g, f_{w}\right)\right|_{w^{o}}=0$. Substituting the definition of KL-divergence, we get

$$
\begin{aligned}
\left.\nabla_{w} \mathcal{D}\left(g, f_{w}\right)\right|_{w^{o}} & =\nabla_{w}\left[\mathbb{E}_{g}\left[\log g(\boldsymbol{x})-\log e^{w^{\top} \phi(\boldsymbol{x})-Z(w)}\right]\right]_{w^{o}} \\
& =\nabla_{w}\left[\mathbb{E}_{g}\left[\log g(\boldsymbol{x})-\left(w^{\top} \phi(\boldsymbol{x})-Z(w)\right)\right]\right]_{w^{o}} \\
& =-\mathbb{E}_{g}[\phi(\boldsymbol{x})]+\left.\nabla_{w} Z(w)\right|_{w^{o}} \\
& =\mathbb{E}_{f_{w^{o}}}[\phi(\boldsymbol{x})]-\mathbb{E}_{g}[\phi(\boldsymbol{x})]=0
\end{aligned}
$$

where the last step used the relationship $\nabla_{w} Z(w)=\mathbb{E}_{f_{w}}[\phi(\boldsymbol{x})]$ from (4.23). From (4.28), it is clear that the expectation of the sufficient statistics $\phi(x)$ under the distribution $g(x)$ is equal to the expectation of $\phi(x)$ under $f_{w^{o}}(x)$ :

$$
\mathbb{E}_{f_{w^{o}}}[\phi(\boldsymbol{x})]=\mathbb{E}_{g}[\phi(\boldsymbol{x})]
$$

Although equivalence (4.29) is promising, it does not hold for multiple iterations of the belief update (4.12). For instance, suppose that we projected $g_{i-1}$ onto the parametric NEF family (4.17) and obtained $f_{w_{i-1}^{o}}$. If we aim to use $f_{w_{i-1}^{o}}$ in (4.12), then the belief update becomes:

$$
g_{i}^{\prime}(x)=\frac{h(J(x)) f_{w_{i-1}^{o}}(x)}{\int h(J(z)) f_{w_{i-1}^{o}}(z) d z}
$$

which is different from (4.12), i.e., $g_{i}^{\prime} \neq g_{i}$ since $f_{w_{i-1}^{o}} \neq g_{i-1}$. Thus, the projection introduces some error that is accumulated along the iterations. Reference [340] proposed to correct this error by considering the surrogate distribution as an importance density in the importance sampling framework. Introduce the importance weights:

$$
\pi_{i}(x)=\frac{g_{i}(x)}{f_{w_{i}^{o}}(x)}
$$

where we assume that any $x$ that makes $g_{i-1}(x)>0$ will lead to $f_{w_{i-1}^{o}}(x)>0$. In the next subsection, we show that $\pi_{i}$ could be efficiently computed.

Using (4.31), we can express the actual belief $g_{i}$ given by (4.12) in terms of the surrogate distribution, $f_{w_{i-1}^{o}}$ :

$$
g_{i}(x)=\frac{h(J(x)) f_{w_{i-1}^{o}}(x) \pi_{i-1}(x)}{\int h(J(z)) f_{w_{i-1}^{o}}(z) \pi_{i-1}(z) d z}
$$


In Section 4.5, we show that (4.32) asymptotically concentrates on the optimal set $\mathbb{X}^{\star}$. Nevertheless, regarding a practical implementation, we still have the problem that we do not know the exact form of $h(J(\boldsymbol{x}))$ (recall that $J$ is unknown, and even if it were known, the distribution of the transformation $J(\boldsymbol{x})$ could be hard to deduce in general). Fortunately, once we have expressed the Bayesian filter as a function of the parametric surrogate sampling distribution, we can derive an efficient particle filter approximation.

\subsubsection{Particle filter optimization (PFO) with projected impor- tance re-sampling step}

We first show that the projection operation (4.27) could be efficiently implemented. Since $f_{w}(x)$ is in NEF and is completely characterized by its mean parameter $\mathbb{E}_{f_{w}}\{\phi(x)\}$, which is the same as $\mathbb{E}_{g}\{\phi(x)\}$ when $w$ satisfies (4.27), we could compute the projected $f_{w_{i}^{o}}(x)$ by computing $\mathbb{E}_{g_{i}}[\phi(x)]$. Multiplying both sides of (4.32) by $\phi(x)$ and integrating over the solution space, we obtain:

$$
\int \phi(x) g_{i}(x) d x=\frac{\int h(J(x)) \pi_{i-1}(x) \phi(x) f_{w_{i-1}^{o}}(x) d x}{\int h(J(z)) \pi_{i-1}(z) f_{w_{i-1}^{o}}(z) d z}
$$

From (4.29) and (4.33), we obtain:

$$
\mathbb{E}_{f_{w_{i}^{o}}}[\phi(\boldsymbol{x})]=\frac{\mathbb{E}_{f_{w_{i-1}^{o}}}\left[h(J(\boldsymbol{x})) \pi_{i-1}(\boldsymbol{x}) \phi(\boldsymbol{x})\right]}{\mathbb{E}_{f_{w_{i-1}^{o}}}\left[h(J(\boldsymbol{x})) \pi_{i-1}(\boldsymbol{x})\right]}
$$

Since we assume that $f_{w_{i}^{o}}$ belongs to the NEF, it is completely characterized by its sufficient statistic $\mathbb{E}_{f_{w_{i}^{o}}}[\phi(\boldsymbol{x})]$. Once we have expressed the belief update as a recursion on the sampling distribution, we can build a particle filter approximation of (4.34) as follows. At every iteration $i$, we draw a set of $S_{i}$ samples $\left\{x_{i, s}\right\}_{s=1}^{S_{i}}$ from our current model $f_{w_{i-1}^{o}}$. These samples are evaluated (in a black-box fashion) to obtain their corresponding output values, so that we end up with a set of candidate solution and output value pairs, denoted by $\Lambda_{i} \triangleq\left\{x_{i, s}, y_{i . s}\right\}_{s=1}^{S_{i}}$. Then, we use $\Lambda_{i}$ to estimate the two expected values in the right side of (4.34) and compute the new sufficient statistics, which determines the new sampling model $f_{w_{i}^{o}}$. We repeat this process until some stop condition is satisfied.

The exact shape of the likelihood has to be learned from samples during the optimization process. Let $h_{i}$ denote the estimate of the likelihood at time $i$. For the smooth indicator likelihood function, the elite-threshold $\gamma_{i}$ can be estimated as the $q$-th 
highest output value obtained so far, which can be seen as the Monte Carlo estimate of the true $q / S_{i}$-th quantile of the distribution of output values $(\boldsymbol{y}=J(\boldsymbol{x}))$ :

$$
h_{i}^{\mathrm{smo}}\left(y_{i, s}\right) \propto \frac{1}{1+e^{-\epsilon\left(y_{i, s}-\gamma_{i}\right)}}
$$

In order to estimate the importance weight $\pi_{i-1}$ from samples, note that it depends on the belief distribution $g_{i-1}$, which is not explicitly known. However, since the belief has been generated recursively, we can express the importance weights in terms of the initial distribution. Assume that the initial belief is uniformly distributed over the initial search space, $\mathbb{X}$, and zero everywhere else, i.e., $g_{0}(x)=1 / Z_{0}, \forall x \in \mathbb{X}$, where $Z_{0}=\int_{\mathfrak{X}} x d x$ is the normalization constant. Then, we can roll out the belief update recursion (4.12) to obtain $g_{i-1}$ as a function of $g_{0}$ :

$$
\begin{aligned}
g_{i}(x) & =\frac{h(J(x)) g_{i-1}(x)}{\int h(J(z)) g_{i-1}(z) d z} \\
& =\frac{h(J(x)) \frac{h(J(x)) g_{i-2}(x)}{\int h(J(u)) g_{i-2}(u) d u}}{\int h(J(z)) \frac{h(y \mid z) g_{i-2}(z)}{\int h\left(J(u) g_{i-2}(u) d u\right.} d z} \\
& =\frac{h(J(x))^{2} g_{i-2}(x)}{\int h(J(z))^{2} g_{i-2}(z) d z} \\
& \vdots \\
& =\frac{h(J(x))^{i} g_{0}(x)}{\int h(J(z))^{i} g_{0}(z) d z} \\
& =\frac{\frac{1}{Z_{0}} h(J(x))^{i}}{\frac{1}{Z_{0}} \int h(J(z))^{i} d z} \\
& =\frac{h(J(x))^{i}}{\int h(J(z))^{i} d z}
\end{aligned}
$$

were we have introduced $z, u \in \mathbb{R}^{M}$ in the normalization terms to see the cancellation of factors more clearly. Hence, we can express the importance weight as

$$
\pi_{i-1}(x)=\frac{g_{i-1}(x)}{f_{w_{i-1}^{o}}(x)}=\frac{\frac{h(J(x))^{i-1}}{\int h(J(z))^{i-1} d z}}{f_{w_{i-1}^{o}}(x)}=\frac{h(J(x))^{i-1}}{f_{w_{i-1}^{o}}(x) \int h(J(z))^{i-1} d z}
$$

Since the importance weight $\pi_{i-1}$ appears in both numerator and denominator of (4.32), we can avoid the normalization term $\int h(J(z))^{i-1} d z$ in (4.37) and estimate another term, $\hat{\pi}_{i-1}$, that is proportional to the actual importance weight and only depends on 
known values:

$$
\hat{\pi}_{i-1}\left(x_{i, s}\right) \triangleq \frac{h\left(J\left(x_{i, s}\right)\right)^{i-1}}{f_{w_{i-1}^{o}}\left(x_{i, s}\right)} \propto \pi_{i-1}\left(x_{i, s}\right)
$$

We remark that we have to use a sample estimate of the likelihood parameter in (4.38), so that the likelihood function is time varying. Thus, the proportional importance weight should be actually defined as:

$$
\pi_{i-1}^{\prime}\left(x_{i, s}\right) \triangleq \frac{\prod_{j=1}^{i-1} h_{j}\left(J\left(x_{i, s}\right)\right)}{f_{w_{i-1}^{o}}\left(x_{i, s}\right)}
$$

For the smooth indicator likelihood (4.15) with large $\epsilon$, we have

$$
\prod_{j=1}^{i-1} h_{j}\left(J\left(x_{i, s}\right)\right) \approx h^{\mathrm{smo}}\left(J\left(x_{i, s}\right) ; \gamma_{\max }, \epsilon\right)
$$

where $\gamma_{\max }=\max \left[\gamma_{1}, \ldots, \gamma_{i}\right]$. For simplicity, we will continue using $\pi_{i-1}(x)$ for the theoretical derivations of this chapter, without worrying about how to estimate it from samples.

Let $\eta_{i}$ denote the Monte Carlo estimate of (4.34), such that

$$
\eta_{i}=\mathbb{E}_{f_{w_{i}^{o}}}[\phi(\boldsymbol{x})]+\xi_{i}^{o}
$$

where $\xi_{i}^{o}$ is some Monte Carlo noise. In theory, we could obtain $\eta_{i}$ at every iteration by computing the Monte Carlo estimate of (4.34) from i.i.d. samples drawn from our current sampling model $f_{w_{i-1}^{o}}$ :

$$
\eta_{i}=\frac{\sum_{s=1}^{S_{i}} h_{i}\left(J\left(x_{i, s}\right)\right) \pi_{i-1}\left(x_{i, s}\right) \phi\left(x_{i, s}\right)}{\sum_{s=1}^{S_{i}} h_{i}\left(J\left(x_{i, s}\right)\right) \pi_{i-1}\left(x_{i, s}\right)}
$$

where $h_{i}$ can be replaced by (4.35). However, in practice, due to the iterative nature of the process, the true sufficient statistics, $\mathbb{E}_{f_{w_{i-1}^{o}}}[\phi(\boldsymbol{x})]$, are unknown and we only know its approximation $\eta_{i-1}$, so that we have to draw samples from an approximate sampling model, $f_{w_{i-1}^{\eta}}$, with sufficient statistics given by our latest Monte Carlo estimate:

$$
\mathbb{E}_{f_{w_{i-1}^{\eta}}}[\phi(\boldsymbol{x})]=\eta_{i-1} \neq \mathbb{E}_{f_{w_{i-1}^{o}}}[\phi(\boldsymbol{x})]
$$

Therefore, due to Monte Carlo noise at every iteration, the particle filter produces a sequence of sampling models, $\left\{f_{w_{i}^{\eta}}\right\}$, which is different from the sequence generated 
by the Bayesian filter, $\left\{f_{w_{i}^{o}}\right\}$. In practice, this error can make the belief density to converge to a local maximum, instead to the desired optimal solution set. Reference [152] proposed a simple smoothing rule for updating (4.42) in little steps, so that the belief distribution moves in the right direction while is also able to compensate the error in the sequence $\left\{f_{w_{i}}^{\eta}\right\}$. Recently, reference [128] introduced a more principled and efficient approach. In the following section, we introduce a fully distributed diffusion-based implementation of CE that generalizes the single-agent SACE algorithm proposed by [128] to a network of cooperative agents.

One final remark before moving to the distributed algorithm, object of this chapter. Note that we are implicitly assuming that the Monte Carlo estimate in (4.43) remains in the interior of the mean parameter set at every iteration, i.e., $\eta_{i} \in \operatorname{int}(\Theta)$. In general, this is a non-trivial condition. Fortunately, in our case, this assumption is less restrictive than it may seem. For example, if $f_{w}$ is a multivariate normal distribution, the mean set is $\mathbb{R}^{M}$ (i.e., unconstrained). Moreover, the Monte Carlo approximation provides covariance matrices in the positive semidefinite cone by construction, and we can achieve non-singular covariance matrices (i.e., positive definite matrices in the interior of the positive semidefinite cone) by simply increasing the sample size.

\subsection{Distributed Cross-Entropy (DCE) Algorithm}

In this section, we propose the distributed-cross-entropy (DCE) algorithm. In order to derive DCE, we first introduce a distributed estimate of (4.42), cooperatively computed by a network of agents, and show that such network estimate reduces the variance of the local estimates that each agent would achieve without cooperation. Then, inspired by the single-agent stochastic-approximation SACE algorithm due to [128], we propose a distributed stochastic approximation, where each agent in the network runs its own algorithm but cooperates with other agents to explore the solution space more efficiently. By reducing Monte Carlo noise and improving the exploration capabilities, the proposed DCE greatly enhances robustness against local maxima, outperforming a SACE agent that evaluates as many samples per iteration as the entire network of DCE agents.

Similar to the distributed algorithms presented in Chapters $2-3$, the proposed algorithm is fully distributed in the sense that cooperation among agents is achieved through only one-hop communications with their neighbors (i.e., multi-hop transmissions or packet forwarding are prohibited). Thus, the communication cost per agent scales with the size of the neighborhood, instead of with the network size. Moreover, the 
agents only transmit their estimates of the belief distribution, not their data samples, preserving their privacy and reducing the communication cost.

\subsubsection{Distributed surrogate sampling distribution}

For the introduction of the particle filter optimization framework, we have considered a single belief distribution $g_{i}$ per iteration. Now, we have to consider as many distributions as agents in the network. Let $g_{k, i}, h_{k, i}$ and $y_{k, i}$ denote the belief, likelihood and sample evaluation, respectively, observed at time $i$ by every agent $k=1, \ldots, N$. Using these variables in (4.12), the belief update for every agent is given by:

$$
g_{k, i}(x)=\frac{h_{k, i}(J(x)) g_{k, i-1}(x)}{\int h_{k, i}(J(x)) g_{k, i-1}(x) d z}
$$

Similar to the single agent algorithm, each agent will sample from a surrogate distribution. In order to make each agent's sampling distribution as similar as possible to its belief distribution, we define the optimal parameters $\left\{w_{k, i}^{o}\right\}_{k=1}^{N}$, such that

$$
w_{k, i}^{o} \triangleq \arg \min _{w \in \mathbb{W}} \mathcal{D}\left(g_{k, i}, f_{w}\right)
$$

Similar to (4.29), the sufficient statistics of an agent's closest sampling distribution equal the statistics of its local belief:

$$
\mathbb{E}_{f_{w_{k, i}^{o}}}[\phi(\boldsymbol{x})]=\mathbb{E}_{g_{k, i}}[\phi(\boldsymbol{x})]
$$

Moreover, from (4.34), we obtain each agent's recursion for the sufficient statistics:

$$
\mathbb{E}_{f_{w_{k, i}^{o}}}[\phi(\boldsymbol{x})]=\frac{\mathbb{E}_{f_{w_{k, i-1}^{o}}}\left[h_{k, i}(J(\boldsymbol{x})) \pi_{k, i-1}(\boldsymbol{x}) \phi(\boldsymbol{x})\right]}{\mathbb{E}_{f_{w_{k, i-1}^{o}}}\left[h_{k, i}(J(\boldsymbol{x})) \pi_{k, i-1}(\boldsymbol{x})\right]}
$$

where importance weight for agent $k$ is given by

$$
\pi_{k, i-1}(x) \triangleq \frac{g_{k, i-1}(x)}{f_{w_{k, i-1}^{o}}(x)}
$$

Let $\Lambda_{k, i} \triangleq\left\{x_{k, i, s}, J\left(x_{k, i, s}\right)\right\}_{s=1}^{S_{k, i}}$ be the set of input (i.e., candidate solution) and output value pairs evaluated by agent $k$ at time $i$, and $S_{k, i}$ denotes its number of samples. Similar to the single-agent formulation $(4.42)$, the term $\mathbb{E}_{f_{w_{k, i}^{o}}}[\phi(\boldsymbol{x})]$ in $(4.47)$ 
is approximated by:

$$
\eta_{k, i} \triangleq \frac{\sum_{s=1}^{S_{k, i}} h_{k, i}\left(J\left(x_{k, i, s}\right)\right) \pi_{k, i-1}\left(x_{k, i, s}\right) \phi\left(x_{k, i, s}\right)}{\sum_{s=1}^{S_{k, i}} h_{k, i}\left(J\left(x_{k, i, s}\right)\right) \pi_{k, i-1}\left(x_{k, i, s}\right)}
$$

It is clear that when the number of samples tends to infinity, this estimate tends to the actual expected value:

$$
\lim _{S_{k, i} \rightarrow \infty} \eta_{k, i}=\mathbb{E}_{f_{w_{k, i}^{o}}}[\phi(\boldsymbol{x})]
$$

Similar to the single-agent case and due to the iterative nature of the process, since $\mathbb{E}_{f_{w_{k, i}^{o}}}[\phi(\boldsymbol{x})]$ is unknown, every agent has to produce its new sampling model from the instantaneous realization $\eta_{k, i}$. In particular, we consider each agent to produce a sequence of sampling models, $\left\{f_{w_{k, i}^{\eta}}\right\}$, such that

$$
\mathbb{E}_{f_{w_{k, i}^{\eta}}}[\phi(\boldsymbol{x})]=\eta_{k, i}
$$

Obviously, the instantaneous realization of the random noise, $\xi_{k, i}^{o}$, makes this sequence different from the sequence that would have been generated by the Bayesian filter, $\left\{f_{w_{k, i}^{o}}\right\}$. We can expect that the smaller the Monte Carlo noise, the more similar these sequences will be. In the following, we show that the agents can cooperate to reduce the noise.

Instead of locally solving (4.45), we propose a collaborative projection step, in which the agents minimize a convex combination of KL divergences across the network:

$$
\underset{w \in \mathbb{W}}{\operatorname{minimize}} S_{i}(w) \triangleq \sum_{k=1}^{N} \tau_{k} \mathcal{D}\left(g_{k, i}, f_{w}\right)
$$

where $\tau \triangleq\left(\tau_{k}\right)_{k=1}^{N}$ is the vector of weights, such that $\mathbb{1}^{\top} \tau=1$ and $\tau_{k} \geq 0$ for $k=1, \ldots, N$. Introduce the optimal parameter that minimizes (4.52):

$$
\bar{w}_{i}^{o} \triangleq \arg \min _{w \in \mathbb{W}} S_{i}(w)
$$

such that $\nabla_{w} S_{i}\left(\bar{w}_{i}^{o}\right)=0$. From (4.52), we have:

$$
\nabla_{w} S_{i}\left(\bar{w}_{i}^{o}\right)=\nabla_{w}\left[\sum_{k=1}^{N} \tau_{k}\left(\mathbb{E}_{g_{k, i}}\left[\log g_{k, i}(\boldsymbol{x})\right]+Z\left(\bar{w}_{i}^{o}\right)-\mathbb{E}_{g_{k, i}}^{\top}[\phi(\boldsymbol{x})] \bar{w}_{i}^{o}\right)\right]
$$




$$
\begin{aligned}
& =\sum_{k=1}^{N} \tau_{k}\left(\mathbb{E}_{f_{\bar{w}_{i}^{o}}}[\phi(\boldsymbol{x})]-\mathbb{E}_{g_{k, i}}[\phi(\boldsymbol{x})]\right) \\
& =\mathbb{E}_{f_{\bar{w}_{i}^{o}}}[\phi(\boldsymbol{x})]-\sum_{k=1}^{N} \tau_{k} \mathbb{E}_{g_{k, i}}[\phi(\boldsymbol{x})] \\
& =0
\end{aligned}
$$

Combining (4.46) and (4.54), we have:

$$
\mathbb{E}_{f_{\bar{w}_{i}^{o}}}[\phi(\boldsymbol{x})]=\sum_{k=1}^{N} \tau_{k} \mathbb{E}_{g_{k, i}}[\phi(\boldsymbol{x})]=\sum_{k=1}^{N} \tau_{k} \mathbb{E}_{f_{w_{k, i}^{o}}}[\phi(\boldsymbol{x})]
$$

Hence, the optimal sufficient statistics of the sampling distribution that solves (4.52) equal the weighted average of the statistics of the agents' actual beliefs. Let $\bar{\eta}_{i}$ denote the Monte Carlo estimate of (4.55) such that:

$$
\bar{\eta}_{i} \triangleq \sum_{k=1}^{N} \tau_{k} \eta_{k, i}
$$

It is clear that for large number of samples, this network estimate approximates to the actual network expected value:

$$
\lim _{S_{k, i} \rightarrow \infty} \bar{\eta}_{i}=\mathbb{E}_{f_{\bar{w}_{i}^{o}}}[\phi(\boldsymbol{x})]
$$

Thus, by solving the collaborative projection problem (4.52), the agents effectively combine their local estimates in (4.47). In the next subsection, we introduce a distributed stochastic approximation that enhances the exploration capabilities of the network and enhance the robustness against local maxima with respect to the single agent formulation.

\subsubsection{Distributed stochastic approximation}

In order to enhance robustness against local maxima for the single agent case, reference [152] proposed a useful heuristic that consists in including damping in the update-rule, such that the new sampling distribution is obtained as a convex combination of the particle filter update and the current sampling distribution. This damping allows to exploit the new information, while also gives chance to draw more samples from the the latest distribution. Recently, reference [128] extended the same idea (again for the single-agent case) and proposed to perform stochastic gradient descent to minimize 
the KL divergence (4.16), leading to a more principled smoothed update and better numerical results. The method proposed by reference [128] can be also seen as using a Robbins-Monro stochastic approximation of the form:

$$
\theta_{i}=\theta_{i-1}-\alpha_{i}\left(\theta_{i-1}-\eta_{i}\right)
$$

where $\alpha_{i}$ denotes the (possibly) time-varying step-size. Now, the sequence of sampling models, $\left\{f_{w_{i}}\right\}$, is determined by taking $\theta_{i}$ as sufficient statistics at every iteration:

$$
\mathbb{E}_{f_{w_{i}}}[\phi(\boldsymbol{x})]=\theta_{i}
$$

We remark this is different from the non-smoothed particle filter (4.42), where the sampling model was built from $\eta_{i}$, as given by (4.43). Again, we remark that we are implicitly assuming $\theta_{i} \in \operatorname{int}(\Theta)$. In the stochastic approximation literature, this is usually satisfied by projecting the iterates onto int $(\Theta)$. But, as explained above, we can avoid the projection step in our particular case (recall that for multivariate normal sampling distributions, the mean set is unconstrained and the sample covariance matrix lies in the positive semidefinite cone by construction, and can be ensured to lie in the interior of the cone by increasing the number of samples).

In this work, we extend (4.58) by proposing a diffusion-based multi-agent distributed stochastic approximation. In particular, we use the same adapt-then-combine (ATC) pattern discussed in Chapter 3. The proposed distributed algorithm is as follows. At every iteration, each agent draws a set of candidate solutions from its current sampling model, evaluates them to obtain a local dataset of candidate solution and output value pairs, and performs the estimation update (4.58) in isolation from this local dataset. Then, the agents share and combine their updated estimates with their neighbors. The resulting distributed recursion is as follows:

$$
\begin{aligned}
\widehat{\theta}_{k, i} & =\theta_{k, i-1}-\alpha_{i}\left(\theta_{k, i-1}-\eta_{k, i}\right) \\
\theta_{k, i} & =\sum_{l \in \mathcal{N}_{k}} c_{l k} \widehat{\theta}_{l, i}
\end{aligned}
$$

where we recall that $\mathcal{N}_{k}$ denotes the neighborhood of agent $k$ (i.e., the set of agents that can share information with agent $k$, including itself), $c_{l k}$ is the combination weight given by agent $k$ to information coming from agent $l$ and, for simplicity, we choose the same time-dependent step-size $\alpha_{i}$ for every agent. Once each agent has performed (4.60b), it builds a new sampling model, $f_{w_{k, i}}$, from the weighted average of the estimated 
sufficient statistics in its neighborhood:

$$
\mathbb{E}_{f_{w_{k, i}}}[\phi(\boldsymbol{x})]=\theta_{k, i}
$$

Note that no data sample is exchanged in (4.60b), rather only the current estimates are shared among neighbors, allowing the agents to preserve the privacy of their local datasets. In addition, mote that, since only within neighborhood communication is required, the communication cost for every agent scales with the size of its neighborhood, rather than with the total number of agents in the network. Thus, the algorithm can be efficient even in very large networks with communication cost per iteration for every agent $k$ in the order of $\mathcal{O}\left(\left|\mathcal{N}_{k}\right|\right)$.

The combination weights used in $(4.60 \mathrm{~b})$ are required to satisfy the same conditions (3.48)-(3.50) from Chapter 3, which we recall here for convenience:

$$
\begin{aligned}
& c_{l k} \geq 0, \quad \sum_{l \in \mathcal{N}_{k}} c_{l k}=1 \\
& c_{k k}>0 \text { for at least one agent } k \\
& c_{l k}=0 \text { if } l \notin \mathcal{N}_{k}
\end{aligned}
$$

We recall that conditions (4.62) make the combination matrix $C=\left[c_{l k}\right]$ left stochastic, such that

$$
C^{\top} \mathbb{1}=\mathbb{1}
$$

and that conditions (4.62)-(4.63), together wit the assumption that the graph is connected (i.e., there is a path between every pair of agents) make the combination matrix irreducible and aperiodic, so that we will be able apply Perron-Frobenius theory. Condition (4.63) is imposed by the network topology, meaning that only single-hop within neighborhood communication is allowed. As already discussed in previous chapters, there are several methods to obtain valid combination matrices (see, e.g., [246, Ch. 14]), even in a distributed manner, with different criteria, like leading to faster convergence speed [320] or to smaller variance of the estimates [336]. In Section 4.6.2, we will assume another rather technical condition on the combination matrix.

For a practical implementation of (4.60a)-(4.60b), we specialize the generic NEF statistics $\phi(x)$ for the multivariate Normal distribution. Introduce the mean and correlation matrix for each agent's sampling model $f_{w_{k, i}}$, respectively.:

$$
\mu_{k, i}=\mathbb{E}[\boldsymbol{x}], \quad R_{k, i}=\mathbb{E}\left[\boldsymbol{x} \boldsymbol{x}^{\top}\right]
$$


From (4.24) and (4.61), we get:

$$
\theta_{k, i}=\left[\begin{array}{c}
\mathbb{E}[\boldsymbol{x}] \\
\mathbb{E}\left[\boldsymbol{x} \boldsymbol{x}^{\top}\right]
\end{array}\right]=\left[\begin{array}{c}
\mu_{k, i} \\
R_{k, i}
\end{array}\right]
$$

Introduce the locally adapted intermediate estimates $\widehat{\mu}_{k, i}$ and $\widehat{R}_{k, i}$, such that

$$
\widehat{\theta}_{k, i}=\left[\begin{array}{c}
\widehat{\mu}_{k, i} \\
\widehat{R}_{k, i}
\end{array}\right]
$$

Introduce the components of the Monte Carlo estimate of sufficient statistics:

$$
\begin{gathered}
\eta_{k, i}(x) \triangleq \frac{\sum_{s=1}^{S_{k, i}} h_{k, i}\left(J\left(x_{k, i, s}\right)\right) \pi_{k, i-1}\left(x_{k, i, s}\right) \cdot x_{k, i, s}}{\sum_{s=1}^{S_{k, i}} h_{k, i}\left(J\left(x_{k, i, s}\right)\right) \pi_{k, i-1}\left(x_{k, i, s}\right)} \\
\eta_{k, i}\left(x x^{\top}\right) \triangleq \frac{\sum_{s=1}^{S_{k, i}} h_{k, i}\left(J\left(x_{k, i, s}\right)\right) \pi_{k, i-1}\left(x_{k, i, s}\right) \cdot x_{k, i, s} x_{k, i, s}^{\top}}{\sum_{s=1}^{S_{k, i}} h_{k, i}\left(J\left(x_{k, i, s}\right)\right) \pi_{k, i-1}\left(x_{k, i, s}\right)}
\end{gathered}
$$

such that

$$
\eta_{k, i}=\left[\begin{array}{c}
\eta_{k, i}(x) \\
\eta_{k, i}\left(x x^{\top}\right)
\end{array}\right]
$$

From (4.60a), (4.68) and (4.69)-(4.71), we have

$$
\begin{aligned}
& \widehat{\mu}_{k, i}=\left(1-\alpha_{i}\right) \mu_{k, i-1}+\alpha_{i} \eta_{k, i}(x) \\
& \widehat{R}_{k, i}=\left(1-\alpha_{i}\right) R_{k, i-1}+\alpha_{i} \eta_{k, i}\left(x x^{\top}\right)
\end{aligned}
$$

From (4.60b), (4.67) and (4.68), we have

$$
\mu_{k, i}=\sum_{l \in \mathcal{N}_{i}}^{N} c_{l k} \widehat{\mu}_{l, i}, \quad R_{k, i}=\sum_{l \in \mathcal{N}_{i}}^{N} c_{l k} \widehat{R}_{l, i}
$$

The covariance matrix can be obtained from (4.74) as:

$$
\Sigma_{k, i}=R_{k, i}-\mu_{k, i} \mu_{k, i}^{\top}
$$


From (4.72)-(4.75), every agent updates its sampling model as $f_{w_{k, i}}(\cdot)=\mathcal{N}\left(\cdot \mid \mu_{k, i}, \Sigma_{k, i}\right)$. We can rearrange the equations:

$$
\begin{aligned}
\widehat{\mu}_{k, i}= & \mu_{k, i-1}-\alpha_{i}\left(\mu_{k, i-1}-\frac{\sum_{s=1}^{S_{k, i}} h_{k, i}\left(J\left(x_{k, i, s}\right)\right) \pi_{k, i-1}\left(x_{k, i, s}\right) \cdot x_{k, i, s}}{\sum_{s=1}^{S_{k, i}} h_{k, i}\left(J\left(x_{k, i, s}\right)\right) \pi_{k, i-1}\left(x_{k, i, s}\right)}\right) \\
\widehat{R}_{k, i}= & \left(1-\alpha_{i}\right)\left(\sum_{k, i-1}+\mu_{k, i-1} \mu_{k, i-1}^{\top}\right) \\
& +\alpha_{i}\left(\frac{\sum_{s=1}^{S_{i}} h_{k, i}\left(J\left(x_{k, i, s}\right)\right) \pi_{k, i-1}\left(x_{k, i, s}\right) \cdot x_{k, i, s} x_{k, i, s}^{\top}}{\sum_{s=1}^{S_{i}} h_{k, i}\left(J\left(x_{k, i, s}\right)\right) \pi_{k, i-1}\left(x_{k, i, s}\right)}\right) \\
\mu_{k, i}= & \sum_{l \in \mathcal{N}_{k}} c_{l k} \widehat{\mu}_{l, i} \\
\Sigma_{k, i}= & \sum_{l \in \mathcal{N}_{k}} c_{l k} \widehat{R}_{l, i}-\mu_{k, i} \mu_{k, i}^{T}
\end{aligned}
$$

Equations (4.76a)-(4.76d) constitute the distributed Cross-Entropy algorithm (DCE), whose detailed description is given in Algorithm 8.
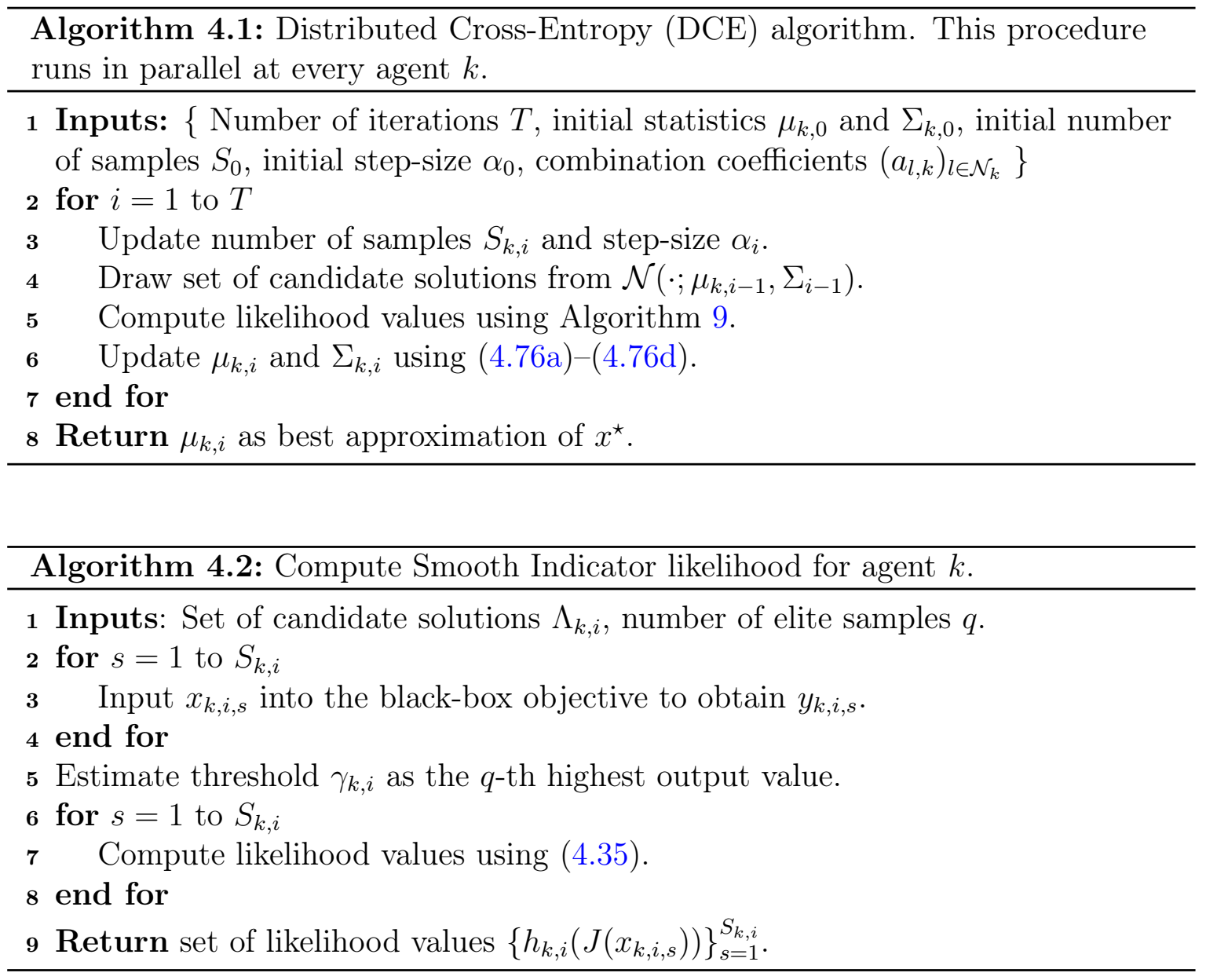


\subsection{Convergence Analysis for the Single Agent Case}

In this section we provide novel convergence results for the single agent particle filter optimization framework. We start analyzing the exact Bayesian filter optimization given by (4.12) and (4.32). Then, we extend the results to a smoothed Bayesian update. The distributed stochastic-approximation particle-filter optimization (4.60a)-(4.60b) (which includes the DCE algorithm as a special case) will be studied in Section 4.6.

\subsubsection{Convergence of single agent Bayesian filter optimization}

Based on the work by [107] on genetic algorithms, reference [108] showed for combinatorial optimization problems that, if the candidate solutions are weighted proportionally to their output value - which is similar to proportional likelihood given by (4.14)-, then the CE algorithm - which can be seen as a particle filter implementation of (4.12) with multinomial sampling distribution (as explained in Section 4.4.1, [340] and [153]) — would asymptotically find the optimal solution, and conjectured that the result could be extended to other distributions as well. Our first contribution is that, by using the Bayesian filtering framework, we are able to extend the result from $[107,108]$ to a large family of sampling and likelihood distributions, proving that their conjecture was correct. Moreover, our result generalizes another asymptotic convergence analysis of the Bayesian filtering optimization framework due to [340] that required to evaluate the filter over a monotonically increasing sequence of likelihood parameters, $\left\{\gamma_{i}\right\}_{i=0}^{\infty}$, such that $\gamma_{i} \geq \gamma_{i-1}$ for all $i$. In particular, our analysis imposes the milder condition that the ratio of the likelihood at any point over the likelihood at the optimal value to be upper bounded (which translates in $\gamma_{i}$ being lower-bounded for the smooth indicator likelihood). We require the following assumptions, easily satisfied in practice.

Assumption 4.1 (Initial belief). The support of the initial belief distribution $g_{0}$ contain the set of optimal solutions $\mathbb{X}^{\star}$, such that $g\left(x^{\star}\right)>0 \forall x^{\star} \in \mathbb{X}^{\star}$.

Assumption 4.2 (Likelihood). The likelihood is upper-bounded by the output value of any point in the optimal set: $h(J(x))<h\left(J\left(x^{\star}\right)\right), \forall x \in \mathbb{R} \backslash \mathbb{X}^{\star}, \forall x^{\star} \in \mathbb{X}^{\star}$.

Assumption 4.1 holds if the initial belief contains the whole initial search set $\mathbb{X}$ (recall that $\mathbb{Z}^{\star} \subset \mathbb{X}$ ). We can ensure that Assumption 4.2 holds by requiring $h(J(x)$ ) to be a strictly increasing function of $J(x)$. This condition is satisfied by the smooth indicator function (4.15). 
Theorem 4.1 (Bayesian filter). Let Assumptions 4.1 and 4.2 hold. Then, the belief distribution $g_{i}$ given by the Bayesian filter recursion (4.12) asymptotically concentrates on the set of optimal solutions $\mathbb{X}^{\star}$ :

$$
\lim _{i \rightarrow \infty} \int_{\mathbb{X}^{\star}} g_{i}(x) d x=1
$$

Proof: The proof extends the argument due to [107, Theorem 4.1], originally proposed for multinomial distributions and for a proportional likelihood function. Note that we can express (4.12) as

$$
g_{i}(x)=\frac{g_{i-1}(x) h(J(x))}{\int g_{i-1}(z) h(J(z)) d z}=\frac{g_{i-1}(x)}{\int g_{i-1}(z) \frac{h(J(z))}{h(J(x))} d z}
$$

Rewrite (4.78) as a function of the initial distribution, $g_{0}(x)$. By induction, we have

$$
\begin{aligned}
g_{1}(x) & =\frac{g_{0}(x) h(J(x))}{\int g_{0}(z) h(J(z)) d z} \\
g_{2}(x) & =\frac{g_{1}(x) h(J(x))}{\int g_{1}(z) h(J(z)) d z}=\frac{\frac{g_{0}(x) h(J(x))}{\int g_{0}(u) h(J(u)) d u} h(J(x))}{\int \frac{g_{0}(z) h(J(z))}{\int g_{0}(u) h(J(u)) d u} h(J(z)) d z}=\frac{g_{0}(x)}{\int g_{0}(z)\left(\frac{h(J(z))}{h(J(x))}\right)^{2} d z} \\
& \vdots \\
g_{i}(x) & =\frac{g_{0}(x)}{\int g_{0}(z)\left(\frac{h(J(z))}{h(J(x))}\right)^{i} d z}
\end{aligned}
$$

Now, we are ready to study the following limit:

$$
\lim _{i \rightarrow \infty} \int_{\mathfrak{X}^{\star}} g_{i}(x) d x=\lim _{i \rightarrow \infty} \int_{\mathbb{X}^{\star}} \frac{g_{0}(x)}{\int g_{0}(z)\left(\frac{h(J(z))}{h(J(x))}\right)^{i} d z} d x
$$

We will use the bounded convergence theorem (see, e.g., [63]) to take the limit under the integral sign. First let us study the convergence of the function inside the integral $\forall x^{\star} \in \mathbb{X}^{\star}$. From Assumption 4.1, we know that $\int_{\mathbb{K}^{\star}} g_{0}(x) d x>0$. Then, from basic properties of limits (e.g., [64, Sec. 2.5]), we have:

$$
\lim _{i \rightarrow \infty} \frac{g_{0}\left(x^{\star}\right)}{\int g_{0}(z)\left(\frac{h(J(z))}{h\left(J\left(x^{\star}\right)\right)}\right)^{i} d z}=\frac{g_{0}\left(x^{\star}\right)}{\lim _{i \rightarrow \infty} \int g_{0}(z)\left(\frac{h(J(z))}{h\left(J\left(x^{\star}\right)\right)}\right)^{i} d z}
$$

Hence, we have to study the convergence of the function inside the integral in the denominator for all $z \in \mathbb{R}^{M}$. From Assumption 4.2, we have that $\frac{h(J(z))}{h\left(J\left(x^{\star}\right)\right)}<1 \forall z \in$ 
$\mathbb{R}^{M} \backslash \mathbb{X}^{\star}$ and $\forall x^{\star} \in \mathbb{X}^{\star}$. Hence, we have:

$$
\lim _{i \rightarrow \infty} g_{0}(z)\left(\frac{h(J(z))}{h\left(J\left(x^{\star}\right)\right)}\right)^{i}= \begin{cases}0 & \text { if } z \notin \mathbb{X}^{\star} \\ g_{0}(z) & \text { if } z \in \mathbb{X}^{\star}\end{cases}
$$

It is clear that the initial distribution $g_{0}(z)$ must be bounded $\forall z \in \mathbb{X}^{\star}$; otherwise, there is no reason to search the solution at all (i.e., $\int g_{0}(z) d z>\int_{\not^{*}} g_{0}(z) d z$ ). Since the function converges and it is bounded, we can use the bounded convergence theorem to state that:

$$
\begin{aligned}
\lim _{i \rightarrow \infty} \int g_{0}(z)\left(\frac{h(J(z))}{h\left(J\left(x^{\star}\right)\right)}\right)^{i} d z & =\int \lim _{i \rightarrow \infty} g_{0}(z)\left(\frac{h(J(z))}{h\left(J\left(x^{\star}\right)\right)}\right)^{i} d z \\
& =\int_{\mathbb{X}^{\star}} g_{0}(z) d z
\end{aligned}
$$

From (4.83), it follows that (4.81) also converges and it is bounded. Hence, we can use the bounded convergence theorem once more and move the limit inside the integral sign in (4.80) and conclude:

$$
\begin{aligned}
& \lim _{i \rightarrow \infty} \int_{{\chi^{\star}}^{\star}} g_{i}(x) d x=\lim _{i \rightarrow \infty} \int_{\mathbb{K}^{\star}} \frac{g_{0}(x)}{\int g_{0}(z)\left(\frac{h(J(z))}{h(J(x))}\right)^{i} d z} d x \\
& =\int_{\not^{\star}} \frac{g_{0}(x)}{\lim _{i \rightarrow \infty} \int g_{0}(z)\left(\frac{h(J(z))}{h\left(J\left(x^{\star}\right)\right)}\right)^{i} d z} \\
& =\frac{\int_{\mathfrak{Z}^{\star}} g_{0}(x) d x}{\int_{\mathfrak{Z}^{\star}} g_{0}(z) d z} \\
& =1
\end{aligned}
$$

The same asymptotic convergence to the optimal belief distribution can be proved when we replace the belief by a surrogate sampling distribution corrected by importance sampling weights and when the likelihood function changes with time.

Corollary 4.1 (Bayesian filter with surrogate). Let Assumptions 4.1 and 4.2 hold. Then, the belief recursion (4.32) asymptotically concentrates on $\mathbf{X}^{\star}$.

Proof: By substituting (4.31) into (4.32), we obtain the original belief and, therefore, the same recursion (4.79).

Consider the following assumption on a time-varying likelihood function. 
Assumption 4.3 (Time-varying likelihood). The time-varying likelihood satisfies the following conditions $\forall x \in \mathbb{R} \backslash \mathbb{X}^{\star}$ and $\forall x^{\star} \in \mathbb{X}^{\star}$ :

$$
\begin{gathered}
h_{i}(J(x))<h_{i}\left(J\left(x^{\star}\right)\right), \quad i=1, \ldots, \infty \\
\exists m \in \mathbb{N}: \frac{h_{m}(J(x))}{h_{m}\left(J\left(x^{\star}\right)\right)} \geq \frac{h_{i}(J(x))}{h_{i}\left(J\left(x^{\star}\right)\right)}, \quad i=m+1, \ldots, \infty .
\end{gathered}
$$

Condition (4.85) states that all likelihood functions in the sequence are upper bounded by their value in the optimal set. Condition (4.86) states that the ratios are upper bounded. This is the case, e.g., for the smooth indicator likelihood (4.15) when the sequence of thresholds $\left\{\gamma_{i}\right\}_{i=1}^{\infty}$ is lower-bounded, as shown by the following proposition.

Proposition 2 (Bounded smooth indicator ratio). For the smooth indicator likelihood (4.15), suppose there exists $0<\gamma_{\text {low }}<J_{\max }$ such that $\gamma_{i} \geq \gamma_{\text {low }}$ for all $i=m+1, \ldots, \infty$. Then, condition (4.86) holds.

Proof: We only have to show that the ratio:

$$
r(\gamma, x) \triangleq \frac{h_{i}(J(x))}{h_{i}\left(J\left(x^{\star}\right)\right)}
$$

is a decreasing function of $\gamma_{i}$. Introduce shorthands:

$$
\begin{aligned}
& a \triangleq e^{-\epsilon J(x)} \\
& b \triangleq e^{-\epsilon J\left(x^{\star}\right)}
\end{aligned}
$$

Then, we can rewrite

$$
r(\gamma, x)=\frac{1+b e^{\epsilon \gamma}}{1+a e^{\epsilon \gamma}}
$$

Since $J\left(x^{\star}\right)>J(x), \forall x \in \mathbb{R}^{M} \backslash \mathbb{K}^{\star}$, we have $a>b$. Hence, taking the derivative of the ratio with respect to $\gamma$, we obtain:

$$
\frac{\partial r(\gamma, x)}{\partial \gamma}=(b-a) \frac{\epsilon e^{\epsilon \gamma}}{\left(1+a e^{\epsilon \gamma}\right)^{2}}<0, \quad \forall x \in \mathbb{R}^{M} \backslash \boldsymbol{K}^{\star}
$$

Having negative derivative, we conclude that the ratio is a decreasing function of $\gamma$.

Proposition 2 shows that Assumption 4.3 is easily satisfied in practice. This is an important remark, since the previous result on BFO given by [340, Lemma 1] required a strictly increasing threshold sequence of the form $\gamma_{i}>\gamma_{i+1}$, for all $i=1, \ldots, \infty$, 
which is a strong assumption, difficult to ensure in practice since it implies to design an increasing schedule that converges to an unknown $J_{\max }$.

Corollary 4.2 (Time-varying likelihood). Let Assumption 4.1 hold. Suppose the likelihood changes with time, such that $h_{i}(J(x))$ satisfies Assumption 4.3. Then, the belief distribution given by:

$$
g_{i}(x)=\frac{f_{w_{i-1}^{o}}(x) \pi_{i-1}(x) h_{i}(J(x))}{\int f_{w_{i-1}^{o}}(z) \pi_{i-1}(z) h_{i}(J(z)) d z}
$$

asymptotically concentrates on $\mathfrak{X}^{\star}$.

Proof: Following the same argument as for Corollary 4.1, we have

$$
\begin{aligned}
g_{1}(x) & =\frac{f_{w_{0}^{o}}(x) \pi_{0}(x) h_{1}(J(x))}{\int f_{w_{0}^{o}}(z) \pi_{0}(z) h_{1}(J(z)) d z}=\frac{g_{0}(x) h_{1}(J(x))}{\int g_{0}(z) h_{1}(J(z)) d z} \\
g_{2}(x) & =\frac{f_{w_{1}^{o}}(x) \pi_{1}(x) h_{2}(J(x))}{\int f_{w_{1}^{o}}(z) \pi_{1}(z) h_{2}(J(z)) d z}=\frac{g_{1}(x) h_{2}(J(x))}{\int g_{1}(z) h_{2}(J(z)) d z}=\frac{\frac{g_{0}(x) h_{1}(J(x))}{\int g_{0}(u) h_{1}(J(u)) d u} h_{2}(J(x))}{\int \frac{g_{0}(z) h_{1}(J(z))}{\int g_{0}(u) h_{1}(J(u)) d u} h_{2}(J(z)) d z} \\
& =\frac{g_{0}(x) h_{1}(J(x)) h_{2}(J(x))}{\int g_{0}(z) h_{1}(J(z)) h_{2}(J(z)) d z}=\frac{g_{0}(x)}{\int g_{0}(z) \frac{h_{1}(J(z)) h_{2}(J(z))}{h_{1}(J(x)) h_{2}(J(x))} d z} \\
g_{i}(x) & =\frac{g_{0}(x)}{\int g_{0}(z) \prod_{j=1}^{i} \frac{h_{j}(J(z))}{h_{j}(J(x))} d z}
\end{aligned}
$$

From Assumption 4.3, there exists some $m$ such that:

$$
\begin{aligned}
\lim _{i \rightarrow \infty} \prod_{j=1}^{i} \frac{h_{j}(J(z))}{h_{j}(J(x))} & =\prod_{l=1}^{m-1} \frac{h_{m}(J(z))}{h_{m}(J(x))} \lim _{i \rightarrow \infty} \prod_{j=m}^{i} \frac{h_{j}(J(z))}{h_{j}(J(x))} \\
& \leq \prod_{l=1}^{m-1} \frac{h_{l}(J(z))}{h_{l}(J(x))} \lim _{i \rightarrow \infty}\left(\frac{h_{m}(J(z))}{h_{m}(J(x))}\right)^{i}
\end{aligned}
$$

Since the first term in the right side of (4.94) is non-negative and upper-bounded, we conclude:

$$
\lim _{i \rightarrow \infty} \prod_{j=1}^{i} \frac{h_{j}(J(z))}{h_{j}(J(x))}= \begin{cases}0 & \forall z \in \mathbb{R}^{M} \backslash \mathbb{X}^{\star}, \forall x \in \mathbb{X}^{\star} \\ 1 & \forall z, x \in \mathbb{X}^{\star}\end{cases}
$$

Therefore, we can follow the same argument used in Theorem 4.1. 
Now, we study the smoothed Bayesian update:

$$
g_{i}(x)=(1-\alpha) g_{i-1}(x)+\alpha \frac{g_{i-1}(x) h_{i}(J(x))}{\int g_{i-1}(z) h_{i}(J(z)) d z}
$$

The following theorem establishes convergence of (4.96) to the optimal belief.

Theorem 4.2 (Smoothed belief). Let Assumptions 4.1 and 4.3 hold. Let the stepsize satisfy $0<\alpha<1$. Then, the belief resulting from the smoothed update (4.96) asymptotically concentrates on $\mathbf{X}^{\star}$.

Proof: We study the following limit:

$$
\lim _{i \rightarrow \infty} \int_{\mathbb{Z}^{\star}} g_{i}(x) d x=\lim _{i \rightarrow \infty}\left[(1-\alpha) \int_{\mathbb{Z}^{\star}} g_{i-1}(x) d x+\alpha \int_{\mathbb{Z}^{\star}} \frac{g_{i-1}(x) h_{i}(J(x))}{\int g_{i-1}(z) h_{i}(J(z)) d z} d x\right]
$$

Let us rewrite (4.96) as follows:

$$
g_{i}(x)=\beta_{i-1}(x) \frac{g_{i-1}(x) h_{i}(J(x))}{\int g_{i-1}(z) h_{i}(J(z)) d z}
$$

where

$$
\beta_{i-1}(x)=(1-\alpha) \frac{\int g_{i-1}(z) h_{i}(J(z)) d z}{h_{i}(J(x))}+\alpha
$$

Use (4.98) and apply induction to express the term inside the rightmost integral of (4.97) as a function of $g_{0}$ :

$$
\begin{aligned}
\frac{g_{i-1}(x) h_{i}(J(x))}{\int g_{i-1}(z) h_{i}(J(z)) d z} & =\frac{\beta_{i-2}(x) \frac{g_{i-2}(x) h_{i-1}(J(x))}{\int g_{i-2}(u) h_{i-1}(J(u)) d u} h_{i}(J(x))}{\int \beta_{i-2}(z) \frac{g_{i-2}(z) h_{i-1}(J(z))}{\int g_{i-2}(u) h_{i-1}(J(u)) d u} h_{i}(J(z)) d z} \\
& =\frac{\beta_{i-2}(x) g_{i-2}(x) h_{i-1}(y \mid x) h_{i}(J(x))}{\int \beta_{i-2}(z) g_{i-2}(z) h_{i-1}(y \mid z) h_{i}(J(z)) d z} \\
& =\frac{\beta_{i-2}(x) \beta_{i-3}(x) \frac{g_{i-3}(x) h_{i-2}(J(x))}{\int g_{i-3}(u) h_{i-2}(J(u)) d u} h_{i-1}(J(x)) h_{i}(J(x))}{\int \beta_{i-2}(z) \beta_{i-3}(z) \frac{g_{i-3}(z) h_{i-1}(J(z))}{\int g_{i-3}(u) h_{i-1}(J(u)) d u} h_{i-1}(J(z)) h_{i}(J(z)) d z} \\
& =\frac{\beta_{i-2}(x) \beta_{i-3}(x) g_{i-3}(x) h_{i-2}(J(x)) h_{i-1}(J(x)) h_{i}(J(x))}{\int \beta_{i-2}(z) \beta_{i-3}(z) g_{i-3}(z) h_{i-2}(J(z)) h_{i-1}(J(z)) h_{i}(J(z)) d z} \\
& \vdots \quad \prod_{j=0}^{i-2} \beta_{j}(x) g_{0}(x) \prod_{l=1}^{i} h_{l}(J(x)) \\
& =\frac{\prod_{j=0}^{i-2} \beta_{j}(z) g_{0}(z) \prod_{l=1}^{i} h_{l}(J(z)) d z}{}
\end{aligned}
$$




$$
=\frac{\prod_{j=0}^{i-2} \beta_{j}(x) g_{0}(x)}{\int \prod_{j=0}^{i-2} \beta_{j}(z) g_{0}(z) \prod_{l=1}^{i} \frac{h_{l}(J(z))}{h_{l}(J(x))} d z}
$$

From (4.95), (4.100) and Corollary 4.2, we can evaluate the limit of the rightmost term of (4.97):

$$
\lim _{i \rightarrow \infty} \alpha \int_{\mathbb{K}^{\star}} \frac{g_{i-1}(x) h_{i}(J(x))}{\int g_{i-1}(z) h_{i}(J(z)) d z} d x=\alpha \lim _{i \rightarrow \infty} \frac{\int_{\mathbb{Z}^{\star}} \prod_{j=0}^{i-2} \beta_{j}(x) g_{0}(x) d x}{\int_{\mathbb{Z}^{\star}} \prod_{j=0}^{i-2} \beta_{j}(z) g_{0}(z) d z}=\alpha
$$

Now, let us use the result from (4.101) in (4.97). By applying induction, we obtain

$$
\begin{aligned}
& \lim _{i \rightarrow \infty} \int_{\mathbb{K}^{\star}} g_{i}(x) d x=\lim _{i \rightarrow \infty}(1-\alpha) \int_{\mathbb{Z}^{*}} g_{i-1}(x) d x+\alpha \\
& =\lim _{i \rightarrow \infty}(1-\alpha)\left((1-\alpha) \int_{\mathbb{K}^{*}} g_{i-2}(x) d x+\alpha\right)+\alpha \\
& =\lim _{i \rightarrow \infty}(1-\alpha)^{2} \int_{\mathbb{X}^{\star}} g_{i-2}(x) d x+(1-\alpha) \alpha+\alpha \\
& =\lim _{i \rightarrow \infty}(1-\alpha)^{2}\left((1-\alpha) \int_{\not^{\star}} g_{i-3}(x) d x+\alpha\right)+(1-\alpha) \alpha+\alpha \\
& =\lim _{i \rightarrow \infty}(1-\alpha)^{3} \int_{\mathfrak{Z}^{\star}} g_{i-3}(x) d x+(1-\alpha)^{2} \alpha+(1-\alpha) \alpha+\alpha \\
& =\lim _{i \rightarrow \infty}\left((1-\alpha)^{i} \int_{\mathfrak{X}^{\star}} g_{0}(x) d x+\sum_{j=0}^{i-1}(1-\alpha)^{j} \alpha\right)
\end{aligned}
$$

Since $0<\alpha<1$, we have

$$
\begin{aligned}
\lim _{i \rightarrow \infty}(1-\alpha)^{i} & =0 \\
\lim _{i \rightarrow \infty} \sum_{m=0}^{i-1}(1-\alpha)^{j} \alpha & =\frac{\alpha}{1-(1-\alpha)}=1
\end{aligned}
$$

By replacing (4.103) and (4.104) in (4.102), we conclude that $\lim _{i \rightarrow \infty} \int_{\swarrow^{\star}} g_{i}(x)=1$.

\subsection{Convergence Analysis for a Network of Agents}

The novel asymptotic results established above are appealing and show that Bayesian filtering optimization is a promising approach. However, studying a distributed recursion is more challenging because the optimization process at each agent is influenced 
by the optimization processes at other agents. In this section, we analyze asymptotic convergence of the $N$ coupled sequences (one per agent) of smoothed distributions, as well as the convergence of the sequences of estimates resulting from the distributed stochastic approximation (4.60a)-(4.60b).

\subsubsection{Convergence of diffusion damping BFO}

The analysis is more complicated but follows the same scheme as for the single agent case. Introduce $N$ coupled smoothed Bayesian updates that follow the ATC diffusion pattern:

$$
\begin{aligned}
\widehat{g}_{k, i}(x) & =(1-\alpha) g_{k, i-1}(x)+\alpha \frac{g_{k, i-1}(x) h_{k, i}(J(x))}{\int g_{k, i-1}(z) h_{k, i}(J(z)) d z} \\
g_{k, i}(x) & =\sum_{l \in \mathcal{N}_{k}} c_{l k} \widehat{g}_{l, i}(x)
\end{aligned}
$$

The following result states convergence of the beliefs $g_{k, i}$ to the optimal distribution.

Theorem 4.3 (Distributed smoothed belief). Let the initial belief $g_{k, 0}$ and the likelihood $h_{k, i}$ at every time step $i=1, \ldots, \infty$ satisfy Assumptions 4.1 and 4.3, respectively, for every agent $k=1, \ldots, N$. Let the step-size satisfy $0<\alpha<1$. Then, the belief $g_{k, i}$ resulting from $(4.105)-(4.106)$ asymptotically concentrates on $\mathbf{X}^{\star}$ for every agent $k=1, \ldots, N$.

Proof: Similar to Theorem 4.2, for the single agent case, we study the following limit:

$$
\begin{aligned}
\lim _{i \rightarrow \infty} \int_{\mathbb{X}^{\star}} g_{k, i}(x) d x= & \lim _{i \rightarrow \infty} \sum_{l \in \mathcal{N}_{k}} c_{l k}\left[(1-\alpha) \int_{\mathbb{K}^{\star}} g_{l, i-1}(x) d x\right. \\
& \left.+\alpha \int_{\mathbb{Z}^{\star}} \frac{g_{l, i-1}(x) h_{l, i}(J(x))}{\int g_{l, i-1}(z) h_{l, i}(J(z)) d z} d x\right]
\end{aligned}
$$

Let us rewrite (4.105) as follows:

$$
\widehat{g}_{k, i}(x)=\beta_{k, i-1}(x) \frac{g_{k, i-1}(x) h_{k, i}(J(x))}{\int g_{k, i-1}(z) h_{k, i}(J(z)) d z}
$$

where

$$
\beta_{k, i-1}(x)=(1-\alpha) \frac{\int g_{k, i-1}(z) h_{k, i}(J(z)) d z}{h_{k, i}(J(x))}+\alpha
$$


Introduce the shorthand:

$$
b_{l k, i-1}(x) \triangleq c_{l k} \beta_{l, i-2}(x) \prod_{m \in \mathcal{N}_{l}, m \neq l} \int g_{m, i-2}(x) h_{m, i-1}(J(x)) d x
$$

Use (4.108) and (4.110) and apply induction to express the term inside the rightmost integral of (4.107) as a function of the product of local likelihoods:

$$
\begin{aligned}
& \frac{g_{k, i-1}(x) h_{k, i}(J(x))}{\int g_{k, i-1}(z) h_{k, i}(J(z)) d z}=\frac{\sum_{l \in \mathcal{N}_{k}} c_{l k} \frac{\beta_{l, i-2}(x) g_{l, i-2}(x) h_{l, i-1}(J(x))}{\int g_{l, i-2}(u) h_{l, i-1}(J(u)) d u} h_{k, i}(J(x))}{\int \sum_{l \in \mathcal{N}_{k}} c_{l k} \frac{\beta_{l, i-2}(z) g_{l, i-2}(z) h_{l, i-1}(J(z))}{\int g_{l, i-2}(u) h_{l, i-1}(J(u)) d u} h_{k, i}(J(z)) d z} \\
& =\frac{\sum_{l \in \mathcal{N}_{k}} b_{l k, i-1}(x) g_{l, i-2}(x) h_{l, i-1}(J(x)) h_{k, i}(J(x))}{\int \sum_{l \in \mathcal{N}_{k}} b_{l k, i-1}(z) g_{l, i-2}(z) h_{l, i-1}(J(z)) h_{k, i}(J(z)) d z} \\
& =\frac{\sum_{l \in \mathcal{N}_{k}} b_{l k, i-1}(x) \sum_{m \in \mathcal{N}_{l}} a_{m l} \frac{\beta_{m, i-3}(x) g_{m, i-3}(x) h_{m, i-2}(J(x))}{\int \beta_{m, i-3}(u) g_{m, i-3}(u) h_{m, i-2}(J(u)) d u} h_{l, i-1}(J(x)) h_{k, i}(J(x))}{\int \sum_{l \in \mathcal{N}_{k}} b_{l k, i-1}(z) \sum_{m \in \mathcal{N}_{l}} a_{m l} \frac{\beta_{m, i-3}(z) g_{m, i-3}(z) h_{m, i-2}(J(z))}{\int \beta_{m, i-3}(u) g_{m, i-3}(u) h_{m, i-2}(J(u)) d u} h_{l, i-1}(J(z)) h_{k, i}(J(z)) d z} \\
& =\frac{\sum_{l \in \mathcal{N}_{k}} b_{l k, i-1}(x) \sum_{m \in \mathcal{N}_{l}} b_{m l, i-2}(x) g_{m, i-3}(x) h_{m, i-2}(J(x)) h_{l, i-1}(J(x)) h_{k, i}(J(x))}{\int \sum_{l \in \mathcal{N}_{k}} b_{l k, i-1}(z) \sum_{m \in \mathcal{N}_{l}} b_{m l, i-2}(z) g_{m, i-3}(z) h_{m, i-2}(J(z)) h_{l, i-1}(J(z)) h_{k, i}(J(z)) d z} \\
& \quad \vdots \\
& =\frac{\psi_{k, i-1}(x)}{\int \psi_{k, i-1}(z) d z}
\end{aligned}
$$

where we used the shorthand:

$$
\begin{aligned}
\psi_{k, i-1}(x) \triangleq & \sum_{l \in \mathcal{N}_{k}} b_{l k, i-1}(x) \sum_{m \in \mathcal{N}_{l}} b_{m l, i-2}(x) \ldots \sum_{j \in \mathcal{N}_{n}} b_{j n, 1}(x) \\
& \cdot g_{0}(x) h_{j, 1}(J(x)) \ldots h_{m, 1-2}(J(x)) h_{l, 1-1}(J(x)) h_{k, i}(J(x))
\end{aligned}
$$

From Corollary 4.2, we have

$$
\begin{aligned}
\lim _{i \rightarrow \infty} \frac{h_{j, 1}(J(z)) \cdots h_{m, i-2}(J(z)) h_{l, i-1}(J(z)) h_{k, i}(J(z))}{h_{j, 1}(J(x)) \cdots h_{m, i-2}(J(x)) h_{l, i-1}(J(x)) h_{k, i}(J(x))} \\
= \begin{cases}0 & \forall z \in \mathbb{R}^{M} \backslash \mathbb{K}^{\star}, \forall x \in \mathbb{K}^{\star} \\
1 & \forall z, x \in \mathbb{K}^{\star}\end{cases}
\end{aligned}
$$

From (4.111) and (4.113), we can use the bounded convergence theorem to evaluate the limit of the rightmost term of (4.107), obtaining:

$$
\lim _{i \rightarrow \infty} \alpha \int_{\not^{\star}} \frac{g_{l, i-1}(x) h_{l, i}(J(x))}{\int g_{l, i-1}(z) h_{l, i}(J(z)) d z} d x=\alpha \lim _{i \rightarrow \infty} \int_{\not_{\aleph^{\star}}} \frac{\psi_{l, i-1}(x)}{\int \psi_{l, i-1}(z) d z} d x
$$




$$
\begin{aligned}
& =\alpha \lim _{i \rightarrow \infty} \frac{\int_{\mathfrak{Z}^{\star}} \psi_{l, i-1}(x) d x}{\int_{\mathfrak{Z}^{\star}} \psi_{l, i-1}(z) d z} \\
& =\alpha
\end{aligned}
$$

This result extends (4.101) to a network of agents.

In order to study the first term in the right side of (4.107), we introduce the network variable $\delta \in \mathbb{R}^{M N}$ and the network vector of local distributions $\mathcal{G}_{i}: \mathbb{R}^{M N} \rightarrow \mathbb{R}^{N}$ :

$$
\begin{gathered}
\delta \triangleq\left(x_{k}\right)_{k=1}^{N} \\
\mathcal{G}_{i}(\delta) \triangleq\left(g_{k, i}\left(x_{k}\right)\right)_{k=1}^{N}
\end{gathered}
$$

Now, using (4.114), we can express (4.107) for all agents in compact form:

$$
\lim _{i \rightarrow \infty} \int_{\mathbb{K}^{\star}} \mathcal{G}_{i}(\delta) d x=\lim _{i \rightarrow \infty}\left(C^{\top} \otimes I_{M}\right)\left((1-\alpha) \int_{\not^{\star}} \mathcal{G}_{i-1}(\delta) d x+\mathbb{1} \alpha\right)
$$

By induction, we obtain an expression similar to (4.102):

$$
\begin{aligned}
\lim _{i \rightarrow \infty} \int_{\mathbb{K}^{\star}} \mathcal{G}_{i}(\delta) d x= & \lim _{i \rightarrow \infty}\left(C^{\top} \otimes I_{M}\right)\left((1-\alpha) \int_{\mathbb{Z}^{\star}} \mathcal{G}_{i-1}(\delta) d x+\mathbb{1} \alpha\right) \\
= & \lim _{i \rightarrow \infty}\left(C^{\top} \otimes I_{M}\right) \\
& \left((1-\alpha)\left(C^{\top} \otimes I_{M}\right)\left((1-\alpha) \int_{\mathbb{Z}^{\star}} \mathcal{G}_{i-2}(\delta) d x+\mathbb{1} \alpha\right)+\mathbb{1} \alpha\right) \\
= & \lim _{i \rightarrow \infty}\left(C^{\top} \otimes I_{M}\right)^{2}(1-\alpha)^{2} \int_{\mathbb{K}^{\star}} \mathcal{G}_{i-2}(x) d x \\
& +\left(C^{\top} \otimes I_{M}\right)^{2} \mathbb{1}(1-\alpha) \alpha+\left(C^{\top} \otimes I_{M}\right) \mathbb{1} \alpha \\
= & \lim _{i \rightarrow \infty}\left(C^{\top} \otimes I_{M}\right)^{2}(1-\alpha)^{2} \int_{\mathbb{Z}^{\star}} \mathcal{G}_{i-2}(x) d x+\mathbb{1}((1-\alpha) \alpha+\alpha) \\
\vdots & \\
= & \lim _{i \rightarrow \infty}\left(C^{\top} \otimes I_{M}\right)^{i}(1-\alpha)^{i} \int_{\mathbb{K}^{\star}} \mathcal{G}_{0}(x) d x+\mathbb{1} \sum_{j=0}^{i-1}(1-\alpha)^{j} \alpha
\end{aligned}
$$

where we used (4.65), so that $\left(C^{\top} \otimes I_{M}\right) \mathbb{1}=\mathbb{1}$. Conditions (4.62)-(4.63) make $C$ primitive (see, e.g., [124, Theorem 8.5.3]). Moreover, since it is left stochastic, with simple algebra, we can see that its power converges to a bounded matrix (see, e.g., [124, Theorem 8.5.1]):

$$
\lim _{i \rightarrow \infty}\left(C^{\top} \otimes I_{M}\right)^{i}=\lim _{i \rightarrow \infty}\left(C^{\top}\right)^{i} \otimes I_{M}=\mathbb{1} \tau \otimes I_{M}
$$


where $\tau$ is the right eigenvector of $C$ associated with its Perron eigenvalue (i.e., its unique maximum eigenvalue of value one): $C \tau=\tau$. Hence, we can simplify (4.118) by using the single agent expressions (4.103)-(4.104) to obtain

$$
\lim _{i \rightarrow \infty} \int_{\mathbb{K}^{\star}} \mathcal{G}_{i}(\delta) d x=\mathbb{1}
$$

This is equivalent to state that $\lim _{i \rightarrow \infty} \int_{\mathfrak{K}^{\star}} g_{k, i}(x) d x=1, k=1, \ldots, N$.

\subsubsection{Almost sure agreement among DCE agents}

It is convenient to express the estimates $\eta_{k, i}$ in terms of the actual smoothed statistics $w_{k, i}$ and Monte Carlo noise:

$$
\begin{aligned}
\mathbb{E}_{f_{w_{k, i}}}[\phi(\boldsymbol{x})] & \triangleq \frac{\mathbb{E}_{f_{w_{k, i-1}}}\left[h_{k, i}(J(\boldsymbol{x})) \pi_{k, i-1}(\boldsymbol{x}) \phi(\boldsymbol{x})\right]}{\mathbb{E}_{f_{w_{k, i-1}}}\left[h_{k, i}(J(\boldsymbol{x})) \pi_{k, i-1}(\boldsymbol{x})\right]} \\
\xi_{k, i} & \triangleq \mathbb{E}_{f_{w_{k, i}}[\phi(\boldsymbol{x})]-\frac{\sum_{s=1}^{S_{k, i}} h_{k, i}\left(J\left(x_{k, i, s}\right)\right) \pi_{k, i-1}\left(x_{k, i, s}\right) \phi\left(x_{k, i, s}\right)}{\sum_{s=1}^{S_{i}} h_{k, i}\left(J\left(x_{k, i, s}\right)\right) \pi_{k, i-1}\left(x_{k, i, s}\right)}}
\end{aligned}
$$

so that we have:

$$
\eta_{k, i}=\mathbb{E}_{f_{w_{k, i}}}[\phi(\boldsymbol{x})]+\xi_{k, i}^{\bullet}
$$

Now, we can consider the quantities appearing in (4.60a)-(4.60b) as instantaneous realizations of random variables and express the random iterates in terms of true statistics and random noise:

$$
\begin{aligned}
\widehat{\boldsymbol{\theta}}_{k, i} & =\boldsymbol{\theta}_{k, i-1}-\alpha_{i}\left(\boldsymbol{\theta}_{k, i-1}-\mathbb{E}_{f_{\boldsymbol{w}_{k, i}}}[\phi(\boldsymbol{x})]-\boldsymbol{\xi}_{k, i}^{\bullet}\right) \\
\boldsymbol{\theta}_{k, i} & =\sum_{l \in \mathcal{N}_{k}} c_{l k} \widehat{\boldsymbol{\theta}}_{l, i}
\end{aligned}
$$

where the initial parameters $\boldsymbol{\theta}_{k, 0}=\theta_{k, 0}$ are given for $k=1, \ldots, N$. We remark that, from (4.61), each parameter $\boldsymbol{w}_{k, i-1}$ is a random variable too. Therefore, parameter $\boldsymbol{w}_{k, i}^{\bullet}$ has to be considered random too.

In this and following subsections, we study almost sure convergence of the distributed stochastic approximation (4.124a)-(4.124b). Instead of using standard stochastic approximation techniques, which would be difficult for a network of agents because the equations are coupled among them, we do two steps. First, in this subsection, we show that distributed recursion $(4.124 \mathrm{a})-(4.124 \mathrm{~b})$ converges to a common recursion for all 
agents. Second, in the following subsection, once we have a single common recursion, we follow the almost sure convergence analysis for the single-agent scenario due to [128] and prove convergence to a local maximum of (4.215).

Reference [36] proved for general distributed stochastic approximations with doublystochastic combination matrices that every agent converges to the average of all agents' recursions almost surely. We extend and simplify the arguments from [36] to the case of left-stochastic combination matrices and show convergence to a weighted average recursion.

In order to study the behavior of the coupled recursions (4.124a)-(4.124b) for all agents, it is convenient to collect them into a single network recursion. Introduce the network vectors of length $M N$ that gather variables of every agent across the network:

$$
\begin{aligned}
\boldsymbol{\vartheta}_{i} & \triangleq\left(\boldsymbol{\theta}_{k, i}\right)_{k=1}^{N} \\
\boldsymbol{\varphi}_{i} & \triangleq\left(\mathbb{E}_{f_{\boldsymbol{w}_{k, i}}}[\phi(\boldsymbol{x})]\right)_{k=1}^{N} \\
\boldsymbol{\zeta}_{i} & \triangleq\left(\boldsymbol{\xi}_{k, i}\right)_{k=1}^{N}
\end{aligned}
$$

Collect (4.124a)-(4.124b) for all agents into the following network recursion:

$$
\boldsymbol{\vartheta}_{i}=\left(C^{\top} \otimes I_{M}\right)\left(\boldsymbol{\vartheta}_{i-1}-\alpha_{i}\left(\boldsymbol{\vartheta}_{i-1}-\boldsymbol{\varphi}_{i}-\boldsymbol{\zeta}_{i}\right)\right)
$$

Introduce the weighted average estimate:

$$
\overline{\boldsymbol{\theta}}_{i} \triangleq \sum_{k=1}^{N} \tau_{k} \boldsymbol{\theta}_{k, i}
$$

Introduce the following pair of complementary projectors:

$$
\begin{aligned}
P & \triangleq \mathbb{1} \tau^{\top} \otimes I_{M} \\
P_{\perp} & \triangleq I_{M N}-P=I_{M N}-\mathbb{1} \tau^{\top} \otimes I_{M}
\end{aligned}
$$

where we recall that $\tau$ is the right eigenvector of $C$. Let us use these projectors to define the agreement and disagreement network vectors:

$$
\begin{gathered}
\boldsymbol{\vartheta}_{i}^{\mathrm{agr}} \triangleq P \boldsymbol{\vartheta}=\left(\mathbb{1} \tau^{\top} \otimes I_{M}\right) \boldsymbol{\vartheta}=\left(\mathbb{1} \otimes I_{M}\right) \sum_{k=1}^{N} \tau_{k} \boldsymbol{\theta}_{k, i}=\left(\mathbb{1} \otimes I_{M}\right) \overline{\boldsymbol{\theta}}_{i} \\
\boldsymbol{\vartheta}_{i}^{\mathrm{dis}} \triangleq P_{\perp} \boldsymbol{\vartheta}_{i}=\left(I_{M N}-P\right) \boldsymbol{\vartheta}_{i}=\boldsymbol{\vartheta}_{i}-\boldsymbol{\vartheta}_{i}^{\mathrm{agr}}=\boldsymbol{\vartheta}_{i}-\left(\mathbb{1} \otimes I_{M}\right) \overline{\boldsymbol{\theta}}_{i}
\end{gathered}
$$


Since projectors (4.130)-(4.131) are complementary, we can decompose the network estimate vector as:

$$
\boldsymbol{\vartheta}_{i}=\boldsymbol{\vartheta}_{i}^{\text {agr }}+\boldsymbol{\vartheta}_{i}^{\text {dis }}
$$

We aim to show that all agents converge almost surely to the agreement vector $\overline{\boldsymbol{\theta}}_{i}$. This is clearly the case when:

$$
\lim _{i \rightarrow \infty} \boldsymbol{\vartheta}_{i}=\lim _{i \rightarrow \infty} \boldsymbol{\vartheta}_{i}^{\text {agr }} \quad \text { a.s. }
$$

A sufficient condition for (4.135) is

$$
\lim _{i \rightarrow \infty}\left\|\boldsymbol{\vartheta}_{i}^{\text {dis }}\right\|^{2}=0
$$

in the sense that (4.136) implies that: $\lim _{i \rightarrow \infty} \boldsymbol{\vartheta}_{i}^{\text {dis }}=0$ with probability one, otherwise the Hilbert space $L_{2}$ would not be complete; recall from previous chapter, equations (3.82)-(3.83) that we are using the 2-norm given by:

$$
\left\|\boldsymbol{\vartheta}_{i}^{\mathrm{dis}}\right\|^{2} \triangleq \mathbb{E}\left[\boldsymbol{\vartheta}_{i}^{\mathrm{dis}^{\top}} \boldsymbol{\vartheta}_{i}^{\mathrm{dis}}\right]
$$

In order to prove (4.136), we require the following assumptions.

Assumption 4.4 (Bounded error). The approximation error is bounded for every agent at every iteration: $\left\|\boldsymbol{\xi}_{k, i}\right\|^{2}=\mathbb{E}\left[\boldsymbol{\xi}_{k, i}^{\top} \boldsymbol{\xi}_{k, i}\right]<\infty, k=1, \ldots, N, i=1, \ldots, \infty$.

Assumption 4.5 (Step-size sequence). The sequence $\left\{\alpha_{i}\right\}_{i=0}^{\infty}$ is the same for every agent and satisfies: $\alpha_{i}>0, i=1, \ldots, \infty, \sum_{i=0}^{\infty} \alpha_{i}=\infty$ and $\sum_{i=0}^{\infty} \alpha_{i}^{2}<\infty$.

Assumption 4.6 (Positive sampling). It holds that $\mathbb{E}_{f_{w_{k, i-1}}}\left[h_{k, i}(J(\boldsymbol{x})) \pi_{k, i-1}(\boldsymbol{x})\right]>0$, $k=1, \ldots, N, i=1, \ldots, \infty$.

Assumption 4.7 (Combination matrix). In addition to conditions (4.62)-(4.64), the combination matrix satisfies:

$$
\left\|C^{\top}-\mathbb{1} \tau^{\top}\right\|<1
$$

where $\tau$ is the right Perron eigenvector of $C$.

Assumptions 4.4 and and 4.5 are standard when studying convergence of stochastic approximations. Assumption 4.6 is a very mild and rather technical condition. In 
particular, from the definition of importance weights, we have

$$
\mathbb{E}_{f_{w_{k, i-1}}}\left[h_{k, i}(J(\boldsymbol{x})) \pi_{k, i-1}(\boldsymbol{x})\right] \propto \int h_{k, i}(J(\boldsymbol{x})) g_{k, i-1}(x) d x
$$

Thus, we are assuming that we must give positive belief to at least one region where the likelihood is positive. But this is precisely what the algorithm does: to shift our belief to the regions with higher likelihood. In addition, for the smooth indicator likelihood (4.15), this assumption is automatically satisfied since $h^{\text {smo }}(y)>0, \forall y \in\left(-\infty, J_{\max }\right]$.

Assumption 4.7 is milder than the conditions used by [36] for the analysis of general distributed stochastic approximation. In particular, if $C$ is doubly stochastic, as assumed by [36], then we will prove that Assumption 4.7 is automatically satisfied, as explained by Proposition 4 below. On the other hand, if $C$ is only left stochastic, we have observed that the norm of the deflated combination matrix, $C^{\top}-\mathbb{1} \tau^{\top}$, is related to the network connectivity. In particular, for dense matrices, we have seen that the condition is very easily satisfied (when $1 / N \sum_{k=1}^{N}\left|\mathcal{N}_{k}\right| \approx N / 2$, the assumption held in near $100 \%$ of $10^{6}$ randomly generated primitive left-stochastic matrices, with $N \in\{20,40\})$; however, for very sparse left stochastic matrices, the assumption does not hold in general. Thus, we remark that Assumption 4.7 is more general than the assumptions used by [36], since it always holds for doubly stochastic matrices, and we have observed that it also holds for dense left-stochastic matrices.

Proposition 3. Let $A$ and $B$ be real matrices. The following facts on the induced 2-norm hold:

$$
\begin{aligned}
\|A\| & =\sigma_{\max }(A) \\
\|A\| & \leq\|A\|_{1}\|A\|_{\infty} \\
\sigma_{\max }(A \otimes B) & =\sigma_{\max }(A) \sigma_{\max }(B)
\end{aligned}
$$

Proposition 4. If $C$ is primitive and doubly stochastic, then Assumption 4.7 holds.

Proof: We have the following equivalence:

$$
\begin{aligned}
P_{\perp}\left(C^{\top} \otimes I_{M}\right) & =\left(I_{M N}-\mathbb{1} \tau^{\top} \otimes I_{M}\right)\left(C^{\top} \otimes I_{M}\right) \\
& =\left(\left(I_{N}-\mathbb{1} \tau^{\top}\right) \otimes I_{M}\right)\left(C^{\top} \otimes I_{M}\right) \\
& =\left(I_{N}-\mathbb{1} \tau^{\top}\right) C^{\top} \otimes I_{M}
\end{aligned}
$$


From Proposition 3, we have:

$$
\begin{aligned}
\left\|P_{\perp}\left(C^{\top} \otimes I_{M}\right)\right\| & =\left\|\left(I_{N}-\mathbb{1} \tau^{\top}\right) C^{\top} \otimes I_{M}\right\| \\
& =\left\|\left(I_{N}-\mathbb{1} \tau^{\top}\right) C^{\top}\right\| \\
& =\left\|C^{\top}-\mathbb{1} \tau^{\top}\right\|
\end{aligned}
$$

Now, we upper bound the one and infinity norms so that we can also upper bound (4.144). Proposition 3. Let $\lambda_{n}, u_{n}$ and $v_{n}$ denote the $n$-th eigenvalue, right and left eigenvectors of $C^{\top}$, respectively, such that:

$$
C^{\top}=\mathbb{1} \tau^{\top}+\sum_{n=2}^{N} \lambda_{n} u_{n} v_{n}^{\top}
$$

From conditions 4.62-4.64, we know that $C$ is primitive. Then, the Perron-Frobenius theorem states that all entries of $\tau$ are positive (see, e.g., [124, Theorem 8.5.1]). Since we have assumed that $C$ is doubly stochastic, by replacing the 2 -norm with the 1 -norm in (4.144), we have:

$$
\left\|C^{\top}-\mathbb{1} \tau^{\top}\right\|_{1}=\left\|\sum_{n=2}^{N} \lambda_{n} u_{n} v_{n}^{\top}\right\|_{1}<\left\|\mathbb{1} \tau^{\top}+\sum_{n=2}^{N} \lambda_{n} u_{n} v_{n}^{\top}\right\|_{1}=\left\|C^{\top}\right\|_{1}=1
$$

For the infinity norm, we obtain exactly the same bound. Thus, we conclude that:

$$
\left\|\left(I_{N}-\mathbb{1} \tau^{\top}\right) C^{\top}\right\| \leq\left\|\left(I_{N}-\mathbb{1} \tau^{\top}\right) C^{\top}\right\|_{1}\left\|\left(I_{N}-\mathbb{1} \tau^{\top}\right) C^{\top}\right\|_{\infty}<1
$$

Proposition 5. For projector (4.131), it holds that:

$$
P_{\perp}\left(C^{\top} \otimes I_{M}\right) P_{\perp}=P_{\perp}\left(C^{\top} \otimes I_{M}\right)
$$

Proof: We extend the result from [36, Lemma 1] to the case of left-stochastic matrices. From the properties of the Kronecker product, we have

$$
\left(\tau^{\top} \otimes I_{M}\right)\left(C^{\top} \otimes I_{M}\right)=\left(\tau^{\top} C^{\top} \otimes I_{M}\right)=\left(\tau^{\top} \otimes I_{M}\right)
$$

The proof follows from standard linear algebra:

$$
P_{\perp}\left(C^{\top} \otimes I_{M}\right) P_{\perp}=\left(I_{M N}-\mathbb{1} \tau^{\top} \otimes I_{M}\right)\left(C^{\top} \otimes I_{M}\right)\left(I_{M N}-\mathbb{1} \tau^{\top} \otimes I_{M}\right)
$$




$$
\begin{aligned}
& =\left(C^{\top} \otimes I_{M}-\mathbb{1} \tau^{\top} C^{\top} \otimes I_{M}\right)\left(I_{M N}-\mathbb{1} \tau^{\top} \otimes I_{M}\right) \\
& =\left(C^{\top} \otimes I_{M}-\mathbb{1} \tau^{\top} \otimes I_{M}\right)\left(I_{M N}-\mathbb{1} \tau^{\top} \otimes I_{M}\right) \\
& =C^{\top} \otimes I_{M}-\mathbb{1} \tau^{\top} \otimes I_{M}-C^{\top} \mathbb{1} \tau^{\top} \otimes I_{M}+\mathbb{1} \tau^{\top} \mathbb{1} \tau^{\top} \otimes I_{M} \\
& =C^{\top} \otimes I_{M}-\mathbb{1} \tau^{\top} \otimes I_{M}-\mathbb{1} \tau^{\top} \otimes I_{M}+\mathbb{1} \tau^{\top} \otimes I_{M} \\
& =C^{\top} \otimes I_{M}-\mathbb{1} \tau^{\top} \otimes I_{M} \\
& =C^{\top} \otimes I_{M}-\mathbb{1}\left(\tau^{\top} \otimes I_{M}\right)\left(C^{\top} \otimes I_{M}\right) \\
& =\left(I_{M N}-\mathbb{1} \tau^{\top} \otimes I_{M}\right) C^{\top} \otimes I_{M} \\
& =P_{\perp}\left(C^{\top} \otimes I_{M}\right)
\end{aligned}
$$

Proposition 6. The following equivalence holds:

$$
\boldsymbol{\theta}_{k, i-1}-\mathbb{E}_{f_{\boldsymbol{w}_{k, i}}[}[\phi(\boldsymbol{x})]=-\left.\nabla_{w} \log \mathbb{E}_{f_{w}}\left[h_{k, i}(J(\boldsymbol{x})) \rho_{k, i-1}(\boldsymbol{x})\right]\right|_{w=\boldsymbol{w}_{k, i-1}}
$$

Proof: This equivalence was already included in [128] for the single agent case (without demonstration). We give a detailed derivation here for completeness.

For an NEF distribution $f_{w}=\exp \left(w^{\top} \phi(x)-Z(w)\right)$, we have:

$$
\begin{aligned}
\nabla_{w} Z(w) & =\nabla_{w} \log \int e^{w^{\top} \phi(x)} d x \\
& =\frac{\int \phi(x) e^{w^{\top} \phi(x)} d x}{\int e^{w^{\top} \phi(x)} d x} \\
& =\int \phi(x) e^{w^{\top} \phi(x)-Z(w)} d x \\
& =\mathbb{E}_{f_{w}}[\phi(\boldsymbol{x})], \\
\nabla_{w} f_{w}(x) & =\nabla_{w} e^{w^{\top} \phi(x)-Z(w)} \\
& =e^{w^{\top} \phi(x)-Z(w)}\left(\phi(x)-\nabla_{w} Z(w)\right) \\
& =f_{w}(x)\left(\phi(x)-\mathbb{E}_{f_{w}}[\phi(\boldsymbol{x})]\right)
\end{aligned}
$$

Hence, we have

$$
\begin{aligned}
\nabla_{w} \log \mathbb{E}_{f_{w}}\left[h_{k, i}(J(\boldsymbol{x})) \rho_{k, i-1}(\boldsymbol{x})\right] & =\frac{\nabla_{w} \mathbb{E}_{f_{w}}\left[h_{k, i}(J(\boldsymbol{x})) \rho_{k, i-1}(\boldsymbol{x})\right]}{\mathbb{E}_{f_{w}}\left[h_{k, i}(J(\boldsymbol{x})) \rho_{k, i-1}(\boldsymbol{x})\right]} \\
& =\frac{\nabla_{w} \int h_{k, i}(J(x)) \rho_{k, i-1}(x) f(x) d x}{\mathbb{E}_{f_{w}}\left[h_{k, i}(J(\boldsymbol{x})) \rho_{k, i-1}(\boldsymbol{x})\right]} \\
& =\frac{\int h_{k, i}(J(x)) \rho_{k, i-1}(x) \nabla_{w} f(x) d x}{\mathbb{E}_{f_{w}}\left[h_{k, i}(J(\boldsymbol{x})) \rho_{k, i-1}(\boldsymbol{x})\right]}
\end{aligned}
$$




$$
\begin{aligned}
& =\frac{\int h_{k, i}(J(x)) \rho_{k, i-1}(x)\left(\phi(x)-\mathbb{E}_{f_{w}}[\phi(\boldsymbol{x})]\right) f_{w}(x) d x}{\mathbb{E}_{f_{w}}\left[h_{k, i}(J(\boldsymbol{x})) \rho_{k, i-1}(\boldsymbol{x})\right]} \\
& =\frac{\mathbb{E}_{f_{w}}\left[h_{k, i}(J(\boldsymbol{x})) \rho_{k, i-1}(\boldsymbol{x})\left(\phi(\boldsymbol{x})-\mathbb{E}_{f_{w}}[\phi(\boldsymbol{x})]\right)\right]}{\mathbb{E}_{f_{w}}\left[h_{k, i}(J(\boldsymbol{x})) \rho_{k, i-1}(\boldsymbol{x})\right]} \\
& =\frac{\mathbb{E}_{f_{w}}\left[h_{k, i}(J(\boldsymbol{x})) \rho_{k, i-1}(\boldsymbol{x}) \phi(\boldsymbol{x})\right]}{\mathbb{E}_{f_{w}}\left[h_{k, i}(J(\boldsymbol{x})) \rho_{k, i-1}(\boldsymbol{x})\right]}-\mathbb{E}_{f_{w}}[\phi(\boldsymbol{x})]
\end{aligned}
$$

Recall that $\boldsymbol{\theta}_{k, i-1}=\mathbb{E}_{f_{\boldsymbol{w}_{k, i-1}}}[\phi(\boldsymbol{x})]$. Hence, by evaluating (4.154) for $w=\boldsymbol{w}_{k, i-1}$, we have

$$
\begin{aligned}
\left.\nabla_{w} \log \mathbb{E}_{f_{w}}\left[h_{k, i}(J(\boldsymbol{x})) \rho_{k, i-1}(\boldsymbol{x})\right]\right|_{w=\boldsymbol{w}_{k, i-1}} & =\frac{\mathbb{E}_{f_{\boldsymbol{w}_{k, i-1}}}\left[h_{k, i}(J(\boldsymbol{x})) \rho_{k, i-1}(\boldsymbol{x}) \phi(\boldsymbol{x})\right]}{\mathbb{E}_{f_{\boldsymbol{w}_{k, i-1}}}\left[h_{k, i}(J(\boldsymbol{x})) \rho_{k, i-1}(\boldsymbol{x})\right]}-\mathbb{E}_{f_{\boldsymbol{w}_{k, i-1}}}[\phi(\boldsymbol{x})] \\
& =\mathbb{E}_{f_{\boldsymbol{w}_{k, i}}[\phi(\boldsymbol{x})]-\boldsymbol{\theta}_{k, i-1}}
\end{aligned}
$$

Introduce the time-varying function $G_{i}: \mathbb{R}^{M N} \rightarrow \mathbb{R}^{M N}$ as a shorthand

$$
\begin{aligned}
G_{i}\left(\boldsymbol{\vartheta}_{i-i}\right) & \triangleq \boldsymbol{\vartheta}_{i-1}-\boldsymbol{\varphi}_{i} \\
& =\left(\boldsymbol{\theta}_{k, i-1}-\mathbb{E}_{f_{\boldsymbol{w}_{k, i}}}[\phi(\boldsymbol{x})]\right)_{k=1}^{N} \\
& =\left(\mathbb{E}_{f_{\boldsymbol{w}_{k, i-1}}}[\phi(\boldsymbol{x})]-\mathbb{E}_{f_{\boldsymbol{w}_{k, i}^{\bullet}}}[\phi(\boldsymbol{x})]\right)_{k=1}^{N} \\
& =-\left(\left.\nabla_{w} \log \mathbb{E}_{f_{w}}\left[h_{k, i}(J(\boldsymbol{x})) \rho_{k, i-1}(\boldsymbol{x})\right]\right|_{w=\boldsymbol{w}_{k, i-1}}\right)_{k=1}^{N} \\
& =\left(G_{k, i}\left(\boldsymbol{\theta}_{k, i-1}\right)\right)_{k=1}^{N}
\end{aligned}
$$

with components defined as:

$$
G_{k, i}\left(\boldsymbol{\theta}_{k, i-1}\right) \triangleq \boldsymbol{\theta}_{k, i-1}-\mathbb{E}_{f_{\boldsymbol{w}_{k, i}}}[\phi(\boldsymbol{x})]
$$

We remark that $G_{i}$ is time-varying because the likelihood functions $h_{k, i}$ are time-varying. We show that (4.156) is bounded.

Proposition 7. Let the likelihood $h_{k, i}$ satisfy Assumption 4.2 for every agent $k=$ $1, \ldots, N$ at every iteration $i=1, \ldots, \infty$. Then, $\left\|G_{i}\left(\vartheta_{i-1}\right)\right\|<\infty, \forall \vartheta_{i-1} \in \mathbb{R}^{M N}$.

Proof: We show that all components $G_{k, i}$ are bounded. From Proposition 6, we have

$$
G_{k, i}\left(\theta_{k, i-1}\right)=\frac{\mathbb{E}_{f_{w_{k, i-1}}}\left[h_{k, i}(J(\boldsymbol{x})) \rho_{k, i-1}(\boldsymbol{x}) \phi(\boldsymbol{x})\right]}{\mathbb{E}_{f_{w_{k, i-1}}}\left[h_{k, i}(J(\boldsymbol{x})) \rho_{k, i-1}(\boldsymbol{x})\right]}-\mathbb{E}_{f_{w_{k, i-1}}}[\phi(\boldsymbol{x})]
$$


Recall from (4.21) that $\mathbb{E}_{f_{w_{k, i-1}}}[\phi(\boldsymbol{x})]<\infty, \forall w \in \mathbb{W}$. From Assumption 4.2, we know the likelihood is bounded by

$$
h_{k, i}^{\max } \triangleq h_{k, i}\left(J\left(x^{\star}\right)\right), \quad \forall x^{\star} \in \mathbb{X}^{\star}
$$

In addition, since $g_{k, i-1}(x)>0$ implies $f_{w_{k, i-1}}(x)>0$, we know that the importance weights are the ratio of two positive numbers, which must be bounded by some $\rho_{k, i}^{\max } \in \mathbb{R}$. Therefore, we have

$$
\mathbb{E}_{f_{w_{k, i-1}}}\left[h_{k, i}(J(\boldsymbol{x})) \rho_{k, i-1}(\boldsymbol{x}) \phi(\boldsymbol{x})\right] \leq h_{k, i}^{\max } \rho_{k, i}^{\max } \mathbb{E}_{f_{w_{k, i-1}}}[\phi(\boldsymbol{x})]<\infty
$$

From Assumption 4.6, we conclude that all terms in (4.158) are bounded.

The following theorem shows that all agents converge to their weighted average.

Theorem 4.4. Under Assumptions 4.2-4.7, the estimates obtained from distributed recursion (4.124a)-(4.124b) converge almost surely to their weighted average:

$$
\mathbb{P}\left(\lim _{i \rightarrow \infty} \boldsymbol{\theta}_{k, i}=\overline{\boldsymbol{\theta}}_{i}\right)=1, \quad k=1, \ldots, N
$$

Proof: We show that $\boldsymbol{\vartheta}_{i}^{\text {dis }}$ converges to zero, so that $\boldsymbol{\vartheta}_{i}$ converges to $\boldsymbol{\vartheta}_{i}^{\text {agr }}$. Rewrite (4.128) using (4.156):

$$
\boldsymbol{\vartheta}_{i}=\left(C^{\top} \otimes I_{M}\right)\left(\boldsymbol{\vartheta}_{i-1}-\alpha_{i}\left(G_{i}\left(\boldsymbol{\vartheta}_{i-1}\right)-\boldsymbol{\zeta}_{i}\right)\right)
$$

Multiply both sides of (4.162) by $P_{\perp}$ and use Proposition 5:

$$
\begin{aligned}
P_{\perp} \boldsymbol{\vartheta}_{i} & =P_{\perp}\left(C^{\top} \otimes I_{M}\right)\left(\boldsymbol{\vartheta}_{i-1}-\alpha_{i}\left(G_{i}\left(\boldsymbol{\vartheta}_{i-1}\right)-\boldsymbol{\zeta}_{i}\right)\right) \\
& =P_{\perp}\left(C^{\top} \otimes I_{M}\right) P_{\perp}\left(\boldsymbol{\vartheta}_{i-1}-\alpha_{i}\left(G_{i}\left(\boldsymbol{\vartheta}_{i-1}\right)-\boldsymbol{\zeta}_{i}\right)\right)
\end{aligned}
$$

Let us upper bound the norm of (4.163) as follows:

$$
\begin{aligned}
\left\|\boldsymbol{\vartheta}_{i}^{\text {dis }}\right\| & =\left\|P_{\perp}\left(C^{\top} \otimes I_{M}\right)\left(\boldsymbol{\vartheta}_{i-1}^{\text {dis }}-\alpha_{i} P_{\perp}\left(G_{i}\left(\boldsymbol{\vartheta}_{i-1}\right)-\boldsymbol{\zeta}_{i}\right)\right)\right\| \\
& \leq\left\|P_{\perp}\left(C^{\top} \otimes I_{M}\right)\right\|\left\|\boldsymbol{\vartheta}_{i-1}^{\text {dis }}-\alpha_{i} P_{\perp}\left(G_{i}\left(\boldsymbol{\vartheta}_{i-1}\right)-\boldsymbol{\zeta}_{i}\right)\right\| \\
& <\left\|\boldsymbol{\vartheta}_{i-1}^{\text {dis }}-\alpha_{i} P_{\perp}\left(G_{i}\left(\boldsymbol{\vartheta}_{i-1}\right)-\boldsymbol{\zeta}_{i}\right)\right\| \\
& \leq\left\|\boldsymbol{\vartheta}_{i-1}^{\text {dis }}\right\|+\alpha_{i}\left\|P_{\perp}\left(G_{i}\left(\boldsymbol{\vartheta}_{i-1}\right)-\boldsymbol{\zeta}_{i}\right)\right\|
\end{aligned}
$$

where the first inequality is due to the submutiplicative property of the norm, the second is due to Assumption 4.7, and the third is the Minkowski's inequality (which 
can be thought as the triangle inequality for random variables). By rearranging terms, we get:

$$
\left\|\boldsymbol{\vartheta}_{i}^{\text {dis }}\right\|-\left\|\boldsymbol{\vartheta}_{i-1}^{\text {dis }}\right\|<\alpha_{i}\left\|P_{\perp}\left(G_{i}\left(\boldsymbol{\vartheta}_{i-1}\right)-\boldsymbol{\zeta}_{i}\right)\right\|
$$

Depending on the sign of the left side of (4.165), we consider two cases. First, if $\left\|\boldsymbol{\vartheta}_{i}^{\text {dis }}\right\| \leq\left\|\boldsymbol{\vartheta}_{i-1}^{\text {dis }}\right\|$, then we conclude that the sequence $\left\{\left\|\boldsymbol{\vartheta}_{i}^{\text {dis }}\right\|\right\}_{i=0}^{\infty}$ converges since it is decreasing and bounded below by zero. Second, if $\left\|\boldsymbol{\vartheta}_{i}^{\text {dis }}\right\|>\left\|\boldsymbol{\vartheta}_{i-1}^{\text {dis }}\right\|$, then we can square both sides of (4.165) without changing the sign of the inequality:

$$
\left(\left\|\boldsymbol{\vartheta}_{i}^{\text {dis }}\right\|-\left\|\boldsymbol{\vartheta}_{i-1}^{\text {dis }}\right\|\right)^{2}<\alpha_{i}^{2}\left\|P_{\perp}\left(G_{i}\left(\boldsymbol{\vartheta}_{i-1}\right)-\boldsymbol{\zeta}_{i}\right)\right\|^{2}
$$

Under Assumption 4.2, Proposition 7 shows that there exists $L \in \mathbb{R}$ such that $\left\|G_{i}\left(\boldsymbol{\vartheta}_{i-1}\right)\right\|^{2} \leq L$. Assumption 4.4 states that there exists $L^{\prime} \in \mathbb{R}$ such that $\left\|\boldsymbol{\zeta}_{i}\right\|^{2} \leq L^{\prime}$. Hence, from Assumption 4.5, we have:

$$
\begin{aligned}
\lim _{i \rightarrow \infty}\left(\left\|\boldsymbol{\vartheta}_{i}^{\text {dis }}\right\|-\left\|\boldsymbol{\vartheta}_{i-1}^{\text {dis }}\right\|\right)^{2} & <\lim _{i \rightarrow \infty} \alpha_{i}^{2}\left\|P_{\perp}\left(G_{i}\left(\boldsymbol{\vartheta}_{i-1}\right)-\boldsymbol{\zeta}_{i}\right)\right\|^{2} \\
& \leq \lim _{i \rightarrow \infty} \alpha_{i}^{2}\left\|P_{\perp}\right\|^{2}\left(\left\|G_{i}\left(\boldsymbol{\vartheta}_{i-1}\right)\right\|^{2}+\left\|\boldsymbol{\zeta}_{i}\right\|^{2}\right) \\
& \leq \lim _{i \rightarrow \infty} \alpha_{i}^{2}\left(L+L^{\prime}\right) \\
& =0
\end{aligned}
$$

where the second inequality is due to the submutiplicative property of the norm, and Minkowski's (triangle) inequality; and the third inequality uses the upper bounds mentioned above, as well as the fact that the norm of a projector is upper bounded by one. Since the leftmost side must be non-negative, it cannot be less than zero, so we have achieved a contradiction. Thus, this second case will never happen. We conclude that the sequence $\left\{\left\|\boldsymbol{\vartheta}_{i}^{\text {dis }}\right\|\right\}_{i=0}^{\infty}$ converges, so that we can introduce a shorthand for the limit:

$$
\left\|\boldsymbol{\vartheta}_{\infty}^{\text {dis }}\right\| \triangleq \lim _{i \rightarrow \infty}\left\|\boldsymbol{\vartheta}_{i}^{\text {dis }}\right\|=\lim _{i \rightarrow \infty}\left\|\boldsymbol{\vartheta}_{i-1}^{\text {dis }}\right\|
$$

Take the limit in both sides of (4.164):

$$
\begin{aligned}
& \lim _{i \rightarrow \infty}\left\|\boldsymbol{\vartheta}_{i}^{\mathrm{dis}}\right\|=\lim _{i \rightarrow \infty}\left\|P_{\perp}\left(C^{\top} \otimes I_{M}\right)\left(\boldsymbol{\vartheta}_{i-1}^{\mathrm{dis}}-\alpha_{i} P_{\perp}\left(G_{i}\left(\boldsymbol{\vartheta}_{i-1}\right)-\boldsymbol{\zeta}_{i}\right)\right)\right\| \\
& \quad \leq \lim _{i \rightarrow \infty}\left\|P_{\perp}\left(C^{\top} \otimes I_{M}\right)\right\|\left\|\boldsymbol{\vartheta}_{i-1}^{\mathrm{dis}}-\alpha_{i} P_{\perp}\left(G_{i}\left(\boldsymbol{\vartheta}_{i-1}\right)-\boldsymbol{\zeta}_{i}\right)\right\| \\
& \leq\left\|P_{\perp}\left(C^{\top} \otimes I_{M}\right)\right\|\left(\lim _{i \rightarrow \infty}\left\|\boldsymbol{\vartheta}_{i-1}^{\mathrm{dis}}\right\|+\lim _{i \rightarrow \infty} \alpha_{i}\left\|P_{\perp}\left(G_{i}\left(\boldsymbol{\vartheta}_{i-1}\right)-\boldsymbol{\zeta}_{i}\right)\right\|\right)
\end{aligned}
$$


where, again, the first inequality is due to the submutiplicative property of the norm, and the second is the Minkowski's. By rearranging terms in (4.169) and using (4.168), we obtain:

$$
\begin{aligned}
\left\|\boldsymbol{\vartheta}_{\infty}^{\text {dis }}\right\|(1 & \left.-\left\|P_{\perp}\left(C^{\top} \otimes I_{M}\right)\right\|\right) \\
& \leq\left\|P_{\perp}\left(C^{\top} \otimes I_{M}\right)\right\|\left(\lim _{i \rightarrow \infty} \alpha_{i}\left\|P_{\perp}\left(G_{i}\left(\boldsymbol{\vartheta}_{i-1}\right)-\boldsymbol{\zeta}_{i}\right)\right\|\right)
\end{aligned}
$$

Again, since $\left\|G_{i}\left(\boldsymbol{\vartheta}_{i-1}\right)\right\|$ and $\left\|\boldsymbol{\zeta}_{i}\right\|$ are bounded, from the properties of limits (see, e.g., [64, Theorem 3.1.4]) and Assumption 4.5, we have:

$$
\lim _{i \rightarrow \infty} \alpha_{i}\left\|P_{\perp}\left(G_{i}\left(\boldsymbol{\vartheta}_{i-1}\right)-\boldsymbol{\zeta}_{i}\right)\right\| \leq \lim _{i \rightarrow \infty} \alpha_{i}\left(\sqrt{L}+L^{\prime}\right)=0
$$

so that (4.170) becomes:

$$
\left\|\boldsymbol{\vartheta}_{\infty}^{\mathrm{dis}}\right\|\left(1-\left\|P_{\perp}\left(C^{\top} \otimes I_{M}\right)\right\|\right) \leq 0
$$

From Assumption 4.7, we know that $\left\|P_{\perp}\left(C^{\top} \otimes I_{M}\right)\right\|<1$, so that the right factor must be positive:

$$
1-\left\|P_{\perp}\left(C^{\top} \otimes I_{M}\right)\right\|>0
$$

Hence, the other factor must be non-positive: $\left\|\boldsymbol{\vartheta}_{\infty}^{\text {dis }}\right\| \leq 0$. Since the norm is nonnegative, we have the following inequality

$$
0 \leq\left\|\vartheta_{\infty}^{\text {dis }}\right\| \leq 0
$$

Therefore, we conclude that

$$
\left\|\boldsymbol{\vartheta}_{\infty}^{\text {dis }}\right\|=0
$$

Taking the square of (4.175) and using (4.125), (4.129) and (4.132), we have:

$$
\left\|\boldsymbol{\vartheta}_{\infty}^{\mathrm{dis}}\right\|^{2}=\lim _{i \rightarrow \infty}\left\|\boldsymbol{\vartheta}_{i}-\boldsymbol{\vartheta}_{i}^{\mathrm{agr}}\right\|^{2}=\lim _{i \rightarrow \infty} \sum_{k=1}^{N}\left\|\boldsymbol{\theta}_{k, i}-\overline{\boldsymbol{\theta}}_{i}\right\|^{2}=0
$$

Therefore, all terms in the right side of (4.176) must be zero. Due to the completeness of the $L_{2}$ space, we conclude that that all agents converge almost surely to their average 
vector:

$$
\lim _{i \rightarrow \infty} \boldsymbol{\theta}_{k, i}=\lim _{i \rightarrow \infty} \overline{\boldsymbol{\theta}}_{i} \quad \text { a.s. }, \quad k=1, \ldots, N
$$

\subsubsection{Almost sure convergence of DCE agents}

Theorem 4.4 ensures almost sure convergence of the network estimate, $\vartheta_{i}$, to the agreement network vector, $\boldsymbol{\vartheta}_{i}^{\text {agr }}$. Hence, in order to study the convergence of $\boldsymbol{\vartheta}_{i}$, it is sufficient to study the convergence of $\boldsymbol{\vartheta}_{i}^{\text {agr }}$.

Let us build a recursion on $\boldsymbol{\vartheta}_{i}^{\text {agr }}$. By multiplying both sides of (4.162) by the agreement projector $P$, given by (4.130), we get

$$
\boldsymbol{\vartheta}_{i}^{\mathrm{agr}}=\boldsymbol{\vartheta}_{i-1}^{\mathrm{agr}}-\alpha_{i}\left(P G_{i}\left(\boldsymbol{\vartheta}_{i-i}\right)-P \boldsymbol{\zeta}_{i}\right)
$$

where we used the relationship

$$
P\left(C^{\top} \otimes I_{M}\right)=\left(1 \tau^{\top} \otimes I_{M}\right)\left(C^{\top} \otimes I_{M}\right)=1 \tau^{\top} C^{\top} \otimes I_{M}=P
$$

Note that (4.178) is not a true recursion on $\boldsymbol{\vartheta}_{i-1}^{\mathrm{agr}}$ because the term $P G_{i}\left(\boldsymbol{\vartheta}_{i-i}\right)$ still depends on $\boldsymbol{\vartheta}_{i-i}$. Introduce the error term:

$$
\boldsymbol{r}_{i} \triangleq P\left(G_{i}\left(\boldsymbol{\vartheta}_{i-1}^{\mathrm{agr}}\right)-G\left(\boldsymbol{\vartheta}_{i-1}\right)\right)
$$

Now, we can rewrite (4.178) as:

$$
\boldsymbol{\vartheta}_{i}^{\mathrm{agr}}=\boldsymbol{\vartheta}_{i-1}^{\mathrm{agr}}-\alpha_{i}\left(P G_{i}\left(\boldsymbol{\vartheta}_{i-i}^{\mathrm{agr}}\right)-\boldsymbol{r}_{i}+P \zeta_{i}\right)
$$

Introduce the following shorthand:

$$
\begin{aligned}
\bar{G}_{i}\left(\overline{\boldsymbol{\theta}}_{i-1}\right) & \triangleq \sum_{k=1}^{N} \tau_{k} G_{k, i}\left(\overline{\boldsymbol{\theta}}_{i-1}\right) \\
\overline{\boldsymbol{\zeta}}_{i} & \triangleq \sum_{k=1}^{N} \tau_{k} \boldsymbol{\xi}_{k, i} \\
\overline{\boldsymbol{r}}_{i} & \triangleq \sum_{k=1}^{N} \tau_{k} \boldsymbol{r}_{i}
\end{aligned}
$$


where $\overline{\boldsymbol{\theta}}_{i-1}$ is the weighted average estimate given by (4.129). Then, we can rewrite (4.181) as:

$$
\left(\mathbb{1} \otimes I_{M}\right) \overline{\boldsymbol{\theta}}_{i}=\left(\mathbb{1} \otimes I_{M}\right)\left(\overline{\boldsymbol{\theta}}_{i-1}-\alpha_{i}\left(\bar{G}_{i}\left(\overline{\boldsymbol{\theta}}_{i-i}\right)-\overline{\boldsymbol{r}}_{i}+\overline{\boldsymbol{\zeta}}_{i}\right)\right)
$$

Note that (4.185) has stacked multiple copies of the same equations. Thus, in order to study convergence, we can equivalently study

$$
\overline{\boldsymbol{\theta}}_{i}=\overline{\boldsymbol{\theta}}_{i-1}-\alpha_{i}\left(\bar{G}_{i}\left(\overline{\boldsymbol{\theta}}_{i-i}\right)-\overline{\boldsymbol{r}}_{i}+\overline{\boldsymbol{\zeta}}_{i}\right)
$$

In order to transform (4.186) into a true recursion, we follow [36, Proposition 1] and show that $\lim _{i \rightarrow \infty}\left\|\overline{\boldsymbol{r}}_{i}\right\|=0$. By using the Minkowski's (triangle) inequality, we have

$$
\begin{aligned}
\left\|\overline{\boldsymbol{r}}_{i}\right\| & =\left\|\sum_{k=1}^{N} \tau_{k}\left(G_{k, i}\left(\overline{\boldsymbol{\theta}}_{i-1}\right)-G_{k, i}\left(\boldsymbol{\theta}_{k, i-1}\right)\right)\right\| \\
& \leq \sum_{k=1}^{N} \tau_{k}\left\|G_{k, i}\left(\overline{\boldsymbol{\theta}}_{i-1}\right)-G_{k, i}\left(\boldsymbol{\theta}_{k, i-1}\right)\right\|
\end{aligned}
$$

We need the following proposition.

Proposition 8. The functions $G_{k, i}$ given by (4.155) are locally Lipschitz for every agent $k=1, \ldots, N$ at every iteration.

Proof: From (4.155)-(4.156), we have:

$$
G_{k, i}\left(\boldsymbol{\theta}_{k, i-1}\right)=\mathbb{E}_{f_{\boldsymbol{w}_{k, i}}}[\phi(\boldsymbol{x})]-\mathbb{E}_{f_{\boldsymbol{w}_{k, i-1}}}[\phi(\boldsymbol{x})]
$$

Recall that $\nabla_{w} Z(w)=\mathbb{E}_{f_{w}}[\phi(\boldsymbol{x})]$. Thus, we can express

$$
\begin{aligned}
\nabla_{w} Z\left(\boldsymbol{w}_{k, i}^{\bullet}\right) & =\mathbb{E}_{f_{\boldsymbol{w}_{k, i}}}[\phi(\boldsymbol{x})] \\
\nabla_{w} Z\left(\boldsymbol{w}_{k, i-1}\right) & =\mathbb{E}_{f_{\boldsymbol{w}_{k, i-1}}}[\phi(\boldsymbol{x})]
\end{aligned}
$$

It is well known (see, e.g., [153, App. D.1]) that $Z(w) \in \mathbb{C}^{\infty}$ so that it can be differentiated any number of times $\forall w \in \mathbb{W}$. Hence, the sum of two smooth functions is also smooth. Any smooth function is locally Lipschitz, since there is a convex neighborhood around every $\theta$ where $\nabla_{w} G_{k, i}(\theta)$ exists and is bounded; being the bound on $\nabla_{w} G_{k, i}(\theta)$ the Lipschitz constant in this neighborhood [182, p. 188].

Since $G_{k, i}$ is locally Lipschitz, for each $\theta \in \Theta$, there is $B>0$ and $A>0$ such that $\left\|\theta-\theta^{\prime}\right\|<A$ implies $\left\|G_{k, i}(\theta)-G_{k, i}\left(\theta^{\prime}\right)\right\| \leq B\left\|\theta-\theta^{\prime}\right\|$, where $A$ depends on $\theta^{\prime}$, and $B$ 
depends on $\theta^{\prime}$ and $A$. From Theorem 4.4, we know that $\lim _{i \rightarrow \infty}\left\|\boldsymbol{\theta}_{k, i-1}-\overline{\boldsymbol{\theta}}_{i-1}\right\|=0$. Hence, we have

$$
\lim _{i \rightarrow \infty}\left\|G_{k, i}\left(\overline{\boldsymbol{\theta}}_{i-1}\right)-G_{k, i}\left(\boldsymbol{\theta}_{k, i-1}\right)\right\| \leq B \lim _{i \rightarrow \infty}\left\|\boldsymbol{\theta}_{k, i-1}-\overline{\boldsymbol{\theta}}_{i-1}\right\|=0
$$

It follows that $\lim _{i \rightarrow \infty}\left\|\overline{\boldsymbol{r}}_{i}\right\|=0$. Thus, we can simplify (4.186) and study the convergence of

$$
\overline{\boldsymbol{\theta}}_{i}=\overline{\boldsymbol{\theta}}_{i-1}-\alpha_{i}\left(\bar{G}_{i}\left(\overline{\boldsymbol{\theta}}_{i-i}\right)+\overline{\boldsymbol{\zeta}}_{i}\right)
$$

Recursion (4.192) is still difficult to be studied with standard stochastic approximation techniques because $\bar{G}_{i}$ depends on the likelihood functions, which depend on the sample-quantiles, which change at every iteration. The following assumptions were used by [128, Lemma 3.1] to prove convergence of the sample-quantile to a stationary estimate, $\gamma_{\infty}$, for the single-agent recursion. We will use them here in a similar manner to ensure that $\bar{G}_{i}$ converges to some stationary function $G_{\infty}$.

Assumption 4.8 (Unique quantile). The quantile $q \in(0,1)$ of $\left\{J(\boldsymbol{x}), \boldsymbol{x} \sim f_{w}(\boldsymbol{x})\right\}$ is unique for each $w \in \mathbb{W}$.

Assumption 4.9 (Number of samples). The number of samples per iteration grows with time for every agent, such that it satisfies $S_{k, i} \geq i^{\delta_{k}}$, for some $\delta_{k}>1$.

Assumption 4.8 is required to show convergence of the sample quantile to a single value [128, Lemma 4.1] (see also [123, Lemma 1]). Assumption 4.9 states that the number of samples per iteration grows with time. This is required as the distance between the sample and true quantiles is inversely proportional to the number of samples. Thus, by increasing the number of samples, we can ensure that the sample quantile converges to the true quantile.

Lemma 4.1. Let the likelihood functions $h_{k, i}$ be the smooth indicator function (4.35) with threshold $\gamma_{k, i}$ estimated as the sample quantile of the set of output values $\left\{y_{k, i, s}\right\}_{s=1}^{S_{k, i}}$. Then, under Assumptions 4.2-4.9, the function $\bar{G}_{i}$ converges to a stationary function $\bar{G}_{\infty}$ almost surely.

Proof: Theorem 4.4 states that all agents converge to (4.192) almost surely, so that they will end up sampling from the same distribution $f_{\overline{\boldsymbol{w}}_{i}}$, where $\overline{\boldsymbol{w}}_{i}=F\left(\overline{\boldsymbol{\theta}}_{i}\right)$. From [128, Lemma 4.1] (see also [123, Lemma 1] and [127, Lemma 7]), we conclude that the sample quantile of output values will be the same for every agent and converges to a stationary value, $\gamma_{\infty}$, almost surely. Thus, the likelihoods $h_{k, i}$ will converge to 
some $h_{\infty}$, the same for every agent, so that there exists some function $\bar{G}_{\infty}$ such that $\lim _{i \rightarrow \infty} \bar{G}_{i}=\bar{G}_{\infty}$.

Lemma 4.1 allows us simplify (4.192) further so that we can equivalently study the almost sure convergence of the following stationary recursion:

$$
\overline{\boldsymbol{\theta}}_{i}=\overline{\boldsymbol{\theta}}_{i-1}-\alpha_{i}\left(\bar{G}_{\infty}\left(\overline{\boldsymbol{\theta}}_{i-i}\right)+\overline{\boldsymbol{\zeta}}_{i}\right)
$$

In order to study (4.193), we need the following proposition.

Proposition 9. The function $\bar{G}_{i}: \mathbb{R}^{M} \rightarrow \mathbb{R}^{M}$ given by (4.182) is continuous at every iteration.

Proof: Similar to Proposition 8, we know that the terms $G_{k, i}$ are smooth and so continuous. Hence, from [64, Prop. 6.1.2 \& Th. 5.3.3], we conclude that $\bar{G}_{i}$ is also continuous.

Recall from (4.57) that the term $\overline{\boldsymbol{\zeta}}_{i}$ is asymptotically unbiased (when the number of samples per iteration grows to infinity). Recall from (4.182) and Proposition 6 that $\bar{G}_{\infty}$ can be seen as the gradient of some function. Since it is also continuous (Proposition 9 ), we can see $\overline{\boldsymbol{\theta}}_{i}$ in (4.193) as a noisy discrete approximation of the following ODE:

$$
\dot{\theta}=\bar{G}_{\infty}(\theta)
$$

We are ready to state the main results of this section:

Theorem 4.5 (Almost sure convergence of agreement recursion). Let Assumptions 4.3-4.8 hold. Assume that the solution set of (4.194) is composed of isolated equilibrium points. Then, the average sequence of Monte Carlo estimates $\left\{\overline{\boldsymbol{\theta}}_{k, i}\right\}_{i=0}^{\infty}$ converges almost surely to some equilibrium point, $\theta_{\infty}$ :

$$
\lim _{i \rightarrow \infty} \boldsymbol{\theta}_{k, i}=\theta_{\infty} \quad \text { a.s., } \quad k=1, \ldots, N
$$

Proof: We can use the single agent result due to [128, Theorem 3.1] that states that a single-agent equation similar to (4.193) converges almost surely to an equilibrium point of (4.194), denoted $\theta_{\infty}$, such that $\bar{G}_{\infty}\left(\theta_{\infty}\right)=0$. Therefore, from Theorem 4.4, we have:

$$
\lim _{i \rightarrow \infty} \boldsymbol{\theta}_{k, i}=\lim _{i \rightarrow \infty} \overline{\boldsymbol{\theta}}_{i}=\theta_{\infty} \quad \text { a.s. }, \quad k=1, \ldots, N
$$




\subsection{Numerical Experiments}

In this section, we illustrate the performance of the proposed DCE algorithm in a difficult benchmark that consists of 10 different nonconvex problems for which we know the global solution. The set of benchmark problems has been mainly taken from $[152,127]$, and includes a collection of standard objective functions that are widely used in the global optimization literature (see, e.g., [323, 152, 127, 322, 165, 312, 37]) and that are representative of different challenges, like severe multimodality, large plateaus, sharp peaks and input spaces ranging from 2 to 100 dimensions.

For the single agent case, model-based methods have already been tested in difficult benchmarks, achieving competitive or even state-of-the art performance [128, 127, 153, 341]. Thus, our purpose here is not to compare the results of DCE with other global optimization methods (e.g., simulated annealing, ant colony optimization, genetic algorithms, etc.). Instead, we aim to compare DCE with the single-agent SACE presented in [128] to investigate two issues. On one hand, we aim to see whether splitting the computational load among multiple agents penalize the performance with respect to a single agent that has the same sample-evaluation budget as the whole network. On the other hand, we aim to check whether having multiple agents searching cooperatively can enhance the exploration capabilities of the network (as a whole) and be more robust against local maxima than the single-agent SACE algorithm.

The set of benchmark problems has been mainly taken from $[152,127]$, and includes a collection of standard objective functions that are widely used in the global optimization literature (see, e.g., $[323,322,165,37])$ and representative of different challenges, like severe multimodality, large plateaus, sharp peaks and input spaces ranging from 2 to 100 dimensions. The description of these functions is as follows (some of them are illustrated in Figure 4.3 for their first two dimensions), where we use parenthesis notation to denote the elements of a vector (e.g., $x(m)$ and $b_{m}(j)$ denote the $m$-th and $j$-th components of vectors $x$ and $b_{m}$, respectively):

1. Dejong 5 th function $(M=2)$

$$
J^{1)}(x)=-\left[0.002+\sum_{j=1}^{25} \frac{1}{j+\sum_{m=1}^{2}\left(x(m)-b_{m}(j)\right)^{6}}\right]^{-1}
$$

where $b_{1}=[-32,-16,0,16,32,-32,-16,0,16,32,-32,-16,0,16,32,-32,-16,0$, $16,32,-32,-16,0,16,32]^{\top}$ and $b_{2}=[-32,-32,-32,-32,-32,-16,-16,-16,-16$, $-16,0,0,0,0,0,16,16,16,16,16,32,32,32,32,32]^{\top}$. This function has 24 
local maxima and one global maximum at $x^{\star}=[-32,-32]^{\top}$ and optimal value $J^{1)}\left(x^{\star}\right) \approx-0.998$

2. Shekel function $(M=4)$

$$
J^{2)}(x)=-\sum_{j=1}^{5} \frac{1}{\left\|x-b_{j}\right\|^{2}+c(j)}
$$

where $b_{1}=[4,4,4,4]^{\top}, b_{2}=[1,1,1,1]^{\top}, b_{3}=[8,8,8,8]^{\top}, b_{4}=[6,6,6,6]^{\top}$, $b_{5}=[3,7,3,7]^{\top}$, and $c=[0.1,0.2,0.2,0.4,0.4]^{\top}$. The solution is $x^{\star} \approx[4,4,4,4]^{\top}$ and $J^{2)}\left(x^{\star}\right) \approx 10.153$.

3. Rosenbrock function $(M=10)$

$$
J^{3)}(x)=-\sum_{m=1}^{M-1} 100\left(x(m+1)-x(m)^{2}\right)^{2}+(x(m)-1)^{2}
$$

This function is unimodal, and the global minimum lies in a narrow, parabolic valley. Although finding this valley is easy, its almost flat parabolic shape makes convergence to the global minimum challenging. The solution is $x^{\star}=[1,1,1,1]^{\top}$ and $J^{3)}\left(x^{\star}\right)=0$.

4. Rastrigin function $(M \in\{30,100\})$

$$
J^{4)}(x)=-10 M-\sum_{m=1}^{M} x(m)^{2}-10 \cos (2 \pi x(m))
$$

This function has several local maxima, which are regularly distributed. The solution is $x^{\star}=\mathbb{O}_{M}$ and $J^{4)}\left(x^{\star}\right)=0$.

5. Zakharov function $(M=50)$

$$
J^{5)}(x)=-\left(\sum_{m=1}^{M} x(m)^{2}\right)-\left(\sum_{m=1}^{M} m 0.5 x(m)\right)^{2}-\left(\sum_{m=1}^{M} m 0.5 x(m)\right)^{4}
$$

This function is also unimodal but plate shaped. The solution is $x^{\star}=\mathbb{O}_{M}$ and $J^{5)}\left(x^{\star}\right)=0$.

6. Ackley function $(M \in\{50,100\})$

$$
J^{6)}(x)=20 e^{-0.2 \sqrt{\frac{1}{M} \sum_{m=1}^{M} x(m)^{2}}}+e^{\frac{1}{M} \sum_{m=1}^{M} \cos (2 \pi x(m))}-20-e
$$


where $e$ is the Euler's number. This function is highly multimodal but most of the local maxima are small and located around a much larger global maximum. The solution is $x^{\star}=\mathbb{O}_{M}$ and $J^{6)}\left(x^{\star}\right)=0$.

7. Levy function $(M=50)$

$$
\begin{aligned}
J^{7)}(x)= & -\sin ^{2}\left(\pi b_{1}\right)-\left(b_{M}-1\right)^{2}\left(1+\sin ^{2}\left(2 \pi b_{M}\right)\right) \\
& -\sum_{m=1}^{M-1}\left(b_{m}-1\right)^{2}\left(1+10 \sin ^{2}\left(\pi b_{m}+1\right)\right)
\end{aligned}
$$

where $b_{m}=1+(x(m)-1) / 4, m=1, \ldots, M$. This function has multiple local maxima, with sharp canyons and valleys. The solution is $x^{\star}=\mathbb{1}_{M}$ and $J^{7)}\left(x^{\star}\right)=0$.

8. Trigonometric function $(M=50)$

$$
\begin{aligned}
J^{8)}(x)= & -1-\sum_{m=1}^{M} 8 \sin ^{2}\left(7(x(m)-0.9)^{2}\right)-6 \sin ^{2}\left(14(x(m)-0.9)^{2}\right) \\
& -(x(m)-0.9)^{2}
\end{aligned}
$$

This function has several abrupt local peaks around the global maximum. The solution is $x^{\star}=[0.9, \ldots, 0.9]^{\top}$ and $J^{8)}\left(x^{\star}\right)=-1$.

9. Griewank function $(M=50)$

$$
J^{9)}(x)=\prod_{m=1}^{M} \cos \left(\frac{x(m)}{\sqrt{m}}\right)-\frac{1}{4000} \sum_{m=1}^{M} x^{2}(m)-1
$$

This function has many widespread local minima, which are regularly distributed. The solution is $x^{\star}=\mathbb{O}_{M}$ and $J^{9)}\left(x^{\star}\right)=0$.

10. Pinter function $(M \in\{50,100\})$

$$
\begin{aligned}
J^{10)}(x)= & \sum_{m=1}^{M}-20 m \sin ^{2}(x(m-1) \sin (x(m))-x(m)+\sin (x(m+1))) \\
& -m \log _{10}\left(1+m\left(x^{2}(m-1)-2 x(m)+3 x(m+1)-\cos (x(m))+1\right)^{2}\right) \\
& -m x^{2}(m)
\end{aligned}
$$


where we artificially extended the dimensionality of the input vector with the convention that $x(0)=x(M)$ and $x(M+1)=x(1)$. The solution is $x^{\star}=\mathbb{O}_{M}$ and $J^{10)}\left(x^{\star}\right)=0$.

\subsubsection{Split computational load vs. performance}

In this first experiment, we compare the performance of SACE with DCE, using the same parameters for both every DCE agent in the network and the single agent performing SACE, with the exception that the networked agents draw $S_{k, i}$ samples per iteration each, and the SACE agent draws $S_{i}$ samples per iteration, such that

$$
\begin{aligned}
S_{k, i} & =S_{i} / N \\
S_{i} & =\text { floor }\left[\max \left[S_{0}, i^{1.01}\right]\right]
\end{aligned}
$$

where we recall that $N$ is the number of agents performing DCE, $S_{0}$ is the initial number of samples and floor[·] is the round-toward-negative-infinity operator. The idea underlying (4.207) is to compare the performance of DCE and SACE with the same total budget of function evaluations for both settings. Thus, the main goal with this experiment is to show that we can split the computational load among all the agents and still achieve performance similar to the centralized algorithm. We remark that (4.208) satisfies Assumption 4.9 so that the sample quantile converges to a stationary quantile. Note that by using an exponent of 1.01, the increment is slow though.

The parameters used in this first experiment are summarized in Table 4.1. We have tried to use similar parameters for all objectives, with as little tuning as possible. Nevertheless, it is clear that the percent of discarded samples (denoted $q$ ) is critical for the performance and should take into account the shape of the function, although the manner to accomplish this is not clear yet. The meaning of each column of the heading row of Table 4.1 is as follows:

- $M$ denotes the dimensionality of the objective.

- $X$ is the search space at the first iteration, and $[-50,50]^{M}=\prod_{m=1}^{M}[-50,50]$, i.e., a hyper-box with same length in every dimension.

- $q(\%)$ is the percent of discarded samples, i.e., each DCE agent takes floor $\left.\left[q S_{k, i} / 100\right]\right)$ elite samples for computing $\eta_{k, i}$ in (4.71), while the SACE agent takes floor $\left[q S_{i} / 100\right]$ elite samples.

- $\epsilon$ is the slope parameter of the smooth indicator function (4.15). 
Dejong 5 th, $J^{1)}$

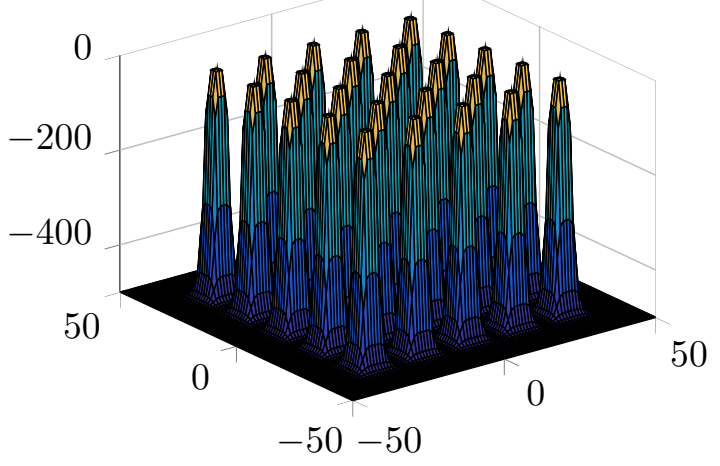

Zakharov, $J^{5)}$

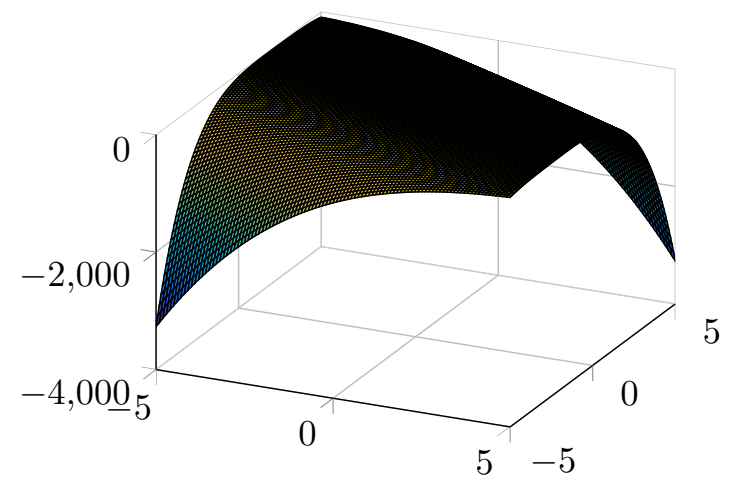

Ackley, $J^{6)}$

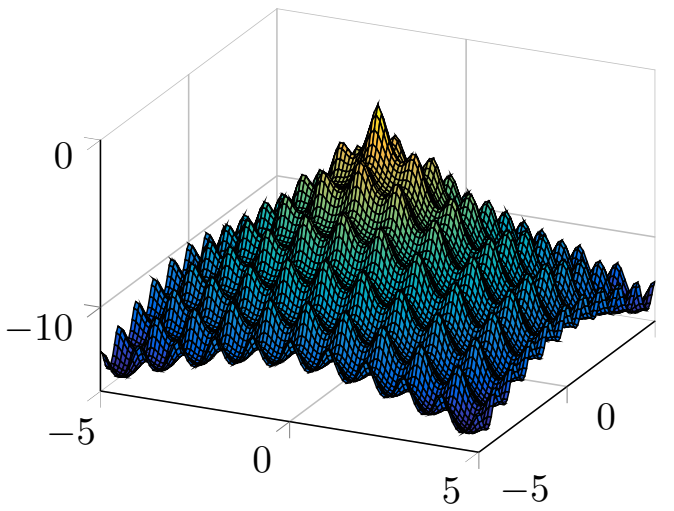

Rosenbrock, $J^{3)}$

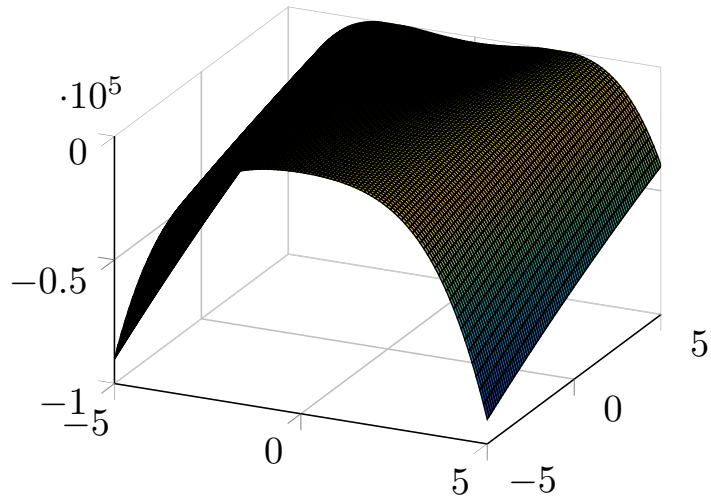

Rastrigin, $J^{4)}$

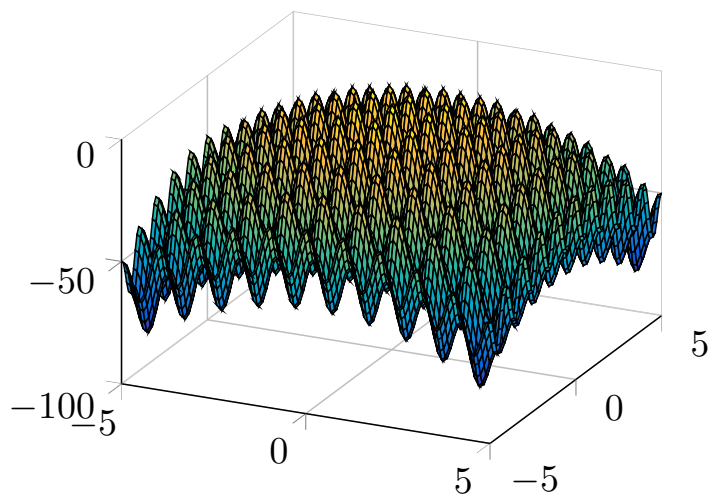

Pinter, $J^{10)}$

$5-100$

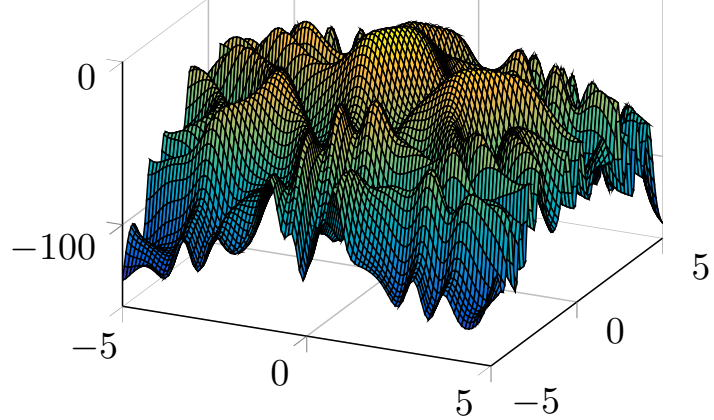

Figure 4.3 Illustration of the shape of some of the test objective functions for their first two dimensions, $x(1)$ and $x(2)$. 
Table 4.1 Simulation parameters for each objective function.

\begin{tabular}{|l|c|c|c|c|c|c|c|c|}
\hline Objective & $M$ & \multicolumn{1}{|c|}{ X } & $q(\%)$ & $\epsilon$ & $\rho$ & $S_{0}$ & $T$ & $S^{\text {total }}$ \\
\hline Shekel & 4 & {$[-50,50]^{M}$} & 90 & $10^{5}$ & $10^{-3}$ & 400 & 1000 & $6.1 \cdot 10^{5}$ \\
\hline Rosenbrock & 10 & {$[-50,50]^{M}$} & 99 & $10^{5}$ & $10^{-4}$ & 400 & 3000 & $4.93 \cdot 10^{6}$ \\
\hline Rastrigin & 30 & {$[-50,50]^{M}$} & 90 & $10^{5}$ & $10^{-9}$ & 400 & 1500 & $1.281 \cdot 10^{5}$ \\
\hline Zakharov & 30 & {$[-50,50]^{M}$} & 90 & $10^{5}$ & $10^{-9}$ & 400 & 1500 & $1.281 \cdot 10^{5}$ \\
\hline Ackley & 50 & {$[-50,50]^{M}$} & 90 & $10^{5}$ & $10^{-9}$ & 400 & 1500 & $1.281 \cdot 10^{5}$ \\
\hline Levy & 50 & {$[-50,50]^{M}$} & 90 & $10^{5}$ & $10^{-3}$ & 400 & 1000 & $6.1 \cdot 10^{5}$ \\
\hline Trigonometric & 50 & {$[-50,50]^{M}$} & 75 & $10^{5}$ & $10^{-3}$ & 400 & 2000 & $2.224 \cdot 10^{6}$ \\
\hline Griewank & 50 & {$[-50,50]^{M}$} & 90 & $10^{5}$ & $10^{-9}$ & 400 & 1000 & $6.1 \cdot 10^{5}$ \\
\hline Pinter & 50 & {$[-50,50]^{M}$} & 90 & $10^{5}$ & $10^{-9}$ & 400 & 1000 & $6.1 \cdot 10^{5}$ \\
\hline
\end{tabular}

- $\rho$ is some regularization noise added for convenience to avoid singular covariance matrices when drawing samples and improve numerical stability for the batch of simulations. Otherwise, if the algorithm converges to a very narrow distribution before getting to the maximum number of iterations, the routine for drawing samples from the multivariate Normal distribution can throw an exception. This is especially relevant for DCE, since the agents can draw fewer samples per iteration than the dimensionality of the objective. Nevertheless, since the accuracy of the solution can be limited by the order of the regularization noise, we have included this noise in the centralized too for the sake of fair comparison.

- $S_{0}$ is the initial number of samples for the SACE agent. For each DCE agent, the initial number of samples is given by: $S_{k, 0}=$ floor $\left[S_{0} / N\right]$.

- $T$ is the maximum number of iterations, i.e., $i=0, \ldots, T$.

- $S^{\text {total }} \triangleq \sum_{i=0}^{T} S_{i}$ is the total number of samples evaluated for each objective function.

In addition, the step-size sequence in this and the following experiments is given by:

$$
\alpha_{i}=\frac{2}{(i+100)^{0.501}}
$$


The network topology used in all simulations of this subsection is a regular graph of $N=20$ agents to show that large performance gains due to parallelization are possible. We recall that a regular graph has the property that all agents have the same number of neighbors [188]. Since $N=20$, each DCE agent draws just $S_{k, 0}=400 / 20=20$ samples for the first iteration and remains taking $S_{k, i}=S_{k, 0}=20$ samples for $i=0, \ldots, 377$ iterations. At iteration $i=1000$, the agents draw $S_{k, 1000}=53$ samples each, from which each one only takes 5 elite samples for building its Monte Carlo estimates. These are very low sample-sizes for building reliable Monte Carlo estimates for $M \in\{4,10,30,50\}$. Therefore, we have a clear tradeoff between the number of agents, $N$, which gives the performance gain due to parallelization; the sample rejection ratio, $\gamma$, which should be high to detect the elite samples; and the number of samples that each agent can use per iteration, $S_{k, i}$, which should be high enough for modeling the posterior distribution.

In addition, the number of neighbors also influence the quality of the Monte Carlo estimates, since by combining its neighbors' estimates, each agent can reduce the variance of its own estimate. A regular graph has the property that all agents have the same number of neighbors. To get an idea of the influence of the degree in the performance, we have run DCE in a set of networks, each one being a regular graph with $N=20$ agents and degree $\mathcal{N}_{k}=2, \ldots, 19$. We have tested the performance after convergence for the Trigonometric and Pinter functions, for two rejection ratio $\gamma \in\{0.75,0.9\}$ (the rest of parameters are given by Table 4.1). Figure 4.4 shows the worst-case (dashed red), average (solid black) and best-case (dotted blue) results averaged over 30 independent trials for each point. We can see that there is a trend such that the higher the degree, the more accurate results. In addition, the agents achieve near optimal performance for $\mathcal{N}_{k} \in\{5, \ldots, 19\}$, which supports the conclusion that DCE is robust for multiple degrees of connectivity. Based on these results, we have chosen $\mathcal{N}_{k}=8$ for the rest of experiments.

Now, we proceed to discuss the main result of this section, which consists in showing that the diffusion-form of cooperation embedded in the proposed DCE algorithm is able to combine the local estimates in an effective manner that matches the performance of the centralized solution. Figures 4.5-4.7 show the convergence of SACE (left column) and DCE (right column) with simulation parameters listed in Table 4.1. The graphs display the worst (solid top), average (dashed) and best (solid down) results over all simulations. The green area represents the area between the best and worst cases. For both SACE and DCE, the results in the figures have been averaged over 100 independent simulations in each case. In addition, for DCE, results have been averaged over all agents. Table 4.2 summarizes the figures with the number of experiments 

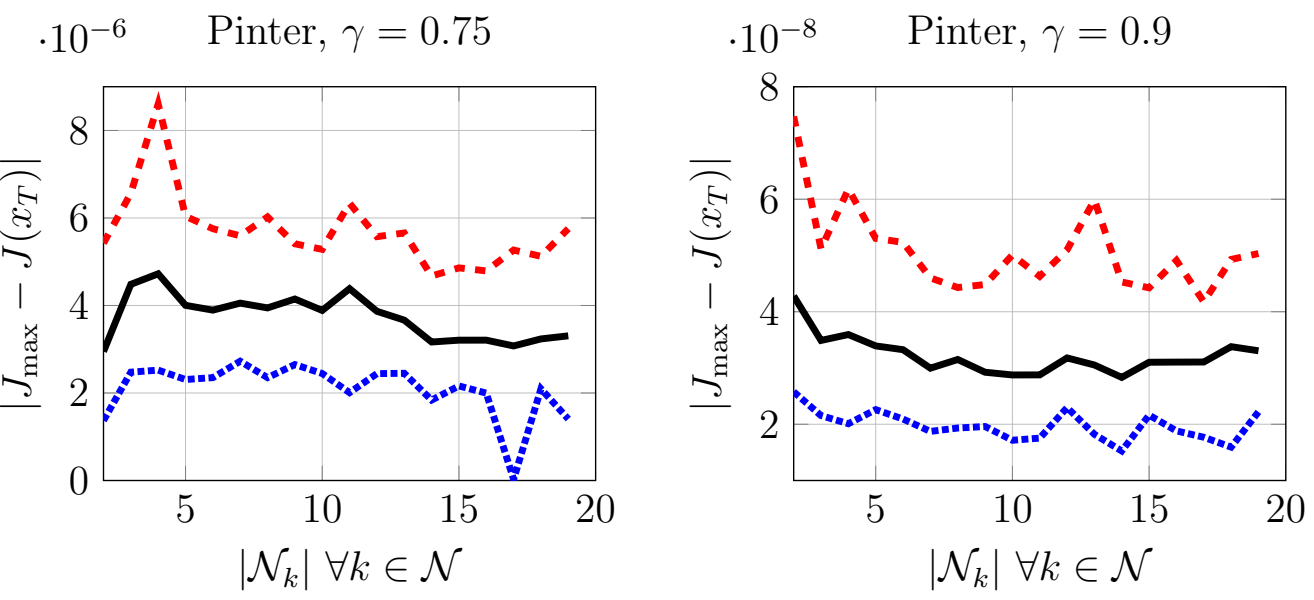

$\cdot 10^{-4}$ Trigonometric, $\gamma=0.75$

$$
\cdot 10^{-4} \text { Trigonometric, } \gamma=0.9
$$
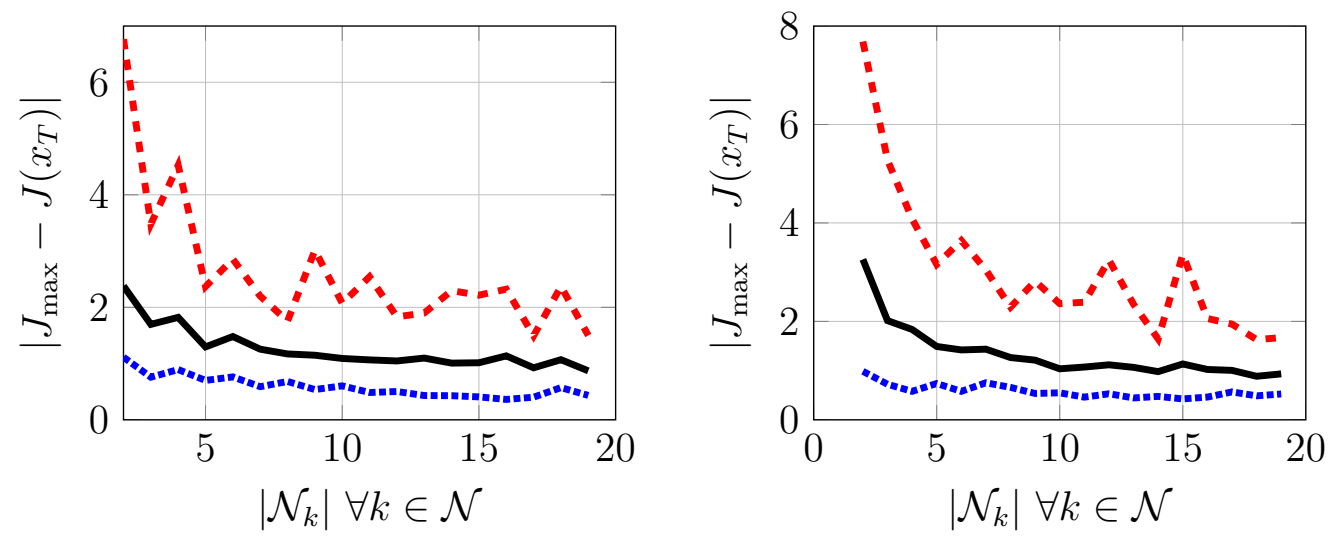

Figure 4.4 Influence of the degree on the performance of DCE. We can see worst-case (dashed red), average (solid black) and best-case (dotted blue) over 30 independent trials for each neighborhood size. 
Table 4.2 Simulation results for SACE and DCE with simulation parameters listed in Table 4.1. Results are in number of experiments over 100 where the convergence error was less than $\Delta$.

\begin{tabular}{|l|c|c|c|}
\hline & $\Delta$ & SACE (\%) & DCE (\%) \\
\hline Shekel & $10^{-3}$ & 100 & 100 \\
\hline Rosenbrock & $3 \cdot 10^{-3}$ & 100 & 100 \\
\hline Zakharov & $10^{-7}$ & 100 & 100 \\
\hline Rastrigin & $10^{-5}$ & 98 & 98 \\
\hline Ackley & $10^{-4}$ & 91 & 100 \\
\hline Levy & $10^{-3}$ & 100 & 100 \\
\hline Trigonometric & $10^{-3}$ & 100 & 100 \\
\hline Griewank & $10^{-7}$ & 100 & 100 \\
\hline Powell & $10^{-7}$ & 100 & 100 \\
\hline Pinter & $10^{-7}$ & 100 & 100 \\
\hline
\end{tabular}

over 100 independent trials where the convergence error was less than some threshold (denoted $\Delta$ ); the results in the DCE column were averaged over all agents. We can see that the results are practically equal for SACE and every agent running DCE, meaning that the proposed algorithm is able to split the computational load among the $N=20$ agents with no performance loss when tested over these objective functions. The only cost is associated to within neighborhood communication cost, which scales with the number of neighbors, rather than with the total number of agents. For Ackley (Figure 4.6-top), it seems that DCE even outperforms SACE. But the results of this table are not statistically significant. In Section 4.7.2, we explore this possibility (of DCE offering enhanced exploration with respect to SACE) further.

So far, we have avoided comparing the Dejong 5th function. The reason is that this function is more sensitive to the accuracy of the local estimators than the other objectives. Dejong 5th is very abrupt, with multiple peaks of local maxima that are close to the global maximum value. We have observed that a very high sample rejection ratio of about $q=99 \%$ is required to obtain high performance consistently. Since the agents take only one percent of elite samples, we require larger number of samples per iteration in order to build more reliable local Monte Carlo estimates.

Table 4.4 shows simulation results for Dejong 5 th function, $J^{1)}$, for multiple initial number of samples, $S_{0} \in\{400,600,800,1000\}$ and simulation parameters listed in 
SACE - Shekel, $J^{2)}$
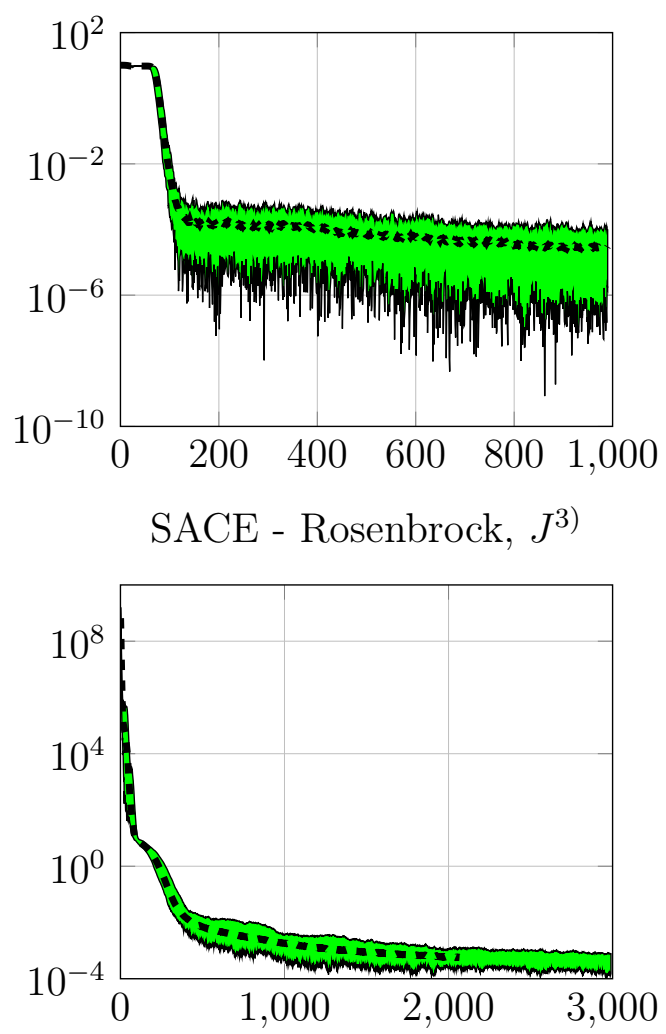

SACE - Zakharov, $J^{5)}$

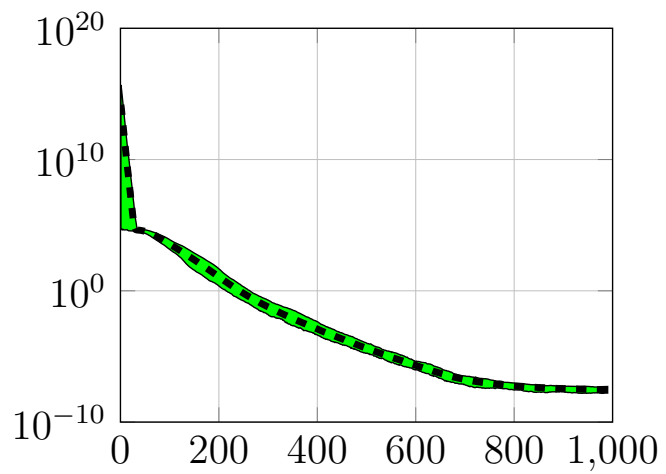

SACE - Rastrigin, $J^{4)}$

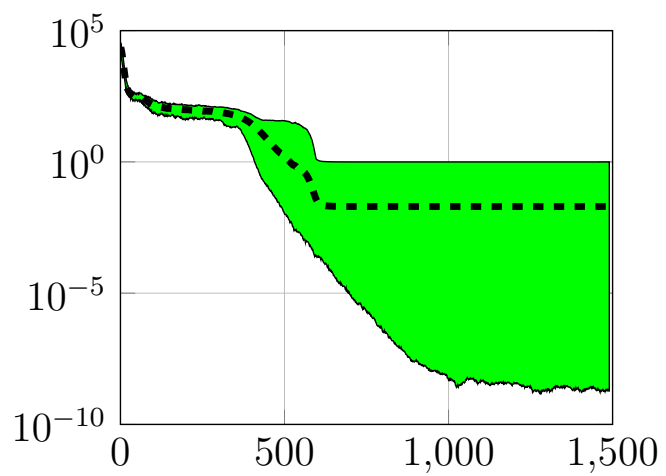

DCE - Shekel, $J^{2)}$
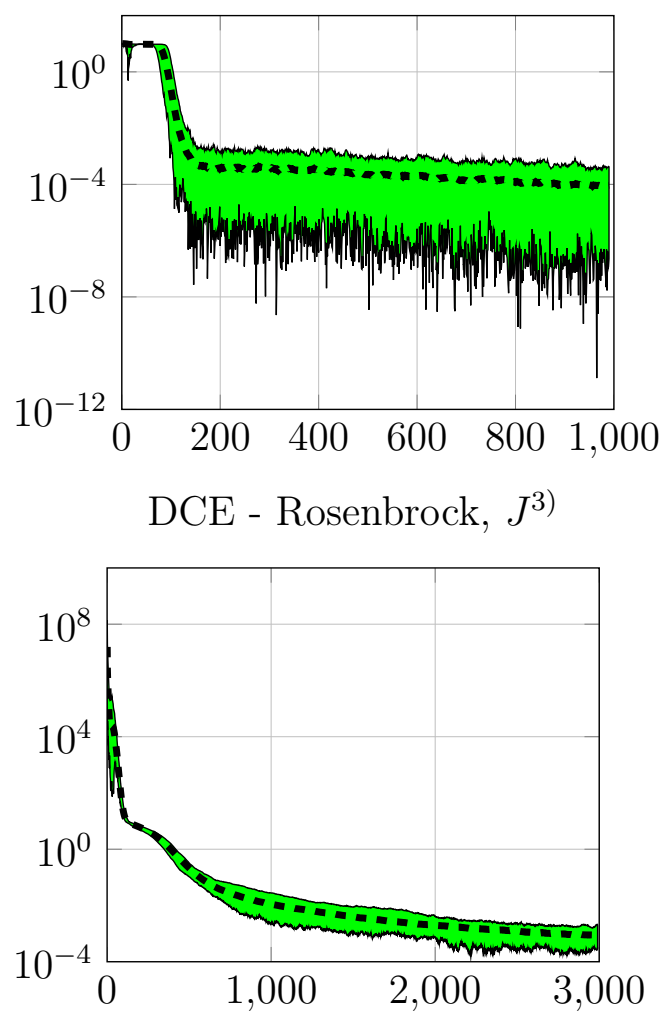

DCE - Zakharov, $J^{5)}$
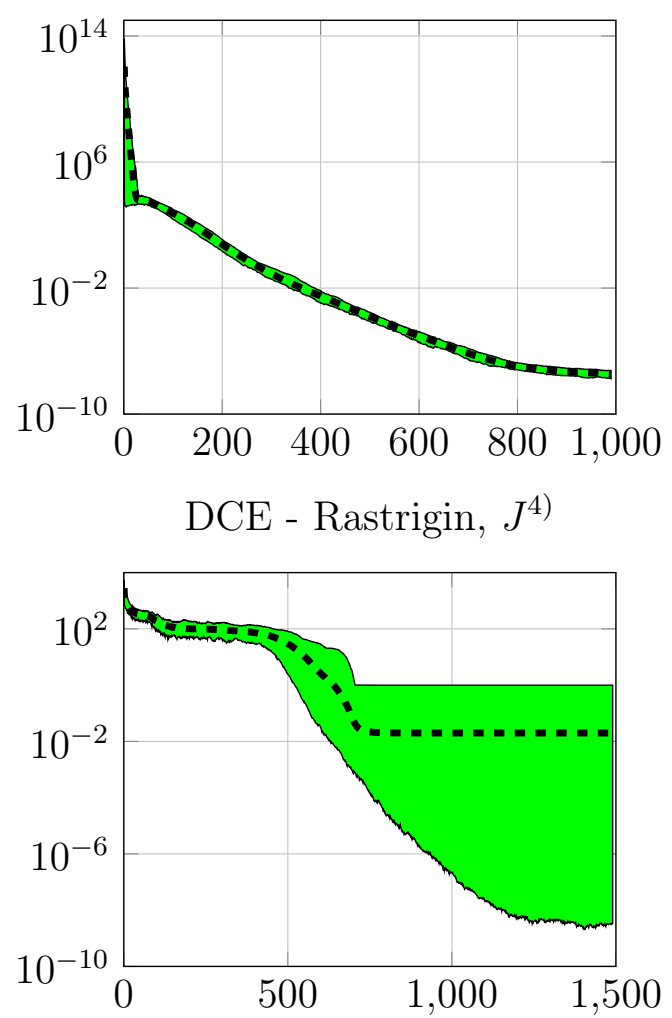

Figure 4.5 Convergence of SACE (left column) and DCE (right column) with simulation parameters listed in Table 4.1. The graphs display the worst (solid top), average (dashed) and best (solid down) results among all trials (and all agents for DCE). Results have been averaged over 200 independent trials (and over all agents for DCE) in each case. 
SACE - Ackley, $J^{6)}$
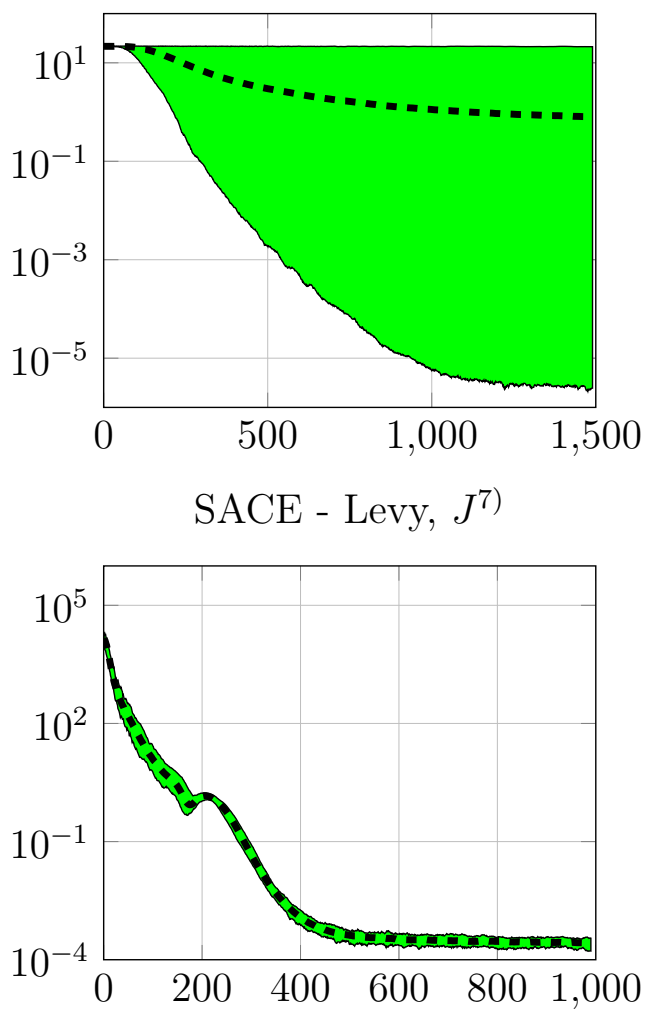

SACE - Trigonometric, $J^{8)}$
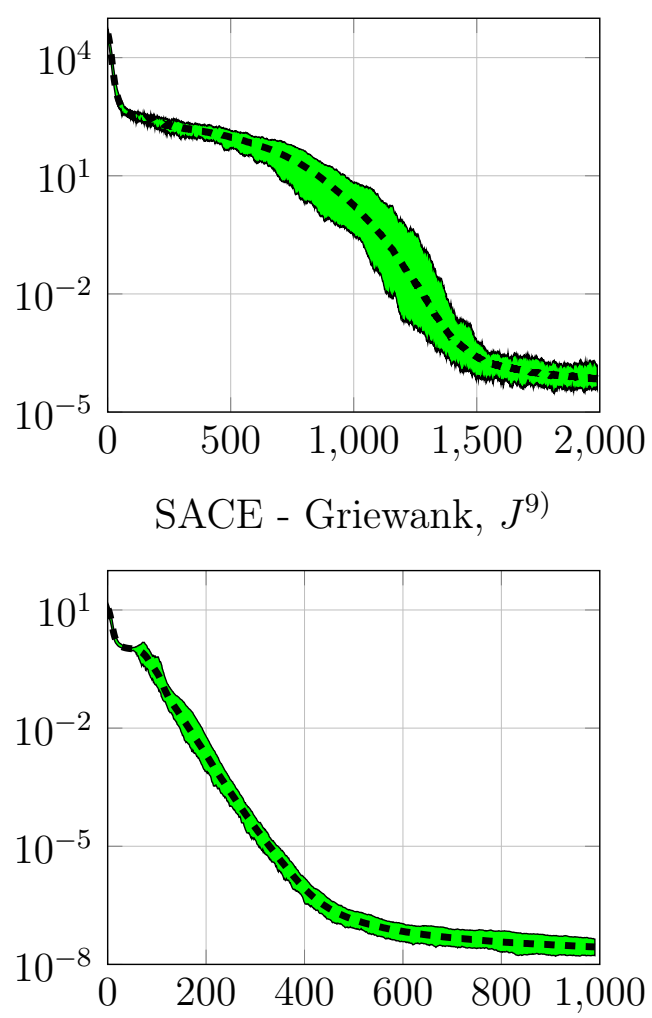

DCE - Ackley, $J^{6)}$
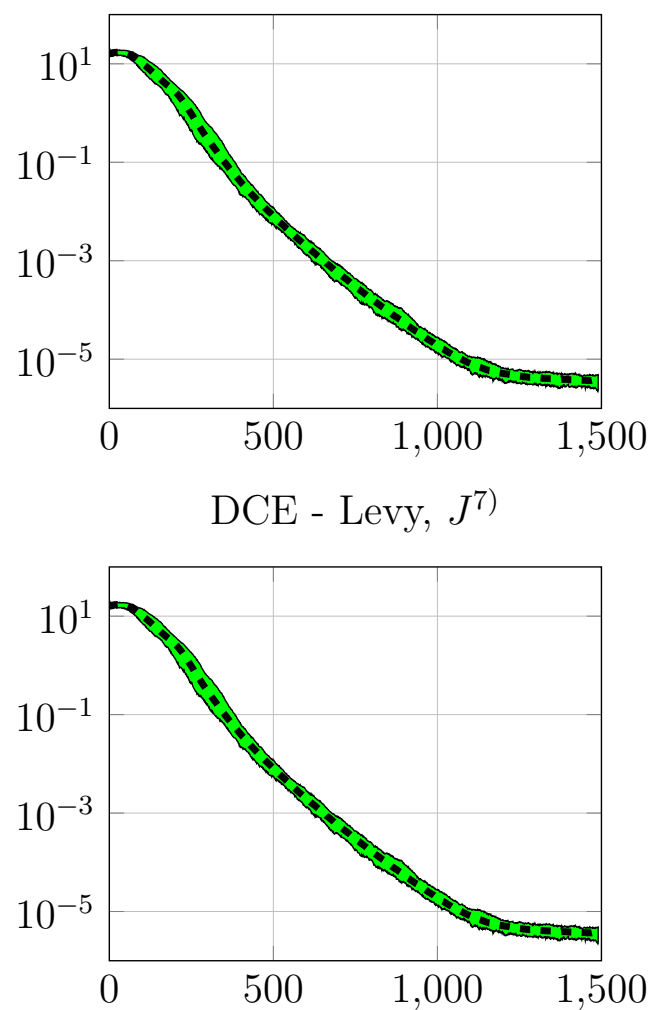

DCE - Trigonometric, $J^{8)}$
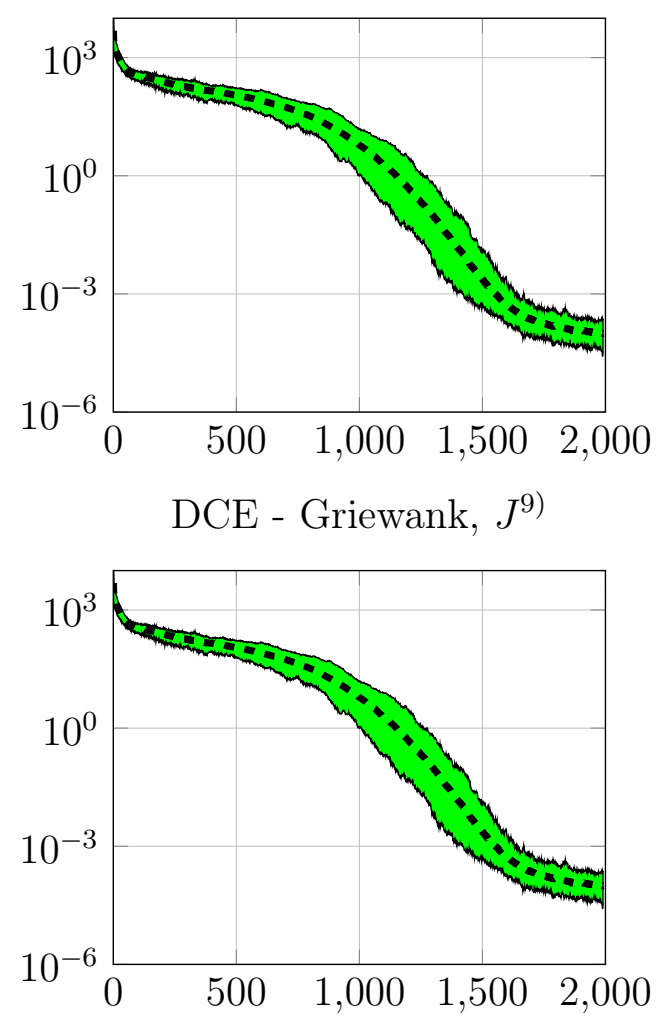

Figure 4.6 Continues Figure 4.5. 

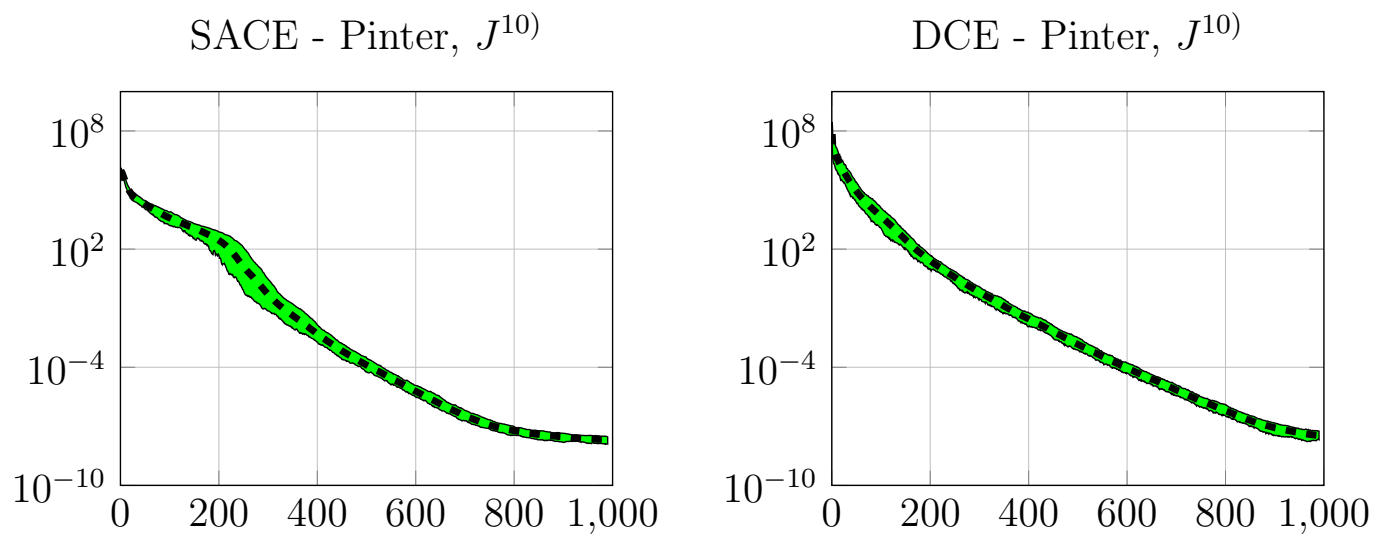

Figure 4.7 Continues Figure 4.6.

Table 4.3 Simulation parameters for Dejong 5th.

\begin{tabular}{|l|c|c|c|c|c|c|}
\hline Objective & $M$ & $\mathbb{X}$ & $q(\%)$ & $\epsilon$ & $\rho$ & $T$ \\
\hline Dejong 5th & 2 & {$[-50,50]^{M}$} & 99 & $10^{5}$ & $10^{-3}$ & 300 \\
\hline
\end{tabular}

Table 4.3, after convergence of SACE and DCE with different network topologies. The results are displayed as the percentage of trials that converged to an error smaller than $\Delta=10^{-8}$. First row includes results averaged over 200 independent trials for SACE algorithm. Second row, shows average results for $N=10$ agents running DCE over 4 random Euclidean graphs with average degree 4 (i.e., $\frac{1}{10} \sum_{k=1}^{10} \mathcal{N}_{k}=4$ ); we have repeated 50 independent trials for each graph, adding up to 200 trials in total. Third row, includes results for a set of $N=20$ connected with a regular graph of degree $\mathcal{N}_{k}=8$ (for all $k=1, \ldots, 20$ ), averaged over 200 independent trials. For $S_{0}=400$, we can see that even SACE cannot achieve perfect performance. For $N=10$, each agent has $S_{k, 0}=40$ samples, so that rejecting the $99 \%$ is not possible. In practice, at most, each agent can only reject $97.5 \%$ (39 out of 40) samples. For $N=20$ the situation is worse, having an effective $95 \%$ rejection ratio (19 out of 20). By increasing the number of samples per iteration, we see that the effective $q$ grows and the DCE agents eventually match the SACE performance. In addition, we have observed that when the agents failed to converge to the optimal solution, the DCE algorithm took longer to converge. 
Table 4.4 Percentage of trials that converged to an error smaller than $\Delta=10^{-8}$ for SACE and DCE (with multiple topologies) for Dejong 5th function, $J^{1 \text { ) }}$, and for multiple number of initial samples, $S_{0}$. Results have been averaged over 200 independent trials with simulation parameters listed in Table 4.3.

\begin{tabular}{|l|c|c|c|c|}
\hline & $S_{0}=400$ & $S_{0}=600$ & $S_{0}=800$ & $S_{0}=1000$ \\
\hline SACE & $98.5 \%$ & $100 \%$ & $100 \%$ & $100 \%$ \\
\hline DCE $N=10$ & $68.5 \%$ & $92.5 \%$ & $100 \%$ & $100 \%$ \\
\hline DCE $N=20$ & $1.5 \%$ & $62.5 \%$ & $92 \%$ & $99 \%$ \\
\hline
\end{tabular}

\subsubsection{Enhanced exploration of DCE}

In Chapter 3, we have seen that a set of networked agents can use a distributed algorithm to fuse their learning experience in a form that increased their exploratory capabilities, even beyond the original single-agent algorithm. For instance, recall from (3.135) that the agents were able to approximate the on-policy solution, while a single-agent could not. Similar to previous chapter, we are interested in testing whether the DCE agents have novel exploratory capabilities, not present in the SACE agent.

One main challenge for particle-filter algorithms, in general, is dealing with high dimensional distributions, since the number of samples required to approximate the distributions grow exponentially with the dimensionality (see, e.g., [149, 233, 33, 77] for recent discussions on this problem). Fortunately, we have seen that for our optimization setting, using a stochastic approximation to smooth the posterior update turns to be an effective approach for surmounting this problem. Nevertheless, we assume that there might be a limit for the effectiveness of such approach.

In this section, we test the behavior of SACE and DCE for Ackley, Rastrigin and Pinter objective functions, when $M=100$ (the rest of simulation parameters are listed in Table 4.5). We could see in Table 4.2 that SACE was not able to achieve the desired performance threshold in $100 \%$ of the cases for Rastrigin and Ackley functions, so that we aim to perform more extensive tests with these two functions. In addition, we have included the Pinter function in this experiment since it has been typically considered one of the most difficult objectives for large $M$ (e.g., references [127, 128, 130, 341] only considered $M$ equal to 20 or 50 ). We have chosen a regular graph of $N=80$ agents with degree $\mathcal{N}_{k}=25$ (for all $k=1, \ldots, 80$ ).

Figure 4.8 and Table 4.6 show the results of this experiment. For Rastrigin function, SACE performs badly, being able to achieve an error smaller than $\Delta=10^{-3}$ in just $2.5 \%$ of the trials; DCE does not perform well either, but it outperforms SACE, being 
Table 4.5 Simulation parameters for $M=100$-dimensional problems.

\begin{tabular}{|l|c|c|c|c|c|c|c|c|}
\hline Objective & $M$ & $\mathbb{X}$ & $q(\%)$ & $\epsilon$ & $\rho$ & $S_{0}$ & $T$ & $S^{\text {total }}$ \\
\hline Rastrigin & 100 & {$[-50,50]^{M}$} & 90 & $10^{5}$ & $10^{-9}$ & 1000 & 1700 & $2.02 \cdot 10^{6}$ \\
\hline Ackley & 100 & {$[-50,50]^{M}$} & 90 & $10^{5}$ & $10^{-9}$ & 1000 & 2000 & $2.67 \cdot 10^{6}$ \\
\hline Pinter & 100 & {$[-50,50]^{M}$} & 90 & $10^{5}$ & $10^{-9}$ & 1000 & 1000 & $10^{6}$ \\
\hline
\end{tabular}

Table 4.6 Percentage of trials that converged to an error smaller than $\Delta=10^{-3}$ for SACE and DCE for $M=100$-dimensional problems. Results have been averaged over 200 independent trials with simulation parameters listed in Table 4.5.

\begin{tabular}{|l|c|c|c|c|}
\hline & SACE & DCE & Difference & $95 \%$ CI \\
\hline Rastrigin & $2.5 \%$ & $20.13 \%$ & 17.63 & $11.67-23.59$ \\
\hline Ackley & $80 \%$ & $100 \%$ & 20 & $14.46-25.54$ \\
\hline Pinter & $100 \%$ & $100 \%$ & 0 & - \\
\hline
\end{tabular}

able to achieve the desired accuracy in $20.13 \%$ of the trials. Interestingly, we have observed that when SACE converges to the global solution, it does faster than DCE, achieving a lower minimum error of $10^{-6}$; while DCE achieves $10^{-3}$ more consistently. For Ackley function, although SACE performs well in $80 \%$ of the cases, it is again outperformed by DCE, which achieves a $100 \%$ success. For Pinter function, both algorithms are able to achieve $100 \%$ success. Again, we observe that SACE converges to a smaller error, but in this case it seems a matter of convergence rate, being SACE faster than DCE (it is known that centralized stochastic gradient descent converge faster than diffusion-based gradient descent with constant step-size for strongly convex problems [246], so we may be observing a similar effect here). Results have been averaged over 200 independent trials and we can conclude that are statistical significant (see, e.g., [191, Sec. 9.3]).

The results discussed in this section seem very promising and support the idea that DCE is able to perform well even when SACE is not. We conjecture that this improvement is due to better exploratory capabilities of DCE. We provide some arguments supporting such idea in the following section. 

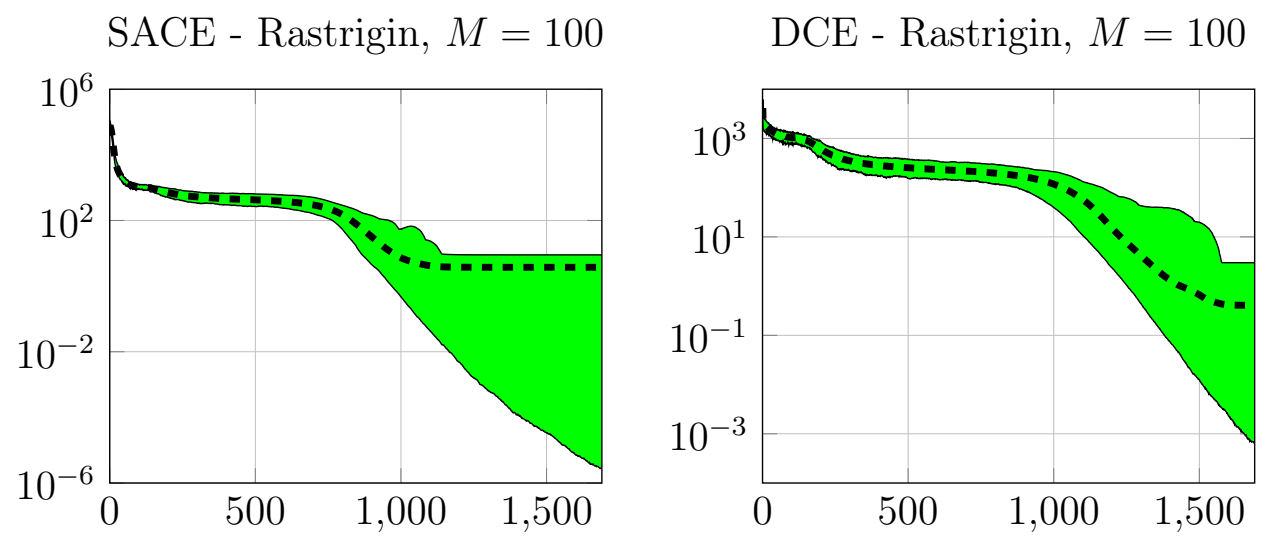

SACE - Ackley, $M=100$

DCE - Ackley, $M=100$
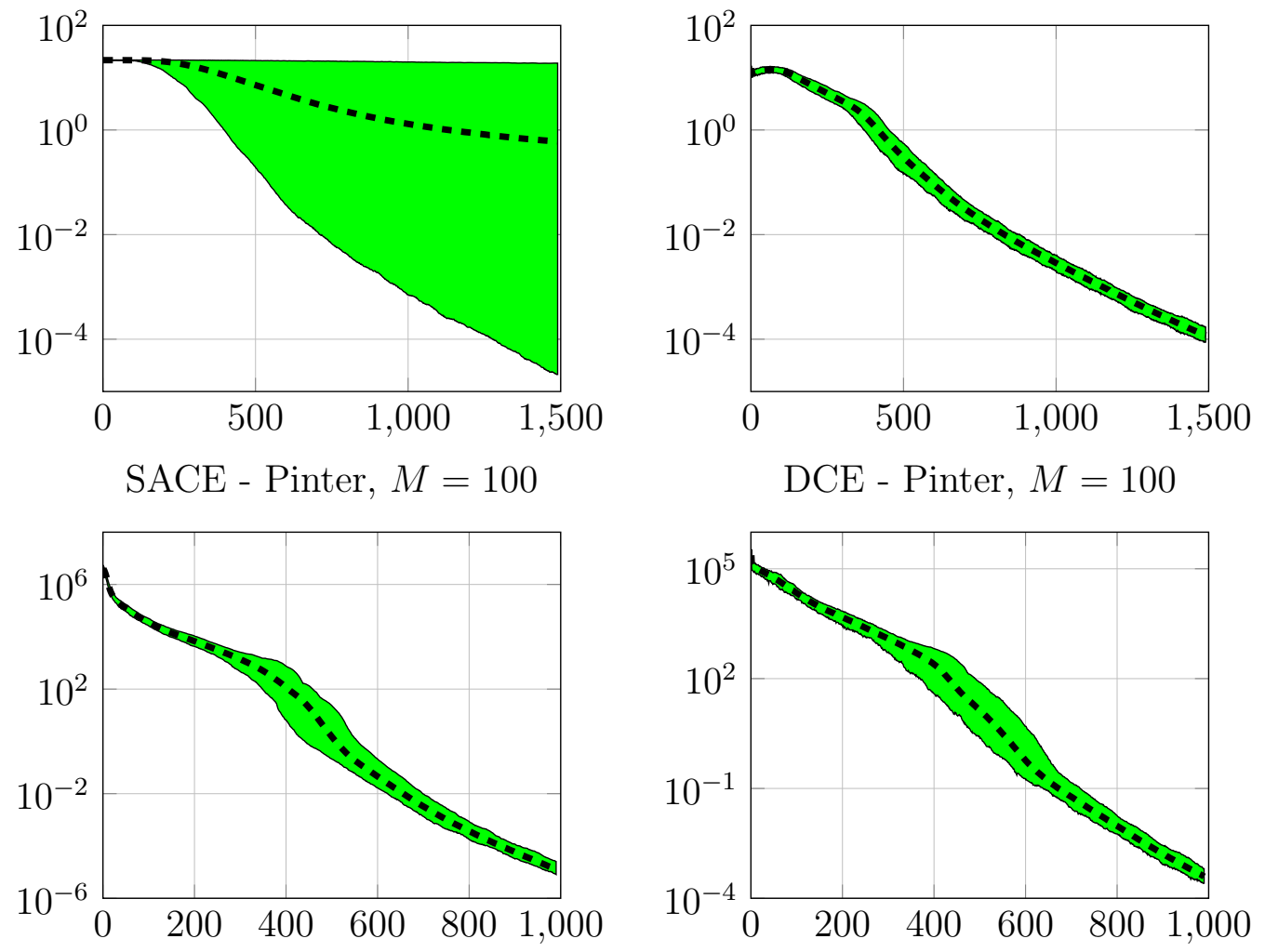

Figure 4.8 Convergence for $M$-dimensional Rastrigin, Ackley and Pinter functions. Similar to Figures 4.5-4.7, the graphs display the worst (solid top), average (dashed) and best (solid down) results among all trials (and all agents for DCE). Results have been averaged over 200 independent trials in each case. For DCE, results have been also averaged over all agents. 


\subsection{Discussion on the robustness of DCE}

In numerical experiments of Section 4.7.2, we have seen that DCE is able to outperform SACE when the problem dimensionality grows. In particular, we have observed that DCE is more robust against local maximum. In this section we provide some arguments supporting the idea that this is actually the expected behavior. We approach the comparison from two points of view: i) explaining the origin of the Monte Carlo approximation error and ii) looking at the algorithms as performing stochastic gradient ascent.

\subsubsection{Monte Carlo approximation error}

Consider a scenario where a network of $N$ agents perform DCE and each agent evaluates $S_{k, i}$ samples at each iteration $i$. Consider also another scenario where a single agent performing DCE evaluates $S_{i}=\sum_{k=1}^{N} S_{k, i}$ samples at each iteration. From Lemma 4.1 , we know that the sample quantile of both the single agent and the network will converge to the true quantile of their respective asymptotic distributions of output values resulting from evaluating candidate solutions drawn from some asymptotic sampling distribution. In the single agent case, the sampling distribution will follow a single path before concentrating its density on some region. This path depends on the initial statistics $\theta_{0}$ and on the Monte Carlo noise. Now consider that the network of agents is initialized with a different $\theta_{k, 0}$ each. Then, the agents will follow different paths each before convergence to the same asymptotic sampling distribution. Thus, the agents will be able to sample from different regions of the solution space - think in a multimodal distribution, as opposed to the unimodal single-agent distribution - being more likely that they approximate the true quantile.

\subsubsection{Gradient ascent on the space of distributions}

The DCE algorithm offers three important features for nonconvex optimization, namely i) sequence of subproblems with increasing difficulty, ii) swarm exploration and iii) enhanced robustness against sample noise.

The first feature is inherited from the single-agent algorithm introduced by [128]. Note that (4.60a)-(4.60b) performs a stochastic approximation in the space of sufficient statistics. If the sampling distribution is initialized with high variance, we are averaging over a large portion of the search space, hence, the model-based objective, $\mathbb{E}_{f_{w}}[J(\boldsymbol{x})]$, will be almost flat. The smaller the variance of the sampling distribution, the less 
smoothing of the objective. Hence, by assigning a small step-size $\alpha_{i}$ to the filter update, the real shape of the deterministic objective $J$ will be slowly revealed, giving time to the estimates to ascend through its envelope, avoiding local peaks (see Figure 4.9). In other words, the Cross Entropy method solves a sequence of subproblems that result from smoothing the objective with a sequence of sampling distributions. It starts from a smooth, with very few extrema subproblem, for which it is easy to find the region around its global maximum (under some conditions, large smoothing leads to a convex function [171]). Then, the optimum of each subproblem is used as starting point of the next subproblem. We find this property very related to continuation or graduated optimization methods [119, 194].
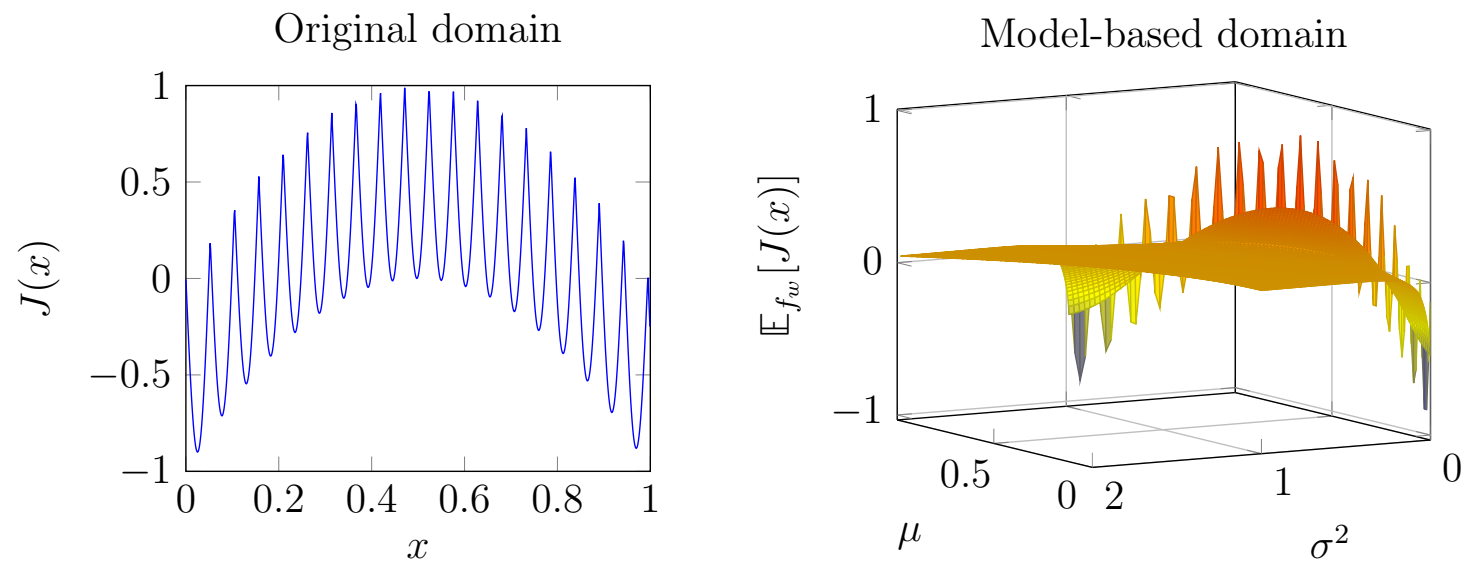

Figure 4.9 Original vs. model-based domain. The left side shows the Garland function $J(x)$, which has multiple extrema such that algorithm prone to get trapped in local minima. The right side shows the model-based function $\mathbb{E}_{f_{w}}[J(x)]$ for a Normal surrogate sampling distribution $f_{w}(\cdot)=\mathcal{N}\left(\cdot ; \mu, \sigma^{2}\right)$. Note that the bigger the variance, the smoother the mode-based objective.

The second feature is the enhanced exploration capabilities of a swarm of agents. Local search algorithms (e.g., SPSA [267, Ch. 7], [99] or simulated annealing [267, Ch. 8]) usually get stuck in a local optimum when applied to nonconvex problems, especially when their parameters are tuned for fast convergence. A common approach to escape from a local optimum is to restart the algorithm when no improvement is observed [183], starting every time from a different initial condition. Another approach is to start multiple instances of the local search algorithm and allocate computational resources (e.g., number of samples evaluated) to the instances depending on their behavior $[112,126]$. DCE proposes a novel approach, where each agent starts at a different initial condition and run an instance of the local search algorithm, then the 
multiple instances are combined at every iteration in a manner that allows them to explore the solution space efficiently.

In order to understand this form of swarm exploration, let us think first in a single agent maximizing the deterministic objective $J$ with gradient ascent. Suppose $J$ is multi-extremal with a set of $C$ local maxima $\left\{x_{c}^{\text {loc }}\right\}_{c=1}^{C}$, such that

$$
\nabla_{w} J\left(x_{c}^{\mathrm{loc}}\right)=0, \quad c=1, \ldots, C
$$

Introduce the attracting region of a local maximum, as the connected set that contains such local maximum as unique critical point (it includes neither saddle points, nor any other extrema). Let $\mathbb{A}_{c}$ be the attracting region of $x_{c}^{\text {loc }}$. Then, we have

$$
x_{c}^{\mathrm{loc}}=\arg \max _{x \in \mathbb{A}_{c}} J(x)
$$

Depending on the initial condition, each agent will lie in one of these attracting regions, and will converge to the local maximum without being able to explore any point beyond the attractive region. Indeed, this situation is equivalent to the agent maximizing an objective, $J_{c}^{\mathrm{loc}}(x)$, given by

$$
J_{c}^{\mathrm{loc}}(x)= \begin{cases}J(x) & \text { if } x \in \mathbb{A}_{c} \\ -\infty & \text { otherwise }\end{cases}
$$

such that $x_{c}^{\text {loc }}$ is the global maximum:

$$
x_{c}^{\mathrm{loc}}=\arg \max _{x \in \mathbb{K}} J_{c}^{\mathrm{loc}}(x)
$$

and such that $J_{c}^{\text {loc }}(x)=J(x)$ if $x \in \mathbb{A}_{c}$ and $J_{c}^{\text {loc }}\left(x^{\prime}\right)<J^{\text {loc }}(x) \forall x^{\prime} \in \mathbb{X} \backslash \mathbb{A}_{c}, \forall x \in \mathbb{A}_{c}$.

Now, consider a network of agents, each one seeking to minimize its own strongly convex objective, with different solution each. Reference [57] showed that when the agents run a diffusion gradient descent algorithm, under some conditions, they will find the point that optimizes the weighted sum of their objectives, with weights given by the left eigenvector of the combination matrix $A$ associated with its unique unit eigenvalue. Our case is a-priori different from the problem studied by [57], since all agents aim to optimize a nonconvex objective. Depending on their initial conditions, the local adaptation step (4.60a) will push each agent toward its own local maximum. Thus, similar to (4.213), when, at time $i$, one agent $k$ is attracted by some maximum, we can think that it is maximizing its own quasiconcave objective function $J_{k, i}^{\text {loc }}(x)$ with global 
maximum $x_{k, i}^{\text {loc }}$ (we remark that these local objectives are actually equal to the objective in the attractive regions where they are defined). However, the diffusion step (4.60b) will pull each agent toward a combination of its neighbors' ascent directions. Hence, we can say that the network aims to solve the following multi-objective optimization problem:

$$
\underset{x \in \mathcal{K}}{\operatorname{maximize}}\left(J_{1, i}^{\mathrm{loc}}(x), \ldots, J_{N, i}^{\mathrm{loc}}(x)\right)
$$

Suppose that the locally attractive objectives, $J_{k, i}^{\text {loc }}(x)$, are strongly concave. Hence, we can use the result from [57] to state that the agents will maximize the weighted sum of their objectives:

$$
\underset{x \in \mathfrak{K}}{\operatorname{maximize}} \sum_{k=1}^{N} \tau_{k} J_{k, i}^{\mathrm{loc}}(x)
$$

where $\tau=\left(\tau_{k}\right)_{k=1}^{N}$ is the weight vector such that $C^{\top} \tau=\tau$. Problem (4.215) is the scalarization of the multi-objective problem (4.214). Therefore, the solution to (4.215) is a Pareto optimal point for (4.214) (see, e.g., [48, Ch. 4.7]). This means that the agents will converge to a Pareto optimal point of their local objectives, being able to escape from their attractive local maxima. Note also that when some agent escapes from its local maximum, it will likely enter into the attracting region of another maximum. In other words, each agent's attracting local maximum changes with time. Moreover, they can find new local maxima that were not in their initial attractive regions. Indeed, we have observed in numerical experiments (see Section 4.7) that DCE achieves the global maximum more consistently than a (centralized) single-agent algorithm that takes as many samples per iteration as the whole network of agents (i.e., $S_{\text {cent }, i}=\sum_{k=1}^{N} S_{k, i}$ ).

The third feature consists in being more robust against Monte Carlo noise. Even though the agents perform the Robbins-Monro update (4.60a) locally (without exchanging any data), and estimates are only exchanged among neighbors during the combination step (4.60b), the information is diffused in a manner that allows them to learn from every other agent in the network. In particular, in Section 4.6, we show conditions for the agents to asymptotically approximate (4.55). 


\subsection{Conclusion and Future Work}

We have introduced a diffusion-based $\mathrm{CE}$ algorithm where a network of agents cooperate for optimizing an unknown nonconvex function. We developed a detailed convergence analysis, showing convergence of the distributed smoothed Bayesian update to the optimal distribution, and providing conditions that ensure almost sure convergence of DCE. Numerical experiments have shown that the diffusion-form of cooperation embedded in the proposed DCE algorithm is usually able to combine the local estimates in an effective manner that matches - and sometimes even improve - the performance of the centralized solution.

We could use the DCE for policy search in the space of parametric policies. In particular it would be interesting to seek the policy parameter from a single stream of experience, using the distributed off-policy DGTD algorithm presented in Chapter 3. The idea would consist in having a network of DCE agents searching the parameter in the policy space that maximizes the value function, such that each DCE agent has to evaluate multiple policies. Then, we could connected a network of DGTD agents to each DCE agent, in two different layers, such that each DGTD network evaluates the policy parameters required by its corresponding DCE agent.

We have argued that the robustness against local maximum is caused by some enhanced exploratory capabilities of the networked solution, not present in the singleagent case. Nevertheless, it would be desirable to guide the agents towards the most promising regions. For a strongly connected topology and a given $\tau$, it is possible to build a matrix $C$ such that $C^{\top} \tau=\tau$. It would be interesting to find a sequence of meaningful weight vectors $\left\{\tau_{i}\right\}_{i=1}^{\infty}$ that effectively guide the agents to the higher maximum.

Our analysis shows that the ideal BFO converges to a distribution that satisfies (4.6). It turns out that there may be several (or even infinite) of such optimal distributions. It would be interesting to study if searching for a particular solution could be beneficial. For instance: Could searching for a high variance optimal distribution benefit the exploration stage? Could a low variance distribution improve convergence rate? Which regularization should be included in each case? Responding these questions could lead to new variants of the DCE algorithm, hopefully with improved performance.

In addition, there are several techniques that improve the performance of vanilla stochastic gradient descent, like acceleration and momentum (see, e.g., [204, 230, 44] or adaptive step-size (see, e.g.., $[83,146])$. It would be interesting to try these techniques in our simple damping scheme. 
We believe the connection of DCE and, more generally the particle filter optimization framework with graduated optimization is novel and should be explored further. In particular, strong theoretical guarantees (e.g., finite time performance bounds) have been obtained for graduated optimization methods [194, 119], and similar ideas could be useful for Cross Entropy.

This chapter finishes our journey on cooperative algorithms. 



\section{Chapter 5}

\section{Dynamic potential games with constraints}

\subsection{Introduction}

Game theory is a field of mathematics that studies conflict and cooperation between intelligent decision makers (also known as players or more generally as agents) when the strategies followed by the agents influence each other (see, e.g., [113] and references therein). It has become a useful tool for modeling many engineering problems. In particular, we are interested in communication and networking problems, such as power control and resource sharing (see, e.g.,[257]). wherein the decision made by any individual user affect others' performance. However, one main assumption of classic game theory is that the users operate in a static environment, which is not influenced by the users' actions. This assumption is unrealistic in many communication and networking problems. For instance, wireless devices have to maximize throughput while facing time-varying fading channels, and mobile devices may have to control their transmitter power while saving their battery level. These time-varying scenarios can be better modeled by dynamic games.

In a noncooperative dynamic game, the agents compete in a time-varying environment, which we assume can be characterized by a discrete-time dynamical system equipped with a set of states and a state-transition equation. These state-transitions can be deterministic or stochastic. Each agent has its reward function, which depends on the current state of the system and the agents' current actions. Both the state and action sets might be subject to constraints, which is an important feature, since constraints are ubiquity in real physical systems; for instance, in wireless communications, the battery (state) must be non-negative and the maximum transmitter power 
(action) is upper-bounded. Moreover, constraints could be coupled among players; for instance, in networking, the users aim to maximize their own throughput, but the sum of all the data from all users that flow through a router cannot exceed the maximum capacity allowed by such router. We consider the general case wherein rewards, state-transition function and constraints can be nonstationary. A dynamic game starts at an initial state. Then, the agents take some action, based on the current state of the game, and receive some reward values. Then, the game moves to another state. This sequence of state-transitions is repeated at every time step over a (possibly) infinite time horizon. We consider the case in which the aim of each agent is to find the strategy (i.e., sequence of actions that could depend on time or on the system state) that maximizes its long term cumulative reward, given other agents' strategies. Thus, a game can be represented as a set of coupled optimal-control-problems (OCPs). In other words, an infinite-horizon dynamic game can be seen as a multiobjective OCP, which is difficult to solve in general.

Fortunately, there is a class of dynamic games, named dynamic potential games (DPGs), that can be efficiently solved through a related OCP. Thus, the benefit of a DPG is that solving a single OCP is generally simpler than solving a set of coupled OCPs (see [122] for a recent survey on DPGs). In other words, the potentiality condition of DPGs allows us to solve the multiobjective OCP through a related single-objective OCP.

The pioneering work in the field of DPGs is that of [68], later extended by [69] and [70]. There have been two main approaches to study DPGs: the Euler-Lagrange equations and the Pontryagin's maximum (or minimum) principle. Recent analysis by [122] and [105] used the Euler-Lagrange with DPGs in its reduced form, that is when it is possible to isolate the action from the state-transition equation, so that the action is expressed as a function of the current and future (i.e., after transition) states. Consider, for example, that the future state is linear in the current action; then, it is easy to invert the state-transition function and rewrite the problem in reduced form, with the action expressed as a linear function of the current and future states. However, in many cases, it is not possible to find such reduced form of the game (i.e., we cannot isolate the action) because the state-transition function is not invertible (e.g., when the state transition function is quadratic in the action variable). The more general case of DPGs in nonreduced form was studied with the Pontryagin's maximum principle approach by [69] and [81] for discrete and continuous time models, respectively. However, in all these studies [122, 68-70, 105, 81], the games have been analyzed without explicitly considering constraints for the state and action sets. 
Other works that consider potential games with state-dynamics include $[181,163$, 164]. However, these references study the myopic problem in which the agents aim to maximize their immediate reward. This is different from DPGs, where the agents aim to maximize their long term reward.

OCPs are usually analyzed from two different approaches: open loop (OL) or closed loop (CL). In the OL analysis, we seek an optimal sequence of actions that will be executed in order without any feedback, i.e., the action is a function of time. In a CL approach, we seek a mapping from states to actions, which we refer as feedback policy or simply policy, and the optimal action is obtained as a function of the current state. For deterministic systems, both OL and CL solutions can be optimal. But for stochastic systems, any OL precomputed sequence of actions is unlikely to be optimal. Thus, CL are usually preferred over OL solutions.

For dynamic games, the situation is more involved even for deterministic systems, since OL and CL refer to the information available to the agents for making their decisions (see, e.g., [15]). In an OL dynamic game, agents' strategies depend on time only, so that they compete with predefined action sequences to obtain an equilibrium trajectory in the state-reward space, so that no agent has incentive to change its action sequence unilaterally. In a CL dynamic game, agents' strategies depend on the current state variable, so that they have to consider how their opponents would react to deviations from the equilibrium trajectory that they have followed so far, i.e., a CL equilibrium can be seen as a tree of trajectories. Hence, the sets of OL and CL Nash equilibria are generally different even for deterministic systems (see, e.g., [156, 93, 317]). The OL analysis of dynamic games has, in general, more tractable analysis than the CL analysis. Indeed, there are only few CL known solutions for simple games, such as the fish war example presented in [4], oligopolistic Cournot games [78] or quadratic games [156].

The main theoretical contribution of this chapter is to analyze deterministic and stochastic DPGs with constrained state-action sets. We start by an OL analysis of deterministic DPG, then we extend the OL results to stochastic DPG and, finally, we provide a simplified CL analysis of stochastic DPG, where we assume that the CL strategies can be expressed as smooth functions of some parameter that lies in a finite-dimensional real vector space. Up to the best of our knowledge, this is the first time that a practical method is proposed to approximate CL equilibrium in potential games with continuous state-action sets. The key idea underlying these analysis is to apply the Euler-Lagrange equation to the Lagrangian (as it is customary in the OCPs literature [242]), rather than to the reward function (as done by earlier works [122] 
and [105]). Using the Lagrangian, we can formulate the optimality condition in the general nonreduced form (i.e., it is not necessary to isolate the action in the transition equation). In addition, we establish the existence of a suitable conservative vector field as an easily verifiable condition for a dynamic game to be of the potential type. This is an extension of the conditions established for static games by [264] and [196]. We remark the importance of dealing with constraints, as they are required for many applications (e.g., in a network flow problem, the aggregated throughput of multiple users is bounded by the maximum link capacity; or in cognitive radio, the aggregated power of all secondary users is bounded by the maximum interference allowed by the primary users). We also remark the relevance of including a CL analysis, since CL policies can yield optimal performance in stochastic settings.

The second main contribution of this chapter is to show that the proposed framework can be applied to several communication and networking problems in a unified manner. We present four examples with increasing complexity level. First, we propose an optimal network flow problem, in which there are two levels of relay nodes equipped with finite batteries. The users aim to maximize their flow while optimizing the use of the relay batteries. This problem illustrates that, when the reward functions have some separable form, it is straightforward to establish that the problem is a DPG. However, the analytical solution for this problem is unknown and we have to solve it numerically. It turns out that, since all batteries will eventually deplete, the game will get stuck in this depletion-state. Hence, we can approximate the infinite-horizon OCP by an effective finite-horizon problem, which simplifies the numerical computation. The second example is an uplink multiple access channel wherein the users' devices are also equipped with batteries (this example was introduced by reference [334]). Again, the simple - but more realistic - extension of battery-usage optimization makes the game dynamic. In this example, instead of rewriting the reward functions in a separable form, we perform a general analysis to establish that the problem is a DPG. The third example studies two decentralized scheduling problems: proportional fair and equal rate scheduling, where multiple users share a time-varying channel. This example shows how to use the proposed framework in its most general form: the problems are nonconcave and nonstationary, with state-transition equation changing with time; and there is no reason that justifies a finite horizon approximation of the problem, so we have to use optimal control methods (e.g., dynamic programming) to solve it numerically.

The outline of this chapter is as follows. Sec. 5.2 introduces the problem setting for dynamic games, its solution concept and the assumptions on which we base our analysis. Sec. 5.3 reviews deterministic static potential games together with the 
instrumental notion of conservative vector field. Sec. 5.4 provides sufficient conditions for a deterministic dynamic game with constrained state and action sets to be a DPG, and shows that an OL solution of a DPG can be found through a related OCP. Sec. 5.5 extend the OL analysis to the more general case of stochastic DPGs. Sec. 5.6 extends the methodology to the CL analysis of stochastic DPGs, when the state-dependent policies are smooth parametric functions of a finite dimensional parameter vector. Sections 5.7-5.9 deal with application examples, the methods for solving them, and some illustrative simulations. Section 5.10 shows that, once we have found an equivalent OCP, we can learn a CL Nash equilibrium from interaction with the environment, without prior knowledge of the model, using reinforcement learning methods. Sec. 5.11 provides some conclusions.

Publications. The results of this chapter were included in [333, 303, 300].

Notation. We use similar notation to Chapter 3 to indicate states, actions, rewards and policies, which is different from Chapter 4.

\subsection{Problem setting}

Let the set of agents be denoted by

$$
\mathcal{N} \triangleq\{1, \ldots, N\}
$$

In this chapter we assume that the set of states is a subset of a real vector space. Hence, we find convenient to slightly change notation with respect to Chapter 3 . In particular, let $\mathbb{X} \subseteq \mathbb{R}^{S}$ denote the set of states of the game, where $S$ denotes now the dimensionality of the state set. Note that dimensionality of the state set can be different from the number of agents (i.e., $S \neq N$ ). Let $x_{i}$ denote the state-vector of the game at every time step $i$, with components $x_{i}(s)$ :

$$
x_{i} \triangleq\left(x_{i}(1), \ldots, x_{i}(S)\right) \in \mathbb{X}
$$

Every agent $k \in \mathcal{N}$ can be influenced by a subset of states $\mathbb{Z}_{k} \subseteq \mathbb{X}$ only. In particular, we assume that the partition of the state space among agents is done in the component domain. We define $\mathcal{X}_{k} \subseteq\{1, \ldots, S\}$ as the subset of indexes of state-vector components that influence agent $k$. Then, the state-vector for agent $k$ at time $i$ is given by:

$$
x_{k, i} \triangleq\left(x_{i}(m)\right)_{m \in \mathcal{X}_{k}} \in \mathbb{X}_{k}
$$


This generality allows for games in which multiple agents are affected by some common components of the state vector (e.g., when they share a common resource), and includes the extreme cases where they share all components and when no components are shared. Let $S_{k} \triangleq\left|\mathcal{X}_{k}\right|$ denote the number of components of $x_{k, i}$. We also introduce $x_{-k, i}$ as the vector of components that do not influence agent $k$, which lies in some subset $\mathbb{X}_{-k} \subset \mathbb{X}$ :

$$
x_{-k, i} \triangleq\left(x_{i}(l)\right)_{l \notin \mathcal{X}_{k}} \in \mathbb{X}_{-k}
$$

Let $\mathbb{A}_{k} \subseteq \mathbb{R}^{A_{k}}$ stand for the actions of agent $k$. Let $\mathbb{A} \triangleq \prod_{k \in \mathcal{N}} \mathbb{A}_{k}$ denote the set of actions of all agents, such that $\mathbb{A} \subseteq \mathbb{R}^{A}$, where $A=\sum_{k \in \mathcal{N}} A_{k}$. We write $a_{k, i} \in \mathbb{A}_{k}$ the action variable of agent $k$ at time $i$, with components $a_{k, i}(n)$ :

$$
a_{k, i} \triangleq\left(a_{k, i}(1), \ldots, a_{k, i}\left(A_{k}\right)\right) \in \mathbb{A}_{k}
$$

Thus, the vector that contains the actions of all agents is given by

$$
a_{i} \triangleq\left(a_{1, i}, \ldots, a_{N, i}\right) \in \mathbb{A}
$$

We also define the vector of all agents' actions except that of agent $k$ :

$$
a_{-k, i} \triangleq\left(a_{1, i}, \ldots, a_{k-1, i}, a_{k+1, i}, \ldots, a_{N, i}\right) \in \mathbb{A}_{-k} \triangleq \prod_{j \in \mathcal{N}, j \neq k} \mathbb{A}_{j}
$$

Hence, by slightly abusing notation, we can rewrite:

$$
a_{i}=\left(a_{k, i}, a_{-k, i}\right)
$$

The state transitions can be either deterministic or stochastic. For simplicity, we only consider the deterministic case in this section, and we leave the stochastic case for Sections 5.5 and 5.6. Thus, we consider that the state transitions are determined by some function $f: \mathbb{X} \times \mathbb{A} \times \mathbb{N} \rightarrow \mathbb{X}$, such that the nonstationary dynamic equation of the game is

$$
x_{i+1}=f\left(x_{i}, a_{i}, i\right)
$$

which can be split among components:

$$
x_{i+1}(s)=f_{s}\left(x_{i}, a_{i}, i\right), \quad s=1, \ldots, S
$$


such that

$$
f=\left(f_{s}\right)_{s=1}^{S}
$$

Note that we are assuming that the transition to state $x_{i+1}$ depends only on the current state-action pair $\left(x_{i}, a_{i}\right)$, rather than on the whole history of state-action pairs $\left\{\left(x_{0}, a_{0}\right), \ldots\left(x_{i}, a_{i}\right)\right\}$. We remark that $f$ corresponds to a nonreduced form, so that there may be no function $\varphi$ such that

$$
a_{i}=\varphi\left(x_{i}, x_{i+1}, i\right)
$$

We include a vector of $C$ nonstationary constraints $g \triangleq\left(g^{c}\right)_{c=1}^{C}$, as it is required for many applications, and define the constraint sets for $i=0$ :

$$
\mathbb{C}_{0} \triangleq \mathbb{A} \cap\left\{a_{0}: g\left(x_{0}, a_{0}, 0\right) \leq 0\right\}
$$

and for $i=1, \ldots, \infty$ :

$$
\begin{aligned}
\mathbb{C}_{i} \triangleq & \left\{\left\{\mathbb{X} \cap\left\{x_{i}: x_{i}=f\left(x_{i-1}, a_{i-1}, i-1\right)\right\}\right\} \times \mathbb{A}\right\} \\
& \cap\left\{\left(x_{i}, a_{i}\right): g\left(x_{i}, a_{i}, i\right) \leq 0\right\}
\end{aligned}
$$

Every agent $k$ has its own nonstationary reward function $r_{k}: \mathbb{X}_{k} \times \mathbb{A} \times \mathbb{N} \rightarrow \mathbb{R}$, such that, at every time $i$, it receives a reward value equal to: $r_{k}\left(x_{k, i}, a_{k, i}, a_{-k, i}, i\right)$. The aim of every agent is to find the sequence of actions $\left\{a_{k, 0}, \ldots, a_{k, i}, \ldots\right\}$ that maximizes its long term cumulative reward, given the sequence of other agents' actions $\left\{a_{-k, 0}, \ldots, a_{-k, i}, \ldots\right\}$. Note that this is an OL approach to the game. Thus, a discretetime infinite-horizon noncooperative nonstationary dynamic game can be represented as a set of $N$ coupled OCPs:

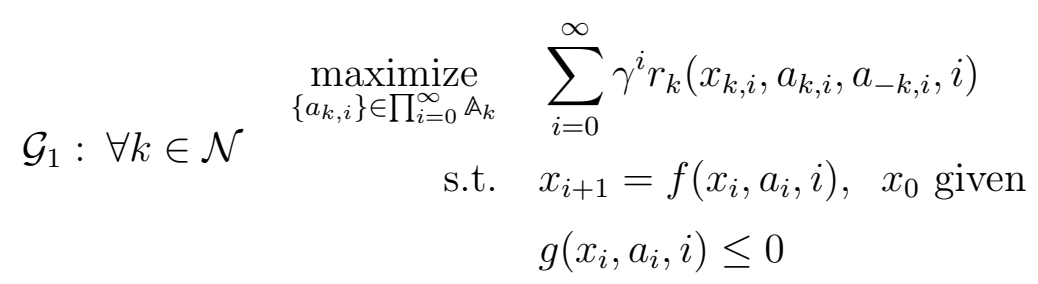

where $0<\gamma<1$ is the discount factor that bounds the cumulative reward (for simplicity, we define the same $\gamma$ for every agent). We say that game (5.15) is infinite-horizon because the reward is accumulated over infinite time steps. Note that, since the agents can share state-vector components, the constraints may affect every agent's feasible 


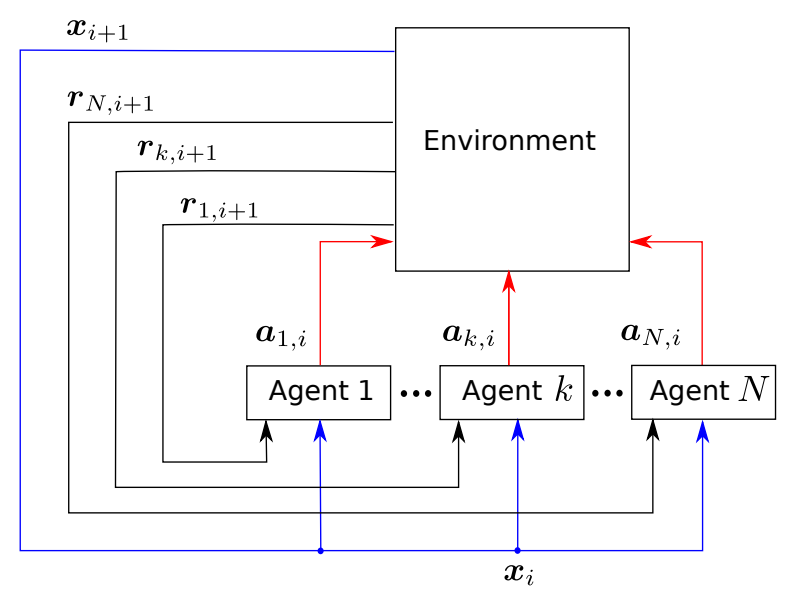

Figure 5.1 Open loop dynamic games. All agents operate in the same environment and influence each other. The problem can be seen as a multiobjective optimal control problem with coupled constraints.

region. In Section 5.5, we consider the stochastic extension of (5.15), in which both the state transitions and rewards are random variables; but let us focus on the deterministic case for now.

The set of $N$ coupled OCPs described by game (5.15) can be seen as a multiobjective variational problem, where the adjective variational refers to the fact that the strategies that we are seeking are functions of time (in the CL analysis, the strategies will be also functions but they depend on the system state rather than on time). As discussed in Sec. 3.6.2, most multiobjective problems have no optimal solution and other solution concepts are needed. The Pareto optimal solutions described in Chapter 3, Definitions 3.2-3.3, establish a tradeoff between the weight given to each agent's individual objective. This tradeoff implies some form of agreement or cooperation among the agents in the sense that they have to accept that the weight given to their objectives is less than one. However, when the agents compete among them and are unwilling to establish any tradeoff, other solution concepts are required. In this chapter, the solution concept of game (5.15) in which we are interested is the Nash Equilibrium (NE) of the game.

Definition 5.1 (Open Loop Nash Equilibrium). An open-loop Nash-equilibrium (OL$N E)$ of (5.15) is a sequence of actions $\left\{a_{i}^{\star}\right\}_{i=0}^{\infty}$ and its induced state trajectory $\left\{x_{i}^{\star}\right\}_{i=0}^{\infty}$ that satisfy the following condition for a given $x_{0} \in \mathcal{X}$ and for every agent $k \in \mathcal{N}$ :

$$
\begin{gathered}
\sum_{i=0}^{\infty} \gamma^{i} r_{k}\left(x_{k, i}^{\star}, a_{k, i}^{\star}, a_{-k, i}^{\star}, i\right) \geq \sum_{i=0}^{\infty} \gamma^{i} r_{k}\left(x_{k, i}, a_{k, i}, a_{-k, i}^{\star}, i\right) \\
\forall a_{k, 0} \in \mathbb{A}_{k}:\left(a_{k, 0}, a_{-k, 0}^{\star}\right) \in \mathbb{C}_{0}, \quad \forall a_{k, i} \in \mathbb{A}_{k}:\left(x_{i},\left(a_{k, i}, a_{-k, i}^{\star}\right)\right) \in \mathbb{C}_{i}
\end{gathered}
$$


We consider the following assumptions:

Assumption 5.1. The reward functions $r_{k}$ are twice continuously differentiable in $\mathcal{X} \times \mathbb{A}, \forall k \in \mathcal{N}$.

Assumption 5.2. The state and action sets, $\mathbb{X}$ and $\mathbb{A}$, are open and convex.

Assumption 5.3. The state-transition function $f$ and the constraints $g$ are continuously differentiable in $\mathbb{X} \times \mathbb{A}$ and satisfy some regularity conditions.

In general, finding an NE of game (5.15) is a difficult task because the reward functions, dynamic equation and constraints of each individual OCP are coupled among agents. However, when (5.15) is a DPG, we can solve it through a related OCP - as opposed to a set of coupled OCPs. We use Assumptions 5.1 and 5.2 to obtain a verifiable condition for problem (5.15) to be a DPG. Assumption 5.3 is required to introduce the conditions that guarantee equivalence between the solution of the OCP and an NE of the original DPG. In particular, since we derive the KKT optimality conditions for both problems (namely the DPG and the OCP), some regularity conditions (such as Slater's, the linear independence of gradients or the Mangasarian-Fromovitz constraint qualifications) are required to ensure that the KKT conditions hold at the optimal points and that feasible dual variables exist (see, e.g., [26, Sec. 3.3.5], [311]). Finally, we introduce one further assumption in Sec. 5.4 to ensure existence of a solution to the OCP and, hence, existence of an NE of the DPG.

This connection between DPGs and single OCPs generalizes the well studied but simpler case of static potential games [264, 196], which is reviewed in the following section.

\subsection{Overview of static potential games}

Static games are a simplified version of dynamic games in the sense that there are neither states, nor system dynamics. The aim of each agent $k$, given other agents' actions $a_{-k}$, is to choose an action $a_{k} \in \mathbb{A}_{k}$ that maximizes its reward function:

$$
\begin{aligned}
\mathcal{G}_{2}: \forall k \in \mathcal{N} \quad & r_{k}\left(a_{k}, a_{-k}\right) \\
\text { s.t. } & g(a) \leq 0
\end{aligned}
$$

where (similar to dynamic games but removing the time-dependence subscript) $a_{k} \triangleq$ $\left(a_{k}(\ell)\right)_{l=1}^{A_{k}} \in \mathbb{A}_{k} \subseteq \mathbb{R}^{A_{k}}$ refers to the action of agent $k$, and $a_{-k} \triangleq\left(a_{j}\right)_{j \in \mathcal{N}: j \neq k}$ is the 
vector of actions of the rest of agents; such that $a \triangleq\left(a_{k}, a_{-k}\right) \in \mathbb{A}$ denotes the vector of all agents' actions.

In general, finding or even characterizing the set of equilibrium points (e.g., in terms of existence or uniqueness) of problem (5.17) is difficult. Fortunately, there are particular cases of this problem for which the analysis is greatly simplified. Potential games is one of these cases.

Definition 5.2. Let Assumptions 5.1-5.2 hold. Then, problem (5.17) is called an exact (static) potential game if there is a function $J: \mathbb{A} \rightarrow \mathbb{R}$, named a potential, that satisfies the following condition for every agent [196]:

$$
\begin{gathered}
r_{k}\left(a_{k}, a_{-k}\right)-r_{k}\left(a_{k}^{\prime}, a_{-k}\right)=J\left(a_{k}, a_{-k}\right)-J\left(a_{k}^{\prime}, a_{-k}\right) \\
\forall a_{k}, a_{k}^{\prime} \in \mathbb{A}_{k}, \quad \forall a_{-k} \in \mathbb{A}_{-k}, \quad \forall k \in \mathcal{N}
\end{gathered}
$$

Definition 5.2 means that there exists some potential function, $J$, shared by all players, such that if some player $k$ changes its action unilaterally, the change in its reward $r_{k}$ equals the change in $J$.

Under Assumptions 5.1-5.2, it can be shown (see, e.g., [196, Lemma 4.4]) that a necessary and sufficient condition for a static game to be potential is the following:

$$
\nabla_{a_{k}} r_{k}(a)=\nabla_{a_{k}} J(a), \quad \forall k \in \mathcal{N}
$$

where $\nabla$ is the gradient.

We can gain insight on potential games by relating (5.19) to the concept of conservative vector field. The following lemma will be useful to this end.

Lemma 5.1. Let $F(a)=\left(F_{1}(a), \ldots, F_{N}(a)\right)$ be a vector field with continuous derivatives defined over an open convex set $\mathbb{A} \subseteq \mathbb{R}^{A}$. The following conditions on $F$ are equivalent:

1. There exists a scalar potential function $J(a)$ such that $F(a)=\nabla J(a)$.

2. The partial derivatives satisfy

$$
\frac{\partial F_{k}(a)}{\partial a_{j}}=\frac{\partial F_{j}(a)}{\partial a_{k}}, \quad \forall a \in \mathbb{A}, \quad k, j=1, \ldots, N
$$

3. Let $u$ be a fixed point of $\mathbb{A}$. For any piecewise smooth path $\xi$ joining a with $u$, we have $J(a)=\int_{u}^{a} F(\xi) \cdot d \xi$. 
A vector field satisfying these conditions is called conservative.

Proof: See, e.g., [6, Theorems 10.4, 10.5 and 10.9].

Let us define a vector field with components the partial derivatives of the agents' reward functions:

$$
F(a) \triangleq\left(\nabla_{a_{1}} r_{1}(a), \ldots, \nabla_{a_{N}} r_{N}(a)\right)
$$

Let us rewrite (5.21) as the gradient of some function $J$, i.e., $F(a)=\nabla_{a} J(a)$, so that Lemma 5.1.1 holds. Then, we have that

$$
\nabla_{a_{k}} r_{k}(a)=\nabla_{a_{k}} J(a), \quad \forall k \in \mathcal{N}
$$

Note that this is exactly the condition given by (5.19). It follows from Lemma 5.1.2 that a necessary, sufficient and also easily verifiable condition for game (5.17) to be a static potential game is given by:

$$
\frac{\partial^{2} r_{k}(a)}{\partial a_{j} \partial a_{k}}=\frac{\partial^{2} r_{j}(a)}{\partial a_{k} \partial a_{j}}, \quad \forall k, j \in \mathcal{N}
$$

Finally, Lemma 5.1.3 is useful since we can find a potential function $J$ by solving the line integral of the field:

$$
J(a)=\int_{0}^{1} \sum_{k=1}^{N} \sum_{\ell=1}^{A_{k}} \frac{\left.\partial r_{k}\left(\xi_{k}(z), a_{-k}\right)\right)}{\partial a_{k}(\ell)} \frac{d \xi_{k}(z)}{d z} d z
$$

where $\xi \triangleq\left(\xi_{k}\right)_{k \in \mathcal{N}}$ is a piecewise smooth path in $\mathbb{A}$ that connects the initial and final conditions, $\xi(0)$ and $\xi(1)=a$, respectively.

Once we have found $J$, it can be seen [264] that necessary conditions for $a^{\star}$ to be an equilibrium of the game (5.17) are also necessary conditions for the following optimization problem:

$$
\begin{aligned}
\mathcal{P}_{1}: \quad \underset{a \in \mathbb{A}}{\operatorname{maximize}} & J(a) \\
\text { s.t. } & g(a) \leq 0
\end{aligned}
$$

Indeed, optimization theorems concerning existence, uniqueness and algorithmic convergence can now be applied to game (5.17). In particular, reference [264] showed that the local maxima of the potential $J$ are a subset of the NE of the game. Furthermore, 
in the case that all agents' reward functions are quasi-concave, the maximum is unique and coincides with the stable equilibrium of the game.

This same approach can be extended to dynamic games. Nevertheless, instead of obtaining an analogous optimization problem, a DPG will yield an analogous OCP.

\subsection{Open loop dynamic potential games}

This section introduces the main theoretical contribution of this chapter: we establish conditions under which we can find an NE of (5.15) by solving an alternative OCP, instead of having to solve the set of coupled OCP with coupled constraints. First, we introduce the definition of a DPG and show conditions for (5.15) to belong to this class. Then, we introduce a related OCP and prove that its solution is an NE of the DPG.

Definition 5.3. Game (5.15) is called a DPG if there is a function $J: \mathbb{X} \times \mathbb{A} \times \mathbb{N} \rightarrow \mathbb{R}$, named potential, that satisfies the following condition for every agent $k \in \mathcal{N}$ :

$$
\begin{array}{r}
\sum_{i=0}^{\infty} \gamma^{i}\left(r_{k}\left(x_{k, i}, a_{k, i}, a_{-k, i}, i\right)-r_{k}\left(x_{k, i}, u_{k, i}, a_{-k, i}, i\right)\right) \\
=\sum_{i=0}^{\infty} \gamma^{i}\left(J\left(x_{i}, a_{k, i}, a_{-k, i}, i\right)-J\left(x_{i}, u_{k, i}, a_{-k, i}, i\right)\right) \\
\forall x_{k, i} \in \mathbb{X}_{k}, \quad \forall a_{k, i}, u_{k, i} \in \mathbb{A}_{k}, \quad \forall a_{-k, i} \in \mathbb{A}_{-k}
\end{array}
$$

The following three lemmas give conditions under which game (5.15) is a DPG (i.e., it satisfies Definition 5.3). It is convenient to recall that the only variables to agent $k$ are its local state and action vectors, $x_{k, i}$ and $a_{k, i}$, (i.e., other agents' actions are considered given data), so that the gradient vector of the reward functions can be decomposed into an infinite sequence of two vectors $(i=1, \ldots, \infty)$ :

$$
\begin{aligned}
& \nabla_{x_{k, i}} r_{k}\left(x_{k, i}, a_{i}, i\right) \triangleq\left(\frac{\partial r_{k}\left(x_{k, i}, a_{i}, i\right)}{\partial x_{k, i}(m)}\right)_{m \in \mathcal{X}_{k}} \\
& \nabla_{a_{k, i}} r_{k}\left(x_{k, i}, a_{i}, i\right) \triangleq\left(\frac{\partial r_{k}\left(x_{k, i}, a_{i}, i\right)}{\partial a_{k, i}(\ell)}\right)_{\ell=1, \ldots, A_{k}}
\end{aligned}
$$

Lemma 5.2. Game (5.15) is a DPG if there exists some function $J\left(x_{i}, a_{i}, i\right)$ that satisfies for all $k \in \mathcal{N}$ and $i=0, \ldots, \infty$ :

$$
\begin{aligned}
& \nabla_{x_{k, i}} r_{k}\left(x_{k, i}, a_{i}, i\right)=\nabla_{x_{k, i}} J\left(x_{i}, a_{i}, i\right) \\
& \nabla_{a_{k, i}} r_{k}\left(x_{k, i}, a_{i}, i\right)=\nabla_{a_{k, i}} J\left(x_{i}, a_{i}, i\right)
\end{aligned}
$$


Proof: We simply extend to dynamic games the argument for static games due to [264, Prop. 1]. From (5.29)-(5.34) and Assumption 5.1, we have:

$$
\begin{aligned}
& \nabla_{x_{k, i}}\left[r_{k}\left(x_{k, i}, a_{i}, i\right)-J\left(x_{i}, a_{i}, i\right)\right]=0_{S_{k}} \\
& \nabla_{a_{k, i}}\left[r_{k}\left(x_{k, i}, a_{i}, i\right)-J\left(x_{i}, a_{i}, i\right)\right]=0_{A_{k}}
\end{aligned}
$$

This means that the difference between a potential and each agent's reward depends neither on $x_{k, i}$, nor on $a_{k, i}$. Thus, we can express this difference as

$$
J\left(x_{i}, a_{k, i}, a_{-k, i}, i\right)-r_{k}\left(x_{k, i}, a_{k, i}, a_{-k, i}, i\right)=\Theta_{k}\left(x_{-k, i}, a_{-k, i}, i\right), \quad \forall a_{k, i} \in \mathbb{A}_{k}
$$

for some function $\Theta_{k}: \mathbb{X}_{-k} \times \mathbb{A}_{-k} \times \mathbb{N} \rightarrow \mathbb{R}$. Since (5.33) is satisfied for every $a_{k, i} \in \mathbb{A}_{k}$, we can subtract two versions of (5.33) with actions $a_{k}$ and $u_{k}$ in $\mathbb{A}_{k}$. Then, by arranging terms and summing over all $i$, we obtain (5.26).

Conditions (5.29)-(5.34) are usually difficult to check in practice because we do not know $J$ beforehand. Fortunately, there are cases in which the agent's reward function have some separable structure that allows us to easily deduce that the game is a DPG, as it is explained in the following lemma.

Lemma 5.3. Game (5.15) is a DPG if the reward function of every agent $k \in \mathcal{N}$ can be expressed as the sum of a term that is common to all agents plus another term that depends neither on its own action, nor on its own state-components:

$$
r_{k}\left(x_{k, i}, a_{k, i}, a_{-k, i}, i\right)=J\left(x_{i}, a_{k, i}, a_{-k, i}, i\right)+\Theta_{k}\left(x_{-k, i}, a_{-k, i}, i\right)
$$

Proof: By taking the partial derivative of (5.34) we obtain (5.29)-(5.34), Hence, we apply Lemma 5.2 (see also [264, Prop. 1]).

Sometimes, posing the reward in the separable structure (5.34) may be nonobvious. The following lemma allows us to check whether (5.3) is a DPG in such case.

Lemma 5.4. Game (5.15) is a DPG if the agents' reward functions satisfy the following conditions, $\forall k, j \in \mathcal{N}$ :

$$
\begin{aligned}
\nabla_{a_{j, i}}\left[\nabla_{x_{k, i}} r_{k}\left(x_{k, i}, a_{i}, i\right)\right] & =\nabla_{a_{k, i}}\left[\nabla_{x_{j, i}} r_{j}\left(x_{j, i}, a_{i}, i\right)\right] \\
\nabla_{x_{j, i}}\left[\nabla_{x_{k, i}} r_{k}\left(x_{k, i}, a_{i}, i\right)\right] & =\nabla_{x_{k, i}}\left[\nabla_{x_{j, i}} r_{j}\left(x_{j, i}, a_{i}, i\right)\right] \\
\nabla_{a_{j, i}}\left[\nabla_{a_{k, i}} r_{k}\left(x_{k, i}, a_{i}, i\right)\right] & =\nabla_{a_{k, i}}\left[\nabla_{a_{j, i}} r_{j}\left(x_{j, i}, a_{i}, i\right)\right]
\end{aligned}
$$


Proof: Under Assumption 5.1, we can introduce the following vector field:

$$
\begin{array}{r}
F\left(x_{i}, a_{i}, i\right) \triangleq\left(\nabla_{x_{1, i}} r_{1}\left(x_{1, i}, a_{i}, i\right)^{\top}, \ldots, \nabla_{x_{N, i}} r_{N}\left(x_{N, i}, a_{i}, i\right)^{\top},\right. \\
\left.\nabla_{a_{1, i}} r_{1}\left(x_{1, i}, a_{i}, i\right)^{\top}, \ldots, \nabla_{a_{N, i}} r_{N}\left(x_{N, i}, a_{i}, i\right)^{\top}\right)
\end{array}
$$

From Lemma 5.2, we can express (5.38) as

$$
F\left(x_{i}, a_{i}, i\right)=\nabla_{\left(x_{i}, a_{i}\right)}\left[J\left(x_{i}, a_{i}, i\right)\right]
$$

From Assumption 5.2 and Lemma 5.1.1, we know that $F$ is conservative. Hence, Lemma 5.1.2 establishes that the second partial derivatives must satisfy (5.35)-(5.37).

Now, introduce the following deterministic OCP:

$$
\begin{aligned}
& \operatorname{\mathcal {P}}_{\left\{a_{i}\right\} \in \prod_{i=0}^{\infty} \mathbb{A}} \sum_{i=0}^{\infty} \gamma^{i} J\left(x_{i}, a_{i}, i\right) \\
& \text { s.t. } \quad x_{i+1}=f\left(x_{i}, a_{i}, i\right), \quad x_{0} \text { given } \\
& g\left(x_{i}, a_{i}, i\right) \leq 0
\end{aligned}
$$

Let us consider the following assumption, which is needed for establishing the connection between a DPG and a single OCP of the form (5.40)

Assumption 5.4. The OCP (5.40) has a nonempty solution set.

Sufficient - and easily verifiable - conditions to satisfy Assumption 5.4 are given by the following lemma, which is a standard result in optimal control theory.

Lemma 5.5. Let $J: \mathbb{X} \times \mathbb{A} \times \mathbb{N} \rightarrow[-\infty, \infty)$ be a proper continuous function. And let any one of the following conditions hold for $i=1, \ldots, \infty$ :

1. The constraint sets $\mathbb{C}_{i}$ are bounded.

2. $J\left(x_{i}, a_{i}, i\right) \rightarrow-\infty$ as $\left\|\left(x_{i}, a_{i}\right)\right\| \rightarrow \infty$ (coercive).

3. There exists a scalar $L$ such that the level sets $\left\{\left(x_{i}, a_{i}, i\right): J\left(x_{i}, a_{i}, i\right) \geq L\right\}_{i=0}^{\infty}$ are nonempty and bounded.

Then, $\forall x_{0} \in \mathbb{X}$, there exists an optimal sequence of actions $\left\{a_{i}^{\star}\right\}_{i=0}^{\infty}$ that is solution to the OCP (5.40). Moreover, there exists an optimal policy $\pi^{\star}: \mathbb{X} \times \mathbb{N} \rightarrow \mathbb{A}$, which is a 
mapping from states to optimal actions, such that when applied over any state $\left\{x_{i}\right\}_{i=0}^{\infty}$, it provides an optimal sequence of actions $\left\{a_{i}^{\star}=\pi^{\star}\left(x_{i}, i\right)\right\}_{i=0}^{\infty}$.

Proof: First, we remark that being proper means (see, e.g., [29]) that $J\left(x_{i}, a_{i}, i\right)>$ $-\infty$ for at least one $\left(x_{i}, a_{i}\right) \in \mathbb{C}_{i}$ and $J\left(x_{i}, a_{i}, i\right)<\infty$, for all $\left(x_{i}, a_{i}\right) \in \mathbb{C}_{i}$ and $i=1, \ldots, \infty$ and for $a_{0} \in \mathbb{C}_{0}$ for $i=0$. Thus, $J$ has some nonempty level set. In addition, since $J$ is proper, its level sets are bounded. Moreover, since $J$ is continuous, its (bounded) level sets are compact. Now, we extend this idea to our control problem with discounted cumulative sum. Let $L$ define a nonempty compact level set of $J$. Since $J\left(x_{i}, a_{i}, i\right) \geq L$ and $\gamma<1$, we have

$$
\sum_{i=0}^{\infty} \gamma^{i} J\left(x_{i}, a_{i}, i\right) \geq L \sum_{i=0}^{\infty} \gamma^{i}=\frac{L}{1-\gamma}
$$

Hence, the following level sets are also nonempty:

$$
\left\{\left(x_{i}, a_{i}, i\right): \sum_{i=0}^{\infty} \gamma^{i} J\left(x_{i}, a_{i}, i\right) \geq \frac{L}{1-\gamma}\right\}_{i=0}^{\infty}
$$

In addition, since $J$ is proper, there exists some scalar $U$, such that

$$
\sum_{i=0}^{\infty} \gamma^{i} J\left(x_{i}, a_{i}, i\right) \leq U \sum_{i=0}^{\infty} \gamma^{i}=\frac{U}{1-\gamma}
$$

Since $L \leq U$, we have that

$$
\frac{L}{1-\gamma} \leq \frac{U}{1-\gamma}
$$

Therefore, the level sets (5.42) are bounded. Finally, since $J$ is continuous, we conclude that these level sets are also compact. Thus, we can use [28, Prop. 3.1.7] (see, also [28, Sec. 1.2 and 3.6]) to ensure existence of an optimal policy.

The main theoretical result of this section is that we can find an OL-NE of a DPG by solving the OCP (5.40). This is proved in the following theorem.

Theorem 5.1. Suppose that (5.15) is a DPG (as in Definition 5.3) and, in addition, Assumptions 5.1-5.4 hold. Then, a solution to the OCP (5.40) is also an NE for (5.15). In particular, the function

$$
J\left(x_{i}, a_{i}, i\right)=\int_{0}^{1} \sum_{k \in \mathcal{N}}\left(\sum_{m \in \mathcal{X}_{k}} \frac{\partial r_{k}\left(\eta_{k}(z), a_{k, i}, a_{-k, i}, i\right)}{\partial x_{k, i}(m)} \frac{d \eta_{k, m}(z)}{d z}\right.
$$




$$
\left.+\sum_{\ell=1}^{A_{k}} \frac{\partial r_{k}\left(x_{k, i}, \xi_{k}(z), a_{-k, i}, i\right)}{\partial a_{k, i}(\ell)} \frac{d \xi_{k, \ell}(z)}{d z}\right) d z
$$

is an instantaneous reward function for the $O C P$, where $\eta(z) \triangleq\left(\eta_{k}(z)\right)_{k \in \mathcal{N}}$ and $\xi(z) \triangleq$ $\left(\xi_{k}(z)\right)_{k \in \mathcal{N}}$ are piecewise smooth paths in $\mathbb{X}$ and $\mathbb{A}$, respectively, with components $\eta_{k}(z) \triangleq$ $\left(\eta_{k, m}(z)\right)_{m \in \mathcal{X}_{k}}$ and $\xi_{k}(z) \triangleq\left(\xi_{k, \ell}(z)\right)_{\ell=1}^{A_{k}}$, such that the initial and final state-action conditions are given by $(\eta(0), \xi(0))$ and $\left(\eta_{k}(1)=x_{k, i}, \forall k \in \mathcal{N}, \xi(1)=a_{i}\right)$, respectively.

The usefulness of Theorem 5.1 is that, in order to find an NE of (5.15), instead of solving several coupled control problems, we can check whether (5.15) is a DPG (i.e., anyone of Lemmas 5.2-5.4 holds) and, if so, we can find an NE by computing a potential function (5.45) and solving the related OCP (5.40).

Proof: The proof is structured in five steps. First, we compute the EulerLagrange equations of the Lagrangian of the game and derive the KKT optimality conditions. Assumption 5.3 is required to ensure that the KKT conditions hold at the optimal point and that there exist feasible dual variables [26, Prop. 3.3.8]. Second, we study when the necessary optimality conditions of the game become equal to those of the OCP. Third, we show that having the same necessary optimality conditions is sufficient condition for the dynamic game to be a DPG. Fourth, we show that the solution to the OCP (whose existence is guaranteed by Assumption 5.4) is also an NE of the DPG. Finally, we derive the per stage reward function of the OCP as a potential function of a suitable vector field. We proceed to explain the details.

Step 1: for game (5.15), introduce each agent's Lagrangian $\forall k \in \mathcal{N}$ :

$$
\begin{aligned}
\mathcal{L}_{k}\left(x_{0: \infty}, a_{0: \infty}, \lambda_{k, 0: \infty}, \mu_{k, 0: \infty}\right)= & \sum_{i=0}^{\infty} \gamma^{i} r_{k}\left(x_{k, i}, a_{i}, i\right) \\
& +\lambda_{k, i}^{\top}\left(f\left(x_{i}, a_{i}, i\right)-x_{i+1}\right)+\mu_{k, i}^{\top} g\left(x_{i}, a_{i}, i\right)
\end{aligned}
$$

where we used subscript $\bullet_{0: \infty}$ as a shorthand to indicate the whole sequence of variables for $i=0, \ldots, \infty$ :

$$
\begin{aligned}
x_{0: \infty} & \triangleq\left(x_{i}\right)_{i=0}^{\infty} \\
a_{0: \infty} & \triangleq\left(a_{i}\right)_{i=0}^{\infty} \\
\lambda_{k, 0: \infty} & \triangleq\left(\lambda_{k, i}\right)_{i=0}^{\infty} \\
\mu_{k, 0: \infty} & \triangleq\left(\mu_{k, i}\right)_{i=0}^{\infty}
\end{aligned}
$$


and where $\lambda_{k, i} \in \mathbb{R}^{S}$ and $\mu_{k, i} \in \mathbb{R}^{C}$ are the corresponding vectors of multipliers at time $i$. It will be convenient to decompose $\lambda_{k, i}$ into $S$ components, denoted $\lambda_{k, s, i}$, that correspond with the $S$ state-components:

$$
\lambda_{k, i} \triangleq\left(\lambda_{k, s, i}\right)_{s=1}^{S}
$$

Introduced a shorthand for the instantaneous Lagrangian of agent $k$ :

$$
\begin{aligned}
\Phi_{k}\left(x_{i}, x_{i+1}, a_{i}, i, \lambda_{k, i}, \mu_{k, i}\right) \triangleq & \gamma^{i} r_{k}\left(x_{k, i}, a_{i}, i\right) \\
& +\lambda_{k, i}^{\top}\left(f\left(x_{i}, a_{i}, i\right)-x_{i+1}\right)+\mu_{k, i}^{\top} g\left(x_{i}, a_{i}, i\right)
\end{aligned}
$$

The discrete time Euler-Lagrange equations and the associated transversality conditions for each agent's Lagrangian are given by ([242, Sec. 6.1]:

$$
\begin{aligned}
\nabla_{x_{k, i}}\left[\Phi_{k}\left(x_{i-1}, x_{i}, a_{i-1}, i-1, \lambda_{k, i-1}, \mu_{k, i-1}\right)+\Phi_{k}\left(x_{i}, x_{i+1}, a_{i}, i, \lambda_{k, i}, \mu_{k, i}\right)\right] & =0_{S_{k}} \\
\nabla_{a_{k, i}}\left[\Phi_{k}\left(x_{i-1}, x_{i}, a_{i-1}, i-1, \lambda_{k, i-1}, \mu_{k, i-1}\right)+\Phi_{k}\left(x_{i}, x_{i+1}, a_{i}, i, \lambda_{k, i}, \mu_{k, i}\right)\right] & =0_{A_{k}} \\
\lim _{i \rightarrow \infty} \nabla_{x_{k, i}} \Phi_{k}\left(x_{i}, x_{i+1}, a_{i}, i, \lambda_{k, i}, \mu_{k, i}\right) & =0_{S_{k}} \\
\lim _{i \rightarrow \infty} \nabla_{a_{k, i}} \Phi_{k}\left(x_{i}, x_{i+1}, a_{i}, i, \lambda_{k, i}, \mu_{k, i}\right) & =0_{A_{k}}
\end{aligned}
$$

Equations (5.53)-(5.54) are the Euler-Lagrange equations in non-reduced form. They basically state that no gain can be achieved by deviating from an optimal stateaction trajectory for one time-step (see, e.g., [137], [272, Sec. 4.5], [2, Sec. 6.5]). As mentioned in Sec. 5.2 (see also, e.g., [242, Sec. 6.1], [122]), in the standard reduced form, the current action can be posed as a function of the current and future states: $a_{i}=\varphi\left(x_{i}, x_{i+1}, i\right)$, for some function $\varphi: \mathbb{K} \times \mathbb{X} \times \mathbb{N} \rightarrow \mathbb{A}$. The reason why we introduced this general nonreduced form of the Euler-Lagrange equations is that such function $\varphi$ may not exist for an arbitrary state-transition function $f$. Equations (5.55)-(5.56) are the transversality conditions and state that nothing can be gained by deviating from the optimal path and never coming back to it (see [137]). Since (5.52) has no dependence on $a_{i-1}$, the gradient of the first term of (5.54) becomes zero. Hence, the second transversality condition (5.56) is included in the second term of (5.54). In economy, the state is usually considered some stock or resource and, therefore, it must be fully spent at the end of the game (i.e., there is no reason to save something that cannot be used, since we would not be maximizing the reward otherwise). In this case, the transversality conditions can be slightly generalized as follows:

$$
\lim _{i \rightarrow \infty} x_{k, i}^{\top} \nabla_{x_{k, i}} \Phi_{k}\left(x_{i}, x_{i+1}, a_{i}, i, \lambda_{k, i}, \mu_{k, i}\right)=0
$$


By substituting (5.52) into (5.53)-(5.56), and by adding the corresponding constraints, we obtain the KKT conditions for the game, for every agent $k \in \mathcal{N}$ and all time steps $i=1, \ldots, \infty$ :

$$
\begin{aligned}
\nabla_{x_{k, i}}\left[\gamma^{i} r_{k}\left(x_{k, i}, a_{i}, i\right)+\lambda_{k, i}^{\top} f\left(x_{i}, a_{i}, i\right)+\mu_{k, i}^{\top} g\left(x_{i}, a_{i}, i\right)\right]-\lambda_{\mathcal{X}_{k}, i-1} & =0_{S_{k}} \\
\nabla_{a_{k, i}}\left[\gamma^{i} r_{k}\left(x_{k, i}, a_{i}, i\right)+\lambda_{k, i}^{\top} f\left(x_{i}, a_{i}, i\right)+\mu_{k, i}^{\top} g\left(x_{i}, a_{i}, i\right)\right] & =0_{A_{k}} \\
\lim _{i \rightarrow \infty} \nabla_{x_{k, i}}\left[\gamma^{i} r_{k}\left(x_{k, i}, a_{i}, i\right)+\lambda_{k, i}^{\top} f\left(x_{i}, a_{i}, i\right)+\mu_{k, i}^{\top} g\left(x_{i}, a_{i}, i\right)\right] & =0_{S_{k}} \\
x_{i+1}=f\left(x_{i}, a_{i}, i\right), \quad g\left(x_{i}, a_{i}, i\right) & \leq 0_{C} \\
\mu_{k, i} \leq 0_{C}, \quad \mu_{k, i}^{\top} g\left(x_{i}, a_{i}, i\right) & =0
\end{aligned}
$$

where $\lambda_{\mathcal{X}_{k}, i-1}$ denotes the vector of multipliers related to the state of agent $k$ :

$$
\lambda_{\mathcal{X}_{k}, i-1}=\left(\lambda_{k, m, i-1}\right)_{m \in \mathcal{X}_{k}}
$$

Recall that, in general, we have to include all constraints in each agent's Lagrangian because there may be shared state-components among agents. In the case that there are disjoint state-components, the corresponding partial derivatives with respect to the variables that do not influence the agent's transition function and/or constraint will be zero.

Step 2: we find the KKT conditions for the OCP. To do so, we obtain the Lagrangian of $(5.40)$ :

$$
\begin{aligned}
\mathcal{L}^{\mathrm{OCP}}\left(x_{0: \infty}, a_{0: \infty}, \beta_{0: \infty}, \delta_{0: \infty}\right)= & \sum_{i=0}^{\infty} \gamma^{i} J\left(x_{i}, a_{i}, i\right) \\
& +\beta_{i}^{\top}\left(f\left(x_{i}, a_{i}, i\right)-x_{i+1}\right)+\delta_{i}^{\top} g\left(x_{i}, a_{i}, i\right)
\end{aligned}
$$

where $\beta_{i} \triangleq\left(\beta_{s, i}\right)_{s} \in \mathbb{R}^{S}$ and $\delta_{i} \in \mathbb{R}^{C}$ are the corresponding multipliers. Again, from (5.64) we derive the Euler-Lagrange and transversality condition equations, which, together with the corresponding constraints, yield the KKT system of optimality conditions for $k=1, \ldots, N$ and all time steps $i=1, \ldots, \infty$ :

$$
\begin{aligned}
\nabla_{x_{k, i}}\left[\gamma^{i} J\left(x_{i}, a_{i}, i\right)+\beta_{i}^{\top} f\left(x_{i}, a_{i}, i\right)+\delta_{i}^{\top} g\left(x_{i}, a_{i}, i\right)\right]-\beta_{\mathcal{X}_{k}, i-1} & =0_{S_{k}} \\
\nabla_{a_{k, i}}\left[\gamma^{i} J\left(x_{i}, a_{i}, i\right)+\beta_{i}^{\top} f\left(x_{i}, a_{i}, i\right)+\delta_{i}^{\top} g\left(x_{i}, a_{i}, i\right)\right] & =0_{A_{k}} \\
\lim _{i \rightarrow \infty} \nabla_{x_{k, i}}\left[\gamma^{i} J\left(x_{i}, a_{i}, i\right)+\beta_{i}^{\top} f\left(x_{i}, a_{i}, i\right)+\delta_{i}^{\top} g\left(x_{i}, a_{i}, i\right)\right] & =0_{S_{k}} \\
x_{i+1}=f\left(x_{i}, a_{i}, i\right), \quad g\left(x_{i}, a_{i}, i\right) & \leq 0_{C} \\
\delta_{i} \leq 0_{C}, \quad \delta_{i}^{\top} g\left(x_{i}, a_{i}, i\right) & =0
\end{aligned}
$$


where $\beta_{\mathcal{X}_{k}, i-1}$ denotes the vector of multipliers related to $x_{k, i}$ :

$$
\beta_{\mathcal{X}_{k}, i-1}=\left(\beta_{m, i-1}\right)_{m \in \mathcal{X}_{k}}
$$

In order for the OCP (5.40) to have the same optimality conditions as the game (5.15), we conclude, by comparing (5.58)-(5.62) with (5.65)-(5.69), that the following conditions must be satisfied $\forall k \in \mathcal{N}$ and $i=1, \ldots, \infty$ :

$$
\begin{aligned}
& \nabla_{x_{k, i}} r_{k}\left(x_{k, i}, a_{i}, i\right)=\nabla_{x_{k, i}} J\left(x_{i}, a_{i}, i\right) \\
& \nabla_{a_{k, i}} r_{k}\left(x_{k, i}, a_{i}, i\right)=\nabla_{a_{k, i}} J\left(x_{i}, a_{i}, i\right) \\
& \lambda_{k, i}=\beta_{i}, \quad \mu_{k, i}=\delta_{i}
\end{aligned}
$$

Step 3: when conditions (5.71)-(5.72) are satisfied, Lemma 5.2 states that (5.15) is a DPG. This means that if condition (5.73) holds, then any DPG has the same optimality conditions as OCP (5.40).

Step 4: note that condition (5.73) represents a feasible point of the game. The reason is that if there exists an optimal primal variable, then the existence of dual variables in the OCP is guaranteed by suitable regularity conditions. Since the existence of optimal primal variables of the OCP is ensured by Assumption 5.4, the regularity conditions established by Assumption 5.3 guarantee that there exist some $\beta_{i}$ and $\delta_{i}$ that satisfy the KKT conditions of the OCP. Substituting these dual variables of the OCP in place of the individual $\lambda_{k, i}$ and $\mu_{k, i}$ in (5.58)-(5.62) for every $k \in \mathcal{N}$ results in a system of equations where the only unknowns are the user strategies. This system has exactly the same structure as the one already presented for the OCP in the primal variables. Therefore, the OCP primal solution also satisfies the KKT conditions of the DPG. Indeed, it is easy to see that an optimal solution of the OCP is also an NE of the game. Let $\left\{a_{i}^{\star}\right\}_{i=0}^{\infty}$ be the OCP solution, so that it satisfies the following inequality for a given $x_{0} \in \mathbb{X}$ and $\forall k \in \mathcal{N}$ :

$$
\begin{gathered}
\sum_{i=0}^{\infty} \gamma^{i} J\left(x_{i}^{\star}, a_{k, i}^{\star}, a_{-k, i}^{\star}, i\right) \geq \sum_{i=0}^{\infty} \gamma^{i} J\left(x_{i}, a_{k, i}, a_{-k, i}^{\star}, i\right) \\
\forall a_{k, 0} \in \mathbb{A}_{k}:\left(a_{k, 0}, a_{-k, 0}^{\star}\right) \in \mathbb{C}_{0}, \quad \forall a_{k, i} \in \mathbb{A}_{k}:\left(x_{i},\left(a_{k, i}, a_{-k, i}^{\star}\right)\right) \in \mathbb{C}_{i}
\end{gathered}
$$

where $\left\{x_{i}^{\star}\right\}_{i=0}^{\infty}$ is the optimal state trajectory induced by $\left\{a_{i}^{\star}\right\}_{i=0}^{\infty}$. From Definition 5.3, we conclude that the OCP optimal solution is also an NE of game (5.15). The opposite may not be true in general. Indeed, this solution, in which dual variables are shared between agents, is only a subset of the possible NEs of the game. 
Step 5: although we have shown that we can find an NE of the DPG by solving a OCP, we still need to find the objective for the OCP. In order to find $J$, we deduce from $(5.71),(5.72),(5.38)$ and (5.39) that the vector field (5.38) can be expressed as

$$
F\left(x_{i}, a_{i}, i\right) \triangleq \nabla J\left(x_{i}, a_{i}, i\right)
$$

Lemma 5.1 establishes that $F$ is conservative. Thus, the objective of the OCP is a potential of the field, which can be computed through the line integral (5.45).

We remark that Theorem 5.1 proves that any policy that solves OCP (5.40) is also an NE for DPG (5.15).

In the next section, we generalize the OL analysis of deterministic DPGs to stochastic games.

\subsection{Open loop stochastic dynamic potential games}

In this section, we consider stochastic dynamic games, in which the state transitions and instantaneous rewards are random variables, and the goal of each agent is to maximize the expected value of its long term cumulative reward, given other agents' action sequences. First, we introduce the problem setting for stochastic dynamic games. Then, we extend results from Sec. 5.4, deriving sufficient conditions for a stochastic dynamic game to be a stochastic-DPG (or SDPG for short) and showing that it can be solved through a related OCP.

We remark that open-loop solutions are likely to be subpotimal in a practical stochastic setting. The reason is that the agents aim to maximize the expected cumulative reward over all possible state-action trajectories (as we will see in (5.80) below), and the problem is that these trajectories could have large variance. Nevertheless, the analysis is novel and interesting (up to the best of our knowledge no previous work have explicitly considered constraints when studying stochastic dynamic games) and exposes the reader to some concepts that will be useful for the CL analysis of Sec. 5.6.

\subsubsection{Problem setting for open loop stochastic dynamic games}

The state transitions are determined by some (possibly nonstationary) probability distribution $p_{\boldsymbol{x}}$ over the future state, conditioned on the current state and current action:

$$
\boldsymbol{x}_{i+1} \sim p_{\boldsymbol{x}}\left(\cdot \mid \boldsymbol{x}_{i}, a_{i}, i\right)
$$


We remark the use of boldface notation for random variables. These transitions can be equivalently expressed from a state dynamics function, $f: \mathbb{X} \times \mathbb{A} \times \Theta \times \mathbb{N} \rightarrow \mathbb{X}$, that now depends on some random variable $\boldsymbol{\theta}_{i} \in \Theta$, such that

$$
\boldsymbol{x}_{i+1}=f\left(\boldsymbol{x}_{i}, a_{i}, \boldsymbol{\theta}_{i}, i\right)
$$

where $\boldsymbol{\theta}_{i} \sim p_{\boldsymbol{\theta}}\left(\cdot \mid \boldsymbol{x}_{i}, a_{i}, i\right)$, for some time varying distribution $p_{\boldsymbol{\theta}}$.

The instantaneous reward of each agent, $\boldsymbol{r}_{k, i}$, is also a random variable with time-varying distribution $p_{\boldsymbol{r}_{k}}$ :

$$
\boldsymbol{r}_{k, i} \sim p_{\boldsymbol{r}_{i}}\left(\cdot \mid \boldsymbol{x}_{k, i}, a_{i}, i\right)
$$

Again, by introducing some random variable $\boldsymbol{\sigma}_{k, i} \in \Sigma_{k}$ with time-varying distribution $p_{\boldsymbol{\sigma}_{k}}$, we can define the reward function $r_{k}: \mathbb{X}_{k} \times \mathbb{A} \times \Sigma_{k} \times \mathbb{N} \rightarrow \mathbb{R}$ and rewrite (5.78) as:

$$
\boldsymbol{r}_{k, i}=r_{k}\left(\boldsymbol{x}_{k, i}, a_{i}, \boldsymbol{\sigma}_{k, i}, i\right)
$$

where $\boldsymbol{\sigma}_{k, i} \sim p_{\boldsymbol{\sigma}_{k}}\left(\cdot \mid \boldsymbol{x}_{i}, a_{i}, i\right)$. We assume that $\boldsymbol{\theta}_{i}$ and $\boldsymbol{\sigma}_{k, i}$ are independent of any earlier variable $\boldsymbol{\theta}_{j}$ and $\boldsymbol{\sigma}_{k, j}$, respectively, given $\boldsymbol{x}_{i}$ when $0 \leq j<i$.

Every agent $k \in \mathcal{N}$ aims to find the sequence of actions $\left\{a_{k, i}\right\}_{i=1}^{\infty}$ that maximizes its long term expected cumulative reward, given the sequence of other agents' actions $\left\{a_{-k, i}\right\}_{i=0}^{\infty}$. Thus, the stochastic game can be expressed as a set of $N$ coupled OCP:

$$
\begin{aligned}
& \underset{\left\{a_{k, i}\right\} \in \prod_{i=0}^{\infty} \mathbb{A}_{k}}{\operatorname{maximize}} \mathbb{E}\left[\sum_{i=0}^{\infty} \gamma^{i} r_{k}\left(\boldsymbol{x}_{k, i}, a_{k, i}, a_{-k, i}, \boldsymbol{\sigma}_{k, i}, i\right)\right] \\
& \mathcal{G}_{3}: \forall k \in \mathcal{N} \quad \text { s.t. } \quad \boldsymbol{x}_{i+1}=f\left(\boldsymbol{x}_{i}, a_{i}, \boldsymbol{\theta}_{i}, i\right), \quad \boldsymbol{x}_{0}=x_{0} \text { given } \\
& g\left(\boldsymbol{x}_{i}, a_{i}, i\right) \leq 0 \\
& \boldsymbol{\theta}_{i} \sim p_{\boldsymbol{\theta}}\left(\cdot \mid \boldsymbol{x}_{i}, a_{i}, i\right), \quad \boldsymbol{\sigma}_{k, i} \sim p_{\boldsymbol{\sigma}_{k}}\left(\cdot \mid \boldsymbol{x}_{i}, a_{i}, i\right)
\end{aligned}
$$

where the expectation is taken at every time step over $p_{\boldsymbol{\theta}}$ on the state transition equation and over $p_{\sigma_{k}}$ on the reward function.

The OL-NE given by Definition 5.1 can be easily extended for stochastic game (5.80) by taking the expected value over the state transition and reward distributions at every time step.

Definition 5.4 (Expected Open Loop Nash Equilibrium). An expected-open-loop NashEquilibrium (EOL-NE) of stochastic game (5.80) is a sequence of actions $\left\{a_{i}^{\star}\right\}_{i=0}^{\infty}$ that 
satisfies the following condition for a given $\boldsymbol{x}_{0}=x_{0} \in \mathbb{X}$ and for every agent $k \in \mathcal{N}$ :

$$
\begin{gathered}
\mathbb{E}\left[\sum_{i=0}^{\infty} \gamma^{i} r_{k}\left(\boldsymbol{x}_{k, i}^{\star}, a_{k, i}^{\star}, a_{-k, i}^{\star}, \boldsymbol{\sigma}_{k, i}, i\right)\right] \geq \mathbb{E}\left[\sum_{i=0}^{\infty} \gamma^{i} r_{k}\left(\boldsymbol{x}_{k, i}, a_{k, i}, a_{-k, i}^{\star}, \boldsymbol{\sigma}_{k, i}, i\right)\right] \\
\forall a_{k, 0} \in \mathbb{A}_{k}:\left(a_{k, 0}, a_{-k, 0}^{\star}\right) \in \mathbb{C}_{0}, \quad \forall a_{k, i} \in \mathbb{A}_{k}:\left(\boldsymbol{x}_{i},\left(a_{k, i}, a_{-k, i}^{\star}\right)\right) \in \mathbb{C}_{i}
\end{gathered}
$$

We consider similar assumptions as for the deterministic case, taking into account that Assumptions 5.1-5.3 refer to $r_{k}\left(x_{i}, a_{i}, \boldsymbol{\sigma}_{i}, i\right)$ and $f\left(x_{i}, a_{i}, \boldsymbol{\theta}_{i}, i\right)$, instead of $r_{k}\left(x_{i}, a_{i}, i\right)$ and $f\left(x_{i}, a_{i}, i\right)$; and that Assumption 5.4 refers to the OCP (5.40) presented below.

\subsubsection{Open Loop analysis of SDPGs}

In this section we extend the analysis of Section 5.4. In particular, we give conditions for the constrained stochastic game (5.15) to be potential, and prove that it can be solved through a related OCP under such conditions. We start by formally introducing SDPGs.

Definition 5.5. Game (5.80) is called a SDPG if there is a function $J: \mathbb{X} \times \mathbb{A} \times \Sigma \times \mathbb{N} \rightarrow$ $\mathbb{R}$ that satisfies the following condition $\forall k \in \mathcal{N}$ :

$$
\begin{gathered}
\sum_{i=0}^{\infty} \gamma^{i}\left(r_{k}\left(\boldsymbol{x}_{k, i}, a_{k, i}, a_{-k, i}, \boldsymbol{\sigma}_{k, i}, i\right)-r_{k}\left(\boldsymbol{x}_{k, i}, u_{k, i}, a_{-k, i}, \boldsymbol{\sigma}_{k, i}, i\right)\right) \\
=\sum_{i=0}^{\infty} \gamma^{i}\left(J\left(\boldsymbol{x}_{i}, a_{k, i}, a_{-k, i}, \boldsymbol{\sigma}_{i}, i\right)-J\left(\boldsymbol{x}_{i}, u_{k, i}, a_{-k, i}, \boldsymbol{\sigma}_{i}, i\right)\right) \\
\quad \forall \boldsymbol{x}_{k, i} \in \mathbb{X}_{k}, \quad \forall a_{k, i}, u_{k, i} \in \mathbb{A}_{k}, \quad \forall a_{-k, i} \in \mathbb{A}_{-k}
\end{gathered}
$$

Introduce a stochastic OCP:

$$
\begin{aligned}
\underset{\left\{a_{i}\right\} \in \prod_{i=0}^{\infty} \mathbb{A}}{\operatorname{maximize}} & \mathbb{E}\left[\sum_{i=0}^{\infty} \gamma^{i} J\left(\boldsymbol{x}_{i}, a_{i}, \boldsymbol{\sigma}_{i}, i\right)\right] \\
\mathcal{P}_{3}: \quad & \boldsymbol{x}_{i+1}=f\left(\boldsymbol{x}_{i}, a_{i}, \boldsymbol{\theta}_{i}, i\right), \quad \boldsymbol{x}_{0}=x_{0} \text { given } \\
& g\left(\boldsymbol{x}_{i}, a_{i}, i\right) \leq 0 \\
& \boldsymbol{\theta}_{i} \sim p_{\boldsymbol{\theta}}\left(\cdot \mid \boldsymbol{x}_{i}, a_{i}, i\right), \quad \boldsymbol{\sigma}_{i} \sim p_{\boldsymbol{\sigma}}\left(\cdot \mid \boldsymbol{x}_{i}, a_{i}, i\right)
\end{aligned}
$$

where $J$ denotes the instantaneous random reward; $\left\{\sigma_{i}\right\}_{i=0}^{\infty}$ is a sequence of random variables, such that $\sigma_{i}$ is independent of $\sigma_{j}$ given $\boldsymbol{x}_{i}$ for $0 \leq j<i$; and the rest of variables are the same as for game (5.80). Again, the expectation in (5.83) is taken at every time step over the state transition and reward distributions. 
The following result extends Theorem 5.1 to SDPG.

Theorem 5.2. Let Assumptions 5.2-5.4 referred to problems (5.80) and (5.83) hold. Let all agents' reward functions satisfy the following conditions $\forall k, j \in \mathcal{N}$ :

$$
\begin{array}{r}
\mathbb{E}\left[\nabla_{a_{j, i}}\left[\nabla_{x_{k, i}} r_{k}\left(x_{k, i}, a_{i}, \boldsymbol{\sigma}_{k, i}, i\right)\right]\right]=\mathbb{E}\left[\nabla_{a_{k, i}}\left[\nabla_{x_{j, i}} r_{j}\left(x_{j, i}, a_{i}, \boldsymbol{\sigma}_{j, i}, i\right)\right]\right] \\
\mathbb{E}\left[\nabla_{x_{j, i}}\left[\nabla_{x_{k, i}} r_{k}\left(x_{k, i}, a_{i}, \boldsymbol{\sigma}_{k, i}, i\right)\right]\right]=\mathbb{E}\left[\nabla_{x_{k, i}}\left[\nabla_{x_{j, i}} r_{j}\left(x_{j, i}, a_{i}, \boldsymbol{\sigma}_{j, i}, i\right)\right]\right] \\
\mathbb{E}\left[\nabla_{a_{j, i}}\left[\nabla_{a_{k, i}} r_{k}\left(x_{k, i}, a_{i}, \boldsymbol{\sigma}_{k, i}, i\right)\right]\right]=\mathbb{E}\left[\nabla_{a_{k, i}}\left[\nabla_{a_{j, i}} r_{j}\left(x_{j, i}, a_{i}, \boldsymbol{\sigma}_{j, i}, i\right)\right]\right]
\end{array}
$$

where the expected value is taken component-wise. Then, problem (5.80) is a SDPG that has an EOL-NE equal to the solution of OCP (5.83). In particular, the function

$$
\begin{aligned}
J\left(x_{i}, a_{i}, \boldsymbol{\sigma}_{i}, i\right)=\int_{0}^{1} \sum_{k \in \mathcal{N}} & \left(\sum_{m \in \mathcal{X}_{k}} \frac{\partial r_{k}\left(\eta_{k}(z), a_{k, i}, a_{-k, i}, \boldsymbol{\sigma}_{i}, i\right)}{\partial x_{k, i}(m)} \frac{d \eta_{k, m}(z)}{d z}\right. \\
& \left.+\sum_{\ell=1}^{A_{k}} \frac{\partial r_{k}\left(x_{k, i}, \xi_{k}(z), a_{-k, i}, \boldsymbol{\sigma}_{i}, i\right)}{\partial a_{k, i}(\ell)} \frac{d \xi_{k, \ell}(z)}{d z}\right) d z
\end{aligned}
$$

is an instantaneous reward function for the $O C P$, where $\eta(z) \triangleq\left(\eta_{k}(z)\right)_{k \in \mathcal{N}}$ and $\xi(z) \triangleq$ $\left(\xi_{k}(z)\right)_{k \in \mathcal{N}}$ are piecewise smooth paths in $\mathbb{X}$ and $\mathbb{A}$, respectively, with components $\eta_{k}(z) \triangleq$ $\left(\eta_{k, m}(z)\right)_{m \in \mathcal{X}_{k}}$ and $\xi_{k}(z) \triangleq\left(\xi_{k, \ell}(z)\right)_{\ell=1}^{A_{k}}$, such that the initial and final state-action conditions are given by $(\eta(0), \xi(0))$ and $\left(\eta_{k}(1)=x_{k, i}, \forall k \in \mathcal{N}, \xi(1)=a_{i}\right)$, respectively.

Proof: The proof is similar to Theorem 5.1. First, we derive two sets of KKT optimality conditions. For game (5.80), each agent's Lagrangian is given for some given $x_{0}$ and $\forall k \in \mathcal{N}$ by

$$
\begin{array}{r}
\mathcal{L}_{k}\left(\boldsymbol{x}_{0: \infty}, a_{0: \infty}, \boldsymbol{\sigma}_{k, 0: \infty}, \boldsymbol{\theta}_{0: \infty}, \boldsymbol{\lambda}_{k, 0: \infty}, \boldsymbol{\mu}_{k, 0: \infty}\right)=\mathbb{E}\left[\sum_{i=0}^{\infty} \gamma^{i} r_{k}\left(\boldsymbol{x}_{k, i}, a_{i}, \boldsymbol{\sigma}_{k, i}, i\right)\right. \\
\left.+\boldsymbol{\lambda}_{k, i}^{\top}\left(f\left(\boldsymbol{x}_{i}, a_{i}, \boldsymbol{\theta}_{i}, i\right)-\boldsymbol{x}_{i+1}\right)+\boldsymbol{\mu}_{k, i}^{\top} g\left(\boldsymbol{x}_{i}, a_{i}, i\right)\right]
\end{array}
$$

where $\boldsymbol{\lambda}_{k, i} \in \mathbb{R}^{S}$ and $\boldsymbol{\mu}_{k, i} \in \mathbb{R}^{C}$ are the corresponding vectors of multipliers at time $i$. Note that $\boldsymbol{\lambda}_{k, i}$ and $\boldsymbol{\mu}_{k, i}$ are random since they apply to the state transition and constraint functions, which depend on $\boldsymbol{\theta}_{i}$ and $\boldsymbol{x}_{i}$. Note that, although the state transitions are random, at any given time-step, the state is already known when we evaluate the random state transition and reward. In other words, at time $i$, the future state $\boldsymbol{x}_{i+1}$ is a random variable but the current state $\boldsymbol{x}_{i}=x_{i}$ and, hence, the multiplier 
$\boldsymbol{\mu}_{k, i}=\mu_{k, i}$ are not. Introduced the shorthand:

$$
\begin{aligned}
\Phi_{k}\left(x_{i}, \boldsymbol{x}_{i+1}, a_{i}, \boldsymbol{\sigma}_{k, i}, \boldsymbol{\theta}_{i}, i, \boldsymbol{\lambda}_{k, i}, \mu_{k, i}\right) \triangleq \mathbb{E}\left[\gamma^{i} r_{k}\left(x_{k, i}, a_{i}, \boldsymbol{\sigma}_{k, i}, i\right)\right. \\
\left.+\boldsymbol{\lambda}_{k, i}^{\top}\left(f\left(x_{i}, a_{i}, \boldsymbol{\theta}_{i}, i\right)-\boldsymbol{x}_{i+1}\right)+\mu_{k, i}^{\top} g\left(x_{i}, a_{i}, i\right)\right]
\end{aligned}
$$

where the expected value is taken only over the distributions of $\boldsymbol{\sigma}_{k, i}$ and $\boldsymbol{\theta}_{i}$ (since for any given value $\boldsymbol{\theta}_{i}=\theta_{i}$, the variables $\boldsymbol{x}_{i+1}=x_{i+1}$ and $\boldsymbol{\lambda}_{k, i}=\lambda_{k, i}$ are given too). In a manner similar to (5.53)-(5.54), we can derive the stochastic Euler-Lagrange equations and transversality conditions for (5.89) and obtain the KKT conditions for every agent $k \in \mathcal{N}$ and all time steps $i=1, \ldots, \infty$ :

$$
\begin{aligned}
& \mathbb{E}\left[\nabla_{x_{k, i}}\left[\gamma^{i} r_{k}\left(x_{k, i}, a_{i}, \boldsymbol{\sigma}_{k, i}, i\right)+\boldsymbol{\lambda}_{k, i}^{\top} f\left(x_{i}, a_{i}, \boldsymbol{\theta}_{i}, i\right)\right]\right] \\
& +\nabla_{x_{k, i}}\left[\mu_{k, i}^{\top} g\left(x_{i}, a_{i}, i\right)\right]-\lambda_{\mathcal{X}_{k}, i-1}=0_{S_{k}} \\
& \mathbb{E}\left[\nabla_{a_{k, i}}\left[\gamma^{i} r_{k}\left(x_{k, i}, a_{i}, \boldsymbol{\sigma}_{k, i}, i\right)+\boldsymbol{\lambda}_{k, i}^{\top} f\left(x_{i}, a_{i}, \boldsymbol{\theta}_{i}, i\right)\right]\right] \\
& +\nabla_{a_{k, i}}\left[\mu_{k, i}^{\top} g\left(x_{i}, a_{i}, i\right)\right]=0_{A_{k}} \\
& \lim _{i \rightarrow \infty} \mathbb{E}\left[\nabla_{x_{k, i}}\left[\gamma^{i} r_{k}\left(x_{k, i}, a_{i}, \boldsymbol{\sigma}_{k, i}, i\right)+\boldsymbol{\lambda}_{k, i}^{\top} f\left(x_{i}, a_{i}, \boldsymbol{\theta}_{i}, i\right)\right]\right] \\
& +\nabla_{x_{k, i}}\left[\mu_{k, i}^{\top} g\left(x_{i}, a_{i}, i\right)\right]=0_{S_{k}} \\
& \boldsymbol{x}_{i+1}=f\left(x_{i}, a_{i}, \boldsymbol{\theta}_{i}, i\right), \quad g\left(x_{i}, a_{i}, i\right) \leq 0_{C} \\
& \mu_{k, i} \leq 0_{C}, \quad \mu_{k, i}^{\top} g\left(x_{i}, a_{i}, i\right)=0
\end{aligned}
$$

where $\lambda_{\mathcal{X}_{k}, i-1}$ is given by (5.63) and known at time $i$, so it is a deterministic variable.

Now, we derive the Lagrangian for OCP (5.83):

$$
\begin{aligned}
\mathcal{L}^{\mathrm{OCP}}\left(\boldsymbol{x}_{0: \infty}, a_{0: \infty},\right. & \left.\boldsymbol{\sigma}_{k, 0: \infty}, \boldsymbol{\theta}_{0: \infty}, \boldsymbol{\beta}_{0: \infty}, \boldsymbol{\delta}_{0: \infty}\right)=\mathbb{E}\left[\sum_{i=0}^{\infty} \gamma^{i} J\left(\boldsymbol{x}_{i}, a_{i}, \boldsymbol{\sigma}_{k, i}, i\right)\right. \\
& \left.+\boldsymbol{\beta}_{i}^{\top}\left(f\left(\boldsymbol{x}_{i}, a_{i}, \boldsymbol{\theta}_{i}, i\right)-\boldsymbol{x}_{i+1}\right)+\boldsymbol{\delta}_{i}^{\top} g\left(\boldsymbol{x}_{i}, a_{i}, i\right)\right]
\end{aligned}
$$

where $\boldsymbol{\beta}_{i} \in \mathbb{R}^{S}$ and $\boldsymbol{\delta}_{i} \in \mathbb{R}^{C}$ are the corresponding random multipliers. Again, we can obtain the Euler-Lagrange and transversality first-order conditions from (5.95) and use them to derive the following KKT system of optimality conditions $\forall k \in \mathcal{N}$ and $i=1, \ldots, \infty$ :

$$
\begin{aligned}
\mathbb{E}\left[\nabla_{x_{k, i}}\left[\gamma^{i} J\left(x_{i}, a_{i}, \boldsymbol{\sigma}_{i}, i\right)+\boldsymbol{\beta}_{i}^{\top} f\left(x_{i}, a_{i}, \boldsymbol{\theta}_{i}, i\right)\right]\right] \\
+\nabla_{x_{k, i}}\left[\delta_{i}^{\top} g\left(x_{i}, a_{i}, i\right)\right]-\beta_{\mathcal{X}_{k}, i-1}=0_{S_{k}}
\end{aligned}
$$




$$
\begin{aligned}
\mathbb{E}\left[\nabla_{a_{k, i}}\left[\gamma^{i} J\left(x_{i}, a_{i}, \boldsymbol{\sigma}_{i}, i\right)+\boldsymbol{\beta}_{i}^{\top} f\left(x_{i}, a_{i}, \boldsymbol{\theta}_{i}, i\right)\right]\right] & \\
+\nabla_{a_{k, i}}\left[\delta_{i}^{\top} g\left(x_{i}, a_{i}, i\right)\right] & =0_{A_{k}} \\
\lim _{i \rightarrow \infty} \mathbb{E}\left[\nabla_{x_{k, i}}\left[\gamma^{i} J\left(x_{i}, a_{i}, \boldsymbol{\sigma}_{i}, i\right)+\boldsymbol{\beta}_{i}^{\top} f\left(x_{i}, a_{i}, \boldsymbol{\theta}_{i}, i\right)\right]\right] & \\
+\nabla_{x_{k, i}}\left[\delta_{i}^{\top} g\left(x_{i}, a_{i}, i\right)\right] & =0_{S_{k}} \\
\boldsymbol{x}_{i+1}=f\left(x_{i}, a_{i}, \boldsymbol{\theta}_{i}, i\right), \quad g\left(x_{i}, a_{i}, i\right) & \leq 0_{C} \\
\delta_{i} \leq 0_{C}, \quad \delta_{i}^{\top} g\left(x_{i}, a_{i}, i\right) & =0
\end{aligned}
$$

where $\beta_{\mathcal{X}_{k}, i-1}$ is known (at time $i$ ) and given by (5.70).

By comparing (5.90)-(5.94) and (5.96)-(5.100), we conclude that both KKT systems are equal if the following holds $\forall k \in \mathcal{N}$ and $i=1, \ldots, \infty$ :

$$
\begin{gathered}
\mathbb{E}\left[\nabla_{x_{k, i}} r_{k}\left(x_{k, i}, a_{i}, \boldsymbol{\sigma}_{k, i}, i\right)\right]=\mathbb{E}\left[\nabla_{x_{k, i}} J\left(x_{i}, a_{i}, \boldsymbol{\sigma}_{i}, i\right)\right] \\
\mathbb{E}\left[\nabla_{a_{k, i}} r_{k}\left(x_{k, i}, a_{i}, \boldsymbol{\sigma}_{k, i}, i\right)\right]=\mathbb{E}\left[\nabla_{a_{k, i}} J\left(x_{i}, a_{i}, \boldsymbol{\sigma}_{i}, i\right)\right] \\
\boldsymbol{\lambda}_{k, i}=\boldsymbol{\beta}_{i}, \quad \mu_{k, i}=\delta_{i}
\end{gathered}
$$

These are the stochastic extension of (5.71)-(5.73). Thus, we can apply the same reasoning as for the deterministic case. We repeat the argument briefly here for completeness. Under Assumptions 5.3-5.4, condition (5.103) ensures that the optimal solution of the OCP also satisfies the KKT system of the game. Hence, using the same argument as for the deterministic case, we conclude that the solution of the OCP is also a EOL-NE of the game. In order to obtain $J$, introduce the vector field

$$
F\left(x_{i}, a_{i}, \boldsymbol{\sigma}_{i}, i\right) \triangleq \nabla_{\left(x_{i}, a_{i}\right)} J\left(x_{i}, a_{i}, \boldsymbol{\sigma}_{i}, i\right)
$$

From Lemma 5.1, we know that $F$ is conservative, so that Conditions (5.101)-(5.102) are equivalent to $(5.84)-(5.86)$. Since $J$ is the potential of $F$, we can calculate $J$ through the line integral (5.87). Moreover, it is straightforward to see that Lemma 5.3 holds, so that $J$ satisfies a relationship similar to (5.34). Thus, we conclude that game (5.80) is a SDPG.

In the following section, we extend these results to a CL analysis when the agents use parametric policies. 


\subsection{Closed loop stochastic dynamic potential games with parametric policies}

In this section, we consider a CL analysis of SDPGs. The solution in which we are interested is not a predefined sequence of actions anymore but a mapping from states to actions, which we refer as feedback policy or simply policy. In other words, an OL policy is a function of time while a CL policy is a function of the system state. Thus, an optimal policy provides the optimal action to be taken by the every agent when the system is at any state. We are interested in policies that can be (perfectly) expressed as a function of a finite-dimensional parameter vector, which we refer as parametric policies. This constraint in the policy space makes derivations simpler, so that we are able to extend the results from the OL analysis and prove that a CL equilibrium for the game can be obtained by solving a related OCP. Specifically, we show that the optimal parametric policy found for the OCP is a CL Nash equilibrium (to be formally defined below) for the game if the agents are constrained to play parametric policies. This parametric policy assumption has been used by a large body of methods for approximating the solution of OCPs with large state-action sets (see, e.g., $[207,150,186,35,226,306,313,315,168,120,255])$, so that this extension to dynamic games should be useful too. When solving a practical problem, we remark that the policy parametric class should be rich enough to represent the complexities of the problem under study. Up to the best of our knowledge, there are no previous CL analysis of continuous potential games.

Similar to the OL case, we start introducing the CL setting for general games. Then, we show that when the games satisfy some potentiality condition, they can be solved through a related OCP.

\subsubsection{Problem setting for closed loop stochastic dynamic games}

Introduce the policy as a mapping from states to actions: $\pi: \mathbb{X} \rightarrow \mathbb{A}$; so that the action at time $i$ is obtained deterministically from the policy:

$$
a_{i}=\pi\left(x_{i}\right)
$$

Let $\pi_{k}: \mathbb{X} \rightarrow \mathbb{A}_{k}$ denote the policy of agent $k$, such that $a_{k, i}=\pi_{k}\left(x_{i}\right)$, and

$$
\pi \triangleq\left(\pi_{k}\right)_{k \in \mathcal{N}}
$$


Note that we are considering that each agent's policy could depend on the whole state vector. The reason is that defining $\mathbb{X}_{k}$ is a little bit more tricky now. Note that as long as one agent's reward depends on the policy of other agents, it will implicitly depend on their state components. Thus, for the sake of generality, we write that $\pi_{k}$ is a mapping from $\mathbb{X}$ to $\mathbb{A}_{k}$, although in practice it can be a mapping of a subset of $\mathbb{X}$ (later we will consider the special case where each agent's policy depends on a subset of components of the state vector and these subsets are disjoints for all agents). Let $\pi_{-k}: \mathbb{X} \rightarrow \mathbb{A}_{-k}$ denote the policy of all agents' policies except that of agent $k$. Then, by slightly abusing notation, we also write:

$$
\pi=\left(\pi_{k}, \pi_{-k}\right), \quad \forall k \in \mathcal{N}
$$

Let $\Omega_{k}$ be the policy space for agent $k$ and let $\Omega$ the policy space for all agents, such that $\pi_{k} \in \Omega_{k}, \pi \in \Omega$ and $\Omega \triangleq \prod_{k \in \mathcal{N}} \Omega_{k}$. Note that $\Omega(\mathbb{X})=\mathbb{A}$.

For simplicity, let us consider reward function, state transition function, constraint functions and probability distributions stationary. Then, the general CL stochastic dynamic game problem consists in finding the set of stationary policies that maximize the expected discounted cumulative reward for all $\boldsymbol{x}_{0}=x_{0} \in \mathbb{X}$ :

$$
\begin{aligned}
& \underset{\pi_{k} \in \Omega_{k}}{\operatorname{maximize}} \mathbb{E}\left[\sum_{i=0}^{\infty} \gamma^{i} r_{k}\left(\boldsymbol{x}_{i}, \pi_{k}\left(\boldsymbol{x}_{i}\right), \pi_{-k}\left(\boldsymbol{x}_{i}\right), \boldsymbol{\sigma}_{k, i}\right)\right] \\
& \mathcal{G}_{4}: \forall k \in \mathcal{N} \quad \text { s.t. } \quad \boldsymbol{x}_{i+1}=f\left(\boldsymbol{x}_{i}, \pi\left(\boldsymbol{x}_{i}\right), \boldsymbol{\theta}_{i}\right) \\
& g\left(\boldsymbol{x}_{i}, \pi\left(\boldsymbol{x}_{i}\right)\right) \leq 0 \\
& \boldsymbol{\theta}_{i} \sim p_{\boldsymbol{\theta}}\left(\cdot \mid \boldsymbol{x}_{i}, \pi\left(\boldsymbol{x}_{i}\right)\right), \quad \boldsymbol{\sigma}_{k, i} \sim p_{\boldsymbol{\sigma}_{k}}\left(\cdot \mid \boldsymbol{x}_{i}, \pi\left(\boldsymbol{x}_{i}\right)\right)
\end{aligned}
$$

where $r_{k}: \mathbb{X} \times \Omega \times \Sigma_{k} \rightarrow \mathbb{R}$ is the reward for agent $k$, and $\left\{\sigma_{k, i}\right\}_{i=1}^{\infty}$ and $\left\{\theta_{i}\right\}_{i=1}^{\infty}$ are sequences of i.i.d. random variables. Note that (5.108) is a multiobjective variational problem with design space $\Omega$ and objective space $\mathbb{R}^{N}$. In other words, the goal is to maximize a vector-valued functional, which is a mapping from a set of functions (i.e., feedback policies of the current state) to a real vector space (i.e., $\mathbb{R}^{N}$ ).

The standard approach for tackling CL dynamic games, when no explicit constrained are considered, is as follows (see, e.g., [104]). For simplicity, consider the following CL deterministic dynamic game:

$$
\begin{aligned}
\mathcal{G}_{5}: \forall k \in \mathcal{N} & \underset{\pi_{k} \in \Omega_{k}}{\operatorname{maximize}_{i=0}} \sum^{\infty} \gamma^{i} r_{k}\left(x_{i}, \pi_{k}\left(x_{i}\right), \pi_{-k}\left(x_{i}\right)\right) \\
\text { s.t. } & x_{i+1}=f\left(x_{i}, \pi\left(x_{i}\right)\right)
\end{aligned}
$$


The first of the standard approach consists in expressing each agent's policy in reduced form:

$$
\pi_{k}\left(x_{i}\right)=h\left(x_{i}, x_{i+1}, \pi_{-k}\left(x_{i}\right)\right)
$$

where we are implicitly assuming that such function $h: \mathbb{X} \times \mathbb{X} \times \prod_{j \neq k} \Omega_{j} \rightarrow \mathbb{A}$ exists, which as we have argued may not be the case if the state-transition function is not invertible. Next, $\pi_{k}$ is replaced with (5.110) in the reward:

$$
\begin{aligned}
r_{k}\left(x_{i}, \pi_{k}\left(x_{i}\right), \pi_{-k}\left(x_{i}\right)\right) & =r_{k}\left(x_{i}, h\left(x_{i}, x_{i+1}, \pi_{-k}\left(x_{i}\right)\right), \pi_{-k}\left(x_{i}\right)\right) \\
& =r_{k}^{\prime}\left(x_{i}, x_{i+1}, \pi_{-k}\left(x_{i}\right)\right)
\end{aligned}
$$

where the apostrophe in the reduced-form reward function $r_{k}^{\prime}: \mathbb{X} \times \mathbb{X} \rightarrow \mathbb{R}$ simply denotes that this is a different function from $r_{k}$. Then, an Euler equation is obtained for each $r_{k}^{\prime}$. When $r_{k}^{\prime}$ are concave for all agents and $\mathbb{X} \subseteq \mathbb{R}^{+}$(i.e., $\mathbb{X}=\left\{x_{i}: x_{i} \geq 0, x_{i} \in \mathbb{R}^{S}\right\}$ ), the reduced-form of the Euler and transversality equations become sufficient condition for equilibrium, as explained by [104, Theorem 4.2]. Hence, under this concavity and non-negative state assumptions, the standard method for finding an NE consists in guessing policies from the space of functions $\Omega$ and check whether any of them satisfies the Euler equations. Let us illustrate this procedure with a well known simple problem named "the great fish war" due to [160] (we follow [104, Sec. 4.3]).

Example 5.1. Let $x_{i}$ be the stock of fish at time $i$, in some fishing area. Suppose there are $N$ countries obtaining reward from fish consumption. More precisely, the countries aim to solve the following game:

$$
\begin{aligned}
\mathcal{G}_{6}: \forall k \in \mathcal{N} & \sum_{\pi_{k} \in \Omega_{k}}^{\infty} \gamma^{i} \log \left(\pi_{k}\left(x_{i}\right)\right) \\
\text { s.t. } & x_{i+1}=\left(x_{i}-\sum_{k \in \mathcal{N}} \pi_{k}\left(x_{i}\right)\right)^{\alpha} \\
& x_{i} \geq 0, \quad \pi_{k}\left(x_{i}\right) \geq 0, \quad i=0, \ldots, \infty
\end{aligned}
$$

where $x_{0} \geq 0$ and $0<\alpha<1$ are given. The action taken by any agent $k$ can be expressed in reduced form:

$$
\pi_{k}\left(x_{i}\right)=x_{i}-x_{i+1}^{1 / \alpha}-\sum_{j \in \mathcal{N}: j \neq k} \pi_{j}\left(x_{i}\right)
$$


So that the reward can be also expressed in reduced form as:

$$
r_{k}^{\prime}\left(x_{i}\right)=\log \left(x_{i}-x_{i+1}^{1 / \alpha}-\sum_{j \in \mathcal{N}: j \neq k} \pi_{j}\left(x_{i}\right)\right)
$$

Thus, the Euler equations for every agent $k \in \mathcal{N}$ and all $t=0, \ldots, \infty$ become:

$$
\frac{-x_{i}^{1 / \alpha-1} / \alpha}{x_{i-1}-x_{i}^{1 / \alpha}-\sum_{j \in \mathcal{N}: j \neq k} \pi_{j}\left(x_{i-1}\right)}+\gamma \frac{1-\sum_{j \in \mathcal{N}: j \neq k} \partial \pi_{j}\left(x_{i}\right) / \partial x_{i}}{x_{i}-x_{i+1}^{1 / \alpha}-\sum_{j \in \mathcal{N}: j \neq k} \pi_{j}\left(x_{i}\right)}=0
$$

Now, the standard method consists in trying to replace the policy with a family of parametric functions and checking whether such parametric policy satisfies (5.115) for some parameter vector. Let us try with policies that are linear mappings of the state, with linear parameter vector $u=\left(u_{k}\right)_{k \in \mathcal{N}}$ :

$$
\pi_{k}\left(x_{i}\right)=u_{k} x_{i}
$$

By replacing (5.116) in (5.115), we obtain the following set of equations:

$$
\alpha \gamma\left(1+u_{k}-\sum_{j \in \mathcal{N}} u_{j}\right)=1-\sum_{j \in \mathcal{N}} u_{j}, \quad \forall k \in \mathcal{N}
$$

Hence, the parameters can be obtained as:

$$
u_{k}=\frac{1-\alpha \gamma}{\alpha \gamma+N(1-\alpha \gamma)}, \quad \forall k \in \mathcal{N}
$$

Since $\alpha<1$ and $\gamma<1$, it is apparent that $u_{k}>0$ and the constraint $\pi_{k}\left(x_{i}\right) \geq 0$ holds for all $x_{i} \geq 0$. Moreover, since $\sum_{k \in \mathcal{N}} a_{k}<1$, we have that $x_{i+1} \geq 0$ for any $x_{0} \geq 0$. In addition, since $x_{i}$ is a resource and the actions must be positive, it follows that $\lim _{i \rightarrow \infty} x_{i}=0$ (there is no reason to save some resource). Therefore, the transversality condition for economic problems given by (5.57) holds. Since the rewards are concave, the states are non-negative and the linear policies with these coefficients satisfy the Euler and transversality equations, they constitute an equilibrium [104, Theorem 4.2].

We observe some handicaps in the standard approach illustrated with Example 5.1:

1. We have to be able to express the dynamics in reduced form.

2. We cannot handle constraints easily and we had to rely in an ad hoc argument. 
3. Guessing policies to check whether they satisfy the Euler and transversality conditions is a hard problem.

4. The reward functions have to be concave for all agents in order to guarantee that the policies that satisfy the conditions constitute an equilibrium.

In order to surmount these issues, we propose a practical method to approximate the Nash equilibrium, which consists in constraining the set of policies to some parametric family. Similar to previous cases, we will extend the Euler-Lagrange methodology to check whether the game is of the potential kind and, if so, to derive a related OCP whose solution is also an equilibrium of the game. In principle, our analysis will show that we can find such related OCP for any parametric family. However, we remark that when we choose a specific family to actually solve the game, it may happen that the optimal policies of the original game could not be expressed exactly as an instance of the parametric family of our preference. In this case, we will still find a solution, but this solution could be far from the solution of the original game. In other words, by imposing this parametric-policy constraint, we basically forget the original game and focus in solving a relaxed version. Fortunately, we can always choose a parametric family with arbitrary expressive capacity (e.g., a neural network with enough neurons in the hidden layers, a Chebyshev polynomial with enough coefficients, etc.) In this case, we can expect that the solution provided by the proposed relaxed game approximates arbitrarily close the solution of the original game. We proceed to introduce the approximate parametric game, the associated concept solution and the required assumptions.

Let $\pi_{u}$ denote a parametric policy with parameter $u \in \mathbb{U} \subseteq \mathbb{R}^{U}$, where $U$ is the length of the parameter vector (similar to Sec. 3.6.2). Introduce the set of parametric policies, $\Omega^{u}$, as a finite-dimensional function space:

$$
\Omega^{u} \triangleq\left\{\pi_{u}: u \in \mathbb{U}\right\}
$$

Note that for a given parameter $u \in \mathbb{U}$, the parametric policy is still a mapping from states to actions: $\pi(\cdot, u): \mathbb{X} \rightarrow \mathbb{A}$. Let $u_{k} \in \mathbb{U}_{k} \subseteq \mathbb{R}^{U_{k}}$ denote the parameter for the $k$-th agent's individual parametrized policy $\pi_{u_{k}}$, which lies in the finite-dimensional space $\Omega^{u_{k}}$ given by:

$$
\Omega^{u_{k}} \triangleq\left\{\pi_{u_{k}}: u_{k} \in \mathbb{U}_{k}\right\}
$$


such that $\Omega^{u} \triangleq \prod_{k \in \mathcal{N}} \Omega^{u_{k}}, \mathbb{U} \triangleq \prod_{k \in \mathcal{N}} \mathbb{U}_{k}, U \triangleq \sum_{k \in \mathcal{N}} U_{k}$, and

$$
\begin{aligned}
u & \triangleq\left(u_{k}\right)_{k \in \mathcal{N}} \\
\pi_{u}\left(x_{i}\right) & \triangleq\left(\pi_{u_{k}}\left(x_{i}\right)\right)_{k \in \mathcal{N}}
\end{aligned}
$$

Let $u_{-k}$ denote the parameters of all agents except that of agent $k$ :

$$
u_{-k} \triangleq\left(u_{j}\right)_{j \in \mathcal{N}, j \neq k}
$$

Although slightly abusing notation, we sometimes find convenient to write

$$
\begin{aligned}
u & =\left(u_{k}, u_{-k}\right) \\
\pi_{u}\left(x_{i}\right) & =\left(\pi_{u_{k}}\left(x_{i}\right), \pi_{u_{-k}}\left(x_{i}\right)\right), \quad \forall x_{i} \in \mathbb{X}
\end{aligned}
$$

In addition, we will find useful to refer to the $\ell$-th components of $u_{k}$ as $u_{k}(\ell)$, such that

$$
u_{k} \triangleq\left(u_{k}(\ell)\right)_{\ell=1}^{U_{k}}
$$

If we constraint the individual policies of problem (5.108) to lie in the finitedimensional parametric set $\Omega^{u_{k}}$, then game (5.108) is transformed into a multiobjective OCP with design space $\mathbb{U}$ :

$$
\begin{aligned}
& \underset{u_{k} \in \mathbb{U}_{k}}{\operatorname{maximize}} \mathbb{E}\left[\sum_{i=0}^{\infty} \gamma^{i} r_{k}\left(\boldsymbol{x}_{i}, \pi_{u_{k}}\left(\boldsymbol{x}_{i}\right), \pi_{u_{-k}}\left(\boldsymbol{x}_{i}\right), \boldsymbol{\sigma}_{k, i}\right)\right] \\
& \mathcal{G}_{5}: \forall k \in \mathcal{N} \\
& \text { s.t. } \quad \boldsymbol{x}_{i+1}=f\left(\boldsymbol{x}_{i}, \pi_{u}\left(\boldsymbol{x}_{i}\right), \boldsymbol{\theta}_{i}\right) \\
& g\left(\boldsymbol{x}_{i}, \pi_{u}\left(\boldsymbol{x}_{i}\right)\right) \leq 0 \\
& \boldsymbol{\theta}_{i} \sim p_{\boldsymbol{\theta}}\left(\cdot \mid \boldsymbol{x}_{i}, \pi_{u}\left(\boldsymbol{x}_{i}\right)\right), \quad \boldsymbol{\sigma}_{k, i} \sim p_{\boldsymbol{\sigma}_{k}}\left(\cdot \mid \boldsymbol{x}_{i}, \pi_{u}\left(\boldsymbol{x}_{i}\right)\right)
\end{aligned}
$$

The solution concept of game (5.127) in which we are interested is the parametric closed-loop Nash equilibrium (PCL-NE), defined as follows.

Definition 5.6 (Parametric Closed Loop Nash Equilibrium). A parametric closed-loop Nash-Equilibrium (PCL-NE) of game (5.127) is a parameter vector $u^{\star}=\left(u_{k}^{\star}, u_{-k}^{\star}\right)$ that satisfies the following condition for all $\boldsymbol{x}_{0}=x_{0} \in \mathbb{X}$ and for every agent $k \in \mathcal{N}$ :

$$
\mathbb{E}\left[\sum_{i=0}^{\infty} \gamma^{i} r_{k}\left(\boldsymbol{x}_{i}, \pi_{u_{k}^{\star}}\left(\boldsymbol{x}_{i}\right), \pi_{u_{-k}^{\star}}\left(\boldsymbol{x}_{i}\right), \boldsymbol{\sigma}_{k, i}\right)\right]
$$




$$
\begin{aligned}
& \geq \mathbb{E}\left[\sum_{i=0}^{\infty} \gamma^{i} r_{k}\left(\boldsymbol{x}_{i}, \pi_{u_{k}}\left(\boldsymbol{x}_{i}\right), \pi_{u_{-k}^{\star}}\left(\boldsymbol{x}_{i}\right), \boldsymbol{\sigma}_{k, i}\right)\right] \\
& \forall u_{k} \in \mathbb{U}_{k}:\left(\boldsymbol{x}_{i},\left(\pi_{u_{k}}\left(\boldsymbol{x}_{i}\right), \pi_{u_{-k}^{\star}}\left(\boldsymbol{x}_{i}\right)\right)\right) \in \mathbb{C}_{i}
\end{aligned}
$$

We consider similar assumptions as for the OL stochastic case, but taking into account that the functions depend on parameter $u$.

Assumption 5.5 (Replaces Assumption 5.1). The reward functions $r_{k}$ are twice continuously differentiable in $\mathbb{X} \times \mathbb{U}, \forall k \in \mathcal{N}$.

Assumption 5.6 (Replaces Assumption 5.2). The state and parameter sets, $\mathbb{X}$ and $\mathbb{U}$, are open and convex.

Assumption 5.7 (Replaces Assumption 5.3). The state-transition function $f$ and the constraints $g$ are continuously differentiable in $\mathbb{X} \times \mathbb{U}$ and satisfy some regularity conditions.

\subsubsection{Closed loop analysis of SDPGs with parametric policies}

In this section we extend the OL analysis of previous sections, giving conditions for the CL problem (5.127) to be a potential game, so that it can be solved through a related OCP. Parametric CL stochastic-dynamic-potential-games (CL-SDPG) can be defined very similar to the OL case (see Definition 5.5); the only difference is that we have to consider the variation in the individual policy parameter, rather than in the individual action sequence.

Definition 5.7. Given a policy family $\pi_{u} \in \Omega^{u}$, game (5.127) is called a CL-SDPG if there is a function $J: \mathbb{X} \times \mathbb{U} \times \Sigma \rightarrow \mathbb{R}$ that satisfies the following condition $\forall k \in \mathcal{N}$ :

$$
\begin{gathered}
\sum_{i=0}^{\infty} \gamma^{i}\left(r_{k}\left(\boldsymbol{x}_{i}, \pi_{u_{k}}\left(\boldsymbol{x}_{i}\right), \pi_{u_{-k}}\left(\boldsymbol{x}_{i}\right), \boldsymbol{\sigma}_{k, i}\right)-r_{k}\left(\boldsymbol{x}_{i}, \pi_{u_{k}^{\prime}}\left(\boldsymbol{x}_{i}\right), \pi_{u_{-k}}\left(\boldsymbol{x}_{i}\right), \boldsymbol{\sigma}_{k, i}\right)\right) \\
\left.=\sum_{i=0}^{\infty} \gamma^{i}\left(J\left(\boldsymbol{x}_{i}, \pi_{u_{k}}\left(\boldsymbol{x}_{i}\right), \pi_{u_{-k}}\left(\boldsymbol{x}_{i}\right)\right), \boldsymbol{\sigma}_{i}\right)-J\left(\boldsymbol{x}_{i}, \pi_{u_{k}^{\prime}}\left(\boldsymbol{x}_{i}\right), \pi_{u_{-k}}\left(\boldsymbol{x}_{i}\right), \boldsymbol{\sigma}_{i}\right)\right) \\
\forall \boldsymbol{x}_{i} \in \mathbb{X}, \quad \forall u_{k}, u_{k}^{\prime} \in \mathbb{U}_{k}
\end{gathered}
$$


Rewrite the single-objective OCP (5.83) in closed-loop formulation and assume the existence of its solution (see Lemma 5.5):

$$
\begin{aligned}
\underset{u \in \mathbb{U}}{\operatorname{maximize}} & \mathbb{E}\left[\sum_{i=0}^{\infty} \gamma^{i} J\left(\boldsymbol{x}_{i}, \pi_{u}\left(\boldsymbol{x}_{i}\right), \boldsymbol{\sigma}_{i}\right)\right] \\
\mathcal{P}_{4}: \quad & \boldsymbol{x}_{i+1}=f\left(\boldsymbol{x}_{i}, \pi_{u}\left(\boldsymbol{x}_{i}\right), \boldsymbol{\theta}_{i}\right) \\
& g\left(\boldsymbol{x}_{i}, \pi_{u}\left(\boldsymbol{x}_{i}\right)\right) \leq 0 \\
& \boldsymbol{\theta}_{i} \sim p_{\boldsymbol{\theta}}\left(\cdot \mid \boldsymbol{x}_{i}, \pi_{u}\left(\boldsymbol{x}_{i}\right)\right), \quad \boldsymbol{\sigma}_{i} \sim p_{\boldsymbol{\sigma}}\left(\cdot \mid \boldsymbol{x}_{i}, \pi_{u}\left(\boldsymbol{x}_{i}\right)\right)
\end{aligned}
$$

Assumption 5.8. OCP (5.130) has a nonempty solution set.

The following result extends Theorem 5.2 to CL-SDPG.

Theorem 5.3. Let Assumptions 5.5-5.8 hold. Let all agents' reward functions satisfy the following conditions $\forall k, j \in \mathcal{N}$ :

$$
\begin{aligned}
\mathbb{E}\left[\nabla_{u_{j}}\left[\nabla_{x_{i}} r_{k}\left(x_{i}, \pi_{u}\left(x_{i}\right), \boldsymbol{\sigma}_{k, i}\right)\right]\right] & =\mathbb{E}\left[\nabla_{u_{k}}\left[\nabla_{x_{i}} r_{j}\left(x_{i}, \pi_{u}\left(x_{i}\right), \boldsymbol{\sigma}_{j, i}\right)\right]\right] \\
\mathbb{E}\left[\nabla_{x_{i}}\left[\nabla_{x_{i}} r_{k}\left(x_{i}, \pi_{u}\left(x_{i}\right), \boldsymbol{\sigma}_{k, i}\right)\right]\right] & =\mathbb{E}\left[\nabla_{x_{i}}\left[\nabla_{x_{i}} r_{j}\left(x_{i}, \pi_{u}\left(x_{i}\right), \boldsymbol{\sigma}_{j, i}\right)\right]\right] \\
\mathbb{E}\left[\nabla_{u_{j}}\left[\nabla_{u_{k}} r_{k}\left(x_{i}, \pi_{u}\left(x_{i}\right), \boldsymbol{\sigma}_{k, i}\right)\right]\right] & =\mathbb{E}\left[\nabla_{u_{k}}\left[\nabla_{u_{j}} r_{j}\left(x_{i}, \pi_{u}\left(x_{i}\right), \boldsymbol{\sigma}_{j, i}\right)\right]\right]
\end{aligned}
$$

where the expected value is taken component-wise. Then, game (5.127) is a CL-SDPG that has a PCL-NE equal to the solution of the OCP (5.130). In particular, the function

$$
\begin{gathered}
J\left(x_{i}, \pi_{u}\left(x_{i}\right), \boldsymbol{\sigma}_{i}\right)=\int_{0}^{1} \sum_{k \in \mathcal{N}}\left(\sum_{m=1}^{S} \frac{\partial r_{k}\left(\eta(z), \pi_{u_{k}}(\eta(z)), \pi_{u_{-k}}(\eta(z)), \boldsymbol{\sigma}_{k, i}\right)}{\partial x_{k, i}(m)} \frac{d \eta_{m}(z)}{d z}\right. \\
\left.+\sum_{\ell=1}^{U_{k}} \frac{\partial r_{k}\left(x_{i}, \pi_{\xi_{k}(z)}\left(x_{i}\right), \pi_{u_{-k}}\left(x_{i}\right), \boldsymbol{\sigma}_{k, i}\right)}{\partial u_{k}(\ell)} \frac{d \xi_{k, \ell}(z)}{d z}\right) d z
\end{gathered}
$$

is an instantaneous reward function for the $O C P$, where $\eta(z) \triangleq\left(\eta_{m}(z)\right)_{m=1}^{S}$ and $\xi(z) \triangleq\left(\xi_{k}(z)\right)_{k \in \mathcal{N}}$ are piecewise smooth paths in $\mathbf{X}$ and $\mathbb{U}$, respectively, the latter with components $\xi_{k}(z) \triangleq\left(\xi_{k, \ell}(z)\right)_{\ell=1}^{U_{k}}$, such that the initial and final state-action conditions are given by $(\eta(0), \xi(0))$ and $\left(\eta(1)=x_{i}, \xi(1)=u\right)$, respectively.

Proof: The proof follows the same template as Theorems 5.1 and 5.2, namely we show that conditions (5.131)-(5.133) guarantee that that the game satisfies (5.129) so that it is a CL-SDPG, and that any solution to the OCP (5.130) with objective given by (5.87) is also a PCL-NE of game (5.128). 
Let us build the KKT systems for the game and the OCP with parametric policies. For game (5.127), each agent's Lagrangian is given $\forall k \in \mathcal{N}$ by

$$
\begin{aligned}
& \mathcal{L}_{k}\left(\boldsymbol{x}_{0: \infty}, u, \boldsymbol{\sigma}_{k, 0: \infty}\right.\left.\boldsymbol{\theta}_{0: \infty}, \boldsymbol{\lambda}_{k, 0: \infty}, \boldsymbol{\mu}_{k, 0: \infty}\right)=\mathbb{E}\left[\sum_{i=0}^{\infty} \gamma^{i} r_{k}\left(\boldsymbol{x}_{i}, \pi_{u}\left(\boldsymbol{x}_{i}\right), \boldsymbol{\sigma}_{k, i}\right)\right. \\
&\left.+\boldsymbol{\lambda}_{k, i}^{\top}\left(f\left(\boldsymbol{x}_{i}, \pi_{u}\left(\boldsymbol{x}_{i}\right), \boldsymbol{\theta}_{i}\right)-\boldsymbol{x}_{i+1}\right)+\boldsymbol{\mu}_{k, i}^{\top} g\left(\boldsymbol{x}_{i}, \pi_{u}\left(\boldsymbol{x}_{i}\right)\right)\right]
\end{aligned}
$$

Introduce a shorthand for the instantaneous Lagrangian at time $i$ :

$$
\begin{aligned}
\Phi_{k}\left(x_{i}, \boldsymbol{x}_{i+1}, u, \boldsymbol{\sigma}_{k, i}, \boldsymbol{\theta}_{i}, \boldsymbol{\lambda}_{k, i}, \mu_{k, i}\right) \triangleq \mathbb{E}\left[\gamma^{i} r_{k}\left(x_{i}, \pi_{u}\left(x_{i}\right), \boldsymbol{\sigma}_{k, i}\right)\right. \\
\left.+\boldsymbol{\lambda}_{k, i}^{\top}\left(f\left(x_{i}, \pi_{u}\left(x_{i}\right), \boldsymbol{\theta}_{i}\right)-\boldsymbol{x}_{i+1}\right)+\mu_{k, i}^{\top} g\left(x_{i}, \pi_{u}\left(x_{i}\right)\right)\right]
\end{aligned}
$$

The discrete time stochastic Euler-Lagrange equations applied to each agent's Lagrangian are slightly different from the OL case, since we only take into account the variation with respect to the state:

$$
\begin{aligned}
& \mathbb{E}\left[\nabla_{x_{i}} \Phi_{k}\left(x_{i}, \boldsymbol{x}_{i+1}, u, \boldsymbol{\sigma}_{k, i}, \boldsymbol{\theta}_{i}, \boldsymbol{\lambda}_{k, i}, \mu_{k, i}\right)\right] \\
& \quad+\nabla_{x_{i}}\left[\Phi_{k}\left(x_{i-1}, x_{i}, u, \sigma_{k, i-1}, \theta_{i-1}, \lambda_{k, i-1}, \mu_{k, i-1}\right)\right]=0_{S}, \quad i=1, \ldots, \infty
\end{aligned}
$$

In addition, we have an optimality condition for the policy parameter $u_{k}$ :

$$
\mathbb{E}\left[\nabla_{u_{k}} \Phi_{k}\left(x_{i}, \boldsymbol{x}_{i+1}, u, \boldsymbol{\sigma}_{k, i}, \boldsymbol{\theta}_{i}, \boldsymbol{\lambda}_{k, i}, \mu_{k, i}\right)\right]=0_{U_{k}}
$$

From these first-order optimality conditions and the corresponding transversality condition, we obtain the KKT conditions for every agent $k \in \mathcal{N}$ and all time steps $i=1, \ldots, \infty$ :

$$
\begin{aligned}
& \mathbb{E}\left[\nabla_{x_{i}}\left[\gamma^{i} r_{k}\left(x_{i}, \pi_{u}\left(x_{i}\right), \boldsymbol{\sigma}_{k, i}\right)+\boldsymbol{\lambda}_{k, i}^{\top} f\left(x_{i}, \pi_{u}\left(x_{i}\right), \boldsymbol{\theta}_{i}\right)\right]\right] \\
&+\nabla_{x_{i}}\left[\mu_{k, i}^{\top} g\left(x_{i}, \pi_{u}\left(x_{i}\right)\right)\right]-\lambda_{k, i-1}=0_{S} \\
& \mathbb{E}\left[\nabla_{u_{k}}\left[\gamma^{i} r_{k}\left(x_{i}, \pi_{u}\left(x_{i}\right), \boldsymbol{\sigma}_{k, i}\right)+\boldsymbol{\lambda}_{k, i}^{\top} f\left(x_{i}, \pi_{u}\left(x_{i}\right), \boldsymbol{\theta}_{i}\right)\right]\right]+\nabla_{u_{k}}\left[\mu_{k, i}^{\top} g\left(x_{i}, \pi_{u}\left(x_{i}\right)\right)\right] \\
& \lim _{i \rightarrow \infty} \mathbb{E}\left[\nabla_{x_{i}}\left[\gamma^{i} r_{k}\left(x_{i}, \pi_{u}\left(x_{i}\right), \boldsymbol{\sigma}_{k, i}\right)+\boldsymbol{\lambda}_{k, i}^{\top} f\left(x_{i}, \pi_{u}\left(x_{i}\right), \boldsymbol{\theta}_{i}\right)\right]\right] \\
&+\nabla_{x_{i}}\left[\mu_{k, i}^{\top} g\left(x_{i}, \pi_{u}\left(x_{i}\right)\right)\right]=0_{S} \\
& \boldsymbol{x}_{i+1}=f\left(x_{i}, \pi_{u}\left(x_{i}\right), \boldsymbol{\theta}_{i}\right), \quad g\left(x_{i}, \pi_{u}\left(x_{i}\right)\right) \leq 0_{C} \\
& \mu_{k, i} \leq 0_{C}, \quad \mu_{k, i}^{\top} g\left(x_{i}, \pi_{u}\left(x_{i}\right)\right)=0
\end{aligned}
$$


where $\lambda_{k, i-1}$ is known at time $i$.

Now, we derive the KKT system of optimality conditions for the OCP (5.130). The Lagrangian for (5.130) is given by:

$$
\begin{array}{r}
\mathcal{L}^{\mathrm{OCP}}\left(\boldsymbol{x}_{0: \infty}, u, \boldsymbol{\sigma}_{k, 0: \infty}, \boldsymbol{\theta}_{0: \infty}, \boldsymbol{\beta}_{0: \infty}, \boldsymbol{\delta}_{0: \infty}\right)=\mathbb{E}\left[\sum_{i=0}^{\infty} \gamma^{i} J\left(\boldsymbol{x}_{i}, \pi_{u}\left(x_{i}\right), \boldsymbol{\sigma}_{i}\right)\right. \\
\left.+\boldsymbol{\beta}_{i}^{\top}\left(f\left(\boldsymbol{x}_{i}, \pi_{u}\left(x_{i}\right), \boldsymbol{\theta}_{i}\right)-\boldsymbol{x}_{i+1}\right)+\boldsymbol{\delta}_{i}^{\top} g\left(\boldsymbol{x}_{i}, \pi_{u}\left(x_{i}\right)\right)\right]
\end{array}
$$

where $\boldsymbol{\beta}_{i} \in \mathbb{R}^{S}$ and $\boldsymbol{\delta}_{i} \in \mathbb{R}^{C}$ are the corresponding multipliers, which are random variables since they depend on $\boldsymbol{x}_{i}$. Again, introduce a shorthand for the instantaneous Lagrangian:

$$
\begin{aligned}
\Phi^{\mathrm{OCP}}\left(x_{i}, \boldsymbol{x}_{i+1}, u, \boldsymbol{\sigma}_{i}, \boldsymbol{\theta}_{i}, \boldsymbol{\beta}_{i}, \delta_{i}\right) \triangleq \mathbb{E}\left[\gamma^{i} J\left(x_{i}, \pi_{u}\left(x_{i}\right), \boldsymbol{\sigma}_{i}\right)\right. \\
\left.+\boldsymbol{\beta}_{i}^{\top}\left(f\left(x_{i}, \pi_{u}\left(x_{i}\right), \boldsymbol{\theta}_{i}\right)-\boldsymbol{x}_{i+1}\right)+\delta_{i}^{\top} g\left(x_{i}, \pi_{u}\left(x_{i}\right)\right)\right]
\end{aligned}
$$

Taking the discrete time stochastic Euler-Lagrange equations, the transversality condition and the optimality condition from (5.145) with respect to the policy parameter, we obtain a KKT system of optimality conditions for $i=1, \ldots, \infty$ :

$$
\begin{aligned}
& \mathbb{E}\left[\nabla_{x_{i}}\left[\gamma^{i} J\left(x_{i}, \pi_{u}\left(x_{i}\right), \boldsymbol{\sigma}_{i}\right)+\boldsymbol{\beta}_{i}^{\top} f\left(x_{i}, \pi_{u}\left(x_{i}\right), \boldsymbol{\theta}_{i}\right)\right]\right] \\
&+\nabla_{x_{i}}\left[\delta_{i}^{\top} g\left(x_{i}, \pi_{u}\left(x_{i}\right)\right)\right]-\beta_{i-1}=0_{S} \\
& \mathbb{E}\left[\nabla_{u_{k}}\left[\gamma^{i} J\left(x_{i}, \pi_{u}\left(x_{i}\right), \boldsymbol{\sigma}_{i}\right)+\boldsymbol{\beta}_{i}^{\top} f\left(x_{i}, \pi_{u}\left(x_{i}\right), \boldsymbol{\theta}_{i}\right)\right]\right] \\
&+\nabla_{u_{k}}\left[\delta_{i}^{\top} g\left(x_{i}, \pi_{u}\left(x_{i}\right)\right)\right]=0_{U_{k}}, \forall k \in \mathcal{N} \\
& \lim _{i \rightarrow \infty} \mathbb{E}\left[\nabla_{x_{i}}\left[\gamma^{i} J\left(x_{i}, \pi_{u}\left(x_{i}\right), \boldsymbol{\sigma}_{i}\right)+\boldsymbol{\beta}_{i}^{\top} f\left(x_{i}, \pi_{u}\left(x_{i}\right), \boldsymbol{\theta}_{i}\right)\right]\right] \\
&+\nabla_{x_{i}}\left[\delta_{i}^{\top} g\left(x_{i}, \pi_{u}\left(x_{i}\right)\right)\right]=0_{S} \\
& \boldsymbol{x}_{i+1}=f\left(x_{i}, \pi_{u}\left(x_{i}\right), \boldsymbol{\theta}_{i}\right), \quad g\left(x_{i}, \pi_{u}\left(x_{i}\right)\right) \leq 0_{C} \\
& \delta_{i} \leq 0_{C}, \quad \delta_{i}^{\top} g\left(x_{i}, \pi_{u}\left(x_{i}\right)\right)=0
\end{aligned}
$$

where $\beta_{i-1}$ is known at time $i$.

By comparing (5.139)-(5.143) and (5.146)-(5.150), we conclude that both KKT systems are equal if the following holds $\forall k \in \mathcal{N}$ and $i=1, \ldots, \infty$ :

$$
\begin{aligned}
\mathbb{E}\left[\nabla_{x_{i}} r_{k}\left(x_{i}, \pi_{u}\left(x_{i}\right), \boldsymbol{\sigma}_{k, i}\right)\right] & =\mathbb{E}\left[\nabla_{x_{i}} J\left(x_{i}, \pi_{u}\left(x_{i}\right), \boldsymbol{\sigma}_{i}\right)\right] \\
\mathbb{E}\left[\nabla_{u_{k}} r_{k}\left(x_{i}, \pi_{u}\left(x_{i}\right), \boldsymbol{\sigma}_{k, i}\right)\right] & =\mathbb{E}\left[\nabla_{u_{k}} J\left(x_{i}, \pi_{u}\left(x_{i}\right), \boldsymbol{\sigma}_{i}\right)\right]
\end{aligned}
$$




$$
\boldsymbol{\lambda}_{k, i}=\boldsymbol{\beta}_{i}, \quad \mu_{k, i}=\delta_{i}
$$

We repeat the same argument as for the OL case. From conditions (5.151)-(5.152), we conclude that game (5.127) is a CL-SDPG. Then, under Assumptions 5.3-5.4, we can see that the optimal solution of the OCP is also a PCL-NE of the CL-SDPG. Now, introduce the vector field

$$
F\left(x_{i}, u, \boldsymbol{\sigma}_{i}\right) \triangleq \nabla_{\left(x_{i}, u\right)} J\left(x_{i}, \pi_{u}\left(x_{i}\right), \boldsymbol{\sigma}_{i}\right)
$$

Since $F$ is conservative by construction (Lemma 5.1), conditions (5.151)-(5.152) are equivalent to (5.131)-(5.133) and we can calculate the potential $J$ through the line integral (5.134).

Under Assumptions 5.5-5.8, Theorem 5.3 proves that any deterministic or stochastic policy that is solution to OCP (5.130) is also a PCL-NE of game (5.127). From optimal control theory we know that under mild conditions there exists at least one deterministic policy that is solution to the OCP (see Lemma 5.5). We remark that there may be other equilibrium points for the game, beyond the OCP solutions, which correspond to the case where the dual variables are different for different agents.

In the following example, we use our approach to solve the war fish problem introduced in Example 5.1 and compare the result with the standard approach.

Example 5.2. Consider the war fish problem described in Example 5.1. In order to use our approach, we replace the generic policy with the specific policy mapping of our preference. We choose the linear mapping to be able to compare the results with those of the standard approach. Thus, we have the following problem:

$$
\begin{aligned}
& \underset{\mathcal{G}_{7}: \forall k \in \mathcal{N}}{\operatorname{maximize}_{\pi_{k} \in \Omega_{k}}} \quad \sum_{i=0}^{\infty} \gamma^{i} \log \left(u_{k} x_{i}\right) \\
\text { s.t. } & x_{i+1}=\left(x_{i}-\sum_{k \in \mathcal{N}} u_{k} x_{i}\right)^{\alpha} \\
& x_{i} \geq 0, \quad \pi_{k}\left(x_{i}\right) \geq 0, \quad i=0, \ldots, \infty
\end{aligned}
$$

Let us verify conditions (5.151)-(5.152). For all $k, j \in \mathcal{N}$ we have:

$$
\begin{aligned}
r_{k}\left(x_{i}, \pi_{u}\left(x_{i}\right)\right) & =\log \left(u_{k} x_{i}\right) \\
\nabla_{x_{i}} r_{k}\left(x_{i}, \pi_{u}\left(x_{i}\right)\right) & =\frac{1}{x_{i}} \\
\nabla_{u_{k}} r_{k}\left(x_{i}, \pi_{u}\left(x_{i}\right)\right) & =\frac{1}{u_{k}}
\end{aligned}
$$




$$
\begin{aligned}
\nabla_{x_{i}} \nabla_{x_{i}} r_{k}\left(x_{i}, \pi_{u}\left(x_{i}\right)\right) & =\nabla_{x_{i}} \nabla_{x_{i}} r_{j}\left(x_{i}, \pi_{u}\left(x_{i}\right)\right)=\frac{-1}{x_{i}^{2}} \\
\nabla_{u_{j}} \nabla_{x_{i}} r_{k}\left(x_{i}, \pi_{u}\left(x_{i}\right)\right) & =\nabla_{u_{k}} \nabla_{x_{i}} r_{j}\left(x_{i}, \pi_{u}\left(x_{i}\right)\right)=0 \\
\nabla_{u_{j}} \nabla_{u_{k}} r_{k}\left(x_{i}, \pi_{u}\left(x_{i}\right)\right) & =\nabla_{u_{k}} \nabla_{u_{j}} r_{j}\left(x_{i}, \pi_{u}\left(x_{i}\right)\right)=0
\end{aligned}
$$

Since conditions (5.151)-(5.152) hold, we conclude that the game is potential. By applying the line integral (5.134), we obtain:

$$
J\left(x_{i}, u_{i}\right)=\log \left(x_{i}\right)+\sum_{k \in \mathcal{N}} \log \left(u_{k}\right)
$$

Now, we can solve OCP (5.130) with potential function (5.162). For this particular problem, it is easy to solve the KKT system in closed form. Introduce a shorthand:

$$
\bar{u} \triangleq \sum_{k \in \mathcal{N}} u_{k}
$$

The Euler-Lagrange equation (5.146) for this problem becomes:

$$
\gamma^{i}+\beta_{i} \alpha x_{i}^{\alpha}(1-\bar{u})^{\alpha}-\beta_{i-1} x_{i}=0
$$

The optimality condition (5.147) with respect to the policy parameter becomes:

$$
\gamma^{i}-\beta_{i} \alpha x_{i}^{\alpha}(1-\bar{u})^{\alpha-1} u_{k}=0
$$

Let us solve for $\beta_{i}$ in (5.165):

$$
\beta_{i}=\frac{\gamma^{i}}{\alpha x_{i}^{\alpha}(1-\bar{u})^{\alpha-1} u_{k}}
$$

Replacing (5.166) and the state-transition dynamics in (5.164), we obtain the following set of equations:

$$
\alpha \gamma\left(1+u_{k}-\bar{u}\right)=1-\bar{u}, \quad \forall k \in \mathcal{N}
$$

Hence, the parameters can be obtained as:

$$
u_{k}=\frac{1-\alpha \gamma}{\alpha \gamma+N(1-\alpha \gamma)}, \quad \forall k \in \mathcal{N}
$$

This is exactly the same solution that we obtained in Example 5.1 with the standard approach. We remark that for the standard approach, we were able to obtain the 
policy parameters since we put the correct parametric form of the policy in the Euler equation. If we had used another parametric family without a linear term, the Euler equations (5.115) might have no solution and we would have got stuck. In contrast, with our approach, we could freely choose any other form of the parametric policy, and always solve the KKT system of the approximate game. Broadly speaking, we can say that the more expressive the parametric family, the more likely that the optimal policy of the original game will be accurately approximated by the optimal solution of the approximate game.

The main point of Theorem 5.3 is that, once we have the potential function, we can formulate and solve the related OCP for any specific parametric policy family. Thus, the main drawback with Theorem 5.3 is precisely that the potential function given by (5.134) is obtained through a line integral that depends on the parametric form of the policy, which could be involved (e.g., for a neural-network approximation). Thus, it would be more convenient to have a result similar to Lemma 5.3 for CL, in which we can find the potential function by inspection for any generic parametric policy, as a separable term of the agents' reward functions. To do so, we are going to assume the particular case where each agent's policy depends on disjoint subsets of components of the state vector. More formally, introduce $\mathcal{X}_{k}^{\pi}$ as the set of state-vector components that influence the policy of agent $k$ and introduce a new state vector, $x_{k}^{\pi}$, with components in $\mathcal{X}_{k}^{\pi}$ :

$$
x_{k, i}^{\pi} \triangleq\left(x_{i}(m)\right)_{m \in \mathcal{X}_{k}^{\pi}}
$$

Introduce also $x_{-k, i}^{\pi}$ as the vector of components that do not influence the policy of agent $k$ :

$$
x_{-k, i}^{\pi} \triangleq\left(x_{i}(l)\right)_{l \notin \mathcal{X}_{k}^{\pi}}
$$

In addition, introduce $\mathcal{X}_{k}^{r}$ as the set of components of the state vector that influence the reward of agent $k$ directly (not indirectly through any other agent's policy), and define the state vectors:

$$
\begin{aligned}
x_{k, i}^{r} & \triangleq\left(x_{i}(m)\right)_{m \in \mathcal{X}_{k}^{r}} \\
x_{-k, i}^{r} & \triangleq\left(x_{i}(l)\right)_{l \notin \mathcal{X}_{k}^{r}}
\end{aligned}
$$


Finally, introduce the union of these two subsets: $\mathcal{X}_{k}^{\Theta}=\mathcal{X}_{k}^{\pi} \cup \mathcal{X}_{k}^{r}$, and its corresponding vectors:

$$
\begin{aligned}
x_{k, i}^{\Theta} & \triangleq\left(x_{i}(m)\right)_{m \in \mathcal{X}_{k}^{\Theta}} \\
x_{-k, i}^{\Theta} & \triangleq\left(x_{i}(m)\right)_{m \notin \mathcal{X}_{k}^{\Theta}}
\end{aligned}
$$

Let us illustrate these definitions with a simple example.

Example 5.3. Consider a game with $N=2$ players, $S=4$ and rewards given by

$$
\begin{aligned}
& r_{1}\left(x_{i}, \pi_{u}\left(x_{i}\right)\right)=x_{i}(1)+x_{i}(2)+\pi_{u_{1}}\left(x_{i}(1), x_{i}(2)\right)-\pi_{u_{2}}\left(x_{i}(3)\right) \\
& r_{2}\left(x_{i}, \pi_{u}\left(x_{i}\right)\right)=x_{i}(3)+x_{i}(4)+\pi_{u_{2}}\left(x_{i}(3)\right)-\pi_{u_{1}}\left(x_{i}(1), x_{i}(2)\right)
\end{aligned}
$$

Then, we have

$$
\begin{aligned}
& \mathcal{X}_{1}^{\pi}=\{1,2\}, \quad \mathcal{X}_{1}^{r}=\{1,2\}, \quad \mathcal{X}_{1}^{\Theta}=\{1,2\} \\
& \mathcal{X}_{2}^{\pi}=\{3\}, \quad \mathcal{X}_{2}^{r}=\{3,4\}, \quad \mathcal{X}_{2}^{\Theta}=\{3,4\}
\end{aligned}
$$

Note that $\mathcal{X}_{1}^{\Theta} \cap \mathcal{X}_{2}^{\Theta}=\emptyset$.

Now, we make the assumption that all agents have disjoint state-component subsets (as in the previous example). This is convenient since it allows us to obtain the potential function by inspection, as formally expressed by the following theorem.

Theorem 5.4. Let Assumptions 5.5-5.8 hold. Assume that the subsets of statecomponents are disjoint for all players, i.e.:

$$
\mathcal{X}_{k}^{\Theta} \cap \mathcal{X}_{j}^{\Theta}=\emptyset, \forall(k, j) \in\{\mathcal{N} \times \mathcal{N}: k \neq j\}
$$

Suppose that the reward function of every agent can be expressed as the sum of a term that is common to all agents plus another term that depends neither on its own state-components, nor on its policy parameter, i.e.:

$$
\begin{aligned}
r_{k}\left(x_{k, i}^{r}, \pi_{u_{k}}\left(x_{k, i}^{\pi}\right), \pi_{u_{-k}}\left(x_{-k, i}^{\pi}\right), \boldsymbol{\sigma}_{k, i}\right)= & J\left(x_{i}, \pi_{u}\left(x_{i}\right), \boldsymbol{\sigma}_{i}\right) \\
& +\Theta_{k}\left(x_{-k, i}^{r}, \pi_{u_{-k}}\left(x_{-k, i}^{\pi}\right), \boldsymbol{\sigma}_{i}\right), \quad \forall k \in \mathcal{N}
\end{aligned}
$$

Hence, game (5.127) is a CL-SDPG, and the common term, $J$, is a potential function equivalent to (5.134). 
Proof: We can rewrite game (5.127) by making explicit that the actions result from the policy mapping, which yields an expression that reminds the OL problem but with extra constraints:

$$
\begin{aligned}
\underset{u_{k} \in \mathbb{U}_{k},\left\{\boldsymbol{a}_{k, i}\right\}_{0}^{\infty} \in \prod_{i=0}^{\infty} \mathbb{A}_{k}}{\operatorname{maximize}} & \mathbb{E}\left[\sum_{i=0}^{\infty} \gamma^{i} r_{k}\left(\boldsymbol{x}_{k, i}^{r}, \boldsymbol{a}_{k, i}, \boldsymbol{a}_{-k, i}, \boldsymbol{\sigma}_{k, i}\right)\right] \\
\text { s.t. } & \boldsymbol{a}_{k, i}=\pi_{u_{k}}\left(\boldsymbol{x}_{k, i}^{\pi}\right), \quad \boldsymbol{a}_{-k, i}=\pi_{u_{-k}}\left(\boldsymbol{x}_{-k, i}^{\pi}\right) \\
& \boldsymbol{x}_{i+1}=f\left(\boldsymbol{x}_{i}, \boldsymbol{a}_{i}, \boldsymbol{\theta}_{i}\right) \\
& g\left(\boldsymbol{x}_{i}, \boldsymbol{a}_{i}\right) \leq 0 \\
& \boldsymbol{\theta}_{i} \sim p_{\boldsymbol{\theta}}\left(\cdot \mid \boldsymbol{x}_{i}, \boldsymbol{a}_{i}\right), \quad \boldsymbol{\sigma}_{k, i} \sim p_{\boldsymbol{\sigma}_{k}}\left(\cdot \mid \boldsymbol{x}_{i}, \boldsymbol{a}_{i}\right)
\end{aligned}
$$

where, from (5.125), it is clear that:

$$
\boldsymbol{a}_{i} \triangleq\left(\boldsymbol{a}_{k, i}, \boldsymbol{a}_{-k, i}\right)=\pi_{u}\left(\boldsymbol{x}_{i}\right)
$$

Rewrite also OCP (5.130) with explicit dependence on the actions:

$$
\begin{aligned}
& \underset{u \in \mathbb{U},\left\{\boldsymbol{a}_{i}\right\}_{0}^{\infty} \in \prod_{i=0}^{\infty} \mathbb{A}}{\operatorname{maximize}} \mathbb{E}\left[\sum_{i=0}^{\infty} \gamma^{i} J\left(\boldsymbol{x}_{i}, \boldsymbol{a}_{i}, \boldsymbol{\sigma}_{i}\right)\right] \\
& \mathcal{P}_{5}: \\
& \text { s.t. } \boldsymbol{a}_{i}=\pi_{u}\left(\boldsymbol{x}_{i}\right) \\
& \boldsymbol{x}_{i+1}=f\left(\boldsymbol{x}_{i}, \boldsymbol{a}_{i}, \boldsymbol{\theta}_{i}\right) \\
& g\left(\boldsymbol{x}_{i}, \boldsymbol{a}_{i}\right) \leq 0 \\
& \boldsymbol{\theta}_{i} \sim p_{\boldsymbol{\theta}}\left(\cdot \mid \boldsymbol{x}_{i}, \boldsymbol{a}_{i}\right), \quad \boldsymbol{\sigma}_{k, i} \sim p_{\boldsymbol{\sigma}_{k}}\left(\cdot \mid \boldsymbol{x}_{i}, \boldsymbol{a}_{i}\right)
\end{aligned}
$$

By following the Euler-Lagrange approach described in Theorem 5.3 (and in previous sections), we have that the KKT systems for game and OCP are equal if the dual variables are equal (including new extra dual variables for the equality constraints that relate the action and the policy) and the following first-order conditions hold $\forall k \in \mathcal{N}$ and $i=1, \ldots, \infty$ :

$$
\begin{aligned}
\mathbb{E}\left[\nabla_{x_{k, i}^{r}} r_{k}\left(x_{k, i}^{r}, a_{k, i}, a_{-k, i}, \boldsymbol{\sigma}_{k, i}\right)\right] & =\mathbb{E}\left[\nabla_{x_{k, i}^{r}} J\left(x_{i}, a_{i}, \boldsymbol{\sigma}_{i}\right)\right] \\
\mathbb{E}\left[\nabla_{a_{k, i}} r_{k}\left(x_{k, i}^{r}, a_{k, i}, a_{-k, i}, \boldsymbol{\sigma}_{k, i}\right)\right] & =\mathbb{E}\left[\nabla_{a_{k, i}} J\left(x_{i}, a_{i}, \boldsymbol{\sigma}_{i}\right)\right]
\end{aligned}
$$

The benefit of this reformulation is that the gradient in (5.184) is taken with respect to the components in $\mathcal{X}_{k}^{r}$ only (instead of the whole set $\mathcal{X}$ ), at the cost of replacing (5.152) with the sequence of conditions (5.185). Although (5.185) looks similar to the OL case (5.102), we have to realize that $a_{k, i}$ is indeed a function of variables $x_{k, i}^{\pi}$ and 
$u_{k}$. In order to understand the influence of this variable change, we use the identity $a_{k, i}=\pi_{u_{k}}\left(x_{k, i}^{\pi}\right)$ and apply the chain rule to both sides of (5.185), obtaining:

$$
\begin{aligned}
\mathbb{E}\left[\nabla_{x_{k, i}^{\pi}} r_{k}\right] & =\mathbb{E}\left[\nabla_{x_{k, i}^{r}} r_{k}\right]^{\top} \nabla_{x_{k, i}^{\pi}} x_{k, i}^{r}+\mathbb{E}\left[\nabla_{a_{k, i}} r_{k}\right]^{\top} \nabla_{x_{k, i}^{\pi}} a_{k, i} \\
\mathbb{E}\left[\nabla_{u_{k}} r_{k}\right] & =\mathbb{E}\left[\nabla_{a_{k, i}} r_{k}\right]^{\top} \nabla_{u_{k}} a_{k, i} \\
\mathbb{E}\left[\nabla_{x_{k, i}^{\pi}} J\right] & =\mathbb{E}\left[\nabla_{x_{k, i}^{r}} J\right]^{\top} \nabla_{x_{k, i}^{\pi}} x_{k, i}^{r}+\mathbb{E}\left[\nabla_{a_{k, i}} J\right]^{\top} \nabla_{x_{k, i}^{\pi}} a_{k, i} \\
\mathbb{E}\left[\nabla_{u_{k}} J\right] & =\mathbb{E}\left[\nabla_{a_{k, i}} J\right]^{\top} \nabla_{u_{k}} a_{k, i}
\end{aligned}
$$

From (5.184)-(5.185), it is clear that the right side of (5.186) and (5.188) are equal. Similarly, from (5.185), the right side of (5.187) and (5.189) are equal, so that their left side must be also equal. Hence, we can replace (5.185) with the two following conditions:

$$
\begin{aligned}
\mathbb{E}\left[\nabla_{x_{k, i}^{\pi}} r_{k}\left(x_{k, i}^{r}, \pi_{u_{k}}\left(x_{k, i}^{\pi}\right), a_{-k, i}, \boldsymbol{\sigma}_{k, i}\right)\right] & =\mathbb{E}\left[\nabla_{x_{k, i}^{\pi}} J\left(x_{i}, \pi_{u_{k}}\left(x_{k, i}^{\pi}\right), a_{-k, i}, \boldsymbol{\sigma}_{i}\right)\right] \\
\mathbb{E}\left[\nabla_{u_{k}} r_{k}\left(x_{k, i}^{r}, \pi_{u_{k}}\left(x_{k, i}^{\pi}\right), a_{-k, i}, \boldsymbol{\sigma}_{k, i}\right)\right] & =\mathbb{E}\left[\nabla_{u_{k}} J\left(x_{i}, \pi_{u_{k}}\left(x_{k, i}^{\pi}\right), a_{-k, i}, \boldsymbol{\sigma}_{i}\right)\right]
\end{aligned}
$$

Moreover, we can combine (5.184) and (5.190) in one single equation:

$$
\mathbb{E}\left[\nabla_{x_{k, i}^{\Theta}} r_{k}\left(x_{k, i}^{r}, \pi_{u_{k}}\left(x_{k, i}^{\pi}\right), a_{-k, i}, \boldsymbol{\sigma}_{k, i}\right)\right]=\mathbb{E}\left[\nabla_{x_{k, i}^{\Theta}} J\left(x_{i}, \pi_{u_{k}}\left(x_{k, i}^{\pi}\right), a_{-k, i}, \boldsymbol{\sigma}_{i}\right)\right]
$$

By using the identity $a_{-k, i}=\pi_{u_{-k}}\left(x_{-k, i}^{\pi}\right)$ in (5.191)-(5.192), we have:

$$
\begin{aligned}
\mathbb{E}\left[\nabla_{x_{k, i}^{\ominus}} r_{k}\left(x_{k, i}^{r}, \pi_{u_{k}}\left(x_{k, i}^{\pi}\right), \pi_{u_{-k}}\left(x_{-k, i}^{\pi}\right), \boldsymbol{\sigma}_{k, i}\right)\right] & =\mathbb{E}\left[\nabla_{x_{k, i}^{\ominus} J} J\left(x_{i}, \pi_{u}\left(x_{i}\right), \boldsymbol{\sigma}_{i}\right)\right] \\
\mathbb{E}\left[\nabla_{u_{k}} r_{k}\left(x_{i}, \pi_{u_{k}}\left(x_{k, i}^{\pi}\right), \pi_{u_{-k}}\left(x_{-k, i}^{\pi}\right), \boldsymbol{\sigma}_{k, i}\right)\right] & =\mathbb{E}\left[\nabla_{u_{k}} J\left(x_{i}, \pi_{u}\left(x_{i}\right), \boldsymbol{\sigma}_{i}\right)\right]
\end{aligned}
$$

Thus, by following the same argument used in Lemma 5.2, under the disjoint-statecomponents assumption given by (5.179), conditions (5.193)-(5.194) are sufficient for game (5.127) to be a CL-SDPG, with potential function $J$. Moreover, by following the same conservative-vector-field argument described above, we can obtain second-order conditions similar to (5.131)-(5.133), but where the gradients are taken with respect to $x_{k, i}^{\Theta}$, instead of $x_{i}$.

Now, we show that the separable common term in (5.180) is actually the objective of the OCP. Since the sets $\mathcal{X}_{k}^{\Theta}$ are assumed disjoint (i.e., $x_{k, i}^{\Theta}$ shares no components with $x_{-k, i}^{\pi}$ or $x_{-k, i}^{r}$, we have:

$$
\mathbb{E}\left[\nabla_{x_{k, i}^{\Theta}} \Theta_{k}\left(x_{-k, i}^{r}, \pi_{u_{-k}}\left(x_{-k, i}^{\pi}\right), \boldsymbol{\sigma}_{i}\right)\right]=0
$$


By taking the gradient with respect to $x_{k, i}^{\Theta}$ and $u_{k}$ in both sides of $(5.180)$, we obtain (5.193)-(5.194).

Again, we remark that Theorem 5.4 is of practical importance, since it is usually much simpler to find $J$ by identifying the terms in (5.180) than by solving the line integral (5.134), especially if the policy is an expressive nonlinear parametric mapping of the state (e.g., a neural network). Note also that other separable cases are possible beyond the disjoint state-components assumption. For instance, the war-fish problem with linear policies described in Example 5.2 can be easily written in separable form, with the peculiarity that the independent term depends on no state-component at all but only the linear policy parameters of other agents.

From this analysis we extract another interesting conclusion. Consider two games with the same reward functions, dynamics and constraints, and where their only difference is that one is open-loop (i.e., the agents optimize over the set of action sequences) and the other is closed-loop (i.e., the agents optimize over the set of parametric policies). You could also imagine that we have an OL game and obtain another CL game by simply replacing every action as a mapping of the state (i.e., $\boldsymbol{a}_{i}=\pi_{u}\left(\boldsymbol{x}_{i}\right)$ ). Loosely speaking, we could say that we have the OL and CL versions of a game. In this case, it could happen that the OL version of the game is potential, while the (parametric) CL version is not. First, we illustrate this with a simple example. Then, we formalize this idea with a lemma.

Example 5.4. Consider an OL game with $N=2$ players and rewards given by

$$
\begin{aligned}
& r_{1}\left(x_{i}, a_{1}\right)=a_{1, i} x_{i} \\
& r_{2}\left(x_{i}, a_{2}\right)=a_{2, i} x_{i}
\end{aligned}
$$

The game is clearly an OL-DPG, with separable functions:

$$
J\left(x_{i}, a_{i}\right)=\left(a_{1, i}+a_{2, i}\right) x_{i}, \quad \Theta_{1}\left(a_{2, i}\right)=-a_{2, i} x_{i}, \quad \Theta_{2}\left(a_{1, i}\right)=-a_{1, i} x_{i}
$$

Now, suppose that the actions are replaced by linear policies of the form: $\pi_{u_{k}}=u_{k} x_{i}$, so that we obtain a parametric CL version of the same game, with reward functions:

$$
\begin{aligned}
& r_{1}\left(x_{i}, \pi_{u_{1}}\left(x_{i}\right)\right)=u_{1} x_{i}^{2} \\
& r_{2}\left(x_{i}, \pi_{u_{2}}\left(x_{i}\right)\right)=u_{2} x_{i}^{2}
\end{aligned}
$$


In order to check whether the CL version of the game is potential, we take second partial derivatives and observe that:

$$
\nabla_{x_{i}} \nabla_{x_{i}} r_{1}\left(x_{i}, \pi_{u_{1}}\left(x_{i}\right)\right)=2 u_{1} \neq \nabla_{x_{i}} \nabla_{x_{i}} r_{2}\left(x_{i}, \pi_{u_{2}}\left(x_{i}\right)\right)=2 u_{2}
$$

Since condition (5.132) does not hold, we conclude that the CL version of the game is not potential.

Lemma 5.6. Let $\mathcal{G}^{\mathrm{OL}}$ be an $O L$ game. Let $\mathcal{G}^{\mathrm{CL}}$ be the $C L$ game obtained by replacing every action in $\mathcal{G}^{\mathrm{OL}}$ with a parametric policy of the state (i.e., $\boldsymbol{a}_{i}=\pi_{u}\left(\boldsymbol{x}_{i}\right)$, for all $i=1, \ldots, \infty)$. If $\mathcal{G}^{\mathrm{CL}}$ is an $C L-S D P G$, then $\mathcal{G}^{\mathrm{OL}}$ is an $O L-S D P G$ (this is sufficient but not necessary condition).

Proof: To prove sufficiency, note that if we replace $\pi_{u}\left(x_{i}\right)$ with $a_{i}$, for all $i=1, \ldots, \infty$, in (5.151), the equality still holds and we obtain (5.84). Moreover, if we do the same change of variables in (5.152), we obtain (5.85). Counter-example 5.4 shows that the condition is not necessary.

With this result, we have finished the theoretical contributions of this chapter. In the following sections, we show how to apply these ideas to different practical problems.

\subsection{Network flow control}

Several works (see, e.g., $[173,193,310,60]$ ) have considered network flow control as an optimization problem wherein each source is characterized by a utility function that depends on the transmission rate, and the goal is to maximize the aggregated utility. We generalize the standard model by considering that the nodes are equipped with batteries that are depleted proportionally to the outgoing flow. In addition we consider several layers of relay nodes, each one being able to receive data from multiple sources, so there are several paths between source and destination (see Figure 5.2). When the relay batteries are completely depleted, no more transmissions are allowed and the game is over. Hence, although we formulate the problem as an infinite horizon dynamic game, the effective time horizon - before the batteries deplete - is finite. This problem has no known analytical solution, but the utilities are concave. Therefore, the finite horizon approximation is convenient because we can solve an equivalent concave optimization problem, significantly reducing the computational load with respect to other optimal control algorithms (e.g., dynamic programming). 


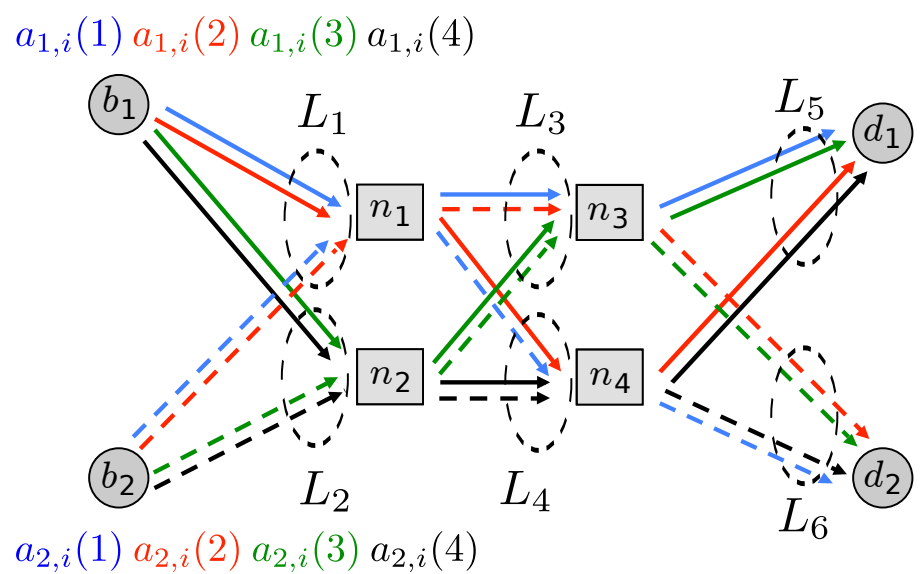

Figure 5.2 Network scenario for two users and two layers of relay nodes. agent $b_{1}$ aims to transmit to destination $d_{1}$, while $b_{2}$ aims to transmit to destination $d_{2}$. They have to share relay nodes $n_{1}, \ldots, n_{4}$. We denote the $L=6$ aggregated flows as $L_{1}, \ldots, L_{6}$.

\subsubsection{Network flow dynamic game and related OCP}

Let $a_{k, i}(\ell)$ denote the flow along path $\ell$ for user $k$ at time $i$. Suppose there are $A_{k}$ possible paths for each user $k$, so that $a_{k, i} \triangleq\left(a_{k, i}(\ell)\right)_{\ell=1}^{A_{k}}$ denotes the $k$-th agent's action vector. Let $A=\sum_{k \in \mathcal{N}} A_{k}$ denote the total number of available paths, and let $a_{i} \triangleq\left(a_{k, i}\right)_{k \in \mathcal{N}}$ be the vector of flows over all available paths.

Suppose there are $S$ relay nodes. Let $x_{i}(s)$ denote the battery level of the $s$-th relay node. The state of the game is given by $x_{i} \triangleq\left(x_{i}(s)\right)_{s=1}^{S}$, such that all agents share all components of the state-vector (i.e., $\mathbb{X}_{k}=\mathbb{X}$ and $\mathcal{X}_{k}=\{1, \ldots, S\}, \forall k \in \mathcal{N}$ ). The battery level evolves with the following state-transition equation for all components $s=1, \ldots, S$ :

$$
x_{i+1}(s)=x_{i}(s)-\delta \sum_{k \in \mathcal{N}} \sum_{\ell \in \mathcal{F}_{s}} a_{k, i}(\ell), \quad x_{0}(s)=B_{s, \max }
$$

where $\mathcal{F}_{s}$ denotes the subset of flows through relay node $s$ (i.e., the components of the action vector that influence the $s$-th component of the state vector), $B_{s, \max }$ is a positive scalar that stands for the maximum battery level of node $s$, and $\delta$ is a proportional depletion factor.

There is some capacity constraint of the maximum aggregated rate at every relay and destination node. Let $c_{\max } \in \mathbb{R}^{C}$ denote the vector with maximum capacities, where $C$ is the number of relay nodes plus destination nodes. Let $M=\left[m_{c \ell}\right]$ denote the matrix of size $C \times A$ that define the aggregated flows for each relay and destination 
node, such that element $m_{c \ell}=1$, if flow node $\ell$ is aggregated in node $c$, and $m_{c \ell}=0$ otherwise.

Similar to the standard static flow control problem, we assume that each agent intends to maximize a concave function $\Gamma: \mathbb{A}_{k} \rightarrow \mathbb{R}$ of the sum of rates across all available paths. This function $\Gamma$ can take different forms depending on the scenario under study, like the square root [202] or a capacity form. In addition to the transmission rate, we include the battery levels of the relays in each agent's utility, weighted by some positive parameter $\alpha$. The combination of these two objectives can be understood as the agent aiming to maximize its total transmission rate, while saving the batteries of the relays. Since multiple agents can share multiple links, the agents have to compete for the capacity and battery of the relays, which are limited resources.

The dynamic network flow control game is given by the following set of coupled OCP:

$$
\begin{aligned}
& \underset{\left\{a_{k, i}\right\} \in \prod_{i=0}^{\infty} \mathbb{A}_{k}}{\operatorname{maximize}} \sum_{i=0}^{\infty} \gamma^{i}\left(\Gamma\left(\sum_{\ell=1}^{A_{k}} a_{k, i}(\ell)\right)+\alpha \sum_{s=1}^{S} x_{i}(s)\right) \\
& \mathcal{G}_{7}: \forall k \in \mathcal{N} \\
& \text { s.t. } \quad x_{i+1}(s)=x_{i}(s)-\delta \sum_{k \in \mathcal{N}} \sum_{\ell \in \mathcal{F}_{s}} a_{k, i}(\ell) \\
& x_{0}(s)=B_{s, \max }, \quad 0 \leq x_{i}(s) \leq B_{s, \max }, \quad s=1, \ldots, S \\
& M a_{i} \leq c_{\max }, \quad a_{i} \geq 0
\end{aligned}
$$

Note that each agent's utility can be expressed in separable form:

$$
\begin{aligned}
r_{k}\left(x_{k, i} a_{i}, i\right) & \triangleq \Gamma\left(\sum_{\ell=1}^{A_{k}} a_{k, i}(\ell)\right)+\alpha \sum_{s=1}^{S} x_{i}(s) \\
& =\sum_{k \in \mathcal{N}} \Gamma\left(\sum_{\ell=1}^{A_{k}} a_{k, i}(\ell)\right)+\alpha \sum_{s=1}^{S} x_{i}(s)-\sum_{j \in \mathcal{N}: j \neq k} \Gamma\left(\sum_{\ell=1}^{A_{j}} a_{j, i}(\ell)\right)
\end{aligned}
$$

where the first two terms are common for all users and the second term depends on actions of all users except user $k$. Therefore, Lemma 5.3 establishes that problem (5.203) is a DPG, with potential function given by:

$$
J\left(x_{i}, a_{i}, i\right)=\sum_{k \in \mathcal{N}} \Gamma\left(\sum_{\ell=1}^{A_{k}} a_{k, i}(\ell)\right)+\alpha \sum_{s=1}^{S} x_{i}(s)
$$

Before applying Theorem 5.1, we have to check whether Assumptions 5.1-5.4 are satisfied. We follow [202] and choose $\Gamma(\cdot) \triangleq \sqrt{(\epsilon+\cdot)}$ (where $\epsilon>0$ is only added to 
avoid differentiability issues when $\left.a_{k, i}(\ell)=0\right)$. Let $\mathbb{X}$ and $\mathbb{A}_{k}$ be open convex sets containing the Cartesian products of intervals $\left[0, B_{s, \max }\right]$ and $[0, \infty)$, respectively. It follows that Assumptions 5.1-5.2 hold. Moreover, since $\Gamma$ is concave and problem (5.203) has linear equality constraints and concave inequality constraints, Slater's condition holds, i.e., Assumption 5.3 is satisfied. Finally, since the constraint set in (5.203) is compact, Lemma 5.5.1 states that Assumption 5.4 holds. Hence, Theorem 5.1 establishes that we can find an NE of (5.203) by solving the following OCP:

$$
\begin{aligned}
\underset{\left\{a_{k, i}\right\} \in \prod_{i=0}^{\infty} \mathbb{A}_{k}}{\operatorname{maximize}} & \sum_{i=0}^{\infty} \gamma^{i}\left(\sum_{k \in \mathcal{N}} \Gamma\left(\sum_{\ell=1}^{A_{k}} a_{k, i}(\ell)\right)+\alpha \sum_{s=1}^{S} x_{i}(s)\right) \\
\text { s.t. } \quad & x_{i+1}(s)=x_{i}(s)-\delta \sum_{k \in \mathcal{N}} \sum_{\ell \in F_{k}} a_{k, i}(\ell) \\
& x_{0}(s)=B_{s, \max }, \quad 0 \leq x_{i}(s) \leq B_{s, \max }, \quad s=1, \ldots, S \\
& M a_{i} \leq c_{\max }, \quad a_{i} \geq 0
\end{aligned}
$$

\subsubsection{Finite horizon approximation and simulation results}

We remark that there is not known closed form solution for problem (5.206). Thus, we have to rely on numerical methods to solve the OCP. Suppose that we set the weight parameter $\alpha$ in $J$ low enough to incentivize some positive transmission. Eventually, the nodes' batteries will be depleted and the system will get stuck in a steady-state, with no further state transitions. Thus, we can approximate the infinite-horizon problem (5.206) as a finite-horizon problem, with horizon bounded by the time-step at which all batteries have been depleted. Moreover, in our setting, we have assumed $\Gamma$ to be concave. Therefore, we can effectively solve (5.206) with convex optimization solvers (we use the software described in [109]). The benefit of using a convex optimization solver is that standard optimal control algorithms are computationally demanding when the state and action spaces are subsets of vector spaces.

For our numerical experiment, we consider $N=2$ agents that share a network of $S=4$ relay nodes, organized in two layers, plus 2 destination nodes (see Figure 5.2). Thus, we have $L=6$ receiving points, with vector of maximum capacities $c_{\max }=[0.5,0.15,0.5,0.15,0.4,0.4]^{\top}$. In this particular setting, each agent is allowed to use four paths, $A_{1}=A_{2}=4$. The connectivity matrix $M \in \mathbb{R}^{L=6 \times S=8}$ can be obtained 
from Figure 5.2:

$$
M=\left(\begin{array}{llllllll}
1 & 1 & 0 & 0 & 0 & 1 & 1 & 0 \\
0 & 0 & 1 & 1 & 0 & 0 & 1 & 1 \\
1 & 0 & 1 & 0 & 0 & 1 & 1 & 0 \\
0 & 1 & 0 & 1 & 1 & 0 & 0 & 1 \\
1 & 1 & 1 & 1 & 0 & 0 & 0 & 0 \\
0 & 0 & 0 & 0 & 1 & 1 & 1 & 1
\end{array}\right)
$$

The battery is initialized to $B_{s, \max }=B_{\max }=1$ for the four relay nodes $(s=1, \ldots, 4)$. We set depleting factor $\delta=0.05$, discount factor $\gamma=0.9$, weight $\alpha=1$ and $\epsilon=0.001$.

We remark that the solution obtained is an NE based on an OL analysis. Figure 5.3 shows (top) the evolution of the $L=6$ aggregated flows, (middle) the $A=8$ paths, and (bottom) the battery of each of the $S=4$ relay nodes. Since we have included the battery level of the relay nodes in the users' utilities (i.e., $\alpha>0$ ), the users have an extra incentive to limit their flow rate. Thus, there are two effective reasons to limit the flow rate: satisfy the problem constraints and save battery. Let $L_{j}, j=1, \ldots, 6$, denote the capacity through each receiving point at time $i$. We can see that the receiving points with higher maximum capacity are not saturated $\left(L_{1}<0.5, L_{3}<0.5, L_{5}<0.4\right.$, and $\left.L_{6}<0.4\right)$. The reason is that the users have limited their individual flow rates in order to save relays' batteries. On the other hand, the receiving points with lower maximum capacity are saturated $\left(L_{2}=L_{4}=0.15\right)$ because the capacity constraint is more restrictive than the self-limitation incentive. When the batteries of the nodes with higher maximum capacity $(s=1, s=3$ ) are depleted (around $i=70$ ), the flows through these nodes stop. This allows the other flows $\left(a_{1, i}(4), a_{2, i}(4)\right)$ to transmit at the maximum rate allowed by the capacity constraints (note that $L_{2}=L_{4}=0.15$ remains constant). When the battery of every node is depleted, none of the users is allowed to transmit any more and the system enters in a final steady-state with no more state transitions.

The results shown in Figure 5.3 have been obtained with a centralized convex optimization algorithm, meaning that it should be run off-line by the system designer, before deploying the real system. Alternatively, we could have used the distributed algorithms proposed by reference [88], enabling the agents to solve the finite horizon approximation of problem (5.206) in a decentralized manner, even with the coupled capacity constraints. 


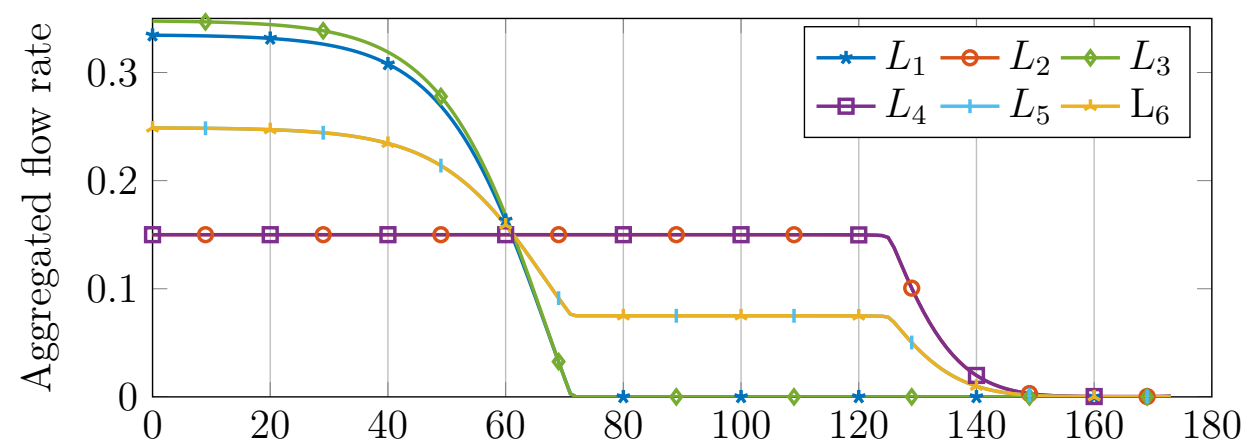

Time
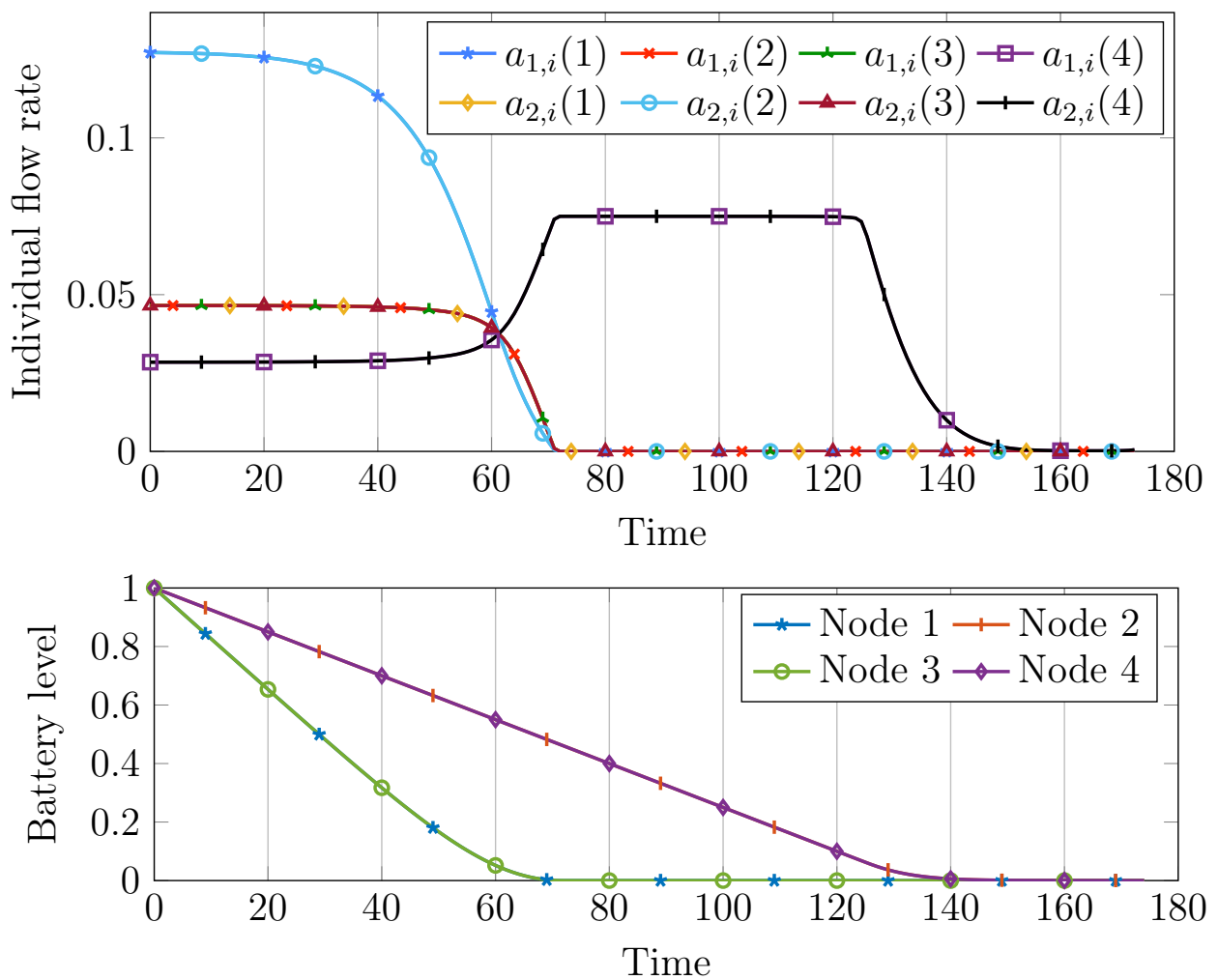

Figure 5.3 Network flow control with $N=2$ agents, $S=4$ relay nodes and $A_{1}=A_{2}=4$ available paths per node, so that $A=A_{1}+A_{2}=8$. (Top) Aggregated flow rates at $L_{1}, \ldots, L_{6}$. (Middle) Flow for each of the $A=8$ available paths. (Bottom) Battery level in each of the $S=4$ relay nodes. 


\subsection{Dynamic Multiple Access Channel}

In this section, we consider an uplink scenario in which every user $k \in \mathcal{N}$ independently chooses its transmitter power, $a_{k, i}$, aiming to achieve the maximum rate allowed by the channel [334]. If multiple users transmit at the same time, they will interfere each other, which will decrease their rate, so that they have to find an equilibrium. Let $R_{k, i}$ denote the rate achieved by user $k$ at time $i$ with normalized noise:

$$
R_{k, i} \triangleq \log \left(1+\frac{\left|h_{k}\right|^{2} a_{k, i}}{1+\sum_{j \in \mathcal{N}: j \neq k}\left|h_{j}\right|^{2} a_{j, i}}\right)
$$

where $h_{k}$ denotes the fading channel coefficient for user $k$.

\subsubsection{Multiple access channel DPG and related OCP}

Let $x_{k, i} \in\left[0, B_{k, \max }\right] \triangleq \mathbb{X}_{k}$ denote the battery level for each agent $k \in \mathcal{N}$, which is discharged proportionally to the transmitted power $a_{k, i} \in\left[0, P_{k, \max }\right] \triangleq \mathbb{A}_{k}$, where $P_{k \text {,max }}$ and $B_{k, \text { max }}$ denote the maximum transmitter power and maximum available battery level for node $i$, respectively. The state of the system is given by the vector with all individual battery levels: $x_{i}=\left(x_{k, i}\right)_{k \in \mathcal{N}} \in \mathbb{X}$. Thus, each agent is only affected by its own battery, such that $S=N$ and all state vector components are unshared, i.e., $\mathbb{X}=\prod_{k \in \mathcal{N}} \mathbb{X}_{k} \subset \mathbb{R}^{N}$, and $\mathcal{X}_{k}=\{k\}$. Suppose the agents aim to maximize its transmission rate, while also saving their battery. This scenario yields the following dynamic game:

$$
\mathcal{G}_{8}: \forall k \in \mathcal{N} \begin{aligned}
\underset{\left\{a_{k, i}\right\} \in \prod_{i=0}^{\infty} \mathbb{A}_{k}}{\operatorname{maximize}} & \sum_{i=0}^{\infty} \gamma^{i}\left(R_{k, i}+\alpha x_{k, i}\right) \\
\text { s.t. } \quad & x_{k, i+1}=x_{k, i}-\delta a_{k, i}, \quad x_{k, 0}=B_{k, \max } \\
& 0 \leq a_{k, i} \leq P_{k, \max }, \quad 0 \leq x_{k, i} \leq B_{k, \max }
\end{aligned}
$$

where $\alpha$ is the weight given for saving the battery and $\delta$ is the discharging factor. Problem (5.209) is an OL dynamic infinite-horizon extension of the static problem proposed in [256].

Instead of looking for a separable structure in the agents' utilities, we show that Lemma 5.4 holds:

$$
\frac{\partial^{2} r_{k}\left(x_{k, i}, a_{i}, i\right)}{\partial x_{j, i} \partial a_{k, i}}=\frac{\partial^{2} r_{j}\left(x_{j, i}, a_{i}, i\right)}{\partial x_{k, i} \partial a_{j, i}}=0
$$




$$
\begin{aligned}
& \frac{\partial^{2} r_{k}\left(x_{k, i}, a_{i}, i\right)}{\partial x_{j, i} \partial x_{k, i}}=\frac{\partial^{2} r_{j}\left(x_{j, i}, a_{i}, i\right)}{\partial x_{k, i} \partial x_{j, t}}=0 \\
& \frac{\partial^{2} r_{k}\left(x_{k, i}, a_{i}, i\right)}{\partial a_{j, i} \partial a_{k, i}}=\frac{\partial^{2} r_{j}\left(x_{k, i}, a_{i}, i\right)}{\partial a_{k, i} \partial a_{j, i}}=\frac{-\left|h_{k}\right|^{2}\left|h_{j}\right|^{2}}{\left(1+\sum_{k \in \mathcal{N}}\left|h_{k}\right|^{2} a_{k, i}\right)^{2}}
\end{aligned}
$$

Hence, problem (5.209) is a DPG. In order to find a related OCP, let us define $\mathbb{X}_{k}$ and $\mathbb{A}_{k}$ as open convex sets containing the closed intervals $\left[0, B_{k, \max }\right]$ and $\left[0, P_{k, \max }\right]$, respectively, so that Assumptions 5.1-5.2 hold. Derive a potential function from (5.45):

$$
J\left(x_{i}, a_{i}, i\right)=\log \left(1+\sum_{k \in \mathcal{N}}\left|h_{k}\right|^{2} a_{k, i}\right)+\alpha \sum_{k \in \mathcal{N}} x_{k, i}
$$

Since (5.213) is concave and all equality and inequality constraints in (5.209) are linear, Assumption 5.3 is satisfied through Slater's condition. Moreover, since the constraint set is compact and the potential (5.213) is continuous, Lemma 5.5.1 establishes that Assumption 5.4 holds. Therefore, Theorem (5.1) states that we can find an NE of (5.209) by solving the following OCP:

$$
\begin{aligned}
\operatorname{Paximize}_{\left\{a_{i}\right\} \in \prod_{i=0}^{\infty} \mathbb{A}} & \sum_{i=0}^{\infty} \gamma^{i}\left(\log \left(1+\sum_{k \in \mathcal{N}}\left|h_{k}\right|^{2} a_{k, i}\right)+\alpha \sum_{k \in \mathcal{N}} x_{k, i}\right) \\
\text { s.t. } \quad & x_{k, i+1}=x_{k, i}-\delta a_{k, i}, \quad x_{k, 0}=B_{k, \max } \\
& 0 \leq a_{k, i} \leq P_{k, \max }, \quad 0 \leq x_{k, i} \leq B_{k, \max }, \quad \forall k \in \mathcal{N}
\end{aligned}
$$

\subsubsection{Simulation results}

Similar to Section 5.7.2, the system reaches a steady-state when the batteries have been depleted. Thus, we can find the solution by solving a finite horizon problem. Moreover, since the problem is concave, we can use convex optimization software (e.g., [109]) - alternatively, we could find the solution by solving the KKT system with an efficient ad-hoc distributed algorithm, like in [334].

We simulated a scenario with $N=4$ users. We set the maximum battery level $B_{\max }=B_{k, \text { max }}=33, \forall k \in \mathcal{N}$, the maximum power allowed per user $P_{\max }=P_{k, \max }=5$, $\forall k \in \mathcal{N}$, the weight battery utility factor $\alpha=0.001$, the transmitter power battery depletion factor $\delta=1$, and the discount factor $\gamma=0.95$. The simulated channel gains are $\left|h_{1}\right|^{2}=2.019,\left|h_{2}\right|^{2}=1.002,\left|h_{3}\right|^{2}=0.514$ and $\left|h_{4}\right|^{2}=0.308$.

Figure 5.4 shows appealing results: the solution of the OCP - which is an NE of the game - is similar to a time-division-multiple-access (TDMA) scheme. In other words, 

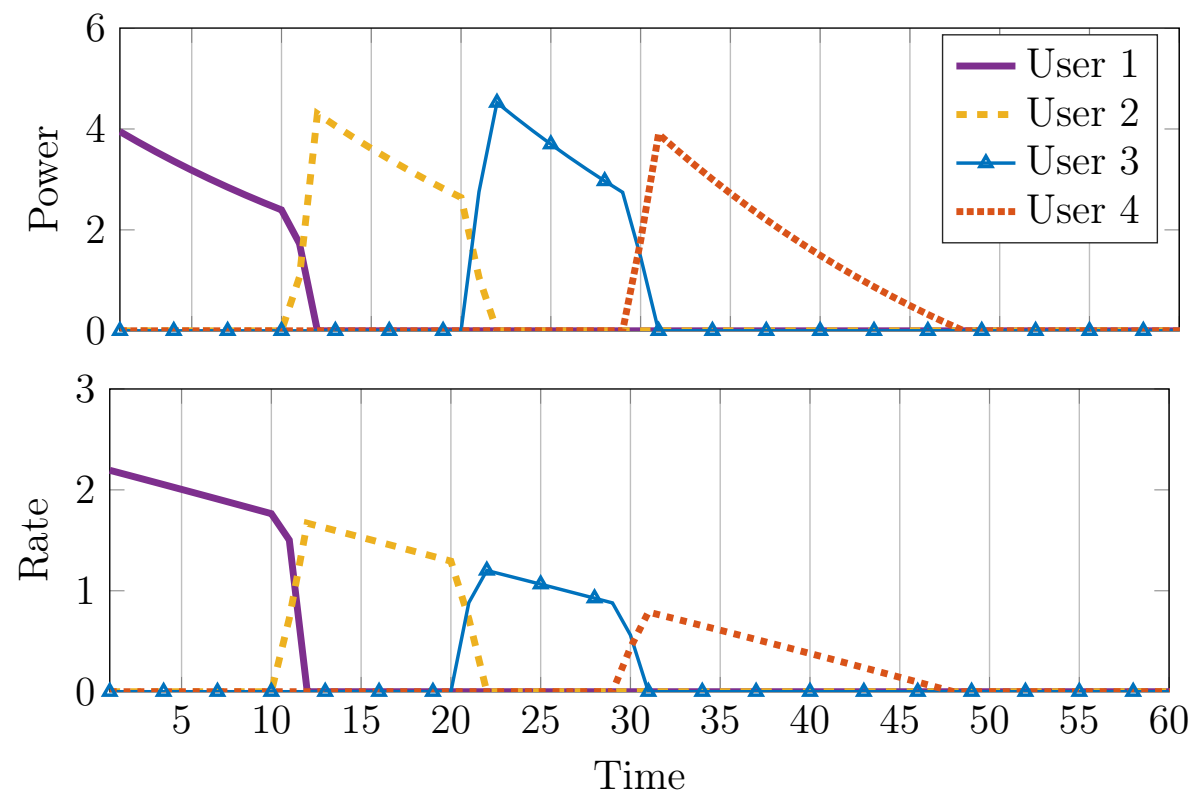

Figure 5.4 Dynamic multiple access scenario with $N=4$ users. (Top) Evolution of transmitter power. (Bottom) Transmission rate for every user. The equilibrium is a schedule with order determined by each user's channel gain.

instead of creating interference among users, they wait until the users with higher channel-gain have depleted their batteries.

\subsection{Optimal Scheduling}

In this section we present the most general form of the proposed framework, and show its applicability to two scheduling problems. Neither the related OCP can be approximated by a finite horizon problem, nor the utilities are concave. Thus, we cannot rely upon convex optimization software and we have to use optimal control methods, like dynamic programming [28]. Finally, we consider a nonstationary scenario, in which the channel coefficients evolve with time. This makes the state-transition equations (and the utility for the equal rate problem) depend not only on the current state, but also on time. 


\subsubsection{Proportional fair and equal rate games and related OCP}

Let us redefine the rate achieved by user $k$ at time $i$, so that we consider time varying channel coefficients:

$$
R_{k, i} \triangleq \log \left(1+\frac{\left|h_{k, i}\right|^{2} a_{k, i}}{1+\sum_{j \in \mathcal{N}: j \neq k}\left|h_{j, i}\right|^{2} a_{j, i}}\right)
$$

where $a_{k, i}$ is the transmitter power of agent $k$, and $\left|h_{k, i}\right|$ is its time-varying channel coefficient.

We propose two different scheduling games, namely, proportional fair and equal rate scheduling.

\section{Proportional fair scheduling}

Proportional fair is a compromise-based scheduling algorithm. It aims to maintain a balance between two competing interests: trying to maximize total throughput while, at the same time, guaranteeing a minimal level of service for all users [142, 143, 342].

In order to achieve this tradeoff, we propose the following game:

$$
\mathcal{G}_{9}: \forall k \in \mathcal{N} \begin{aligned}
\underset{\substack{\left\{a_{k, i}\right\} \in \prod_{i=0}^{\infty} \mathbb{A}_{k} \\
\text { maximize }}}{\text { s.t. }} & \sum_{i=0}^{\infty} \gamma^{i} x_{k, i+1}=\left(1-\frac{1}{i}\right) x_{k, i}+\frac{R_{k, i}}{i} \\
& x_{k, 0}=0,0 \leq a_{k, i} \leq P_{k, \max }
\end{aligned}
$$

where the state of the system is the vector of all agents' average rates $x_{i}=\left(x_{k, i}\right)_{k \in \mathcal{N}} \in \mathbb{X}$. Since each agent aims to maximize its own average rate, the state-components are unshared among agents: $S=N, \mathcal{X}_{k}=\{k\}$ and $\mathbb{X}=\prod_{k \in \mathcal{N}} \mathbb{X}_{k}$.

In order to show that problem (5.216) is a DPG, we evaluate Lemma 5.3 with positive result, and obtain the potential function $J$ from (5.34):

$$
J\left(x_{i}, a_{i}, i\right)=\sum_{k \in \mathcal{N}} x_{k, i}
$$

Now, we show that we can derive a related OCP. It is clear that Assumptions 5.1-5.2 hold. By taking the gradient of the constraints of (5.216) and building a matrix with the gradients of the constraints (i.e., the gradient of each constraint is a column of this matrix), it is straightforward to show that the matrix is full rank. Hence, the linear independence constraint qualification holds (see, e.g., [26, Sec. 3.3.5], [311]), meaning 
that Assumption 5.3 is satisfied. Finally, since $R_{k, i} \geq 0$ and $x_{k, 0}=0$, we conclude that there exists some scalar $M \geq 0$ for which the level set $\left\{x_{i}: \sum_{k \in \mathcal{N}} x_{k, i} \geq M\right\}$ is nonempty and bounded. Hence, Lemma 5.5.3 establishes that Assumption 5.4 is satisfied. Thus, from Theorem 5.1, we can find an NE of DPG (5.216) by solving the following OCP:

$$
\begin{aligned}
& \operatorname{maximize}_{\left\{a_{i}\right\} \in \prod_{i=0}^{\infty} \mathbb{A}} \sum_{i=0}^{\infty} \gamma^{i} \sum_{k \in \mathcal{N}} x_{k, i} \\
& \mathcal{P}_{8}: \quad \text { s.t. } \quad x_{k, i+1}=\left(1-\frac{1}{i}\right) x_{k, i}+\frac{R_{k, i}}{i} \\
& x_{k, i}=0, \quad 0 \leq a_{k, i} \leq P_{k, \max }, \quad \forall k \in \mathcal{N}
\end{aligned}
$$

\section{Equal rate scheduling}

In this problem, the aim of each user is to maximize its rate, while at the same time keeping the users' cumulative rates as close as possible. Let $x_{k, i}$ denote the cumulative rate of user $k$. The state of the system is the vector of all users' cumulative rate $x_{i}=\left(x_{k, i}\right)_{k \in \mathcal{N}}$. Again $S=N$ and $\mathcal{X}_{k}=\{k\}$. This problem is modeled by the following game:

$$
\mathcal{G}_{10}: \forall k \in \mathcal{N} \begin{aligned}
\underset{\left\{a_{k, i}\right\} \in \prod_{i=0}^{\infty} \mathbb{A}_{k}}{\operatorname{maximize}} & \sum_{i=0}^{\infty} \gamma^{i}\left((1-\alpha) R_{k, i}-\alpha \sum_{j \in \mathcal{N}: j \neq k}\left(x_{k, i}-x_{j, i}\right)^{2}\right) \\
\text { s.t. } \quad & x_{k, i+1}=x_{k, i}+R_{k, i} \\
& x_{k, 0}=0, \quad 0 \leq a_{k, i} \leq P_{k, \max }
\end{aligned}
$$

where parameter $\alpha$ weights the contribution of both terms.

It is easy to verify that conditions (5.35)-(5.37) are satisfied. Hence, from Lemma 5.4, we know that problem (5.219) is a DPG. Let us define $\mathbb{X}_{k}$ and $\mathbb{A}_{k}$ as open convex sets that contain the intervals $[0, \infty)$ and $\left[0, P_{k, \max }\right]$, respectively. It follows that Assumptions 5.1-5.2 hold. Similar to the proportional fair scheduling problem (5.216), Assumption 5.3 holds through the linear independence constraint qualification. Then, we only have to show that Assumption 5.4 holds to state that the game is a DPG. Let us proceed as follows. We derive a potential $J$ by integrating (5.45), obtaining:

$$
J\left(x_{i}, a_{i}, i\right)=(1-\alpha) \log \left(1+\sum_{k \in \mathcal{N}}\left|h_{k, i}\right|^{2} a_{k, i}\right)-\alpha \sum_{k \in \mathcal{N}}^{N-1} \sum_{j=k+1}^{N}\left(x_{k, i}-x_{j, i}\right)^{2}
$$


In order to study the existence of solution of the related OCP, we distinguish two extreme cases:

1. All agents have exactly the same rate (i.e., $x_{k, i}=x_{j, i}, \forall k, j \in \mathcal{N}$ ).

2. Each agent's rate is different from any other agent's rate (i.e., $x_{k, i} \neq x_{j, i}, k \neq j$, $k, j \in \mathcal{N})$.

When all agents have exactly the same rate, the terms $\left(x_{k, i}-x_{j, i}\right)^{2}$ vanish for all $(k, j)$ pairs, and (5.220) only depends on the actions (the state becomes irrelevant). Since the action constraint set is compact, existence of solution is guaranteed by Lemma 5.5.1. When each agent's rate is different from any other agent's rate, the term $-\left(x_{k, i}-x_{j, i}\right)^{2}$ is coercive, so that (5.220) becomes coercive too (since the constraint action set is compact, the term depending on $a_{k, i}$ is bounded). Thus, existence of optimal solution is guaranteed by Lemma 5.5.2. Finally, the case where some agent's rate are equal and some are different is a combination of the two cases already mentioned. so that the equal terms vanish and the different terms make (5.220) coercive. Therefore, Theorem 5.1 states that we can find an NE of DPG (5.219) by solving the following OCP:

$$
\begin{aligned}
\underset{\left\{a_{i}\right\} \in \prod_{i=0}^{\infty} \mathbb{A}}{\operatorname{maximize}} & \sum_{i=0}^{\infty} \gamma^{i}\left((1-\alpha) \log \left(1+\sum_{k \in \mathcal{N}}\left|h_{k, i}\right|^{2} a_{k, i}\right)\right. \\
& \left.-\alpha \sum_{k=1}^{N-1} \sum_{j=k+1}^{N}\left(x_{k, i}-x_{j, i}\right)^{2}\right) \\
\text { s.t. } & x_{k, i+1}=x_{k, i}+R_{k, i}, \quad x_{k, 0}=0 \\
& 0 \leq a_{k, i} \leq P_{k, \max }, \quad \forall k \in \mathcal{N}
\end{aligned}
$$

\subsubsection{Solving the OCP with dynamic programming}

Although Lemma 5.5 establishes existence of optimal solution to these OCPs, these problems are nonconcave and cannot be approximated by finite horizon problems. Thus, we cannot rely on efficient convex optimization software. We remark that we are assuming that every problem parameter, the state transition and potential functions are known. Hence, in order to numerically solve these problems, we can use dynamic programming methods [28].

Standard dynamic programming methods assume that the OCP is stationary. One standard method to cope with nonstationary OCPs is to augment the state set so that it includes the time as an extra dimension for some time length $T$. Let the augmented state-vector at time $i$ be denoted by $\widetilde{x}_{i}=\left(x_{i}, i\right) \in \widetilde{\mathbb{K}} \triangleq \mathbb{K} \times\{0, \ldots, T\}$. The 
state-transition equation in the augmented state space becomes $\tilde{f}: \widetilde{\mathbb{X}} \times \mathbb{A} \rightarrow \widetilde{\mathbb{X}}$. Since we are tackling an infinite horizon problem, when augmenting the state space with the time dimension, it is convenient to impose a periodic time variation:

$$
\widetilde{f}\left(\widetilde{x}_{i}, a_{i}\right) \triangleq\left[\begin{array}{c}
f\left(x_{i}, a_{i}, i\right) \\
i+1 \quad \text { if } i<T) \text { or } 0(\text { if } i=T)
\end{array}\right]
$$

One further difficulty for solving OCPs with continuous state and action sets is that dynamic programming methods are mainly derived for discrete state-action sets. Two common approaches to overcome this limitation are:

1. Discretize the continuous variables, so that the value function is approximated in a set of points (see, e.g., how we solved the problem in Section 5.9.2).

2. Use a parametric approximation of the value function (see discussion in Section 3.2 .2 , or the tutorial [98]).

For simplicity, we follow the discretization approach here, since the goal of this examples are to illustrate the general approach. We remark that it may be problematic to finely discretize the state-action spaces in high-dimensional problems though, since the computational load increases exponentially with the number of states. Indeed, these and other approximation techniques, usually known as approximate dynamic programming, are still an active area of research (see, e.g., [28, Ch. 6], [226]).

Introduce the optimal value function for the time-augmented state set:

$$
V^{\star}\left(\widetilde{x}_{0}\right) \triangleq \max _{\left\{a_{i}\right\} \in \prod_{i=0}^{\infty} \mathbb{A}} \sum_{i=0}^{\infty} \gamma^{i} J\left(\widetilde{x}_{i}, a_{i}\right)=\sum_{i=0}^{\infty} \gamma^{i} J\left(\widetilde{x}_{i}, a_{i}^{\star}\right)
$$

Let $\pi^{\star}: \widetilde{\mathbb{K}} \rightarrow \mathbb{A}$ be the optimal policy that provides the sequence of actions $\left\{a_{i}^{\star} \triangleq\right.$ $\left.\pi^{\star}\left(\widetilde{x}_{i}\right)\right\}_{i=0}^{\infty}$ that is the solution to the OCP, as explained by Lemma 5.5. Then, the Bellman optimality equation is given by

$$
V^{\star}\left(\widetilde{x}_{i}\right)=J\left(\widetilde{x}_{i}, \pi^{\star}\left(\widetilde{x}_{i}\right)\right)+\gamma V^{\star}\left(\widetilde{f}\left(\widetilde{x}_{i}, \pi^{\star}\left(\widetilde{x}_{i}\right)\right)\right)
$$

where the time dependence of $J$ is implicit in $\widetilde{x}_{i}$ (i.e., $J\left(\widetilde{x}_{i}, \pi^{\star}\left(\widetilde{x}_{i}\right)\right)=J\left(x_{i}, \pi^{\star}\left(\widetilde{x}_{i}\right), i\right)$ ).

Let $\mathbb{Q} \triangleq\left\{\widetilde{x}_{1}^{\diamond}, \ldots, \widetilde{x}_{Q}^{\diamond}\right\}$ denote the discrete grid of that discretizes the state set $\mathbb{X}$ into $Q$ points. Let $\lceil\widetilde{x}\rceil$ denote a operator that returns the point in $\mathbb{Q}$ that is closest to $\widetilde{x} \in \tilde{\mathbb{X}}$. Moreover, let $\widetilde{x}_{q}^{\diamond} \in \mathbb{Q}$ denote the $q$-th point in $\mathbb{Q}$.

Among the available dynamic programming methods, we choose value iteration (VI) for its reduced complexity per iteration with respect to policy iteration (PI), which is 
especially relevant when the state-grid has fine resolution (i.e., large number of states). VI is obtained by turning the Bellman optimality equation (5.224) into an update rule, so that it generates a sequence of value function estimates, $\left\{V_{t}\right\}_{t=1}^{\infty}$, that converges to the optimal value: $\lim _{t \rightarrow \infty} V_{t}=V^{\star}$, for arbitrary $V_{0}$. In particular, at every iteration $t$, we obtain the policy $\pi_{t}$ that maximizes $V_{t}$ (policy improvement). Then, we update the value function $V_{t+1}$ for the latest policy (policy evaluation). VI is summarized in Algorithm 5.1.

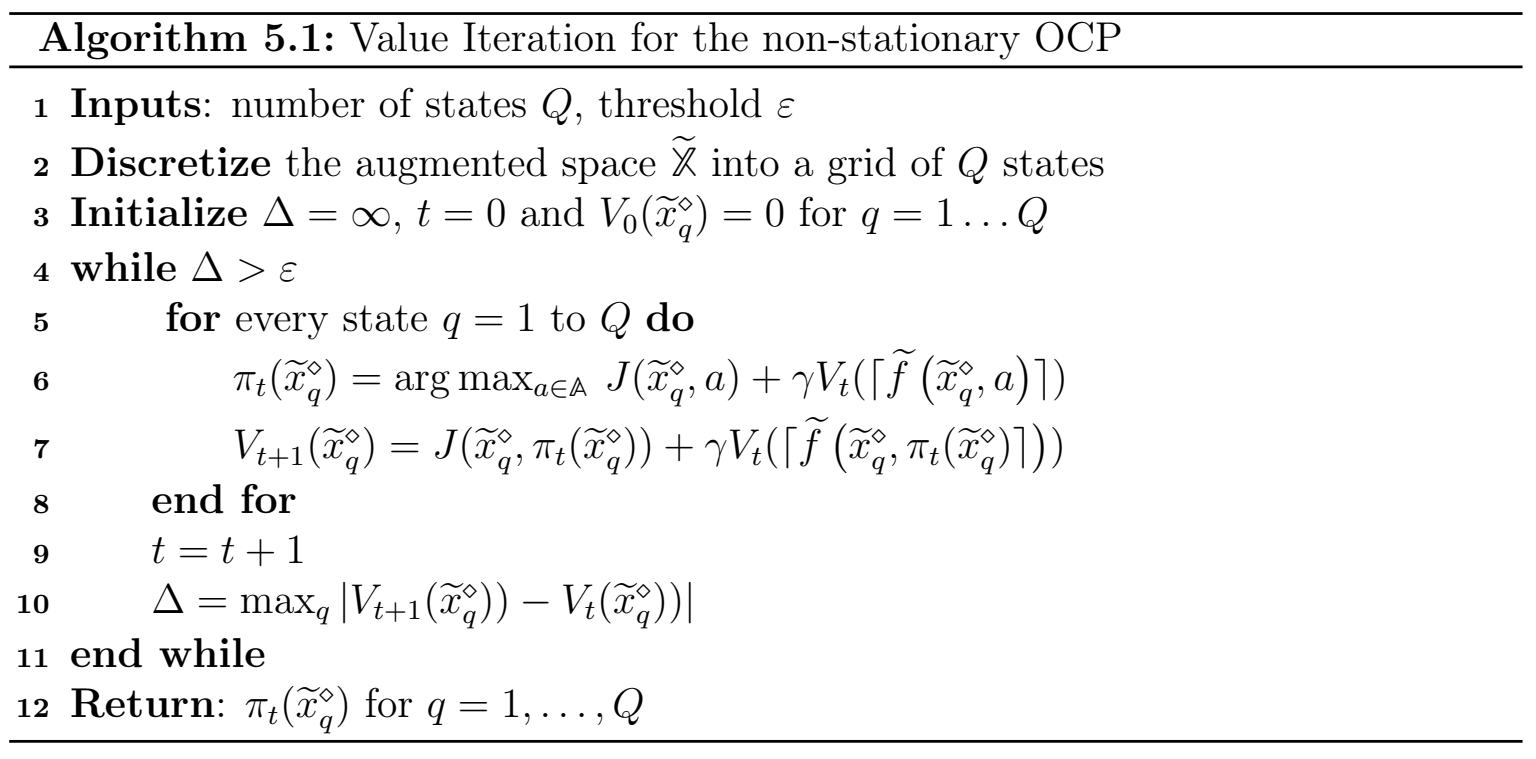

Note that, in order to improve the policy, we have to perform an optimization problem (i.e., we have to maximize over the action set $\mathbb{A}$ ). This optimization problem is usually nonconvex, meaning that this step will be usually impractical. When using the state discretization approach, it is common to discretize also the action set.

Note that the output of the value iteration algorithm is a policy (i.e., a function), rather than a sequence of actions. This is mandatory when there is no reason to expect that the game will enter in steady state after a finite number of steps.

We simulate a simple scenario with $N=2$ users. The channel coefficients are sinusoids with different frequency and different amplitude for each user (see Figure 5.5). The maximum transmitter power is $P_{1, \max }=P_{2, \max }=5$, with 20 possible power levels per user, which amounts to $Q=400$ possible actions (i.e., we assume the action set to be finite). We discretize the state-space (i.e., the users' rates) into a grid of 30 points per user. The nonstationarity of the environment is surmounted by augmenting the state-space with $T=20$ time steps. Hence, the augmented state space has a total of $Q=30^{2} \times 20=18.000$ states. The discount factor is set $\gamma=0.9$. For the equal-rate problem, the utility function uses $\alpha=0.9$. 


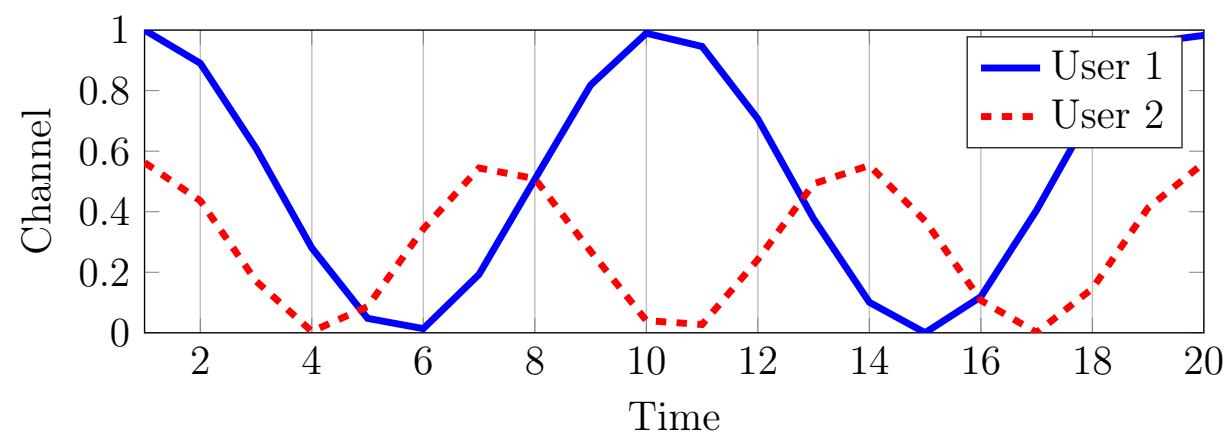

Figure 5.5 Periodic time variation of the channel coefficients $\left|h_{k, t}\right|^{2}$ for $N=2$ users. All possible combination of coefficients are included in a window of $T=20$ time steps.

The solution of the proportional fair game leads to an efficient scheduler (see Figure 5.6 ), in which both users try to minimize interference so that they approach their respective maximum rates.

For the equal rate problem, we observe that the agents achieve much lower average rate, but very similar between them (see Figure 5.7). The trend is that the user with a channel with less gain (User 2, red-dashed line) tries to achieve its maximum rate, while the user with higher gain channel (User 1, blue-continuous line) reduces its transmitter power to match the rate of the other user. In other words, the user with poorest channel sets a bottleneck for the other user.

Finally, note that Algorithm 5.1 is centralized, such that the results displayed in Figures 5.6-5.7 have been obtained assuming the existence of a central unit that knows the channel coefficients, transmission power and average rate for all users, so that it can update the value and policy functions for all states. We remark that the design and analysis of distributed dynamic programming algorithms when multiple agents share state-vector components and/or have coupled constraints is a nontrivial task. Nevertheless, when the agents share no state-vector components and they have uncoupled constraints, there are distributed implementations of VI and PI that converge to the optimal solution $[25,27,133,32]$. This is indeed the case for problems (5.218) and (5.221), where each agent $k$ has a unique state-vector component $x_{k, i}$ and the constraints are uncoupled. Thus, the agents could solve these problems in a decentralized manner, where each agent updates the value function for its own state-variables, assuming that it can observe the objective and value function for the complete state vector. 

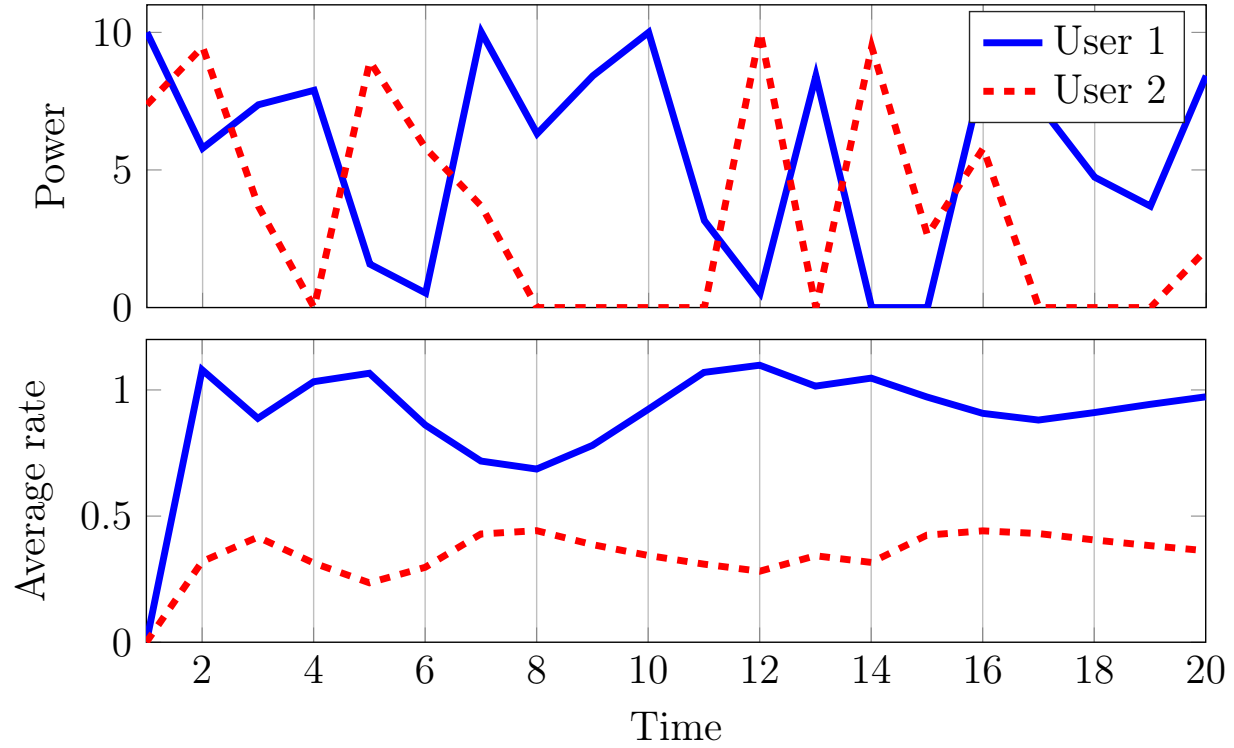

Figure 5.6 Proportional fair scheduling problem for $N=2$ users. (Top) Transmitter power $u_{k, i}$. (Bottom) Average rate $x_{k, i}$ given by (5.216). Both users achieve near maximum average rates for their channel coefficients $\left|h_{k, i}\right|^{2}$.
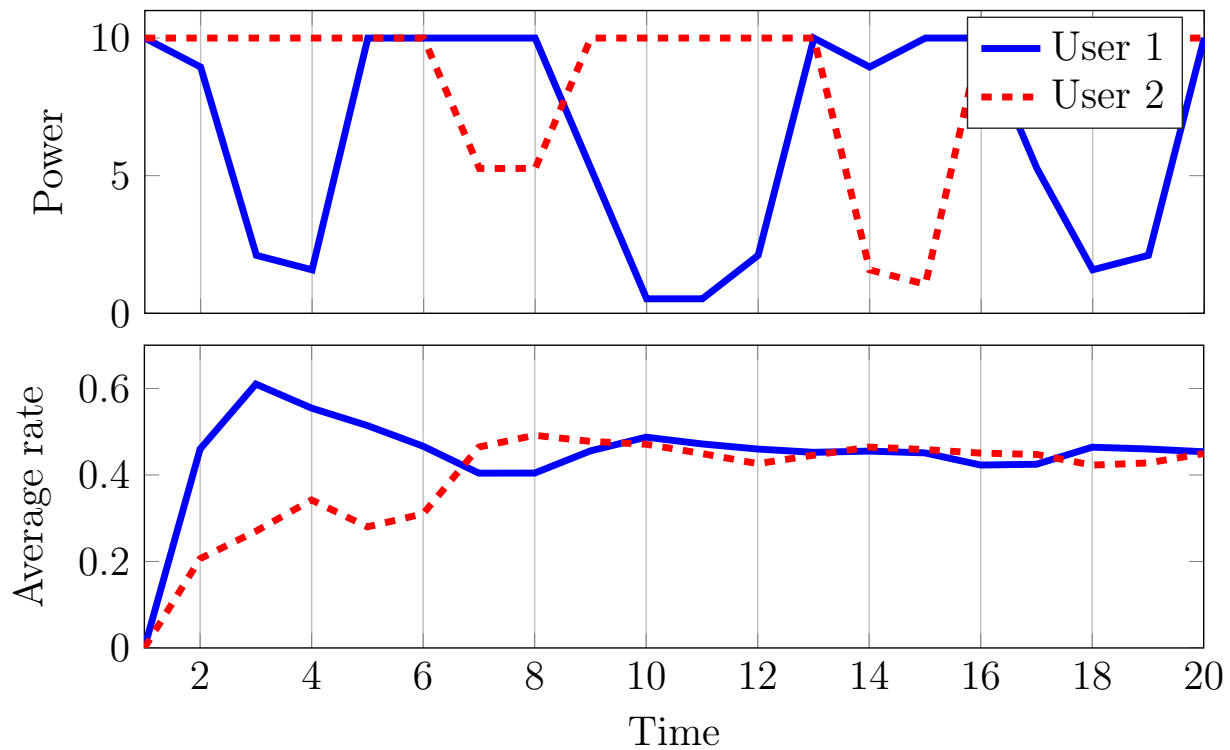

Figure 5.7 Equal rate problem for $N=2$ users. (Top) Transmitter power $u_{k, i}$. (Bottom) Average rate $x_{k, i} / i$ (recall that $x_{k, i}$ given by (5.219) denotes accumulated rate). User 1 reduces its average rate to match that of User 2, regardless of having higher channel coefficient. 


\subsection{Reinforcement learning for DPG}

Having established a relationship between a DPG (5.127) and an OCP (5.130) is a significant step for finding an NE, since we can apply standard optimal control techniques. However, there are cases in which the reward and/or the state-transition distributions are unknown. Rather, we have access only to the real system that we want to control or, at most, to a simulator. Thus, we have to learn the optimal policy by interacting with the real or simulated environment. At every time step, the learning agent observes the state of the environment - or some features that represent the state-, takes an action, and observes the instant reward. By repeating this process, the agent obtains a sequence of tuples $\left\{\left(x_{i}, a_{i}, r_{i}\right)\right\}_{i=0}^{T}$, where $T$ is the maximum number of samples. As we have already seen in Chapter 3, reinforcement learning (RL) is a field that studies this kind of problems and its solution methods (see, e.g., [274, 31, 278, 51]).

Traditional RL algorithms work well in environments with finite state-action sets. However, for DPGs (5.127), the state set and action sets are subsets of $\mathbb{R}^{S}$ and $\mathbb{R}^{\sum_{i=1}^{Q} A^{i}}$, respectively. As we have seen in Section 5.9.2, discretizing state-action variables is only acceptable for very small problems, since the number of discrete states grows exponentially with the number of dimensions. A preferred approach for dealing with continuous state-action sets is to map the state-action variables to a vector of features and learn a parametric approximation of the value function and/or the policy from these features (see, e.g., [5, 307, 186, 9] and Sections 3.2.3 and 5.6 and references therein). However, learning parametric approximations presents also some drawbacks. In particular, appropriate features have to be hand-crafted, automatically discovered (see, e.g., [179, 266]) or learned end-to-end (see, e.g., [168, 255]). We remark that there are also non-parametric approaches, which might be especially useful when standard parametric methods are difficult to learn or cannot approximate the problem well (see, e.g., [190, 157, 184, 206, 219, 308]).

Another issue is that standard RL algorithms do not usually consider constrained state-action sets of the form $g\left(x_{i}, \pi\left(x_{i}\right)\right) \leq 0$. Indeed, none of the referenced RL methods have considered constrained state-actions sets explicitly. One way to deal with constrained sets is to consider any state-action pair that violates the constraint as a terminal state. Hence, if the agent violates a constraint, it receives some penalty and the system restarts at the initial state (see, e.g., [237]). In order to set the penalty, inspired by convex optimization methods (see, e.g., [16, Ch. 9]), we define a penalty function, $H: \mathbb{X} \times \mathbb{A} \rightarrow \mathbb{R}$, that gives negative reward to solutions that fall outside the 
feasible set. A suitable penalty function is of the form:

$$
H\left(x_{i}, a_{i}\right) \triangleq \max \left[0, g\left(x_{i}, a_{i}\right)\right]^{\rho}, \quad \rho \geq 1
$$

This way, we transform the original problem into a new unconstrained OCP:

$$
\begin{aligned}
& \left.\underset{u \in \mathbb{U}}{\operatorname{maximize}} \mathbb{E}\left[\sum_{i=0}^{\infty} \gamma^{i}\left(J\left(\boldsymbol{x}_{i}, \pi_{u}\left(\boldsymbol{x}_{i}\right), \boldsymbol{\sigma}_{i}\right)\right)-H\left(\boldsymbol{x}_{i}, \pi_{u}\left(\boldsymbol{x}_{i}\right)\right)\right)\right] \\
& \mathcal{P}_{10} \text { : } \quad \text { s.t. } \quad \boldsymbol{x}_{i+1}=f\left(\boldsymbol{x}_{i}, \pi_{u}\left(\boldsymbol{x}_{i}\right), \boldsymbol{\theta}_{i}\right) \\
& \boldsymbol{\theta}_{i} \sim p_{\boldsymbol{\theta}}\left(\cdot \mid \boldsymbol{x}_{i}, \pi_{u}\left(\boldsymbol{x}_{i}\right)\right), \quad \boldsymbol{\sigma}_{i} \sim p_{\boldsymbol{\sigma}}\left(\cdot \mid \boldsymbol{x}_{i}, \pi_{u}\left(\boldsymbol{x}_{i}\right)\right)
\end{aligned}
$$

In the remaining of this section, we show how by using RL algorithms it is possible to learn a PCL-NE of a CL-SDPG. We assume that the unconstrained OCP (5.226) related to the CL-SDPG under study is available through a simulator. In particular, we are going to study a network flow problem similar to the one used in Section 5.7, with some little variations.

\subsubsection{Learning parametric policies for MAC problem}

The closed-loop medium access channel game used for the numerical experiments in this section is given by the following set of coupled OCP:

$$
\begin{aligned}
\mathcal{G}_{11}: \underset{u_{k} \in \mathbb{U}}{\operatorname{maximize}} & \sum_{i=0}^{\infty} \gamma^{i}\left(\log \left(1+\frac{\left|\boldsymbol{h}_{k, i}\right|^{2} \pi_{u_{k}}\left(\boldsymbol{x}_{k, i}\right)}{1+\sum_{j \in \mathcal{N}: j \neq k}\left|\boldsymbol{h}_{j}\right|^{2} \pi_{u_{j}}\left(\boldsymbol{x}_{j, i}\right)}\right)+\alpha \boldsymbol{x}_{k, i}\right) \\
\forall k \in \mathcal{N} \quad & \boldsymbol{x}_{k, i+1}=\boldsymbol{x}_{k, i}-\boldsymbol{\delta}_{i} \pi_{u_{k}}\left(\boldsymbol{x}_{k, i}\right), \quad x_{k, 0}=B_{k, \max } \\
& 0 \leq \pi_{u_{k}}\left(\boldsymbol{x}_{k, i}\right) \leq P_{k, \max }, \quad 0 \leq \boldsymbol{x}_{k, i} \leq B_{k, \max }, \quad i=0, \ldots, \infty
\end{aligned}
$$

where the only difference with $\mathcal{G}_{8}$ in (5.209) is that the channel coefficients and depletion factor are random variables now. We choose the same setting with $N=4$ agents.

The main novelty of this section is that we learn a PCL-NE of (5.227) with no prior knowledge of the reward or state-dynamic functions, just by interaction with a black-box that simulates the system. At every time step, we observe state-sample $x_{i}$ and take $a_{i}=\pi_{u}\left(x_{i}\right)$, then the system returns new stochastic battery values and reward.

First of all, note that each agent's policy and reward depend only on its own battery, $\boldsymbol{x}_{k, i}$. Therefore, we can apply Theorem 5.4 and establish that the game is potential. 
Similar to Section 5.8.2, the optimal policy will make the battery deplete in finite time. Moreover, since $\boldsymbol{\delta}_{k, i} \geq 0$, the episode gets into a stationary (i.e., terminal) state once the battery has been completely depleted. Thus, we can consider a finite time-horizon problem and solve the OCP with standard convex optimization solvers (we use Matlab CVX [109]) and use the result as our benchmark for comparing with RL algorithms. Nevertheless, standard convex optimization solvers do not allow to include random variables. To surmount this issue, we have generated 100 independent sequences of random variables $\boldsymbol{h}_{k, i}$ and $\boldsymbol{\delta}_{k, i}$, for $i=1, \ldots, 100$ and $k=1, \ldots, 4$; and use them as deterministic variables for solving 100 convex optimization problems. In particular, we set $\left|\boldsymbol{h}_{k, i}\right|^{2}=\left|h_{k}\right|^{2} \boldsymbol{v}_{k, i}$ where $\left|h_{k}\right|^{2}$ is the deterministic value used in Sec. 5.8 .2 (i.e., $\left|h_{1}\right|^{2}=2.019,\left|h_{2}\right|^{2}=1.002,\left|h_{3}\right|^{2}=0.514$ and $\left|h_{4}\right|^{2}=0.308$ ) and $\boldsymbol{v}_{k, i}$ is a uniform random variable in the interval $[0.5,1]$. Similarly, we set $\boldsymbol{\delta}_{k, i}=\delta \boldsymbol{w}_{k, i}$, where $\delta=1$ is the value used in Sec. 5.8.2 and $\boldsymbol{w}_{k, i}$ is uniformly distributed in $[0.7,1.3]$.

The batteries depleted at a level $x_{T}<10^{-6}$ in all realizations. Then, we averaged the discounted sum of rewards over the 100 problems, so that we obtained an estimator of the value function. This average estimate of the value function obtained with the convex solver is $V_{\mathrm{cvx}}^{\star}=34.90$.

We have performed experiments with two state of the art RL algorithms that have proved to be able to handle continuous state-action sets, namely deep-deterministicpolicy-gradient (DDPG) [168] and trust-region-policy-optimization (TRPO) [255]. Both algorithms approximate the value function and the policy with a parametric approximations. The description of these algorithm is beyond the scope of this section. We use the implementations of both algorithms available at the RLLAB library [214] (see [82] for the description of some implementation details). Here, we just mention the parameters used during simulations:

- For DDPG, the policy and the value function are approximated with a multilayer perceptron (MLP) with two and three hidden layers, respectively, of size 32 units each and "rectified linear units" (RELU); the policy network uses a "tanh" nonlinear output, while the value network has linear output. Each iteration of DDPG takes 1000 simulation steps (i.e., state transition, action and rewards). The step-sizes are 0.001 for learning both the value function and the policy.

- TRPO uses a similar architecture for the policy (MLP with three hidden layers of size 32 and RELU output), with the difference that the output of the MLP maps each state to the mean of a Gaussian distribution, rather than to a deterministic value; the value function is approximated empirically as the discounted sum of 


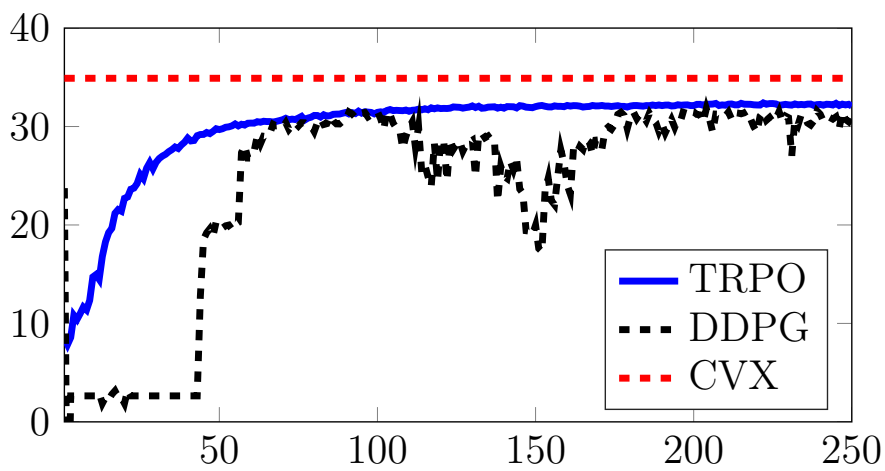

Figure 5.8 Results for the mac problem (5.227). We display the convergence of the TRPO and DDPG algorithms as well as the result obtained with the convex optimization solver).

the actual sample rewards, and it uses a linear function of a time-varying feature vector (obtained from a polynomial kernel of the state and reward) as baseline function for reducing variance of the optimization process. Each iteration of TRPO uses a batch of size 4000 simulation steps (i.e., state transition, action and rewards). The step-size is 0.01 .

Figure 5.8 shows the results. We can see that both DDPG and TRPO achieve similar results after convergence: about $84.6 \%$ and $92.7 \%$ of the optimal solution obtained with the convex optimization solver, respectively. More precisely, after 400 iterations, we obtain $V_{\text {ddpg }}^{\star}=29.53$, and $V_{\text {trpo }}^{\star}=32.34$. However, DDPG is less stable than TRPO.

We remark that the idea with this experiment is to show that RL is possible for DPGs, rather than achieving optimal performance. Hence, we avoid the cost of tuning the cited RL algorithms for this specific environment.

\subsection{Conclusions}

DPGs provide a useful framework for competitive multiagent applications under timevarying environments, when the agents compete for a common resource. We have considered a general problem setting with reward, state-transition and constraint functions that can be deterministic or stochastic and even change with time (i.e., nonstationary scenarios). We started analyzing open-loop deterministic DPGs in nonreduced form, with time-varying transition, reward and constrain functions and with explicit constrained state-action sets. Then, we extended the setting to stochastic state-transitions and rewards. Finally, we provided the closed-loop analysis of the latter stochastic case when the policies belong to any parametric class. To the best of our 
knowledge, previous works have neither dealt with DPGs with constraints explicitly in nonreduced form, nor provided closed-loop analysis with this generality. In all cases, we followed the same methodology, consisting in applying the Euler equation to the Lagrangian, so that we found conditions for finding an NE of the DPG through the solution of a related OCP.

In addition, we have shown that it is possible to learn its solution with an RL approach, even when the reward and state transition models are unknown. However, this is a difficult problem for standard RL algorithms because the state and action are continuous vector variables that lie in constrained sets. We have reviewed the RL literature and found algorithms that can work for continuous variables. Then, we included a penalty function when the solution does not satisfy the constraints. As an example, we applied this framework to a network flow optimization problem, obtaining satisfactory results.

We also introduced a range of communication and networking examples: energy demand control in a smart-grid network, network flow with relays that have bounded link capacity and limited battery life, multiple access communication in which users have to optimize the use of their batteries, and two optimal scheduling games with nonstationary channels. Although these problems have different features, the proposed framework allowed us to analyze and solve them in a unified manner.

As future work, it would be interesting to extend the current analysis to the most general case of stochastic policies. 



\section{Chapter 6}

\section{Conclusions}

We started this thesis introducing philosophical and practical motivations for studying multiagent scenarios.

Consider, for instance, neural networks, which are living a renaissance in the last years. The standard approach is to consider the neurons as non-smart parts of the system, but out of the interaction of these simple (non-intelligent) parts it emerges some adaptive behavior at the whole network level that has yielded state of the art results in supervised, unsupervised and reinforcement learning. This point of view makes sense since, at the end of the day, neurons only perform a linear combination and a simple nonlinear mapping, and the network is usually seen as a box that only interacts with the world through the minimization of some loss function that relates its input/output layers. However, most of the intelligent networks that we observe in our daily life consists of elements that are already intelligent by themselves (e.g., a basketball team, a group of students, a dolphin pack, etc.). Thus, our main philosophical motivation has been to study networks of adaptive agents, and see whether they could exhibit more intelligent behavior than each of the constituent elements (as it is the case of other successful systems, like the mentioned neural networks, where the constituent elements seem little or non intelligence at all). Our main conclusion in this regard is that cooperative consensus and diffusion based algorithms are able to combine the exploratory capabilities of the agents, including their input data distributions (e.g., different regions of the space of block of a vectorized image for distributed-PCA, or different behavior policies for distributed-GTD reinforcement learning) and their trajectories in the parameter space (e.g., the evolution of the distributed stochastic approximation performed by distributed-CE). This fusion of the exploratory capabilities of the agents yields two interesting features: 
- A form of transfer learning, where the agents learn from each other and are able to generalize to a global data distribution that each one has only observed partially. This feature can usually be theoretically guaranteed through the convergence analysis of each distributed algorithm.

- A form of regularization, where the agents provide their local information to their neighbors so that they can, e.g., avoid getting trapped in local optima in non-convex optimization. This is a promising empirical observation that deserves further investigation.

Further research of both features seem very promising for, e.g., transfer learning of optimal policies for families of problems in reinforcement learning, and as a novel form of regularization for deep-learning consisting in combining multiple networks with overlapping or distributed datasets.

In addition, recent advances in communications and sensing and computing technologies are yielding novel application domains, like cloud computing or the Internetof-Things, where large number of devices can gather and process huge amounts of data. Although centralized architectures, in which a central base-station (or fusion center) coordinates multiple devices, are useful (and even preferred) for small to mid-size applications, they might not be appropriate for coordinating myriads of devices or processing the huge amounts of data that they can provide. Thus, it would be desirable to have suitable distributed algorithmic frameworks that can tackle these challenging scenarios. We can extract two main conclusions in this regard:

- Cooperative consensus and diffusion based algorithms can be applied to many machine learning problems while bringing multiple benefits: they are robust against partial observability of the global dataset; they are able to distribute the computational load, while the communication cost per node scales with the number of neighbors, rather than with the network size); they are robust against agent or link failure; and they preserve the privacy of local data in distributed datasets.

- Competitive environments where the agents have to compete for a common resource can be usually solved in a very efficient manner, even in the most general case of dynamic environments with stochastic transitions and rewards, with coupled constraints and with closed-loop information structure, by checking whether they are potential and, if so, by solving a related single-objective problem.

More specifically, in this thesis we have studied the following cooperative and competitive scenarios. 
First, in Chapter 2, we use average-consensus algorithms to propose distributed implementations of PCA, PPCA, FA and LDA, and illustrated the performance of our algorithms on simulated image compression and distributed classification scenarios.

In Chapter 3, we applied diffusion optimization to off-policy reinforcement learning. In particular, we proposed a distributed algorithm, named DGTD, for policy evaluation in a scenario where the agents explore the environment with different behavior policies but cooperate to predict a common target policy. We provided conditions that guarantee convergence of the proposed DGTD and derived performance bounds for sufficiently small step-sizes. These results generalize the asymptotic results of the original single agent algorithm, in which we inspired.

We finished our journey on cooperative algorithms in Chapter 4, where we introduced a diffusion-based CE algorithm where a network of agents cooperate for optimizing an unknown nonconvex function. We developed detailed convergence analysis of DCE and showed convergence of the smoothed Bayesian recursion to the global solution. Numerical experiments have shown that the diffusion-form of cooperation embedded in the proposed DCE algorithm is usually able to combine the local estimates in an effective manner that matches - and sometimes even improve - the performance of the centralized solution.

In Chapter 5, we moved to competitive scenarios and considered a set of agents that compete to maximize their individual rewards in a time-varying environment, equipped with a some given state-transition equation. This is formally stated as a dynamic game, and we focused on a specific class of games, named dynamic potential games (DPG) that appear often in applications where the agents share some common resource. We were able to generalize previous analysis by considering the general case of dealing with constraints in nonreduced form and even to provide a preliminary CL study. In the studied cases, we introduced a related single-objective control problem and showed that the solution of this single-objective problem is a Nash equilibrium of the game.

In addition, we included some interesting lines of future and on-going research. 



\section{Bibliography}

[1] (2007). Learning principal directions: Integrated-squared-error minimization. Neurocomputing, 70(7-9):1372-1381.

[2] Acemoglu, D. (2009). Introduction to Modern Economic Growth. Princeton University Press.

[3] Al-Naffouri, T. Y. and Sayed, A. H. (2003). Transient analysis of data-normalized adaptive filters. IEEE Transactions on Signal Processing, 51(3):639-652.

[4] Amir, R. and Nannerup, N. (2006). Information structure and the tragedy of the commons in resource extraction. Journal of Bioeconomics, 8(2):147-165.

[5] Antos, A., Szepesvari, C., and Munos, R. (Vancouver, Canada, Dec. 2007). Fitted Q-iteration in continuous action-space MDPs. In Advances in Neural Information Processing Systems 20 (NIPS).

[6] Apostol, T. (1969). Calculus: Multi-variable Calculus and Linear Algebra, with Applications to Differential Equations and Probability. Wiley.

[7] Arfken, G., Weber, H., and Harris, F. (2013). Mathematical Methods for Physicists: A Comprehensive Guide. Elsevier.

[8] Arrow, K. J., Hurwicz, L., and Uzawa, H. (1958). Studies in Linear and Non-linear Programming. Stanford University Press.

[9] Azar, M. G., Lazaric, A., and Brunskill, E. (Beijing, 2014). Online stochastic optimization under correlated bandit feedback. In Proc. Int. Conf. on Machine Learning (ICML). JMLR Workshop and Conference Proceedings.

[10] Bai, Z., Chan, R. H., and Luk, F. T. (2005). Principal Component Analysis for Distributed Data Sets with Updating. In In Proceedings of International Workshop on Advanced Parallel Processing Technologies (APPT), pages 471-483.

[11] Baird, L. (Tahoe City, CA, USA, 1995). Residual algorithms: Reinforcement learning with function approximation. In Proc. Int. Conf. on Machine Learning $(I C M L)$, pages $30-37$.

[12] Bajovic, D., Jakovetic, D., Xavier, J., Sinopoli, B., and Moura, J. M. F. (2011). Distributed Detection via Gaussian Running Consensus: Large Deviations Asymptotic Analysis. IEEE Transactions on Signal Processing, 59(9):4381-4396. 
[13] Baldi, P. and Hornik, K. (1989). Neural networks and principal component analysis: learning from examples without local minima. Neural Netw., 2(1):53-58.

[14] Barbarossa, S. and Scutari, G. (2007). Bio-inspired sensor network design. IEEE Signal Processing Magazine, 24(3):26-35.

[15] Basar, T. and Olsder, G. J. (1999). Dynamic Noncooperative Game Theory. Society for Industrial and Applied Mathematics.

[16] Bazaraa, M. S., Sherali, H. D., and Shetty, C. M. (2006). Nonlinear programming: theory and algorithms. Wiley-Interscience, 3rd ed edition.

[17] Bear, M., Connors, B., and Paradiso, M. (2015). Neuroscience: Exploring the Brain. Wolters Kluwer.

[18] Béjar, B., Belanovic, P., and Zazo, S. (2010). Distributed gauss-newton method for localization in ad-hoc networks. In Asilomar Conf. on Signals, Systems and Computers (ASILOMAR), pages 1452-1454.

[19] Belanovic, P., Valcarcel Macua, S., and Zazo, S. (2012a). Distributed primary user identification from imprecise location information. In Int. Conference on Systems, Signals and Image Processing (IWSSIP), pages 89-92.

[20] Belanovic, P., Valcarcel Macua, S., and Zazo, S. (2012b). Distributed static linear gaussian models using consensus. Neural Networks, 34:96-105.

[21] Belanovic, P., Valcarcel Macua, S., and Zazo, S. (2012c). Location-aided distributed primary user identification in a cognitive radio scenario. In IEEE Int. Conference on Acoustics, Speech and Signal Processing (ICASSP), pages 3761-3764.

[22] Bellemare, M. G., Veness, J., and Bowling, M. (2012). Sketch-based linear value function approximation. In Proc. Advances in Neural Information Processing Systems 25 (NIPS), pages $2222-2230$.

[23] Benzi, M., Golub, G. H., and Liesen, J. (2005). Numerical solution of saddle point problems. Acta Numerica, 14:1-137.

[24] Bertrand, A. and Moonen, M. (2014). Distributed adaptive estimation of covariance matrix eigenvectors in wireless sensor networks with application to distributed PCA. Signal Processing, 104:120-135.

[25] Bertsekas, D. (1982). Distributed dynamic programming. IEEE Transactions on Automatic Control, 27(3):610-616.

[26] Bertsekas, D. (1999). Nonlinear Programming. Athena Scientific.

[27] Bertsekas, D. and Tsitsiklis, J. (1997). Parallel and Distributed Computation: Numerical Methods. Athena Scientific.

[28] Bertsekas, D. P. (2007). Dynamic Programming and Optimal Control, volume 2. Athena Scientific, 3rd edition. 
[29] Bertsekas, D. P. (2009). Convex Optimization Theory. Athena Scientific.

[30] Bertsekas, D. P. (2012). Dynamic Programming and Optimal Control, volume 2. Athena Scientific, 4rd edition.

[31] Bertsekas, D. P. and Tsitsiklis, J. N. (1996). Neuro-Dynamic Programming. Athena Scientific.

[32] Bertsekas, D. P. and Yu, H. (2010, Allerton, IL, USA). Distributed asynchronous policy iteration in dynamic programming. In IEEE Allerton Conf. on Communication, Control, and Computing, pages 1368-1375.

[33] Beskos, A., Crisan, D., Jasra, A., Kamatani, K., and Zhou, Y. (2014). A stable particle filter in high-dimensions. arXiv preprint arXiv:1412.3501.

[34] Bhatnagar, S. (2011). The Borkar-Meyn theorem for asynchronous stochastic approximations. Systems and Control Letters, 60(7):472-478.

[35] Bhatnagar, S., Sutton, R. S., Ghavamzadeh, M., and Lee, M. (2009). Natural actor-critic algorithms. Automatica, 45(11):2471-2482.

[36] Bianchi, P. and Jakubowicz, J. (2013). Convergence of a multi-agent projected stochastic gradient algorithm for non-convex optimization. IEEE Transactions on Automatic Control, 58(2):391-405.

[37] Bingham, D. (2015). Optimization test problems. https://www.sfu.ca/ ssurjano/ optimization.html.

[38] Bishop, C. M. (2006). Pattern Recognition and Machine Learning (Information Science and Statistics). Springer.

[39] Blatt, D., Hero, A. O., and Gauchman, H. (2007). A convergent incremental gradient method with a constant step size. SIAM Journal on Optimization, 18(1):2951 .

[40] Blondel, V., Hendrickx, J., Olshevsky, A., and Tsitsiklis, J. (Seville, Spain, 2005). Convergence in multiagent coordination, consensus, and flocking. In Proc. IEEE Conf. on Decision and Control, and European Control Conf. (CDC-ECC), pages 2996-3000.

[41] Bohm, D. (2005). Wholeness and the Implicate Order. Taylor \& Francis.

[42] Boots, B. and Gordon, G. J. (2010). Predictive state temporal difference learning. In Proc. Advances in Neural Information Processing Systems 23 (NIPS), pages 271-279.

[43] Borkar, V. S. and Meyn, S. (1999). The O.D.E. method for convergence of stochastic approximation and reinforcement learning. SIAM Journal on Control and Optimization, 38:447-469.

[44] Botev, A., Lever, G., and Barber, D. (2016). Nesterov's Accelerated Gradient and Momentum as approximations to Regularised Update Descent. ArXiv e-prints. 
[45] Boutsidis, C. and Woodruff, D. P. (2015). Communication-optimal distributed principal component analysis in the column-partition model. CoRR, abs/1504.06729.

[46] Boyd, S., Ghosh, A., Prabhakar, B., and Shah, D. (2006). Randomized gossip algorithms. IEEE/ACM Transactions on Networking (TON), 14(SI):2508-2530.

[47] Boyd, S., Parikh, N., Chu, E., Peleato, B., and Eckstein, J. (2011). Distributed optimization and statistical learning via the alternating direction method of multipliers. Foundations and Trends in Machine Learning, 3(1).

[48] Boyd, S. and Vandenberghe, L. (2004). Convex Optimization. Cambridge University Press.

[49] Brewer, J. A., Worhunsky, P. D., Gray, J. R., Tang, Y.-Y., Weber, J., and Kober, H. (2011). Meditation experience is associated with differences in default mode network activity and connectivity. Proceedings of the National Academy of Sciences, 108(50):20254-20259.

[50] Brochu, E., Cora, V. M., and de Freitas, N. (2010). A tutorial on bayesian optimization of expensive cost functions, with application to active user modeling and hierarchical reinforcement learning. CoRR, abs/1012.2599.

[51] Busoniu, L., Babuska, R., Schutter, D., and Ernst, D. (2010). Reinforcement Learning and Dynamic Programming Using Function Approximators. CRC Press.

[52] Cannelli, L., Facchinei, F., Kungurtsev, V., and Scutari, G. (2016). Asynchronous parallel algorithms for nonconvex big-data optimization: Model and convergence. arXiv preprint arXiv:160\%.04818.

[53] Cattivelli, F. S. and Sayed, A. H. (2010). Diffusion LMS Strategies for Distributed Estimation. IEEE Transactions on Signal Processing, 58(3):1035-1048.

[54] Chamley, C., Scaglione, A., and Li, L. (2013). Models for the diffusion of beliefs in social networks: An overview. IEEE Signal Processing Magazine, 30(3):16-29.

[55] Chang, T.-H., Nedic, A., and Scaglione, A. (2014). Distributed constrained optimization by consensus-based primal-dual perturbation method. IEEE Transactions on Automatic Control, 59(6):1524-1538.

[56] Chen, J. and Sayed, A. H. (2012a). Diffusion adaptation strategies for distributed optimization and learning over networks. IEEE Transactions on Signal Processing, 60(8):4289-4305.

[57] Chen, J. and Sayed, A. H. (2013a). Distributed Pareto optimization via diffusion strategies. IEEE Journal of Selected Topics in Signal Processing, 7(2):205-220.

[58] Chen, J. and Sayed, A. H. (Monticello, IL, USA, October 2012b). On the limiting behavior of distributed optimization strategies. In Proc. Annual Allerton Conference on Communication, Control, and Computing, pages 1535-1542.

[59] Chen, J. and Sayed, A. H. (submitted for publication. Also available as arXiv:1312.7581, Dec. 2013b). The learning behavior of adaptive networks - Part I: Transient analysis. 
[60] Chiang, M., Low, S., Calderbank, A., and Doyle, J. (2007). Layering as optimization decomposition: a mathematical theory of network architectures. Proceedings of the IEEE, 95(1):255-312.

[61] Chopra, D. and Mlodinow, L. (2011). War of the Worldviews: Where Science and Spirituality Meet - and Do Not. Three Rivers Press.

[62] Cortés, J. (2008). Distributed algorithms for reaching consensus on general functions. Automatica, 44(3):726-737.

[63] Cunningham, F. (1967). Taking limits under the integral sign. Mathematics Magazine, 40(4):179-186.

[64] Davidson, K. and Donsig, A. (2009). Real Analysis and Applications: Theory in Practice. Springer.

[65] Dawkins, R. (2016). The Extended Selfish Gene. Oxford University Press.

[66] De la Torre, F. (2012). A least-squares framework for component analysis. IEEE Transactions Pattern Analysis and Machine Intelligence, 34(6):1041-1055.

[67] De la Torre, F. and Kanade, T. (2006). Discriminative cluster analysis. In Proc. of Int. Conf. on Machine Learning, pages 241-248. ACM.

[68] Dechert, W. D. (1978). Optimal control problems from second-order difference equations. Journal of Economic Theory, 19(1):50-63.

[69] Dechert, W. D. (1997). Non cooperative dynamic games: a control theoretic approach. Technical report.

[70] Dechert, W. D. and Brock, W. A. (2000). The lake game. Technical report.

[71] Degris, T. and Modayil, J. (Palo Alto, CA, USA, 2012). Scaling-up knowledge for a cognizant robot. In Notes AAAI Spring Symposium Series.

[72] Degris, T., White, M., and Sutton, R. S. (Edinburgh, Scotland, 2012). Off-policy actor-critic. In Proc Int. Conf. on Machine Learning (ICML), pages 457-464, New York, NY, USA. ACM.

[73] DeGroot, M. H. (1974). Reaching a consensus. Journal of American Statistical Association, 69(345):118-121.

[74] del Val, J., Zazo, S., Valcarcel Macua, S., Zazo, J., and Parras, J. (2016). Optimal attack and defence of large scale networks using mean field theory. In European Signal Processing Conference (EUSIPCO), pages 973-977.

[75] Deng, L. and Yu, D. (2014). Deep Learning: Methods and Applications. Foundations and trends in signal processing. Now Publishers.

[76] Di Lorenzo, P. and Scutari, G. (2016). NEXT: In-network nonconvex optimization. IEEE Transactions on Signal and Information Processing over Networks, 2(2):120136. 
[77] Djuric, P. M. and Bugallo, M. F. (Brisbane, Australia, Apr. 2015). Multiple particle filtering with improved efficiency and performance. In Proc. IEEE Int. Conf. on Acoustics, Speech and Signal Processing (ICASSP).

[78] Dockner, E. (1988). On the relation between dynamic oligopolistic competition and long-run competitive equilibrimn. European Journal of Political Economy, $4(1): 47-64$.

[79] Dopico, N. I., Bejar, B., Valcarcel Macua, S., Belanovic, P., and Zazo, S. (2011). Improved animal tracking algorithms using distributed kalman-based filters. In European Wireless - Sustainable Wireless Technologies, pages 1-8.

[80] Dorigo, M. and Gambardella, L. M. (1997). Ant colony system: a cooperative learning approach to the traveling salesman problem. IEEE Transactions on Evolutionary Computation, 1(1):53-66.

[81] Dragone, D., Lambertini, L., Leitmann, G., and Palestini, A. (2015). Hamiltonian potential functions for differential games. Automatica, 62:134-138.

[82] Duan, Y., Chen, X., Houthooft, R., Schulman, J., and Abbeel, P. (New York, USA, 2016). Benchmarking deep reinforcement learning for continuous control. In Proc. Int. Conf. on Machine Learning (ICML).

[83] Duchi, J., Hazan, E., and Singer, Y. (2011). Adaptive subgradient methods for online learning and stochastic optimization. Journal of Machine Learning Research, $12(\mathrm{Jul}): 2121-2159$.

[84] Dyson, F. (1990). Infinite in All Directions. Penguin Books.

[85] Dyson, F. (2000). Progress in religion : A talk by freeman dyson. Acceptance speech for the Templeton Prize, Washington National Cathedral.

[86] Ehrgott, M. (2005). Multicriteria Optimization. Springer.

[87] Evans, D. (2011). The internet of things: How the next evolution of the internet is changing everything. Technical report, Cisco.

[88] Facchinei, F., Piccialli, V., and Sciandrone, M. (2011). Decomposition algorithms for generalized potential games. Computational Optimization and Applications, $50(2): 237-262$.

[89] Fellus, J., Picard, D., and Gosselin, P.-H. (2015). Asynchronous gossip principal components analysis. Neurocomputing, 169:262 - 271.

[90] Fernandez-Bes, J., Arenas-Garcia, J., and Sayed, A. H. (Florence, Italy, May 2014). Adjustment of combination weights over adaptive diffusion networks. In Proc. IEEE Int. Conf. on Acoustics, Speech and Signal Processing (ICASSP), pages 6409-6413.

[91] Forero, P. A., Cano, A., and Giannakis, G. B. (2010). Consensus-based distributed support vector machines. Journal of Machine Learning Research, 11(May):1663-1707. 
[92] Frank, A. and Asuncion, A. (2010). UCI Machine Learning Repository. http: //archive.ics.uci.edu/ml.

[93] Fudenberg, D. and Levine, D. K. (1988). Open-loop and closed-loop equilibria in dynamic games with many players. Journal of Economic Theory, 44(1):1-18.

[94] Fukunaga, K. (1990). Introduction to statistical pattern recognition (2nd ed.). Academic Press Professional, Inc.

[95] Garin, F. and Schenato, L. (2011). A survey on distributed estimation and control applications using linear consensus algorithms. In Networked Control Systems, volume 406, pages 75-107. Springer.

[96] Geist, M. and Pietquin, O. (Paris, France, 2011). Parametric value function approximation: A unified view. In IEEE Symp. on Adaptive Dynamic Programming And Reinforcement Learning (ADPRL), pages 9-16.

[97] Geramifard, A., Walsh, T. J., Tellex, S., Chowdhary, G., Roy, N., and How, J. P. (2013a). A tutorial on linear function approximators for dynamic programming and reinforcement learning. Foundations and Trends in Machine Learning, 6(4):375-451.

[98] Geramifard, A., Walsh, T. J., Tellex, S., Chowdhary, G., Roy, N., and How, J. P. (2013b). A tutorial on linear function approximators for dynamic programming and reinforcement learning. Foundations and Trends in Machine Learning, 6(4):375-451.

[99] Gerencsér, L., Vágó, Z., and Hill, S. D. (2008). The magic of spsa. Proceedings in Applied Mathematics and Mechanics.

[100] Glover, F. and Laguna, M. (1999). Tabu search. In Handbook of combinatorial optimization, pages 2093-2229. Springer.

[101] Goldberg, D. E. (1989). Genetic Algorithms in Search, Optimization, and Machine Learning. Addison-Wesley.

[102] Goleman, D. (2007). Social Intelligence: The New Science of Human Relationships. Bantam Books.

[103] Golub, G. and Van Loan, C. (1996). Matrix Computations. Johns Hopkins University Press.

[104] González-Sánchez, D. and Hernández-Lerma, O. (2013). Discrete-Time Stochastic Control and Dynamic Potential Games: The Euler-Equation Approach. Springer.

[105] González-Sánchez, D. and Hernández-Lerma, O. (2014). Dynamic potential games: The discrete-time stochastic case. Dynamic Games and Applications, 4(3):309-328.

[106] Goodfellow, I., Bengio, Y., and Courville, A. (2016). Deep Learning. MIT Press.

[107] Goschin, S., Littman, M. L., and Ackley, D. H. (2011). The effects of selection on noisy fitness optimization. In Proc. of the 13th annual Conf. on Genetic and Evolutionary Computation, pages 2059-2066. 
[108] Goschin, S., Weinstein, A., and Littman, M. (2013). The cross-entropy method optimizes for quantiles. In Proc. of the 30th International Conference on Machine Learning, volume 28, pages 1193-1201. JMLR Workshop and Conference Proceedings.

[109] Grant, M. and Boyd, S. (2014). CVX: Matlab software for disciplined convex programming, version 2.1. http://cvxr.com/cvx.

[110] Grondman, I., Busoniu, L., Lopes, G. A. D., and Babuska, R. (2012). A survey of actor-critic reinforcement learning: Standard and natural policy gradients. IEEE Transactions on Systems, Man, and Cybernetics, Part C (Applications and Reviews), 42(6):1291-1307.

[111] Guo, Y.-F., Lin, X., Teng, Z., Xue, X., and Fan, J. (2012). A covariancefree iterative algorithm for distributed principal component analysis on vertically partitioned data. Pattern Recognition, 45(3):1211-1219.

[112] György, A. and Kocsis, L. (2011). Efficient multi-start strategies for local search algorithms. Journal of Artificial Intelligence Research, pages 407-444.

[113] Han, Z., Niyato, D., Saad, W., Baar, T., and Hjrungnes, A. (2012). Game Theory in Wireless and Communication Networks: Theory, Models, and Applications. Cambridge University Press.

[114] Hansen, N. and Auger, A. (Dublin, Ireland, 2011). Cma-es: evolution strategies and covariance matrix adaptation. In Proc. ACM Conf. Companion on Genetic and Evolutionary Computation (GECCO), pages 991-1010.

[115] Hansen, N. and Ostermeier, A. (2001). Completely derandomized self-adaptation in evolution strategies. Evolutionary computation, 9(2):159-195.

[116] Hastings, W. K. (1970). Monte Carlo sampling methods using Markov chains and their applications. Biometrika, 57(1):97-109.

[117] Hawkins, J., Ahmad, S., and Dubinsky, D. (2012). Hierarchical temporal memory including htm cortical learning algorithms. Technical report, Numenta, Inc.

[118] Hawkins, J. and Blakeslee, S. (2007). On Intelligence. Henry Holt and Company.

[119] Hazan, E., Levy, K. Y., and Shalev-Swartz, S. (2015). On graduated optimization for stochastic non-convex problems. arXiv preprint arXiv:1503.03712.

[120] Heess, N., Wayne, G., Silver, D., Lillicrap, T., Erez, T., and Tassa, Y. (2015). Learning continuous control policies by stochastic value gradients. In Advances in Neural Information Processing Systems 28 (NIPS), pages 2926-2934.

[121] Heidrich-Meisner, V. and Igel, C. (Dortmund, Germany, 2008). Evolution strategies for direct policy search. Int. Conf. Parallel Problem Solving from Nature (PPSN), pages 428-437.

[122] Hernández-Lerma, O. and González-Sánchez, D. (2013). Discrete Time Stochastic Control and Dynamic Potential Games: The Euler Equation Approach. Springer. 
[123] Homem-de Mello, T. (2007). A study on the cross-entropy method for rare-event probability estimation. INFORMS Journal on Computing, 19(3):381-394.

[124] Horn, R. and Johnson, C. (1990). Matrix Analysis. Cambridge University Press.

[125] Hotelling, H. (1933). Analysis of a Complex of Statistical Variables into Principal Components. Journal of Educational Psychology, 24(6):417-441.

[126] Hu, J., Chang, H., Fu, M. C., and Marcus, S. I. (2011). Dynamic sample budget allocation in model-based optimization. Journal of Global Optimization, 50(4):575-96.

[127] Hu, J., Fu, M. C., and Marcus, S. I. (2007). A model reference adaptive search method for global optimization. Operations Research, 55(3):549-568.

[128] Hu, J., Hu, P., and Chang, H. S. (2012a). A stochastic approximation framework for a class of randomized optimization algorithms. IEEE Transactions on Automatic Control, 57(1):165-178.

[129] Hu, J., Wang, Y., Zhou, E., Fu, M. C., and Marcus, S. I. (2012b). A survey of some model-based methods for global optimization. In Optimization, Control, and Applications of Stochastic Systems, pages 157-179. Springer.

[130] Hu, J., Zhou, E., and Fan, Q. (2014). Model-based annealing random search with stochastic averaging. ACM Transactions on Modeling and Computer Simulation, 24(4):21.

[131] Jadbabaie, A., Lin, J., and Morse, A. S. (2003). Coordination of groups of mobile autonomous agents using nearest neighbor rules. IEEE Transactions on automatic control, 48(6):988-1001.

[132] Jaggi, M., Smith, V., Takác, M., Terhorst, J., Krishnan, S., Hofmann, T., and Jordan, M. I. (2014). Communication-efficient distributed dual coordinate ascent. In Advances in Neural Information Processing Systems (NIPS), pages 3068-3076.

[133] Jalali, A. and Ferguson, M. (1992). On distributed dynamic programming. IEEE Transactions on Automatic Control, 37(5):685-689.

[134] Jin, Z., Yang, J.-Y., Hu, Z.-S., and Lou, Z. (2001). Face recognition based on the uncorrelated discriminant transformation. Pattern recognition, 34(7):1405-1416.

[135] Johansson, B., Rabi, M., and Johansson, M. (2009). A randomized incremental subgradient method for distributed optimization in networked systems. SIAM Journal on Optimization, 20(3):1157-1170.

[136] Jolliffe, I. T. (2002). Principal Component Analysis. Springer, 2nd edition.

[137] Kamihigashi, T. et al. (2006). Transversality conditions and dynamic economic behavior. Technical report, Kobe University, Japan.

[138] Kar, S. and Moura, J. (2011). Convergence rate analysis of distributed gossip (linear parameter) estimation: Fundamental limits and tradeoffs. IEEE Journal of Selected Topics in Signal Processing, 5(4):674-690. 
[139] Kar, S. and Moura, J. M. (2009). Distributed consensus algorithms in sensor networks with imperfect communication: Link failures and channel noise. IEEE Transactions on Signal Processing, 57(1):355-369.

[140] Kar, S., Moura, J. M. F., and Poor, H. V. (2013). QD-learning: A collaborative distributed strategy for multi-agent reinforcement learning through consensus + innovations. IEEE Transactions on Signal Processing, 61(7):1848-1862.

[141] Kargupta, H., Huang, W., Sivakumar, K., and Johnson, E. (2001). Distributed Clustering Using Collective Principal Component Analysis. Knowledge and Information Systems, 3:422-448.

[142] Kelly, F. (1997). Charging and rate control for elastic traffic. European Transactions on Telecommunications, 8(1):33-37.

[143] Kelly, F., Maulloo, A., and Tan, D. (1998). Rate control in communication networks: shadow prices, proportional fairness and stability. Journal of the Operational Research Society, 49:237-252.

[144] Kenyeres, J., Kenyeres, M., and Rupp, M. (2011a). Experimental node failure analysis in WSNs. In Int. Conf. on Systems, Signals and Image Processing, pages $1-5$.

[145] Kenyeres, J., Kenyeres, M., Rupp, M., and Farkas, P. (2011b). Wsn implementation of the average consensus algorithm. In European Wireless, pages 1-8.

[146] Kingma, D. and Ba, J. L. (San Diego, USA, 2015). Adam: A method for stochastic optimization. In Proc. Int. Conf. on Learning Representations (ICLR).

[147] Kirkpatrick, S., Gelatt, C. D., and Vecchi, M. P. (1983). Optimization by simulated annealing. Science, 220(4598):671-680.

[148] Kober, J. and Peters, J. R. (Vancouver, Canada, Dec. 2009). Policy search for motor primitives in robotics. In Advances in neural information processing systems (NIPS), pages 849-856.

[149] Koblents, E. and Miguez, J. (Vancouver, Canada, May 2013). A population monte carlo scheme for computational inference in high dimensional spaces. In Proc. IEEE Int. Conf. on Acoustics, Speech and Signal Processing (ICASSP).

[150] Konda, V. R. and Tsitsiklis, J. N. (2003). On actor-critic algorithms. SIAM Journal on Control and Optimization, 42(4):1143-1166.

[151] Konidaris, G. (Chicago, Illinois, July 2008). Value function approximation in reinforcement learning using the fourier basis. In Proc. AAAI Conf. on Artificial Intelligence.

[152] Kroese, D., Porotsky, S., and Rubinstein, R. (2006). The cross-entropy method for continuous multi-extremal optimization. Methodology and Computing in Applied Probability, 8(3):383-407. 
[153] Kroese, D. P., Taimre, T., and Botev, Z. I. (2011). Handbook for Monte Carlo methods. Wiley.

[154] Kurzweil, R. (2005). The Singularity Is Near: When Humans Transcend Biology. Penguin Publishing Group.

[155] Kurzweil, R. (2012). How to Create a Mind: The Secret of Human Thought Revealed. Penguin Publishing Group.

[156] Kydland, F. (1975). Noncooperative and dominant player solutions in discrete dynamic games. International Economic Review, pages 321-335.

[157] Lazaric, A., Restelli, M., and Bonarini, A. (Vancouver, Canada, Dec. 2007). Reinforcement learning in continuous action spaces through sequential monte carlo methods. In Advances in neural information processing systems (NIPS), pages 833-840.

[158] Le, Q., Ranzato, M., Monga, R., Devin, M., Chen, K., Corrado, G., Dean, J., and $\mathrm{Ng}$, A. (2012). Building high-level features using large scale unsupervised learning. In Proc. Int. Conf. on Machine Learning (ICML), pages 81-88.

[159] Le Borgne, Y.-A., Raybaud, S., and Bontempi, G. (2008). Distributed Principal Component Analysis for Wireless Sensor Networks. Sensors, 8(8):4821-4850.

[160] Levhari, D. and Mirman, L. J. (1980). The great fish war: An example using a dynamic cournot-nash solution. The Bell Journal of Economics, 11(1):322-334.

[161] Levi-Montalcini, R. (2001). La galaxia mente. Critica.

[162] Levin, D. A., Peres, Y., and Wilmer, E. L. (2006). Markov Chains and Mixing Times. American Mathematical Society.

[163] Li, N. and Marden, J. (2013). Designing games for distributed optimization. IEEE Journal of Selected Topics in Signal Processing, 7(2):230-242.

[164] Li, N. and Marden, J. (2014). Decoupling coupled constraints through utility design. IEEE Transactions on Automatic Control, 59(8):2289-2294.

[165] Liang, J., Qu, B., Suganthan, P., and Hernández-Díaz, A. G. (2013). Problem definitions and evaluation criteria for the cec 2013 special session on real-parameter optimization. Technical report, Zhengzhou University, Computational Intelligence Laboratory, and Nanyang Technological University.

[166] Liang, J., Yu, G., Chen, B., and Zhao, M. (2016). Decentralized dimensionality reduction for distributed tensor data across sensor networks. IEEE Transactions on Neural Networks and Learning Systems, 27(11):2174-2186.

[167] Liang, Y., Balcan, M.-F. F., Kanchanapally, V., and Woodruff, D. (2014). Improved distributed principal component analysis. In Advances in Neural Information Processing Systems (NIPS) 27, pages 3113-3121. 
[168] Lillicrap, T. P., Hunt, J. J., Pritzel, A., Heess, N., Erez, T., Tassa, Y., Silver, D., and Wierstra, D. (preprint arXiv:1509.02971v1, 2015.). Continuous control with deep reinforcement learning.

[169] Lindgren, G. (2012). Stationary Stochastic Processes: Theory and Applications. Taylor \& Francis.

[170] Liu, B., Liu, J., Ghavamzadeh, M., Mahadevan, S., and Petrik, M. (New York, USA, 2016). Proximal gradient temporal difference learning algorithms. In Proc. Int. Joint Conference on Artificial Intelligence (IJCAI).

[171] Loog, M., JisseDuistermaat, J., and Florack, L. M. (2001). On the behavior of spatial critical points under gaussian blurring a folklore theorem and scale-space constraints. In Scale-Space and Morphology in Computer Vision, pages 183-192. Springer.

[172] Lopes, C. G. and Sayed, A. H. (2007). Incremental adaptive strategies over distributed networks. Signal Processing, IEEE Transactions on, 55(8):4064-4077.

[173] Low, S. (1999, Edinburgh, UK). Optimization flow control with on-line measurement or multiple paths. In Int. Teletraffic Congress, pages 237-249.

[174] Luders, E., Cherbuin, N., and Kurth, F. (2015). Forever young(er): potential age-defying effects of long-term meditation on gray matter atrophy. Frontiers in Psychology, 5:1551.

[175] Luders, E., Clark, K., Narr, K. L., and Toga, A. W. (2011). Enhanced brain connectivity in long-term meditation practitioners. NeuroImage, 57(4):1308-1316.

[176] Lutz, A., Brefczynski-Lewis, J., Johnstone, T., and Davidson, R. J. (2008). Regulation of the neural circuitry of emotion by compassion meditation: effects of meditative expertise. PloS one, 3(3):e1897.

[177] Maei, H. R. (2011). Gradient Temporal-Difference Learning Algorithms. PhD thesis, University of Alberta.

[178] Maei, H. R. and Sutton, R. S. (Lugano, Switzerland, 2010). GQ( $\lambda$ ): A general gradient algorithm for temporal-difference prediction learning with eligibility traces. In Proc. Conference on Artificial General Intelligence (AGI), volume 1, pages 91-96.

[179] Mahadevan, S. (2009). Learning representation and control in Markov decision processes: New frontiers. Foundations and Trends in Machine Learning, 1(4):403565.

[180] Mannor, S., Rubinstein, R. Y., and Gat, Y. (Washington D.C., USA, 2003). The cross entropy method for fast policy search. In Proc. Int. Conf. Machine Learning (ICML), pages 512-519.

[181] Marden, J. R. (2012). State based potential games. Automatica, 48(12):30753088. 
[182] Marsden, J. and Hoffman, M. (1993). Elementary Classical Analysis. W. H. Freeman.

[183] Martí, R., Moreno-Vega, J. M., and Duarte, A. (2010). Advanced multi-start methods. In Handbook of metaheuristics, pages 265-281. Springer.

[184] Martin H, J. A. and de Lope, J. (2009). Ex $\langle a\rangle$ : An effective algorithm for continuous actions reinforcement learning problems. In IEEE Annual Conf. of Industrial Electronics (IECON), pages 2063-2068.

[185] Mclachlan, G. J. and Krishnan, T. (2007). The EM Algorithm and Extensions. Wiley-Interscience.

[186] Melo, F. S. and Lopes, M. (2008). Fitted natural actor-critic: A new algorithm for continuous state-action mdps. In Machine Learning and Knowledge Discovery in Databases, volume 5212, pages 66-81. Springer.

[187] Menache, I., Mannor, S., and Shimkin, N. (2005). Basis function adaptation in temporal difference reinforcement learning. Annals of Operations Research, 134:215238.

[188] Meringer, M. and Weisstein, E. W. (2017). Regular graph. MathWorld, http: //mathworld.wolfram.com/RegularGraph.html.

[189] Metropolis, N., Rosenbluth, A. W., Rosenbluth, M. N., Teller, A. H., and Teller, E. (1953). Equation of state calculations by fast computing machines. The journal of chemical physics, 21(6):1087-1092.

[190] Millán, J., Posenato, D., and Dedieu, E. (2002). Continuous-action Q-learning. Machine Learning, 49(2-3):247-265.

[191] Milton, J. and Arnold, J. (1995). Introduction to Probability and Statistics: Principles and Applications for Engineering and the Computing Sciences. McGrawHill.

[192] Minsky, M. (1988). Society Of Mind. Simon \& Schuster.

[193] Mo, J. and Walrand, J. (2000). Fair end-to-end window-based congestion control. IEEE/ACM Transactions on Networking, 8(5):556-567.

[194] Mobahi, H. and Fisher III, J. W. (2015). On the link between gaussian homotopy continuation and convex envelopes. In Energy Minimization Methods in Computer Vision and Pattern Recognition, pages 43-56.

[195] Modayil, J., White, A., and Sutton, R. S. (2014). Multi-timescale nexting in a reinforcement learning robot. Adaptive Behavior, 22(2):146-160.

[196] Monderer, D. and Shapley, L. S. (1996). Potential games. Games and Economic Behavior, 14(1):124-143.

[197] Morell, A., Correa, A., Barceló, M., and Vicario, J. L. (2016). Data aggregation and principal component analysis in wsns. IEEE Transactions on Wireless Communications, 15(6):3908-3919. 
[198] Munos, R. (Granada, Spain, Dec. 2011). Optimistic optimization of a deterministic function without the knowledge of its smoothness. In Advances in Neural Information Processing Systems 24 (NIPS).

[199] Nedic, A. and Bertsekas, D. (2001). Incremental subgradient methods for nondifferentiable optimization. SIAM Journal on Optimization, 12(1):109-138.

[200] Nedic, A. and Olshevsky, A. (2015). Distributed optimization over time-varying directed graphs. IEEE Transactions on Automatic Control, 60(3):601-615.

[201] Nedic, A. and Ozdaglar, A. (2009). Distributed subgradient methods for multiagent optimization. IEEE Transactions on Automatic Control, 54(1):48-61.

[202] Nedic, A. and Ozdaglar, A. (2010). Cooperative distributed multi-agent optimization. In Convex Optimization in Signal Processing and Communications. Cambridge University Press.

[203] Nedich, A. et al. (2015). Convergence rate of distributed averaging dynamics and optimization in networks. Foundations and Trends@ in Systems and Control, $2(1): 1-100$.

[204] Nesterov, Y. (1983). A method of solving a convex programming problem with convergence rate o (1/k2). In Soviet Mathematics Doklady, volume 27, pages 372-376.

[205] Neto, E. S. H. and De Pierro, Á. R. (2009). Incremental subgradients for constrained convex optimization: a unified framework and new methods. SIAM Journal on Optimization, 20(3):1547-1572.

[206] Neumann, G. and Peters, J. R. (Vancouver, Canada, Dec. 2009). Fitted qiteration by advantage weighted regression. In Proc. Advances in neural information processing systems (NIPS), pages 1177-1184.

[207] Ng, A. Y., Parr, R., and Koller, D. (Denver, CO, USA, Dec. 1999). Policy search via density estimation. In Advances in Neural Information Processing Systems 12 (NIPS), pages 1022-1028.

[208] Nielsen, F. and Garcia, V. (2009). Statistical exponential families: A digest with flash cards. CoRR, abs/0911.4863.

[209] Olfati-Saber, R. (2005). Distributed kalman filter with embedded consensus filters. In IEEE Conf. on Decision and Control, pages 8179-8184.

[210] Olfati-Saber, R. (2009). Kalman-consensus filter: Optimality, stability, and performance. In Proc. IEEE Conf. on Decision and Control, pages 7036-7042.

[211] Olfati-Saber, R., Fax, J. A., and Murray, R. M. (2007). Consensus and cooperation in networked multi-agent systems. Proceedings of the IEEE, 95(1):215-233.

[212] Olfati-Saber, R. and Murray, R. (2004). Consensus problems in networks of agents with switching topology and time-delays. IEEE Transactions on Automatic Control, 49:1520-1533. 
[213] Olshevsky, A. and Tsitsiklis, J. N. (2009). Convergence speed in distributed consensus and averaging. SIAM Journal on Control and Optimization, 48(1):33-55.

[214] OpenAI (2016). Rllab. https://github.com/openai/rllab.

[215] Păduraru, C., Precup, D., Pineau, J., and Comănici, G. (2012). An empirical analysis of off-policy learning in discrete mdps. In Workshop on Reinforcement Learning, page 89.

[216] Pagliari, R. and Scaglione, A. (2009). Implementation of average consensus protocols for commercial sensor networks platforms. In Grid Enabled Remote Instrumentation, pages 81-95. Springer.

[217] Parr, R., Li, L., Taylor, G., Painter-Wakefield, C., and Littman, M. (Helsinki, Finland, 2008). An analysis of linear models, linear value-function approximation, and feature selection for reinforcement learning. In Proc. Int. Conf. on Machine Learning (ICML), pages 752-759.

[218] Parras, J., del Val, J., Zazo, S., Zazo, J., and Valcarcel Macua, S. (2016). A new approach for solving anti-jamming games in stochastic scenarios as pursuit-evasion games. In IEEE Statistical Signal Processing Workshop (SSP), pages 1-5.

[219] Pazis, J. and Lagoudakis, M. (Paris, France, Apr. 2011). Reinforcement learning in multidimensional continuous action spaces. In IEEE Symp. on Adaptive Dynamic Programming And Reinforcement Learning (ADPRL).

[220] Pearson, K. (1901). On Lines and Planes of Closest Fit to Systems of Points in Space. Philosophical Magazine, 2(6):559-572.

[221] Pelikan, M., Goldberg, D. E., and Lobo, F. G. (2002). A survey of optimization by building and using probabilistic models. Computational Optimization and Applications, 21(1):5-20.

[222] Penna, F. and Stańczak, S. (2015). Decentralized eigenvalue algorithms for distributed signal detection in wireless networks. IEEE Transactions on Signal Processing, 63(2):427-440.

[223] Pentland, A. and Heibeck, T. (2010). Honest Signals: How They Shape Our World. MIT Press.

[224] Peters, J., Mülling, K., and Altun, Y. (Atlanta, USA, July 2010). Relative entropy policy search. In $A A A I$ Conf. on Artificial Intelligence (AAAI), pages $1607-1612$.

[225] Polyak, B. T. (1987). Introduction to Optimization. Optimization Software Inc.

[226] Powell, W. B. and Ma, J. (2011). A review of stochastic algorithms with continuous value function approximation and some new approximate policy iteration algorithms for multidimensional continuous applications. Journal of Control Theory and Applications, 9(3):336-352. 
[227] Precup, D. (2000). Eligibility traces for off-policy policy evaluation. Computer Science Department Faculty Publication Series, page 80.

[228] Puterman, M. L. (1994). Markov Decision Processes: Discrete Stochastic Dynamic Programming. John Wiley \& Sons.

[229] Qi, H. and Wang, T. (2004). Global Principal Component Analysis for Dimensionality Reduction in Distributed Data Mining. In Statistical Data Mining and Knowledge Discovery, pages 327-342. Chapman \& Hall, CRC Press.

[230] Qian, N. (1999). On the momentum term in gradient descent learning algorithms. Neural networks, 12(1):145-151.

[231] Rabbat, M. and Nowak, R. (2005). Quantized incremental algorithms for distributed optimization. IEEE Journal on Selected Areas in Communications, 23(4):798-808.

[232] Ram, S., Nedic, A., and Veeravalli, V. (2009). Asynchronous gossip algorithms for stochastic optimization. In Int. Conf. Game Theory for Networks, pages 80-81.

[233] Rebeschini, P. and Van Handel, R. (2013). Can local particle filters beat the curse of dimensionality? arXiv preprint arXiv:1301.6585.

[234] Ren, W., Chao, H., Bourgeous, W., Sorensen, N., and Chen, Y.-Q. (2007). Experimental implementation and validation of consensus algorithms on a mobile actuator and sensor network platform. In IEEE Int. Conf. on Systems, Man and Cybernetics, pages 171-176.

[235] research, A. (2013). More than 30 billion devices will wirelessly connect to the internet of everything in 2020. https://www.abiresearch.com/press/ more-than-30-billion-devices-will-wirelessly-conne/.

[236] Reyment, R. A. and Jöreskog, K. G. (1996). Applied Factor Analysis in the Natural Sciences. Cambridge University Press, 2 edition.

[237] Riedmiller, M. (2012). 10 steps and some tricks to set up neural reinforcement controllers. In Neural Networks: Tricks of the Trade, pages 735-757. Springer.

[238] Roweis, S. (1998). Em algorithms for PCA and SPCA. In Jordan, M. I., Kearns, M. J., and Solla, S. A. E., editors, Advances in Neural Information Processing Systems, volume 10. The MIT Press.

[239] Roweis, S. and Ghahramani, Z. (1999). A Unifying Review of Linear Gaussian Models. Neural Computation, 11:305-345.

[240] Rubinstein, R. and Kroese, D. (2004). The Cross-Entropy Method: A Unified Approach to Combinatorial Optimization, Monte-Carlo Simulation and Machine Learning. Springer.

[241] Rückstiess, T., Sehnke, F., Schaul, T., Wierstra, D., Sun, Y., and Schmidhuber, J. (2010). Exploring parameter space in reinforcement learning. Paladyn, 1(1):14-24. 
[242] Sage, A. P. and White, C. C. (1977). Optimum Systems Control. Prentice-Hall, 2nd ed. edition.

[243] Sainz, M., Valcarcel Macua, S., and Zazo, S. (2017). dce - distributed (cross-entropy) black-box optimization of non-convex functions. https://github.com/msainz/dce/tree/sergio.

[244] Savic, V., Wymeersch, H., and Zazo, S. (2014). Belief consensus algorithms for fast distributed target tracking in wireless sensor networks. Signal Processing, 95:149-160.

[245] Sayed, A. H. (2008). Adaptive Filters. John Wiley \& Sons.

[246] Sayed, A. H. (2014a). Adaptation, learning, and optimization over networks. Foundations and Trends in Machine Learning, 7(4-5):311-801.

[247] Sayed, A. H. (2014b). Adaptive networks. Proceedings of the IEEE, 102(4):460497.

[248] Sayed, A. H. (2014c). Diffusion adaptation over networks. In Chellapa, R. and Theodoridis, S., editors, Academic Press Library in Signal Processing, volume 3, pages 323-454. Also available as arXiv:1205.4220v1, May 2012. Elsevier.

[249] Sayed, A. H., Tu, S.-Y., Chen, J., Zhao, X., and Towfic, Z. J. (2013). Diffusion strategies for adaptation and learning over networks. IEEE Signal Processing Magazine, 30(3):155-171.

[250] Scaglione, A., Pagliari, R., and Krim, H. (Asilomar, CA, USA, 2008). The decentralized estimation of the sample covariance. In Asilomar Conf. on Signals, Systems and Computers, pages 1722-1726.

[251] Scherrer, B. (Haifa, Israel, 2010). Should one compute the temporal difference fix point or minimize the Bellman residual? The unified oblique projection view. In Proc. Int. Conf. on Machine Learning (ICML), pages 959-966.

[252] Schizas, I. D., Giannakis, G. B., Roumeliotis, S. I., and Ribeiro, A. (2008a). Consensus in ad hoc wsns with noisy links - part II: Distributed estimation and smoothing of random signals. IEEE Transactions on Signal Processing, 56(4):16501666.

[253] Schizas, I. D., Ribeiro, A., and Giannakis, G. B. (2008b). Consensus in ad hoc wsns with noisy links - part I: Distributed estimation of deterministic signals. IEEE Transactions on Signal Processing, 56(1):350-364.

[254] Schneider, J., Wong, W.-K., Moore, A., and Riedmiller, M. (Bled, Slovenia, 1999). Distributed value functions. In Proc. Int. Conf. on Machine Learning (ICML), pages $371-378$.

[255] Schulman, J., Moritz, P., Levine, S., Jordan, M., and Abbeel, P. (2015). Highdimensional continuous control using generalized advantage estimation. arXiv preprint arXiv:1506.02438. 
[256] Scutari, G., Barbarossa, S., and Palomar, D. (2006, Toulouse, France). Potential games: A framework for vector power control problems with coupled constraints. In Proc. IEEE Int. Conf. on Acoustics, Speech and Signal Processing (ICASSP).

[257] Scutari, G., Palomar, D. P., Facchinei, F., and Pang, J.-s. (2010). Convex optimization, game theory, and variational inequality theory. IEEE Signal Processing Magazine, 27(3):35-49.

[258] Seneta, E. (2006). Non-negative Matrices and Markov Chains. Springer.

[259] Shahriari, B., Swersky, K., Wang, Z., Adams, R. P., and de Freitas, N. (2016). Taking the human out of the loop: A review of bayesian optimization. Proceedings of the IEEE, 104(1):148-175.

[260] Shawe-Taylor, J. and Cristianini, N. (2004). Kernel Methods for Pattern Analysis. Cambridge University Press.

[261] Shi, W., Ling, Q., Wu, G., and Yin, W. (2015). Extra: An exact first-order algorithm for decentralized consensus optimization. SIAM Journal on Optimization, 25(2):944-966.

[262] Shoham, Y. and Leyton-Brown, K. (2008). Multiagent Systems: Algorithmic, Game-Theoretic, and Logical Foundations. Cambridge University Press.

[263] Silver, D., Huang, A., Maddison, C. J., Guez, A., Sifre, L., Van Den Driessche, G., Schrittwieser, J., Antonoglou, I., Panneershelvam, V., Lanctot, M., et al. (2016). Mastering the game of go with deep neural networks and tree search. Nature, $529(7587): 484-489$.

[264] Slade, M. E. (1994). What does an oligopoly maximize? The Journal of Industrial Economics, 42(1):45-61.

[265] Socha, K. and Dorigo, M. (2008). Ant colony optimization for continuous domains. European journal of operational research, 185(3):1155-1173.

[266] Song, Z., Parr, R. E., Liao, X., and Carin, L. (2016). Linear feature encoding for reinforcement learning. In Advances in Neural Information Processing Systems 29 (NIPS), pages 4224-4232.

[267] Spall, J. C. (2005). Introduction to stochastic search and optimization: estimation, simulation, and control, volume 65. John Wiley \& Sons.

[268] Srinivas, M. and Patnaik, L. M. (1994). Genetic algorithms: a survey. Computer, $27(6): 17-26$.

[269] Srivastava, K. and Nedic, A. (2011). Distributed asynchronous constrained stochastic optimization. IEEE Journal of Selected Topics in Signal Processing, $5(4): 772-790$.

[270] Stankovic, S., Stankovic, M., and Stipanovic, D. (2011). Decentralized parameter estimation by consensus based stochastic approximation. Automatic Control, IEEE Transactions on, 56(3):531-543. 
[271] Stanković, M. S. and Stanković, S. S. (2016). Multi-agent temporal-difference learning with linear function approximation: Weak convergence under time-varying network topologies. In 2016 American Control Conference (ACC), pages 167-172.

[272] Stokey, N., Lucas, R., and Prescott, E. (1989). Recursive Methods in Economic Dynamics. Harvard University Press.

[273] Stulp, F. and Sigaud, O. (2013). Policy improvement: Between black-box optimization and episodic reinforcement learning. In Journée Francophones de Planification, Decision et Apprentissage.

[274] Sutton, R. S. and Barto, A. G. (1998). Reinforcement Learning: An Introduction. MIT Press.

[275] Sutton, R. S., Maei, H. R., Precup, D., Bhatnagar, S., Silver, D., Szepesvari, C., and Wiewiora, E. (Montreal, Quebec, Canada, 2009). Fast gradient-descent methods for temporal-difference learning with linear function approximation. In Proc. Int. Conf. on Machine Learning (ICML), pages 993-1000.

[276] Sutton, R. S., Modayil, J., Delp, M., Degris, T., Pilarski, P. M., White, A., and Precup, D. (Taipei, Taiwan, 2011). Horde: a scalable real-time architecture for learning knowledge from unsupervised sensorimotor interaction. In Proc. Int. Conf. on Autonomous Agents and Multiagent Systems (AAMAS), vol. 2, pages 761-768.

[277] Sutton, R. S., Szepesvari, C., and Maei, H. R. (Vancouver, British Columbia, Canada, 2008). A convergent $O(n)$ temporal-difference algorithm for off-policy learning with linear function approximation. In Proc. Advances in Neural Information Processing Systems 21 (NIPS), pages 1609-1616.

[278] Szepesvari, C. (2009). Algorithms for Reinforcement Learning. Morgan \& Claypool Publishers.

[279] Thapa, D., Jung, I.-S., and Wang, G.-N. (Changsha, China, Aug. 2005). Agent Based Decision Support System Using Reinforcement Learning Under Emergency Circumstances, pages 888-892.

[280] Theocharous, G., Thomas, P. S., and Ghavamzadeh, M. (Buenos Aires, Argentina, July, 2015). Personalized ad recommendation systems for life-time value optimization with guarantees. In Proc. Int. Conf. on Artificial Intelligence (IJCAI), pages 18061812 .

[281] Theodorou, E., Buchli, J., and Schaal, S. (2010). A generalized path integral control approach to reinforcement learning. Journal of Machine Learning Research, 11(Nov):3137-3181.

[282] Thiery, C. and Scherrer, B. (2009). Improvements on learning tetris with cross entropy. ICGA Journal, 32(1):23-33.

[283] Tian, L. and Gu, Q. (2016). Communication-efficient Distributed Sparse Linear Discriminant Analysis. ArXiv e-prints. 
[284] Tipping, M. E. and Bishop, C. M. (1999). Probabilistic Principal Component Analysis. Journal of the Royal Statistical Society, Series B, 61:611-622.

[285] Towfic, Z. J., Chen, J., and Sayed, A. H. (2013). On distributed online classification in the midst of concept drifts. Neurocomputing, 112:138-152.

[286] Towfic, Z. J. and Sayed, A. H. (2015). Stability and performance limits of adaptive primal-dual networks. IEEE Transactions on Signal Processing, 63(11):2888-2903.

[287] Tsitsiklis, J., Bertsekas, D., and Athans, M. (1986). Distributed asynchronous deterministic and stochastic gradient optimization algorithms. IEEE Transactions on Automatic Control, 31(9):803-812.

[288] Tsitsiklis, J. N. (1993). Decentralized detection. In Advances in Signal Processing, volume 2, pages 297-344. JAI Press.

[289] Tsitsiklis, J. N. and Athans, M. (1984). Convergence and asymptotic agreement in distributed decision problems. IEEE Transactions on Automatic Control, 29(1):4250 .

[290] Tsitsiklis, J. N. and Van Roy, B. (1997). An analysis of temporal-difference learning with function approximation. IEEE Transactions on Automatic Control, 42(5):674-690.

[291] Tu, S.-Y. and Sayed, A. H. (2012). Diffusion strategies outperform consensus strategies for distributed estimation over adaptive networks. IEEE Transactions on Signal Processing, 60(12):6217-6234.

[292] Urmson, C., Anhalt, J., Bagnell, D., Baker, C., Bittner, R., Clark, M., Dolan, J., Duggins, D., Galatali, T., Geyer, C., et al. (2008). Autonomous driving in urban environments: Boss and the urban challenge. Journal of Field Robotics, 25(8):425-466.

[293] Valcarcel Macua, S., Belanovic, P., and Zazo, S. (2010). Consensus-based distributed principal component analysis in wireless sensor networks. In IEEE Int. Workshop on Signal Processing Advances in Wireless Communications (SPAWC), pages $1-5$.

[294] Valcarcel Macua, S., Belanovic, P., and Zazo, S. (2011). Distributed linear discriminant analysis. In IEEE Int. Conference on Acoustics, Speech and Signal Processing (ICASSP), pages 3288-3291.

[295] Valcarcel Macua, S., Belanovic, P., and Zazo Bello, S. (2012). Diffusion gradient temporal difference for cooperative reinforcement learning with linear function approximation. In IEEE Cognitive Information Processing Workshop (CIP).

[296] Valcarcel Macua, S., Chen, J., Zazo, S., and Sayed, A. H. (2013a). Cooperative off-policy prediction of markov decision processes in adaptive networks. In IEEE Int. Conference on Acoustics, Speech and Signal Processing (ICASSP), pages 4539-4543. 
[297] Valcarcel Macua, S., Chen, J., Zazo, S., and Sayed, A. H. (2015. Also available as arXiv:1312.7606, Dec. 2013b). Distributed policy evaluation under multiple behavior strategies. IEEE Transactions on Automatic Control, 60(5):1260-1274.

[298] Valcarcel Macua, S., Leon, C. M., Romero, J. S., Pereira, S. S., Zazo, J., PagesZamora, A., López-Valcarce, R., and Zazo, S. (2014a). COMONSENS UPM wirelesssensor-networks testbed. http://comonsens.org/index.php?name=demonstrators.

[299] Valcarcel Macua, S., Leon, C. M., Romero, J. S., Pereira, S. S., Zazo, J., Pages-Zamora, A., López-Valcarce, R., and Zazo, S. (2014b). How to implement doubly-stochastic matrices for consensus-based distributed algorithms. In IEEE Sensor Array and Multichannel Signal Processing Workshop (SAM), pages 333-336.

[300] Valcarcel Macua, S., Zazo, J., and Zazo, S. (2016a). Closed-loop stochastic dynamic potential games with parametric policies and constraints. In Learning, Inference and Control of Multi-Agent Systems Workshop on Neural Information Processing (NIPS).

[301] Valcarcel Macua, S. and Zazo, S. (2014). Some applications of distributed signal processing. In URSI.

[302] Valcarcel Macua, S., Zazo, S., and Zazo, J. (2015). Distributed black-box optimizaion of nonconvex functions. In IEEE Int. Conference on Acoustics, Speech and Signal Processing (ICASSP).

[303] Valcarcel Macua, S., Zazo, S., and Zazo, J. (2016b). Learning in constrained stochastic dynamic potential games. In IEEE Int. Conference on Acoustics, Speech and Signal Processing (ICASSP), pages 4568-4572.

[304] van de Geijn, R. A. (1997). Using PLAPACK. MIT Press.

[305] van der Meulen, R. (2015). Gartner says 6.4 billion connected "things" will be in use in 2016, up 30 percent from 2015. http://www.gartner.com/newsroom/id/ 3165317.

[306] Van Hasselt, H. (2012). Reinforcement learning in continuous state and action spaces. In Reinforcement Learning, pages 207-251. Springer.

[307] Van Hasselt, H. and Wiering, M. e. a. (Honolulu, Hawaii, Apr. 2007). Reinforcement learning in continuous action spaces. In Proc. IEEE Int. Symp. on Approximate Dynamic Programming and Reinforcement Learning (ADPRL), pages 272-279.

[308] Van Hoof, H., Peters, J., and Neumann, G. (San Diego, California, May 2015). Learning of non-parametric control policies with high-dimensional state features. In Proc. Int. Conf. on Artificial Intelligence and Statistics (AISTATS), pages 995-1003.

[309] Varshavskaya, P., Kaelbling, L., and Rus, D. (2009). Efficient distributed reinforcement learning through agreement. In Asama, H., Kurokawa, H., Ota, J., and Sekiyama, K., editors, Distributed Autonomous Robotic Systems 8, pages 367-378. Springer Berlin Heidelberg. 
[310] Wang, W.-H., Palaniswami, M., and Low, S. H. (2003). Optimal flow control and routing in multi-path networks. Performance Evaluation, 52(2-3):119-132.

[311] Wang, Z., Fang, S.-C., and Xing, W. (2013). On constraint qualifications: motivation, design and inter-relations. Journal of Industrial and Management Optimization, 9(4):983-1001.

[312] Wang, Z., Shakibi, B., Jin, L., and de Freitas, N. (Reykjavik, Iceland, 2014). Bayesian multi-scale optimistic optimization. In Proc. Int. Conf. Artificial Intelligence and Statistics (AISTATS), pages 1005-1014.

[313] Weinstein, A. and Littman, M. L. (São Paulo, Brazil, 2012). Bandit-based planning and learning in continuous-action markov decision processes. In Int. Conf. on Automated Planning and Scheduling (ICAPS).

[314] Wiering, M. and van Otterlo, M. (2012). Reinforcement Learning: State-of-theArt. Springer.

[315] Wierstra, D., Schaul, T., Glasmachers, T., Sun, Y., Peters, J., and Schmidhuber, J. (2014). Natural evolution strategies. Journal of Machine Learning Research, 15(1):949-980.

[316] Wikipedia, T. F. E. (2017). Intelligence. https://en.wikipedia.org/wiki/ Intelligence. [Online; accessed 06-February-2017].

[317] Wiszniewska-Matyszkiel, A. (2014). Open and closed loop nash equilibria in games with a continuum of players. Journal of Optimization Theory and Applications, 160(1):280-301.

[318] Wu, F., Jing, X.-Y., Liu, Q., Wu, S.-S., and He, G.-L. (2015). Large-scale image recognition based on parallel kernel supervised and semi-supervised subspace learning. Neural Computing and Applications, pages 1-16.

[319] Wu, T., Yuan, K., Ling, Q., Yin, W., and Sayed, A. H. (2016). Decentralized Consensus Optimization with Asynchrony and Delays. ArXiv e-prints.

[320] Xiao, L. and Boyd, S. (2004). Fast linear iterations for distributed averaging. Systems \& Control Letters, 53(1):65-78.

[321] Yan, S., Xu, D., Zhang, B., and Zhang, H.-J. (2005). Graph embedding: a general framework for dimensionality reduction. In IEEE Computer Society Conf. on Computer Vision and Pattern Recognition, (CVPR), volume 2, pages 830-837.

[322] Yang, X.-S. (2010). Test Problems in Optimization. ArXiv e-prints.

[323] Yao, X., Liu, Y., and Lin, G. (1999). Evolutionary programming made faster. IEEE Transactions on Evolutionary computation, 3(2):82-102.

[324] Ye, J. (2005). Characterization of a family of algorithms for generalized discriminant analysis on undersampled problems. Journal of Machine Learning Research, 6:483-502. 
[325] Ye, J., Janardan, R., Li, Q., and Park, H. (2006). Feature reduction via generalized uncorrelated linear discriminant analysis. IEEE Tran. on Knowledge and Data Engineering, 18(10):1312 -1322.

[326] Yildiz, M., Ciaramello, F., and Scaglione, A. (2009). Distributed distance estimation for manifold learning and dimensionality reduction. In IEEE Int. Conf. on Acoustics, Speech and Signal Processing (ICASSP), pages 3353-3356.

[327] Yoon, S. and Pavlovic, V. (2012). Distributed probabilistic learning for camera networks with missing data. In Pereira, F., Burges, C. J. C., Bottou, L., and Weinberger, K. Q., editors, Advances in Neural Information Processing Systems 25 (NIPS), pages 2924-2932.

[328] Yu, H. and Bertsekas, D. P. (Nashville, TN, USA, 2009). Basis function adaptation methods for cost approximation in MDP. In Proc. IEEE Symp. on Adaptive Dynamic Programming and Reinforcement Learning (ADPRL), pages 74-81.

[329] Zazo, J., Valcarcel Macua, S., Zazo, S., Pérez, M., Pérez-Álvarez, I., Jiménez, E., Cardona, L., Brito, J. H., and Quevedo, E. (2016a). Underwater electromagnetic sensor networks, part II: Localization and network simulations. Sensors, 16(12):2176.

[330] Zazo, J., Zazo, S., and Valcárcel Macua, S. (2014). Distributed cognitive radio systems with temperature-interference constraints and overlay scheme. In European Signal Processing Conference (EUSIPCO), pages 855-859.

[331] Zazo, J., Zazo, S., and Valcarcel Macua, S. (2016b). Non-monotone quadratic potential games with single quadratic constraints. In IEEE Int. Conference on Acoustics, Speech and Signal Processing (ICASSP), pages 4373-4377.

[332] Zazo, J., Zazo, S., and Valcarcel Macua, S. (2016c). Robust worst-case analysis of demand-side management in smart grids. IEEE Transactions on Smart Grid.

[333] Zazo, S., Valcarcel Macua, S., Sánchez-Fernández, M., and Zazo, J. (2016d). Dynamic potential games with constraints: Fundamentals and applications in communications. IEEE Transactions on Signal Processing, 64(14):3806-3821.

[334] Zazo, S., Zazo, J., and Sánchez-Fernández, M. (2014, Lisbon, Portugal). A control theoretic approach to solve a constrained uplink power dynamic game. In Proc. European Signal Processing Conference (EUSIPCO).

[335] Zhang, Q. and Muhlenbein, H. (2004). On the convergence of a class of estimation of distribution algorithms. IEEE Transactions on Evolutionary Computation, $8(2): 127-136$.

[336] Zhao, X. and Sayed, A. H. (2012). Performance limits for distributed estimation over LMS adaptive networks. IEEE Transactions on Signal Processing, 60(10):51075124 .

[337] Zhao, X. and Sayed, A. H. (2015a). Asynchronous adaptation and learning over networks - part i: Modeling and stability analysis. IEEE Transactions on Signal Processing, 63(4):811-826. 
[338] Zhao, X. and Sayed, A. H. (2015b). Asynchronous adaptation and learning over networks - part ii: Performance analysis. IEEE Transactions on Signal Processing, 63(4):827-842.

[339] Zhao, X. and Sayed, A. H. (2015c). Asynchronous adaptation and learning over networks-part iii: Comparison analysis. IEEE Transactions on Signal Processing, 63(4):843-858.

[340] Zhou, E., Fu, M., and Marcus, S. (2014). Particle filtering framework for a class of randomized optimization algorithms. IEEE Transactions on Automatic Control, 59(4):1025-1030.

[341] Zhou, E. and Hu, J. (2014). Gradient-based adaptive stochastic search for nondifferentiable optimization. IEEE Transactions on Automatic Control, 59(7):18181832 .

[342] Zhou, H., Fan, P., and Li, J. (2011). Global proportional fair scheduling for networks with multiple base stations. IEEE Transactions on Vehicular Technology, 60(4):1867-1879.

[343] Zhu, M. and Martinez, S. (2012). On distributed convex optimization under inequality and equality constraints. IEEE Transactions on Automatic Control, $57(1): 151-164$.

[344] Zlochin, M., Birattari, M., Meuleau, N., and Dorigo, M. (2004). Model-based search for combinatorial optimization: A critical survey. Annals of Operations Research, 131(1):373-395. 